

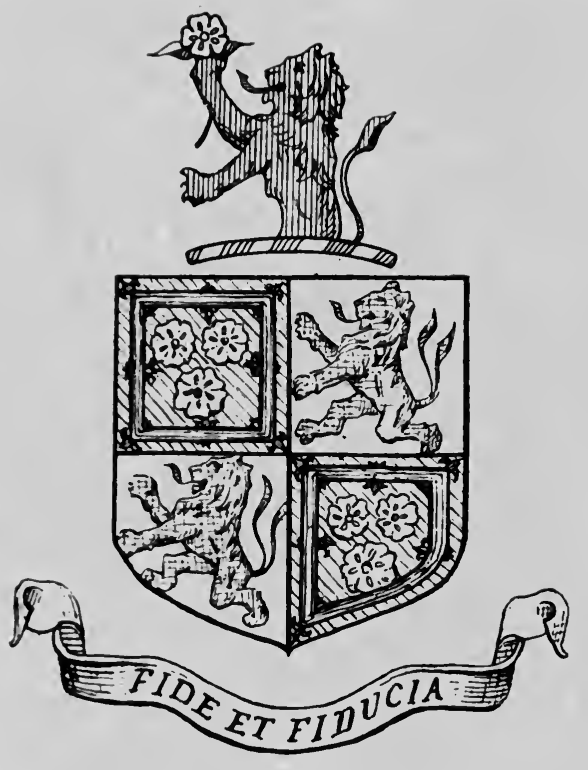

ALEXANDER PRIMROSE C.B. 


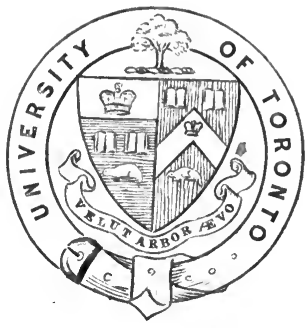

Dresented to

The Tíbraty

of the

University of coronto

by

Department of Surgery 


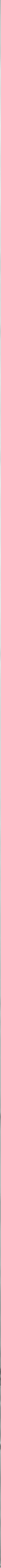



THE MEDICAL DISEASES OF CHILDHOOD 
Thes 
Digitized by the Internet Archive in 2007 with funding from Microsoft Corporation 


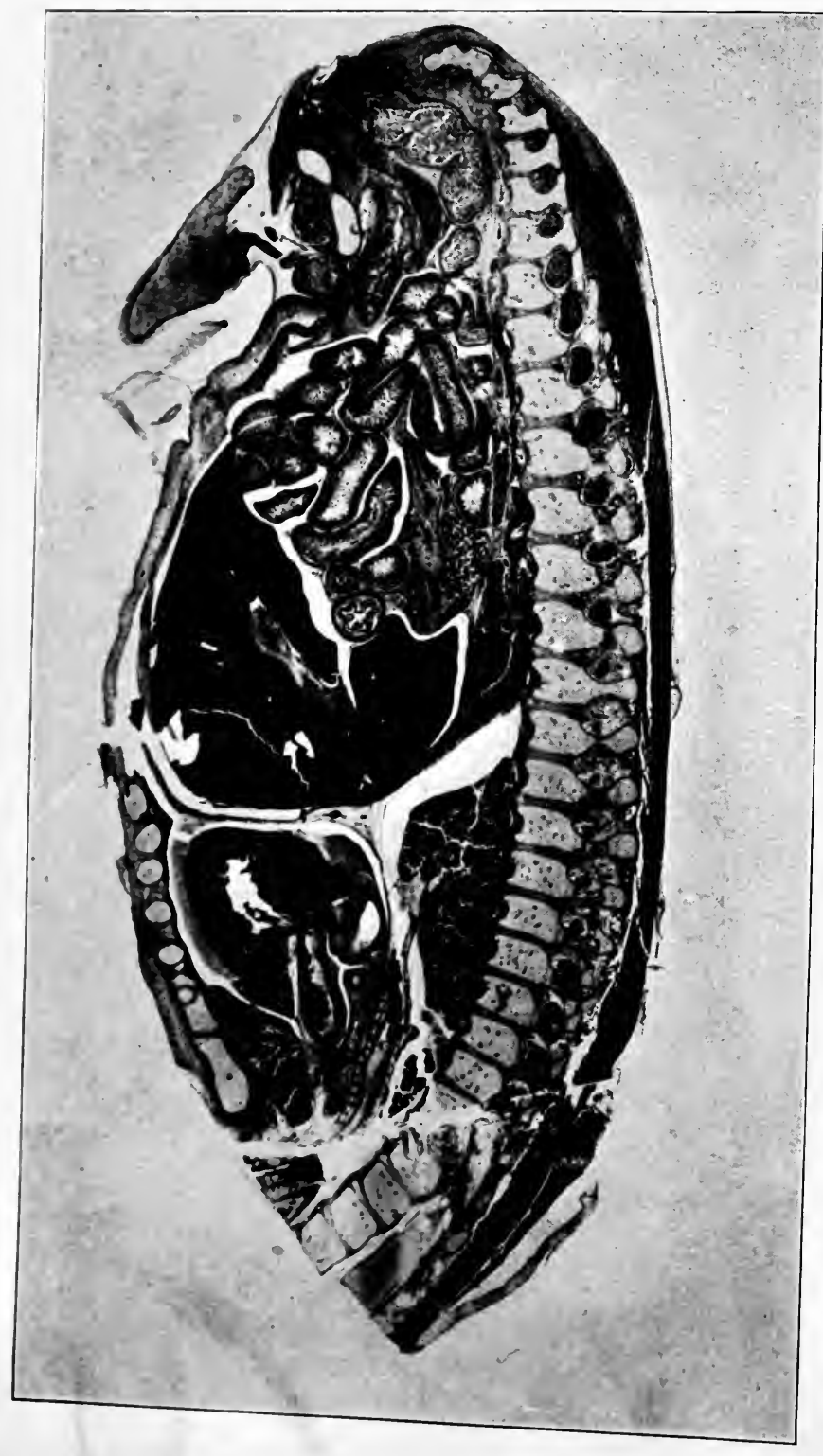

|ญ्ّ. 


\section{THE MEDICAL DISEASES OF CHILDHOOD}

BY

\section{NATHAN OPPENHEIM}

A.B. (HaRv.), M.D. (Coll. P. \& S., N.Y.)

AUTHOR OF "THE DEVELOPMENT OF THE CHILD" attending PHysician to the CHILdRen's Departuent OF MT. SINAI HOSPITAL DISPENSARY

WITH ONE HUNDRED AND ONE ORIGINAL ILLUSTRATIONS IN HALF-TONE, AND NINETEEN CHARTS

\section{0 \\ 19. 4.49}

Nown Wark

THE MACMILLAN COMPANY

LONDON : MACMILLAN \& CO., LTD.

1900

All rights reserved 
Copyright, 1900 ,

By THE MACMILlá COMPANY.

Norwood 3 Bress

J. 8. Cushing \& Co. - Berwick \& Smith

Norwood Mass. U.S.A. 
THE HONORED MEMORY OF fttw 猡arents 



\section{PREFACE}

A wORK on the diseases of children which will tend to promote a greater use of logic and a lessened reliance upon empirical methods than have formerly been common, may always be sure of a place in the medical world. Such improvements in method represent one of the most desirable advances in scientific work and are equally important with the discovery of details in therapeutics or new facts in pathology ; for they all have in view the recognition of diseased conditions and their prevention or successful treatment. The tendency of our thought in the present time is in the direction of the accurate recognition of abnormal conditions; for when this is accomplished, the treatment, so far as it is elaborated, follows as a matter of course. For this reason the greatest stress in modern medicine has been laid upon the necessity of depicting lesions in the most graphic manner possible. Following out this idea, the whole available space for illustrations in this book has been given up to photomicrographs of pathological sections, which have been thought to have a keener and greater didactic value than pictures of instruments, photographs of patients, and representations of surface changes, all of which must, on account of the limitations of fidelity in reproduction, convey either a trivial or one-sided impression. As a resulting consideration, if the student or physician can be made to have a clearer conception of the difference between the healthy and unhealthy condition, if he can in any degree be made to understand better than before the phases of change which occasion interferences with functional activity, he will have made a profitable step in the direction of efficiency and can more thoroughly subserve his patient's interest as well as his own satisfaction.

Another change which has been made in this book is the placing of statistics among matters of minor importance. In collecting the material for the work the compilation of statistical returns received much attention; as research progressed, a feeling of dissatis- 
faction with the results and uncertainty in regard to their reliability grew with an even growth. The author soon recognized that the widely separated sources and varying methods of diagnostication and treatment made more than an approximate approach to the truth almost beyond expectation. As an example, one may cite the case of diphtheria. In this disease, unless a culture and microscopical examination of the bacteria are made, the possibility of mistaken diagnostication is often great. The clinical evidence is commonly insufficient; and since most reports have been founded upon such evidence alone, and since in former times this evidence was the only means of definition possible, the errors in compiling statistics must necessarily be great. There is yet another and even greater source of mistakes: we know that in diphtheria an infection of the lungs by pneumococci or streptococci resulting in a broncho-pneumonia is comparatively common. If death results in spite of the use of antitoxic serum, should it be attributed to the general diphtheritic invasion, the presence of the pneumococcus, or that of the streptococcus? If the diphtheria itself was the primary cause of death, how far was it helped by the pneumococcus or the streptococcus, or both? And it is even probable that in many of these cases the streptococcus was the fatal agent although they are classified in the mortality of diphtheria. The overweening importance of environment and the unreliable nature of statistical reports are shown again by the discrepancy between the general mortality of from 14 per cent to 20 per cent collected from hospitals and boards of health on the one hand, and on the other, the experience of reasonably able and conscientious physicians in private practice, whose results are no more than one-third or one-quarter of these figures. The same or similar doubts arise in almost every other common disease in which the opportunities for careful investigation are abundant. And therefore it has seemed advisable to onit the formidable arrays of figures, which are copied by one author after another, as being far from conclusive, leading to inaccuracies, and bolstering up a spirit that is unscientific.

Another omission, which was made after careful consideration, involves the citing of the various authorities upon whose work many statements have been founded. This is permissible because in the large brotherhood of science there is no distinct ownership. and one man takes up the work of another, completing or merely 
utilizing it, without regard to the responsibility of its beginning or its end. We are all borrowers from one another, and we all live in a condition of joint ownership. The citation of references does not by any means do full justice to the real doers of the work in question; and what is of great importance, it interferes seriously with the narrative quality and easy understanding of the text.

The author wishes to record his thanks to Dr. Charles A. Elsberg for many acts of helpfulness in the preparation of the work and the correction of proof. To Dr. Morris Manges recognition is to be made for preparing the section from which the frontispiece was made. Finally, the author takes pleasure in expressing his obligation to the publishers for their consistently liberal and sympathetic attitude in all the details of printing and publishing the book.

50 East 79th Street, New York. 



\section{TABLE OF CONTENTS}

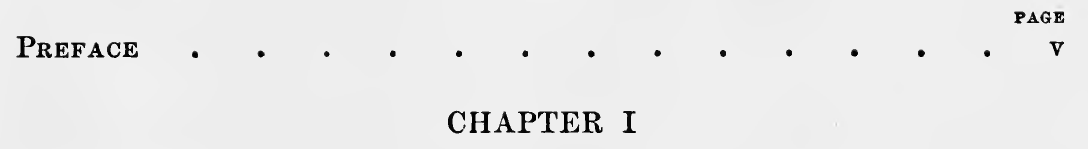

DEVELOPMENT

CHAPTER II

General Hygiene of the New-born Child $\quad$ - $\quad$ - $\quad$ - 24

\section{CHAPTER III}

Method of Examination and Diagnostic Suggestions

Therapeutic Suggestions. Glycosuria. Anuria. Polyuria.

\section{CHAPTER IV}

Congenital Malformations and Deformities •

The Brain. The Spinal Cord. The Larynx and Trachea. The Lungs. The Heart. The Mouth. The Esophagus. The Stomach. The Intestines. The Peritonæum. The Liver. The Spleen: Pancreas. The Suprarenal Capsules. The Kidneys. The Urinary Bladder. The Urethra. The Vulva. The Vagina. The Uterus. The Ovaries. The Fallopian Tubes. The Mamma. The Penis. The Testicles. Congenital Dislocations.

\section{CHAPTER V}

Injuries and Diseases of The New-born . . . . . .

Caput Succedaneum. Cephalhæmatoma. Hæmatoma of the Sternocleido-mastoid Muscle. Visceral Hæmorrhage in the New-born. "Hæmorrhagic Disease" of the New-born. Obstetric Paralyses. Asphyxia. Congenital Atelectasis. Icterus Neonatorum. Acute Pyogenic Infection. Ophthalmia Neonatorum. Infectious Hæmoglobinæmia. Fatty Degeneration of the New-born. Pemphigus in Infants. Umbilical Polypus. Diverticulum Tumor of the Umbilicus. Umbilical Hernia. Mastitis. Intestinal Obstruction in the New-born. Hernia of the Diaphragm. Sclerema Neonatorum. Edema. 


\section{CHAPTER VI}

Feedixg

Substitute Feeding. Infant Foods. Care of Premature Infants. Weaning. Feeding during Second Year and its Continuance. Feeding in Acute Sickness. Dentition.

\section{CHAPTER VII}

Diseases of the Mouth and Cesophagus

Pathological Conditions of the Lips. Hare-lip and Cleft Palate. Gangrene of the Cheek. Simple Erosions of the Mouth. Ulcerative Gingivitis. Simple Acute Stomatitis. Herpetoid Stomatitis. Mycetogenetic Stomatitis. Croupous Stomatitis. Gonorrhœal Stomatitis. Simple Superficial Glossitis. Acute Glossitis. Acute (Esophagitis. Retro-œsophageal Lymph-adenitis.

\section{CHAPTER VIII}

Diseases of the Stomach and Syall -Intestine

Acute Functional Derangement of the Stomach. Acute Gastritis. Croupous (iastritis. Toxic Gastritis. Chronic Gastritis. Dilatation of the Stomach. Ulcer of the Stomach. Hæmorrhagic Erosions of the Gastric Mucous Membrane. Gastralgia. Hæmatemesis. Duodenitis. Chronic Duodenitis.

\section{CHAPTER IX}

Diseases of tire Pancreas, Liver, and Spleen

The Pancreas. Icterus. Functional Derangements of the Liver. Congestion of the Liver. Parenchymatous Hepatitis. Purulent IIepatitis. Interstitial Hepatitis. Acute Yellow Atrophy of the Liver. Fatty Liver. Amyloid Degeneration of the Liver. Echinococcus Infection of the Liver. Wounds of the Liver. Subphrenic Abscess. Biliary Calculi. Congestion of the Spleen. Amyloid Degeneration of the Spleen. Parasites of the Spleen. Wounds of the Spleen.

\section{CIIAPTER $\mathrm{X}$}

Dibeases of the Large Intestine

Acute Ileo-colitis. Chronic Ileo-colitis. Ileo-colitis due to Amœbic Infection. Infectious Derangements of the Intestines. Subacute Intestinal Infection. Chronic Functional Derangement of the Intestines. 


\section{CHAPTER XI}

Diseases of the Large Intestine (Continued) .

Intussusception. Volvulus. Animal Parasites of the Intestines. Intestinal Colic in Infants. Habitual Constipation. Appendicitis. Proctitis. Prolapse of the Anus and Rectum. Rectal Polypus. Fissure of the Anus.

\section{CHAPTER XII}

Diseases of the Peritonaum

Ascites. Acute Peritonitis. Chronic Peritonitis.

\section{CHAPTER XIII}

Diseases of Malnutrition

Laryngismus Stridulus. Simple Atrophy. Rachitis. Infantile Scurvy (Barlow's Disease).

\section{CHAPTER XIV}

Diseases of the Nose and Throat

Acute Rhinitis. Epistaxis. Nasal Polypi. Adenoid Vegetations of the Pharyngeal Vault. Acute Simple Tonsillitis. Acute Follicular Tonsillitis. Chronic Inflammation and Hypertrophy of the Tonsils. Peritonsillar Abscess. Uvulitis and Elongated Uvula. Acute Pharyngitis. Chronic Pharyngitis. Retro-pharyngeal Lymphadenitis. Acute Laryngitis. Chronic Laryngitis. Spasmodic Laryngitis. CEdema of the Glottis.

\section{CHAPTER XV}

Diseases of the Bronchi, Lungs, and Pleura .

Acute Bronchitis. Chronic Bronchitis. Acute Broncho-pneumonia. Chronic Broncho-pneumonia. Interstitial Pneumonia. Secondary Pneumonia. Bronchiectasis. Acute Pleurisy. Atelectasis. Emphysema. Gangrene of the Lungs.

\section{CHAPTER XVI}

Diseases of the Heart .

Functional Cardiac Disorders. Myocarditis. Acute Endocarditis. Chronic Endocarditis. Malignant or Mycotic Endocarditis. Cardiac Hypertrophy and Dilatation. Anæmic Murmurs. Pericarditis. Chronic Pericarditis. 


\section{CHAPTER XVII}

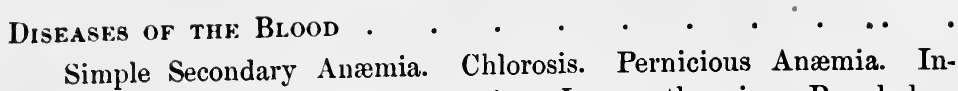
fantile Pseudo-leucæmic Anæmia. Leucocythæmia. Pseudo-leucæmia (Hodgkin's Disease). Hæmophilia. Purpura.

\section{CHAP'TER XVIII}

Diseases of the Genito-Urisary System .

Functional Derangement of the Bladder. Acute Cystitis. Chronic Cystitis Incontinence of Urine. Vesical Calculi. Balanitis. Phimosis. Paraphimosis. Urethritis in Male Children. VulvoVaginitis. Stricture of the Urethra in Boys. Orchitis and Epididymitis. Hydrocele.

\section{CHAPTER XIX}

Diseases of the Genito-Urinary System (Continued). Diseases of THE KidNeYs . • • • • • • • • • •

Acute Congestion of the Kidneys. Acute Degeneration of the Kidneys. Acute Exudative Nephritis. Acute Diffuse Nephritis. Chronic Congestion and Degeneration of the Kidneys. Chronic Diffuse Nephritis without Exudation. Suppurative Nephritis. Chronic Pyelo-nephritis. Amyloid Degeneration of the Kidney. Infarction of the Kidney. Perinephritis. Hydronephrosis. Parasites of the Kidney. New Growths of the Kidney. Renal Calculi.

\section{CHAPTER XX}

The Specific Infectious Diseases

Scarlet Fever. Measles. Rubella. Varicella. Variola. Vaccinia and Varioloid. Diphtheria. Epidemic Infectious Parotitis. Pertussis. Epidemic Influenza. Lobar Pneumonia. Enteric Fever. Erysipelas. Tetanus. Epidemic Cerebro-spinal Meningitis.

\section{CHAPTER XXI}

Tne Specific Ixfectious Diseases (Continued) .

Tuberculosis. Inherited Syphilis. Malaria. Weil's Disease.

\section{CHAPTER XXII}

Nervous Diseases

Acute Ieptomeningitis. Pachymeningitis. Spinal Meningitis. Syringomyelia. Myelitis. Compression of the Spinal Cord. Infantile 
Spinal Paralysis. Multiple Neuritis. Progressive Muscular Atrophies. Hereditary Ataxias (Friedreich's Disease, Marie's Disease, Hereditary Ataxic Paraplegia). Acute Ascending Paralysis. Infantile Cerebral Paralysis. Thrombosis of the Intracranial Arteries. Abscess of the Brain. Tumors of the Brain. Tumors of the Spinal Cord. Hydrocephalus. Asthma. Hay Fever. Sporadic Cretinism. Amaurotic Family Idiocy. Idiocy. Insanity in Childhood. Functional Aphasia. Deficiency of Speech from Peripheral Paralysis. Lisping. Stuttering. Stammering. Backwardness in acquiring Speech. Echolalia. Coprolalia. Disorders of Speech. Adventitious Sucking. Masturbation. Deaf-mutism. Convulsions. Epilepsy. Chorea. Tetany. Hysteria. Headaches.

\section{CHAPTER XXIII}

Diseases of Obscure Origin .

Diabetes Mellitus. Rheumatism. Rheumatoid Arthritis. Acute Arthritis of Infants.

\section{CHAPTER XXIV}

INflammations of the Middle Ear . . . • . . 。

\section{CHAPTER XXV}

Diseases of The Skin

Scleroderma. Seborrhœa. Hyperidrosis. Erythema. Miliaria. Eczema. Simple Herpes. Herpes Zoster. Impetigo Contagiosa. Dermatitis Exfoliativa Neonatorum. Dermatitis Gangrænosa Infantum. Urticaria. Furunculosis. Lentigo. Icthyosis. Verruca. Nævus Pigmentosus. Alopecia Areata. Ringworm. Favus (Tinea Favosa). Scabies. Pediculosis. 



\section{LIST OF ILLUSTRATIONS}

Sagittal Section of Human Fœtus (about $4 \frac{1}{2}$ months)

Frontispiece.

IIG.

1. Diagram of Temporary or "Milk" Teeth in the Order of Eruption . 100

2. Diagram of Permanent Teeth in the Order of Eruption . . $\quad 100$

3. Croupous or Membranous Esophagitis . . . . . . 114

4. Normal Stomach . • • • • • • • • • . 117

5. Acute Gastritis . . . . . . . . . . . 119

6. Acute Catarrhal Gastritis . . . . . . . . . 120

7. Croupous or Membranous Gastritis • • • . . 122

8. Chronic Gastritis . . . . . . . . . . . 125

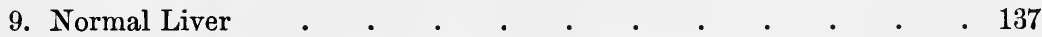

10. Hypertrophic Cirrhosis of the Liver (early stage) . . . . 141

11. Interstitial Hepatitis . . . . . . • . . . 142

12. Acute Yellow Atrophy of the Liver . . . . . . . 144

13. Fatty Liver . . . . . . . . . . . . 145

14. Amyloid Degeneration of the Liver . . . . . . . . 146

15. Normal Spleen . . . . . . . . . . . 149

16. Congested Cavernous Vein of Spleen . . . . . . . 150

17. Chronic Interstitial Splenitis (Sago Spleen) · . . . . 151

18. Normal Intestine .

19. Catarrhal Enteritis . . . . . . . . . . 154

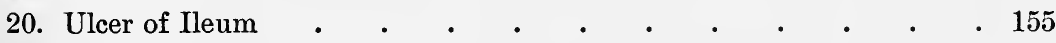

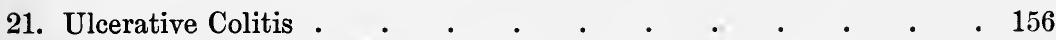

22. Croupous or Membranous Mleo-colitis . . . . . . • . 157

23. Membranous or Croupous Colitis . • . . . . . . 158

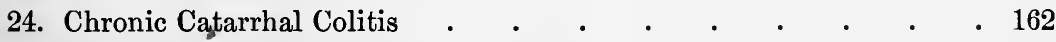

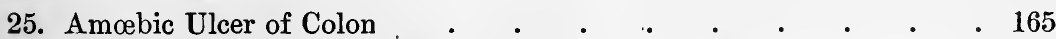

26. Pulse, Respiration, and Temperature Chart of Intestinal Intoxication.

Age, 4 years . . . . . . . . . . . . . 167

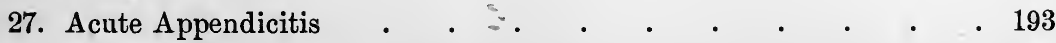

28. Rectal Polypus . . . . . . . . . . . 200

29. Perihepatitis, occurring with Chronic Peritonitis _ . • . 206 
30. Perisplenitis, occurring with Chronic Peritonitis • • • • . 207

31. Normal Bone • • • • • • • • • • • • . 215

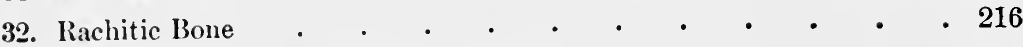

33. Polypus of Nasal Mucous Membrane . . . • • • . 233

34. Acute Tonsillitis . . • • • • • • • • • • 237

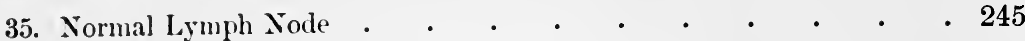

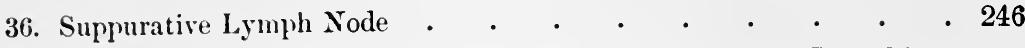

37. Pulse, Respiration, and Temperature Chart of Acute Bronchitis.

Age, 7 months . . . . . . . . . . . 258

38. Normal Lung $\quad$ • $\quad$ •

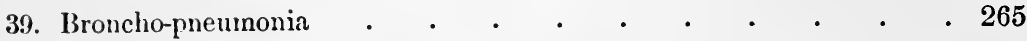

40. Pulse, Respiration, and Temperature Chart of Broncho-pneumonia. Age, 13 months . . . . . . . • • • . 268

41. Chronic Interstitial Pneumonia . . . . . . . . . 275

42. Normal Pleura . . . . . . . . . . . 280

43. Fibrinous (Adhesive) Pleuritis . . . . . . . . 283

44. Pulse, Respiration, and Temperature Chart of Purulent Pleurisy.

Age, 8 months . . . . . . . . . . • . 287

45. Chronic Pleurisy . . . . . . . . . . . 291

46. Atelectasis with Compensatory Emphysema . . . . . 293

47. Emphysema • • • • • • • • • • • . 295

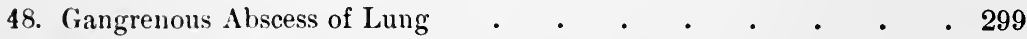

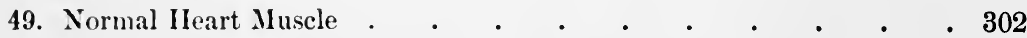

50. Parenchymatous Myocarditis . . . . . . . . 303

51. Interstitial Myocarditis . . . . . . . . . . 304

52. Valve in Acute Endocarditis . . . . . . . . 306

53. Cirrhosis of the Liver. Passive Congestion from Mitral Stenosis . 312

54. Pneumonia of Endocarditis . . . . . . . . . 314

55. Aortic Valve: Acute Mycotic Endocarditis . . . . . . 316

56. Pulse, Respiration, and Temperature Chart of Malignant Endocarditis. Age, 9 years . . . . . . . . . . . . . . 317

57. Normal Pericardium . . . . . . . • • . 322

58. Simple or fibrinous Pericarditis . . . . . . . . 323

59. Acute Sero-fibrinous Pericarditis . . . . . . . . 324

60. Acute Fibrino-purulent Pericarditis . . . . . . . 325

61. Liver in Pernicious Anæmia . • • • • • • . . 336

62. Kidney : in Leucocythæmia . . . . . . . . . 341

63. Normal Kidney.$\quad$ •

64. Acute Diffuse Nephritis, showing Plasma Cell Infiltration . . . 370

65. Chronic Passive Congestion of Kidney (Casts in situ) . . . . 373 
FIA.

PAGE

66. Chronic Diffuse Nephritis without Exudation; Connective Tissue and Atrophied Glomerulus . . . . . . . . 375

67. Chronic Diffuse Nephritis without Exudation . . . . . 376

68. Chronic Diffuse Nephritis with Exudation. Hyaline Glomeruli . 377

69. Chronic Diffuse Nephritis with Exudation . . . . . . 378

70. CEdema of Lung; in Chronic Diffuse Nephritis with Exudation . . 379

71. Amyloid Degeneration of Kidney, showing Glomerulus • • . 382

72. Infarction of the Kidney (Endocarditis and Broncho-pneumonia) . 383

73. Large Round Cell Sarcoma of Kidney . . . . . . . 387

74. Acute Scarlatinal Nephritis . . . . . . . . . 394

75. Acute Hæmorrhagic Scarlatinal Nephritis: Glomerular Thrombosis 395

76. Pulse, Respiration, and Temperature Chart of Scarlet Fever. Age, 5 years . . . . . . . . . . . . 397

77. Pulse, Respiration, and Temperature Chart of Scarlatinal Nephritis.

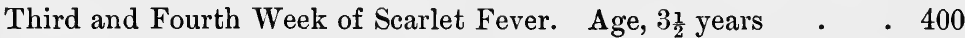

78. Pulse, Respiration, and Temperature Chart of Measles. Age, 4 years 408

79. Pulse, Respiration, and Temperature Chart of Measles. Acute Broncho-pneumonia. Age, 2 years . . . . . . . 410

80. Pulse, Respiration, and Temperature Chart of Varioloid. Age, 4 years 427

81. Diphtheritic Inflammation of Pharynx . . . . . . . 429

82. Diphtheritic Inflammation of Trachea . . . . . . . 430

83. Lymphadenitis of Diphtheria . . . . . . . . 431

84. Pulse, Respiration, and Temperature Chart of Diphtheria. Age, 5 years . . . . . . . . . . . . 434

85. Epidemic Infectious Parotitis (Mumps) . . . . . . . 441

86. Pulse, Respiration, and Temperature Chart of Influenza. Age, 10 years . . . . . . . . . . . . . 450

87. Lobar Pneumonia : First Stage . . . . . . . . 454

88. Lobar Pneumonia: Second Stage . . . . . . . . 455

89. Lobar Pneumonia : Third Stage . . . . . . . . . 456

90. Lobar Pneumonia: Third Stage (Fibrin Threads) • . . $\quad$. 457

91. Pulse, Respiration, and Temperature Chart of Lobar Pneumonia.

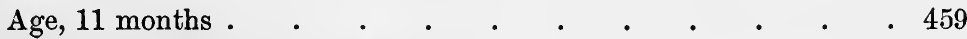

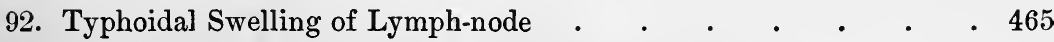

93. Typhoidal Ulceration of Intestine. Healing Stage . . . . 466

94. Pulse, Respiration, and Temperature Chart of Mild Typhoid. Age, 4 years . . . . . . . . . . . . . 468

95. Normal Cerebral Meninges . . . . . . . . . 471

96. Epidemic Cerebro-spinal Meningitis . . . . . . . 480

97. Acute Miliary Tuberculosis of the Lung . . . . . . 488 
rIG.

98. Chronic Tubercular Pneumonia with Cheesy Degeneration

99. Chronic Pulmonary 'Tuberculosis with Conservative Fibrosis

100. Tuberculosis of Lymph-node . . . . . . . . . . 491

101. Tuberculosis of the Kidney . . . . . . . . . . 492

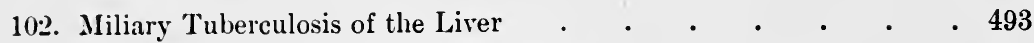

103. Acute Miliary Tuberculosis of the Spleen . . . . . . 494

101. Tubercular Llcer of Ileum . . . . . . . . . 495

10j. Tubercular Meningitis . . . . . . . . . . . . 496

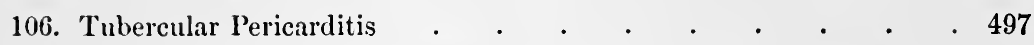

107. Pulse, Respiration, and Temperature Chart of General Miliary Tuberculosis. Age, 7 years . . . . . . . . 500

108. Pulse, Respiration, and Temperature Chart of Tubercular Meningitis, First and Second Weeks. Age, 5 years _ . . . 507

109. Infantile Hereditary Syphilis of Lung . . . . . . 518

110. Gumma of Spleen . . . . . . . . . . . 519

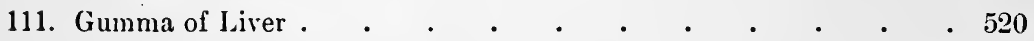

112. Syphilitic Cirrhosis of the Lirer . . . . . . . . 521

113. Induration of Kidney: Hereditary Syphilis . . . . . 522

114. Syphilitic Lymphadenitis . . . . . . • . . 523

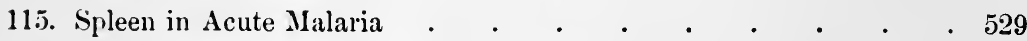

116. Pulse, Respiration, and Temperature Chart of Intermittent Fever. Age, 6 years . . . . . . . . . . . 530

117. Pulse, Respiration, and Temperature Chart of Tertian Intermittent

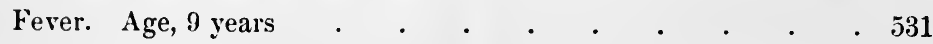

118. Compression of the Spinal Cord: Degeneration of Descending Columns . . . . . . . . . . . . 547

119. Organizing Thrombus in Vein . . . . . . . . 562

120. Neuro-glioma of Brain . . . . . . . . . . 568

121. Pulse, Respiration, and Temperature Chart of Chorea, Lobar Pneumonia, Hyperpyrexia, Death. Age, 6 years . . . . . 599 


\section{THE MEDICAL DISEASES OF CHILDHOOD}





\section{CHAPTER I}

\section{DEVELOPMENT}

THE ideal of the normal child is, and must be, the basis of intelligent appreciation of the pathological processes which occur in his body and mind; for without a distinct idea of an ordinary condition, one is unable to distinguish clearly the character of the innumerable extraordinary states which constitute what is called disease. This is of even more importance in the child than in the adult, since the former does not represent a fixed and definite entity, that is the same yesterday, to-day, and to-morrow, as is the latter, but on the contrary is a mutable being, an organic flux, changing from week to week and month to month in the endeavor to obtain a permanent economy that is sufficient for the work of self-preservation and propagation of kind. This series of changes does not begin and end at birth; rather does it start at the moment of conception, develop with wondrous rapidity, and finally terminate in that dim region where absolute maturity fades away with physical and mental decadence. Many of the stages are unknown or at best partly known; but those which lie open and exposed before us show enough of the truth to indicate what the unknown remainder must necessarily be. The more thoroughly one becomes acquainted with the child, the more one is impressed with the idea that he is a creature of infinite variety who bears to the adult a relation of potentiality, but nothing more. One can go ever farther and not only make a clear distinction between the child and the adult, but also differentiate between the child and the child. Although the study would be endless, nevertheless there is a logical necessity for it, and without it our knowledge of the subject in hand must be partial. This is demonstrated in.Vierordt's table, which, although provisional and even fragmentary, nevertheless has much philosophy hidden in its dry figures. 
Weight of Organs reckoned in Percentage of Weight of Body

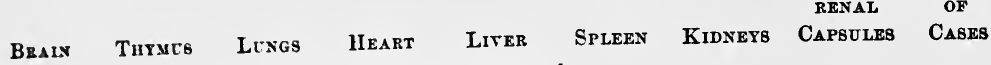
$0-2$ months

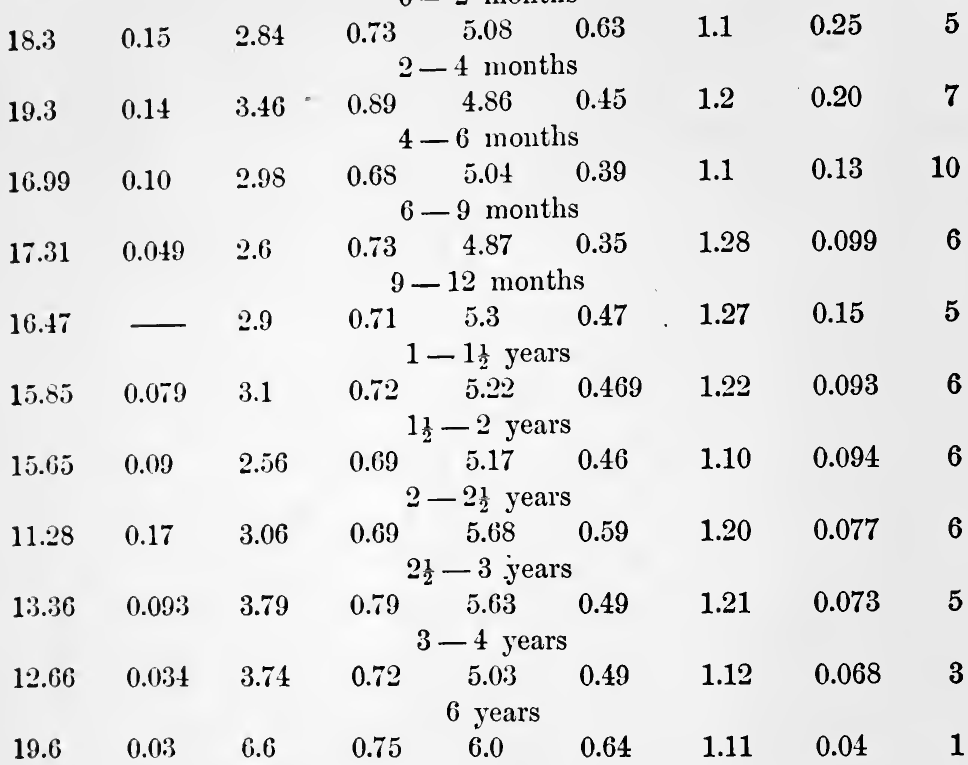

Such a demonstration is interesting in itself, and it becomes still more so when it is considered in relation to a table which shows the difference in percentage of the weight of the various organs in the new-born child and the adult.

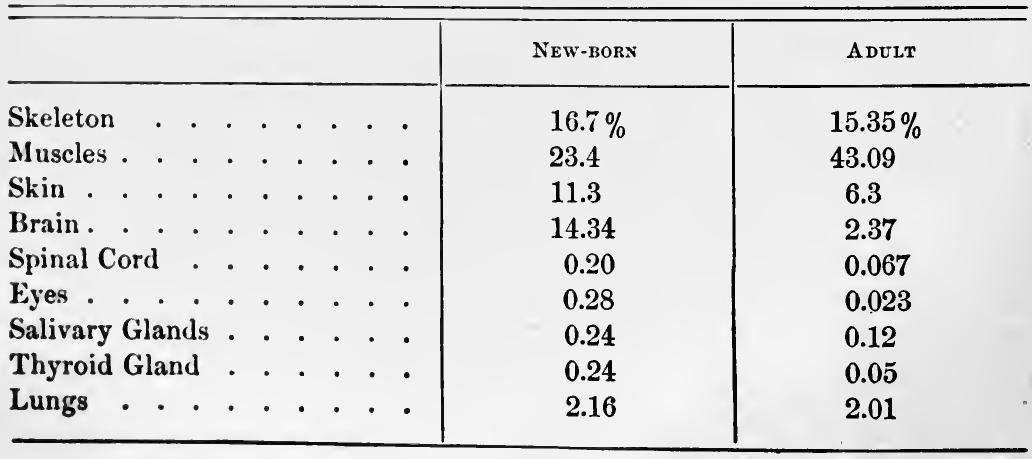

* Vierordt. 


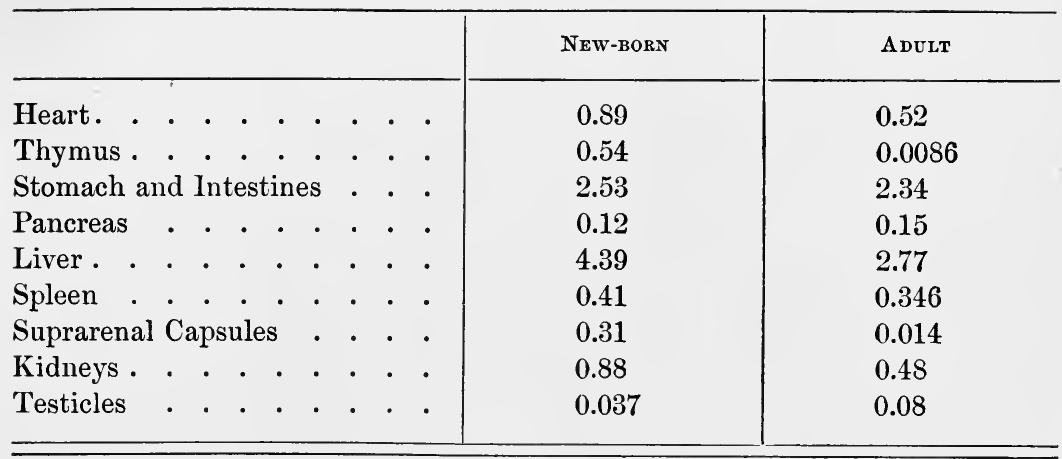

While these variations are truly striking, they could be made even more so if one wished to include the analysis from still more widely separated extremes in life. For example, one might cite the contrasting percentages of water in the very young fœtus, ninety-seven and five-tenths per cent, with that of the new-born child, seventy-four and seven-tenths per cent, and finally with what exists at mature age, fifty-eight and five-tenths per cent. All along the line the most salient differences appear, so that in the most generalized as well as particularized details the child is not a man in small, but to all intents a different being. Thus, if the proportions of the infant were multiplied to equal those of the adult, the huge head, the undeveloped lower face, the apex-like thorax, the dwarfed arms and legs, would make the impression of grotesqueness. Even the respiration is different, for in the infant it is diaphragmatic and does not become costal until after the seventh year, although even at that time it does not assume its fully matured type. In the early times of life it is, moreover, irregular, assuming a rhythmical character only in sleep. A still stranger fact is, that the lungs do not expand equally, and at times one of them may for a variable period be quiescent. The frequency of respiration, decreasing from thirty-five per minute at birth to twenty per minute at twelve years of age and eighteen at maturity, is in itself capable of a wide interpretation. And the notable rapidity of the pulse shortly after birth, the ease with which it is made still faster, and its naturally irregular character would in a person of greater -age be pathological. The same feature of continuous change applies to every part of the organism and at the same time explains characteristics that are peculiar 
to the infant. Thus, for instance, he is more supple than the adult because, comparatively, he has a larger proportion of muscletissue and a smaller proportion of tendon; the muscles contain more water, a smaller amount of myosin, extractives, fats, and inorganic salts. Somewhat similar conditions may be seen in the eartilages, which increase their proportion of mineral salts from two and twenty-four hundredtls per cent at six months of age to seven and twenty-nine hundredths per cent at nineteen years. A more detailed demonstration of the general truth may be made from the analysis of any characteristic bone, such, for instance, as the tibia: -

Phosphate of Calcium . . Carbonate of Calcium . . Phosphate of Magnesia. . Chloride of Sorlium . . Cartilaginous Substance Fatty Matter . . . . Organic Matter . . . . Inorganic Matter.

.

\begin{tabular}{|c|c|c|c|c|}
\hline 2 uos. & 9 sos. & 3 yRs. & 19 YRs. & 25 YRs. \\
\hline 57.54 & 48.55 & 59.74 & 54.84 & 57.18 \\
\hline $6.0^{\circ}$ & 5.79 & 6.00 & 10.82 & 8.95 \\
\hline 1.03 & 1.00 & 1.34 & 1.26 & 1.70 \\
\hline 0.73 & 1.24 & 0.63 & 0.76 & 0.60 \\
\hline 33.861 & 41.50 & 31.34 & 31.37 & 29.54 \\
\hline 0.82 & 1.92 & 0.95 & 0.92 & 1.84 \\
\hline 34.68 & 43.42 & 32.29 & 32.29 & 31.36 \\
\hline 65.32 & 56.36 & 67.71 & 67.71 & 68.42 \\
\hline
\end{tabular}

Not only the bones but the periosteum as well are more vascular in the infant than the adult, and the periosteum is, likewise, thicker in the former. The medullary canals contain a reddish oily fluid that finally develops into mature marrow. Its early color, which is most intense at the centre, is derived from its injected ressels. The change to the normal hue, structure, and consistency begins at the centre and spreads to the periphery, reaching the extremities of the long bones last of all. This development begins in the distal bones and thence advances centripetally. The gradual but radical variations are seen nowhere more plainly than in the skull. At first the base is almost flat; then there is a sudden rise of the basilar process in front of the foramen magnum with the formation of the angle between it and the body of the sphenoid, supplemented finally by the marked descent of the vomer, which originally was almost horizontal. The cause of this flatness of the base is the fact that in the later months of fœtal life this part does not grow as rapidly as the upper part of the cranium, 
which maintains its preponderance for the first few years. In the infant the squamous portion of the temporal bone is, as compared to the parietal, comparatively small, and does not override the latter. As the adult characteristics begin to assert themselves, the squamous suture begins to rise; simultaneously the base attains a higher rate of development than the upper portion of the cranium; the squamous portion approaches the vertical portion and so rapidly increases in size that instead of lying below the outer margin of the parietal it extends so far upward that it reaches and finally overlaps the contiguous edge. All the changes progress, not only gradually, but very irregularly, thus leaving the way open for endless complicating conditions ; and in the midst of the mass of isolated facts it is hard to discern the philosophy of the matter. Thus, while the fontanelles ordinarily disappear by the time a child is four years old, the sphenoid and occipital bones, with the layer of cartilage between them, do not fully unite at their basilar parts until about the age of twenty or twenty-one years. In another part, the orbital plate of the frontal bone, the process of ossification is regularly incomplete even after puberty, and the patency of the petro-squamous suture in the roof of the tympanum in early life is well known, being the path of infection from the lining membrane of the cavity to the dura mater. In still another part of the face and head, the upper and lower jaws, the progress is . very steady, but, nevertheless, the rami do not obtain their proper direction for a long time; and the upper jaw, especially, although it may be regarded as the most important part of the face, does not obtain its full development until puberty or even thereafter. In short, the general dimensions of the infantile and adult skulls are essentially different : in the former the greatest diameter equals or exceeds the combined height of the cranium and face, but in the latter it is only three-quarters of the corresponding height; again, the breadth between the outermost points of the infantile zygomata is to the height of the face as ten to four, but in the adult the proportion is as nine to eight.

Not only does the bony skull but also its contents show a noteworthy distinction between the early and late forms. The first thing that strikes one is the fact that the dura mater in young children is adherent to the skull instead of, as in the mature person, being free, thus avoiding the collection of extravasations between it and the bone; at the same time one notices that the subarachnoid space 
holds more fluid than in the older persons and the brain itself is large but undeveloped; chemically it contains a noticeably large amount of water. Naturally it is softer and of a lower specific gravity. Its morphological development is so slow that the cells of Purkinje, which are recognizable in the fotal cerebellum of five months, do not get all their characteristic structure for a considerable time, and it is only after birth that their inner branches begin to be prolonged into process-like formations extending into the mantel layer. Even then the developed cerebral ganglion cells do not exist, and may require several weeks to get a fair amount of growth. The cerebral convolutions are equally tardy, and for the period directly after the birth are indistinctly mapped out. From that time up to the seventh or eighth year the whole organ shows a rapid, although irregular, growth, the convolutions becoming more distinct and more deeply marked. While it is growing, many of its relations are changing enough to alter wellknown landmarks. Thus the Sylvian fissure, instead of being at the level of the anterior part of the squamous suture between the temporal and parietal bones (as it is in adults), is about threequarters of a centimetre above it. In another place the fissure of Rolando is at the begimning almost transverse in its direction and only after the passage of years does it acquire its true mature inclination; in addition, this fissure extends farther forward and downward in the infant than the adult, because the frontal lobe is relatively small, particularly in the third or inferior frontal convolution, and also, but to a smaller extent, in the middle frontal convolution. Such development is no more remarkable than that the frontal sinuses do not begin to appear until the second year, or the sphenoidal until the third, or that the foramen cæcum is usually closed about the time of puberty. Facts like these are easy to multiply, but would demand more than their comparative share of space and attention, for each one, if logically followed out, means a statement of all its altered relations; but enough has been stated to show in what direction the truth lies.

The minute changes are equally noteworthy with the gross. In the fotal brain the individual cells are entirely distinct and separated from each other. Before they can perform their function in a reliable manner they must undergo a process of elaboration, must bud and branch, and interlace like the fine roots of a large plant. Even after a real growth has begun, the struggle for 
existence among the young nerve elements goes on so sharply that their mere presence does not necessarily mean a coeval degree of function. As an example, one may cite the undeveloped conducting fibres between the immature brain and the spinal cord. We know, as a matter of research, that these cells are not thoroughly elaborated ; likewise, as a matter of experience, we know equally well that the impulses, which they later on conduct, do not exist. The inevitable conclusion to be drawn from this combination is that most of a child's earliest and many of his later movements are purely reflex and not the result of activity in the higher cerebral centres. Experimental researches confirm this idea: Goltz removed the cerebrum of a young dog and then found that the animal was yet able to eat and drink, to bark, to respond to salient stimuli, and, in general, to accomplish the most important of its physical functions and instincts. Longet, having amputated a pigeon's cerebral hemispheres, demonstrated that the bird was able to eat and drink, to perform the excretory functions, to follow a moving light, to blink the eyes, and to accommodate the iris.

As the brain develops, each progressing stage is marked by a distinct increase in the nerve-medullation. Those parts which first obtain their myelin sheaths are likewise the first to functionate ; in this way we have an accurate and satisfactory method of ascertaining developmental progress. Consequently, we know that the fibres of the cord, medulla oblongata, pons Varolii, and corpora quadrigemina, being medullated long before those of higher cerebral centres, first perform their functions. That is one way in which we know that a child's somatic impulses are and must be developed long before the most purely intellectual ones begin to exist. In a similar way we can understand why an infant's movements are incoördinated, uncontrolled, and purposeless, for the peripheral nerves become medullated and therefore efficient long before the inhibitory centres. The same rule holds true not only of the main fibres but of their primary and secondary branches as well. The process of growth by which the nerve cells put out their neurons and dendrons is a most elaborate one, as is conclusively demonstrated by the fact that the cell-bodies alone make up no more than ten per cent by weight of the central nervous system, while the neurons, dendrons, and like bodies constitute the remaining ninety per cent. The position of an animal in the vertebrate series is marked by the number of divisions and 
subdivisions that characterize the branches of the cortical cells. Cajal's figures show that each cell begins with no branches but finally has many ; Vulpius and Kaes have demonstrated the steady and remarkable increase of these bodies, and Howell states that the increase continues up to the age of thirty years, being accompanied by a like growth in medullation. One can easily see how provisional is the mental equilibrium of the child from the facts that the first seven years of life are largely concerned in building up the peripheral system, that the motor and sensory fibres constitute no more than one-third of the whole cortical surface, and that the development of the remaining two-thirds is prolonged over a much greater space of time. This slow elaboration is accompanied by an equally slow appearance of chromatin granules, whose importance in the functional activity of nerve-structures is doubtless great, although at present not clearly defined. Even after the brain has attained a fair degree of efficient formation, the transitory, unstable nature of its functional activity is shown by Peterson when he says: "From studies I have made of hemiplegia in children, I have been led to conclude that during the first years of life (perhaps up to eight or ten or more) the two hemispheres share equally the motor and sensory functions of speech, and that it is only during adolescence that the left hemisphere (in righthanded persons) takes upon itself gradually the greatest part of this burden."

The vertebra show equally interesting changes with the brain and skull. The whole structure at birth is broader and shorter than in the adult, and the cord descends about one vertebra lower than later in life, with the natural result that the cavity of the spinal dura mater usually ends near the top of the third sacral vertebra. The whole column is very pliable and lacks the characteristic cervical and lumbar curves that come into existence with later growth and strength. The proportions between the different portions of the column are not the same as in maturity : in the latter case the cervical is to the lumbar part as two is to three, but in infants they are equal to each other ; the cervical is proportionately longer in infants, while in the late fœtal and early extrauterine life the proportion of the movable part of the column in the neck is greater than that in the loins - the reverse of the adult condition; and in later childhood the lumbar portion grows more rapidly than the cervical, until after puberty the final pro- 
portions begin to be marked out. The vertebral bodies do not begin to consolidate until the fourth year, and progress more or less regularly until the eighth and more; this does not include the epiphyseal plates, which do not get their growth until about the seventeenth year. The coccyx is slowest of all, for its consolidation does not come about until puberty, the third piece is not ossified until the sixteenth, and the fourth until between the eighteenth and nineteenth years.

About two-thirds of the growth of the eye belongs to early infancy; but the recessus opticus is more plainly distinguishable at birth than at maturity. A remarkable fact is shown in the growth of the macula lutea, which is developed only after birth. The lachrymal glands are equally tardy, and for weeks or months are not prepared to shed tears. The ear likewise develops unevenly ; while the parts of the internal ear, the ossicles and tympanic cavity, are comparatively well formed a short time after birth, the external meatus, the mastoid portion, and the Eustachian tube are far from having reached their final form. The annulus tympanicus soon begins to grow outward to make the base and anterior walls of the external meatus and also forward and inward along the outer wall of the tympanum, at the same time reinforcing a small part of the outer wall of the Eustachian tube. The osseous meatus of the ear is not thoroughly well developed until about the fourth year. In infancy the auditory canal has a peculiar course, since it passes inward and downward while the drum membrane is almost horizontal. The development of the mastoid cells is yet later; after birth the external petro-squamosal suture gradually closes up until by the end of the first year the bones are practically consolidated. Slowly then the mastoid process begins to form. From the periosteum and the surface there is a growth of new bone which slowly thickens the external and inferior walls of the antrum. In the new-born this wall is only about one or two millimetres thick, but the increase is so steady that at nine years of age it is nearly one centimetre. The new bone is a fragile and delicate cancellous structure which at the approximate time of puberty is eaten away by a process of absorption so that a number of communicating air cells are formed, each being lined with mucous membrane.

Another part which shows characteristic growth is the Eustachian tube. Its nasal meatus is in the foetus lower than the 
level of the hard palate; shortly after birth the two are in the same plane, while in the adult the meatus is higher by a considerable space. The tube in the new-born is nearly horizontal, but it inclines downward in the adult. In very young children it is shorter than in the mature subject, but it is relatively and absolutely wider at its narrowest portion in the former than the latter. During the period of growth it doubles its length, but the aural meatus maintains its original size. In early infancy the nose has a peculiar growth. The inferior turbinated bone projects slightly into the nasal cavity, but there is no more than a small free space below and none behind it. In this part the greatest development may be expected. The height increases rapidly until dentition, then it becomes slow for a time and again grows rapidly until nearly the eighth year. At this time the breadth increases and the olfactory portion becomes larger. At a very early age the posterior margin of the vomer is noticeably oblique, but gradually with the downward growth of the face assumes a less horizontal slant.

The development of no part of the body is more interesting than that of the teeth. The alveolar processes as early as the seventh futal month show a row of indentations which correspond to the twenty milk teeth. Slowly the crowns become formed and calcified, but at the same time the dental sacs of the permanent incisors, canines, bicuspids, and first molars begin to form in the jaws. The first upper molars lie behind the second temporary molars, but not in alveoli. There is so little free space that the second temporary molars in the upper jaw are set in shallow depressions without posterior walls. In the inferior maxilla the sockets for the second temporary molars extend back to the coronoid processes, underneath which the first permanent molars are stowed. The elements of the second molars are formed before the end of the first year, while those of the third molars do not appear until the fifth. All these teeth have not room to be set evenly side by side, in the small and undeveloped jaws, and therefore we find them packed together, the central overlapping the lateral incisors, the canines being forced over all. The structural composition undergoes divers changes in such items as enamel, dentine, and nerve supply, so that the stored-up elements appear little by little as the corresponding functions grow out into activity. 
During all these changes, the tongue is altering its shape and direction, and is steadily endeavoring to overcome its thinness, its long ribbon-like form. The soft palate lies very close to it, like a curtain, and when the mouth is closed extends backward rather than down, as in the adult. At the same time the uvula is rudimentary rather than merely small, and the follicles at the back of the tongue are so little developed that often they cannot be seen. The same may be said of the pharyngeal or third tonsil, which at the beginning of life is normally very small, although exceptional cases occur in which it is hypertrophied at or before birth. During the early months of infancy the ptyalin-forming glands are entirely inactive, and when the ferment begins to be formed it is weak in character and small in quantity. The salivary glands are likewise of a poor relative development, and at first their functional activity is practically no more than potential.

In this early part of life one of the main characteristics is the unevenness, the irregularity of the growth in the various parts. While the development of most parts is forward, the rule does not apply universally. As an example one may cite the evolution of the thymus gland. At birth it is almost as large as the left lung, and in addition it continues to grow for about three years. Then, mysteriously enough, it stops growing and remains stationary until near the time of puberty, when, in the same unknown way, it gradually atrophies or is replaced by a mass of fat. In infancy it is large enough to lie in both neck and thorax, extending down into the anterior mediastinum and resting in two long lobes on the pericardium; by its relatively large size it holds the right and left lungs with their pleuræ apart. The change by which it so rapidly fades into nothing is certainly remarkable. The thyroid gland has something of the same character on account of having its largest relative size in childhood; although in this case the after-development is not so noteworthy as in the thymus.

The neck has its peculiar evolution which maintains the idea of irregularity. Between birth and maturity the larynx lowers its relative position in the equivalent of two vertebræ and two intervertebral disks. Thus the top of the epiglottis descends from the lower border of the atlas to the centre of the third cervical vertebra or lower. At the same time the absolute size is gradually increasing. In the trachea the bifurcation at birth 
corresponds in position to the third dorsal vertebra, but at maturity it is opposite the fourth. The upper sternal extremity is relatively higher as well as smaller in the infant than the adult. The adjacent ribs go through simultaneous changes which mark off one period of life from another. In the first months the third and fourth costal cartilages are attached in a horizontal direction to the sternum, but in later life they have an upward slope. The lower three true costal cartilages have a more horizontal course, and the angle which they subtend is greater in infancy than in maturity. The clavicles follow the same rule of horizontal attachment, and do not have the upward curve of the outer extremities that exists in adults.

These charges necessarily indicate equally great deviations from the form of the adult chest. In the young child the transverse diameter increases more rapidly than the antero-posterior, having the relation of one to two instead of, as in the adult, one to three. Such reasons control the fact that the infantile thorax is a blunt right cone rather than the mature form of reversed cone. A characteristic fact is that in early life the superior sternal margin is about even with the centre of the second dorsal vertebra, but in the development of later years it sinks to the third dorsal vertebra. Also one may easily explain, in part, the barrel-shape of the child's chest and his abdominal method of breathing by the less oblique position of the ribs, by their being flatter and less hooped up than in the man.

The heart shows some interesting and characteristic alterations in function and size. Before birth it fills up the greater part of the thoracic cavity; at this time its circulation is so different from the later form that it belongs to the same category of noteworthy transitional forms, some of which have already been mentioned. The blood, after passing from the placenta by the umbilical vein, flows to the inferior surface of the liver and there divides into two streams, one going through the ductus venosus to the inferior vena cava, the other entering the portal vein, from which it is distributed through the liver and finally empties into the inferior vena cava. Thence it flows into the right auricle, into which the superior vena cava directs the current from the head, neck, and arms. The two streams do not mix, the latter flowing through the auriculo-ventricular opening, while the former is directed by the Eustachian valve through the 
foramen ovale into the left auricle, thence to the left ventricle, finally to the aorta. The current from the superior vena cava flows in a small part to the lungs through the pulmonary artery, and finally to the left auricle; the remaining and larger part issuing through the ductus arteriosus finds its way into the aorta, where it meets that portion of the current from the inferior vena cava which has not entered the three great vessels of the upper body; thence the two are sent to the trunk and lower extremities, a part reaching the placenta by the umbilical arteries, thence by the umbilical vein to the inferior surface of the liver.

When the child is born and the umbilical vessels are tied, the foramen ovale, the ductus arteriosus and ductus venosus rapidly close. The two currents which flow from the superior and inferior venæ cavæ meet and mingle in the right auricle, from which the common stream enters the right ventricle. Thence by the pulmonary artery it is distributed through the lungs; it then enters the left auricle and finally the left ventricle, from which it is sent to the rest of the body. If the heart is measured at this time, its size will still be found relatively larger than the adult, the comparative dimensions being eighty-nine hundredths of one per cent of the body weight in the new-born infant, and fifty-two hundredths of one per cent in the adult; in spite of this large size the left ventricle is about equal to the right, being as yet without the added growth that eventually comes to it. Examination will show a concomitant alteration in structural relations : if we consider the above-mentioned facts in relation to the known narrowness of the chest in the infant, and the maintenance of a similar vertical extent in childhood and maturity, it naturally follows that the transverse diameter in the child is greater than it later is. As a further result the apex beat is nearer the mammary line, or outside of it, which is quite different from what we see in men and women. Another fact which concerns the position of the organ is the noticeable proximity of the conus arteriosus to the chest wall with the resulting "milk spot" caused by friction. All in all, one may say that the containing space, the relative and absolute positions, and the form of the youthful heart are plainly different from what they are at maturity.

The rate of increase in the size of this organ is so irregular that it bears no definite relationship to the growth of as closely connected a system as the arteries. Thus the heart increases 
rapidly in the first months of life, slowly from the third to the ninth or tenth year, and very rapidly during the general period of puberty. Thus, with the slowly developing heart there is a wide and active arterial system, while at puberty, with its comparatively large and active heart, there is a distinctly narrow arterial system. During this period the heart increases twelve times its original size, while the arteries increase to only three times their original proportions. Stated differently the fact would be that in infancy the relation of the heart's volume to the diameter of the ascending aorta is as twenty-five to twenty, before puberty as one hundred and forty to forty-six and after puberty as two hundred and ninety to sixty-one. A closely associated fact is the difference in blood pressure, for the conditions of early life make for a low tension, which one can see in the action of the abdominal viscera, while at and after puberty the tension is considerably higher. While the blood pressure in the infant body as a whole is low, that in the lungs is high - a fact that is doubtless due in part to the unstable and changing relations of the pulmonary artery and aorta which in childhood bear the relation to each other of forty to forty-six, but at maturity of thirty-five and nine-tenths to thirtysix and two-tenths. As results, there are a heightened excretion of carbonic dioxide, a more rapid respiration, and a greater nitrogen percentage in the early condition. Another example of uneven local development may be seen in the abdominal aorta and common iliac arteries which in the first few days are greater than at any time for the succeeding three months. Yet another interesting fact is contained in the varying rapidity of the circulation; at birth the entire round is made in about half the time required in the adult, but the adjustment progresses so rapidly that at three years of age the rate is only one-third faster. In fact, the whole course of the cardiac development is irregular, so that we cannot give a steady proportional rate between its growth and that of the other viscera. All that is possible is to make generalizing statements, such as, that the size of the heart in childhood is relatively greater than that of the lungs, or that while the heart doubles its size the liver increases only one-half of its volume.

In the liver the changes are fully as noteworthy as those which have been considered. This organ, during the second foetal month, attains a relatively enormous size, in the next month it extends into the hypogastric region and occupies the greater part 
of the abdominal cavity. Its decrease in size is continuous until at birth it constitutes one-eighteenth of the body weight, and at maturity one thirty-sixth. At the same time one should keep in mind that the change is not steady and regular, but rather that it occurs in fits and starts. In the adult, as every one knows, it ought to lie in the right side of the thorax and not extend below the free border of the ribs, but in infancy it is pushed down one, two, or more centimetres below that line, and may invade enough of the left thoracic region to displace both breast and lungs; it may even extend so far as to fill up a noticeable part of the abdominal space. In its minute arrangement there is likewise a characteristic slowness of development. Thus in fœtal life there are two main varieties of hepatic cells : one a polyhedral form that is practically the same as the mature; the other, which is probably a young type of the adult variety, is a small round cell that tends to disappear after birth. A considerable time passes before the hepatic cylinders assume the adult mammalian type. Slowly they become longer and narrower on account of a rearrangement of the cells, until the number of cells, as shown by cross-section, is reduced to two. Simultaneously the gall-bladder changes its relations, for its fundus is farther removed from the anterior wall in children than in adults. The whole system seems to have its own rule in development, for instead of showing a slowly increased absolute size and a stationary relative condition, it exhibits a decreased relative size and an eccentric absolute bulk. In the secretion of bile the organ once more shows its peculiarity; for this fluid appears very early, in some cases as soon as the third month of fotal life. In addition it is produced in relatively greater quantities than in the adult, and its consistency is thinner. While the liver is very large, and evidently very active, the spleen, although it occupies its adult position, is both absolutely and relatively smaller in infancy than in later years. In these young children one can with difficulty make out its form. Likewise, it is very inactive, so that its backwardness in growth accompanies a similar backwardness in functional ability.

While the heart shows but little relation in growth to the liver, it shows no more to the lungs. In the time required to increase its size by one-fifth, the lungs have added five-sevenths of the original volume to their dimensions. Up to the fourteenth 
year these organs come into no closer relationship. The irregularity of the whole connection is shown by the fact that in infancy the bulk of the heart is to that of the lungs as is one to three and one-half or four; but at or shortly after puberty the lungs have so much increased their capacity that they are to the heart as seven and three-tenths are to one. About this latter time the heart in its turn receives a strongly accelerated growth, so that within a short period the proportion is reduced to about one to eighttenths. In a very generalized fashion one would say that the approximate growth of the lungs resembles that of the liver, while the heart develops in a comparatively similar way to the kidneys. The lungs in their minute structure are of decided theoretical and practical interest. During the first two years the walls of the alveoli are thick and their blood-vessels are loosely held. 'It is not until between the fourth and fifth years that the stroma has become tense and binding, with the adult control of the capillaries, and that the developmental relations between the alveoli and bronchi begin to assert themselves. In infancy the underlying loose tissue lining the bronchial tubes slowly binds the mucous membrane to the fibro-muscular wall, and then keeps pace in its growth with the other compact tissues, until in adult life it appears as dense fibrous bands. The area of bronchi and their divisions exceeds that of the air spaces ; the connective tissue of the parts is likewise more abundant, and easily undergoes a proliferation of its cellular elements ; the submucous connective tissue of the bronchi is loose, richly supplied with nuclei, and holds its capillaries in a loose grasp; the alveoli are small, their lining cells are set in a continuous layer, their epithelium proliferates abundantly, and their absorbents act slowly. During this period the blood-vessels play a more important part than later on, permitting congestive disorders with very little resistance. With all this rapid growth the lungs do not attain their forward expansion until seven years of age, and before that time the left lung, on account of the high position of the diaphragm which is characteristic of early childhood, is somewhat higher situated than the right.

In the kidneys lobulation occurs in prenatal life, and continues for a considerable period after birth; then the lobules slowly disappear, giving way to the newly formed pyramids of Malpighi. The organs are comparatively larger than in the adult, and have 
a lower situation. This is the more remarkable since the lumbar spine is relatively small. At birth the two kidneys are of equal size, but during the first year the left one becomes larger than the right, and its location is slightly higher, where it remains until seven or eight years of age. A noteworthy fact may be seen in these organs in the form of infarctions which may exist for a considerable time after birth. These occur normally in fotal life, and sometimes persist in a form which in later years would have a pathological significance. It reminds one of the conical tragus so often seen in early childhood, which regularly occurs in prenatal life, or of hare-lip, which is likewise a fœtal condition carried over into postnatal growth, or a patent foramen ovale.

The calibre of the arterial system in the kidneys bears a somewhat anomalous relation to these organs; the transverse section of the former increases more rapidly than the volume and weight of the latter, with the natural result of increased tension. This fact has a more important bearing upon the pathological conditions of childhood and the great liability of a nephritic involvement. 'This large increase is in distinct contrast to some of the largest vessels, for instance, the carotid, in which the growth is relatively small, or the femoral, whose increase is no more than moderate. The suprarenal capsules present another example of eccentric development, for they are almost as large at birth as they are in the adult life, and in some cases they are yet larger. The question of their function is so obscure that one may not go farther than the mere statement above.

The stomach shortly after birth grows very rapidly, but before many months the rate becomes considerably slower. The shape in infancy is more tubular, the position more vertical, the situation higher, and the œsophageal sphincter less developed than at maturity. With these premises it is easy to understand why a baby vomits on very slight provocation and with very little distress. Microscopically, as well as macroscopically, the structure is peculiar. The characteristic gastric ducts which perform so large a part of the organic work do not attain their full development until maturity; about the time of birth each one of them contains about seven glands. This number is progressively reduced until in the adult there are only three to a duct. Peristalsis is comparatively weak, and the functional secretions are of a special sort, inasmuch as they have little or no corrosive 
power and the capability of breaking up cell-envelopes. On the other hand, they contain a relatively large amount of rennet and a noteworthy faculty for digesting casein. The necessity of restricting an infant's diet to milk may thus easily be seen. The secretions of the pancreas similarly vary between infancy and maturity, for in the former the amylopsin is entirely inert and steapsin and trypsin scarcely active.

The intestines grow in an irregular fashion, at one time much, at another time but little; and they require considerable time to obtain their permanent position and fixed attachments. In adults there is a constriction between the first and second parts of the intestine which in infants is very often absent. The length of the large intestine does not increase during the first four months, but after this time the upper portion begins to grow rapidly and at the expense of the sigmoid flexure, which at birth constitutes about one-half of the whole large intestine. From the fifth month the sigmoid flexure, having obtained its permanent proportions, continues to grow according to the general rate of increase. The transverse colon is at first relatively huge; the ascending colon is, on account of the higher position of the crecum and the large size of the liver, very short; it more often has a mesentery than in the adult and a relatively larger part above the crecum is covered with peritonæum. As a result the gut in this region is free and can change its, position and relations to a surprising extent; in foetal life it lies in the median plane and very high up. In its growth it passes to the right, in front of the second portion of the duodenum, and finally descends into the iliac fossa. The whole sigmoid flexure, even after it has obtained the permanent proportions, is placed so high that barely any of it is found in the pelvis until such later times when the pelvic bones are spread out. In early childhood Brunner's and Lieberkühn's glands are no more than partially developed, and the solitary and agminated glands are unusually rich in lymphoid tissue. The whole lymphatic system, in fact, is highly developed in the first part of life and contains a relatively greater amount of lymph in circulation than what is normal at adult age.

The condition of the rectum may be inferred from what we know of the portion of the large intestine above it. The greater part of the rectal length is in the abdomen instead of the true pelvis; it is nearly straight and occupies a fairly, vertical position 
instead of lying in the lateral and the two antero-posterior curves of the adult form. In children the attachments do not extend high up, and the reflected peritonæum is located farther down than in developed persons. These facts have a strong bearing upon the ease of contracting chronic constipation, prolapse of the rectum, and digestive disorders.

The bladder of the infant is practically an abdominal, and not a pelvic, viscus. Its posterior surface in the course of development becomes covered by peritonæum, but its anterior surface remains without this covering until after puberty. The ovaries, uterus, prostate gland, testicles, and male urethra cannot be expected to develop in any large measure until about the time of puberty. The perinæum varies according to the changes in the neighboring structures. The pelvis increases in size very much, especially about the time of puberty, when the organs which naturally belong in it are allowed to sink into their proper places; the change is naturally most plainly visible in the female whose broad pubic arch and wide transverse diameter (exceeding the antero-posterior) are characteristic of the adult condition.

All these facts should be regarded not as the final and complete statements upon which to form the ideal of the normal child, but rather as isolated and characteristic observations which may be used as the outlines of a true picture that requires the work of many hands for its finishing. A full account of all the slow changes that make the child so widely different from the adult would of necessity bring in practically every element of physical and mental growth; but enough has been recorded and suggested to demonstrate that the child is not a fully formed being, that his development is not a rigidly immovable process, that each progresses toward fruitful maturity slowly and irregularly, and that the unstable equilibrium, which perforce exists, demands intelligent and sympathetic care for its conservation. The medical care of children has this in view as much as the control of immediate pathological conditions. 


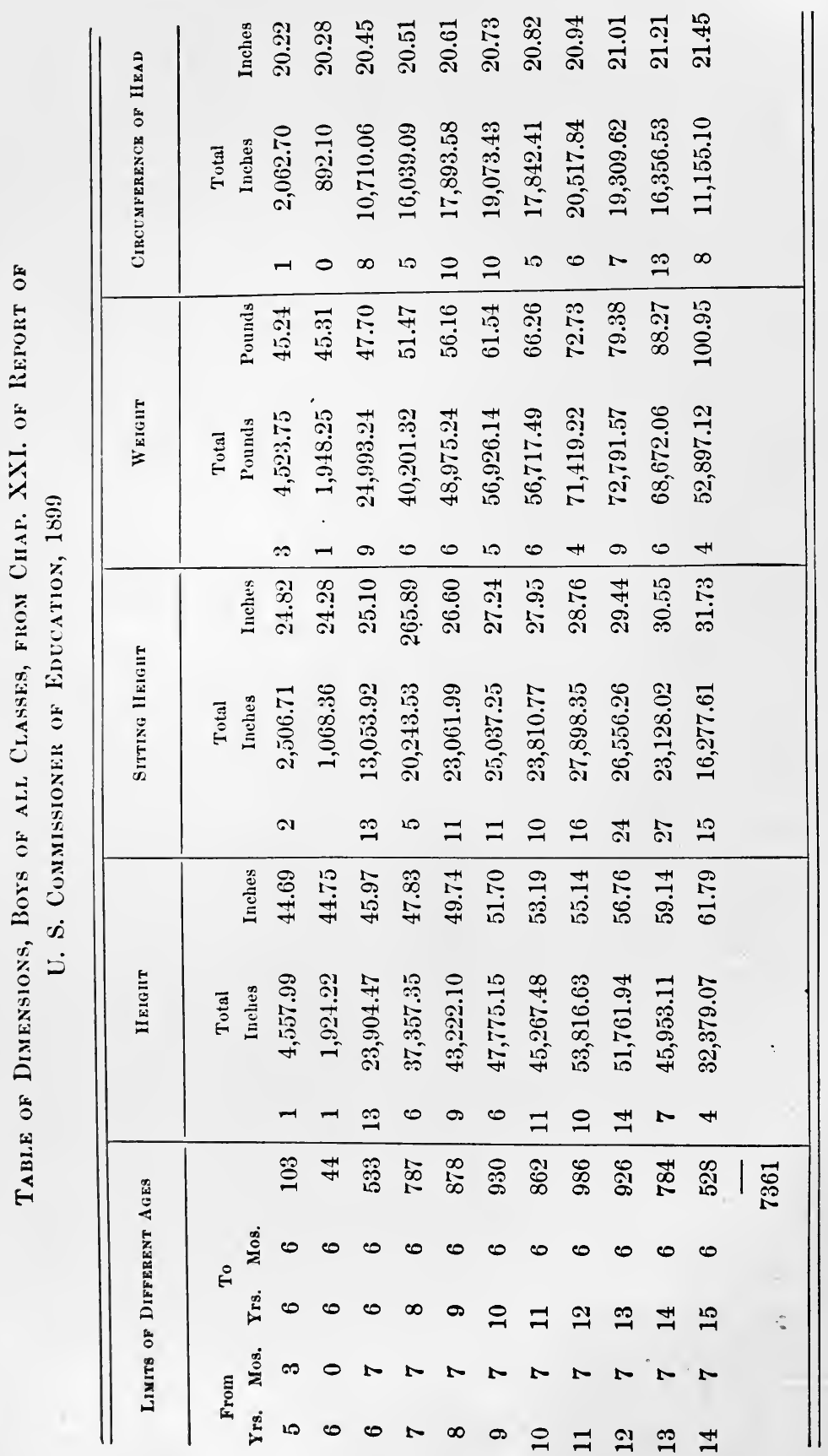




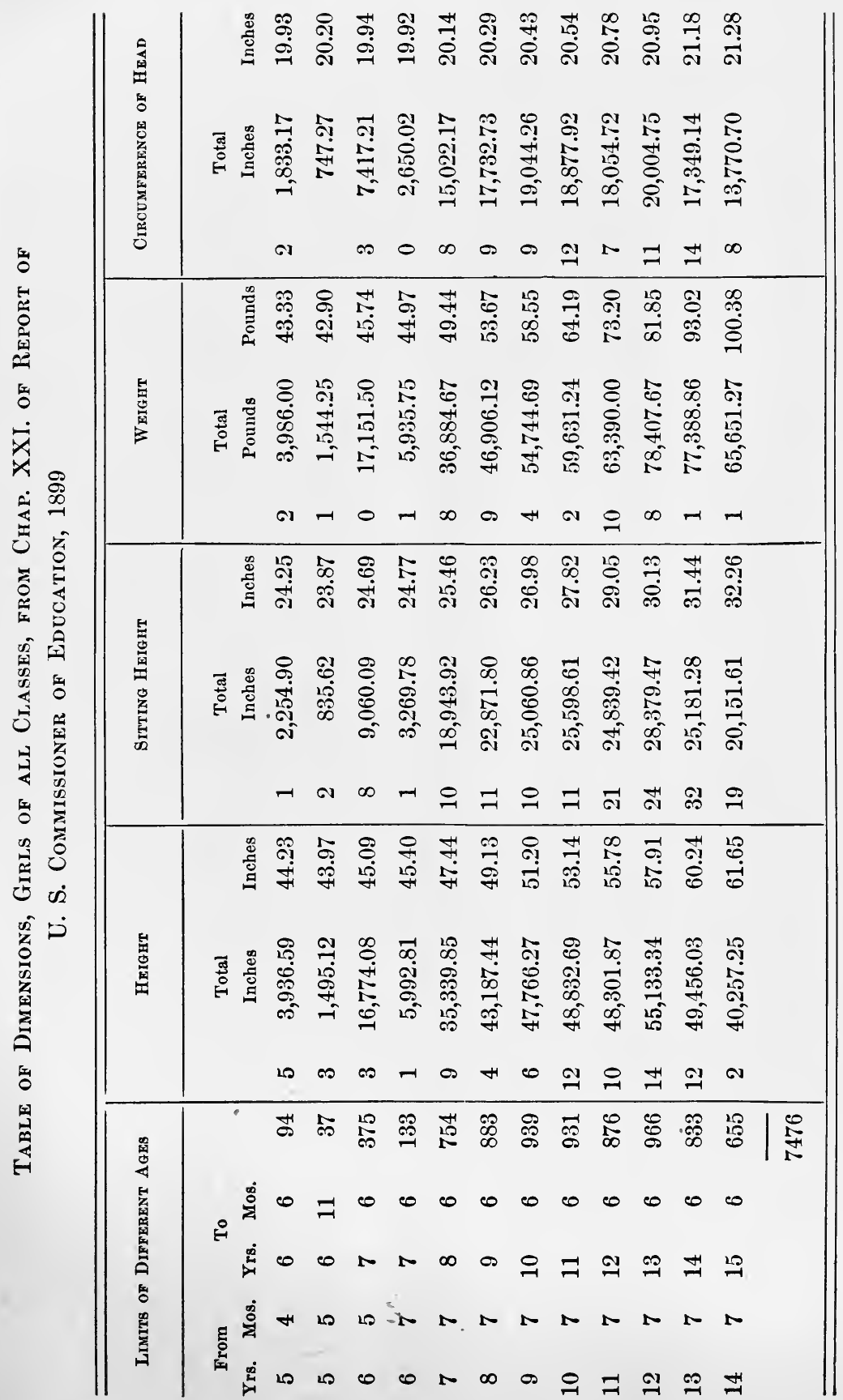


Quetelet's Table of Average Height and Weight, Tex of Each Age and Sex

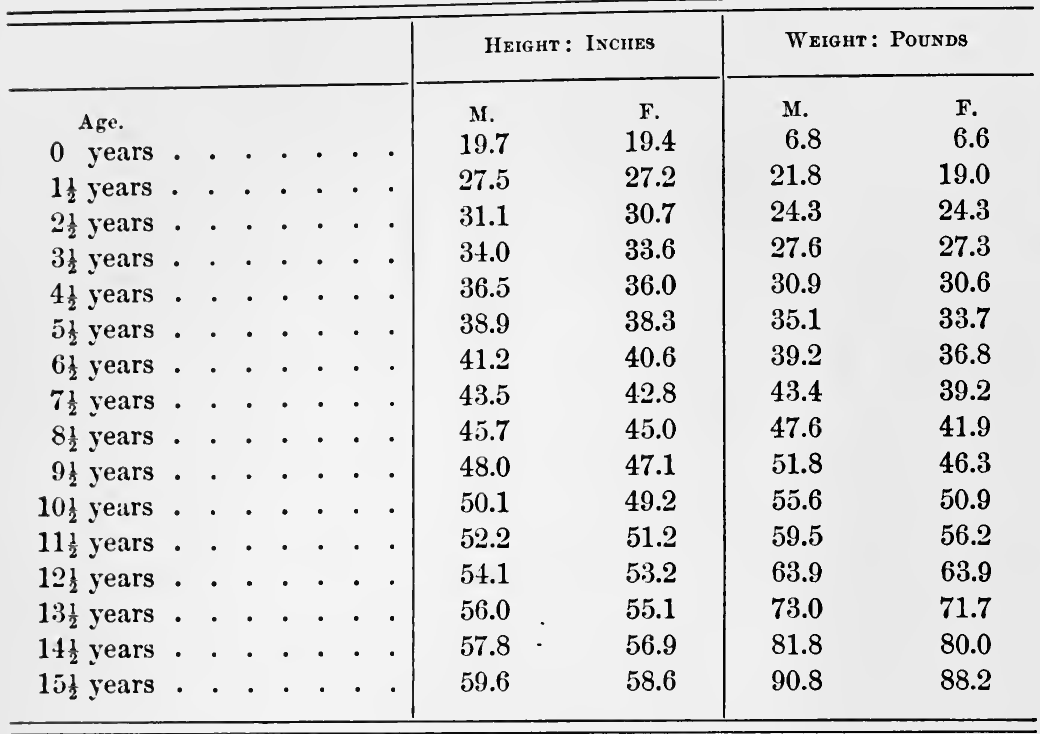

Table showing Weight, IIeight, and Circumference of the Head and Chest fron Birth to the Fourteenth Year.

(Boas in Science, April 12, 1895.)

\begin{tabular}{|c|c|c|c|c|c|c|c|c|c|c|}
\hline & & & \multicolumn{2}{|c|}{ WEIGIT } & \multicolumn{2}{|c|}{ Height } & \multicolumn{2}{|c|}{ Chest } & \multicolumn{2}{|c|}{ Head } \\
\hline & & & Pounds & Kilos & Inches & $\mathrm{Cm}$. & Inches & $\mathrm{Cm}$. & Inches & $\mathrm{Cm}$. \\
\hline & Boys . & & 7.55 & 3.43 & 20.6 & 52.5 & 13.4 & 34.2 & 13.9 & 35.5 \\
\hline Birth & Girls . & & 7.16 & 3.26 & 20.5 & 52.2 & 13.0 & 33.2 & 13.5 & 34.5 \\
\hline & Boys . & & 16.0 & 7.26 & 25.4 & 64.8 & 16.5 & 42.0 & 17.0 & 43.5 \\
\hline 6 months & Girls . & & 15.5 & 7.03 & 25.0 & 64.6 & 16.1 & 41.0 & 16.6 & 42.2 \\
\hline & Boys . & & 20.5 & 9.29 & 29.0 & 73.8 & 18.0 & 45.9 & 18.0 & 45.9 \\
\hline 12 months & Girls . & & 19.8 & 8.84 & 28.7 & 73.2 & 17.4 & 44.4 & 17.6 & 44.6 \\
\hline 18 months & $\begin{array}{l}\text { Boys . } \\
\text { Girls . }\end{array}$ & & $\begin{array}{l}22.8 \\
22.0\end{array}$ & $\begin{array}{r}10.35 \\
9.98\end{array}$ & $\begin{array}{l}30.0 \\
29.7\end{array}$ & $\begin{array}{l}76.3 \\
75.6\end{array}$ & $\begin{array}{l}18.5 \\
18.0\end{array}$ & $\begin{array}{l}47.1 \\
45.9\end{array}$ & $\begin{array}{l}18.5 \\
18.0\end{array}$ & $\begin{array}{l}47.1 \\
45.9\end{array}$ \\
\hline & Boys . & & 26.5 & 12.02 & 32.5 & 82.8 & 19.0 & 48.4 & 18.9 & 48.2 \\
\hline 2 years & Girls . & & 25.5 & 11.56 & 32.5 & 82.8 & 18.5 & 47.0 & 18.6 & 47.2 \\
\hline & Boys . & & 31.2 & 14.14 & 35.0 & 89.1 & 20.1 & 51.1 & 19.3 & 49.0 \\
\hline 3 years & Girls . & & 30.0 & 13.60 & 35.0 & 89.1 & 19.8 & 50.5 & 19.0 & 48.4 \\
\hline
\end{tabular}


Table showing Weight, Height, etc. (continued)

\begin{tabular}{|c|c|c|c|c|c|c|c|c|c|}
\hline & & \multicolumn{2}{|c|}{ WeIGHT } & \multicolumn{2}{|c|}{ HEIGHT } & \multicolumn{2}{|c|}{ Chest } & \multicolumn{2}{|c|}{ Head } \\
\hline & & Pounds & Kilos & Inches & $\mathrm{Cm}$. & Inches & $\mathrm{Cm}$. & Inches & $\mathrm{Cm}$. \\
\hline & Boys . & 35.0 & 15.87 & 38.0 & 96.7 & 20.7 & 52.8 & 19.7 & 50.3 \\
\hline 4 years & Girls. . & 34.0 & 15.41 & 38.0 & 96.7 & 20.5 & 52.2 & 19.5 & 49.6 \\
\hline & Boys . . & 41.2 & 18.71 & 41.7 & 106.8 & 21.5 & 54.8 & 20.5 & 52.2 \\
\hline 5 years & Girls . . & 39.8 & 18.06 & 41.4 & 105.3 & 21.0 & 53.5 & 20.2 & 51.3 \\
\hline & Boys . . & 45.1 & 20.48 & 44.1 & 112.0 & 23.2 & 59.1 & & \\
\hline 6 years & Girls . . & 43.8 & 19.87 & 43.6 & 110.9 & 22.8 & 58.3 & & \\
\hline & Boys . . & 49.5 & 22.44 & 46.2 & 117.4 & 23.7 & 60.6 & & \\
\hline 7 years & Girls . . & 48.0 & 21.78 & 45.9 & 116.7 & 23.3 & 59.5 & & \\
\hline & Boys . . & 54.5 & 24.70 & 48.2 & 122.3 & 24.4 & 62.2 & & \\
\hline 8 years & Girls . . & 52.9 & 24.01 & 48.0 & 122.1 & 23.8 & 60.8 & & \\
\hline & Boys . . & 60.0 & 26.58 & 50.1 & 127.2 & 25.1 & 63.9 & & \\
\hline 9 years & Girls . • & 57.5 & 26.10 & 49.6 & 126.0 & 24.5 & 62.5 & & \\
\hline & Boys . . & 66.6 & 30.22 & 52.2 & 132.6 & 25.8 & 65.6 & 21.0 & 53.5 \\
\hline 10 years & Girls . • & 64.1 & 29.07 & 51.8 & 131.5 & 24.7 & 63.0 & 20.7 & 52.8 \\
\hline 11 years & $\begin{array}{l}\text { Boys . . } \\
\text { Girls . . }\end{array}$ & $\begin{array}{l}72.4 \\
70.3\end{array}$ & $\begin{array}{l}32.83 \\
31.87\end{array}$ & $\begin{array}{l}54.0 \\
53.8\end{array}$ & $\begin{array}{l}137.2 \\
136.6\end{array}$ & $\begin{array}{l}26.4 \\
25.8\end{array}$ & $\begin{array}{l}67.2 \\
65.8\end{array}$ & & \\
\hline 12 years & $\begin{array}{l}\text { Boys . . } \\
\text { Girls . . }\end{array}$ & $\begin{array}{l}79.8 \\
81.4\end{array}$ & $\begin{array}{l}36.21 \\
36.90\end{array}$ & $\begin{array}{l}55.8 \\
57.1\end{array}$ & $\begin{array}{l}141.7 \\
145.2\end{array}$ & $\begin{array}{l}27.0 \\
26.8\end{array}$ & $\begin{array}{l}68.8 \\
68.3\end{array}$ & & \\
\hline 13 years & $\begin{array}{l}\text { Boys . . } \\
\text { Girls . }\end{array}$ & $\begin{array}{l}88.3 \\
91.2\end{array}$ & $\begin{array}{l}40.04 \\
41.36\end{array}$ & $\begin{array}{l}58.2 \\
58.7\end{array}$ & $\begin{array}{l}147.7 \\
149.2\end{array}$ & $\begin{array}{l}27.7 \\
28.0\end{array}$ & $\begin{array}{l}70.6 \\
71.3\end{array}$ & & \\
\hline 14 years & $\begin{array}{l}\text { Boys . } \\
\text { Girls . }\end{array}$ & $\begin{array}{r}99.3 \\
100.3\end{array}$ & $\begin{array}{l}45.03 \\
45.50\end{array}$ & $\begin{array}{l}61.0 \\
60.3\end{array}$ & $\begin{array}{l}155.1 \\
153.2\end{array}$ & $\begin{array}{l}28.8 \\
29.2\end{array}$ & $\begin{array}{l}73.3 \\
74.1\end{array}$ & & \\
\hline & Boys . . & 110.08 & 50.26 & 63.0 & 159.9 & 30.0 & 76.6 & 21.8 & 55.5 \\
\hline 15 years & Girls . . & 108.4 & 49.17 & 61.4 & 155.9 & 30.3 & 76.8 & 21.5 & 54.8 \\
\hline
\end{tabular}




\section{CHAPTER II}

\section{GENERAL HYGIENE OF THE NEW-BORN CHILD}

THE child's care is supposed to begin as soon as the cord has been tied and respiration fully established. The eyes must be thoroughly cleansed with boric acid solution, excepting where the physician has reason to believe that the mother's genital track is the seat of infection, in which case one or two drops of a one-half per cent solution of silver should be instilled into the infant's eyes. Such children must thereafter be carefully watched lest an ophthalmia be allowed to develop. The next step is to ascertain whether the child's mouth and throat are clean and unimpeded. The infant's body may next be anointed with oil or vaseline, he should be wrapped in a blanket and placed in his crib. There is no necessity to bathe him for the next twenty four hours. One must remember that a new-born babe is exceedingly sensitive, that he has before birth been perfectly protected from sudden changes of temperature and exposure of all kinds, that the vernix caseosa covered the skin before birth and cannot possibly do harm for one day more, and that its removal causes unnecessary exposure at a time of greatest susceptibility to injurious impressions. At the end of the first day he may be bathed and clothed with his first garments.

The cord should be lightly covered with a dusting powder composed of boric acid and starch, and then drawn through an aperture in a square piece of soft gauze or lint in which it is to be wrapped. From time to time the cord should be inspected in order to see that no hæmorrhage exists. Attention should likewise be given to the child's heart-action and respiration, and, if necessary, a series of cryings must be provoked to insure full inflation of the lungs. When these items are in satisfactory condition, the child should be kept in the dark and allowed to sleep, and in the succeeding months his room during his sleep should always be darkened. 
During the first day the necessity of feeding is not great. The child should be once put to the breast after the mother has been cleansed and made comfortable, and thereafter considerable latitude may be allowed. Some authorities order nursing every six hours for the first day, every four hours for the second, and on the third every two hours, this last rate being continued during the first weeks. But with many children there is little need of giving more than one or two feedings the first day. Two or three teaspoonfuls of boiled water may be given if the infant is restless. On the second day three or at the most four nursings may be required; and on the third the regular interval of two hours should be instituted. From the first the child should be trained to require the minimum of nursing at night; and even when he is fed every two hours by day, he must be accustomed to an interval of quiet for six hours at night, during which he and his mother may sleep. Another very important matter connected with nursing is that of cleanliness of the mouth. Every day the cavity must be carefully but gently washed out with a solution of boric acid; if this is done lightly enough, the child's mouth may be washed before each feeding without in the least injuring the delicate mucous membrane. This measure will naturally be of no avail unless the mother's nipple is likewise rendered clean. 'This may easily be accomplished by washing it before and after nursing with boric acid solution and at least once a day with alcohol.

His clothing should be sufficiently warm and by all means light. Thin, fine wool gives most satisfaction; the garments must be made sufficiently large to permit free movement, and while the whole body should be covered, there is no reason for elongating the skirts to the extreme length that is fashionable. The little shirts and skirts should be closed by means of tapes rather than pins or buttons, and suspended garments must be hung from the shoulders. Diapers are best made from linen diapering; but if this is objected to on the score of expense a cotton substitute may take its place. The abdominal band is not so necessary as mothers usually believe. There is a reason for it during the first two months when a pad over the navel may be required to prevent a possible hernia, but after that time it is not of much value. From the first days the feet should be covered with woollen "booties."

The daily bath for the first few weeks should have the tem- 
perature of the body ; as the child increases in strength and vigor the warmth of the water may be lessened, so that at the end of the first year it is no more than from $30^{\circ}$ to $33^{\circ} \mathrm{C}$. $\left(86^{\circ}\right.$ to $91.4^{\circ} \mathrm{F}$.). When the child is old enough to stand up with security, it is a good plan to follow the ordinary warm bath with a rapid douche whose temperature is a few degrees lower. Another good plan is to give the cool douche in the morning, the child standing in a few inches of warm water, and follow it with a brisk but not too violent rubbing. The warm bath may then be reserved for the evening before the child retires. If the child does not react properly after the cool douche, it should be discontinued. Young children, before being clothed, may be powdered in all the folds of the body with some bland dusting powder to avoid irritations of the skin to which they are peculiarly liable; if the diapers are not thoroughly clean and dry when they are put on, if the soap used in the bath and laundry is harsh, or if the little one's gastrointestinal system is deranged, the likelihood of these irritations is always imminent and their results are sometimes serious.

The principal business of a new-born baby is to nurse and sleep. In the period immediately after birth he should devote nearly nine-tenths of the day to the latter, and every disturbing factor must be removed. The best position for sleep is lying somewhat on the side, and he should be changed from right to left and left to right in order to avoid a one-sided pressure. $\mathrm{He}$ should, as far as possible, sleep in the dark, the room must be reasonably quiet, thoroughly well ventilated, not draughty, and of a reasonably large size. The night-gown may be made of some light, fine woollen stuff, and after the first months, and in cool weather should be of the so-called union form with the legs continued so as to form stockings. The coverings should be warm but light blankets, and for very young or delicate children a hot water bag placed at the feet is a desirable precaution against chilling. With increasing age less time will be passed in sleep; in the second three months he will be awake about six or seven hours in the twenty-four, in the third from seven to eight, and at one year he will sleep about eleven or twelve hours at night and two during the day. The daily nap, beginning at noon, should be insisted on, until the child is four or five years of age.

Good habits of sleep are easily inculcated provided there is some definite system observed. Artificial "comforters," rubber 
nipples and similar articles for the child to suck on should not be used; singing and rocking the baby to sleep are no more than useless forms of infantile tyranny for which there is rarely any excuse. If the child is not sick, if he is nursed regularly during the day, being roused from a nap whenever the fixed nursing time comes, it will not be a difficult matter to put him asleep promptly. But there is a period when sleep must be insisted upon, when no nursing should be allowed, when quiet must reign in the house not only for the child's sake but also for the mother's. This time is from midnight to six in the morning during the first two months, eleven to six during the next two, ten to six during the fifth and six months and from ten to seven in the succeeding period.

An infant who is well trained in habits of sleep is not only healthier and more amiable than he otherwise would be, but also he is more amenable to other forms of discipline. One of the most important of these is the early formation of habits of cleanliness. He can be taught to control urination and defæcation at a surprisingly early age, within a few months commonly, with the natural result of decreasing the work of caring for him, of promoting the efficient action of the gastro-intestinal track and lessening the liability of skin diseases. It is no difficult matter to place him on the chamber vessel at regular intervals and support him there for a reasonable time; and within a few days or weeks one will undoubtedly begin to see the salutary effects of the procedure.

Outside of the exercise which an infant derives from the unrestrained movements of arms and legs, a very important matter is the regularity as well as the sufficiency of being carried into the open air. In warm weather this may be begun within a week or ten days after birth. In winter it is usual to wait until he is a month old; but the delay should not be longer unless he is premature, extremely weak, or the weather is particularly rough and inclement. Clear cold weather is no bar, for he can easily be made and kept so comfortably warm that it will act as an exhilarating tonic. The length of the airing should be increased from a few minutes at the first outing until, weather permitting, hours are to be spent out of doors. He may be allowed to sleep during these outings whether the temperature is high or low.

The nursery should be one of the largest, lightest, and airiest 
rooms in the house, preferably one with a southern exposure. Its furniture should be of wood and not textile-covered. There should be no heavy curtains or portières, and its walls should be painted or covered with a glazed paper which permits washing. The best sort of floor is of hard or painted wood covered in part by rugs that are not too large to permit of frequent removals and cleansing. Provision for thorough and constant ventilation, excepting in the very coldest weather, should be made in the windows without allowing the formation of draughts. The best method of heating is a coal or wood fire which at the same time insures a fair exchange of air.

One of the most important matters in the care of a child is the selection of a nurse; and the physician can do the child and the family no greater service than to secure the engagement of an intelligent and faithful person. The usual custom of engaging an uninformed and careless peasant, whose habits, lack of discipline, and general inaptitude smack of the fields and the hovel, is most illogical and mischievous. No woman would think of putting in the hands of such a maid the full custody of jewels, fine ornaments, clothing, or furniture; but the responsibility of a child is evidently thought to be of less importance. To the thinking mind the elaboration of such an error is unnecessary. It is likewise unnecessary to demonstrate that more advantage can be obtained from being lavish in the way of hiring the best and most intelligent attendants for a child than from putting an equal outlay on the child's clothes and toys.

A final recommendation should be made to inspect and cleanse the male genital organs, especially the glans penis. If adhesions exist they must be broken up, and under no circumstances ought smegma be allowed to accumulate. If the foreskin is too long and too tight, it should be amputated.

The whole care of an infant calls for the exercise of thoughtfulness, common-sense, and the exercise of those well-known principles of sanitation which constitute the foundation of health. There is nothing peculiarly mysterious about it, nothing that cannot be logically deduced from the recognition of plain facts which an observing eye can see without difficulty. 


\section{CHAPTER III}

\section{METHOD OF EXAMINATION AND DIAGNOSTIC SUGGESTIONS}

\section{Therapeutic Suggestions}

THE physician on entering the sick-room must remember that the patient is very young, lacking in self-control, irritated, and exhausted by illness and pain, easily alarmed, and even more irrational than adult patients. He must try to avoid any clash, any abruptness or harshness, any appearance of severity or impatience. It is often the best plan to begin talking with the mother or nurse, to sit for a few moments without noticing the child at all, in order to give the easily alarmed little mind an opportunity to become familiar with the stranger. Then the story of the sickness should be elicited from the mother as thoroughly as possible: how it began, the order of development of the symptoms, the previous history of the child from birth, the existence of similar cases in the family or neighborhood, the tendency of the family toward one disease or another, and finally, the full story of the child's present condition, including an account of the methods of feeding, dressing, sleeping, and exercise. By this time the child in all likelihood will have become used to the stranger's presence, and the physical examination may then be made. The patient should be entirely undressed and his body narrowly inspected. The amount of information to be obtained depends upon the physician's power of observation. No rules can entirely govern this, for they cannot create a keen eye, nor an experienced faculty of interrogation ; nevertheless, a systematized method is always useful, and gives definite instead of desultory results. The posture will first engage the attention, and the condition of the joints, back, chest, and extremities must be noticed. One must examine the skin, its hue, whether it is dry and hot, or wet with perspiration, the circulation in the extremities, the color of the conjunctivæ, the condition of the cornea and pupil, the facial expression and its character, whether it denotes apathy, restlessness, or pain. Fright 
is what one most commonly sees, and it is in sharp contrast to the apathy of marked prostration or mental disease. An expression of pain will commonly be seen in examining a case of pleurisy, rheumatism, scurvy, rickets, injury, and general hyperæesthesia in meningitis. Mention should be made of the general tenderness that so frequently comes with auto-infections from the gastrointestinal canal.

The respiration may show various conditions: it may be slow or rapid, shallow or dyspnœic (on inspiration or expiration, or both), regular or irregular; it may be impeded by disease of the nose, by hypertrophy of the pharyngeal tonsil, by disease of the throat, larynx, and lungs. The observation of the pulse is always important, and its character is more noteworthy than its rapidity. One should notice its rate, regularity, and tension. The slow and irregular rate of meningitis is sufficiently peculiar to deserve mention. In this connection one may mention the item of temperature. In infants the thermometer should always be inserted in the rectum, thereby often obtaining a reading that is slightly more than $37^{\circ} \mathrm{C}$. $\left(98.6^{\circ} \mathrm{F}\right.$.). In very young infants the normal mark may be as high as $37.5^{\circ} \mathrm{C}$. $\left(99.5^{\circ} \mathrm{F}\right.$.), and in conditions of prostration a subnormal temperature is of frequent occurrence. As a general rule, however, pathological conditions in children give higher temperatures than in adults, the exceptions - as in enteric fever - being very few. Indeed, it is no unusual thing to encounter very high ranges from seemingly trivial causes; thus a simple pharyngitis, rhinitis, or a slight functional disorder of the stomach may be associated with a really great production of fever. Doubtless the marked susceptibility to low grades of intoxication due to bacterial products may be the responsible agent.

The cry may at times be partially diagnostic. That produced by temper is much less often seen than common belief implies. In young infants it is practically non-existent, and in older children it is, as a rule, provoked and encouraged by an unreasoning and flagging discipline. A child that is constantly petted, coaxed, and cajoled, cannot be expected to have a good disposition; and if he has, it is in spite not on account of such treatment. If he has been accustomed to rocking, to sucking on an empty rubber nipple, to being trotted up and down, to the exaggerated grimaces of the attendants which are designed to amuse him, he naturally 
will demand the continuance of such attentions in an increasing variety. When he cries, it will be violently, in a prolonged fashion, with a throwing out of the extremities, stiffening of the body, and sometimes holding of the breath. The cry of hunger is a sharp, fretful cry, often accompanied by sucking of the fingers and an inconsiderable rise of temperature. It is stilled by feeding. The cry of gastric derangement is fretful, irregular, complaining; it becomes quiet when the child is fed, but soon returns. It is accompanied by other signs of impeded function that makes a diagnosis comparatively simple.

A child with colic and abdominal disorders gives a sharp, loud, and frightened cry, that often may be described as a scream; it comes in gusts and paroxysms, permitting him to sleep only for short and scattered intervals. When the pain comes on, he clenches his fists, draws up the legs, hardens the belly, and the face often assumes a strained, bluish appearance. The cry due to pain, such as that caused by a pin, a scratch, a tight band, or offensive button, varies with the severity of the injury and the persistence of the cause. When the baby is exhausted his voice becomes weak, mildly complaining, whimpering. In otitis the cry is sharp, shrill, and cutting, and is commonly accompanied by a motion of the hand to the affected side of the head. In syphilis it is a snuffling, hoarse sound: in pneumonia it is quick and short, suggesting the sound of choking, and is associated with the rapid, impeded respiration ; in chronic tubercular meningitis, we hear the keen nocturnal note, and in marasmus there is a low, exhausted, attenuated moan or whine. Inspection will be completed when we have examined the nose, head, genital organs, glands, and finally the throat.

Auscultation of the chest is an exceedingly important factor. After noting the appearance, symmetry, and development of the chest, the lungs may be examined with a stethoscope rather than the naked ear. The posterior surface of the chest, on account of the closer proximity of the lungs, will give the plainer sounds. The so-called puerile breathing is harsher and louder than the adult form, partly on account of the comparatively large amount of bronchial surface, partly because the chest walls are thin; this is especially apparent between the scapulæ and under the clavicles. The small size of the air vesicles is apt to make a comparatively slight congestion give the same interference with the 
respiration that consolidation does in the adult. And when one portion is affected there is always a strong likelihood of some degree of compensatory emphysema in a neighboring part. Another frequent source of error is the liability to pass over a small area of consolidation whose presence may be masked by the existence of such a transitory patch of emphysema as that referred to. Percussion shows some peculiarities in the way of a crackedpot sound over a normal but superficial bronchus, the hyper-resonant note over comparatively unaffected portions of lung tissue, and the ease with which the flatness due to a small amount of fluid may be overlooked. Fremitus due to bronchial irritation is very easily detected.

In auscultating the heart, one should keep in mind the comparatively large size of the organ in infancy, and its liability to slight irregularities of action. The murmurs in children under two and two and a half years of age are almost always congenital in origin, and the hæmic murmurs are much more likely of occurrence in young children than those of organic disease.

In the abdomen one notices the possible existence of fluid, tympanites, enlarged glands, local or general tenderness, and the retraction of the walls which occur in meningitis. The spleen is not easily made out by the palpating fingers unless it is enlarged, as in typhoid fever, malaria, or tuberculosis, syphilis, anæmia, and leukæmia. The liver, or rather its lower border, is much more readily distinguished below the free margin of the ribs, and any enlargement is more apt to deceive by its large than its small size.

The possibility of involvement of the kidneys, especially as a complicating feature in the acute infectious fevers, is much greater than is usually thought; and if proper attention is given to them the recoveries from these disorders will be quicker and more uneventful than otherwise. It is therefore especially desirable to examine the urine as a routine procedure and with sufficient frequency. In infants there may be some difficulty in collecting it without an admixture of fæces. This can be avoided in the male by tying a condom over the head of the penis, and in the female by fastening a small cup or similar receptacle over the vulva and inside the napkin. Concentrated urine and crystalline uric acid produce brownish yellow stains on the diaper.

Young children excrete a larger quantity of urine, comparatively, than do adults. This is partly due to the character of 
their food, and in part to their active tissue-change. Many authors have given tables of approximate amounts per day, and the average of these, as stated by Holt, are the following : -

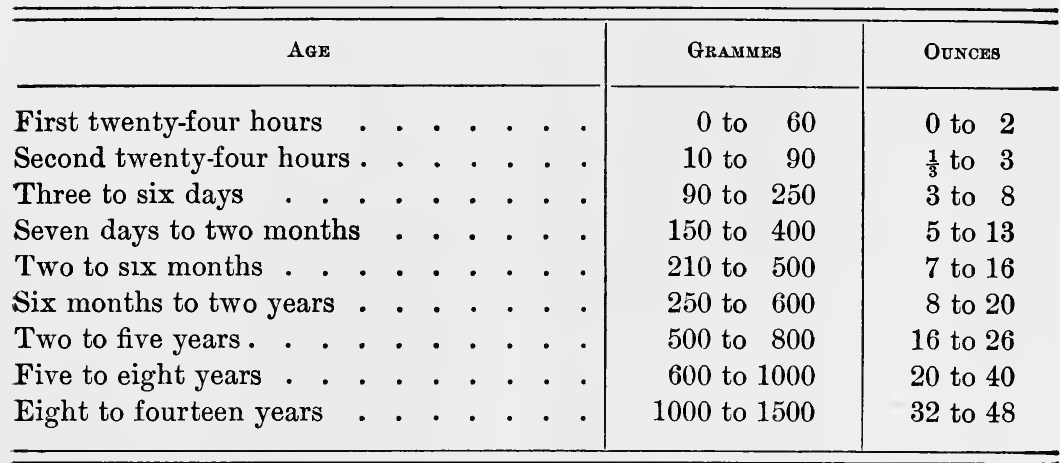

In the first period of life it has in some cases characteristics which are noteworthy. The proportion of uric acid is higher than at any other period during a state of health, its specific gravity is low, the acidity is marked, it sometimes has small amounts of sugar, albumin, hyalin, and granular casts. After the passage of a few months, these seemingly abnormal characters gradually fade away.

\section{GLycosuria}

In children who are being wrongly fed, especially if their food contains too much sugar, the urine may contain a sugar derivative that is readily detected by Fehling's test. A somewhat similar phenomenon has shown itself after attacks of acute sickness. The pathological significance is not great, for with the regulation of the diet and general health, the urine becomes normal.

\section{ANURIA}

In infants who are quite healthy there may be a sudden and seemingly causeless arrest of urinary excretion. The cause is supposed to be nervous and has never been satisfactorily explained. It may continue for a half day, a day, or even longer. It is removed by warm baths, by the drinking of fairly large amounts of water, and by the application of heat to the lumbar region: It is rarely, if ever, necessary to prescribe the ordinary medicinal diuretics. 


\section{Polyuria}

An opposite condition is seen, in rare cases, among older children. Its cause has never been ascertained; but the noteworthy observation has been made that in many instances it follows injury or disease of the brain and spinal cord, and may occur in the convalescence of the acute fevers. The main symptoms are polyuria and polydipsia, nervous and vaso-motor disturbances, and at times some degree of malnutrition, anæmia, and disordered development.

The treatment is directed toward the improvement of the general health and strength by means of a carefully regulated diet, mode of life, exercise, clothing, and rest. General tonics are to be administered, and the amount of fluid consumed is to be restricted to a reasonable amount.

The condition is easily distinguished from diabetes mellitus by the lack of sugar in the urine. While it is not so dangerous as the latter disease, it nevertheless in many cases is incurable and sometimes has a fatal termination.

Indican is occasionally present in the urine when the patient is suffering from intestinal putrefaction and the lack of proteid digestion. It is thus seen in acute and chronic derangements of the intestines and the conditions with which such derangements are concomitant factors, as in chronic constipation, convalescence after the acute fevers, epilepsy, and convulsions.

Acetone and diacetic acid have been found in large amounts in the urine of children who were suffering from the acute diseases with high fever, and in certain nervous conditions. ${ }^{1}$ They are formed by disturbed metabolism and disappear when the ordinary healthy condition returns. Comparatively little is known about them, and the amount of our information does not justify elaboration of the subject. Small quantities of both indican and acetone have been found in the urine of healthy children.

Hæmaturia, pyuria, lithuria, and functional albuminuria occur in children but rarely ; and when they do, the causes and manifestations are the same as in adults. Thus, one may occasionally see a post-febrile albuminuria which generally fades away on the reappearance of health. Hæmoglobinuria is described in another place.

1 For fuller statements concerning these conditions the reader is referred to the researches of Herter, of New York. 


\section{Therapeutic Suggestions}

The practitioner should always keep the fact in mind that children, while they easily fall into abnormal and pathological conditions, react with comparative ease and rapidity to drugs, efficient nursing, and advantageous environment. He must, accordingly, be exceedingly careful about every item of treatment, must make his orders as simple and direct as possible, and must never allow himself the privilege of prescribing measures whose efficiency has not been fairly well demonstrated by experience or reason. There is little excuse for the "shot-gun" prescription in the treatment of adults, but there is absolutely none in that of children.

One of the main indications in the care of these little patients is the promotion of excretory functions. If the attendant will see to it that the gastro-intestinal canal, the urinary system, the glandular system, and the skin are in active and tonic state of efficiency, he will have accomplished a considerable measure of the help that we can give toward eliminating disease. This measure is yet further increased when the food has been so selected and modified that excretion, digestion, and assimilation are reasonably and actively conserved. With these items attended to, the amount of prescribing of drugs is remarkably small. Of the medicines that remain, the principal part is composed of tonics, in the discriminating use of which the physician has ample opportunity to show judgment and resourcefulness.

There are some drugs that should rarely be prescribed for children. The main ones are opium and its derivatives, the coaltar antipyretics, and the more potent depressants. Naturally enough there are circumstances where they must be used; but the quantities must always be as small as will do the desired work, and at the same time one should try as far as possible to counteract their possibly bad effect. For instance, it may be necessary to prescribe fairly large doses of antipyrin in whooping cough, but a heart stimulant must, at the same time, be given in order to avoid collapse; opium may, in rare cases of great pain, as, for instance, in acute rheumatism or peritonitis, be required; then it may be prescribed in the form of deodorated tincture, in Dover's powder, or in the alkaloid, morphine. Under all circumstances, however, one must remember that it disorders the gastro- 
intestinal track, that it temporarily abolishes secretions, and that in many cases its use will prolong the original disease, or delay convalescence. In the cough mixtures for the older children, its place is often well taken by codeine, whose bad effects are much less pronounced. In the younger ones the use of bromide of soda is always much safer. Of all the bromides this one seems to be most easily borne, it has the least irritating effect upon the kidneys and heart, and the doses may with safety be made fairly large.

Of the other antispasmodics, belladonna will be found to be reasonably safe; and in some cases it is not only efficacious, but. also can be tolerated in remarkably large doses. This is especially noticeable in the functional enuresis of children, where its value becomes apparent only when the amount prescribed is large. A slightly related drug that is comparatively well borne is chloral, which is commonly given by rectum in order to avoid the irritation which it so often produces on the stomach.

In the last few years the use of antipyrin in the convulsive or spasmodic diseases of children has been increasing. At first the step was taken with hesitancy, but cautiousness has now given way to a bolder confidence, for remarkably few cases of its ill effects have come to notice. I have used this drug for the past four years in a wide variety of circumstances, and in comparatively large doses, but always with a cardiac stimulant. It has so often given me satisfaction that I take pleasure in stating that I have never had a case of collapse which I could attribute to it.

In prescribing for a child one cannot be guided exactly by his age; and for this reason it is unwise to give or follow any hard and fast scheme of fractional amounts of the adult dose. In children much more than in adults it is necessary to reckon with the size, weight, susceptibility, and vigor of the patient; and therefore each one must receive his special doses. This cannot possibly be laid down in a book; but the practitioner, by a careful knowledge of the means at his command and an equally careful summing up of the physical factors of his patient, will be able, at the expense of not much trouble, to settle the matter of each individual case.

'To summarize, we may say that children do not bear well such drugs as opium, the inorganic preparations of iron, most of the coal-tar products, the corrosive acids, large doses of quinine, 
cocaine, iodoform, alcohol in large doses, and the salicylates. They tolerate well such preparations as the various salts of mercury, the bromides, especially the bromide of sodium, the iodides, moderate doses of chloral, and fairly large doses of antipyrin. Concerning alcohol there is a wide difference of opinion; one is safe, however, in prescribing it during times of pyrexia in small although frequent doses.

There are two methods of external treatment that may well be eliminated: blisters and poultices. The first is altogether too irritating, and often will produce a disorder that is worse than the one which it is intended to help. Its place is well taken by the simple, clean, and cheap mustard leaves that are sold in every drug shop. These leaves are usually superior to the mustard poultices or pastes that formerly were in such extensive use. Poultices likewise may be advantageously abolished, for they are apt to do harm by their weight, their sogginess, their lack of asepsis, and the ease with which they are chilled. In place of them one may use aseptic or antiseptic moist dressings made of clean gauze or similar material, wrung out of hot water, boracic solutions, or turpentine mixtures, and covered by rubber tissue. This method is clean, not troublesome, retains the heat, and is easily prepared.

The use of hydrotherapy is especially to be commended for children. And every physician who has occasion to treat them should make himself thoroughly familiar with the various methods employed. 


\section{CHAPTER IV}

\section{CONGENITAL MALFORMATIONS AND DEFORMITIES}

\section{The BraIN}

Crclopia is the name given to that condition of the brain in which there is no separation into two hemispheres. With it are other deformities, such as a single ventricle and usually a single eye. This eye is located at that part of the face where the root of the orbit ought to be. In other cases there may be two eyes, joined or separate, or no eye at all. There may be two, one, or no optic nerve. Various parts of the bones about the nose or orbits may be lacking, or only present in part.

Anencephalia denotes a partial or total lack of brain. In some cases there is likewise acrania, or absence of the skull bones excepting those at the base. The scalp may completely or only partially cover the open space.

Cephalocele, or hernia of the brain, designates a protrusion of a part of the brain, covered with membranes, through an abnormal opening of the skull. The brain may otherwise be normal or in the condition of anencephalia. If the sac consists of membranes alone, it is called meningocele ; if it is filled with fluid, it is called hydromeningocele; if it contains both brain substance and fluid, it is called hydrencephalocele. Such sacs may be of various sizes and in various situations.

Microcephalia denotes a small-sized brain in a small skull. The condition may sometimes occur from inflammation and disease of the fotal brain. In some cases parts of the brain may be absent, but the mental condition good. In other cases the whole brain is small and rudimentary, the least development being in the cerebral hemispheres. The convolutions may be rudimentary and the cavities filled with serum.

Porencephalus is the name of a deformity which consists of a circumscribed and sharply defined absence of tissue in the brain. The hole may be very small or large, shallow or deep. It occurs 
congenitally, or may be acquired; in the latter case it commonly results from meningeal hemorrhage.

\section{The Spinal Cord}

The spinal cord may be entirely absent, a condition called amyelia; such cases commonly occur in conjunction with anencephalia. When the cord does not exist at all its place may be taken by a cord or sac of connective tissue which may contain fluid. In other cases it may exist in part (atelomyelia), in still others it may be longer than the normal. Very rarely it is double (diplomyelia), either in parts or through its whole length. An abnormal position of gray matter may exist, and constitutes what is known as heteropia.

Hydromyelia is a condition of the cord in which the central canal is dilated by an unusually large amount of fluid. The force of gravity, by regulating the amount of pressure, decides the degree of dilatation and at the same time the intensity of the atrophic process. Hydromyelia externa, or hydrorrachis externa, is a variant of the form above in which the fluid is contained between the spinal meninges; the logical result is an atrophy of the cord due to pressure.

Spina bifida, or hydromyelocele, is a hydromyelia plus a smaller or greater opening of the posterior aspect of the spinal canal; there may be an anterior opening, and the condition would then be called spina bifida occulta - a very rare deformity. Of hydromyelocele there are three varieties which we commonly recognize : meningocele, meningo-myelocele, and syringo-myelocele.

In meningocele there is usually a small aperture leading to the central canal, often situated in the cervical region. The tumor consists of membranes filled with fluid, and may be of variable size. It is covered with a strong, normal skin, and on account of its protection as well as its simple composition offers a good chance of life or even recovery.

Meningo-myelocele is a commoner and more dangerous form, its greater danger lying in the fact that instead of being entirely covered by a strong skin, its centre is protected by nothing more than a thin membrane, to which, or to a near-by part, the cord is attached. The fluid is derived from the anterior arachnoid or subarachnoid space, and produces a moderate-sized sessile tumor 
that is oftenest situated in the lumbar or sacral regions. The thin and easily irritated membrane at the centre of its surface makes the danger of rupture, infection, and inflammation a constant and serious menace, and the presence of the cord in the sac renders operation exceedingly grave.

Syringo-myelocele is the rarest and most deadly form of spina bifida. The tumor is usually in the lower part of the dorsal or the upper part of the lumbar region. The fluid is derived from the central space of the cord, and communicates with the lateral ventricles of the brain. The sac is lined with the crushed and degenerated spinal substance, and offers a direct and unimpeded way to the other parts of the cord and brain. This form commonly occurs in association with hydrocephatus; and with all varieties there may be deformities of the extremities, trunk, and face.

The symptoms in spina bifida vary according to the situation and composition of the tumor. In meningocele they may be very few or none, and treatment may bring about a cure. In meningomyelocele and syringo-myelocele there will be some form of paralysis and focal symptoms that vary with the portions of the cord which are involved. The weaker the covering of the sac is, the greater is the danger of irritation, rupture, draining away of cerebro-spinal fluid, and infection of the cord and brain.

The treatment is purely surgical and should not be conducted by the general practitioner. From the very beginning the tumor must be protected by a pneumatic rubber ring from pressure, irritation, and blows, thereby avoiding the dangers of rupture and infection. The older methods of aspiration, with or without injection of fluids which promote an adhesive inflammation, are gradually giving way to radical operations which seek to remove the defect in a logical and scientific manner. The meningocele gives the best hopes for recovery, the syringo-myelocele the poorest.

\section{The Larynx and Trachea}

Both larynx and trachea may be wanting in acephalic beings, or in other cases they may be unnaturally small or large. In still other instances there is an opening between the trachea and the œsophagus. In some children one or more of the cartilages may be small or absent, the epiglottis in especial being subject to vari- 
ations according to which it is too large or too small. Or the trachea may have too small or too large a number of rings, or may divide into three instead of two bronchi; in the latter case, two lead off to the right and one to the left lung. There may be faulty development of the branchial arches, with the resulting production of fistulæ which communicate with the trachea or pharynx. In some instances the trachea instead of being in front of the œsophagus has been found behind or to the left side of it.

\section{The Lungs}

Cases of partial or complete absence of one or both lungs have been reported. A deformity, which is tantamount to partial absence, has occurred : it consists of a vicious development of pulmonary tissue into small bags or sacs which contain air and serum. These sacs communicate with the bronchi. In other cases the lobes may be subdivided; and a case of supernumerary lobe has - been reported. Transposition of the lungs, with similar relational changes of the heart and abdominal viscera, may occur. And, finally, in the absence of a part of the chest wall, a hernia of the lung may occur.

\section{The Heart}

Monsters have been born in whom there was no heart at all, but they are very rare. Cases of two hearts in one body have been known; the instances of a large-sized heart from obstructive malformations of the great vessels are more frequent. Also, a very small-sized heart may occur, usually associated with small and thin arteries ; such anomalies generally appear in anæmic and hæmorrhagic patients. Unnatural shapes, relations between the different parts, and positions have been noted; one side, or a part thereof, may be over-developed, while the other is hypoplastic; the heart may be transposed from the left to the right side with corresponding changes in its relations; in the absence of a part of the chest wall the heart may project outside and then usually lacks its pericardium. Or the diaphragm may be partly or wholly absent, so that the heart lies in the abdominal cavity. In badly deformed monsters the heart may be in the neck or even in the head.

One or more parts of the heart or its great vessels may be malformed or deficient in various ways. The trunk, from which the 
aorta and pulmonary artery usually spring, may be so developed that the division into these two branches exists only partially or not at all. At the same time the septa between any two of the four cavities may be deficient: thus there may be one ventricle and two auricles, or one ventricle and one auricle, or one ventricle and no auricle at all.

In other cases the trunk of the aorta or pulmonary artery is absent, obliterated, or stenosed. As a result, the septa between the cavities and the course of the blood current are abnormal. Thus the aorta at any part of its course is obliterated or very much narrowed. Then the pulmonary artery branches off from the descending aorta, supplying the subclavian and carotid arteries. To complete the circulation, the foramen ovale is patent or there is no inter-auricular septum ; this condition may be supplemented by an imperfect or absent inter-ventricular septum, and finally the force of the blood stream results in hypertrophy of the right ventricle. In other instances, the pulmonary artery, instead of the aorta, is obliterated, defective, or stenotic. Its branches then are supplied through the ductus arteriosus by the aorta. The inter-ventricular or inter-auricular septum is imperfect and the foramen ovale is patent.

There is another class where the malformations are farther removed from the heart. Thus, the aorta may be very small and narrow at the cluctus arteriosus, or between that point and the left subclavian, or all the branches of the aorta may spring from the arch ; then the descending aorta would be continued from the pulmonary artery. Other abnormalities are transpositions of the arteries and veins, with or without imperfections of the septa.

A commoner set of malformations consists of deficiencies of the septa alone. The foramen ovale, or the ductus arteriosus may be patent or there may be an aperture in the ventricular septum. Or one of the auriculo-ventricular apertures may be closed while communication may be kept up through the foramen ovale, and an imperfect septum between the ventricles.

Another interesting condition consists where one or more of the cardiac openings are defective or do not exist. Or the valves of the aortic or pulmonary orifices may be changed from three to two or four, or the semilunar valves are fenestrated.

The most general symptoms of such organic malformations of the heart as permit life are cyanosis induced by interference with 
pulmonary circulation, cardiac murmurs, clubbing of the distal phalanges of the fingers, dyspnœa, episaxis, hæmoptysis and œdema of the feet and dropsy of the serous cavities. In the large majority of cases the predominant signs are the conjunction of a murmur with cyanosis ; to ascertain the location of the disorder is not by any means an easy matter. There are, however, a few generalizations which may be worth mentioning, even though they do not help the treatment of the case. Thus, the common condition of a systolic murmur heard at the base and combined with cyanosis usually denotes a stenosis of the pulmonary artery with or without abnormalities of the septa. A systolic murmur heard at the base but without accompanying cyanosis commonly denotes a defective ventricular septum. A systolic murmur at the apex may be associated with a deficient auricular septum, open ductus arteriosus, and transposition of the great vessels ; a patent foramen ovale may give a presystolic and pulmonary insufficiency a diastolic murmur. On the other hand there may be aortic or pulmonary stenosis, deficient septa, transposition of the great vessels or irregular branching of them without either murmurs or cyanosis, or with cyanosis alone.

There is no specific treatment for these conditions, and all that one can do is to treat the symptoms. One must therefore try to distinguish between congenital and acquired cardiac disease. The former is characterized by its occurrence, or discovery, at or near birth, its possibly atypical character, the absence of exciting causes in the history, the harsh quality of its sound and probable location at the base. Hæmic murmurs may be distinguished by the facts of their being comparatively soft, being heard over the large vessels and in the neck; and although they are systolic and heard at the base, nevertheless are not connected with the enlarged right heart which so commonly accompanies congenital disease. There are, in addition, the appearances and history of hæmic disorders.

The outlook in congenital disease of the heart is not good; the patient may live for one or more years, but the diseased condition or intercurrent sickness is apt to cause death before childhood has passed. Pulmonary disease is especially liable under these circumstances to bring about a fatal result. But even if death does not immediately occur, the patient's vitality is not strong; and, as a rule, the problem of caring for these cases in an efficient manner is complex. 


\section{The Mouth}

The congenital malformations of the mouth are the result of arrested development; all the parts may be present but in an abnormal condition, or many of them may be absent. Thus the whole lower part of the face below the temporal bones may be absent, and these bones may approach each other in the median line. Cases have occurred in which the mouth is closed from behind, and nothing remains but the opening between the lips; in others the lips are joined completely or so much that only a small round aperture exists. In some instances there is a large irregular cavity which inclucles the location and openings of the mouth and nose and even the orbits as well. Minor malformations may exist, such as imperfectly formed lips, prolongation of the mouth so that it extends almost to the ears, cleft lower lip and undersized lower or upper jaw. The commonest deformity is the so-called hare-lip, a cleft in the upper lip situated under the middle of the nostril, which may exist on one or both sides; it may be so slight as to form no more than an indentation, or so marked as to divide the lip completely. It is commonly accompanied by single or double cleft palate. This consists in a fissure of various size in the soft or hard palate. The soft palate is more frequently so affected than the hard, and the resulting deformity is naturally less serious. With both hare-lip and cleft palate the child may be unable to nurse in a normal manner, and requires feeding with a spoon or a large medicine dropper. These children may give much trouble in the attempt to maintain their nutrition, and on account of their feeble vitality contract many intercurrent diseases that have a more serious prognosis than in perfectly formed children. The deformity of the lip should be corrected as soon as the physical condition of the child permits, while that of the palate should usually be postponed until the age of two or three years.

The tongue may be deformed in the way of a hypoplasia or hyperplasia, a true microglossia or macroglossia; and rare instances of bifid tongue have been reported. The anterior part may be entirely absent, or the whole tongue may be adherent at the bottom, sides, or top to corresponding parts of the mouth. The frenum of the tongue may be so much shortened that full movement and functional activity of the organ may be impeded. In other cases it may extend to the tip of the tongue. 
The deformity is easily rectified by snipping the membrane with a scissors. Another slight and unimportant malformation is bifid uvula. I have noticed in a number of cases the occurrence of this condition in the mother or father as well as in the child. No treatment is required.

\section{The Esophagus}

The whole organ may be absent or may exist only in the lower half, the upper part being replaced by a fibrous cord. In some cases it may be irregularly dilated, may have diverticula, or may be divided in its upper half into two parts.

\section{STOMACH}

In acephalous monsters the stomach may be entirely absent. More often it may be very small or very large, and it has been known to possess a median constriction that practically divides it into two parts. More commonly the pyloric orifice is much decreased in size, and it has been known to be closed. The whole viscus may be forced through a diaphragmatic hernia, or on account of absence of the thoracic wall may be on the exterior of the body. It may be transposed in all its relations in those cases where general visceral transportation exists. It has been found to be adherent to some part of the small intestine, and occasionally its fœtal upright position remains unchanged.

\section{The Intestines}

The intestines may be abnormally short or long; in the small intestines there may be various degrees of stenosis, or there may be blind terminations. There may be diverticula of the intestines in various positions. The most common is where the process is attached by one end only to the convex surface of the gut or its attached border, in the latter case being attached to the mesentery by a band of peritonæum. Such a process is usually from two to ten centimetres in length, and smallest at its free extremity. Sometimes there may be a diverticulum of the ileum; which is attached by a fibrous cord to the umbilicus. Such a one may be the remains of the omphalo-mesenteric duct, and is commonly called Meckel's diverticulum. Occasionally it remains patent throughout its whole length, thus producing a fæcal fistula; at other times a part of its mucous membrane may be 
extruded and form an umbilical or diverticulum tumor. Another variety of diverticulum consists of a patency of the abdominal wall at the umbilicus, which is joined immediately to a similar opening in the ileum. The inferior parts of the ileum and colon are diminished in size or closed. A long diverticulum may, by catching a loop of intestine between its length and the abdominal wall, produce an incarceration.

The rectum or colon may be partially undeveloped; the colon may be absent in part or wholly; the rectum may be entirely absent, its lower half may be replaced by a fibrous cord or a blind pouch, or its anal extremity may be closed by a membrane. These various forms are called atresia ani.

There may be a union of the rectum with the bladder and genital organs, so that a common outlet or cloaca is formed; or the rectum may empty into the bladder, vagina, or urethra. The lower part of the rectum would then be absent. A very rare malformation consists of absence of the anterior abdominal wall, symphysis pubis, and anterior vesical wall; the posterior vesical wall then receives the openings of the ileum, ureters, and vagina.

\section{The Peritonaum}

The peritonæum may be malformed in the way of arrest or excess of development. In the former there may be longitudinal lines of fissure near the median line; if the diaphragm is absent, the peritonæum will be joined with the pleura; or the omentum or mesentery may be deficient in various areas. In other cases, there may be an excess of omentum, mesentery, or other process of the peritoneum. If bands are thus formed, they may produce an incarceration of the intestine.

\section{The Liver}

The liver may be absent, its form may be changed, its lobes may be increased or decreased in number. The gall-bladder or ducts may be absent. The ductus choledochus may empty into the stomach or it may be double, one tube then emptying into the stomach and the other into the duodenum or both into the duodenum. The liver may be transposed to the left side or, on account of a diaphragmatic hernia, may lie in the thoracic cavity; absence of the abdominal wall may cause it to be on the exterior of the body. 


\section{The SpleEN}

The spleen may be absent, may be increased or decreased in size. It may be double, there may be small accessory spleens or it may be divided into lobes. In cases of transposition it may be found on the right side, in the thorax when there is a diaphragmatic hernia, or outside the body when the anterior abdominal wall is lacking.

\section{Pancreas}

The organ may be wanting in monsters and in rare cases of marked umbilical hernia. Its duct may open into the stomach or the biliary duct, and sometimes it may be double. The head may be separated from the body of the organ and have its own separate duct. An accessory pancreas has been reported.

\section{The Suprarenal Capsules}

These organs may be absent in monsters; there are, in addition seemingly normal cases of development in which they have not been found. Occasionally accessory bodies have been seen which grew from the surface of the organ and differed in no way from it in structural composition. The suprarenal capsule may be present and in correct position even if the kidney is absent.

\section{The Kinneys}

Both kidneys may be absent in monstrosities. One is lacking in a comparatively large number of cases; under such circumstances, the remaining organ is larger than the normal. There may be no ureter on the affected side or the kidney may be replaced by a small growth of fat and connective tissue from which a ureter leads to the bladder. Minor malformations may exist, such as disparity in the sizes of the two kidneys, double pelves or double ureters. In other cases the two organs may be somewhat more curved than usual and their extremities connected by a commissure of fibrous or characteristic kidney tissue, the whole structure imitating the shape of a horseshoe; this peculiarity may be present at the top or the bottom. The structures may otherwise be normal or their ureters and vessels may be abnormally arranged. Rare cases of blended kidney have been reported. The two organs may be bound together with a regular or irregular arrangement of the parts. They may lie on one side of the spinal column or in the pelvis. 


\section{The Urinary Bladder}

The bladder has been found lacking in a few cases; oftener it is much smaller than the normal, and occasionally it is constricted in its middle horizontal plane, or divided in its middle vertical plane by a membrane. Sometimes the urachus remains patent if the urethra is congenitally occluded. In other cases the neck of the bladder is closed, or retention of small amounts of urine may occur on account of the existence of diverticula of the vesical wall.

In a few instances the abdominal wall below the umbilicus has been absent, the whole pelvis is then lower and broader than the normal. In the opening the posterior wall of the bladder with the mouths of the ureters may be seen. In such circumstances the genital organs are likewise malformed. In other deformities the bladder itself is normal, but is easily seen and touched on account of an opening in the abdominal wall. In yet other cases, there may be an opening in the bladder while all other organs are normal.

\section{The UrethrA}

The urethra may be closed at any part of its length, the distal parts then being more or less completely undeveloped. In some cases it resists pressure so poorly that it almost immediately becomes irregulately dilated; also there may be valves in the canal that cause dilatation of it and the bladder. At times the opening may be irregularly placed, at the root of the penis or on the dorsum; the meatus on account of malposition of the whole tube may be in the inguinal region, or it may be double or triple. There has been a case reported in which the course of the urethra was paralleled by a tube on the dorsum of the penis and a meatus in the glans.

\section{The Vulva}

There may be an entire absence of the external genitals in the female ; or they may be partially formed, or wholly formed but not divided. The nymphæ may be increased in size or number; the clitoris may be long - so much so as almost to resemble a small penis, it may be divided into two parts, or it may hold the meatus of the urethra. 
The hymen may show irregularities even when the other parts are normal. It may be entirely absent or imperforate. Instead of one perforation it may have several, or the perforation may be eccentrically situated. In other cases the free margin may be fringed or formed into small and irregular processes.

\section{The Vagina}

This organ may be entirely absent, especially when the other genital organs are wanting; not so rarely it is present only in part, the absent portion being sometimes replaced by a fibrous cord. In other cases it may be remarkably small and so remain throughout life. The introitus may be occluded by an imperforate hymen or by a similar membrane in any part of its course.

\section{The Uterus}

The uterus may be wanting, with or without the absence of one or more of the genital parts. It may be abnormally large or abnormally small; and more frequently than is usually supposed it has abnormal flexions at birth. I have seen a few cases where the malposition was so marked as to be comparable to those disorders in the adult that are considered worthy of correction by some form of operative treatment.

Most malformations of this organ represent some manner of persistence of the embryonal division into two cornua. Sometimes these horns make up the whole uterus of the new-born child, sometimes they are connected only at the cervix so that there are two uteri; in other cases the cervix and adjacent part of the uterine body are normal, but the upper portion of the body branches out into two horns; a still other deformity is where only one of the original horns has developed naturally, so that the uterus is normal on one side, but on the other is flattened and without its tube. In some cases the uterus is of normal size and shape on the exterior, but the interior is divided by a complete or incomplete septum into two long canals. A very rare malformation is that of two wholly separate uteri; in such an event there might be separate cervices or a fused cervix and a single or double vagina.

\section{The Ovaries}

The ovaries may be absent when the uterus and tubes are likewise lacking; or one may be wanting in children who are 
otherwise normal. In other cases one or both may be abnormally small or only partially developed. Malpositions may occur even when the organs themselves are quite natural: thus they may be prolapsed into the inguinal canal or the labia majora; they have also been found in the foramen ovale or crural canal.

\section{The Fallopian Tubes}

Both tubes may be wanting when the uterus is lacking; or with an imperfect development of one side of the uterus the corresponding tube may be no more than partially elaborated. In other cases both tubes are only partially developed, especially if the uterus is more or less rudimentary. Either or both extremities may be occluded, or the uterine junctions may be unnaturally situated. Sometimes the abclominal ends may be divided into two or more parts.

\section{The Mamma}

One or both manmæ may be indeveloped or absent (amazia); or the nipples may be absent (athelia). These irregularities rarely occur alone, and almost always in connection with malformations of the thorax or genital organs. In other cases there may be supernumerary mammæ (polymazia) or nipples (polythelia) or both; the extra organs are often potentially active.

\section{The Penis}

The whole organ may be absent, or it may be rudimentary or the prepuce alone may be deficient. Where there is marked insufficiency of the penis, there are usually irregularities of other genital parts, especially the testicles. When it is wanting, the urethra is apt to open into the rectum. Partial malformations, such as hypospadias and epispadias, are not very rare. In the former there is an imperfect development of the penis, which commonly is small, with a mucous-lined furrow on the inferior aspect. Near the root of the penis the urethra opens upon this furrow. The testicles may descend or remain in the abdomen, and the scrotum generally fails to unite in the middle, the two parts being attached in the manner of labia majora. In epispadias the urethra opens on the dorsum of the penis at one point or another between the root and the head.

By some unexplained freak of development, the male and 
female elements may be partly combined in one child, forming the condition known as true hermaphroditism. In the so-called lateral form there is hypospadias, a testicle and spermatic cord on one side, and on the other a vagina, uterus, ovary, and tube. In the bilateral variety there is an ovary on one side and a testicle on the other. In pseudo-hermaphroditism there is a variation in the normal development of the fœtal parts so that the resulting formation is atypical. There may be various grades in which the different parts may be almost entirely of one sex, or may be divided between the two.

\section{The Testicles}

One or both testicles may be absent without necessarily involving the absence of epididymes, seminal vesicles, and spermatic cords. The connection between these various parts does not seem close enough to predicate from the presence or absence of one the presence or absence of any other one or all of them. In some cases the testicles may be undeveloped, but the epididymes may be in a normal state in the scrotum, and in others the spermatic cord may also be present.

A not infrequent abnormality consists of the retention of one or both testicles in the abdomen, called cryptorchidism. Normally these organs should descend into the scrotum about the seventh or eighth month of intra-uterine life; in some few cases they may descend at some later period up to adult age. Usually, however, they remain in the abdomen unless freed by operative interference. The causes are many, and include unusual size or partial development of the testicle, deficient gubernaculum testis, insufficiency of the vaginal process of the peritonæum, narrowness of the inguinal canal, adhesions from fœtal peritonitis, and various malpositions of the testicle. As a rule, one side only is involved, and the retained organ may be found in the inguinal canal, in the crural canal, or in the perinæum. It may remain normal or may undergo some form of degeneration.

\section{Congenital Dislocations}

Congenital dislocations are not often seen ; of those which are recognized about ninety per cent affect the hip. These are oftener seen in girls than in boys, and are located on the two sides in about an equal degree. 
In the vertebral column dislocations may occur between any two bones ; also the cranium may be luxated forward or backward.

The inferior maxilla may be dislocated forward on one or both sides.

The clavicle may be dislocated at either or both extremities.

The shoulder joint may be dislocated on either or both sides. The deformity may be subglenoid, subcoracoid, and subacromial, or subspinous. Speaking in round numbers, one may say that about fifty per cent of the cases are bilateral.

The elbow has been known to be dislocated in various ways : the whole forearm has been luxated backward, the head of the radius has been found above and behind its usual position, the head of the radius has slipped forward, and the head of the radius has been pushed upward and outward.

The dislocations at the wrist constitute "club-hand," and commonly occur in connection with club-foot. The hand may be turned inward, outward, backward, or forward. A similarly large range of deformities has been noticed in the fingers.

At the knee the dislocation may be either forward or backward. The former is by far the more frequently seen. Luxation at the ankle has likewise been reported.

All of these deformities are susceptible of a variable degree of correction, if the child has a fair amount of vitality, by means of orthopædic appliances or operation. Subluxations, likewise, are amenable to treatment, and the prognosis is better than in full dislocation.

There may be supernumerary fingers or toes of various degrees of organic development; or one or more fingers or toes may be congenitally absent. Webbed fingers and toes are a not uncommon deformity. For full details the reader should consult special works. 


\section{CHAPTER V}

\section{INJURIES AND DISEASES OF THE NEW-BORN}

\section{Caput Succedaneum}

Most new-born children have a tumor on the part of the head which has been least pressed upon and squeezed that is known as caput succedaneum. The constant pressure on the part of the scalp which is held, as if in a vise, by the bony pelvis necessarily makes a corresponding congestion in the free portion on the opposite side of the head. The tissues of the scalp and fascia become infiltrated, distended, and finally form a heavy, baggy tumor whose size is in proportion to the duration and intensity of the pressure exerted upon the head. The infiltration is commonly situated over the parietal bone on the side which first presents; or there may be certain natural variations : instead of being unilateral the swelling may cover two sides, as the result of long pressure after the head has come down to the external genitals ; or there may be a primary and secondary tumor, the first of which occurs as described above, while the second originates in a similar fashion, after anterior rotation has occurred, and is situated in the mesial line. The same process may happen to the shoulder in shoulderpresentation, and in the same way the free side, which naturally presents first, bears the tumor.

No treatment is necessary, for in a few days the infiltration will disappear by absorption. Manipulation, bandaging, massage, and inunctions are mischievous, since they are apt to break the skin and change an aseptic to a septic condition. If such a misfortune occurs, the ordinary surgical measures hold good.

\section{Cephalhamatoma}

Children who are weak, anæmic and badly nourished, who are born of debilitated and exhausted mothers, sometimes show at birth a blood-tumor of the head that is called cephalhæmatoma. 
Two sorts are seen : one lies beneath the periosteum and is called a true cephalhæmatoma; the other lies under the aponeurosis of the scalp and is called false cephalhæmatoma. The mysterious alterations in the blood or vessels which make hæmorrhage from debilitated subjects easier than from those in robust health seems to be the active cause in this disorder. When the blood has escaped, it collects between the cranium and pericranium, and so prevents the deposition of new cells from the bone-producing layers of the periosteum; but at the margin, where the cranium and periosteum meet, a hard circle shows where bone-production has gone on without interruption. Occasionally such a tumor may form after birth from injury or clumsy manipulation.

'The distinction between cephalhæmatoma and caput succedaneum is not usually hard to make. In the former the tumor is not on the presenting part as it is in the latter; in the former the swelling is sharply marked off from the rest of the scalp, while in the latter it gradually merges into the surrounding surface; the first has fluctuation at the centrie, but the second has not; the first does not necessarily attend a severe or prolonged labor, while the second does; the one presents no discoloration of the skin, but the other is usually covered by scalp of a dark red or bluishred hue; the first tends to increase after birth, while the second in the same space of time diminishes; the first never occurs over sutures or fontanelles, but the second does. In addition, palpation in cephalhæmatoma furnishes a sensation, caused by pressure upon the particles of newly formed bone, as if one were crackling paper.

The treatment of cephalæmatoma is conservative. Most cases improve under a protective dressing and the application of measures which will develop the child's vitality. If infection occurs, the tumor must be freely opened, drained, and packed.

\section{Hematoma of the Sterno-cleido-mastoid Muscle}

In some weak or prematurely born infants a fibrosis of one or both sterno-cleido-mastoid muscles occurs as the result of a difficult or violent delivery. There may be a tearing of the muscle-fibres with a greater or less amount of hæmorrhage. The head is for a considerable period inclined toward the uninjured side, although the deformity is not necessarily permanent. If, however, healing takes place with the formation of adhesions and 
strong fibrous bands, a torticollis may result, and is with difficulty corrected. These cases usually occur in foot and breech presentations. Such an instance of wry-neck is to be differentiated from a congenital torticollis whose origin is unassignable. No medicinal treatment is required; some advantage in the way of preventing the formation of adhesions may be gained by gentle massage or passive motion ; a somewhat harsher method of obtaining this result is by the use of a well-padded apparatus that is designed to keep the infant's head and neck in a correct position. If, however, firm adhesions have been allowed to form, the usual operative measures are indicated.

\section{Visceral Hamorrhage in the New-Born}

Hæmorrhages may occur at birth or within the next few days in one or more parts of the body. Commonly the condition is impossible to diagnosticate, and is fully recognized only on autopsy. The cause has never been fully understood: in some cases a violent, spontaneous, or artificial delivery seems to be responsible, but in others, where the pelvis was large and the fœtus small, this supposition could not be entertained. Some unknown alteration in the character of the blood or the vessels has been suspected; and we know that children born of mothers who had suffered from syphilis, tuberculosis, or acute infectious diseases during or at the end of pregnancy sometimes become subject to hæmorrhages for little or no ostensible provocation. Some observers have claimed that infection by pathogenic microorganisms, such as streptococci, may cause the bleeding. On the whole, one may say that the rtiology is no more than conjectural.

The loss of blood may be minute or great enough to cause death. The most frequent location is in the brain and meninges; the lesions are not attended by inflammatory processes. In the liver, the suprarenal capsules, the kidney, the lungs, the intestines, and peritonæum the hæmorrhages occur in varying frequency, but rarely, if ever, give symptoms by which one can recognize them. In the lung there may in some cases be the signs of consolidation, with subnormal temperature, blue and cyanotic skin, and marked làck of vitality.

There is no treatment for these conditions. 


\section{"Hemorrhagic Disease" of the New-Born}

This provisional name has been given to a class of cases which are characterized by hremorrhages, usually small in amount, from the surface or viscera of the recently born child. The ætiology has for a long time been a matter of dispute, but probably inheres in a small bacillus, described by Gaertner, which resembles bacterium coli commune. The hæmorrhages occur within the first few days or certainly within the first month of life. No constant lesions, excepting the hiemorrhages, have been found. The whole matter has so little of absolute clearness about it, that one cannot be certain whether most hemorrhages, which occur at or before birth, should not be included in this category.

The loss of blood may occur from the umbilicus, intestines, stomach, mouth, lungs, pleura, kidneys, ureters, the mucous membranes, the meninges, and the skin. Usually these hæmorrhages are multiple. There may be associated symptoms of loss of flesh and strength, high or subnormal temperature, and, in some cases, convulsions. The course of the disease seems to be self-limited and not affected by treatment. In most cases the children die after a few days.

The care of the disorder is symptomatic.

\section{Obstetric Paralyses}

Pressure lesions of the nervous system may occur during parturition to all classes of cases ; but those of weak vitality or impoverished heredity more easily fall victims to such disorders than children who are more fortunately constituted. In almost all cases the active cause is some form of hæmorrhage that inflicts pressure and consequent degeneration upon some part of the cerebral, spinal, or peripheral systems. Such hæmorrhage is not often spontaneous but rather the result of violence, due in most cases to long and difficult labor. The use of forceps is calculated to decrease rather than increase this danger, especially if they are rightly manipulated. Also, it is possible that some form of violence may affect the child, such as a fall or blow upon the mother's abdomen before parturition, or allowing the child to fall while handling him. In this way congestion and concussion of the brain may arise, or even a fracture of the skull. In rare 
cases, a condition of sepsis may favor the tendency toward cerebral bleeding.

Most of these hæmorrhages are cerebral, and of these, the ones at the base are oftener seen than those of the convexity. In such cases, similar lesions are found in other parts of the body as well; one of the commonest of these is the upper spinal cord and its membranes, and then next in frequency come the viscera. The peripheral paralyses are generally caused by the pressure of forceps upon some bony part where a nerve may be injured. This results in partial or total disability of the face or arm. The facial nerve and brachial plexus are thus not in the same class as the cerebrum, and their disabilities as a rule do not last so long. The spinal paralyses occur almost entirely in connection with injuries of the head, for it is rare to find a direct trauma of the cord or vertebræ alone. The symptoms differ widely in various cases, corresponding closely to the amount of pressure produced, as well as its locality. Each case is therefore a study in focal relations. A small hæmorrhage may give rise to nothing more than some degree of dulness with or without rigidity and convulsions. With cortical hæmorrhages there may be monoplegia, hemiplegia, diplegia, or paraplegia. In such cases there may be the accompanying symptoms of slow respiration and pulse, nystagmus, contraction of the pupils, and even opisthotonos. If the amount of blood is very large, it may cause a bulging of some part of the skull or distention at the sutures.

Facial paralysis, when peripheral, is not often a permanent or serious disorder. In this case the whole side may be affected, it looks smooth or swollen, lacking the usual markings and folds; simultaneously, the mouth is pulled toward the other side. A diagnostic sign, which distinguishes the facial paralysis of central origin, is the fact that in such case only the lower half is affected. In very marked cases the electrical reaction of degeneration may be present.

In peripheral paralysis of the arm we have such injuries as can be produced by the margin of the forceps pressing upon the neck, by the impact of a finger which was placed in the axilla for the purpose of traction, or even by a tightly strained cord about the neck. The parts usually affected are those muscles supplied by the fifth and sixth cervical nerves; among them are the biceps, deltoid, supinator longus, brachialis anticus, supraspinatus, and 
infraspinatus. A paralysis of some or all of these muscles constitute the so-called Erb's type. In a characteristic case the arm is helpless, is rotated toward the body so that the palm of the hand is directed away from the body. The severity of the injury decides not only the amount of paralysis but also that of anæsthesia, atrophy, and electrical reaction of degeneration. Usually, the disability is transitory, and at the end of a few days or weeks motion is satisfactorily restored. Nevertheless, cases of permanent effects, such as atrophy, subluxation, and dislocation, occasionally occur.

The treatment in all these disorders is slight. Passive motion and massage of the affected muscles, may, with the help of normal growth and development, hasten a return to natural usefulness. Doubtless some good may be obtained from orthopædic apparatus for the prevention of subluxation and ultimate dislocation; at the sane time, however, too much reliance should not be placed upon such purely mechanical means. The advantages to be derived from the use of electricity are doubtful.

\section{Asphyxia}

Before birth the fœtus is remarkably well supplied with oxygen; when birth takes place the attendant phenomena include cessation of this supply. 'The resulting oxygen-liunger, or relative over-supply of carbonic dioxide, as some authorities prefer to say, causes a stimulation of the respiratory centres, and the child begins to breathe. The anterior edges are first inflated, followed by the superficial parts of the upper lobes, the deeper parts, and thus gradually the air works its way down to the lower lobes, first on the surface and then to the interior. The process is not always perfectly regular nor very rapid, so that complete aëration may not ensue for days.

While most children experience little or no trouble in the transition from prenatal to postnatal breathing, a fairly large proportion suffer some degree of asphyxia. The causes may be personal or maternal, intra-uterine or extra-uterine. The child may be so deficient in muscular and nervous force that he is unable, from sheer inanition, to begin or carry out the necessary movements. Or, as the result of a huge liver, syphilitic inflammation of the lungs, tumors, or anasarca, he may be similarly impotent. Under the intra-uterine causes we include the conditions of pressure upon 
the umbilical cord, the placenta, or fœtal brain, premature freeing of the placenta, and tight wrapping of the cord about the neck ; in other cases there may be a long second stage of labor, uræmia, or death of the mother. The extra-uterine causes are injuries inflicted during or directly after labor, and malformations of the respiratory, circulatory, and cerebral systems.

As the result of these circumstances the lungs may be not at all aërated or only in small part, the brain and thoracic viscera may be congested and studded with hæmorrhages, and in the air passages there may be small amounts of foreign matters that have been aspirated. The blood is dark and thin, the right heart is dilated, the serous cavities may contain serum.

If the asphyxia is slight in degree, the child looks cyanotic and puffy, the attempts at respiration are few and weak, the pulse is slow and fairly strong. The condition may change for the better or may pass into a more serious degree where the heart-action is very weak or inaudible, the face is pale, muscular contractility has ceased, and appreciation of external stimuli is lost.

Treatment. - A routine procedure is to clear the mouth by means of a piece of fine lint soaked in boracic acid of mucus and material that may be taken in from the vagina or uterus. The child may then be held in the inverted position, and if additional stimulus is needed, it may be supplied by sprinkling with cold water, spanking with the hand or a wet towel, or alternate immersion in hot and cold water. If the degree of asphyxiation is too great to be thus relieved, one or more of the various methods of artificial respiration should be used. There are so many of these that one has a large choice; and in making a decision, the very important factor of preserving the body heat should be kept in mind. For this reason the child should, if possible, be kept in a hot bath while the efforts for resuscitation are making. With proper support Sylvester's method can thus be used. This is practised by placing the child on his back with the shoulders slightly raised. The arms are then to be grasped above the elbows and rhythmically extended above the head and depressed against the sides of the thorax at a rate which approximately imitates the frequency of respiration at birth. Forest's practice is also commendable : he first places the child on his left hand, face down, and then clears the obstructions from the mouth and throat by pressure with his right hand on the back. Next the infant is 
placed in a hot bath in a sitting posture, the back being supported by the physician's left hand. With his other he grasps the little hands and raises them on high, at the same time blowing in the child's mouth. Then he brings the arms down, bends the body forward, and compresses the chest. Schultze's method is good but somewhat violent. In practising it, one holds the child to the front, one's thumbs on the breast, index fingers in the axillæ, the other fingers spread around the back, while the infant's head rests upon the upper part of the palms. The infant is then gently swung upward until his head points nearly down and his feet and legs are forward on the abdomen, his chest being held firmly fixed by the operator's thumbs and fingers. In this position the physician's arms are almost straight in front of him. The child is then swung back to the first position, when the whole process is repeated. While this method has many advantages, it has dangers for a weak heart, and may, moreover, if done roughly, result in producing a fracture of a bone. Various other methods such as Dew's, Duke's, Richardson's, Ribemont's laryngeal inflation and Laborde's lingual traction, may be used. The main requirements are freeing the mouth and throat of mucus, continued heat, a satisfactory method of artificial respiration, and the preservation of one's presence of mind, judgment, and tireless patience.

\section{Congenital Atelectasis}

This condition represents a collapsed and unaërated state of any part of the lung. It may be primary, as when a part or all of a lung has not been dilated by air; or it may be secondary, as in those cases where aëration has taken place, but is followed by collapse of the lung tissue in question. Its causes are those of asphyxia, with which it is inseparably connected; for atelectasis is in fact a localized condition of asphyxia.

The parts of the lung most frequently involved are the same as in asphyxia ; namely, the interior, inferior, and posterior portions. The extent may be very small or great, and sometimes there are several minute areas. They are of a dark red color, heavy, hepatoid, non-crepitant, showing lobulations. Even after being in the collapsed condition for days or weeks, they can, without much effort, be artificially aërated. Where inflation has taken place in parts, there is almost always some degree of compensatory 
emphysema near the atelectatic areas. In the pleura small hæmorrhages may sometimes be seen; the right heart is dilated, the liver, spleen, and gastro-intestinal mucous membranes are congested and possibly swollen to a noticeable degree. In some cases there is a cerebral hyperæmia and even minute punctate hæmorrhages. Deformities of the heart are found in some rare instances.

Symptoms. - In the primary form the children die at or shortly after birth. Respiration may practically not begin at all, or it may be weak, shallow, incompetent, and plainly is not produced by the whole lung. There will be cyanosis, weak pulse, possibly convulsions, and finally death. In the secondary or postnatal form the lungs have seemingly become aërated, and then have fallen into a condition of partial collapse. Nevertheless there is a disposition toward cyanosis, of which there may be a number of attacks. The child is weak, the circulation impeded, the temperature at times subnormal. Either the attack may be sudden and rapidly fatal, or there may be one or more periods of improvements. Each exacerbation leaves the patient more exhausted than he formerly was. Intercurrent diseases are feebly withstood and sometimes are the occasion for a new and often fatal attack of atelectasis. When the end comes, it is sometimes preceded by convulsions. The collapsed area may give a diminished percussion note, the breathing is slightly harsh or even bronchial and immediately beyond the borders there may be poorly defined signs of emphysema.

Treatment. - Prophylaxis is a large part of the treatment. The physician should see to it that every child cries enough in the first week or two to insure proper pulmonary aëration. If the attack takes place, heat should be applied to the body and artificial respiration must be practised. The treatment of atelectasis is that of asphyxia.

\section{ICTERUS NEONATORUM}

Infants at any time within the first few days may become jaundiced, the favorite times being the third, fourth, and fifth days. When the jaundice is not due to distinct disease, such as congenital malformations of the bile-ducts, syphilis of the liver, or septic infection, it is called physiological icterus. The cause of it is unknown; some investigators believe that it is an evidence of resorp- 
tion caused by stasis in the bile-ducts, others that it is caused by the swelling of Glisson's capsule and odema of the hepatic vessels, others that it results from the breaking down of red blood corpuscles soon after birth, still others that it is due to the great number of red blood-cells formed in the liver. No one theory has been found to apply to all cases, and the single factor that the majority of investigators agree upon is that it is hepatogenic in origin.

The symptoms consist in the pigmentation which may color every part of the body. Usually it begins on the chest or face or eyes, and then it may spread more and more. The skin and mucous membranes may be stained in various degrees, and practically every internal portion of the body has been found to be similarly colored. The frees are generally unchanged; the urine may be normal, or more or less tinged, occasionally containing bile pigment and an excess of urea and uric acid. No other manifestations are noticeable. It may be of some interest to note that weak children seem to take on a deeper stain than robust ones. The condition may last from a few days to two or two and a half weeks. No treatment beyond the ordinary care is necessary.

\section{Acute Pyogenic Infection}

Acute infections of pathogenic micro-organisms are one of the dangers of very early life which are particularly dangerous on account of the infant's feeble faculty of resistance. The microorganisms which are most commonly found are the streptococcus, staphylococcus pyogenes aureus, and those encountered in the parturient canal of the mother. The infection takes place through the umbilicus, the mouth, the air passages, and possibly through the alimentary tract. Wounds, cracks, and abrasions of the skin can certainly act as the place of admittance. The matter is one of plain pathological contamination such as we are most familiar with in surgical diseases. The umbilical vessels and the umbilicus with the surrounding connective tissue are the commonest regions affected. The arteries are more commonly involved than the vein, and in both structures the infection may be of various degrees of severity and may extend for various distances along their course. Infection of the umbilicus may occur alone, but more often: it is consequent or subsequent to infectious arteritis and phlebitis. In the large majority of cases such a condition marks the beginning of a process which finally involves any part of the body in throm- 
bus-formation and the scattering of pyogenic micro-organisms and their toxines. Thus a localized or general peritonitis frequently follows an omphalitic infection; in some cases an inflammation of the gastro-intestinal track occurs which in all likelihood is communicated from the peritonæum rather than the food. The liver may be invaded, as an extension of the primary infection, in the form of a purulent inflammation of the portal vein or its branches, or an interstitial hepatitis. In the lungs there may be a purulent bronchitis or disseminated broncho-pneumonia with variable involvements of the pleura. From the same source and as a rule following the pleuritis there may be an inflammation of the pericardial sac that should be regarded as a severe and terminal manifestation. Another rare localization is a pseudo-membranous inflammation of the throat that is streptococcic in origin, bearing no relation to true diphtheria. Other forms that occasionally are seen are meningitis, erysipelas, multiple abscesses in the cellular and muscular tissue, arthritis and osteomyelitis. There is nothing peculiar about them excepting the difficulty of obtaining welldefined objective symptoms.

These symptoms are characteristic of nothing in particular excepting general septic infection. There may be progressive loss of flesh and strength, variable temperature, disorders of the gastrointestinal track, hæmorrhage, icterus, nervous and cerebral symptoms. Some of the local signs are hard to distinguish, such as those of the broncho-pneumonia, pericarditis, internal hæmorrhages. The external manifestations, such as abscesses, arthritides, erysipelas and superficial omphalitis, are naturally noticed without delay or trouble; but there is always danger of one of them being regarded as the whole cause of the sickness rather than as a partial and local manifestation of an infection that in all likelihood is sweepingly general.

The treatment of this condition is largely symptomatic ; in addition, the best that one can do is to stimulate the child's physical resources to the utmost and to fight the sepsis by such surgical means as are practicable. The prognosis is always a gloomy one.

\section{Ophthalmi NeONATORUM}

An important percentage of all births, especially in institutions and among ignorant people, show a purulent ophthalmia. The severe and some of the mild cases are due to an infection of the 
conjunctiva with the gonococcus. The source of the infection is the mother's genital track, the child's toilet utensils, the hands, instruments, and dressings of the attendants. The milder cases may be due to irritating discharges of the maternal vagina, other micro-organisms than the gonococcus, and uncleanliness.

Usually the discharge begins within the first week; at first it is slight and with it go swelling and congestion of the lids. There is an infiltration of the bulbar conjunctiva which partially hides the cornea. The whole conjunctiva is bright red, smooth, covered with small whitish patches. The discharge becomes thicker, more profuse, and may be mixed with small amounts of blood and serum. As the disease approaches the end of its course unchecker, the conjunctival mucous membrane becomes dark, relaxed, rough, and necrotic. Under such circumstances the return to health is slow and imperfect. On account of the great chemosis, the integrity of the cornea may be most seriously impaired. As a consequence, total haziness or one or more ulcers may form and finally cause perforation. There may then follow pyramidal cataract, adherent leucoma, or staphyloma. The destruction may vary all the way from slight scars to a panophthalmitis whose natural outcome is atrophy of the bulb.

Treatment. - Prophylaxis should always be carefully practised both in institutions and in private cases where the environment is not favorable to health and cleanliness. An efficient method consists in washing out the child's eyes as soon as possible with a boric acid solution followed by the installation of a few drops of a onehalf per cent solution of argentic nitrate. Even in cases of doubt this should be done, since nothing can be lost and everything gained thereby. If the disease has once begun, the eyes must be thoroughly douched out about every thirty minutes, according to the severity of the inflammation and the amount of discharge, with a copious supply of boric acid solution held in a fountain syringe at no great distance above the patient's head. In the meanwhile the affected eye must be kept covered with iced cloths which are to be changed every two or three minutes, day and night. Some authorities advise the use of the silver solution daily; others caution against its use after the swelling and infiltration are intense, since the cupping about the cornea tends to hold some of the irritating fluid in the depression and so gives rise to epithelial destruction. Both methods have successes and failures in their 
results; and doubtless one can get good results from the boric acid douching if it is thoroughly and frequently done. If, on the other hand, it is not conscientiously carried out, the aid that silver gives is at best doubtful. If only one eye is involved, the other should be protected by means of a watch glass whose margins are kept securely and effectively protected and in place by strips of plaster.

The disease is always a serious one, even when the care is good ; but when it is intermittent or imperfect, the result is some degree of destruction of the eye. In this disease constant watchfulness and work are the price of sight. The non-gonorrhœal cases are naturally apt to be less severe.

\section{INFECTIOUS HÆMOGLOBINAMIA}

In 1873 Charrin, of France, described a disease, and reported fourteen cases of it, which was later investigated by Winckel. In 1879 the latter reported twenty-three cases of it that occurred in a Dresden institution. After this report the disease was known as Winckel's disease or epidemic hæmoglobinuria. Its nature and course seem to be acutely infectious, but what the exact cause is remains unknown. It usually attacks infants in the first week of life and has a short duration of one, two, or three days.

The lesions do not cast much light upon the disease. In most of the viscera, minute hæmorrhages have been found; there is swelling of the liver, spleen, Peyer's patches, and the mesenteric glands. In the heart and liver there are areas of fatty degeneration which doubtless may extend to other parts. The blood contains a high percentage of hæmoglobin which has also been found free in the serum. There is free blood-pigment in the spleen and its blood-vessels. In the kidneys, the cortex is broad, there are streaks and spots of darkened pigmentation in the pyramids, and the straight tubes are filled with hæmoglobin.

The symptoms are abrupt and short-lived. The child becomes weak, fretful, rapidly exhausted and may fall into convulsions before death. The temperature fluctuates about the normal mark, but is not characteristic. In the first few hours the child's complexion becomes cyanotic and then jaundiced; both colors increase, so that the hue is finally a shade of brown. The pulse and breathing are rapid, the stomach and intestines may show functional dis- 
orders. The urine is, on account of the presence of hæmoglobin, brown in color, and contains epithelial debris, blood cells, granular casts, bacteria, and some albumin.

The treatment is symptomatic.

\section{Fatty Degexeration of the New-Born}

This disease, described by and called after Buhl, is a rare condition about which very little is known. It may attack any child, but has been noticed to a greater extent in those who were partially asphyxiated than where birth was quite normal. Its cause is unknown, and it occurs in isolated cases.

Throughout the body there are hæmorrhages of various sizes; they may be found in the skin, in the brain, the viscera, the mucous membranes, and serous cavities. Throughout the viscera. there may be a disseminated parenchymatous inflammation that is finally replaced by a fatty degeneration. This process is not equally diffused in all cases, nor does it proceed in an even degree in all parts. The cord is commonly normal.

The preliminary history is often one of asphyxia, for which no good cause may be found. After a few days the child's complexion is seen to change; it may become somewhat icteric and cyanotic, although these two elements are not necessarily combined in equal degree. In some cases the skin may be œedematous, and in a large percentage of cases it is stained with hæmorrhages. When the cord falls off it may be seen to be normal, although the tissue round about may be the seat of parenchymatous hæmorrhage; the same process may be present in any of the external parts, especially the mucous membranes. There may be functional disorders of the stomach and intestines, the heart is weak and irregular, and the whole economy falls into progressive prostration.

The treatment is merely symptomatic and in severe cases is of no avail. Death usually comes before the disease has run for two weeks.

\section{Pemphigus in Infants}

This term designates a symptom that occurs in more than one disorder. It may be a manifestation of a contagious skin disease which may be communicated from the mother or attendant to the child or vice versâ. Again, it may occur as one of the symptoms 
of hereditary syphilis, or it may result from exposure to a very hot bath.

The disease is characterized by the appearance on the skin of a few or many bullæ or blebs which are filled with clear serum. In syphilis they commonly break out on the palms and soles during the first few days of life, but may extend to other parts. These children are commonly very weak and puny, and in this respect they show a marked difference from the robust infants who contract the infectious disease. In the latter the trunk, especially the buttocks and adjacent areas, and the extremities rather than the palms and soles are involved. Where the eruption is very plentiful it may even attack the mucous surfaces. The blebs are small or large, they may be single or coalesced, and they rest on a pinkish base. After a few days the blebs may rupture and dry up, leaving a pink or reddened scar that after a time disappears. The duration of the eruption has no fixed limit, nor is the number of crops certain.

The treatment consists in general care and the application of boracic acid dusting powders. The syphilitic children have a much poorer chance of recovery than the more vigorous subjects of the infectious disease.

\section{UMBilical Polypus}

The umbilicus may instead of healing promptly assume a polypoid or granulomatous development. The new tissue is tender and friable, and discharges a small quantity of thin fluid. It may be removed by the application of silver nitrate stick. These applications are best made in decreasing circles from day to day, rather than in an attempt to remove the whole structure at one time. After each application the area should be protected by a dressing of a simple cerate, gauze and a roller bandage.

\section{Diverticulum Tumor of the Umbilicus}

This condition is also called adenoma of the umbilicus and congenital mucous polypus of the umbilicus. It occurs in connection with Meckel's diverticulum, a cylindrical process giving off from the ileum about a metre above the ileo-cæcal valve; it is rudimentary, being a retention of a part of the omphalomesenteric duct. In some cases it is no more than a small blind sac connected with the ileum, in others is an open 
passage leading from the intestine to the umbilicus, and thereby producing a fistula. If for any reason the mucous membrane prolapses, a mucous tumor, pierced in the centre, appears at the umbilicus. As more and more of the tube is forced out, the tumor becomes steadily larger, until it may contain one or more folds of gut. As the knuckle of the intestinal wall is forced through the opening there will naturally be two apertures, one leading to the lower, and the other to the upper, part of the bowel. The tumor is irreducible and, as stated above, contains two fistulæ. Between this extreme form and the slight forms consisting of a bit of mucous membrane in a polypoid form there are many gradations. The closing of the slight form is not a difficult matter; the operation on the advanced form is an important one and may be beset with unexpected obstructions and malformations.

\section{Umbilical Hernia}

A small hernia at the umbilicus is a matter of frequent occurrence, in girls oftener than boys, and in poorly nourished girls most of all. The usual cause is straining, due to violent crying, pain and constipation. It is not often larger than one's finger nail and rarely occasions trouble; but when the trouble does occur it takes the serious form of strangulation. The treatment in most cases is merely preventive: after the cord has fallen off, a pad should be held in place over the umbilicus for two, three, or four months. After that time it is unnecessary. If the hernia develops to a noticeable size, it may be held in place by a wooden button which is securely strapped down by strips of adhesive plaster. Another truss that has given me good service is a small truncated cone of beeswax with its blunt apex fixed in the hernia and held in place by adhesive plaster. If such means are insufficient, a radical operation then comes under consideration. In most cases, however, little or no treatment is required; but in the presence of any form of pressure the surface must be well covered with a bland dusting powder to avoid the likelihood of irritations of the skin.

\section{MASTITIS}

An inflammation of the breast in very young infants may be either idiopathic or the result of septic infection. The first sort is frequently seen in every large clinic for children. Within the 
first two or three weeks of life the breasts of both male and female infants may secrete a fluid that looks like colostrum and has the chemical composition of ordinary human milk. Such a development has no practical importance and gives merely temporary inconvenience. The breasts are swollen, tender, and on pressure give out a few drops of lacteal secretion. If, however, they are subjected to rough handling, squeezing, massage, or injury of any sort, the delicate skin may be broken and pyogenic germs may enter. The result is an abscess with the ordinary symptoms of pus-formation : fever, malaise, local pain, swelling, and redness. This pus-process may occur in various degrees of severity, and its consequences naturally become of equal moment.

The so-called physiological mastitis requires no especial treatment. The surface must be kept scrupulously clean, the breast may be covered with absorbent cotton which is to be held in place by a soft flannel roller bandage. Care must be exercised to avoid any sort of injury to the affected part. If the mastitis becomes infected and purulent, it must, like any other abscess, be treated by free incision and draining.

\section{Intestinal Obstruction in the New-Born}

Abnormal conditions of the intestines at or shortly after birth may cause partial or complete obstruction. Partial obstruction may be due to neoplasms, to bands and fibres, such as a persistent omphalo-mesenteric duct. In other cases it may be due to a stenosis or partial atresia of the intestine at any point from the pylorus to the anus. Complete obstruction is commonly due to malformations in which some part of the intestinal tract is absent or so undeveloped that it resembles the lash of a whip. Such deformities are oftenest seen in the lower third of the rectum, but they may also occur on any plane.

The symptoms of partial obstruction are great and increasing difficulty in having normal passages, plus a growing tendency to vomit; after a short time one may be able to distinguish a tumor about and above the obstruction, followed by the formation of gases in the abdomen. The child grows weaker and weaker until his condition soon becomes alarming. If the obstruction is total, there will be no movements except the passage of meconium and mucus; the abdomen soon becomes filled with gases, vomiting 
sets in without delay, especially if the obstruction is high in the gut, and the child within a short time collapses. Vomiting is not longer delayed than one or two days, and death results within the week.

Obstructions in the rectum and imperforate anus may often be relieved by immediate operation. When the obstruction is situated above the rectum, an artificial anus may temporarily relieve the child; but malnutrition and severe intestinal disorders are apt to set in and give a fatal result.

\section{Hermia of the Diaphragm}

A congenital absence of the diaphragm to a greater or less extent may occur, usually on the left anterior border. Through the opening a knuckle of gut may force its way, and then draws after it more and more of the intestinal tube. There will be corresponding symptoms of displacement of the heart to the right, imperfect expansion of the lung, tympanic percussion note and, if the intestine in large amount has found its way into the thoracic cavity in intra-uterine life, the lung may be undeveloped. The younger the child, and the larger the loop of escaped intestine, the sooner is death apt to occur.

\section{Sclerema Neoxatorum}

This condition may occur in young children, but also it happens as a congenital affection; between the two there are many gradations. Its origin is unknown, but since the disease appears in premature and weak children, some form of malnutrition is supposed to act as its basis. There are no known lesions excepting the mysterious hardening of the skin and the equally mysterious solidification of the subcutaneous fat. The disorder may involve a part or the whole of the surface. Usually it begins on the lower extremities, and then spreads upward; it is only in the exceptional instances that it starts on the face and head, after which it involves the rest of the body. In the beginning the skin is pale, whitish, and of a cadaveric texture. Later on it takes on a bluish, somewhat cyanotic tint and also may be slightly icteric. The surface becomes progressively hard, thick, and unpliable ; it cannot be indented by the finger, and the skin becomes so adherent that one cannot pick it up. Gradually a pseudo- 
anchylosis supervenes so that the jaws are sufficiently rigid to prevent nursing, and the joints become sufficiently stiff to make motion no more than partial. The temperature falls one, two, or three degrees below normal, the pulse is slow, and the respiration is weak, slow, and shallow.

Congenital cases are usually still-born; in the others the disease within a few days after birth usually ends in death. The older the child and the less general the involvement, the greater are the chances of recovery. Under all circumstances, however, the outlook is grave, since there is no treatment except keeping the infant warm in an incubator, and the most scrupulous attention to the general care.

\section{CEDema}

Edema is a symptom, not a distinct disease, and may occur in any part of life. It is very liable to happen, in greater or less degree, in very young infants who suffer from cardiac disease, especially when the right heart is involved, in conditions of malnutrition, anæmia, and after loss of blood. There are no distinctive appearances that mark it off from the œdema of adults when they are free from disease of the kidney. The puffiness is first observed in the eyelids and feet, and may then extend to the legs, arms, and body. The diagnostic sign is the pitting on pressure and the presence of the concomitant nutritional, cardiac, and hæmic disturbances.

Treatment is general : the infant must receive wise and good care, the body heat if abnormally low must be kept to or slightly above normal, and such drugs as heart stimulants with diuretics should be administered. If the œdema passes away, general tonics must be prescribed. 


\section{CHAPTER VI}

\section{FEEDING}

THE standard food for infants is human milk, which is a secretion, not an exudation, from the mammary gland. It is a bluish white, sweet fluid, of an alkaline or neutral reaction, and an average specific gravity of 1.031 at $15.5^{\circ} \mathrm{C} .\left(60^{\circ} \mathrm{F}.\right)$, the extremes being 1.027 and 1.032. The average composition according to a number of authorities, such as König, Pfeiffer, Leeds, Harrington, Forster, and Meigs, is

\begin{tabular}{|c|c|c|c|c|c|c|c|}
\hline Fat & . & - & • & . & •. & 3 to 4 & \\
\hline Sugar & . & - & - & - & $\therefore$ & 6 to 7 & \\
\hline Proteids & . & . & . & . & . & 1 to 2 & \\
\hline Salts & . & - & - & • & . & 0.1 to 0.2 & \\
\hline \multirow{3}{*}{\multicolumn{2}{|c|}{$\begin{array}{l}\text { Total solids } \\
\text { Water . } \\
\text { Specific gravity }\end{array}$}} & ${ }^{\circ}$ & - & & . & 13 to 12 & \\
\hline & & . & . & - & . & 87 to 88 & \\
\hline & & - & - & • & . & . $\quad$. & 1.028 to 1.034 \\
\hline Reaction & . & . & . & . & . & & Alkaline or neutral \\
\hline
\end{tabular}

While these figures represent the normal average, many variations are possible even where the mothers are healthy and the children are well and increasing in weight. The very instructive table of analyses, made by Harrington, and quoted by Rotch, of fourteen specimens of human milk occurring under such circumstances may be used as a demonstration :-

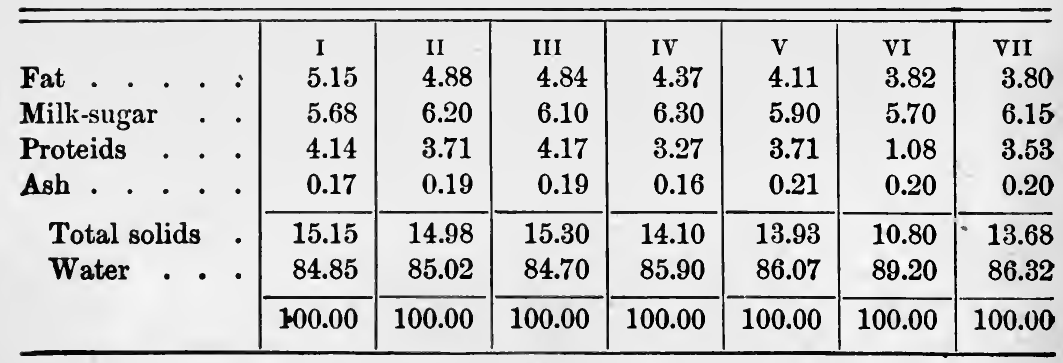




\begin{tabular}{|c|c|c|c|c|c|c|c|c|}
\hline Fat & . & $\begin{array}{l}\text { VIII } \\
3.76\end{array}$ & $\begin{array}{l}\text { IX } \\
3.30\end{array}$ & $\begin{array}{l}\mathrm{x} \\
3.16\end{array}$ & $\begin{array}{l}\text { XI } \\
2.96\end{array}$ & $\begin{array}{l}\text { XII } \\
2.36\end{array}$ & $\begin{array}{c}\text { XIII } \\
2.09\end{array}$ & $\begin{array}{l}\text { XIV } \\
2.02\end{array}$ \\
\hline Milk-sugar & . . & 6.95 & 7.30 & 7.20 & 5.78 & 7.10 & 6.70 & 6.55 \\
\hline Proteids . & . & 2.04 & 3.07 & 1.65 . & 1.91 & 2.20 & 1.38 & 2.12 \\
\hline Ash . . & - & 0.14 & 0.12 & 0.21 & 0.12 & 0.16 & 0.15 & 0.15 \\
\hline \multirow{3}{*}{$\begin{array}{l}\text { Total solid } \\
\text { Water }\end{array}$} & $\mathrm{s}$ & 12.89 & 13.79 & 12.22 & 10.77 & 11.82 & 10.32 & 10.84 \\
\hline & . & 87.11 & 86.21 & 87.78 & 89.23 & 88.18 & 89.68 & 89.16 \\
\hline & & 100.00 & 100.00 & 100.00 & 100.00 & 100.00 & 100.00 & 10() .00 \\
\hline
\end{tabular}

Colostrum, the milk secreted during the first two or three days after parturition, has, according to Pfeiffer, the following composition : -

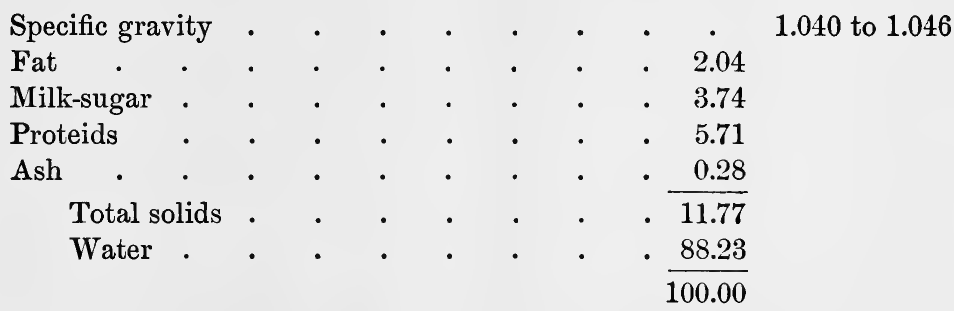

It is a strongly alkaline, yellowish fluid, less sweet than ordinary mother's milk. It coagulates very easily in the presence of heat and sometimes spontaneously. It contains a variable but large number of granular bodies, called colostrum bodies, which are supposed to be degenerated epithelial cells. They are four or five times greater in size than the milk globules of a later period. The function of this fluid is in part nutritional and in part cathartic.

The fat in human milk is a compound of stearin, palmatin and olein, made up in part of the glycerides of butyric, myristic, caproic, and caprylic acids. Its function seems to be threefold; to conserve nutrition, to contribute to the reserve forces of the body, and to act as a cathartic.

The proteids are in process of investigation, so that as yet one may not make a positive statement of their composition. Blythe states that they contain casein, an albumin, and peptone (galactin); Hammarsten gives casein, lacto-albumin and lactoglobulin. In all likelihood, the composition will be found to be even more complex. The casein is suspended in the body of the 
fluid and is not dissolved. The quantity of albumenoids varies between very wide limits in the milk of different women, and of the same women in different conditions of health and functional activity. In colostrum and the milk of the first two weeks they exist in surprisingly large amounts.

The mineral matters, ash or salts exist in large amount in the first two weeks; after that they assume their regular proportion as shown above. They have an important nutritive and stimulating function. The best analysis of their composition is that of Harrington which was made for Dr. Rotch.

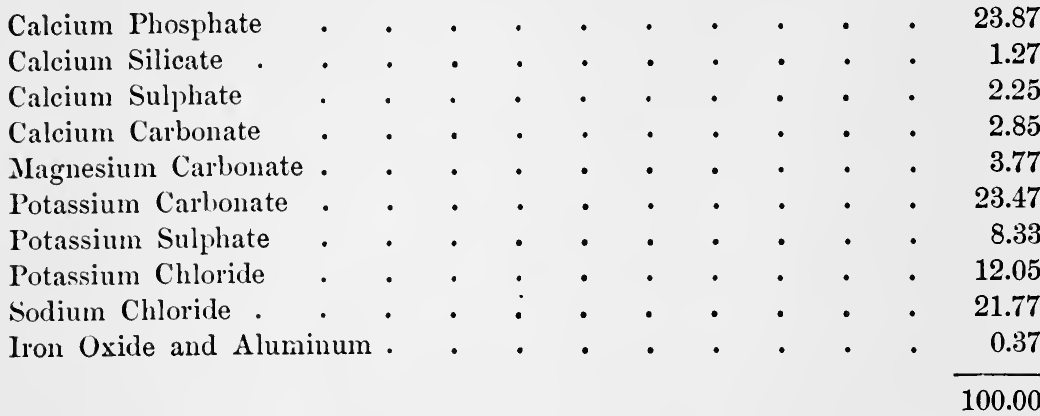

The sugar, in contradistinction to the proteids, is not abundant during the first two weeks; thereafter it begins to assume its permanent proportions which, once attained, undergo very little change. This element of the milk is more nearly constant than most of the other ingredients. It is both easy of digestion and valuable in nutrition.

In those cases where the milk does not agree with the child or his development is unsatisfactory, an exact diagnostication of the trouble and an intelligent attempt to rectify it require a fairly accurate knowledge of the constituent parts of the food. The most thorough method is a full quantitative analysis. This, while not especially difficult, requires considerable technical knowledge, and the expenditure of more time than can usually be given to it. The necessity of obtaining approximately accurate results quickly and easily has brought about many good methods, some of which, while rapid enough, need special apparatus; in this category we may place F. Soxhlet's excellent method. Some of the desired items are easily ascertained : thus the reaction may be ascertained by litmus paper. The quantity 
may be roughly estimated by the amount obtained with a breast pump ; this is so inaccurate that better results will follow weighing the child on a sensitive scales before and after several nursings. The specific gravity may be read off on any reliable hydrometer which has the necessary range. The sugar so rarely varies much from the normal limits that one assumes little risk in supposing it to be regularly constant. The same may be said of the inorganic salts, so that the remaining unknown factors are the fat and the proteids. The fat may be reckoned out by means of one of the various centrifugal machines, such as the Leffman \& Beam or the Lister-Babcock. Another method is by the use of a creamgauge, for instance that of Holt. This device is very simple and very often satisfactory; but it must not be regarded as more than approximately exact. "The glass cylinder holding ten cubic centimetres is filled to the zero mark with freshly drawn milk. This is allowed to stand at the temperature of the room $\left(66^{\circ}\right.$ to $72^{\circ} \mathrm{F}$.) for twenty-four hours and the percentage of cream is then read off. Under these conditions, the relation of the percentage of cream to that of fat is very nearly as 5 to 3 , thus 5 per cent of the cream will indicate that the milk contains 3 per cent of fat, etc." Another procedure that is both rapid and simple, is the Werner-Schmidt method; the apparatus required is a test-tube, whose capacity is $50 \mathrm{cc}$. calibrated in cubic centimetres and tenths. Into this $10 \mathrm{cc}$. of milk and an equal amount of strong hydrochloric acid are poured; the mixture is then boiled, being shaken from time to time, until it assumes a dark brown color. The tube with its contents is then cooled quickly by being plunged into water, after which a known quantity of ether, usually about 25 to 30 cc., is added, thus filling the tube to one of its marked divisions. The tube is then shaken for about half a minute to mix its contents and allowed to stand for about five minutes. The layer of ether is then read off, an aliquot part pipetted off, evaporated to dryness, and weighed. The process is very simple and pleasurably accurate.

The proteids may be calculated in a general fashion from the known specific gravity and percentage of fat. Since we know that large amounts of proteids raise the specific gravity, and large amounts of fat lower it, the result naturally follows that a high specific gravity with a normal quantity of fat indicates a high proteid percentage; and a high fat percentage with a high spe- 
cific gravity means a markedly high proteid percentage. Other variations can easily be worked out. In making these tests, the middle portion of the milk should be used, since the fore-milk is richer in proteids and poorer in fats, while the after-milk or "strippings" is poorer in proteids and richer in fat than the whole quantity combined.

In some cases a bacteriological examination may reveal the presence of staphylococcus pyogenes albus, s. pyogenes aureus, and streptococcus pyogenes. These, although present in small amounts, prevent the fluid from being considered sterile. It is supposed that the micro-organisms penetrate the nipple, and therefore are found in the first quantity withdrawn.

The milk does not necessarily contain the same percentage of its ingredients at all times; indeed, for various reasons, its composition may vary considerably, so much so as to influence seriously the health of the child. Some causes, such as menstruation, passing indispositions, and repeated pregnancies, are commonly supposed to have a more direct importance than they really possess. Others, such as disproportion between diet and exercise, disturbed mental conditions, and certain drugs, may have a very real bearing upon the case. These drugs are most apt to act when the nurse is debilitated, the milk poor in quality, or the child feeble. Those which are most sure to affect the infant are the saline cathartics, opium, and belladonna. The salicylates, the bromides, and the iodides are less certain in their effects, and the mercurial salts are only slightly liable to be carried in the milk. The effect of disturbed mental conditions may be even greater than that of drugs; this is especially apt to occur, and in the most pronounced form, in nervous, sensitive, and irritable women who undergo the shock of great sorrow, overwhelming elation, uncontrollable attacks of temper, or even the pettier annoyances of daily life. In such cases the milk may become not only unfit for the child's nourishment, but also seemingly poisonous.

The effects of diet upon the quality and quantity of milk is at times very great. A woman whose food is poor and not plentiful is apt to produce milk of a lowered fat percentage; at the same time the proteids are deficient, normal, increased in amount, or changed in quality. If, in addition, she has hard work to do, the proteids are more apt to be lessened in quantity than otherwise. A largely nitrogenous diet has the tendency to increase 
both fats and proteids, especially if the amount of fluids consumed is not great and the woman's exercise is insufficient. A vegetable diet has, on the contrary, a tendency to diminish both fat and proteids. The effect of alcohol, especially in the form of beer, ale, porter, and stout, is not a constant one ; in some cases it may increase the quantity of proteids. A nurse who lives upon a rich diet and whose general life is lazy and sedentary is apt to produce a milk that is characterized by too much proteids and fats.

These fats may be cast into a sort of therapeutic table like the following : -

To increase proteids . . . . . decrease exercise and make diet rich. To decrease proteids . . . . . . increase exercise and simplify diet.

To increase fat . . . . . . increase quantity of meat in diet.

To decrease fat . . . . . . . decrease quantity of meat in diet.

To increase total quantity . . . . increase total liquids in diet.

To decrease total quantity . . . d decrease total liquids in diet.

Under normal conditions the nursing should be arranged as follows : -

From birth to the end of the first month, every two hours from 6 A.M. to midnight.

From the end of the first month to the middle of the third, every two and a half hours from 6 A. M. to 10.30 P. M.

From the middle of the third month to the eighth month, every three hours from 6 A.M. to 9 P. M.

From the eighth month to one year, every three and a half hours from 6 A. M. to 8 P. M.

\section{Substitute Feeding}

In the many cases where the mother is unable, for physical or mental reasons, to nurse her child, a substitute for her milk should be provided with as little delay as possible. The practice of waiting in the hopes of some indefinite improvement is apt to result in so pronounced a condition of malnutrition in the infant that months of earnest endeavor may be required to restore a fair amount of health. One of the first resources, fortunately not so common as in former times, is to secure a wet-nurse, who should be between twenty and thirty years of age, healthy, not fat, of good habits and character, whose child is healthy and of the same approximate age as the foster-child. The objections to the ordinary wet-nurse are, in most cases, weighty negations of these qualifications. Usually the woman comes from an unfavorable class, or from distinctly 
unfavorable circumstances. The change from poverty and hardship to ease or even luxury is hard for her to bear. She is very liable to become lazy, to take insufficient exercise, to eat and drink unreasonably and inordinately ; she may even have had a personal history of syphilis or a family history of tuberculosis without being frank enough to acknowledge the fact. She realizes the strength of her position, becomes dictatorial and insubordinate, and interferes with the discipline of the household. Commonly she has vicious habits and vicious associates, and the influence upon the child is not for good. Her manner of life is apt to induce unfavorable conditions of the milk supply, and in many instances it is difficult to make her care for her charge in a thorough fashion. In spite of the parent's willingness to submit to her extravagances of conduct, frequent changes are inevitable; and a succession of wet-nurses is tantamount to a succession of tribulations. I have endured so much unnecessary care, anxiety, and labor from these women that their value seems to me, at the best, doubtful. Nevertheless, if a healthy, well-mannered, and tractable woman can be secured, some of these objections may not hold good; such a person is potentially very useful. Many practitioners prefer a wet-nurse to any other method of substitute feeding, and give as their reason that her milk is the natural food for a child, that it is what the mother herself would have given if she were able, and that by the close association between child and nurse an intimate bond of interest and affection may arise that must necessarily result in advantage to the baby. As a matter of theory this is excellent; and if the practical results were in any way commensurate with the favorable statement of her expected virtues, there would be no doubt of what the best method of substitute feeding is. Unfortunately the demands of the situation are considerable, and a woman must really be a fairly superior person to fulfil them. Such a person is hard to find, and according to common report it is becoming harder and harder to find her. The main trouble lies in the discrepancy between her theoretical value and her actual efficiency.

With the wet-nurse out of the way, the problem consists in adapting the milk of a domestic animal for the digestive and assimilative capacities of a human infant. The choice may be made from the milk of the mare, the ass, the goat, and the cow. The respective analyses are, according to Blythe, as follows : - 


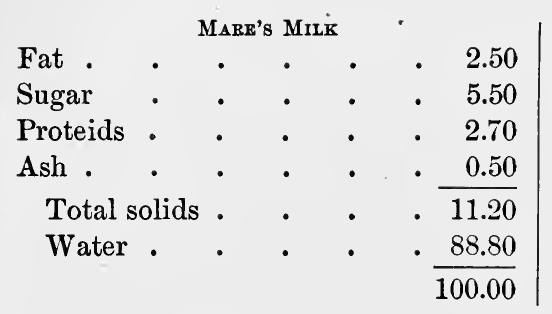

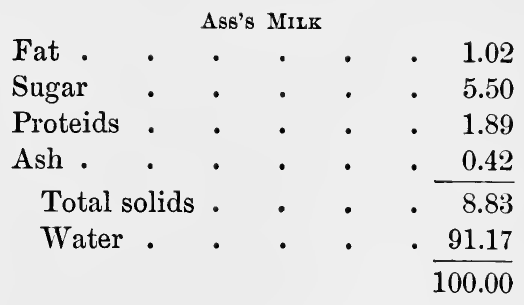

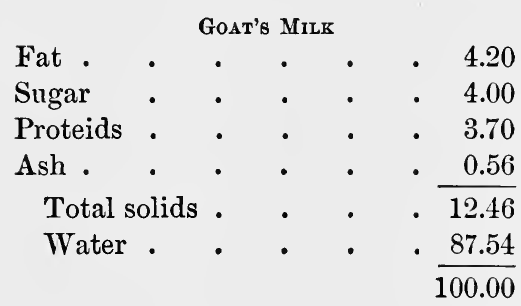

Cow's MrLK

Average (G. E. Gordon's Compilation)

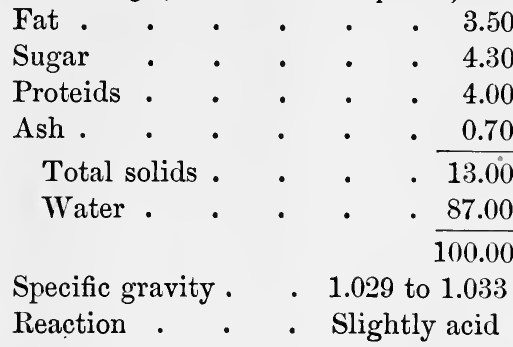

The different breeds of cows, such as the Jersey, Durham, Bretonne, Ayrshire, Holstein, Holstein-Friesian, and the various American strains, have each their peculiarities; but besides being not radically dissimilar, they come to us mixed, as in the common dairy product. Moreover, we have very little assurance that, if we wished for the milk of a particular breed, our wish would be respected. The responsibility would be more than the ordinary dairy conscience is supposed to withstand. The greatest measure of safety and satisfaction is to be obtained from the collective milk of a general well-kept herd.

While the milk of all these different animals may be used for infant-feeding, the only one that produces a large enough quantity to supply the demand is the cow. At almost regular intervals plans to collect sufficient herds of goats or mares or asses are formed, with the idea of substituting their milk for that of the cow. Hitherto, on account of the great cost and the small amount of milk obtained, each one has ended in failure. The mere fact that the ass produces no more than one and one-quarter litres of milk per day is enough to demonstrate the futility of these efforts. Nevertheless, in spite of the satisfactory quantity of the cow's milk, the quality leaves something to be desired. The differences consist in the greater amount of inorganic salts, 
especially phosphate of calcium, and the equal excess of proteids in cow's milk. In addition, these proteids vary in quality, having much more casein than lactalbumin, while in human milk the reverse is true. There is twice as much coagulation of the albumenoids in cow's as in human milk, and the curd is much harder and more tenacious.

In preparing cow's milk for an infant's use, the tendency in these days is in the direction of modification - of such manipulation as will make the cow's milk the approximate equivalent of the human secretion, or in case of weak and delicate children of attenuating it to such a degree that the work of digestion is appropriately lessened. The main difficulty resides in the modification of the proteids, which ought commonly to be reduced below the level of the percentage in the human milk; and then as the child shows ability to digest the food, we may increase the quantity to the normal limit. In the beginning the wisest plan in practically all cases is to give a small proportion of albumenoids largely diluted rather than a more concentrated mixture. Thus it is commonly wiser to begin with less than one per cent instead of more. The dilution is naturally made by the addition of water.

The element of fat does not usually give as much difficulty as the proteids. It exists in the cream, which is no more than milk with a large percentage of fat. In the less rich specimens the other ingredients are almost the same as in ordinary milk; and it is only in the richest that these ingredients are noticeably - and even then only to a moderate degree - reduced. The ordinary cream that results from the effect of gravity when the milk is allowed to stand is equivalent to sixteen per cent. By the use of a centrifugal machine a forty per cent cream may be obtained or any degree less. Since an eight per cent or twelve per cent cream is most useful in modification, one of them is generally selected for this purpose; and they may readily be obtained by placing a jar of milk on ice for five hours when the lighter cream will be formed, or for six hours when the heavier will be prepared. It is estimated that one quart of milk produces two and five-tenths grammes of the former and one and five-tenths grammes of the latter. In modification it is best, as with the proteids, to begin with a small quantity and gradually to approximate to the normal. The cream may be separated by decanting or drawing off the milk through a siphon. The percentage of sugar may be put at or near the normal in 
comparatively early infancy; milk-sugar is used for this purpose, since it exists in normal human milk, and in addition it has a lesser tendency to undergo fermentation than cane-sugar. The fact that it is not so sweet has little bearing upon the matter, since the function of this ingredient is rather one of supplying nutrition than of creating a sweet taste. It is usually employed in the dry state or in a filtered solution of from five to twenty per cent, which should be made anew each day. The mineral salts, being relatively of the same excess as the albumenoids, are reduced to their proper amount when the proteids are diluted. The reaction may, of course, be influenced by the addition of an alkali, the usual one being lime water. About four or five per cent will satisfy the requirements. It is supposed that the milk used for modification is as clean and as pure as the best care will provide. The cow's udders, the utensils, and the hands and clothes of the dairymen must be thoroughly clean; and in cases of doubt the fluid may be filtered through absorbent cotton, although a small part of the fat may thus be lost.

A convenient, rapid, and ingenious method of combining these ingredients is that published by Holt. He uses the 12 per cent and the 8 per cent creams mixed with various quantities of suitable sugar solutions, as in the following formulæ:-

1. To make 2 per cent fat, 6 per cent sugar, and 0.60 per cent proteids, use one part of 12 per cent cream and five parts of 6 per cent sugar solution.

2. 'To make 2.5 per cent fat, 6 per cent sugar, and 0.8 per cent proteid, use one part of 12 per cent cream and four parts of 6 per cent sugar solution.

3. To make 3 per cent fat, 6 per cent sugar, and 1 per cent proteids, use one part of 12 per cent cream with three parts of 7 per cent sugar solution.

4. To make 3.5 per cent fat, 6 per cent sugar, and 1.2 per cent proteids, use one part of 12 per cent cream with two and onehalf parts of 7 per cent sugar solution.

5. To make 4 per cent fat, 6 per cent sugar, and 1.3 per cent proteids, use one part of 12 per cent cream with two parts of 7 per cent sugar solution. "In all these formulæ it will be seen that the ratio of the fat to the proteids is 3 to 1 . Not only these formulæ, but any intermediate ones with this ratio may be derived by varying the dilution. The sugar may be easily modi- 
fied, if desired, by using weaker or stronger solutions than those mentioned."

A more concentrated or more attenuated milk mixture may be made by using the 8 per cent cream.

6. To make 4 per cent fat, 7 per cent sugar, and 2 per cent proteids, use equal parts of 8 per cent cream and 10 per cent sugar solution.

7. To make 3 per cent fat, 6 per cent sugar, and 1.5 per cent proteids, use one part of 8 per cent cream with one and one-half parts of 7 per cent sugar solution.

8. To make 2 per cent fat, 6 per cent sugar, and 1 per cent proteids, use one part of 8 per cent cream with three parts of 7 per cent sugar solution.

9. To make 1 per cent fat, 5 per cent sugar, and 0.5 per cent proteids, use one part of 8 per cent cream with seven parts of 5 per cent sugar solution. Other formulæ in which the fat and proteids stand in the relation of 2 to 1 can easily be reckoned out by the quoted method.

Formulæ giving small fat percentages may be filled by using plain milk instead of cream.

10. To make 1.8 per cent fat, 6 per cent sugar, and 2 per cent proteids, use equal parts of plain milk and 8 per cent sugar solution.

11. To make 0.9 per cent fat, 5 per cent sugar, and 1 per cent proteids, use one part of plain milk with three parts of 5 per cent sugar solution.

12. To make 0.45 per cent fat, 4 per cent sugar, and 0.50 per cent proteids, use one part of plain milk with seven parts of 4 per cent sugar solution.

13. To make 0.30 per cent fat, 4 per cent sugar, and 0.34 per cent proteids, use one part of plain milk with eleven parts of 4 per cent sugar solution.

The reckoning of the separate quantities that correspond to these proportions is a simple matter of arithmetic which hardly justifies elaboration. Allowance must be made for the lime water that is necessary to give the required alkaline reaction. Since 5 per cent of this ingredient is called for, the corresponding quantity must replace an equal amount of the boiling water in which the milk-sugar is dissolved. The process of combining is simple and rapid; the ascertained quantity of boiling water minus the bulk of lime water is to be mixed with the milk-sugar and strained 
through filter-paper, or aseptic absorbent cotton. The cream or milk and lime water are then added, and the whole mixture is well shaken and divided into the required number of bottles. These are immediately to be closed with plugs of aseptic absorbent cotton, chilled, and put in an ice-chest.

A simple method of modifying milk at home has been devised by Baner. It certainly has the merits of easy manipulation and plainness of statement. He has reduced the relation of the component parts of milk to the form of equations, and by arranging them he arrives at his result. His method is "based on the understanding that good cow's milk contains on the average 4 per cent of fat, 4 per cent of proteids, thus regarding cream as simply a superfatted milk, containing practically the same amount of proteids as milk itself. So long as the cream is fresh and clean we may use either a 12 per cent cream, obtained by allowing milk to stand over ice in a tall vessel for six hours, and removing the upper fifth; a 16 per cent cream, obtained by skimming, or a 20 per cent cream obtained by the separator.

Given: Quantity desired (in ounces) $=\mathrm{Q}$

Desired percentage of fat $=\mathrm{F}$

Desired percentage of sugar $=\mathrm{S}$

Desired percentage of proteids $=\mathbf{P}$

To find (in ounces) :

$$
\begin{array}{ll}
\text { Cream (16 per cent) } & =\frac{2}{12} \times(\mathrm{F}-\mathrm{P}) \\
\text { Milk } & =\frac{\mathrm{Q} \times \mathrm{P}}{4}-\mathrm{C} \\
\text { Water } & =\mathrm{Q}-(\mathrm{C}+\mathrm{M}) \\
\text { Dry milk-sugar } & =\frac{(\mathrm{S}-\mathrm{P}) \times \mathrm{Q}}{100}
\end{array}
$$

If 20 per cent centrifugal cream is used, the denominator of the cream formula will be 16 instead of 12 . If 12 per cent cream is used, it will be 8 instead of 12 .

Example. - Suppose we want $40 \mathrm{oz}$. of a mixture to contain 4 per cent of fat, 7 per cent of sugar, and 2 per cent of proteids : -

$$
\begin{aligned}
& \text { Cream }=\frac{40}{12} \times 2=6 \frac{2}{3} \text { oz. } \\
& \text { Milk }=\frac{40 \times 2}{4}-6 \frac{2}{3}=13 \frac{1}{3} \mathrm{oz} . \\
& \text { Water }=40-20=20 \mathrm{oz} . \\
& \text { Sugar }=\frac{5 \times 40}{100}=2 \mathrm{oz},
\end{aligned}
$$


An apparatus has recently been devised for the home-modification of milk and was presented before the New York Academy of Medicine, by Dr. L. Emmett Holt, in January, 1899. It is a seven-panelled glass jar upon the various faces of which are marks which indicate quantity of the constituent parts of the milk. It is called the "Materna " milk modifying apparatus.

In November, 1899, Dr. H. D. Chapin of New York reported yet another method of home modification of milk. The following quotation is from his paper and gives his plan in full: "By mixing cream and skimmed milk, as it were in situ, in certain proportions, there is a certain relation between the fats and proteids that may be used in feeding the infant. In the home modification of milk by means of this bottled milk, the cream is readily and accurately separated from the under milk by means of a dipper measuring exactly one fluid ounce. Quart milk bottles all have substantially the same kind of neck, and the dipper has been made to easily fit into any of these bottles, after an inspection of a large number. The very top layer of cream is taken off with a teaspoon, and the dipper thus filled and the first ounce removed; otherwise the milk would spill over when the dipper is let down. The successive ounces of cream are then easily removed without jarring, siphoning, or other manipulation. While it is not easy to test the proteids in milk, we are taught on good authority that they nearly equal the fat in milks up to four and a half per cent fat. Avoiding small fractions, cream with 12 per cent fat would have three times as much fat as proteids (12 per cent fat and 4 per cent proteids); ten-per-cent cream would have two times and a half as much fat as proteids (ten per cent fat and four per cent proteids); eight-per-cent cream would have twice as much fat as proteids (eight per cent fat and four per cent proteids); six-percent cream would have one time and a half as much fat as proteids (six per cent fat and four per cent proteids). Almost any desired percentage of fat or proteids can be procured by diluting these creams, using the cream that contains the desired ratio between fat and proteids. Having at hand a bottle of milk which has separated into two layers, one very rich in fat and the other very poor in fat, the problem of getting a cream of any desired percentage of fat consists in mixing the very rich milk with enough poor milk to reduce its percentage of fat to the point desired. In practice, cream containing twelve per cent and eight 
per cent fat are most useful. If it were possible completely to separate the fat for a quart of four-per-cent-fat milk, nearly eleven fluid ounces of twelve-per-cent-fat cream could be had, or sixteen fluid ounces of eight-per-cent-fat cream. In the 'deep-setting' process of creaming, about ten per cent of the total fat is left in the skimmed milk, so we would naturally expect to get only ninetenths of the theoretical quantity of fat in the creamy layer. In practice, where it is desired to have fat three times the proteids, make a twelve-per-cent-fat cream by taking the first nine dippers of cream and milk from a quart bottle on which the cream has risen . . . and mix. Result, nine fluid ounces cream - about twelve fat, four per cent proteids, four per cent sugar. What is not needed of the nine fluid ounces can be put back into the bottle. For any quantity of food containing fat three times the proteids, use this formula with twelve-per-cent cream :-

"Dilution of twelve-per-cent cream = twelve per cent divided by desired percentage of fat.

"Fluid ounces twelve-per-cent cream = desired fluid ounces food divided by dilution.

" Sugar = desired fluid ounces food divided by twenty.

"Diluent $=$ desired fluid ounces food minus fluid ounces twelve-per-cent cream.

" Example. - Desired twenty-four fluid ounces containing fat, three per cent; proteids, one per cent; sugar, six per cent.

"Twelve per cent $\div$ three per cent $=$ four dilution.

"Twenty-four $\div$ four $=$ six fluid ounces twelve-per-cent cream.

"Twenty-four $\div$ twenty $=$ one ounce and a fifth sugar.

"Twenty-four - six = eighteen fluid ounces diluent.

"When it is desired to have fat two times the proteids, take the first sixteen dipperfuls of cream and milk out of a quart bottle and mix. Result, sixteen fluid ounces cream - about eight per cent fat, four per cent proteids, five per cent sugar. What is not needed of the sixteen ounces can be put back in the bottle. For any quantity of food containing fat two times the proteids, use this formula with eight-per-cent cream :-

"Dilution of eight-per-cent cream = eight per cent divided by desired percentage of fat.

"Fluid ounces eight-per-cent eream = desired fluid ounces divided by dilution.

"Sugar = desired fluid ounces food divided by twenty. 
"Diluent $=$ desired fluid ounces food minus fluid ounces eightper-cent cream.

"Example. - Desired forty fluid ounces food containing fat, four per cent; proteids, two per cent; sugar, seven per cent.

" Eight per cent $\div$ four per cent $=$ two dilution.

"Forty $\div$ two $=$ twenty fluid ounces eight-per-cent cream.

"Forty $\div$ twenty $=$ two ounces sugar.

"Forty - twenty = twenty ounces diluent."

This work of adapting cow's milk to the needs of a young child, especially a weak or ailing one, is most accurately done in the so-called milk laboratories which have been recently instituted through the initiative of Dr. T. M. Rotch of Boston. The undoubted advance in infant-feeding which the enterprise has accomplished is of the greatest possible value, for it has changed the preparation of the food from a matter of guesswork, to one of commendable certainty. The ingredients which they commonly use are a 20 per cent milk-sugar solution, a 16 per cent fat, and a "separated" milk from which the cream has been removed by a centrifugal machine. The one objection to the centrifugalizing process is the liability which the violent motion has to break up the fat-globules with the resulting formation on the surface, when the milk is heated, of a layer of oil. Some chemists believe that this method of separating and recombining the elements of milk does not permit of a suitable mixture, that especially the fatty portion is in a poor state of emulsion. While the same, or similar objections can be urged against any artificial combination, the main fact holds good that a properly modified milk represents the best manufactured infant's food which is at our command.

The fact that milk is usually not sterile suggests to the physician the possibility of infecting a healthy child, while attempting to nourish him, with a serious disease, such as tuberculosis, diphtheria, enteric fever, scarlet fever, and many gastro-intestinal disorders. To reduce this danger the oldest plan was to boil the milk. This was found to make it sterile, but at the same time the physiological character of the food was so much changed that a large proportion of infants could not digest it. The situation therefore changed from the dangers of infection to those of equally grave malnutrition. When this was fully recognized a change was made to the process of sterilization, the continued heating at a temperature of $100^{\circ} \mathrm{C}$. $\left(212^{\circ} \mathrm{F}\right.$.) for an hour and a half. The 
pathogenic micro-organisms were thereby destroyed, although their spores were not. At the same time, as was later on ascertained, the value of the milk itself was seriously affected. The children thus fed, instead of being afflicted with microgenic disorders, suffered from constipation, scurvy, and disturbances of nutrition that were equally noteworthy. An examination of the milk showed that the lactalbumin was sufficiently coagulable, that the fat-globules lost their individuality and became blended with each other and the insoluble albuminous matter, that the sugar was injured, and that in general the quality of the food became lowered. To obviate these disadvantages the plan of partial sterilization, called pasteurizing or heating the milk at a temperature of $75^{\circ} \mathrm{C}$. $\left(167^{\circ} \mathrm{F}\right.$.) for twenty minutes, was devised. This was also found to destroy most pathogenic germs, although the spores were less affected than in sterilization. A feeling has been steadily growing that pasteurized milk is open to similar objections as those produced by the more prolonged heating; and while in many circumstances some such procedure is at present desirable, since the milk sold by even the better class of dairymen is far from clean, the rational and most desirable protection comes from such rigorous asepsis in the collection and handling of milk that any form of sterilization will be unnecessary.

It is doubtful whether absolute asepsis is practically possible; and likewise it is doubtful whether it is necessary. We all know that within a few hours after birth the gastro-intestinal contents give cultures of various micro-organisms; and in addition, many samples of breast-milk have been shown to be nonsterile. The main indication for preventing disease is the adoption of such measures of cleanliness as will prevent contamination of the milk by too great a number of bacteria, and, most of all, to exclude those specific germs that are communicated by direct or indirect contact with the well-known diseases. This can unquestionably be done by rigid cleanliness, in which every farmhand must be conscientiously instructed. In this work the physician has as large a responsibility as the dairyman.

After the milk has been prepared it must be divided into as many bottles as there are feedings in the twenty-four hours, thereby obviating one source of infection in filling a bottle whenever the time for feeding has arrived. This may be easily arranged each morning according to the schedule appended. 
Scheme for Feeding, with Intervals and Quantities During First Year

\begin{tabular}{|c|c|c|c|c|}
\hline & $\begin{array}{l}\text { INTERVALS } \\
\text { BETWEEN } \\
\text { FEEDINGS }\end{array}$ & Amount in Each Bottle & $\begin{array}{c}\text { Number } \\
\text { of } \\
\text { FeEdings } \\
\text { Per Day }\end{array}$ & Total amount Per Dax \\
\hline 1 wk. & 2 hours & 30 gram. (1 oz.) & 10 & 300 gram. (10 oz.) \\
\hline 2 wks. & 2 hours & 45 gram. ( $1 \frac{1}{2}$ oz. $)$ & 10 & 450 gram. (15 oz.) \\
\hline 3 wks. & 2 hours & 60 gram. (2 oz.) & 10 & 600 gram. $(20$ oz. $)$ \\
\hline 2 mos. & 2 hours & 75 gram. ( $2 \frac{1}{2}$ oz.) & 10 & 750 gram. (25 oz.) \\
\hline $2 \frac{1}{2}$ mos. & $2 \frac{1}{2}$ hours & 90 gram. (3 oz.) & 9 & 810 gram. ( 27 oz. $)$ \\
\hline 3 mos. & $2 \frac{1}{2}$ hours & 105 gram. ( $3 \frac{1}{2}$ oz. $)$ & 8 & 840 gram. (28 oz.) \\
\hline 4 mos. & 3 hours & 124 gram. (41 oz.) & 7 & 868 gram. (29 oz.) \\
\hline 5 mos. & 3 hours & 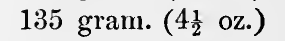 & 7 & 945 gram. ( $31 \frac{1}{2}$ oz. $)$ \\
\hline 6 mos. & 3 hours & 165 gram. ( $5 \frac{1}{2}$ oz.) & 6 & 990 gram. (33 oz.) \\
\hline 7 mos. & 3 hours & 195 gram. (6 $\frac{1}{2}$ oz.) & 6 & 1170 gram. (39 oz.) \\
\hline 8 mos. & $3 \frac{1}{2}$ hours & 210 gram. (7 oz.) & 6 & 1260 gram. $(42 \mathrm{oz})$. \\
\hline 9 mos. & $3 \frac{1}{2}$ hours & 210 gram. (7 oz.) & 6 & 1260 gram. (42 oz.) \\
\hline 10 mos. & $3 \frac{1}{2}$ hours & 255 gram. ( $8 \frac{1}{2}$ oz.) & 5 & 1275 gram. ( $42 \frac{1}{2}$ oz.) \\
\hline 11 mos. & $3 \frac{1}{2}$ hours & 264 gram. ( $8 \frac{3}{4}$.oz.) & 5 & 1320 gram. (44 oz.) \\
\hline 12 mos. & $3 \frac{1}{2}$ hours & 270 gram. (9 oz.) & 5 & 1350 gram. (45 oz.) \\
\hline
\end{tabular}

It must be kept in mind that such a plan is general and that there must be exceptions to it. The weight of the child has almost as much to do with the amount of food, the intervals between the feedings, and the chemical composition, as his age. Thus, an infant of five months who has the size, weight, and development of one of three must be regarded as being less than five months. A hard and fast rule must not take the place of judgment and individual consideration.

The care of the bottles and rubber nipples is a matter of the first importance, for on their cleanliness depends, to a large extent, the freedom from infection. In selecting them the ease with which they can be kept clean is always the deciding factor. For this reason the plain cylindrical bottles are the best, and if they are graduated, the measuring of the desired quantity becomes simple. The nipple should be made of soft black rubber, cone-shaped like a "dunce's cap," and having an aperture that is not tơ large. The bottles must be thoroughly scrubbed directly after being used, scalded, and then put in a solution of borax or washing soda. Before being again used they must be thoroughly rinsed. The nip- 
ples after being used must be scrubbed on both sides with a brush, scalded, and then kept in the borax or soda solution. Before being put on the bottle they also must be rinsed. When the bottle is filled for nursing, it must be properly heated by being placed in hot water. Besides the regularity of feeding, the process should be uninterrupted, should cover from twelve to fifteen minutes, and must receive the active attention of the mother or nurse. The child should recline in the attendant's arms, and the bottle must be held, rather than be propped up by pillows, in order to regulate the flow of food and exclude air from the nipple. If a portion of food remains in the bottle, it must not be warmed over for a second feeding, but should be thrown away.

If these recommendations concerning feeding are not followed by an increase in weight and the development of a quiet, happy general condition, the physician must examine the symptoms to see whether the milk is faulty, and thereby get at the root of the trouble. One of the commonest errors is an excess of proteids, which is indicated by constipation or diarrhœea, vomiting of curdy masses, colic, or the presence in the fæces of uncligested curds. In these cases either the proportion of the albumenoids, or the absolute amount due to excessive quantity of milk, or both, may be too great. Both should be reduced, and after toleration is established they may gradually be brought up to their permissible limit. In other cases there may be an excess of fat, followed by too great a number of stools, by the presence of masses of fat in the fæces, vomiting regurgitation of food after nursing, or by colic. On the other hand, a deficiency of fat will commonly cause constipation and hardening of the fæces. In still other instances the quantity of sugar may be too small. The consequence may be the production of gastric and intestinal fermentation, regurgitation or vomiting, an excess of movements which are foul-smelling, thin, acrid, and of abnormal color. If the milk is unduly attenuated in all or most of its constituents, there will be deficient weight and development. In most cases, by carefully following such indications, one can discover in a very short time both the cause of the child's disorder and the indicated remedy. The children that will in all likelihood give most trouble are the very delicate ones whose digestive capabilities are feeble. From the first they suffer from all the symptoms of intestinal derangement, although the food has been very carefully prepared. Under these circumstances 
the reduction of the ingredients, especially the proteids, must be immediate and sufficient. The albumenoids may be decreased until they are no more than one per cent or even a fraction thereof ; the fats may also be decreased, but not often to as low a point. No more particular rules can well be formulated; each case must be carefully considered, and the changes may have to follow each other in rapid and wearying order. For the flagging assimilative abilities of a delicate child seem to have peculiar idiosyncrasies and fluctuations that must be patiently and tirelessly followed. Occasionally a partial peptonization of the milk, after it has been modified, will give relief; but it must be remembered that this device is of temporary value and may not be continued for a long time. In other cases the stimulating properties of beef juice, especially where the indications call for a very small percentage of proteids, may give some aid when added in amounts of eight to sixteen grammes (one-quarter to one-half ounce). But no surer rule can be made than the one of consistently adapting the food to the expressed symptoms until relief has been obtained. When toleration has once been established, the gradual development of the nourishment to a normal composition and amount is often no more than a matter of intelligent patience.

\section{Infant Foods}

A very large and increasing number of these foods are on the market, and although they have many faults, nevertheless their ease of preparation and cheapness retain for them a large popularity. If it were possible to make an artificial food that imitated very closely the composition and physiological structure of human milk, its production would undoubtedly be of great value. So far this has not been done, although the foods which are commonly employed could be replaced by products which are superior to those now sold. The Liebig foods, such as Horlick's food, Mellin's food, malted milk, Hawley's food, represent a combination of flours which has been treated with diastase until the starch has been converted into maltose and dextrin. The milk foods, such 'as Nestlé's, the various Swiss foods and Gerber's food represent condensed milk which has been sweetened and supplemented with dextrinized flour. The farinaceous foods, such as Ridge's food, Imperial Granum, Robinson's Patent Barley, Hubbell's Prepared 
Wheat, usually consist of combined flours whose starch is partly changed into sugar. Carnrick's soluble food consists for the most part of carbohydrates; Lacto-preparata has about the same composition, but in-it milk-sugar replaces a portion of the starch. Lactated food is composed mostly of carbohydrates. The Peptogenic Milk Powder of Fairchild consists of pancreatin, lactose and alkaline milk salts. The condensed milks represent ordinary cow's milk which has been evaporated in vacuo, sterilized, and sweetened with cane-sugar. The unsweetened variety can also be obtained.

The best way to examine these foods is to compare them, when prepared for use, to normal milk, as in the compilation made by Holt : -

\begin{tabular}{|c|c|c|c|c|c|c|c|c|c|}
\hline & 荡 & 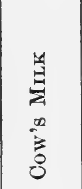 & 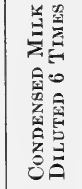 & 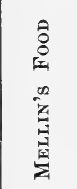 & 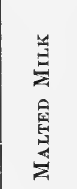 & 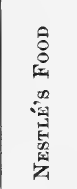 & 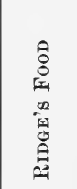 & 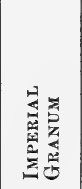 & 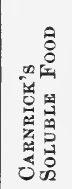 \\
\hline & $\begin{array}{l}\text { Per } \\
\text { cent }\end{array}$ & $\begin{array}{l}\text { Per } \\
\text { cent }\end{array}$ & $\begin{array}{l}\text { Per } \\
\text { cent }\end{array}$ & $\begin{array}{l}\text { Per } \\
\text { cent }\end{array}$ & $\begin{array}{l}\text { Per } \\
\text { cent }\end{array}$ & $\begin{array}{l}\text { Per } \\
\text { cent }\end{array}$ & $\begin{array}{l}\text { Per } \\
\text { cent }\end{array}$ & $\begin{array}{l}\text { Per } \\
\text { cent }\end{array}$ & $\begin{array}{l}\text { Per } \\
\text { cent }\end{array}$ \\
\hline Fat & 4.00 & 3.50 & 0.99 & 0.04 & 0.39 & 0.76 & 0.16 & 0.14 & 1.12 \\
\hline Proteids & 1.50 & 4.00 & 1.20 & 1.50 & 2.28 & 1.54 & 1.67 & 1.98 & 1.35 \\
\hline $\begin{array}{c}\text { Soluble carbohydrates } \\
\text { (sugars) }\end{array}$ & 7.00 & 4.30 & 7.23 & 11.56 & 10.18 & 6.38 & 0.41 & 0.25 & 4.06 \\
\hline $\begin{array}{c}\text { Insoluble carbohydrates } \\
\text { (starch) }\end{array}$ & & & & & & 4.19 & 10.91 & 10.65 & 5.61 \\
\hline Inorganic salts & 0.20 & 0.70 & 0.17 & 0.45 & 0.50 & 0.24 & 0.07 & 0.06 & 0.56 \\
\hline Water & 87.30 & 87.50 & 90.41 & 86.45 & 86.65 & 86.89 & 86.78 & 86.92 & 87.30 \\
\hline
\end{tabular}

A special mention should be made of the Gaertner mothermilk, which is cow's milk that has been so treated in a centrifugal machine that one half of its casein, sugar, and inorganic salts has been removed. The needed quantity of sugar must naturally then be added. The resulting percentage of fat is 3.1, and that of casein is 1.7. The manufacturers claim that the process eliminates bacteria and dirt, thus making a physiological sterilization.

\section{The Care of Premature Infants.}

The task of rearing a child that is born before term, involves the application of artificial heat, protection from exposure, and the 
suitable regulation of the food. Such children may weigh as little as two pounds, and in addition are usually undersized; they are extremely weak, flabby, without enough vitality to suck or to breathe in a regular and sufficient manner. The pulse is very small, weak, rapid, and often irregular ; the cry is weak, the general condition is one of apathy, and the temperature is commonly subnormal.

Children who are premature by one month only may be well protected by an oil inunction of the body which is to be followed by a swathing in cotton batting. About the buttocks and genitals absorbent cotton may be tucked, thus doing away with the necessity of using diapers, which are very apt to cause excoriations. Blankets may be wrapped outside of the cotton, and hot water bottles should be placed about the legs and body. These children instead of being washed may be cleaned with sweet oil, in much the same fashion as before soap was invented.

Feeding should be accomplished by means of a large medicine dropper that is made for the purpose. Breast milk is best, but lacking it a properly modified milk should be substituted. The intervals between feeding should be reduced to one and a half hours.

When the child is under eight months, an incubator will in all likelihood be required. One of the best of the elaborate types is that of Rotch, and almost every instrument maker has one or more specimens of his own. The simpler types are easily made by any one who has a fair amount of mechanical ingenuity. The requirements consist of a glass-covered box in which there is a bed made up of cotton batting or absorbent cotton. Underneath is a chamber containing.hot water, the heat of which is maintained by gas, alcohol, or oil flame. A thermometer for registering the temperature is placed inside, and in the walls are apertures for ventilation. A desirable amount of dampness in the atmosphere is maintained by means of a wet sponge. Naturally the glass lid is movable so that the child may be reached for purposes of manipulation, cleaning, and feeding. The cleaning is done with oil, not water. The food is administered at intervals of an hour or hour and a half by means of a large medicine dropper or a tube which has been passed into the stomach (gavage). The length of the residence in the incubator depends upon the child's development and vitality. 


\section{WEANING}

A child, under ordinary circumstances, should be weaned when he is one year of age, and the process should be a gradual rather than a sudden one, to avoid the dangers of gastric and intestinal derangements that are apt to follow an abrupt transition from one to another sort of food. Under some circumstances, such as pregnancy, wearing out of milk supply, or acute sickness of the mother, the change may have to be made at some time previous to that stated; and if these conditions arise, there should not be too long a delay with a consequent impoverishment of the infant's vitality. In some cases of acute sickness, where the necessity of the artificial food is no more than temporary, the milk may be prevented from drying up by the consistent use of the breast-pump. On the other hand, when the normal nursing period closes, in the latter half or at the end of the summer, the child may be kept at the breast, supposing that the quality and quantity of the milk are sufficient, for a short additional period, in order to reduce the dangers of infection, that are greatest during hot weather. This prolongation of breast-feeding must be made with the full knowledge that the quality of milk is apt to deteriorate, as well as the fact that in the beginning of the second year the diet normally calls for a food composition and food bulk which human milk cannot supply. In some cases, where the robustness of the child is not quite complete, a course of mixed feeding may be advised; while this has some advantages, the objections are very apt to be weighty. For in many instances the mother's milk is not only deficient but also unstable, and the attempt to compensate with an artificial mixture will involve a burdensome frequency of analysis of the mother's milk, and an undesirable frequency of change in the artificial food, in the attempt to maintain an equilibrium.

\section{Feeding During the Second Year and its Continuance}

During this period the foundation of the food must be good, wholesome milk. Many robust children are able to digest it in its undiluted form. If a difficulty exists, it is, as a rule, on account of the excessive quantity of proteids which may need no more than a trivial reduction. A good formula for the first two months is 


\section{Milk}

Cream (12 per cent)

Boiled Water

Milk Sugar
540 grammes ( $18 \mathrm{oz}$.

115 grammes ( $\left.3 \frac{3}{4} \mathrm{oz}.\right)$

270 grammes $(9$ oz.)

$120-150$ grammes (4-5 oz.)

This will make about one quart of milk. From the fourteenth or fifteenth month the child should be able to digest plain milk. An additional element of food may be supplied in the form of various cereals which have been reduced to a thin gruel or jelly, strained, and mixed with milk. Clear soups may form an occasional meal, and baked apples, stewed prunes, and orange juice are eagerly received and well borne. A piece of zwieback, well toasted stale wheat bread, or toasted biscuit dipped in milk may be added to the soup or milk. When the child has shown his ability to digest these articles, he may, in addition, receive poached or soft-boiled eggs, rare scraped beef, and stale bread ; very often he may derive satisfaction and some benefit from being allowed to pick shreds of meat from the bone of a lamb chop from which the bulk of the meat has been cut. In the second half of the year it is unnecessary to strain the cereals as it was before that time.

From the third year the diet may be very much enlarged, and includes milk and cream, meat broths and soups, zwieback, toast, stale bread, biscuits (crackers), poached and soft-boiled eggs, broiled beefsteak and lamb chops, roast beef and lamb, underdone mutton, roast or fricassé chicken (preferably the white meat), boiled or broiled fish, especially those which are not too fatty. In the way of vegetables, one may use baked or creamed potatoes, spinach, asparagus tops, fresh peas and string beans, young and tender carrots. Stale bread, biscuits, and zwieback may of course be given every day, as likewise may all the cereals which must be well cooked. For dessert, the child may have plain rice pudding, custards, junket, clear jellies, apple sauce, stewed prunes, oranges, and such mixtures as prunes or apples with rice, tapioca, or the other cereals.

No cakes, pies, and similar pastries, nor fresh bread should be allowed, nor may fried foods of any description. These are especially mentioned since mothers and nurses are frequently tempted to give them. The other interdicted foods and drinks are all those not included in the list given above. 


\section{Feeding IN Acute Sickness}

When a child is struggling with an acute disease, his food must be as simple and as easily digested as possible and at the same time reduced in quantity. A child at breast should be allowed to nurse no longer than two-thirds or one-half of his regular time, and one who is past that period should have his food sufficiently attenuated to preclude the possibility of added digestive derangements. All of these little patients require a liberal quantity of pure water, the infant being satisfied with teaspoonfuls while the half-grown child demands ounces. In some diseases, such as pneumonia, the amount of food must be reduced in order that the suddenly enlarged digestive tract may not take up too much of the already curtailed visceral space. In all these cases the object in view is merely to sustain the patient's vitality until convalescence sets in, rather than to attempt to fulfil all the needs of the healthy organism.

In many cases milk, which is commonly the basis of food in sickness, is not easily digested, especially the proteids. To overcome this difficulty it may be predigested or peptonized. The process consists of mixing with each pint of milk a peptonizing powder composed of one gramme ( $\mathrm{gr} . \mathrm{xv}$ ) of bicarbonate of soda and three-tenths of a gramme (gr. v) of pancreatic extract. The jar containing the milk is then placed in water of the approximate temperature of $43^{\circ} \mathrm{C}$. $\left(110^{\circ} \mathrm{F}\right.$.) and occasionally agitated. The object of the procedure is to convert the proteids into peptones. The thoroughness and completeness with which this is done depends upon the length of time during which the digestion is allowed to continue. As short a time as five or six minutes has an appreciable effect upon assimilability of the food, while the limit of the conversion takes place in two hours. The use of the peptonized milk should not be continued for too long a time under penalty of reducing the tonicity of the gastro-intestinal tube. In some cases one may use kumyss instead of the plain or peptonized milk. This preparation was originally made by the Tartars from mare's milk which had undergone fermentation through the agency of kefir grains. At the present time cow's milk is used as a body. The particular value of kumyss comes from the breaking up of the casein, with the resulting formation of alcohol, lactic acid, carbon dioxide, and some other less thoroughly identified products. An- 
other similar preparation is matzoon. The manufacture of this article was brought from Asia Minor where, from the beginning, the basis of the product was cow's milk which had gone through a course of fermentation. Both proteids and milk-sugar are thereby changed and split up into alcohol, lactic acid, and carbon dioxide, as in kumyss, but the amounts formed are not the same in both. For very young children they may be diluted.

Some other articles of diet for acutely sick children are beef juice and beef extract. The first may be made by lightly broiling a piece of beef (top round, for example) and then expressing the juice with a wooden lemon squeezer. The second is prepared by cutting the meat into small cubes after having removed all the fat. The pieces are then placed in a Mason jar, which is to be tightly closed, and placed on the fire in a kettle of water. The water is allowed to simmer until the red color of the meat turns into a brownish gray. The juice is then expressed as in making beef juice. The beef extract has more nourishment and is therefore more of a true food than the juice which may be classified under the heading of stimulants. The meat broths, made from beef, mutton, or veal, are likewise valuable. They are the result of steally cooking for two or more hours. Before being served they should be freed from their fat and seasoned with salt. Farinaceous food in the form of barley, rice, and oatmeal water may be of decided use for children over the age of infancy. In preparing them the grain must be allowed to stand in water until it is cleansed and softened. It must next be boiled from two and a half to three hours and then strained. Older children when convalescent may enjoy junket, curds and whey, wine-whey and custards.

\section{Dentition}

The subject of dentition has furnished a never-ending source of discussion. Expert opinion has decided on a fairly uniform opinion about it which in almost all other cases would be sufficient to settle the matter once and for all. But this subject is different from others, both in the liberal exceptions which the profession makes to the strict application of the standard opinion enunciated by experts, and the tenacity with which laymen hold to ideas which were formerly held concerning it. Therefore a 
real need exists for endeavoring to settle the question in a simple and final way.

In former times the eruption of the teeth was supposed to bring with it a necessary train of concurrent diseases. Not only were attacks of bronchitis, gastritis, and otitis referred to it, but likewise any disability which happened to appear while the teeth were forcing their way through the gums. Such an rtiology was so indiscriminate, so clearly lacking in the elemental relations of cause and effect, that even cursory observers became dissatisfied with it. A large proportion of children, by uninterruptedly going through the period of dentition, seemed to be an active protest against such a rule. And as medical knowledge increased, more and more of the diseases which were formerly attributed to dentition were shown positively to have quite other causes. This experience rendered observers bolder in declaring the innocuousness of the dentition-process.

In spite of all this, the subconscious belief in the dangerous tendencies which children have in the time of teething is very strong. In spite of all attempts to dissipate the old beliefs, parents notice that during the period from six months to two and one-half years of age children are commonly subject to all manner of gastro-intestinal and pulmonary disorders, of earache and nervous irritations. And until these are satisfactorily explained, their proper connection with dentition cannot be properly understood, nor, in consequence, will they be adequately treated.

It is unquestionably a fact that any physiological process, as long as it is in no way abnormal, should complete its formation with practically no disturbance. Following this rule, dentition, in its natural evolution, gives rise to no appreciable symptoms. Very often the process is not absolutely normal, and often the variation should be classified as functional rather than organic. One of the penalties of civilization is the notable complexity and sensitiveness of nerve functions which cause so many of our disabilities. On account of such considerations, various processes which are purely "physiological" come to have a character that is strikingly allied to the pathological. Thus parturition, which, in a perfectly natural state is a matter of inconsiderable disturbance, under the conditions of civilized life becomes burdened at times with the most striking possibilities of pain and sickness. Menstruation is another example of a similar sort; and others 
will suggest themselves on a fair amount of thought. In a like manner one should reason about teething, and at the same time recognize the limitations that control the possibilities of acquired nervous irritability. Thus, for example, we know that, in the presence of the comparatively slight congestion and swelling which attend the irruption of the teeth, the accompanying pain may be out of proportion to the ostensible cause. Also we know that the blood currents supplying the jaws and the middle ear are closely related. Consequently one can readily see how earache should at times accompany a difficult dentition which is attended by an unusual nervous reflex like that mentioned above. But one should remember that between such a state and that of a purulent otitis media is a far cry.

Following out this train of thought one can easily understand how the impaired nervous innervation of the young child would render him an easier victim to disorders of any kind than before the irritation existed, merely because his power of resistance is lessened. In this way he becomes an easy victim of gastric and pulmonary disorders, and not because teething and disease are naturally bound together. Any other condition which brought with it similarly acute accompaniment of nervous wear and tear would be followed by an equal number of coughs, colds, diarrhœas, and similar troubles. Therefore the relation between dentition and the sicknesses that are supposed to accompany it is at best an indirect one. Also, one may, with equal assurance, say that the cases of convulsions of which one so commonly hears as the result of teething are in almost all cases due to intestinal fermentation or other well-known causes. The cases of convulsions due to direct irritation from the gums are so rare that the ordinary practitioner is not apt to see more than one or two of them in a life-time.

It follows from all this that the habit of lancing the gums to facilitate the irruption of a tooth is in almost all cases useless. In fact, one would be quite conservative in stating that lancing a gum never helps a tooth to appear. One should remember that the teeth are not prepared to spring forth whenever the superimposed gum is divided; moreover, when the gum is cut the aperture does not become larger, as it would if it were made in an elastic membrane stretched over a pushing object. On the contrary, it remains unchanged, the tooth is not at all hurried in its 
progress, and all that one has accomplished by the operation is a partial relief of the local congestion. In extremely rare cases this may be of some value; but over against it one should set the tenderness and soreness which arise from the cutting of the gum. A child who has been irritated and worn out by the pain, the sleeplessness, and the nervous stress which follow difficult dentition is in no condition to withstand the added discomfort and possible infection that may be associated with this operation.

In this connection it is worth while to recall the manner in which the teeth develop. Long before the seventh month of age, the average time for the appearance of the first tooth, they are working their way into their final place and position. ${ }^{1}$ "Even as soon as the seventh month of fœtal life, the alveolar processes contain a series of crypts, corresponding to the twenty milk teeth, for which they later on furnish lodgment. Soon the crowns of all these teeth are partially calcified. In addition to the milk teeth, the jaws contain the dental sacs of the permanent incisors, canines, bicuspids, and first molars. The first upper molars lie behind the second temporary molars, but are not lodged in alveoli or sockets; indeed, at this period of life, the crypts for the second temporary molars have no posterior walls, and so resemble mere depressions rather than clearly cut pockets. In the lower jaw the crypts for the second temporary molars extend as far back as the basis of the coronoid processes, while the first permanent molars lie underneath these processes. . . . Thus one sees that the development of the permanent teeth, except the second and third molars, begins early in fœtal life and continues for some years. The germs of the second molars appear a little before the end of the first year, but those of the third molars as late as the fifth year. ... An interesting fact is that there is not room in the young jaws for the teeth before their irruption to lie in a series; for this reason the central incisors overlap the lateral ones, and the canines are pushed up above the other teeth."

The appearance of the teeth in time and order follows no invariable rule. At times one sees a child with one or more teeth at birth; again, in other children, especially the poorly nourished and rachitic ones, they may not appear until some time in the second year. A plan giving the average in time and order of appearance is appended, although its importance is not marked.

1 "The Development of the Child," by Nathan Oppenheim, Macmillan Co. 


\section{Temporary OR MiLK Teeth}

(1) Lower central incisors

(2) Upper central incisors

(3) Upper lateral incisors

(4) Lower lateral incisors

(5) Molars (first) . .

(6) Canines

(7) Second molars
- $\quad 7$ th month

- 9-16 months

. 10-16 months

. 13-17 months

- 16-21 months

. 16-25 months

. 24-30 months

Total 20
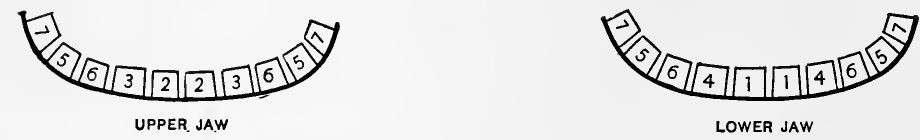

Fig. 1-Diagram of temporary or "milk" teeth in the order of eruption.

\section{Permanent Teeth}
(1) First molar
$\begin{array}{lll}\cdot & \cdot & . \\ . & . & . \\ . & . & . \\ . & . & .\end{array}$
6 years
(2) Middle incisors.
7 years
(3) Lateral incisors.
8 years
(4) First bicuspids
9 years
(5) Second bicuspids . . . . . 10 years
(6) Canines . . . . . . . . 11-13 years
(7) Second molars . . . . . . . 12-14 years
(8) Third molars . . . . . . . . 17-25 years
Total 32
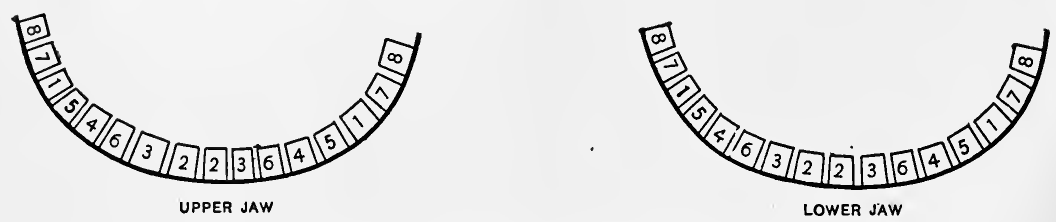

Fig. 2-Diagram of permanent teeth in the order of eruption. 


\section{CHAPTER VII}

\section{DISEASES OF THE MOUTH AND ESOPHAGUS}

\section{Pathological Conditions of the Lips}

IN children the lips are rarely the seat of a primary inflammation. Injuries are responsible for a large proportion of the secondary inflammatory disorders; here infection may take place and result in an acute or subacute cheilitis of varying depth. The treatment of this condition is surgical. Not often one sees a furuncle; but when it occurs and is not properly treated, it may give rise to a deep-seated inflammation. It is merely necessary to mention the fact, for there is no peculiarity in it that appertains to childhood. In like fashion one should mention the occurrence of herpes of the lip. Such an eruption may be seen in poorly nourished children, especially those who are suffering from disorders of the gastro-intestinal track, or from the acute infectious diseases. Still more frequently an eczema of the lips, especially the upper one, results after a rhinitis. A chronic inflammation of the nasal mucous membrane, such as that which accompanies the presence of adenoid fungations, is very apt by the action of its acrid discharge to cause an irritation of the skin and mucous membrane of the lip. Cure follows removal of the rhinitis and the application of protective and emollient ointments. Before healing has resulted, the abrasions and cracks in the surface act as a fertile culture ground for erysipelas and lymphangitis. The mere mention of these possibilities is enough to show the importance of guarding against them.

In rare cases one sees in connection with macroglossia an accompanying macrocheilia.

\section{Hare-Lip and Cleft Palate}

Hare-lip and cleft palate are congenital deformities, due to deficient development, which on account of their frequency of 
occurrence and the marked influence that they exert on a child's health, logically belong in the list of active diseases. The deformity occurs in various degrees, the least of which is a slightly bifid uvula, while the greatest is seen in the complete cleft palate and double hare-lip. The bifid uvula has no more than an academic interest; but the others possess a decided practical importance. The method by which the upper lip develops and closes in front of the mandibular fissure, and the palate by the inward growth and fusing of the palatine processes, is a matter of embryology, and therefore calls for no extended description in this place. Noreover, the radical treatment, which is purely surgical, must be ascertained from works upon surgery, and should be undertaken only by a skilled surgeon. But it is distinctly pertinent to mention some of the important facts concerning the course of the troubles which arise from the deformities.

The effects of these disorders are in proportion to the amount of functional disability which they impose upon the acts of feeding and swallowing. A partial or one-sided hare-lip prevents the efficient closing of the buccal cavity and the making of a vacuum, and therefore renders sucking difficult. If the hare-lip is double, the inefficiency is much increased. If cleft palate exists, the nose and mouth are practically turned into one cavity, food regurgitates through the nostrils, suction is correspondingly lessened, and the child literally starves if he has no other method of obtaining nourishment. For this reason, it may be necessary to feed him with a teaspoon or, if he is very weak, by means of a large medicine dropper. Such children are apt to be congenitally deficient in general vitality, and therefore the problem of rearing them becomes a difficult and complicated one. The question of impaired phonation comes up later on, when the child should begin to talk; but it is suggested by the thin, nasal cry, which will be noticed at birth, if the cleft in the palate is at all considerable.

It is not hard to deduce the other common sequels to hare-lip and cleft palate: the defective development is often a local manifestation of a general condition, and outside of it we are apt to find other deficiencies present. On account of the imperfect closure of the mouth and nose, the mucous membrane is exposed and readily falls into a condition of subacute or chronic irritation and inflammation. The air which passes through the nose is not sufficiently warmed and filtered, and this may occasion disorders 
of the respiratory track. Disorders of the stomach and intestines may follow insufficient feeding and the swallowing of unclean matters which may accumulate in the open mouth.

The time of operation for hare-lip depends upon the child's vitality. If there is sufficient strength to withstand the slight loss of blood and the effects of the anæsthetic, the hare-lip may be corrected within the first month or two. No more definite rule than this can be laid down, for there may be the greatest variation in strength as well as the amount of deformity between various cases. If the operation has been successfully completed, a gradual change for the better may be expected in the shape of the superior maxilla, and therefore in the cleft palate. The operation for the latter deformity may, therefore, be postponed until some time in the second or third year.

A certain amount of prophylactic treatment is possible. These disorders seem to occur in families, and the hereditary element should not be attributed to the direct laws of descent which tend to hand on a definite characteristic to succeeding generations, but rather to the weakened tissues of the parents. The offspring of asthenic organisms are, naturally enough, apt to be defective. "Maternal impressions," as a factor in the ætiology, have very little importance. Therefore, if parents are unhealthy or if there has been a case of hare-lip and cleft palate in the family, they should receive the tonic treatment whose aim is to supply the strength and vitality which they lack. The father as well as the mother needs such attention; and some observers believe that weakness in the former is a more telling factor than in the latter. The indicated treatment is the use of the compound syrup of hypophosphites plus the careful regulation of the diet, exercise, and general mode of life.

\section{Gangrene of the Cheek}

(Cancer Aquaticus, Cancrum Oris. Noma. Stomatitis Gangrcenosa)

Atrophic and degenerative conditions in young children are common. But as a rule they are easily controlled. When sufficient vitality is restored, the patient, to all appearances, enjoys as good health as ever. In some cases, however, the process becomes so marked as to result in a profound destruction of tissue. This may occur in almost any part of the body. The location is often 
decided by some injury, possibly a small one. No matter how small it is, it is large enough to admit micro-organisms which thereafter are able to flourish in proportion to the patient's debility. As the disease occurs in children under nine years old, the resistance which they offer is not very great.

Causes. - Since the mouth is very liable to injury on account of the habit of young children of putting all sorts of things into it, it is oftener than any other part of the body the seat of gangrene. The disease rarely happens in robust children; on the contrary, almost invariably one finds a weak and poorly nourished patient, usually with a history of a recent sickness such as measles, pertussis, or other acute infectious fevers, whose general atony invites disaster. Sometimes one finds a previous stomatitis, although this is not necessarily of pathological importance. Some observers believe that the abuse of mercury is sufficient to start in motion a stomatitis of such severity that gangrene must certainly follow. In the diseased area various pathogenic micro-organisms may be found.

Lesions. - The pathological changes begin on the mucous membrane of the cheek, usually near the teeth and not far from the corner of the mouth. The mucous membrane becomes devitalized, dark, and hard. The tissue then begins to break down, and the gangrenous process is fairly started. It may spread in all directions and attack muscular and osseous tissue as well as the more delicate mucous membrane. Soon a perforation of the cheek is formed which spreads at first laterally and then in all directions.

Symptoms. - Almost always the patient has been suffering from a stomatitis and general debility. One's attention is drawn to the bad odor of the child's mouth, and a small spot of severe inflammation in the cheek. Given these conditions, one is almost sure of the diagnosis. About the inflamed spot the tissues become swollen and heavy, the natural hue changes to an unnaturally dark one, which becomes more pronounced as the process develops. These stages become exaggerated in proportion to the child's atony, until the cheek begins to break down. The progress of the disease is now rapid, and is accompanied by an offensive discharge, falling out of the teeth, and an involvement of the deeper bony structures. Although the blood-vessels are destroyed, nevertheless one rarely sees extensive hæmor- 
rhages, doubtless because they are filled with thrombi and plugs of coagulation material. The temperature fluctuates, being influenced by the marked prostration and sepsis which accompany the disease. These factors at the same time are doubtless responsible for the frequent involvement of the lungs, stomach, intestines, and kidneys which are so apt to occur in this disease. Curiously enough, although there is such an extensive destruction of tissue, nevertheless there is but little pain, doubtless on account of the local paralysis of sensory nerves due to profound inanition.

It is not easy to confound this disease with another, even an ulcerative process. For the symptoms are so marked and the progress toward a fatal termination so impressive, that even an inexperienced attendant very soon recognizes the nature of the disease. Occasionally one sees or hears of a case where the process limited itself to a small area which sloughed away and allowed spontaneous recovery. Such cases are, however, very exceptional, since the child's lack of vitality is generally sufficient to bring death. This may happen in a few days or a few weeks.

Treatment. - Outside of the cleanliness and general hygiene of the mouth which may prevent the gangrene, there is no palliative treatment. As soon as a diagnosis is made, the offending area must be cut out with a liberal incision and the seemingly healthy periphery burned with the Paquelin or galvano-cautery. In incipient cases some practitioners advise the application to the mucous membrane of a saturated solution of chloride of zinc on cotton for five or ten minutes. The less delay in adopting these measures, the less will be the ultimate destruction of tissue and resulting deformity. Every effort should be made by means of hygiene, diet, stimulants, and tonics to increase the patient's vitality. Thus, combinations of iron and strychnine may be of some use, and alcohol in the form of good wines, whiskey or brandy is well tolerated. Food must be given as liberally as the digestive faculty permits, and although the separate amounts may be small, the collective quantities must be large. Especial pains must be taken to keep the mouth scrupulously clean.

Prognosis. - The prognosis is bad, and a large majority of the cases go on uninterruptedly to a fatal termination. 


\section{Simple Erosions of the Mouth}

Young children are apt, as a result of injuries from rubber nipples, their finger nails, or hard substances which they put into their mouths, to wear away and destroy the mucous membrane of some portion of the mouth. The habit of tongue-sucking may cause these erosions to appear in the hard palate. In the ordinary cases the injury is small and superficial, and it becomes serious only when neglect permits the cause to continue. When they appear in new-born children on the hard palate about the middle line and near the velum, or upon the soft palate, they are called Bednar's aphthæ. They are very superficial ulcers, with a grayish or yellow-grayish base.

Usually these children are poorly nourished. Proper care of the patient's general condition, plus a removal of the cause and cleanliness of the mouth, will bring about a healing of the injured mucous membrane. A useful mouth wash for this condition is a combination of saturated solution of boric acid and peroxide of hydrogen in equal parts.

\section{Ulcerative Gingivitis}

Causes. - This disease, commonly known as ulcerative stomatitis, is really a disorder of the gums. The process begins on the gums, about one or more teeth, and the pathological changes are for the most part confined to this place. It occurs in poorly nourished and poorly cared for children of from three to nine years of age. Given poor local and general conditions, any one of a number of causes can set the characteristic inflammation in motion. Residence in hospitals and asylums, the presence of pathogenic bacteria, the administration of mercury, arsenic, lead, and phosphorus are some of them. In infantile scorbutus it is often seen as well as in weak children who suffer from carious teeth. At times it occurs in localized epidemics, most of all where hygienic conditions are unfavorable.

Lesions. - This disorder is first seen on the outside mucous margin of the lower jaw, usually on the lateral aspect. Thence it may spread in all directions, but only in severe or uncared-for cases does it extend to the upper gums and the rest of the mouth. The gums become swollen, tender, and easily abraded. Then a grayish membrane with a tinge of yellow in it begins to appear. 
This is composed of mucus, epithelial debris, and bacteria. this membrane forms, the tissue of the gums is destroyed, especially about the teeth. These may fall out, and about their cavities as well as in the ulcers which appear in the continuity of the gum, a necrobiotic process may start which results in a localized destruction of the underlying periosteum.

Symptoms. - At the onset of the disease one can plainly notice an offensive odor from the child's mouth. Thus one's attention is immediately drawn to the seat of the disorder. It is easy to recognize the large indented tongue and the swollen, coated gums in the mild cases, and the bleeding foci, the ulcers, the loose teeth, and the osteo-necrosis of the severer ones. Manual examination will demonstrate the swollen, tender glands under the jaw. One is sure to find more or less constitutional, as well as temporary, depression and malaise, with a loss of restfulness, appetite, flesh, and strength.

Treatment. - The treatment depends in the first place on the cause, and then upon the condition. Whatever the active cause may be; one must carefully and assiduously seek it out and remove it. Where, as in the case of infantile scurvy, it requires a definite treatment and diet, such measures should immediately be begun. In addition one should use a spray or mouth wash of equal parts of peroxide of hydrogen and saturated solution of boracic acid. In case there are loose teeth or sequestra of bone, they should be removed, so that infecting cavities and pockets may be exposed and cleansed.

Chlorate of potash is regarded as a specific remedy. A threeyear-old child may take $0.2 \mathrm{gm}$. ( $3 \mathrm{gr}$.) of the drug every three hours. But one must watch for the prostration and cardiac weakness with which it overwhelms some susceptible children.

Every effort must be made to improve the patient's sanitary and hygienic surroundings, and to fortify his general condition by diet and tonics.

Prognosis. - The prognosis varies with the treatment. If the care of the child is intelligent and unremitting, he will rapidly recover. If he lingers for weeks in an unsatisfactory state, the attendants are apt to be at fault. Complications in the way of bone necrosis, and various forms of stomatitis, may delay recovery. 


\section{Simple Acute Stomatitis}

Causes. - One of the most common complaints of children, especially the very young, is a catarrhal inflammation of the mouth. In most cases it occurs in connection with derangements of the gastro-intestinal track associated with the local irritation due to lack of cleanliness, injuries, the excessive use of mercury, chilled or overheated food, and the tendency in the acute eruptive fevers to inflammation of the mucous membranes.

Lesions. - The mucous membrane of the mouth, or any part of it, is swollen and congested. There may be more or less degeneration of the epithelial cells, which, when sufficiently marked, results in death of the tissue and the consequent formation of white patches. The muciferous glands are enlarged and congested, and thus may secrete an unusual amount of discharge. On the other hand, in a few cases the congestive disturbances may be so obstructive that the glandular functions are temporarily obliterated and the mouth becomes hot and dry. Occasionally the obstruction may be so great that the glands become markedly swollen, enlarged, and cystlike. Regularly one may expect to see an accompanying enlargement of the associated glands.

Symptoms. - Although the symptoms are not hard to discover, they are nevertheless frequently overlooked. The child's mother usually believes that they are due to some disorder of the stomach; and as they commonly cause no great constitutional disturbance, she may not at first seek relief. When the case is brought to the physician's notice, he will distinguish a hot, inflamed condition of the mucous membrane, which in marked cases is accompanied with considerable swelling. The saliva is so plainly acrid in its action that the skin of the lower lip and chin may be raw and inflamed. The tongue is coated with a white fur which gradually takes on a darker, dirtier hue. Here and there, piece by piece, this coating disappears, until finally nothing remains. The tongue may then be unusually red and tender, as if covered by a new and very delicate epithelium. Sometimes, as the coating wears off, the surface is seen to be studded with superficial ulcers of varying size. In a large proportion of cases there may be some fretfulness and constitutional disturbance; in other children the disability may be purely local, and in some the little patient may seem not at all incommoded. 
Treatment. - The treatment consists in removing whatever cause one may discover, and then in exercising rigid cleanliness. It is always wise to act as if there were a precedent gastric or gastro-intestinal disorder. This will necessitate a thorough cleansing of the stomach and intestines, and a careful regulation of the food. The mouth should be frequently sprayed with a saturated solution of boric acid. At intervals of two or three hours it should be swabbed out with cotton moistened in the same solution. In exceptional cases the ulcers may require the application of a dilute solution of silver. This measure should not be adopted until one is sure of its absolute necessity.

The outcome of these cases is almost always favorable, although the length of time consumed in treatment is variable.

\section{Herpetoid Stomatitis}

Causes. - This simple disorder has been the cause of much discussion, and at the same time full research in its phenomena has been lacking. At various times it has been differently designated, having had the names follicular, aphthous, vesicular, and herpetic stomatitis. Our knowledge concerning it is mostly negative, so that we are more able to say what it is not than what it actually is. All that one can state is that it never occurs without a simple acute stomatitis, that it probably has some connection with disorders of the stomach and their sequelæ, that it is doubtfully associated with bacteriological life, and that it is not contagious.

Lesions. - The changes are neither many nor important. Outside of those of simple acute stomatitis, one sees here and there a small vesicle lying under the epithelium. After a short time the epithelial layer becomes devitalized and breaks down, leaving a small superficial ulcer. At first the color of these vesicles is creamy white; as soon as the process of devitalization begins it changes to a dirty hue, partly gray, partly white. The eruption is most often seated upon the under surface of the tongue, and in the furrow between the lips and gums.

Symptoms. - In the way of symptoms, outside of the inflammation of the mucous membrane and the appearance of the eruption, there is a varying amount of constitutional disturbance, which, however, is rarely severe and never dangerous. This dis- 
turbance is the same as that of simple stomatitis, with the possible exception of an added degree of intensity. The disease may last from a few days to two weeks.

Treatment. - In ordinary cases no treatment beyond cleansing the mouth is called for. As a matter of routine the stomach and intestines should be thoroughly emptied. Where the superficial ulcers are slow in healing one may touch them with a weak solution of silver nitrate. After all, washing the mouth with boracic acid is the main thing.

Prognosis. - The prognosis is uniformly good.

\section{Mycetogenetic Stomatitis}

Causes. - This form of mouth-infection, commonly called thrush or sprue, is a further example of diseases which, on account of their generally harmless nature, have escaped thorough investigation. It is caused by the lodgement and growth on the mucous membrane of the mouth. of a fungus lately called saccharomyces albicans. In the presence of dirt it can grow on any mucous membrane, especially one which has a neutral or slightly acid reaction; since the mouth receives greater quantities and more kinds of dirt than any other mucous membrane cavity, it is the most frequent seat of infection.

Lesions. - Very soon after the spores of saccharomyces albicans find a lodging place on the mucous membrane they begin their natural growth, sending out great quantities of their thin white mycelium, which, with broken-down epithelial cells, leucocytes, and detritus make up the characteristic whitish fur. The spores find a culture-ground in the first instance in some abrasion or other solution of continuity; then the growth makes its way between the cell elements and gradually splits up the different strata. For this reason of growth the development of the fungus is much more pronounced on squamous than on cylindrical epithelium. As this disintegration of tissue proceeds it is accompanied by variable amounts of congestion, swelling, and tenderness of the affected parts. The capillary vessels become engorged and easily friable, so that when the fur is swept off they may bleed from the slight trauma.

Symptoms. - The symptoms are usually confined to the tongue; in exceptional cases, where there is an extension to the pharynx, 
œsophagus, or stomach, appropriate symptoms will follow. In some of the rare cases of protracted extension, where deep ulcers result, the fungus makes its way through the walls of blood-vessels; in consequence, metastatic deposits in any of the viscera may suddenly exhibit their characteristic signs.

The common experience is, however, quite removed from such startling extensions. One generally sees the beginning and the end of the invasion limited to the mouth. At first it appears in small scattered masses which somewhat resemble bits of coagulated milk. These grow in size until they flow together. They may attack the tongue and the cheeks; if not arrested they may cover the lips, the pharynx, the palate, and so extend gradually to the deeper parts of the alimentary track. The buccal cavity is sometimes seen to be dry, and the surface of the mucous membrane loses the soft texture of its normal condition. With the spread of the growth the color changes from a white to a grayish white. Associated with this one may distinguish the ordinary symptoms of a simple stomatitis. Occasionally one sees the signs of a constitutional depression, but this condition is in all likelihood due to causes which precede rather than follow the appearance of the fungus. As a rule there is little difficulty in making a diagnosis : for the main possibility of error lies in mistaking milk clots for the disease. Such clots may be easily removed by a brush or swab, while the growth can not. The diagnosis can be confirmed by a microscopical examination which will demonstrate spores, mycelium, and degenerated cells.

Rigid cleanliness will remove all traces of the growth. For this purpose repeated washing with a saturated solution of boracic acid, or in severe cases with boracic acid and peroxide of hydrogen, is efficient. Likewise it is advisable to clean out the gastrointestinal track, and in case of general debility to fortify the child's health as far as possible.

Prognosis. - The prognosis is good. The extent of the growth or the child's poor condition may delay a perfect recovery until the bad effects of poor care or constitutional disability are at least partially removed.

\section{Croupous Stomatitis}

Causes. - As the result of local irritants, the mouth may be covered with a membrane whose limits are defined only by the 
spread of the exciting cause. Also it occurs as a complication of the eruptive fevers. In this case its ætiology is doubtless bacterial.

Lesions. - The parts involved are covered with a false membrane which varies in thickness and in color in different cases. The mucous membrane is split up and partially destroyed. It is infiltrated with pus and a small amount of fibrin. The pus, fibrin, and coagulated epithelium make up the false membrane.

Symptoms. - In addition to the formation of membrane, there may be considerable general clisturbance, malaise, lack of appetite and strength, as well as disorders of the gastro-intestinal system. There may be fetor and a foul discharge from the mouth; and commonly the buccal tissues are both tender and painful. Within a few days or a week the false membrane falls off, leaving a bare and sometimes bleeding surface. Very rarely there may be a noteworthy hemorrhage.

Treatment. - The treatment consists in maintaining cleanliness in the mouth by means of repeated washing and spraying with boracic acid solutions. Any precedent abnormal conditions must be carefully looked after and the stomach must be maintained in good condition.

Prognosis. - The prognosis is generally good.

\section{Gonorrhoeal Stomatitis}

This infection is in children almost entirely confined to the new-born whose stomal mucous membrane becomes contaminated through some abrasion. The gonococcus burrows underneath the upper epithelium, producing an acute inflammation. This is followed by the appearance of creamy patches. At the same time the general health is not seriously compromised.

The treatment consists in constant cleansing with a saturated solution of boracic acid; in severe cases, a mixture of boracic acid solution with peroxide of hydrogen is efficient.

These cases are commonly complicated with a gonorrhœal ophthalmia that is much severer and more serious. Syphilitic and diphtheritic stomatitis are treated in their respective places under the headings of the diseases, syphilis and diphtheria. 


\section{Simple Superficial Glossitis}

The superficial epithelium of the tongue is often the seat of disorders which in most cases are of small importance. The transitory catarrhal condition, known as coated tongue, is the accompaniment of most diseases. Here the epithelium becomes weakened and desquamates, and the resulting detritus, when mixed with saliva and particles of food, produces the characteristic coating. This tendency to desquamation exists in some children who in other respects are healthy, so that a coated tongue may be always present.

Another familiar condition may be that of the strawberry tongue of scarlet fever, where the fungiform papillæ are sufficiently swollen to suggest the appearance of a strawberry ; or very rarely one may see a swelling and enlargement of the filiform papillæ constituting the so-called hairy tongue.

More commonly one sees variously shaped, benign patches on the back of the tongue which may result from lack of cleanliness, too much roughness in washing the mouth, or a rough nipple or the sharp edge of a tooth. Outside of the appearance there are no especial symptoms. In the cases where the confluence of the patches, in the centre of which the process of healing is going on, creates a number of irregular lines on the dorsum of the tongue, the name of geographical tongue has been applied. Where the superficial horny epithelium has entirely degenerated and fallen off, the whole tongue may have a strong red color.

For all such conditions no further treatment than washing with boracic acid is needed. If much of the tongue is affected, relief may more rapidly be obtained by local applications of a weak (one-half per cent) solution of argentic nitrate.

\section{Acute Glossitis}

Occasionally a child may, by some accident, hurt or bite his tongue. The great number of micro-organisms in the mouth then renders infection easy. As a result, the tongue may become acutely inflamed, the parenchyma may be swollen and infiltrated with serum and pus, and the child will present the various symptoms which naturally belong to such a condition. These symptoms are rarely serious, for the most part being no more than inconvenient. 
If the organ is much swollen, it may be necessary to feed the child by means of a tube passed through the nostril. Children who are old enough to follow directions can be spared discomfort if they are ordered to keep small pieces of ice in the mouth. In almost all cases, thorough and continued disinfection of the mouth will reduce the disorder. Exceptionally an abscess may result; when it does, one is very apt to overlook it. After opening it, one should cleanse it very often by means of a spray or gargle composed of peroxide of hydrogen and a saturated solution of boracic acid.

\section{Acute Oesophagitis}

Inflammation of the cesophagus in children is rare, and hardly ever does it occur as a primary disorder. The rule is that an

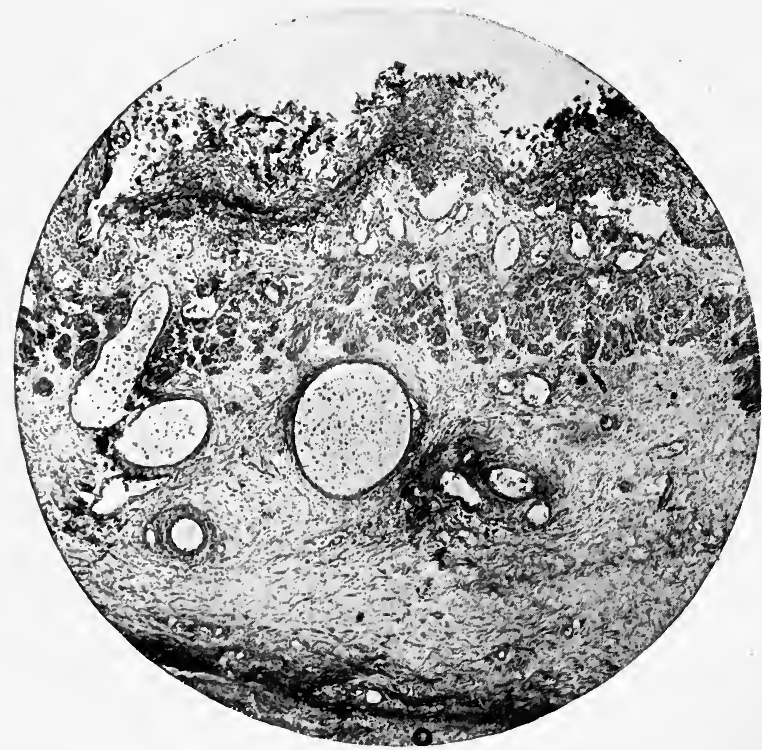

Fig. 3. - Croupous or Membranous Esophagitis. $\times 25$.

acute catarrhal inflammation may follow an extension of a similar disorder in the pharynx or other near-by region, or it may be caused by the passage through the esophagus of some foreign body, which, on account of its size or form, irritates the mucous membrane. Attention to diet and cleanliness of the mouth constitute the treatment. 
As an extension of a croupous inflammation of the pharynx or near-by parts and the stomach, from swallowing irritating chemical substances and foreign bodies, and as a sequel of the acute fevers, a croupous œsophagitis may start up. In case of recovery the injured parts heal by granulation and thus give rise to the possibility of stenosis and contractions of the osophageal tube. These are serious in proportion to the extent of surface involved and the depth of the injury. The consequent deformities follow no special rule and may be of any shape. Following these deformities one may find dilatations which, in turn, may give an opportunity for the creation of a rupture of the wall. The same result may be obtained by an ulcer or localized abscess in the inflamed surface. In consequence, pus or a foreign body may find its way into some neighboring part.

The symptoms of oesophagitis are not easily separated from those of the related parts. The patient suffers from pain, dysphagia, thirst, anorexia, and malaise. The pus, blood, and pieces of membrane which are thrown off in the progress of the disease may be swallowed and then vomited. And the physician should be careful not to ascribe such an occurrence to organic lesions of the stomach.

The treatment is symptomatic.

\section{Retro-CEsophageal LYMph-Adenitis}

Causes. - The causes which produce an inflammation of the retropharyngeal lymph glands may excite a similar inflammation of those behind the cesophagus. The symptoms occur in the form of pain, restlessness, slight dysphagia, fever, and a more or less poorly defined tumor. The swelling may, by pressing upon the pneumogastric nerve, render the condition most critical.

This inflammation may also be tubercular, following a tubercular condition of the cervical vertebræ. In both cases the aid of a surgeon must be sought.

Treatment. - The treatment may be summed up in attention to the symptoms, or tracheotomy. As soon as the case comes under observation one should employ ice and antiseptic sprays in the mouth and ice bags to the neck. The bowels should be thoroughly emptied and the diet restricted to fluids. The patient 
must be confined to bed and protected against the possible irritations which may be involved in eunnecessary exposure and movement.

Prognosis. - The prognosis is bad, especially in the tubercular variety. 


\section{CHAP'TER VIII}

\section{DISEASES OF THE STOMACH AND SMALL INTESTINE}

\section{Acute Functional Derangement of the Stomach}

One of the most frequent ills of children is a disordered condition of the stomach, which is due to wrong feeding. Under this head one includes eating too much, or too rapidly, or eating un-

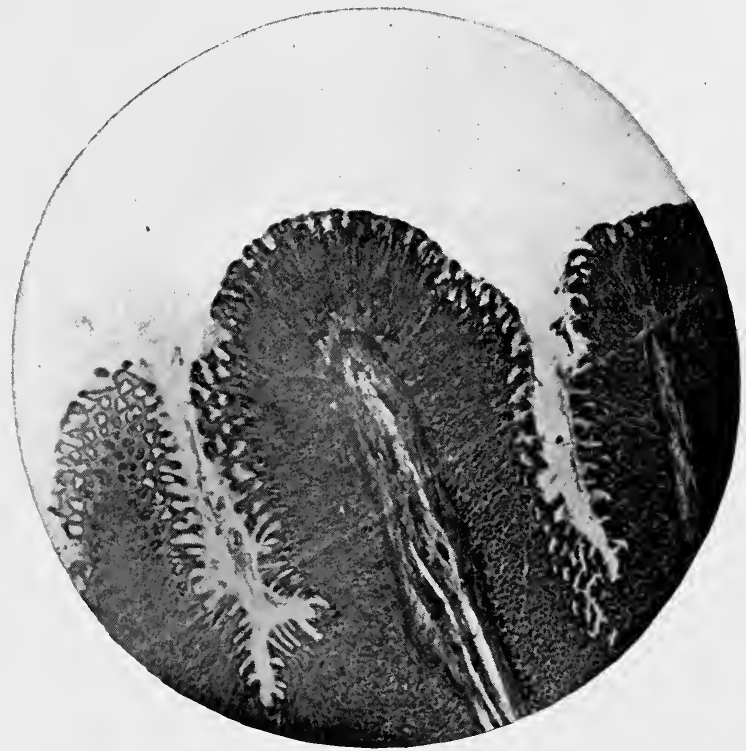

Fig. 4. - Normal Stomach. $\times 10$.

suitable articles of food. Likewise, a sudden nervous shock, an impression of fear, a blow or fall, or even the irritation of dentition, or a fit of temper, may be the starting-point of an arrest of gastric secretions and gastric activity. At the same time, one should not make the mistake of confusing the more serious or longcontinued cases, where a real gastritis may exist, with these instances of nature's attempt to rid the stomach of its undesirable contents. 
Lesions. - There are no characteristic pathological changes, with the possible exception, in individual cases, of transitory congestion, or anæmia of the mucosa. It is only when the abnormal causative conditions from which a distinct gastritis results are continued that characteristic changes occur. We are ignorant of the exact pathology of the nervous depression which at times accompanies this condition. The chemistry of the decomposition of food which at times attends this disorder is likewise a matter of future work.

Symptoms. - The symptoms usually begin with vomiting; in older children it may be accompanied by nausea and pain. The temperature may be slightly or much elevated, and the pulse and respiration may be similarly influenced. The prostration may be variable ; in one case very little, in another very much. Where prostration exists, the picture reminds one at times of a case of poisoning - the possible stupor or delirium, the contracted pupils, the spasmodic vomiting with or without a straining diarrhœa. The tongue is coated, the breath may have a foul odor.

Treatment. - As a rule, these cases recover very rapidly. By emptying the stomach and intestines, providing rest and quiet, and restricting the diet, the evil effects are quick to pass away. For infants, eight or ten quarter-hourly doses of calomel $(0.006 \mathrm{gm}$. $\frac{1}{10} \mathrm{gr}$.) followed by a mild cathartic is efficient. For older children calomel may be used in the same manner, or the citrate of magnesia by the wineglassful every fifteen minutes until the bowels have been thoroughly flushed out. In addition, the stomach should be washed out once or twice daily with plain boiled water until the wash water runs clear. The irritability of the stomach may be quieted by sucking or swallowing bits of ice, by an ice-bag on the epigastrium, or by the counter-irritation of mustard on the same region. After the stomach is quiet, sponge baths or warm full baths have a soothing and refreshing effect. No drugs beside the subgallate of bismuth are required, and this may be used in doses of $0.3 \mathrm{gm}$. (gr. v) every hour or two ; but when convalescence is well started small doses of nux vomica or strychnine, with or without an organic preparation of iron, are often beneficial.

The regulation of the diet is a matter of the first importance. At the beginning all food should be withheld. When the stomach has become quiet, one may begin to give very small quantities of albumin water, diluted milk, or beef extract. 
Infants may begin by taking nourishment by the teaspoonful every hour or two. As the patient shows ability to retain and digest food, the quantity may gradually be increased.

- Every effort should be made to provide rest, good ventilation, proper clothing, and sufficient cleanliness.

Prognosis. - The outlook is good, especially if sufficient care is taken to prevent a relapse or a rapid recurrence of new attacks.

\section{Acute Gastritis}

This disease, formerly spoken of as acute gastric catarrh, acute gastric fever, or acute gastric adenitis, is an acute inflam-

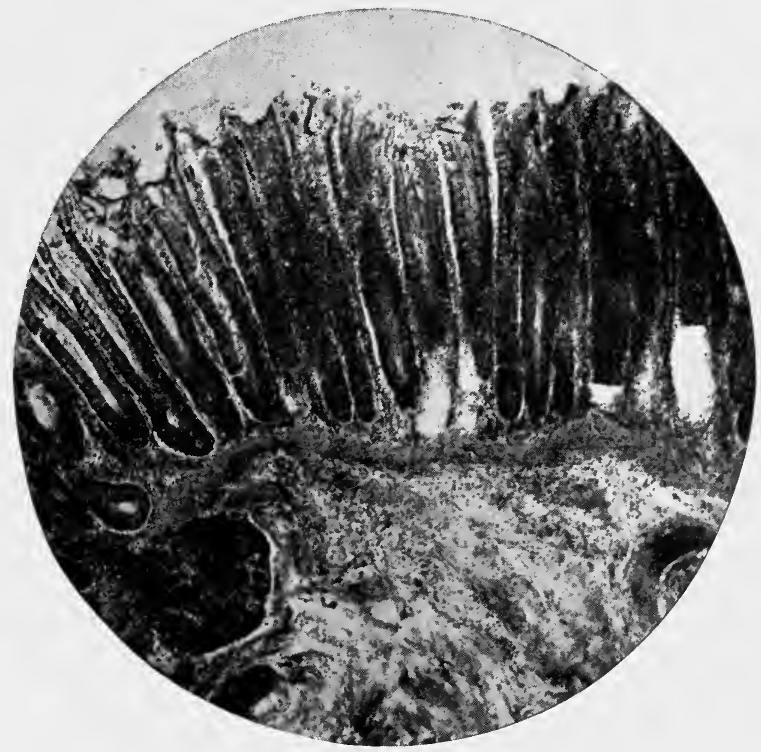

FIG. 5. - Acute Gastritis. $\times 30$.

mation of the glandular parenchyma of the stomach. Outside of the simple form, there are three varieties of inflammation, the croupous, the toxic, and the suppurative. These, on account of their rarity, are of less importance than the simple catarrhal inflammation. They will therefore be spoken of at the end of the section on acute gastritis.

Causes. - The most frequent cause is improper food, improper in chemical composition or purity, or in being excessively heated or cooled; a very common factor is too short an interval between 
the meals, which consequently leads to a congestion of the glandular elements. Likewise, diseases of the intestinal and portal systems, or any disorder, such as diathetic and wasting diseases, which destroys the equilibrium of the economy, may bring about a similar result, especially in hot weather. A factor, at present unestimated, is the irritation which may be produced by bacteria and their toxines as well as by the putrefaction and decomposition of contaminated foods. The chemistry of the subject is so vast that the limits of it no man can now know.

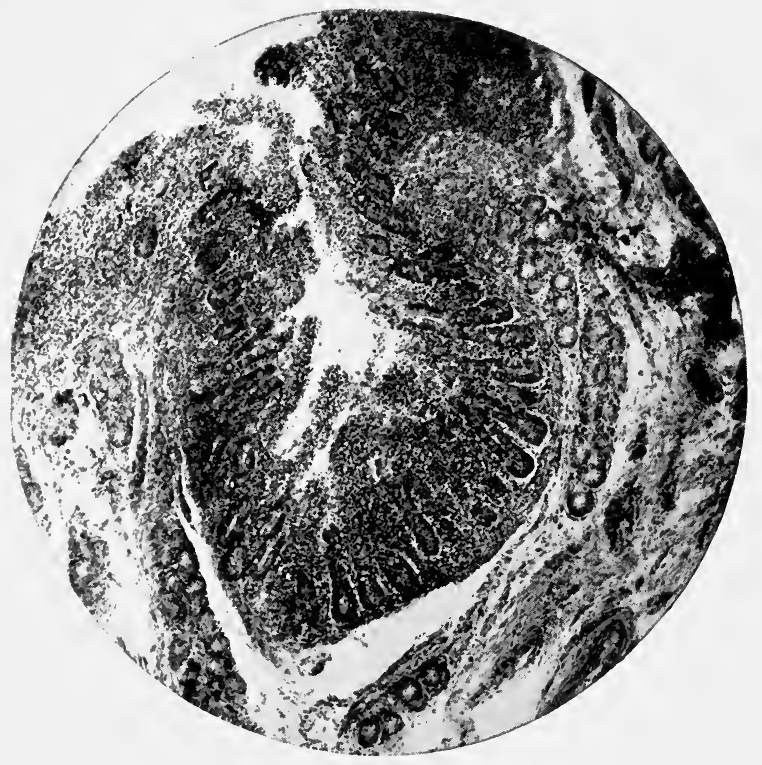

FIG. 6. - Acute Catarrhal Gastritis. $\times 50$.

Lesions. - The gastric surface is a deeper red than usual, although the color is irregularly scattered. The mucosa is swollen, the capillaries injected, the gastric secretions may be increased, decreased, or altered in composition. The epithelial cells are thick, cloudy, and at times eroded here and there. The glands may be swollen, the mucosa and even the submucosa infiltrated with white cells. In very severe cases the parenchyma may have small collections of pus enmeshed in its substance. There is an abnormal amount of mucus present which gravitates to the pyloric portion of the stomach.

Symptoms. - The onset is usually sudden, accompanied by 
varying amounts of pain and fever. The limits between which the fever may run are very wide; in all likelihood the high temperatures, such as $40.5^{\circ}$ and $41^{\circ} \mathrm{C}$. $\left(105^{\circ}\right.$ and $\left.106^{\circ} \mathrm{F}.\right)$, are associated with the absorption of toxines or the products of decomposition rather than a mere catarrhal irritation of the mucosa. The child may suffer much pain, so that the legs may be drawn up and the muscles set. Later on the exhaustion may be so great that the child is prostrated. From the beginning vomiting may be violent and very frequent. The vomitus is sour, usually pale in color, sometimes mixed with bile, rarely streaked with blood. Inspection will occasionally show a slight puffiness of the epigastrium, or on palpation the area may seem hard and tumified. There is naturally some tenderness which, after the lapse of a few hours, may extend to the abdomen. The respiration may be hurried and shallow, and the pulse rapid and weak. In the beginning there may be constipation followed within a short time by diarrhœa, or diarrhœa alone may be present. The urine is high colored, high in specific gravity, and with a strong odor. In severe cases, or in very weak children, it may contain albumin and a large amount of urates.

Complications are commonly seen, often in the way of extensions of the inflammation to other parts of the intestinal track, and frequently, especially in very young children, one sees lesions of the skin, such as erythematous eruptions.

Treatment. - The first step is the withdrawal of all food, until the gastro-intestinal track has been emptied and the stomach has become quiet. In infants one should wash out the stomach as soon as possible and then give small doses of calomel $(0.006 \mathrm{gm}$. $\frac{1}{10} \mathrm{gr}$.) every quarter-hour until thorough catharsis has been obtained or until ten doses have been given. It is considered good practice to follow the calomel with a saline cathartic. After this the lavage may be repeated twice a day until the stomach is in a fairly good condition. The pyrexia may be agreeably and safely controlled by warm baths for infants and graduated baths for older children. When vomiting has fairly ceased one may begin to give food, in the form of albumin water or beef extract, by the teaspoonful. As the child shows ability to retain and digest this, diluted milk may be substituted for it. The return to the ordinary quantity and composition of milk must be gradual and governed by judgment. 
In older children, lavage is not so easily practised, and the procedure of treatment may therefore be somewhat modified. The administration of calomel should be begun as soon as possible, and to make this the more possible the application of an ice-bag or a counter-irritant to the epigastrium, with the sucking of ice, is of use. Then, when practicable, the stomach should be washed out. The diet should be managed in the same careful and conservative manner as with infants; in fact, the more carefully one feels one's return toward an ordinary general diet, the less need

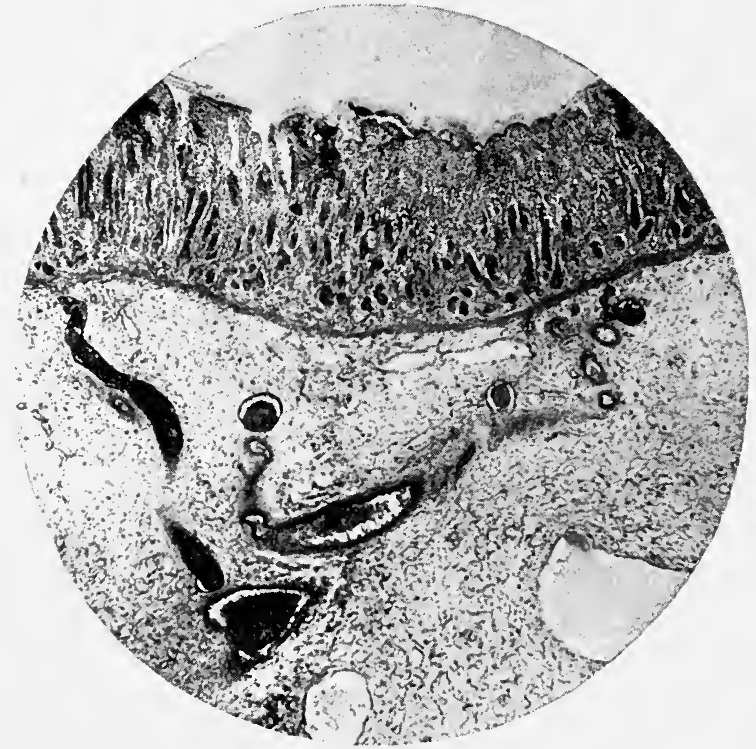

Fig. 7. - Croupous or Membranous Gastritis. $\times 32$.

will there be for medicines. In those cases where medicine is required, the subgallate of bismuth in hourly doses of $0.3 \mathrm{gm}$. (gr. v) will be as unobjectionable as anything. Rarely an alkali before or an acid after meals may be indicated. When convalescence is once established, one may stimulate both secretion and excretion by small doses of nux vomica.

Croupous gastritis is so rare as to be a curiosity; moreover, one is practically not able to diagnosticate it during life unless a piece of membrane should be vomited. Generally it follows a croupous inflammation of the pharynx, cesophagus, or intestines. In some unusual cases it has followed attacks of the exanthemata. 
The symptoms of the original disease cover those of the gastritis, so that we know very little about it except as we see it in autopsies. Usually the membrane is located in scattered areas which may coalesce. The membrane may have a dirty white or a greenish-gray hue, and may be of varying thickness. It is composed of mucus, fibrin, blood, pus, and epithelial cells, epithelial debris, and bacteria. The inflammatory process extends through the mucosa and submucosa.

Toxic gastritis results from the local action of irritant poisons, such as caustic acids and alkalis, salts of mercury, carbolic acid, phosphorus, arsenic, or antimony. There seems to be no limit to the remarkable devices which children are able to contrive by which they may poison themselves. In this way a toxic gastritis may be started by a great number of agents, all the way from matches to green candy and painted toys.

Lesions. - The lesions vary in severity according to the concentration of the poison, its amount, and the length of time which it remains in the stomach; usually they are most marked at the fundus. The effects produced are some of the various degrees of destruction of tissue. They may vary from intense congestive changes to sloughs of the superficial tissues and even to an amorphic, soft, pulp-like condition that easily permits of perforation. After the less serious injuries, contractions and scars may form which materially alter the shape, relation, and functional activity of the organ.

The severe degeneration of tissue caused by a toxic gastritis must be distinguished from gastromalacia or softening of a part of the stomach wall, which has often been seen some hours after death. It is supposed to be caused by a digestion of the part where the gastric secretions gravitate. For this reason almost always the posterior wall is affected. The tissue is soft, faded in color, and of a disagreeable odor.

Treatment. - The treatment of toxic gastritis must often be decided by the conditions of individual cases. In ordinary cases the stomach should be thoroughly washed out and the proper antidote administered. One must keep in mind the possibility of producing a perforation in the injured tissue by means of the stomach tube. Acid poisons should be neutralized by the administration of dilute alkalis, and alkaline poisons by dilute acids. If the child begins to recover, the diet should be most carefully 
regulated so that, until the ability to digest food is assured, no harsher nourishment than albumin water or a weak modified milk should be given. It is often advisable to keep the stomach clear by daily lavage until convalescence is well advanced.

Suppurative gastritis is characterized by infiltrations of pus in the connective tissue of the stomach. It occurs in conditions of marked physical debility, marasmus, and after severe attacks of the acute infectious diseases. The pus-areas are microscopic, although some observers have mentioned cases where they developed sufficiently to be seen by the naked eye. The disease is recognized on autopsy. There is no special treatment for it.

\section{Chronic Gastritis}

Causes. - This is a prolonged inflammation of the gastric mucosa attended in severe cases by more or less permanent changes in the parenchyma. In its ætiology an acute gastritis which is allowed to continue or frequently to recrudesce holds an important place. Other responsible factors are diseases of wasting and malnutrition, atonic conditions following the acute infectious fevers, disorders of other parts of the intestinal track, and diseases of the heart and lungs.

Lesions. - On the whole, the changes are the logical outcome of those in the acute variety. The characteristic structure of the parenchyma is gradually altered. The tubules may become enlarged and obliterated, the cells cloudy, choked, and finally destroyed. The mucosa is seen to be roughened, and in protracted cases this characteristic is contributed to the submucosa. In some severe and long-continued cases among older children, these structures are encroached upon by a deposition of plain connective tissue. There is an infiltration of small cells which in rare cases penetrates as far as the submucosa.

Commonly there is a condition of gastric dilatation. Although this is seen often enough in older children, nevertheless, I believe it is still more frequently seen in the younger patients whose tissues have less power to resist the causes of gastric dilatation. In an undilated organ, the chronic process is severest near the pyloric orifice; where dilatation exists, the whole stomach is more apt to be involved. In such cases there is a greater likelihood of parenchymatous degeneration which is beyond repair. In the subjects 
of extreme wasting an infiltration of fat globules may take place, which likewise is final.

On section the interior is seen to be coated with a thick, sticky mucus which naturally collects in largest quantity at the pylorus. The color of the mucous membrane in the more recent cases may be a dull, heavy red; in those of long duration the hue fades to a dull, poorly defined gray. These colors are not necessarily uniform, so that in one subject we may see many shades that vary from dark to almost white.

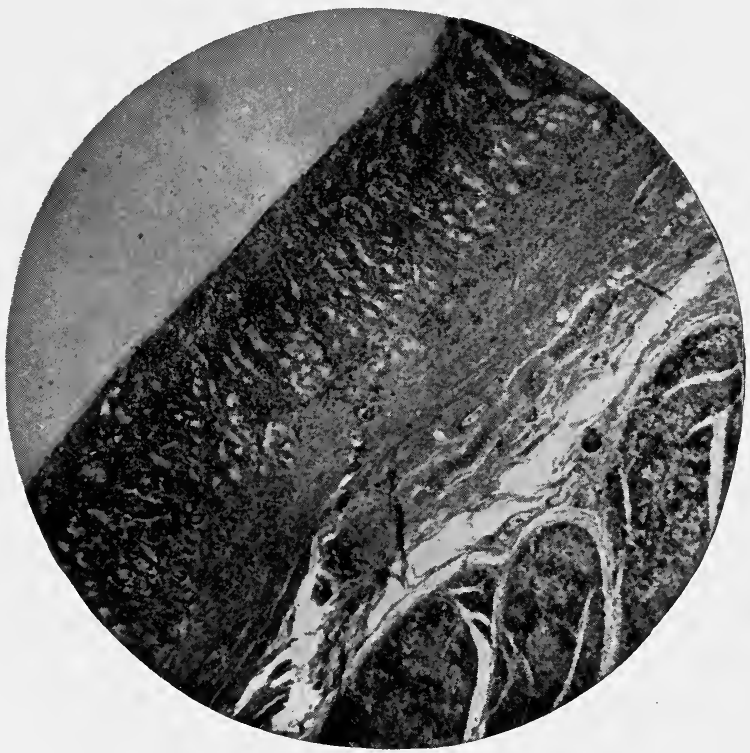

FIG. 8. - Chronic Gastritis. $\times 30$.

Symptoms. - Generally it is easy to demonstrate by light percussion and succussion the tendency to dilatation. The abdomen may be hard to the touch, and pressure over the epigastrium as well as over the sigmoid flexure will often show the presence of tenderness. The child is apt to look flabby and white or emaciated. The eyes are dull, the tongue is coated, and the general condition is poor. On account of this atonic state, the mucous membrane of the throat may become congested and relaxed so that an obstinate pharyngeal cough may result. There may be frontal headache in children who are old enough to express their sensations in words. The appetite is capricious or deficient, and 
at varying times after eating vomiting is apt to ensue. Intestinal action is irregular, so that there are periods of constipation, varied by attacks of diarrhoea. The patient's sleep is restless, fitful, and disturbed.

Some of the main symptoms arise from the imperfect digestion of food. Much of this material remains in the stomach for hours; proteids are only partly changed and some of the albumenoses thus formed are poisonous. Evidence of imperfect digestion, especially of casein, is furnished by the presence of unchanged or partly changed masses of food in the stool. Also, the fats, mucus, and albumins ferment and decompose, with the resulting formation of fatty acids, gases, and a multitude of by-products whose chemistry is hard to follow. Absorption necessarily brings various degrees of low toxic conditions which not merely affect the body but also cause strange and remote subjective mental impressions. This subject of intoxication is worthy of the most serious attention; for not only may it give rise to a multitude of mental symptoms, but also the possibility of permanent physical as well as psychical effects is always present. Certainly the influence upon development has an imminent potentiality of serious results.

Treatment. - The cure of these cases is simple in principle, although individual cases may call for the exercise of much judgment and tact. The main indications look to the rigid regulation of diet, the cleanliness of the gastro-intestinal track, and the support of the patient's vitality. In infants the cutting off of all food for a few hours, followed by the administration of small quantities of albumin water, weak modified milk, or beef extract for a few days is highly desirable. Gradually then, as the condition of the stomach permits, the amount may be increased. In older children food may at first be confined to clear meat soups, milk, soft-boiled eggs, and scraped beef in small quantities. These may be so varied that, with the addition of dry toast as the treatment of the case progresses, no less easily digestible articles of food will be needed for some time.

Cleanliness of the gastro-intestinal track may be promoted by washing the stomach out once or twice a day with plain boiled water. For this operation one needs a soft rubber catheter (18 to 25 French); this is joined by a glass tube to one end of a rubber pipe about $0.5 \mathrm{~m}$. (about 20 inches) in length, while into the other 
end a glass funnel is inserted. The procedure of passing the catheter is very simple. The child's arms are confined to his sides by pinning a towel about them ; he is seated on an attendant's lap and his head is held by attendant's right hand. The operator's left forefinger is laid along the patient's tongue to act as a director, and then with a quick movement of the right hand the catheter is passed through the osophageal opening. When the tube is in the stomach, the funnel is raised high in order to facilitate the escape of gas; this is followed by the introduction of water, which, when the stomach is nearly full, is siphoned out. The operation may be repeated till the wash water runs clear. As it may be necessary to continue this treatment for weeks, the mother or nurse may be instructed in its details.

The medicinal treatment has of late years been growing progressively less important. The drug which will give the most help is nux vomica or its alkaloid, strychnine. This, by promoting peristalsis and muscular tone, may be of real service. It may be combined, in cases of hyperacidity, with an alkali, or where anacidity exists with dilute hydrochloric acid. When there is need for the acid, it must, to be of service, be given in large doses (five drops four times a day, for a child of one year). Outside of these drugs, one may, when convalesence is established, use small doses of an organic preparation of iron as a tonic. All through the sickness, the utmost care must be given to hygiene and control of the patient's general mode of life. Rest, exercise, bathing, and clothing must be carefully reviewed and regulated.

Differential Diagnosis. - In most cases the nature of the disorder is fairly clear. The history of successive acute attacks, which finally merge into a chronic process, or of a chronic disposition from the beginning, is fairly characteristic. One of the best methods of differentiation is by means of a test meal, which is to consist of milk in infants, of bread and milk in somewhat older children, and of milk, bread, and meat in children of eight or more years. After the meal has been in the stomach for an hour, it should be siphoned out with the stomach tube and analyzed by the well-known methods which are given at length in special works. The main objects of information are increased secretion of hydrochloric acid, decreased secretion of the hydrochloric acid, and the quantity of mucus. At the same time one may obtain additional information concerning putrefaction and decomposition 
of food, the formation of gases, and the presence in the stomach of injurious by-products.

In some cases the child's condition is so much depressed that the possibility of tuberculosis or other wasting diseases must be considered. But the absence of characteristic temperature and localized manifestations will bar the first, while the peculiar signs of rickets, scurvy, and similar disorders of nutrition will likewise be wanting. The process of exclusion is surely, although gradually, completed by extended observation.

Prognosis. - The outlook under favorable conditions is good. An ignorant, stupid parent, who does not know how to control his child, is more of an obstacle in the way of the patient's recovery than the disease itself.

\section{Dilatation of the Stomach}

Causes. - Various degrees of gastric dilatation are commonly seen, especially in infants. In such young children any attack of functional derangement or gastritis may bring about a temporary dilatation of the weak muscular walls. It is not often, however, that such a condition takes on a permanent phase. But repeated attacks of acute gastritis or a chronic gastritis are very apt to bring with them a dilatation which may have a marked persistence. This is in part due to the piling up of food in the stomach and the production of gases of decomposition which occurs when peristalsis is deficient. In this way any atonic condition could bring about a similar result. For this reason we are very apt to see this disorder in marasmus, in rachitis, in the wasting diseases. In fact, all conditions, such as stenosis of the pylorus or partial obstruction of the intestine, which are able to dam back the flow of food, must necessarily cause a gastric dilatation.

In some rare cases partial or sacculated dilatations may follow the swallowing of coins, buttons, pieces of wood and similar bodies which are too large to pass through the pylorus.

Symptoms. - Very rarely does dilatation occur without a concomitant chronic inflammation of the mucous membrane. The symptoms of the two disorders almost always coincide, excepting that in marked dilatation the absorption of the products of fermentation and the consequent low forms of poisoning may be prominent. In both the same signs of vomiting, constipation and 
diarrhœea, headache, coated tongue, malnutrition, lack of appetite, and general weakness may be present. In dilatation the vomiting may be more pronounced and in older children the appetite may be some what better, especially at intervals, when the stomach has been emptied. The main differential symptom is furnished by percussion. This, if carefully practised about three hours after eating, gives a tympanic note nearly to or even below the umbilicus. One's opinion in the matter may be verified by filling the stomach with water, percussing anew, and noting the quantity of water used. Carefulness in such ways will prevent confusing a dilated stomach with a dilated transverse colon. There is always a danger of such confusion, because in both conditions, especially if the patient has thin, weak body walls, there may be a prominence.in and below the epigastric region, with tenderness on pressure.

Treatment. - The treatment is likewise similar to that of chronic gastritis. The stomach should be washed out every day, in older children before the principal meal. The diet should be restricted in quantity and variety of food. For infants, albumin water, beef extract, and diluted milk may be given in smaller amounts and at longer intervals than a normal baby demands. Older children may have diluted milk, clear meat soups, scraped beef, and dry toast. As they improve, this diet may be enlarged by the addition of broiled steaks and chops with some easily digested fresh vegetable. Considerable help may be obtained by the liberal use of nux vomica or strychnine with the addition, when improvement sets in, of an organic preparation of iron.

A wise regulation of exercise, of the hygienic and sanitary arrangements as well as the general mode of life is of the utmost importance.

Prognosis. - The outlook is fairly good, excepting where the condition is dependent upon an organic pathological condition which, like stenosis of the pylorus, does not easily permit of cure. The chances of recovery are better in children than in adults.

\section{UlCER OF The Stomach}

Causes.-Although gastric ulcer is rare, it is nevertheless apt to appear at any time, in all classes of patients and at any age. The cause in most cases is obscure. An ulcer may be present at birth; on autopsy it has been found in cases of the hæmorrhagic diseases of 
the new-born ; it may occur in chronic gastritis where a portion of the mucous membrane becomes injured or loses its full blood supply. Or it may be the result of tubercular deposit and degeneration. In other rare cases, the small pus-foci of suppurative gastritis may coalesce and form a large ulcer. The round perforating ulcer has generally a mysterious atiology; the most logical explanation lies in an embolism of the gastric arteries or their branches by which the vitality of any part is so far debased that necrosis may occur; the lesion naturally would be exaggerated by the action of the gastric secretions upon the devitalized spot. In somewhat the same way an embolism of the umbilical vein after birth might indirectly cause an ulcer of the stomach. In all cases one must reckon on having an exhausted physical condition as a precedent fact. Purpura hæmorrhagica may be a predisposing factor.

Lesions. - These vary according to the child's vitality and the individual disposition of the disease. Sometimes the mucous membrane alone is attacked; again, the successive tissues are eaten through so that the opening extends through the peritoneal coats. In some cases the hole looks as if made by an instrument; in others it slopes like a funnel, the large end being in the mucous membrane. These latter have a margin that may be in a condition of intense congestion or even necrosis. The surrounding area is usually much congested, although occasionally it is fairly normal. No especial portion may be assigned as a favorite location.

Symptoms. - The signs are obscure. There may be diarrhœa and vomiting, the latter being the more prominent. In the vomitus may be variable quantities of blood, or, if a large artery breaks down, there may be a vomiting of clear blood. In such cases, as well as in some of those where the hæmorrhage is not so great, blood will be found in the stools. In some cases there are pain, tenderness on pressure, and active signs of disorders of digestion, which are especially prominent shortly after taking food. In others the symptoms may come on suddenly and with few premonitory signs.

The temperature varies so much that no characteristic course can be given; at times it is slightly raised, but at others it is very high. If perforation occurs, it may drop to subnormal; in the presence of shock this may be maintained, but if the child survives long enough to undergo a peritonitis, the fever may again rise very high. In a considerable proportion of the cases a stenosis of the pylorus is apt to result. 
Treatment. - Very little can be done for these cases. One should order complete rest, should give ice, and small doses of opium. Nourishment is best given in the form of peptonized milk or beef extract by rectum. Direct medication of the stomach is of questionable value.

Prognosis. - The outlook is bad.

\section{Hamorrhagic Erosions of the Gastric Mucous}

\section{Membrane}

In debilitated children of any age the mucous membrane, generally in the region of the pylorus, may become eroded. Outside of debility no cause is known. The destruction of tissue is superficial, and the only symptoms are irritability, pain, disordered digestion, and blood-streaked vomitus.

There is no method of ascertaining the existence of this condition except by autopsy. Consequently no treatment can be prescribed.

\section{Gastralgia}

Gastralgia is a functional irritation of the peripheral nerves in and about the stomach, whose main expression is pain in various degrees. What its cause is, outside of local and general atony, has never been fully demonstrated. It may be associated with organic diseases or be entirely divorced from them. The stomach and epigastrium may be hard and contracted. The pain is situated in the epigastrium and may be sharp, heavy, or spasmodic. It is apt to be periodic, in many cases it appears at night, and may occur when the stomach is empty or full. When it exists in a pure form there is no necessary rise of temperature, but there may or may not be considerable depression. It may come suddenly and leave as quickly.

Treatment. - Before adopting means of relief, one should be sure that there is no complicating gastritis or gastric ulcer, whose treatment would deserve consideration. Uncomplicated gastralgia may be relieved by rest in bed, the application of a hot water bag, and the administration of a few doses of antipyrin in combination with aromatic spirits of ammonia. The antipyrin may be given in doses of $0.06 \mathrm{gm}$. ( $1 \mathrm{gr}$.) for each year of age up to $0.3 \mathrm{gm}$. ( $5 \mathrm{gr}$.).

Some persons seem predisposed to successive attacks of this 
disorder. Strict attention to the general health and a wise regulation of daily life are the best preventives.

\section{HeMATEMESIS}

This is a symptom that may occur without discoverable lesions. It is sometimes seen in the newly born, in "bleeders" (hæmophiliacs), in purpura hæmorrhagica, in scorbutus, in malaria, in profound anæmia, in vicarious menstruation, in trauma, in malignant forms of the acute infectious diseases. The blood may or may not appear in the stools.

One should be careful not to be deceived by blood which was swallowed, either in hæmorrhage of the mouth, nose, or throat, or from a cracked nipple. Likewise the distinction between hæmatemesis and hrmoptysis must be made. In the latter the blood is bright red, frothy, mixed with mucus; in the former it has a dark color, is thick, and commonly is accompanied by various quantities and sorts of food.

The treatment includes rest, the use of ice, and at times opium in small doses. Stimulants may be given hypodermically.

The prognosis varies with the cause and circumstances of each case.

\section{DuODENitis}

An inflammation of the duodenum may occur alone, but as a rule it spreads in some further direction, or is the result of a communication from a neighboring part. In this way we may find the stomach and duodenum associated, or the duodenum and common bile-duct, or the duodenum and the rest of the small intestine. As an example, an acute gastritis may be followed by an acute gastro-duodenitis, or ulcer of the stomach by ulcer of the duodenum. An irritant poison will not necessarily confine itself to the stomach, but may affect the whole or any part of the small intestine as well. There are even a few seemingly mysterious cases on record where severe burns of the skin, which destroy the vaso-motor nerve filaments in the skin and thus bring about deep-seated congestions, have been followed by the formation of ulcers in the duodenum, or ileum, or both. As a rule, the inflammation that one finds is catarrhal, with varying degrees of congestion of the mucous membrane, and the over-production of mucus. The only symptoms that one can trace are pain, 
vomiting, constipation or diarrhœa, some elevation of temperature, and a moderate degree of prostration. Usually, since a neighboring organ is involved, there will be present the symptoms of such extension. Thus one may see the jaundice that follows inflammation of the common bile-duct or the swelling of the solitary and agminated glands in the ileum. This last complication constitutes one of the dangers of the duodenitis; for these glands may, after swelling, soften and break down. The ulcers that result may be of any depth, may even perforate the intestinal wall, causing peritonitis and death.

The treatment of duodenitis is usually combined with that of the associated disease. In itself it includes rest in bed, catharsis by means of a saline or calomel followed by a saline, and the restrictions of the diet to fluids. The possibility of a sharp differential diagnosis is limited by the fact that the disease is almost always an extension of a gastritis or enteritis.

The prognosis, except in case of serious involvement of neighboring organs, is good.

\section{Chronic Duodenitis}

This disease is one that is often enough seen, but not as often recognized. It so readily suggests disease of the large intestine, and in the resulting confusion has received so many different names, that many practitioners are not to be blamed for regarding it as somewhat obscure. It has been called chronic gastro-intestinal catarrh, chronic gastro-duodenal catarrh, mucous disease, chronic pseudo-membranous gastro-enteritis, chronic muco-colitis, intestinal desquamative catarrh, and several other names.

Causes. - Its ætiology is hard to determine; no micro-organism has been definitely associated with it, nor has any clearly defined connection been shown with a recognized form of intestinal abuse. Thus one can have much sympathy with the older views which regarded it as the product of an intestinal neurosis. As a matter of fact, we know that it is likely to follow an exhausting sickness or a debilitated condition. Moreover, a child that has a bad diet for weeks and months is liable sooner or later to be afflicted with this disease. In addition, the acidulated chyme of gastric hyperacidity or the alkaline fermentation of gastric anacidity is sufficient to set up an irritation in the duodenum that may run a chronic course. Still there are occasional 
cases which simply baffle an inquiry into their origin. There is the same uncertainty in regard to the age at which the disease usually occurs. Thus, 'as a rule, it is seen in children from four years up to ten or twelve. Nevertheless, one of the most obstinate examples of it that I have seen was in a baby of fifteen months who had had the advantages of reasonably good care.

Lesions. - The changes at first occur in the duodenum. From there they may spread to the rest of the intestine or the stomach. 'The mucous membrane is congested, thick and heavy, and pours out a large amount of mucus. The glands and follicles may be swollen and in marked cases may break down and ulcerate.

Symptoms. - The disease is apt to begin in a subacute manner, and the child suffers a series of intestinal or gastro-intestinal disorders. At the same time the general nutrition is debased, the nervous equilibrium is poor, and invasion by intercurrent diseases is weakly resisted. The temperature never runs very high excepting when an acute exacerbation occurs. The patient looks depressed and run down, his tongue may be somewhat coated, or dotted with shallow aphthous ulcerations, or may merely be red and shiny. The nose and throat may, doubtless as a result of debility, be the seat of catarrhal inflammation. There may be tenderness and pain in the left hypochondrium and abdomen, which run an irregular course.

Occasionally there are gastric disturbances, but not so frequently as disorders of the intestines. These latter appear in the form of constipation or diarrhoea, oftener the latter. Both may be accompanied by the discharge of mucus, whose amount is strikingly and abnormally large. Sometimes this comes in masses, more often it is worked all through the movement; again it constitutes the whole movement, replacing all fæces. The fæcal movements are often characteristically clay-colored.

As the disease progresses, especially in the absence of wise efforts to uphold nutrition, the child becomes emaciated, his skin turns dry and harsh, and nervous symptoms become pronounced. Hysteria and disturbances of the sense-functions present manifold symptoms which may blindly mislead the relatives as well as the physician. Under such circumstances subjective impressions upon the child and temporary moral defects are common. In other cases, attacks of skin diseases - dermatitis, eczema, and herpes of the lips and genitals - add their influence to make confusion.

No matter how many related symptoms exist, if the attention 
is fixed upon the characteristic signs, one will usually make a correct diagnosis. These are the chronic course, the mental irritability, the clay-colored stools and abnormal amounts of mucus, the dry, harsh skin, the abnormal tenderness, pain, and distension, the intestinal irregularity and lack of high temperature. If these are kept in mind, one will not be deceived into naming intestinal parasites, tuberculosis, or nervous disorders as the basis of the disease.

Treatment. - The care of these cases demands the utmost vigilance, judgment, and tact. Intestinal antiseptics are useless, astringents are even harmful. The main reliance should be put upon the stimulants and tonics, and of these nux vomica or strychnine is the chief. Other drugs may from time to time be needed, such as bismuth subgallate for diarrhoea, calomel or citrate of magnesia for constipation, or small doses of opium for pain. The nose and throat may require alkaline douches, the skin may need massage, and every detail of hygienic and sanitary management must be carefully supervised. Whenever the weather permits, the patient should be in the air as long as possible, the bedroom and the nursery must be cheeríul and well ventilated, and the clothing proper in quality and weight. One of the most important items of treatment is the diet. This must as far as possible admit proteids and exclude sugars and starches. Milk, if possible, should be excluded; if it cannot be, then it should at least be diluted enough to make a weak cream and sugar percentage. In addition one may order clear meat soups, beef extract, lean meats, soft-boiled or poached eggs, and dry toast. Much food may not be given at one time; therefore it may be advisable, even for well-advanced children, to have four small, instead of three large, meals per day. The patient each day should have a cool bath, which is to be followed by vigorous friction.

As the child shows improvement, the nux vomica or strychnine may be supplemented by small doses of an organic preparation of iron.

Prognosis. - The outlook must include a fairly long course with a fairly certain recovery. Parents must be warned that infringement of the diet will, in all probability, mean a relapse, and that this diet must be maintained until the child is free from mucous stools. Recovery will be hastened by avoiding a too free or indiscriminate medication. 


\section{CHAPTER IX}

\section{DISEASES OF THE PANCREAS, LIVER, AND SPLEEN}

\section{The Pancreas}

The main pathological condition which one meets in children is the acute parenchymatous pancreatitis which occurs with the acute infectious diseases. This is characterized by congestion and swelling and a degeneration of the epithelial tissue. When convalescence is chronically retarded, there may be a formation of new connective tissue.

There may be a fatty or an amyloid degeneration of the pancreas which occurs when a similar process attacks other viscera, such as the liver or the spleen. The causes and course of the changes are the same in all.

\section{ICTERUS}

Icterus, commonly called jaundice, is a yellow coloration of the sclerotic membrane, mucous membrane, and skin, caused by the absorption of bile pigment. It is a symptom which has in some cases no pathological importance; in others it may accompany serious organic changes. The simple form seen at birth is said to arise from a disturbance of the circulatory equilibrium which accompanies the infant's entrance into life. At the same time congenital obliteration of the cystic and hepatic ducts, either above or below their junction, can bring about the same result. Other varieties of obstruction may be caused, in rare cases, through the plugging of a duct by a round worm, by congestion of the lining membrane of the duct, or by pressure resulting from organic disease and growths in adjacent structures. Outside of these hepatogenous forms, there are some non-obstructive or hæmatogenous cases produced by malaria, pyogenic inflammation of the umbilical vein, gastro-enteritis, pyæmia, yellow fever, Winckel's disease, Buhl's disease, syphilis, the inhalation of large quantities of ether or chloroform, and poisoning by certain metals, such as phosphorus. 
Some animal and vegetable poisons at times bring about the same result, but the action is not regular and inevitable.

The treatment varies according to the cause ; in icterus neonatorum no treatment is necessary. The discoloration occurs within three days after birth and may persist for one or two weeks.

\section{Functional Derangements of the Liver}

These derangements are but little understood, and, moreover, are very hard to investigate. They should not be classed among fatal

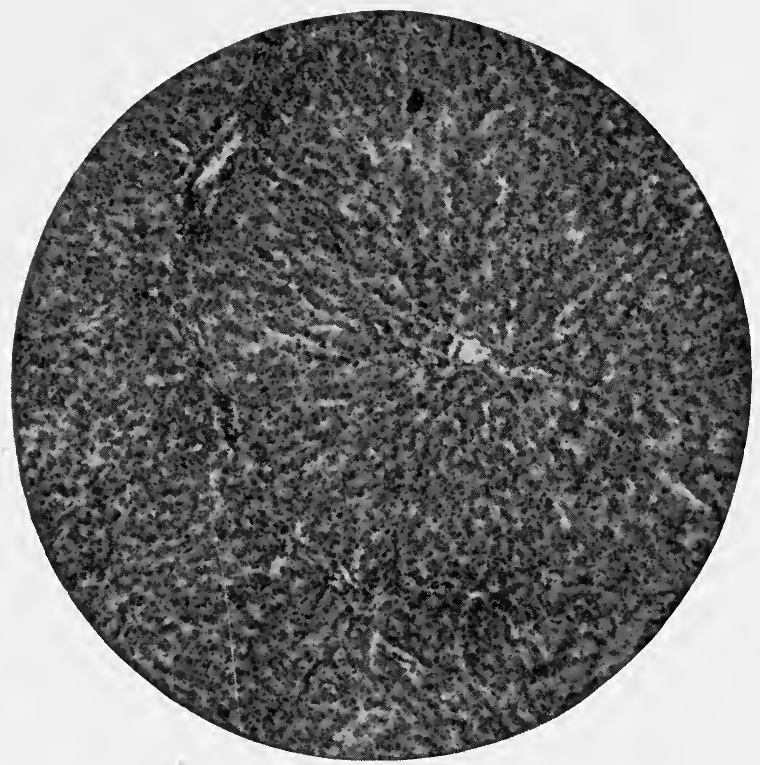

Fig. 9. - Normal Liver. $\times 50$.

diseases, and although they are not rare during life, nevertheless one is not often able to find them in post-mortem examinations, excepting under such circumstances as would at all events interfere with the normal function and structure of the liver.

The usual symiptoms that we associate with hepatic functional derangements are hard to differentiate sharply from those of derangements of the small intestine. The stools are apt to be hard and gray, and there may be evidences of much decomposition of intestinal contents, with the consequent absorption of products of putrefaction, and there may or may not be some degree of jaun- 
dice. Children react quickly and fully to such intoxication, mentally as well as physically. I believe that many abnormal psychical states, many instances of viciousness, of rebellion against control may be - as I have stated in a former publication - traced to some error in nutrition, such as hepatic derangement; and, doubtless, more frequently to it than to any other allied disorder.

The usual treatment consists in free catharsis by means of calomel and a saline cathartic, joined with rest in bed, and a strict confinement of the diet to fluids. Frequent warm baths will prove to be of considerable value.

\section{Congestion of the Liver}

This disorder may occur in two forms which from their ætiology one may call primary and secondary. The first occurs as the result of errors in diet, especially where the child hạs been allowed to have rich and spiced foods or alcohol in comparatively large doses. Malnutrition, or the existence of prostrating heat, renders him still more liable to the disorder. The secondary form follows chronic malarial poisoning, scurvy, syphilis, emphysema, pleurisy with effusion, valvular diseases of the heart, and abnormal conditions producing pressure upon the vena cava.

The result is an enlargement of the liver, except in very longcontinued cases. This enlargement is easily made out by percussion and palpation. The microscopical changes are those of congestion in the acini followed, if the process be protracted, by fatty degeneration.

In addition to the enlargement of the liver, there is generally, although not always, some jaundice. It is not difficult to convince oneself whether the congestion is primary or secondary, although in both cases symptoms of gastro-intestinal derangement are present.

The treatment in both cases requires the careful regulation of diet, cutting off of rich and highly seasoned food, decrease or elimination of alcohols, and the administration of calomel, followed by small and repeated doses of saline cathartics. The later use of acids and intestinal tonics is advisable. In this disorder the compound mixture of rhubarb and soda, one teaspoonful thrice daily for a child of six years, has value. If the congestion is syphilitio, it will yield to the specific treatment as given in the section on syphilis. 


\section{Parenchymatous Hepatitis}

In the presence of certain pathological conditions the liver, as well as other viscera, becomes acutely affected with parenchymatous degeneration. These conditions are, for the most part, the acute infectious fevers, such as diphtheria, scarlet fever, acute lobar pneumonia, typhoid and typhus fevers, measles, chicken-pox, and yellow fever. Other diseases which are characterized by marked toxic effects, such as septicæmia and erysipelas, may bring about the same result.

When this degeneration happens, the organ increases in size and loses something of its characteristic formation. The hepatic cells become congested, swollen, and choked up, and may start in a process of deterioration and breaking down. Under conditions of faulty environment and nutrition, the parenchymatous hepatitis may be followed by a state of fatty infiltration with a consequent destruction of structure.

The symptoms, when they are recognized, are usually classified under disorders of the stomach and small intestine. The treatment, outside of what the original disease demands, consists in the strict regulation of diet and enforced inactivity. The main danger in the disease is the possibility of forming an unlookedfor complication of the original sickness.

\section{Purulent Hepatitis}

Purulent inflammation of the liver may result directly from an injury or indirectly from an antecedent infecting cause. In the latter case many different sources may be responsible for the injury. Even if the original focus of suppuration is far removed from the liver, the general circulation is always able to act as an efficient vehicle. Thus, not only have septic inflammations of the gall-ducts and portal veins been the starting-point, but also septic processes of the umbilical vein, ulcers of the stomach, parasites from the intestine, typhoid fever, ileo-colitis, appendicitis, and other intestinal ulcers have accomplished the same result.

When such a purulent inflammation has been set in motion, it regularly culminates in an abscess. Excepting after traumatism, the abscesses are usually multiple, but by their confluence they form one large mass. As the pus approaches the surface, the re- 
sistance becomes less until perforation occurs into intestines, peritonæum, stomach, lungs, pleura, pericardium, or through the chest wall. The metastatic abscesses of pyæmia have the same natural history as those in other parts of the body.

Symptoms. - Where there is a history of trauma, the child will immediately suffer from pain and possibly from shock. The characteristic signs of suppuration may not show themselves for some days. Then pain is apt to reappear, the child may have a chill, and the temperature will rise. Where the cause depends upon a previous pathological condition, the symptoms of such disease are niaturally first seen, and those due to the involvement appear later as a sequel or complication. At times the condition is confusing, but the main things which one must keep in mind are the local symptoms of pain and tenderness and those of suppuration. There is almost always an increase in the size of the liver, which most often may be noticed below the free border of the ribs. If the increase in size is directed toward other viscera, the pressure will produce corresponding signs in proportion to its extent. In addition there will be pain over the liver, over the abdomen, in the chest, or even in the right shoulder; generally there is tenderness in the hepatic region, although its location does not inevitably define the position of the abscess. The temperature is irregular, intermittent, or remittent. There may be an initial chill or daily chills. The prostration increases, wasting becomes marked, and in many cases jaundice appears in varying degrees. As the sickness becomes more profound, stupor and delirium supervene, and the picture becomes one of pyogenic intoxication and rapid decline.

Differential Diagnosis. - In the event of trauma to or about the liver, the history of the case immediately directs the attention to the possibility of a pus-process in this region. But in the other cases the diagnosis is apt to be more obscure, and at times must be made by exclusion. If there are signs of pus, and if other localities are evidently clear, one should explore the liver with an aspirator or hypodermatic syringe in the attempt to find pus. A failure to find it is no conclusive proof that it does not exist; and one should always remember the intimate relations, as far as symptoms go, that exist between purulent hepatitis, pus in the right pleural cavity, and right diaphragmatic or subdiaphragmatic abscess. In a fair proportion of cases it is practically impossible to 
locate the lesion without definite exploratory evidence. Usually one can without much hesitation distinguish purulent hepatitis from the enlarged liver that occurs with cardiac disease, tumors, syphilis, or hypertrophic cirrhosis. For the combination of chills, local pain, the irregular temperature, and the general picture of developing sepsis prevents confusion.

Treatment. - These cases should be referred to a surgeon as soon as the diagnosis is made. The sooner they are operated upon, the better are their chances for recovery. Under such measures a large majority of the cases may be saved. In all events, the course of the case is apt to be protracted, and the child may not regain his health for weeks or months.

\section{Interstitial Hepatitis}

Causes. - This disease is generally regarded as belonging to adult life; and it is practically never seen in the first half of

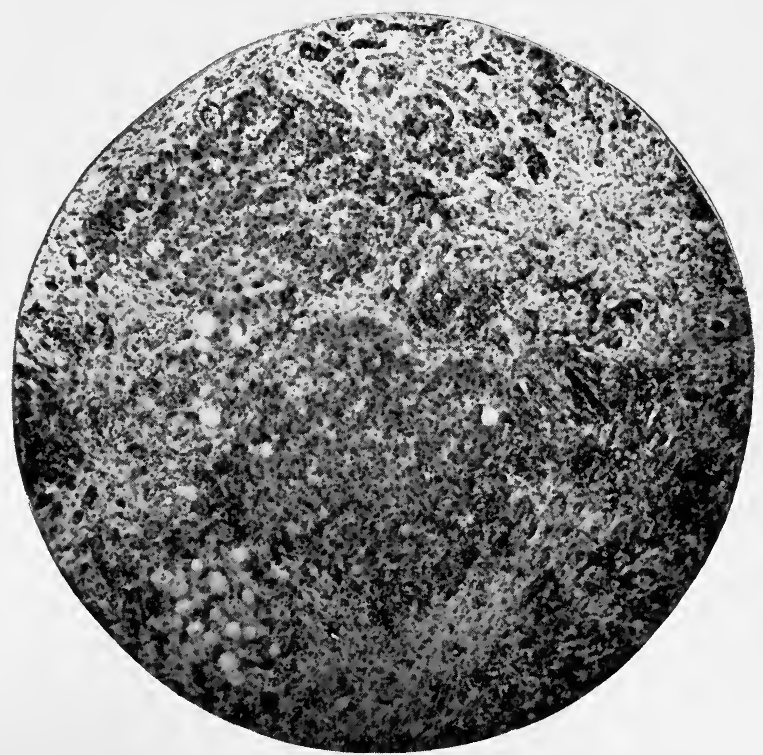

FIG. 10. - Hypertrophic Cirrhosis of the Liver (early stage). $\times 55$.

childhood; nevertheless, as with other rare sicknesses, instances of it occur occasionally, but steadily, in hospital practice. The causation is very obscure, for in children one has not the ready recourse to alcoholic and other excesses to which adults are 
addicted. Thus very few of the children who suffer from cirrhosis give a history of alcoholic excess; a few are congenitally syphilitic, and a few more have suffered from severe or wasting disease. The most that one can say is that this complaint is a vicious form of deflected nutrition whose explanation is at present obscure.

Lesions. - The characteristic feature of the changes is a deposition of connective tissue in the liver. When the process begins, its course is more rapid than in adults, although the main characters are alike. The new tissue grows in various shapes and in all parts of the organ, and by pressure squeezes and chokes the

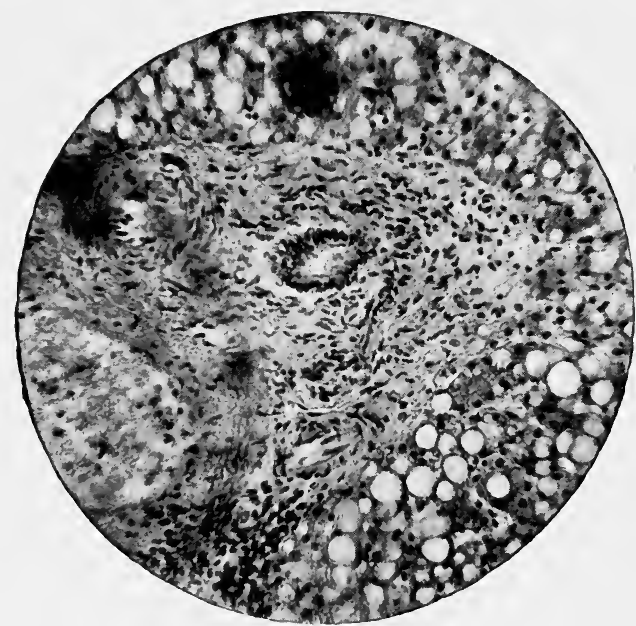

FIG. 11. - Interstitial Hepatitis. $\times 125$.

hepatic cells. The blood-vessels, especially the hepatic and portal veins and their branches, as well as the gall-ducts, later on become attacked, so that their elasticity is sufficiently encroached upon to become progressively functionless, while the lumen regularly grows smaller. In addition, the bile-ducts may fall into a catarrhal inflammation, the hepatic tissue may become subject to amyloid or fatty degeneration, and the whole organ may become bound by adhesions to near-by structures.

Secondary changes occur in the later stages: the veins about the umbilicus, or all the abdominal veins may become enlarged; the spleen may become hypertrophied, the stomach and intestines may show the symptoms of a catarrhal inflammation, or may even 
have considerable hæmorrhages. Ascites and a chronic interstitial nephritis characterize the final stages of the disease.

Symptoms. - The course of the disease is so insidious that, unless the liver is carefully palpated, its true nature is often unsuspected until far advanced. The first enlargement of the liver then changes to an unduly small size, the child begins to waste away, gastro-intestinal disorders of various kinds appear, and the skin may assume a yellow discoloration. As the malnutrition becomes more marked, ascites appears, and usually is accompanied by disturbances of various abdominal and thoracic viscera. These cases are very hard to nourish, and the process of wasting progresses with rapid strides. This disease is a good example of the general truth that degenerative processes in children are much quicker in their course than in adults.

The distinction between this disease and acute yellow atrophy of the liver is at times difficult. In interstitial hepatitis the course is ever so much longer, and one stage fades into the other by insensible degrees. In atrophy the progress is comparatively rapid. In the first the symptoms are more clearly referable to the liver, and therefore less to the general organism than in the second. Also, in the first, the form of the organ can be well discerned as being large in the beginning, then smaller and at all times fairly hard and regular, with a moderately sharp lower margin. In atrophy there is a tendency to softness of consistency, irregular contour and blunted or indistinct lower edge. The extreme rarity of the second disease must always remain a factor of diagnostication.

Treatment. - The most that one can do is to regulate the diet and treat the symptoms. Attention to every detail of the child's hygienic and medicinal care may delay the fatal outcome. The diet must be simple and nutritious; some form of gentle exercise in the open air should be taken as long as the child's condition permits; the action of the bowels and kidneys should be kept as clear as possible; bathing should be regular. In some cases one may use tonics with beneficial effect.

\section{Acute Yellow Atrophy of the Liver}

Acute yellow atrophy is an exceedingly rare disease in children. It is hard to say whether it is primarily a disease of the 
liver or of the general economy. At all events, the lesions are not found alone in the liver but in the other abdominal and thoracic viscera as well. It may be classified under the heading of obscure wasting diseases.

The liver, though at first enlarged, becomes diminished in size, is soft and wrinkled. The characteristic structure fades away, and is replaced by a condition of fatty degeneration. The tissue is colored by the presence of yellow or reddish-yellow pigment that increases in quantity as the disease progresses. Small quantities of leucin and tyrosin may be found in the broken-down cells, and the interstitial tissue wastes and fades away. In the final stage, the liver is practically a shapeless mass.

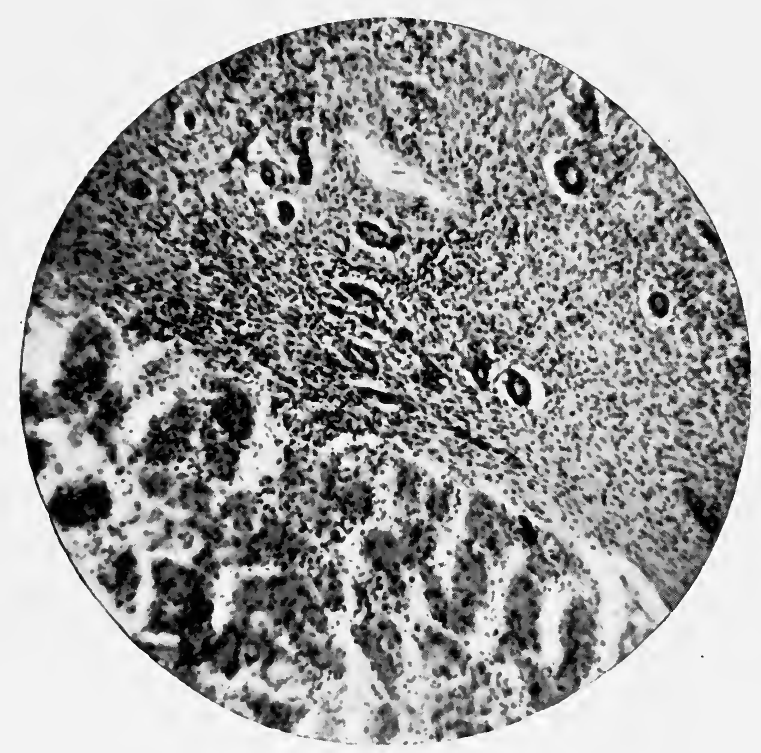

Fig. 12. - Acute Yellow Atrophy of the Liver. $\times 100$.

The symptoms consist in a general way of steady malnutrition, prostration, gradual starvation. Usually there is jaundice, and cerebral disorders may supervene.

The treatment is symptomatic. The outcome is death.

\section{FAtTy Liver}

The normal liver contains some fat which may vary from time to time. In the presence of severe wasting diseases, such as ma- 
rasmus, chronic ileo-colitis, tuberculosis, profound anæmia, and phosphorus poisoning, this amount of fat may be very largely increased. The infiltration begins at the periphery, and requires a considerable time to get to the centre. Thus, on section, the tissue near the surface may be a light yellow, while the centre has a reddish color. The fat may be so plentiful that the cut surface is greasy.

The condition is recognized by the history of previous disease, the discovery of a large liver with rounded edges, and the absence of hepatic symptoms.

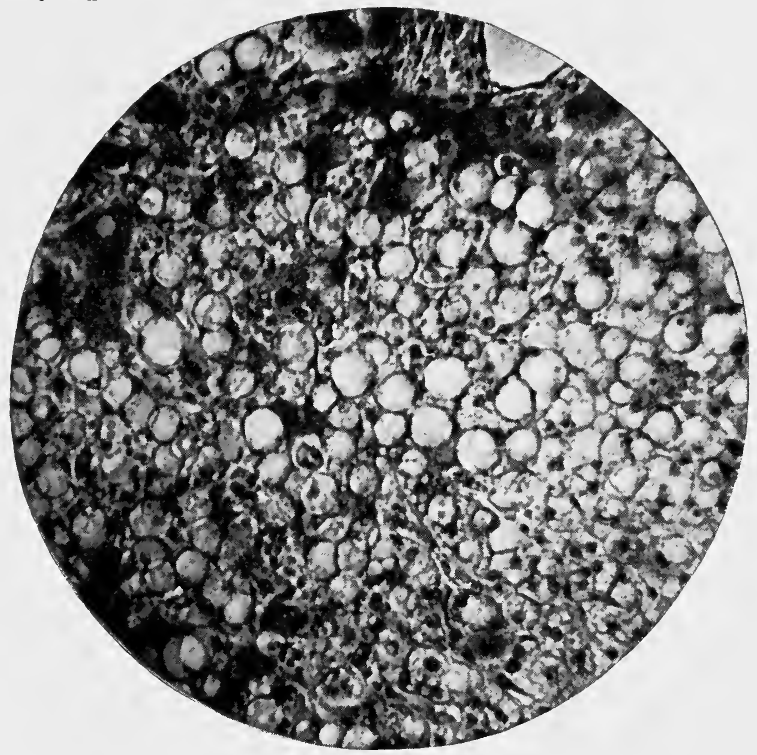

FIG. 13. - Fatty Liver. $\times 180$.

The treatment is that of the primary disease, and the regulation of the diet and hygienic conditions. The patient should be in the air as much as possible, must bathe regularly, and should take tonics.

\section{Amyloid Degeneration of the Liver}

A waxy liver rarely occurs without a concurrent similar condition in the kidneys, spleen, intestines, or other viscera. One's attention is thus drawn to the fact that this degeneration is not a local disease so much as a local expression of a general affection. It arises from conditions of marked exhaustion, from wasting, dia- 
thetic and osseous diseases, from chronic suppurative processes, from such complaints as chronic tuberculosis and congenital syphilis.

The process usually begins in the intralobular arterioles, which become thick and waxy. Later, it attacks other vessels and then the liver tissue. The affected parts lose their characteristic structure, become light brown in color, hard, smooth, and translucent. The size of the organ is much increased, and the margin is hard and sharp. The size may be restrained in its growth if cirrhosis develops to any considerable extent. In addition, there may be areas of fatty infiltration.

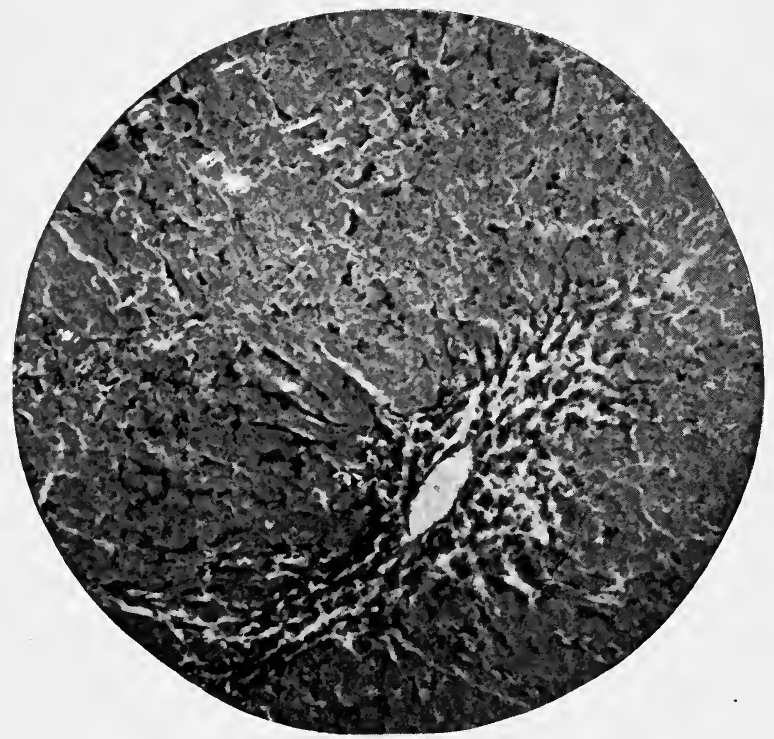

Fig. 14. - Amyloid Degeneration of the Liver. $\times 60$.

The condition practically never appears alone; especially, should one look for amyloid conditions in the kidneys. These symptoms, with the history of the original sickness, are fairly sure to make the diagnosis clear. There are rarely any liver symptoms, and the exceptional cases of jaundice or ascites can generally be explained on the score of pressure. The patient's skin has a peculiar waxy appearance that is noteworthy. The disease does not cause pain.

The treatment is that of the original disease. Although the 
prognosis is not encouraging, nevertheless, I believe that with careful and vigorous treatment of the general condition much can be done for the child's comfort and well-being. By means of a carefully regulated diet, tonics, and whatever exercise is possible, the functional activity of the body may be prolonged for an indefinite time. The treatment of such a case is a matter of details, the aggregation of which makes a general impression. The growth of the disease is so gradual that one usually has months at one's disposal before the case becomes hopeless.

\section{Echinococcus INFECTION OF THE LIVER}

This infection is due to the tænia echinococcus or dog tapeworm. When the eggs of the parasite find their way into the intestinal canal they begin to develop. They then penetrate various organs, but most frequently the liver, where they produce cysts, called hydatids. From them secondary or daughter cysts may grow, and in turn may give off tertiary or grand-daughter cysts. Each cyst has a connective tissue capsule in two layers; and on the lining membrane the heads or scoleces of the young tænia are producerl. Each scolex has a rostellum placed in the middle of two rows of hooklets and four sucking plates. The cysts contain a fluid of low specific gravity, of an alkaline reaction, containing albumin, sugar, sodium chloride, and cholesterin crystals. In the fertile cysts the fluid is made less clear by the pressure of scoleces, hooklets, and fragments of parenchymatous tissue.

Symptoms. - In children the cysts are generally unilocular. Their growth is so slow that months may pass before their pressure attracts attention. This happens the easier because they rarely cause pain, high temperature, or pressure effects. When this last-named symptom exists, it shows itself in whatever direction the growth takes. Thus the right lung and pleural cavity may be encroached upon, or the abdomen or heart and great vessels be affected. In many cases the swelling can be made out by palpation, and in a few, one may distinguish the "hydatid fremitus" by placing the left hand on the tumor and tapping a near-by part of the liver with the right.

Jaundice may occur from pressure; nevertheless, the general health is, as a rule, unaffected. This rule does not hold, of course, if suppuration sets in. 
Treatment. - The treatment is surgical: when properly and thoroughly accomplished, the prognosis is fairly good. Delay means ultimate rupture, either externally or into one of the thoracic or abdominal viscera ; when rupture is internal the result is usually fatal.

Differential Diagnosis. - Hydatids are to be distinguished from syphilitic and carcinomatous diseases of the liver. As a rule this is not difficult, as the latter disorders have characteristic histories, carcinoma especially making general and systemic inroads upon the patient's health. 'The diagnosis is certified by aspirating the tumor and finding hooklets.

\section{WOUNDS OF THE LIVER}

Wounds of the liver may be caused by artificial and very difficult delivery, or by falls or blows. They may be of various degrees of severity, and are usually followed by inflammation or abscess. The most serious cases usually involve a rupture of the capsule as well as the parenchyma. Here there would be a large and fatal hrmorrhage. In all cases of wounds there is more or less bleeding, which in children is always dangerous. Extravasations of blood in the liver may result from hard delivery, scorbutus, the administration of poisonous quantities of phosphorus, and from purpura hæmorrhagica.

The portal vein may be injured by blows and falls. The result may be endophlebitis and the formation of a thrombus; marked changes in the functional equilibrium of the abdominal viscera may make themselves apparent.

\section{Subphrenic Abscess}

As a result of pus infection an abscess may form between the liver and diaphragm. From its position it is called subphrenic. The original focus of pus may be in the liver, intestines, stomach, lungs, pleura, or other near-by viscera ; in another class of cases, which is rarely seen, the pus may come after the rupture of an hydatid cyst of the liver. The infection may in others be communicated through the lymph circulation from a fairly remote source, such as the skin of the glands. Thus, I may cite a case which I saw where a very severe furuncle in the back was followed by a subphrenic abscess. There was much difficulty and several failures before the correct diagnosis was made. 
The symptoms resemble those of purulent pleuritis of the right side. The diagnosis may be confirmed by the aspirating needle.

The treatment is free opening and drainage.

\section{Biliary Calculi}

In childhood gall-stones are so rare as to require no more than a mention. They have been found at all ages, and no matter what the time of life the symptoms are always the same. During the attacks sedatives should be administered in sufficient quantity to quiet pain. After the attack has passed phosphate of sodium in large doses may be prescribed. These children should drink a generous amount of water.

\section{Congestion of the Spleen}

Congestion may occur simply as a hyperæmia, or as an active inflammatory process. This is the condition that accompanies

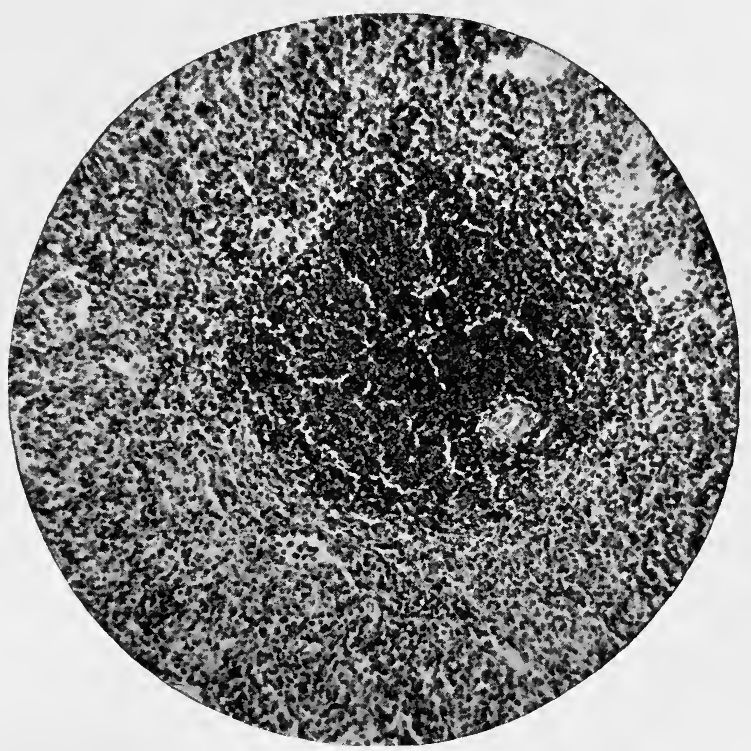

Fig. 15. - Normal Spleen. $\times 100$.

the acute infectious diseases, such as scarlet fever, measles, acute lobar pneumonia, typhoid and typhus fever, diphtheria, and in the severe diseases of pyogenic invasion, as septicæmia and pyæmia. In malaria, especially of a pronounced form, it may be 
present in a marked degree; in diseases of malnutrition, such as rickets, and in systemic disorders, such as syphilis, tuberculosis, and blood diseases, it may be looked for more regularly in children than in adults.

Lesions. - The size of the organ is increased, the cavernous veins surcharged with blood, the pulp tissue has a deep red color, is spongy, soft, and of an unstable consistency. In severe cases, or those characterized by large amounts of toxines, there is a marked increase in the cell tissue, or a noticeable hyperplasia in the glomeruli. Where vitality is deficient there may be a consequent degeneration of this tissue, or even a necrotic breaking

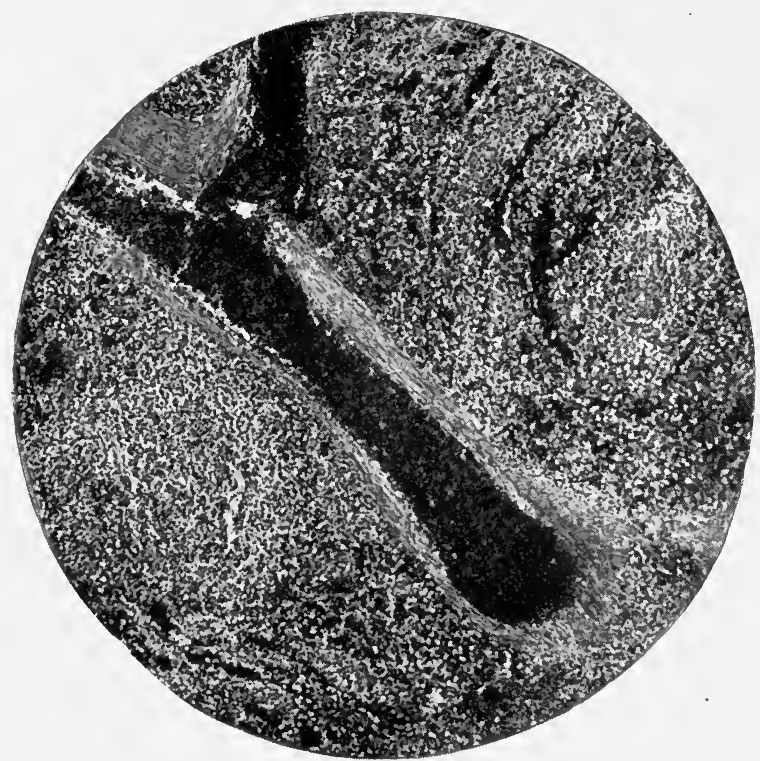

Frg. 16. - Congested Cavernous Vein of Spleen. $\times 70$,

down into small abscesses. When convalescence takes place the congestion fades away, and the products of degeneration are absorbed. When recovery is delayed too long, the small foci of suppuration may increase and flow together, forming an abscess; or the hyperplasia may give way to a formation of connective tissue.

There is no special treatment for these diseases of the spleen outside of the treatment of the original disorders, which must be continued until one is sure that the organ is practically restored to its normal usefulness. For a considerable period the spleen must be regarded as liable to revert to its pathological condition. 


\section{Amyloid Degeneration of the Spleen}

There may be a waxy degeneration of the blood-vessels and reticulum, or of the glomeruli, or of the pulp tissue. Occasionally the process is practically general. The organ is much increased in size, is hard, and the edges are rounded. The process rarely occurs alone, but usually is associated with similar changes in other viscera, especially the liver. The cause seems to be marked malnutrition and lack of systemic vitality. The treatment is that of the general condition and includes a careful diet, gentle exercise, baths and tonics.

\section{Parasites of the Spleen}

Echinococcus sometimes attacks the spleen. And cysticercus, as well as pentastomum denticulatum, is found. Various bacteria have likewise been found when an infectious disease was attacking the body.

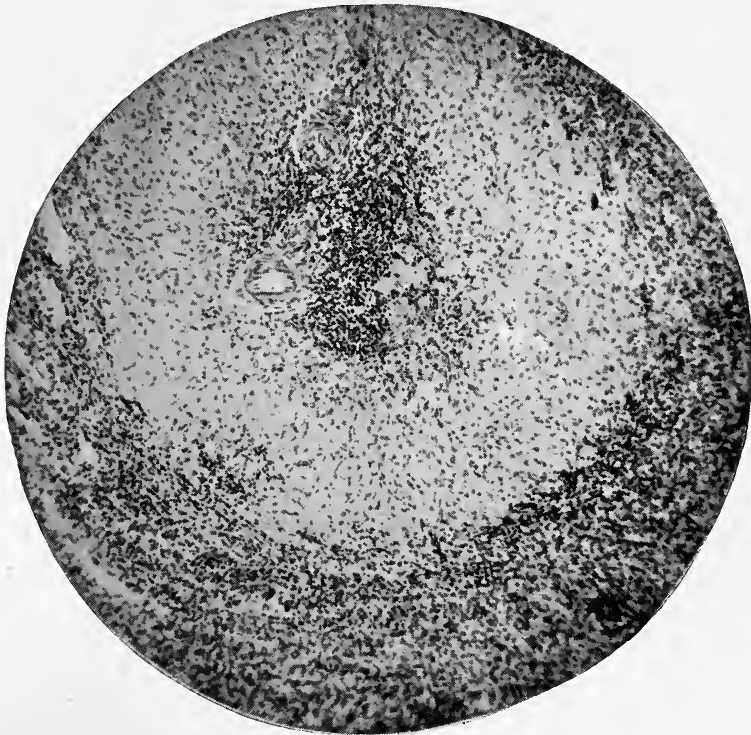

Fig. 17. - Chronic Interstitial Splenitis (Sago Spleen). $\times 60$.

\section{Wounds OF THE SPLEEN}

Wounds of the spleen are always serious, no matter what their extent may be. The peculiar structure of the organ is 
partly responsible for this, since the pulp-tissue serves in place of the blood-vessels which are found in greater quantity in other viscera. Thus, a wound of the spleen involves at one time both the vascular tissue and parenchyma. These wounds may be due to an active agent, such as blows or falls ; or to a passive cause, such as excessive swelling brought on by a general disease. While such a great enlargement is rare, nevertheless minor but noteworthy degrees are very common; for since the organ acts as a sort of filter, it is commonly affected by the acute infectious diseases, chronic and wasting diseases. In this manner a sufficient enlargement may occur to make the danger of wounds possible. 


\section{CHAPTER X}

\section{DISEASES OF THE LARGE INTESTINE}

\section{Acute Ileo-Colitis}

The inflammations of the colon, the ileum, and the large intestine in general have a very unsatisfactory classification and

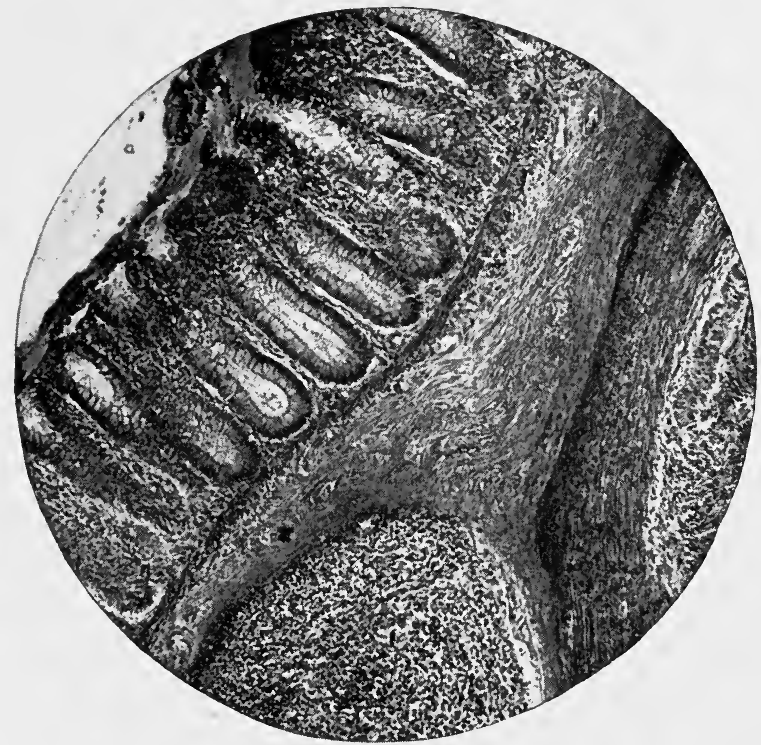

Fig. 18. - Normal Intestine. $\times 70$.

nomenclature. This is due to our imperfect knowledge of the micro-organic life and the chemical changes in the fæces. In former years, when we were less conscious of our ignorance, no attempt was made to arrange or classify the group of symptoms which are characteristic of intestinal organic lesions. With the investigations of men like Escherich and Booker, a knowledge of our limitations came into active existence; and one of the first fruits of this dissatisfaction was the abolition of the term dysen- 
tery. The name colitis and ileo-colitis were elected to fill the vacant place, but not as a necessarily permanent term. The usual classification that is applied to the inflammations of mucous membranes in general was affixed to the disorders in question. Immediately practitioners began to find fault with the difficulty in assigning the clinical appearances to the pathological changes, as well as with the provisional nature of the statements concerning those changes. Even now the dissatisfaction continues; practically the distinction between colitis and ileo-colitis is lost, and the

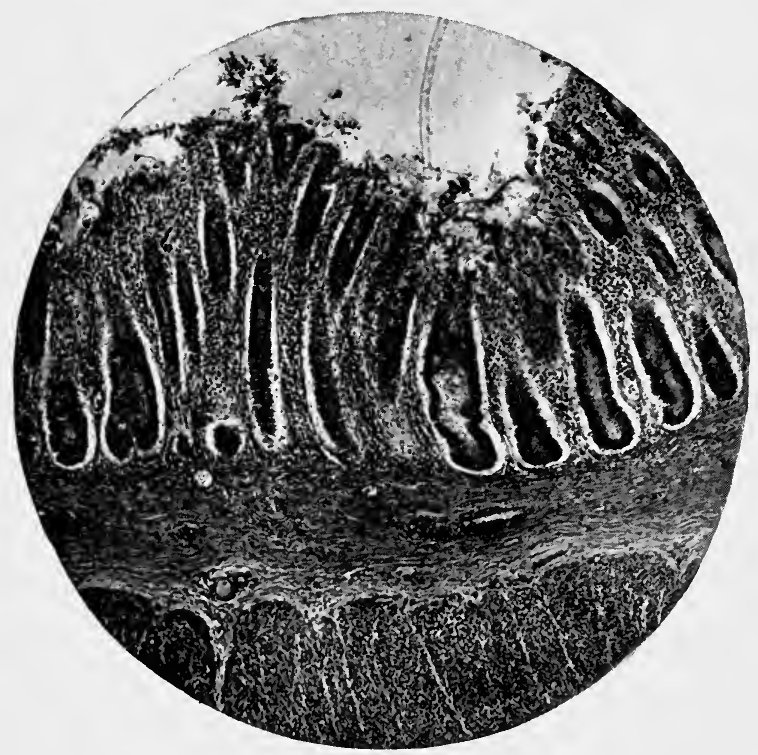

FIG. 19. - Catarrhal Enteritis. $\times 70$.

various forms of the inflammation have come to have an academic rather than a working interest.

Causes. - Ileo-colitis is generally preceded by some form of infection enteritis. The denuded epithelium gives an opening to the attacks of bacteria and infusoria, of whose exact character and life history we are poorly informed. Among them various sorts of streptococci are thought to hold a prominent place. Continued habits of bad feeding are likewise liable to bring about the same result by producing an irritation of the mucous membrane and thus making a good culture ground for micro-organic life. High temperature and great humidity also seem capable of sufficiently 
depressing the vital tone so that resistance to disease is much lessened. The sum of our knowledge is yet merely general; and the field for research remains very wide.

Lesions. - The pathological changes are commonly grouped in the colon and part of the ileum; these limits are not absolutely fixed, and may be extended either as far as the stomach in one direction or the rectum in the other. The degree of inflammation is likewise not constant, varying in different parts of the same in-

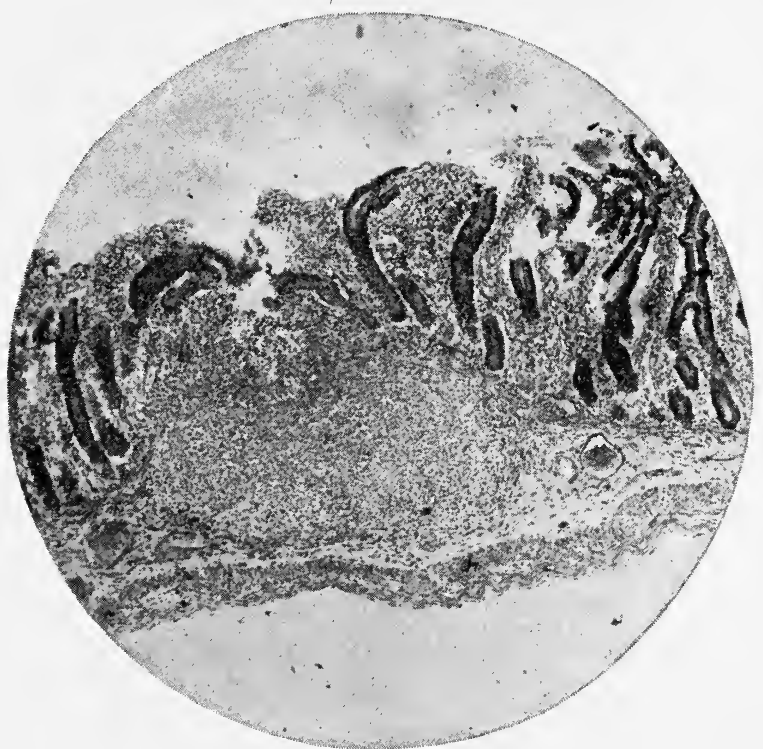

Fig. 20. - Ulcer of Ileum. $\times 50$.

testine. In many post-mortem examinations this exaggeration of local change of tissues is emphasized by the rapid disintegration that follows death.

- In the catarrhal inflammation, the mucous membrane is swollen and congested, producing an unduly large amount of mucus and serum. The capillaries may be so much swollen and congested, so friable, that insignificant agents produce hæmorrhages of greater or less intensity. The epithelium is denuded, in some places for large areas. In these areas more than elsewhere the inflammation extends into the superficial structures through the mucosa, even into the connective tissue. The greatest amount of inflammation carries the greatest quantity of pus cells. Outside 
of these lesions there are swelling and congestion of Peyer's patches, the solitary and agminated glands and the minute glandular structure throughout the gut and mesentery.

Where this inflammatory process is severe, the epithelium over the follicles breaks down, followed by the destruction of the follicular tissue. Thus, small ulcers are formed which, when in close juxtaposition, are apt to merge and make large ulcers with undermined edges. This constitutes the so-called ulcerative variety.

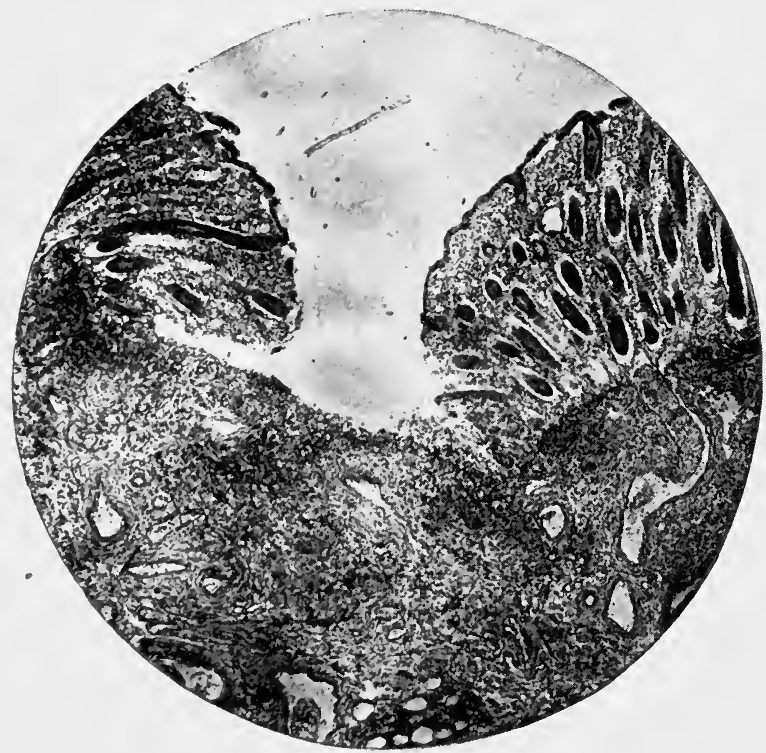

FIG. 21. - Ulcerative Colitis. $\times 30$.

In the croupous or membranous variety, we have the severest form of ileo-colitis. Here the congestion is very intense, the swelling is so marked that the bowel is stiff and hard, and the infiltration is so great that the surface is irregular and even fissured. A pseudo-membrane, composed of fibrin, blood cells, and bacteria, covers scattered portions of the gut. Although the principal seat of this formation is in the colon and the adjacent portion of the ileum, nevertheless other parts of the large intestine from the sigmoid flexure down through the rectum may be similarly invaded. Not only is the mucosa inflamed, but the connective tissue also may be involved. Even the muscular and peritoneal coats may be infiltrated with fibrin and pus. The 
process very rarely extends to the peritonæum except in small areas of plastic inflammation which give scarcely any additional symptoms. On account of the malignant intensity of the worst attacks, one or more of the layers of the intestine may lose sufficient vitality to insure breaking down of the wall and formation of an ulcer. This, however, is not often seen.

Symptoms. - The course of the sickness soon shows that the lesions do not explain the severe symptoms, and that fully to understand them one must place most stress upon the toxines -

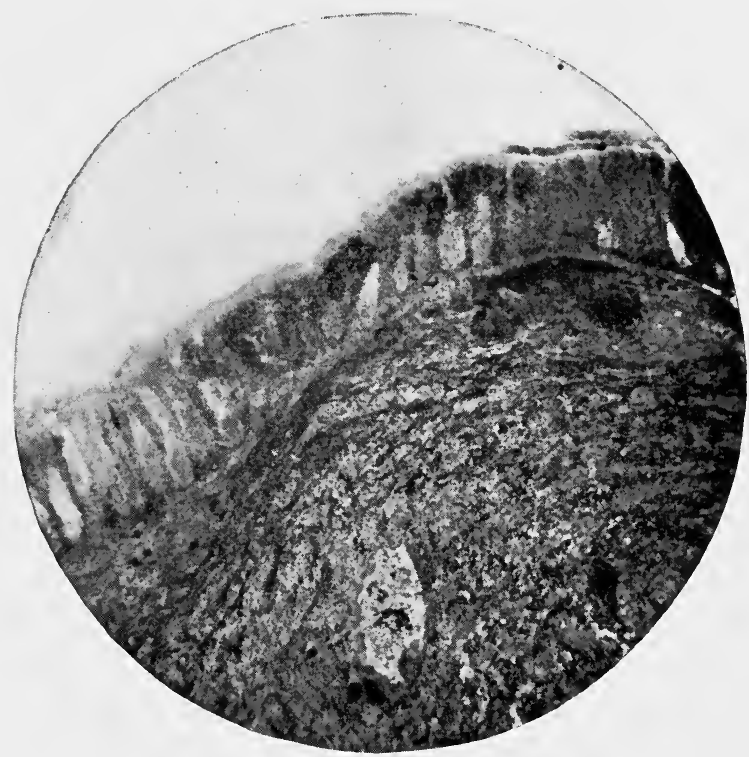

Fig. 22.-Croupous or Membranous Ileo-Colitis. $\times 30$.

whatever they may be - that are elaborated by the micro-organisms in the intestines. Either there may have been a previous gastro-enteritis or the ileo-colitis may begin as a primary disease. At all events one sees at first disturbances of the stomach and intestines with a rise of temperature to $39.5^{\circ}$ or $40^{\circ} \mathrm{C} .\left(103^{\circ}\right.$ or $104^{\circ} \mathrm{F}$.). The fever, excepting in very mild cases, remains fairly high, with intervals of exacerbation and remission, until the end of the sickness sets in. From the first the child is in much pain, which he shows by continual restlessness and crying. At first the movements are characteristic of diarrhœa, being yellow, brown, and green; they are partially formed, and have an un- 
pleasant odor. After the intestinal track has been emptied, the movements are very frequent, small, and composed largely of mucus and small amounts of blood in the form of streaks or little masses. Not often does one find the blood fluid and in considerable quantity. At times there may be attacks of vomiting, especially if the food is not very easily digested. Commonly the urine is diminished in quantity and holds in solution a small amount of albumin. The child is plainly prostrated, the tongue is heavily coated, the loss of strength and flesh is very apparent. The abdomen at first has no remarkable signs, but as the disease

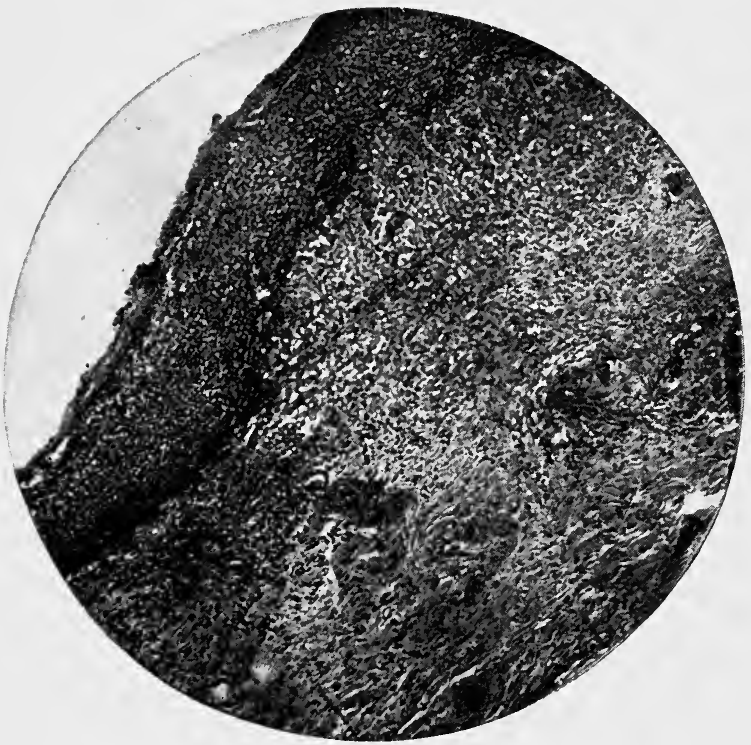

Fig. 23. - Membranous or Croupous Colitis. $\times 70$.

wears on, it may become enlarged, tympanitic, and tender. If the patient is very sensitive, he may suffer from delirium or even convulsions. This is the case in children of all ages, but infants have a marked tendency towards prostration and convulsions. They seem peculiarly unable to withstand the poisons of the disease and show this disposition at an early stage of the illness.

If the inflammation spreads to the rectum, the suffering before and after defæcation is extreme, calling forth shrill cries of pain. At the same time there will be much straining and occasionally a prolapse of the rectum, usually not more than from 3 to $5 \mathrm{~cm}$. 
in extent. The prolapsed gut is always much congested and swollen. The skin around the anus and along the buttocks is commonly excoriated on account of the irritating nature of the movements.

As the child recovers, the temperature subsides, the blood leaves the stools, which gradually become fæcal. A rapid return to health is not to be expected.

The variety of follicular ulceration is not by any means easy to diagnosticate. It is principally known by the small amount of vomiting, the small quantity of blood, the large masses of mucus, the protracted course, and the fact of previous attacks of acute or subacute infectious gastro-enteritis. The movements are apt to be less numerous, less watery, and to have more color. The emaciation is marked, and the physical depression is extreme.

The croupous or membranous variety is exceedingly severe and generally fatal. During its course it is at times apt to be confounded with the severe cases of catarrhal ileo-colitis. It is distinguished by the general intensity of its symptoms, its high fever, delirium, the large quantity of blood in its stools, the more common invasion of the lower rectum, and the consequently increased tenesmus and straining. The principal diagnostic point is the presence of pieces of membrane in the stools or on the surface of a prolapsed rectum.

Treatment. - A safe rule to follow is to give small doses of calomel (0.006 gm. $-\frac{1}{10} \mathrm{gr}$. - every fifteen minutes for ten doses) followed by a dose of castor oil. All milk should be rigorously excluded from the diet, which is to be composed of albumin water, home-made beef extracts, and meat broths. The patient generally suffers from thirst, and will get some relief from drinking cool, sterile water. The fever may be combated by hot or cool packs, by sponging with alcohol and water, or by the graduated cool bath. This last must be given with caution when the child is prostrated. In all cases where it is prescribed, active friction of the surface should accompany it; and when the child is placed in his crib, the nurse should see that his extremities are comfortably warm. The regular bathing must, under all circumstances, be continued every day. Also, an invariable rule is to keep all utensils and clothing about the child clean and sterile.

One of the most useful measures at our command is irrigation of the lower intestine. This, to be efficacious, must be sufficiently 
copious and frequent. The child should be so placed that the buttocks are somewhat higher than the shoulders. By means of a well-greased rectal tube of small calibre from 2 to 4 litres ( 1 to 2 gallons) of warm normal salt solution - salt, $4 \mathrm{gm}$. ( $1 \mathrm{dr}$.) to sterilized water ( 1 pt.) - may be thrown into the bowel. This may be used two, three, or four times a day as the condition of the child demands. When much blood is in the stools, relief may be obtained by enemata of ice water. A wise use of these various irrigations will relieve much of the tenesmus, and thus do away with the necessity of giving opium by mouth, hypodermatic injection, or enema. Nevertheless, in spite of all efforts to the contrary, it may occasionally be necessary to give this drug. If so, it is best, in infants, to give the deodorated tincture in a starch enema or, in older children, a minute dose of morphine, hypodermatically. This step, however, is not commonly necessary.

Another drug that is of some use is the subgallate of bismuth, according to the following prescription :-

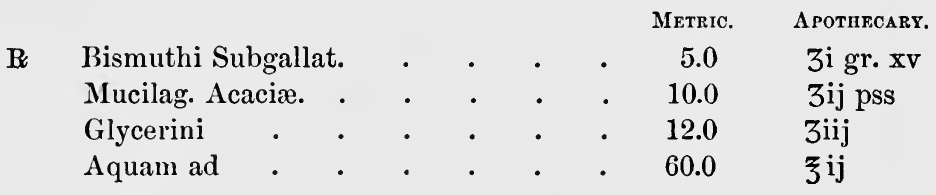

m. S. One teaspoonful every two hours.

This drug, as well as all the bismuth preparations, must be given in large doses to produce a definite result. Therefore in older children the dose máy easily be doubled. One need have no fear of toxic effects, since the drug is not absorbed but rather is intended to coat the mucous membrane of the gut. On the other hand, the use of astringent enemata, which were formerly much employed, is of doubtful value. They are liable to do harm, and generally one can do without them. In the few cases where they may be thought to be indicated, their strength should not be great.

In all these cases an important part of the treatment is to keep up the strength by stimulants. Good whiskey or brandy in small and frequent doses and well diluted may be used; and when prostration is present, a wise and liberal use of nux vomica may be very valuable.

As convalescence sets in, tonics containing nux vomica or strychnine and an organic preparation of iron may be advanta- 
geously prescribed. The return to ordinary diet should be gradual and conservative; and if the disease shows signs of breaking out afresh, the restricted method of feeding must directly be resumed. The patient from the earliest permissible date in the sickness should be kept in the air as much as possible. In warm weather total residence in the open is highly advisable ; and at all events city children should with the least delay be removed to the country.

Differential Diagnosis. - Ileo-colitis is to be distinguished from infectious gastro-enteritis by its frequent small stools which contain much mucus, blood, or membrane, by its continued high temperature and its smaller amount of vomiting. From intussusception it can easily be marked off, as it lacks the normal temperature and small, mucous stools followed by constipation, gas-production, tumor, fæcal vomiting, and collapse that characterize that accident. From typhoid fever it may be diagnosticated by the fever's characteristic temperature, enlargement of the spleen, eruption, tympanites, and greater rarity of occurrence in infants; the absence of Widal's reaction will confirm the opinion.

Prognosis. - In the mild forms and in children who have been fairly well cared for, the outlook is on the whole good. With the severer forms, in very young children, in those who have been badly fed, badly cared for, who have been shut up in tenement houses or in institutions, the prognosis is bad. The personal equation is an important factor in the outcome of the disease. Also those cases where in the course of the disease the patient contracts broncho-pneumonia have a serious complication to face.

\section{Chronic Ileo-Colitis}

The chronic form of ileo-colitis occurs, as a rule, as the sequel of an acute attack, although a few cases have, in the beginning, the slow and gradual characteristics of chronicity. Most of these acute cases are of the mild or medium catarrhal variety, while a few survive a moderate degree of follicular ulceration. Thus the severest examples of both varieties as well as almost all of the croupous are excluded by death.

Lesions. - As a rule only a part of the colon and the large intestine is involved, and in the part affected the changes rarely have the same degree of intensity throughout. Instead of a tem- 
porary inflammatory change, as in the acute form, there is a definite alteration in the minute structure of the intestine. Between the tubules in the large intestine, and to a less extent between the villi in the small intestine, there is a connective tissue which increases the thickness of the whole glandular coat. This thickening may be communicated to the muscular and peritoneal layers. In some places the deposition of connective tissue is so marked as to crush out and atrophy the tubular formation, and then lead to a wasting of the whole stratum. Where the atrophy of the glandular tissue is rapid, the surface may be so weakened that it

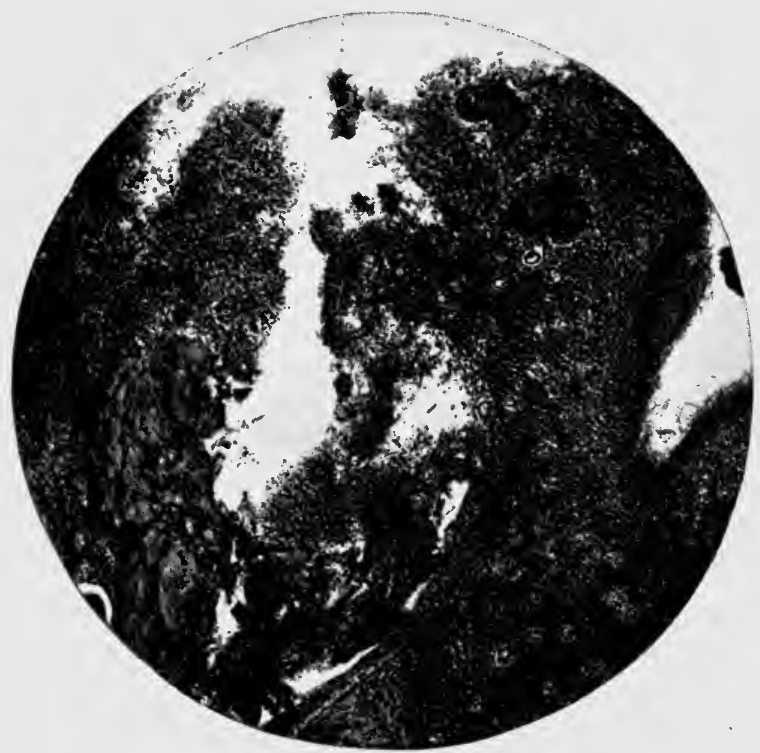

FIG. 24. - Chronic Catarrhal Colitis. $\times 50$.

breaks down into ulcers whose size and shape depend upon the extent and position of the region involved. In the same way, the solitary follicles may be attacked, may break down, and likewise form ulcers. These various ulcer-formations may gradually heal, be replaced by connective tissue, or linger in a granulating condition. Some rare cases of polypoid and cyst-like growth have been reported as resulting from obliteration of the mouths of glands.

Symptoms. - This chronic form, in contradistinction from the acute, does not have many characteristics of a septic infection. 
On the other hand, the main symptoms arise from the great physical depression, the alteration in the glandular structure of the intestine, and the fermentation and putrefaction of food. The course of the disease, if these factors are kept in mind, is fairly easy to understand; especially if one recognizes the great facility with which the exhausted economy succumbs to new forms of disease or acute exacerbations of the old complaint.

When the acute symptoms subside, the child does not materially improve. The stools are less in number, but are green, or greenish brown, or brown, and usually have a foul odor of decomposition. Although the child's appetite may be fairly good, nevertheless he gains almost nothing in weight, and often progressively loses. As this process goes on, the child may seem starved and wasted, the abdomen may be puffed out, the face and body look repulsively distorted and even deformed; the fontanelle may be depressed, the pulse may be weak, thin, and irregular. Various forms of stomatitis, of diseases of the skin and scalp may appear ; and inflammation of the abdominal and thoracic viscera may crop out at any time. Other signs of weakness and malnutrition are the liability to subnormal temperature, prolapse of the rectum, and nervous disorders, such as opisthotonos or convulsions. These cases are very trying to both physician and parents on account of their chronic course, their restlessness, their irritable crying during day and night, and the difficulty which they experience in responding to treatment for anæmia and malnutrition. Here and there a patient recovers or may temporarily improve. But as a rule one must expect a succession of changes and secondary diseases which tax the child's vitality and the physician's resources to the utmost.

Treatment. - In this disease, as in the acute form, the general management of the case is very important, for there is no specific drug to use. The rules of hygiene and sanitation should be rigidly enforced; the child's baths, exercise, and clothing should be carefully supervised. City children should, if possible, be sent to the country, or at all events should be kept as much as possible in the air. The diet will demand an important share in the treatment. Fats should be excluded, starches altered by diastase, and sugars diminished. Milk, with a small part of its cream retained, may often be usefully employed. But the main reliance will have to be put upon meat foods. Meat extracts and meat soups are usually well borne, and the older patients seem to derive benefit from 
scraped beef, broiled steak, and broiled lamb chops finely divided and very thoroughly chewed. As a rule, the case should be fairly well advanced before vegetables may be safely given.

The medicinal treatment is apt to be unsatisfactory. Symptoms will have to be controlled as they arise ; stimulants, such as good whiskey, brandy, or fine old wines that have a good bouquet, will be needed. Nux vomica or strychnine will often do good service, and when convalescence begins an organic preparation of iron may be prescribed. Still later the compound syrup of hypophosphites may be called into play. A measure that will often give fairly satisfactory results is irrigation of the large intestine with cold water. 'The frequency of its application will depend upon individual circumstances; in the ordinary cases once a day will be enough. All in all, the treatment of chronic ileo-colitis must be regulated by the circumstances and environment of each particular case and the ingenuity and resourcefulness of the physician.

Differential Diagnosis. - The main possibility of confusion lies in the division between this disease and tuberculosis. Outside of the low and irregular fever which characterizes the latter complaint, the general course of the two sicknesses will gradually clear up the question. Most of all, the history of the sickness and the peculiar character of the stools will be diagnostic. In addition the involvement of various organs in tuberculosis, with the inevitable train of additional symptoms, will make the situation easily understood.

Prognosis. - This depends upon the severity of the acute attack, the strength of the patient, and the possibility of employing every therapeutic measure. On the whole, the outlook in infants is not especially encouraging. In older children it is somewhat better because they have greater vitality in withstanding the inroads of the disease.

\section{Ileo-Colitis due to Amabic Infection}

A rare form of ileo-colitis, as far as its occurrence in temperate climates is concerned, is caused by amoba coli. Generally it is imported from tropical countries, where it flourishes vigorously.

Lesions. - The pathological changes consist mostly in ulcers of various shapes and sizes, some of which have undermined and others smooth edges. The number of them is variable, but the usual experience shows it to be large. There is a surrounding 
zone of congestion and inflammation, with a central area of necrotic tissue. In this tissue and the adjacent region amoeba coli may be found, as well as in the blood-vessels. Thence it works its way to different organs, especially the liver, from which it may spread, by the breaking of an abscess, to the lung. In these viscera the pathological changes are the same as what one finds in the intestines.

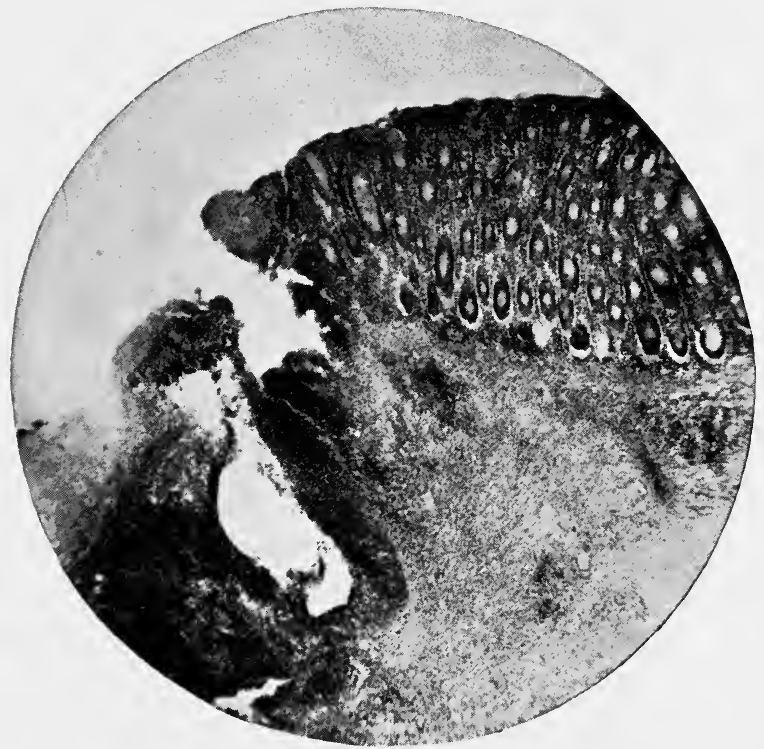

Fig. 25. - Amœbic Ulcer of Colon. $\times 30$.

Symptoms. - The only respects in which the symptoms differ from those of the catarrhal form of ileo-colitis are the low fever or normal temperature, the slow and remittent course of the disease, and the presence of amoboe in the stools.

Treatment. - The treatment is practically the same as in the catarrhal disorder. Various practitioners recommend rectal injections of quinine in solutions of 1-5000 to 1-1000. Especial stress is to be laid upon the necessity of liberal stimulation. Even then the patient will become and remain for a long time much emaciated.

Prognosis. - The outlook is not good, the patient sometimes succumbing to mere exhaustion, even although the characteristic movements have passed away. Even under favorable circumstances the disease lasts for weeks. 


\section{Infectious Derangements of the Intestines}

The intestines of a new-born child are practically sterile; within a few days, however, the fæces begin to show the usual bacteria, although the quantity is smaller than later on in life. While the child is kept at the breast the intestinal micro-organisms, as a rule, do not become pathogenic. Nevertheless, one must keep in mind that even breast milk is not always absolutely sterile; and, in addition, there is always the possibility of communicating infection through the persons, utensils, or toys about the child. In such ways even breast-fed infants become infected by micro-organisms whose toxines may produce various disturbances of the intestines. The most common cases occur in hand-fed babies whose food is contaminated by pathogenic germs. Such children are often, previous to the invasion, in poor general health. Their food has not been carefully regulated in quality and quantity, and commonly the'whole ordering of their attendance has been faulty. Their poor nutrition easily lays them open to attacks of sickness, so that when their food is impure they easily become poisoned.

That these cases should be looked upon as illustrations of poisoning there is little doubt. The efforts of the economy to free itself of offending material by means of prolonged, violent diarrhcea and vomiting, the prostration which follows them, and the lack of organic pathological lesions make a picture of toxicity. The mere fact that we have as yet been unable to isolate and describe all the various bacteria that occur in the diarrhoeal complaints does not at all militate against this conclusion. For these microorganisms are so numerous that their very plenitude forces the belief in a common condition in which one germ may exist as well as another; and it is so difficult to separate the products of one from those of another that any attempt at finding the particular bacillus to bear the burden of responsibility is at present almost certainly bound to fail. For this reason comparatively little has been done in tabulating and describing the life histories of these organisms, or in producing a useful antitoxic serum.

Lesions. - The pathological changes vary with the severity of the infection, the patient's power of resistance, and the length of time during which the disease has continued. Primarily but little impression is made upon the gut; but when a congestion or irritation of the mucous membrane exists antecedently, or the patient's 
general condition is poor, the further excitation of acid fermentation and albuminous putrefaction may cause various degrees of inflammation and molecular degeneration. These effects commonly start in the small intestine; thence they may spread to the large intestine, and also to the stomach. What effect the absorption of products of decomposition causes is, on account of the many forms and the varying degrees of intensity, almost impossible to say.

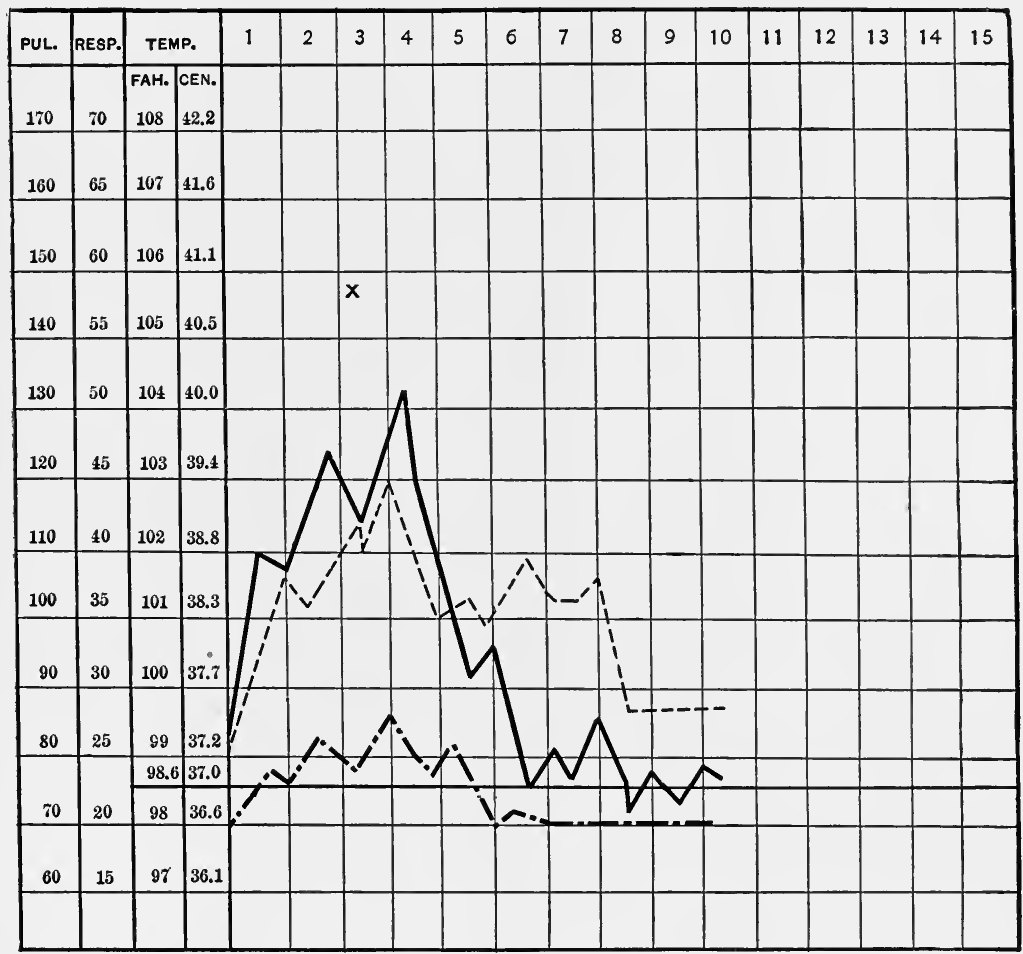

PULSE, RESPIRATION AND TEMPERATURE CHART OF INTESTINAL AUTO-INTOXICATION. $\mathbf{X}=$ CALOMEL

PULSE. TEMPERATURE AGE, 4 YEARS.

FIG. 26.

Symptoms. - The symptoms are best interpreted if one regards them as the signs of irritant poisoning. Usually the onset is rapid and the child immediately begins to fail. The cases of gradual invasion are apt to be those which might be classified as gastritis or enteritis; upon these an infection is easily grafted. Diarrhœa 
sets in rapidly, and in most cases is exhausting. This is evident from the general condition of the child : he loses weight rapidly, the skin hangs loosely where a day or two before it was well filled out; the eyes are heavy and sunken, the complexion is dull and yellow, the face becomes wrinkled and old-looking. At first the movements are solid or semi-solid, but as the sickness progresses they become more and more fluid, until they are merely serous. Their number varies, in some cases being five or six per day, in others one or more per hour. The color in the beginning is yellow, then greenish yellow, and finally green. There is a mawkish odor to the frees that at times may be differentiated from the sour smell of acid fermentation or the putrid smell of albuminous decomposition.

The child is constantly in pain and shows it by continuous crying until he is exhausted. As his strength fails, his former shrieks are replaced by short and irritating moans. If his condition becomes more serious, the eyes become dull, the nostrils and lips retract, and the fontanelle becomes sunken.

The temperature is apt to be elevated, especially in infants, ranging from $38^{\circ}$ to $40^{\circ} \mathrm{C}$. $\left(100.5^{\circ}\right.$ to $104^{\circ} \mathrm{F}$.) 'This statement is merely approximate, for there are so many degrees of intoxication, and the septic agents are evidently so various, that the widest limits of temperature are possible. Even with considerable fever the surface of the body may seem cool and clammy.

In children who have passed the age of infancy, the disorder is apt to assume a less violent form ; and the depression which it produces is neither so alarming nor so dangerous. At the beginning, if there is a gastric irritation, there will be some vomiting. But this symptom is not necessarily a constituent factor in the picture. There will be pain, which is sometimes referred to a definite area. in the abdomen and sometimes to the general region of the belly. At the outset of the disease there will occasionally be one or two movements followed by diarrhœa, but more frequently diarrhwea. will set in immediately. The stools are light-colored, sometimes stained with green; but a uniform green hue is not so common as in infants. The appetite will be poor, thirst marked, the temperature will have a mounting curve, and the urine will be decreased in quantity and high in color. Relief of these symptoms will follow proper treatment much more readily than in younger children. 
Treatment. - The first order should be to stop all food. Then the stomach and lower intestine should be cleansed by lavage and irrigation. This may be followed by small doses of calomel ( 0.006 gm. - $\frac{1}{10} \mathrm{gr}$.) every quarter-hour until $0.06 \mathrm{gm}$. ( $1 \mathrm{gr}$.) has been given. A saline cathartic may then be given to complete the cleansing and prevent the storing up of calomel. If the diarrhœa and vomiting are thus controlled, they may be followed by large doses of the subgallate of bismuth for about two days in order still further to protect and soothe the irritated mucous membrane. But if these symptoms continue, the main reliance must be put upon lavage and irrigation, especially the latter. Attempts to sterilize the gastro-intestinal track by means of antiseptic drugs is a waste of time and opportunity.

No food need be given for the first twelve hours. By that time the stomach ought to be fairly quiet, so that the administration of small quantities of nourishment may be resumed. For this purpose one may use albumin water or beef extract. At first they should be given by the teaspoonful until one is certain that the stomach can retain them; then the quantity may gradually be increased. No milk should be given until the patient, if he is an infant, is out of all danger. An older child need not be treated so rigidly; but even with him carefulness is highly desirable. And if he refuses albumin water and beef extract, the milk which is substituted must be well diluted.

If the temperature is high, it may be safely reduced by means of sponging with cool water and alcohol, the graduated bath or cool packs. These measures have the additional value of refreshing the flagging vitality of the child. At times, still stronger remedies are called for ; in fact, a wise stimulation is always to be kept in mind. For this purpose hypodermatic injections of strychnine do the work most satisfactorily.

The patient should be kept in the air as much as possible, and a trip to the country, seashore, or mountains is of much importance. After convalescence has set in, tonics, in the form of nux vomica or strychnine, combined with small doses of the organic preparations of iron, may be given until the child seems in flourishing health. The return to the ordinary diet must be made slowly and by conservative gradations.

Differential Diagnosis. - The main error that one is apt to fall into is mistaking the diarrhœa, or diarrhœa and vomiting, that 
occur in the course of the acute infectious diseases, especially in the summer, for these derangements. The former disorders are not so violent nor so prolonged, the movements usually do not have the intensely green color that we are familiar with in infectious intestinal derangement, and in addition the fact of their occurrence in the course of the acute infectious diseases makes the likelihood of their being more than a passing complication very small.

Prognosis. - If these cases are treated promptly and vigorously, the outlook is good. Half-hearted measures, either in cleansing the gastro-intestinal track, or in the restriction of food, is as dangerous as the disease itself. As the disease may be easily communicated by contact, it is highly desirable that the child's napkins, clothes, and bed linen, as well as the hands and clothing of the nurse, be thoroughly disinfected as often as possible or convenient.

\section{Subacute Intestinal Infection}

It is difficult to make any ætiological distinction between the acute and subacute forms of intestinal infection. So much remains to be found out concerning the natural history of intestinal germlife, that one is not justified in trying to assign responsibility to specific bacteria. One may say, in short, that when the temperature of the air is $15.5^{\circ} \mathrm{C}$. $\left(60^{\circ} \mathrm{F}\right.$.) or more, the many micro-organisms that may infest a child's food begin to have the proper condition for existence. A condition of malnutrition, and especially a disordered digestion in the stomach or intestines, give the opportunity for them to spring into activity. Whether the difference in the course of the disease from that of the acute form is one of quantity or quality, is a matter that can, with our present knowledge, be discussed indefinitely without necessarily coming to a satisfactory conclusion; for the course and symptoms of subacute intestinal infection vary widely in different cases and thus give an opportunity for much divergence of opinion.

Lesions. - The pathological changes are not confined to one separate part of the intestine. As a rule, they begin in the small intestine, whence they may extend in either or both directions. In some cases they are surprisingly small in extent and severity. In others they include swelling and congestion of the mucous membrane, exfoliation of epithelium, occasional formation of superficial 
ulcers, and the swelling and congestion of the associated glandular structures.

Symptoms. - The disease may, and often does, begin in a gradual fashion. Usually, there is a history of intestinal disorder. The child is fretful, peevish, and shows a loss of flesh and strength. The stools begin to increase in number and change in quality. The food may be seen in them in undigested particles, and small masses of casein and drops of fat may easily be distinguished. At times, these movements may have a disagreeable odor, but it is not so penetrating nor persistent as in chronic functional derangement of the intestines. The stools may vary in number, gradually growing greater from the beginning. Their hue may be various, either yellow, brown, or green, or a mixture of all three. Especially after the beginning of the disease there may be considerable amounts of mucus in the stools; but this is apt to vary from day to day. Associated with these unnatural movements is a production of gas, which, when its amount is appreciably large, causes pain and restlessness. The putrefactive processes, which are at the foundation of gas-formation, are capable of setting up various forms of toxicity that have a very important bearing upon the course and outcome of the disease.

Although the attack may begin with some vomiting and there may be occasional signs of gastric irritability, nevertheless, on the whole, the stomach is fairly free from any marked extension of the disease in the intestines. A broad statement of the case would say that the disease is located in the intestinal track in and below the lower part of the ileum, and that practically all absorption of toxic matter takes place in these portions of the gut. Whatever serious organic changes may take place arise from this cause.

The temperature is variable, rising with any exacerbative influence and dropping when such influence is eliminated. When the temperature is high, the tongue is apt to be more coated than at other times. The respiration and pulse have a tendency to be small, thin and weak.

The possible complications are many and depend for the most part upon the child's debilitated condition. There is a marked liability to skin diseases, especially erythematous inflammations about the buttocks and eczema of the face and head. Or there may be a local or general adenitis. In other cases there may be an cedema of the feet and legs, or a troublesome irruption of 
stomatitis, or an invasion of tuberculosis or hypostatic pneumonia. Most of all, one must keep in mind that children who are suffering from subacute intestinal infection have lost much of their original resistance to disease and therefore easily contract all manner of germ-diseases and, in a lesser degree, diathetic diseases.

Treatment. - The preventive treatment is of the greatest possible importance. A child who is fed on breast-milk of good quality, who enjoys the advantages of rigid cleanliness, of exercise in the air, and of hygienic domestic surroundings, will not, according to ordinary experience, run much danger of intestinal infection. It is of real importance that a family physician should explain to the mother the meaning of infection in general and the ease with which children contract infectious and fermentative disorders of the gastro-intestinal track. She must understand the responsibility that rests upon the child's unboiled napkins and the nurse's infected hand, upon utensils that are not absolutely sterile and toys that are filthy.

As soon as the attack begins, the stomach and intestines should be emptied by repeated fractional doses of calomel followed by castor oil, or, in larger children, citrate of magnesia. After this the lower intestine should be thoroughly irrigated with a sterile normal salt solution, as described in the treatment of acute intestinal infection. This washing may be practised every day until the movements are fairly normal. In some cases, that are marked by much tenesmus, one or two small doses of the deodorated tincture of opium may be needed to give rest and quiet. In more instances a liberal use of the subgallate of bismuth every hour for a day or two will bring about the same result. In all of these children the strength will in part be maintained and recovery facilitated by the liberal use of tonies and stimulants. For this purpose I have used nux vomica or strychnine with considerable satisfaction, sometimes combining it with an organic preparation of iron. Occasionally one may use, to bridge over a crucial period of depression, small and repeated doses of good whiskey or brandy. Whenever the condition of the child permits, and the weather is bearable, he should be taken into the air and kept there as long as possible. Residence in the country or at the seashore is better than in the city, and cool, airy rooms have a distinct remedial advantage over hot and stuffy apartments. In short, 
every aid in the way of sanitary and hygienic regulation has a real value in the effort to regain health.

From the very first, all milk must be excluded from the dietary. This rule is rigid and no advantage can be obtained by trying to modify it. The food may be made up of albumin water, of homemade beef extract and some barley water. These can easily, by the use of judgment and tact, be so manipulated as to satisfy the child's hunger and the parents' anxiety. When convalescence has set in, much good may be obtained from the use of iron, the compound syrup of hypophosphites and occasionally from codliver oil.

Prognosis. - The question whether these cases will live or die depends much upon the degree of wisdom exhibited in the medical and general care of the patient. Poverty and ignorance have destroyed their thousands. If all circumstances are favorable, a large majority recover. The parents must be warned that until recovery is fully established there is a constant danger of recrudescence.

\section{Chronic Functional Derangement of the Intestines}

This disorder is in large part the result of ignorance, therefore it is commonly seen. The majority of its causes are avoidable, but their effects, when once set in motion, are not so easily controlled. These causes are wrong feeding, too frequent feeding, exposure, uncleanliness, repeated attacks of acute derangement which have not been carefully treated, and deficient regulation and control of the general life of the child. It is not often that one sees, in a general and mixed practice, an infant whose diet, if artificial, is really well arranged. Especially are parents not apt to reckon on the general debility that follows acute gastric disorders, the eruptive fevers, and the frequent attacks of bronchitis to which babies are subject. In fact, the subject of infant feeding is not, as a rule, handled with sufficient thoroughness and seriousness by parents, nor even by many medical men. They lose sight of the fact that the milk upon which an infant lives is composed of various elements which depend for their digestion and assimilation upon diverse secretions, and that a congenital or acquired disturbance of equilibrium, as commonly worked out by a method of averages, makes the ordinary artificial food undesirable. 
This statement is true of human milk which varies from the standard; and the mere fact that a mother has a plentiful supply of milk, or that she may have nursed other children with success, does not at all mean that her milk is suited for the patient in question. The continued administration of such a diet is one of the commonest and least appreciated causes of chronic functional derangements in infants. In addition, the deterioration, either bacterial or fermentative, of the food-contents of the intestines is a fruitful cause of various organic lesions and conditions of low intoxication.

In older children an unregulated and too promiscuous diet will accomplish much the same results. Lack of cleanliness has its share of importance in the causality; especially in summer, when the heat and humidity hasten the decomposition of food and make infection easy.

Lesions. - On examination one may find in the less serious cases almost no pathological changes. As the condition becomes graver one may see an irritation and swelling of the mucous membrane, a congestion of the glands and lymph nodes. Not only are the lesions not constant, but also they are not uniform in their location. Thus they may start at any part of the small or large intestine and thence work their way up or down.

Symptoms. - From the first the patient begins to show malaise or even prostration. The mental depression may be noticed before the physical inroads of the disorder are pronounced. The eyes look heavy, the skin dull, the tongue somewhat coated. After a short period, the weight of the child begins to decrease and finally extreme emaciation may result. Although the limbs, neck, and chest may be much wasted, the abdomen is often puffed out and distended; there may be sufficient stretching to make the superficial veins unusually visible as thin blue lines. For the most part this is caused by the presence of various gases of decomposition which the atonic muscular tissue is not able to confine within the ordinary calibre of the intestines. Naturally there will be attacks of pain and discharges of gas.

The movements at the beginning of the attack are disordered, periods of diarrhœa following short times of constipation. After the disorder is well under way an irregular diarrhœa may be expected. As a rule the stools are not profuse, are semi-fluid, and are characterized by the presence of varying amounts of undi- 
gested food. This last item helps to give the clay or putty color that is so often seen. At other times one may notice the shades of green that are present as the product of chromogenetic bacteria or the unusual excretion of the biliary coloring matter, biliverdin. A characteristic feature of the fæces is their offensive odor which is so pronounced as to induce some observers to call these movements "putrid." During an attack of constipation the passage of a large, hard mass of freces may scratch the mucous membrane and bring forth a thread of blood. This must not be confused with a possible ulceration of the intestine which usually is indicated by dark, clotted blood in a dark, watery movement.

The child's appetite is poor, and if he is induced to eat, the process is apt to be followed by diarrhoic movements. Tenesmus, accompanied by pain, may characterize this condition. Exacerbations, such as these, may elevate the temperature slightly; otherwise it does not run high, and at times of prostra. tion it may be subnormal. At such periods the pulse is weak and small, the respiration tends to irregularity. The liver, spleen, and mesenteric glands do not seem to the touch to be enlarged, nor do we find albumin in the urine.

When convalescence sets in, one notices first of all that the mental condition is improved, the restlessness and peevishness decrease, and the face loses its drawn and puckered aspect. In infants the secretion of tears, which in the height of the sickness had been interrupted, is resumed. The body begins slowly to fill out and the movements become fewer. For a long time the foul odor persists in them and the change of color to the normal is gradual. This evolution to health is practically never uninterrupted, even though the treatment and general care be wise. The lapses, however, become progressively shorter. It is noteworthy that humid and bad weather has an unfavorable effect upon these cases, while under favorable meteorological conditions they are much more comfortable.

Treatment. - An important part of the treatment is the general care. The patients, especially infants, must be warmly clothed, and especially must the extremities be kept warm. Care must be exercised that the child be not chilled during or after the daily bath. Cleanliness of the mouth is of especial importance; the ventilation of the nursery, the airing of the bed 
linen, and the cleanliness of the clothes must be carefully supervised. All milk must be forbidden and replaced by albumin water or beef extract. In older children the diet may be composed of clear meat soups, soft-boiled or poached eggs, toast, or zwieback, scraped beef or lamb, with as small a quantity of starchy vegetables as possible. At times wine-whey may be useful.

The medicinal treatment is simple, and on the whole less important than the dietetic. The intestinal track may be emptied by means of calomel in divided doses, followed by a saline cathartic, such as the citrate of magnesia. The subgallate of bismuth may be used, preferably in large doses $(0.3 \mathrm{gm} .-\mathrm{gr}$. v every two hours for a child of three years). The bowel may be thoroughly irrigated every day with a warm normal saline solution. One of the most necessary details to keep in mind is the treatment of the mental and physical depression. This can best be done by the administration of nux vomica or strychnine; the latter, administered hypodermatically, is of the greatest value in the collapse which sometimes attends this sickness. In some cases, especially in older children, small doses of whiskey or brandy diluted may be temporarily required.

When convalescence sets in, these children are often benefited by being taken to the country, where they should be kept until recovery is complete.

Prognosis. - The outlook is fairly good. Nevertheless the parents must be informed that the sickness is apt to be tedious and convalescence prolonged. 


\section{CHAP'TER XI}

\section{DISEASES OF THE LARGE INTESTINE (Continued)}

\section{INTUSSUSCEPTION}

Causes. - Intussusception, or, as it is sometimes called, invagination of the intestine, occurs more frequently during infancy than at any other period of life. Boys seem to be oftener attacked than girls, but the question of sex does not have any influence upon the course or mortality of the disease. The origin of the condition is hard to locate; nevertheless one can obtain a logical idea of it from experimental research. We know that electrical stimulation causes local contraction of a tense, spasmodic nature; when this ends, the portion under it rolls up and over it. In the living subject the process probably starts from the irritation brought on by improper food. This may happen from either an antecedent intestinal derangement or food that is taken in a state of health. Thus one can understand that some cases of severe colic, which eventually recover, are really examples of intussusception that become reduced either spontaneously or by the action of sedatives.

Lesions. - The changes take place, as a rule, in the peristaltic line, from above downward; only exceptional cases reverse the direction. Usually there is no more than one layer of intestine invaginated in another; nevertheless there are unusual occurrences of a series of two or three invaginations, one inside of the other. The condition can be simply and satisfactorily imitated with the finger of a glove, and a more elaborate and satisfactory counterfeit can be made with one or two metres of bullock's gut. It is advisable to make such a reproduction; for one immediately will have a vivid picture of the condition of the three layers, the outer and inner of which run in their normal direction, while the middle is reversed, its mucous membrane lying in contact with that of the outer. The condition may occur in any part of the intestine, but the commonest location, occurring in three-fourths 
of all the cases, is where a part of the ileum descends into the colon; the next common in frequency is in the colon, and after that come invaginations of the small intestine.

As a result of the pulling and squeezing on the mesentery as well as the gut - which is especially liable to happen in infants congestion, hæmorrhage, inflammation, and gangrene may supervene. In fact, it is from one or more of these factors that the serious symptoms of great swelling of the invaginated portion, adhesions of the layers, and sloughing of the constricted part result. With these changes, concomitant lesions of peritonitis may supervene. Difficulty in reduction may be increased by strain from the mesentery, which changes the right shape of the affected part to a concavo-convex outline.

Symptoms. - If there is no previous disorder of the gastrointestinal track, one ought to have no trouble in diagnosis. Generally the onset of paroxysms is sudden and the cries which they elicit are piercing and startling. Almost simultaneously the child vomits and has one or a few loose movements of the bowels. Following these there will be a number of small fluid movements consisting of mucus stained more or less copiously with blood. The temperature is normal or subnormal, the face during the paroxysms is puckered and anxious, the pulse is feeble and often irregular, and a general picture of marked prostration gradually unrolls itself. Shortly one may in more than two-thirds of the cases feel a tumor in the abdomen, most often on the left side in the region of the sigmoid flexure, which increases in size as the invaginated portion slips lower and lower toward the anus. The abdomen is soft and unstretched until tympanites begins; as this happens, the temperature rises and may remain high; the total obstruction is apt to induce stercoraceous vomiting (although this is less frequent in children than in adults), the pain becomes almost unbearable, and the patient is apt to fall into collapse.

A minority of the cases, especially recurrences, begin with a fairly gradual onset. In a short time, however, they present the characteristic picture of the disease. Recurrences are apt to occur, especially if after reduction the child is not kept quiet. Fairly often an examination through the anus, even if the tumor was originally felt high up, will disclose the end of the invaginated part in the rectum; and it is not very rare that this part may protrude for one or two inches through the anus. In other 
and rare cases, the constricted portion may slough off and be passed with a movement. In the subacute and chronic cases, which have a slower onset and last for weeks instead of days, shreds of gut instead of a section may be passed. The ordinary case lasts from a day and a half to a week.

Treatment. - As soon as the diagnosis is made, all food, drinks, and cathartics must be stopped. Opiates should be given in large enough doses to keep the patient as quiet as possible. Usually I prefer to treat these cases by hypodermatic medication and thus avoid any additional strain upon the activity of the stomach. As soon as the child is quiet, one must attempt to reduce the invagination. One had best begin by using hydrostatic pressure. For this purpose an ordinary fountain syringe may be employed, hung about 150 to $200 \mathrm{ctm}$. ( $5 \mathrm{ft}$. to $6 \frac{2}{3} \mathrm{ft}$.) above the bed. While the water is passing into the rectum, the buttocks should be higher than the shoulders. Some practitioners prefer the totally inverted position; but this will be of use only in the first stage before the invagination is much swollen.

Another method consists in the use of air forced high into the bowel through a soft rubber catheter by means of a bellows. When this is done, one usually anæsthetizes the patient. With either treatment, while the intestine is being dilated, the abdomen must be rubbed and lightly kneaded. In both injection and inflation the manipulations must be practised with great care in order to avoid the danger of rupturing the bowel. This accident has been reported as happening after the employment of unusual and, in some instances, moderate force. The physician cannot, therefore, exercise too much caution in these procedures.

If the attempts at reduction are not successful, a surgeon should without delay be called in to open the abdomen. The longer this step is put off, the fewer are the chances of recovery. It is undoubtedly true that delay is far more dangerous than operation, no matter how young the child may be.

Prognosis. - Delay in diagnosis or treatment usually means death. If reduction by hydrostatic pressure or inflation is unsuccessful, delay in operating is almost always fatal. By promptness in measures of relief the mortality can, I believe, be reduced to 50 per cent or even less.

Differential Diagnosis. - The only conditions with which one can confuse intussusception are gastro-enteritis and ileo-colitis. 
In reality there is very little in common between them, and the mere mention of the possible confusion ought to be enough to obviate the probability of it. The history, peculiar stools, early vomiting, and temperature of enteritis and colitis are absent; and unless such a rare event as intussusception complicating an enteritis or colitis occurs, the distinction should be readily made. The history, vomiting, headache, coated tongue, and the usual pains of gastritis mark off this disease very clearly from intussusception. The projection of an invagination may be mistaken for prolapse of the rectum or even hæmorrhoids; but a fairly careful examination will correct the error. 'The possibility of fæcal impaction must also be considered. This occurs in the large intestine, in the crecal or sigmoid region. There is a history of gradually increasing incompetence of the bowel due to over-distention and the accumulation of fæces. The attack is not acute; there may be a swelling which has a large and not clearly defined shape, and when the child is anæesthetized the tumor can be clearly indented by the operator's finger. Finally, the principal symptoms which we must expect to find in intussusception are a tumor which commonly has a cylindrical form, marked abdominal pains that the patient describes as colic, tenesmus which may at first be accompanied by a few small and loose movements, but later on by the passage of blood-stained mucus.

\section{Volvulus}

When the mesentery of the bowel is very long, especially of the sigmoid flexure of the colon and the nearest portion of the ileum, a twisting of the gut upon its axis may occur, which is called volvulus. It may be due to a large mass of hardened intestinal contents or a great collection of Ascarides lumbricoides, or to unknown irritations. The disorder is not by any means a common one, especially in children. The symptoms are much the same as in intussusception, except that there may be no blood or even mucus discharged. Among them will be pain, prostration, a slightly elevated or even subnormal temperature. If the twisting is in the small intestine, vomiting will set in early and be a prominent feature of the symptomatology ; if the sigmoid flexure is the part involved, the vomiting may not appear until the condition is plainly urgent. If one can distinguish Wahl's sign (a well- 
defined tympanitic spot at the site of the volvulus), the certainty of correct diagnosis is much increased. The treatment consists in laparotomy as soon as diagnosis is made. Nothing is gained by delay excepting a greater probability of rapid death. A majority of the cases, when promptly operated upon, recover.

\section{Animal Parasites of the Intestines}

The animal parasites which live or breed in the human intestines belong, for the most part, to the sub-kingdom of worms and are divisible into the two classes, platyhelminthes and nemathelminthes. The first are "worms with a flat, elongated body, very generally provided with hooks or suckers or both; they possess a cerebral ganglion and, as a rule, are hermaphrodite." The second are "roundworms with a tubular or filiform body, the cuticle of which is often ringed; the head end may be provided with hooks or papillæ: the sexes are separate." Of the platyhelminthes two orders are described: Cestoda (tapeworms) and Trematoda (flukes). Of the latter there have been only the fewest instances among children. Of these Cobbold reported a case of infection of a large distome (D. Crassum) in a child who had resided in China. Many species of the platyhelminthes are known to live in the human body, but not all are associated with child life. Of these latter the commonly known appear in the following table:-

\section{Platyhelminthes}

Cestoda,

$\begin{array}{ll}\text { Family_Tæniada } & \\ \text { Tænia nana } & \text { Tænia flavopuncta } \\ \text { Tænia echinococcus } & \text { Tænia solium } \\ \text { Tænia mediocanellata } & \text { Tænia cucumerina } \\ \text { Family - Bothriocephalida } \\ \text { Bothriocephalus latus }\end{array}$

Tapeworms have certain common characteristics: they have a long, flat, whitish body, called strobila, composed of many segments, or proglottides. This body is surmounted by a minute head and neck, termed scolex. Generally there are one.or more rows of hooklets placed aboüt a central rostellum or beak; in all cases there are two or four suckers or discs which aid in attaching the parasite to the intestinal wall of the host. The mode of trans- 
ference and growth is simple. The proglottides, when the eggs which they contain are ripe, leave the intestinal canal. These eggs, in order to infect another host, must be swallowed. This may be done directly if they are expressed by the proglottis ; or in other cases the whole segment is swallowed and when the wall is digested in the stomach the ova are free. The embryo, after shedding its outer covering, works its way by means of its six hooklets to the maturing place. These hooklets are then shed, and from the opposite end of the embryo a scolex begins to grow. In this stage the organism is called a cysticercus, on account of which the type of organism is called cystici ; another type whose cysticercal process is very small is called cistoidei. The echinococcifer are characterized by the so-called echinococcus cysts, from the inner wall of which secondary cysts or brood capsules bud out. In the bothriocephalidæ no cysts are formed; in these parasites the embryo enlarges gradually but directly in the intermediate host and in such form is taken into the system of the definite host.

Ordinarily the cysticercus in the muscles, connective tissue, or viscera of the intermediate host finds its way into the intestines of the final host where it attaches itself and grows into the mature worm.

Tonia mediocanellata (synonyms: T. saginata, T. inermis, Tæniarhynchus mediocanellatus) is the commonest tapeworm that we find in children. They become infected by eating raw or imperfectly cooked beef which contains the cysticerci. The parasite is from four to eight metres long, and has a thousand or more proglottides, the largest of which are from fourteen to eighteen $\mathrm{mm}$. long and twelve to fourteen broad. The head is very small and has four suckers but no rostellum or hooklets. The eggs are only $0.03 \mathrm{~mm}$. in diameter and have an embryo with six hooklets. The cysticerci may live for years without losing their vitality. This parasite is more widely distributed in eastern Europe, Asia, and Africa than in western Europe and the United States. The affected beef is easily recognized by the presence in it of narrow cysts, one ctm. in length.

About two months are required for the development of the cysticercus into the full worm, which may live for many years, the exact number not being known.

Tonia solium finds its way into the intestine in raw or imperfectly cooked pork which is infected with the cysticerci. It is 
found wherever the pig exists, and grows most luxuriantly where the animal is poorly cared for. The parasite is characterized by having twenty-two to twenty-eight hooklets in a double row about the rostellum and by its four suckers. It is from three to three and one-half $\mathrm{m}$. in length. The proglottides are considerably smaller than those of $\mathrm{T}$. mediocanellata. The embryo has six hooklets.

Tonia echinococcus is described under the heading of Echinococcus Cysts of the Liver.

Toenia nana (Cystoidei) is the smallest tapeworm which occurs in human beings. It is from twelve to twenty $\mathrm{mm}$. in length and one-half $\mathrm{mm}$. in breadth. It has from twenty-two to twenty-eight hooklets about a rostellum and has four suckers. Its embryo has six hooklets, and is enclosed in a double shell. It is more apt to occur in warm than in temperate or cold climates, and preferably in children than in adults.

Toenia flavo-punctata is a very rare worm which has been found only in children. It gets its name from a yellow spot that occurs on the proglottides of the fore part of the worm. 'The segments are triangular or trapezoid in form, and measure three $\mathrm{mm}$. in length and four mm. in breadth. The embryo has six hooklets.

Tonia madagascariensis has been seen in Asia and Africa. Very little is known of it.

Tonia cucumerina (synonyms: T. canina, T. elliptica) is said to develop in the dog-louse (Trichodectes canis). From the affected $d o g$ or cat it finds its way into the human subject, who generally is young. It measures from twelve to thirty-five $\mathrm{mm}$. in length and its segments are from one and one-half to two $\mathrm{mm}$. in breadth. The head has from forty to sixty hooklets about its rostellum as well as four suckers.

Bothriocephalus latus has some individual characteristics which differentiate it sharply from those described above. It grows from eight to sixteen $\mathrm{m}$. in length, has from three thousand to four thousand proglottides, the middle ones of which measure from four to five $\mathrm{mm}$. in length and ten to twelve $\mathrm{mm}$. in breadth. Toward both ends the width of the worm decreases markedly, the neck especially being very narrow. This worm has no cysticercus form. The eggs while in water develop six hooklets. They must then be swallowed by some animal as yet unrecognized by which they are transferred to certain fresh-water fish where they 
may lie free or encysted. When such fish is eaten raw, smoked, or partially cooked, the parasite is transferred to the animal eating it.

It is found in Russia, Switzerland, Italy, Japan, Ireland, the United States, and other fish-eating countries. The life of the worm is long, being sometimes twenty-one years or more.

Symptoms. - Unless the proglottides are seen in the stools or about the patient, it is hard, and in many cases impossible, to diagnose the presence of tapeworms. There may be various nervous symptoms, digestive disorders, and possibly anæmia. If the child for any reason fasts or confines himself to a milk diet, the symptoms become more marked. The commonly mentioned signs of teeth-grinding during sleep, and nose-picking are falsely believed to be distinctive of the presence of worms.

Treatment. - Anthelmintics are best given on an empty stomach. The usual method is to give a supper of a glass of milk; in the morning a saline cathartic, given on rising, will empty the intestines. After this the oleoresin of male fern may be given in capsules. The ordinary dose for children of from eight to ten years is 0.75 to $1.0 \mathrm{gm}$. (gr. xii to $\mathrm{xv}$ ) in half-hourly doses four or at the most five times. This should be followed by a brisk dose of castor oil. The child should then lie down until the bowels move again. When the worm is expelled, a careful search must be made for the head; for without it one cannot be sure that the parasite is entirely removed.

Other drugs that have been used for this purpose are chloroform, oil of turpentine, pumpkin seeds, kousso, and pomegranate root. The last-named drug in an improved form is sold as the sulphate of pelletierine ; its use is attended with danger of poisoning and therefore should be omitted in treating children.

I have used thymol advantageously in these cases; it may be prescribed in emulsion or capsules, in doses of $0.06 \mathrm{gm}$. (gr. i) three or four times a day for a child of five years.

Nemathelminthes. - Order, Nematoda. This order is composed of long, cylindrical animals that inhabit the human intestine. The ends of the worm taper off considerably, the tail of the male curving so markedly as readily to serve as a means of identification. The female is larger than the male. All in all, the sexes are quite distinct.

Family, Ascarida. These worms have long, stout bodies; the 
head has one ventral and two dorsal lips. There are two species commonly and one rarely seen in children :-

\section{Ascaris lumbricoides. \\ Oxyuris vermicularis. \\ Ascaris mystax.}

Ascaris lumbricoides, or roundworm, inhabits the upper part of the small intestine. The length of the male is as a rule from fifteen to twenty-five ctm.; that of the female is often twenty ctm. more. The breadth of the male is four ctm., that of the female is six. The body is firm, of a pinkish color, a glittering surface, and has numerous, thin, transverse striations. The ovum is spherical in shape, resists heat and cold well, and requires half a year or more for its development. The eggs are formed in immense quantities, although the mature worm is not often found in any but very small numbers in one host. The embryo, which may exist for years as long as conditions are not too unfavorable, is taken into the stomach where its outer envelope is shed. It then in a few weeks becomes mature. The worm is found in persons of all ages (but most commonly in children), and in all climates.

Symptoms. - The signs which this worm produces are very obscure, and unless the parasite is expelled or the eggs seen in the fæces, the diagnosis is never quite certain. It doubtless can and does produce disturbances in the gastro-intestinal and nervous systems, and in the quality of the blood; but farther than this one may not go. Some of its most noteworthy effects are due to its ability and disposition to wander into more or less remote portions of the body. Thus it has been known to work its way into the hepatic and pancreatic ducts, through the intestinal wall into the peritonæum, into the air passages, through the urethra, Merckel's diverticulum - in fact all of the thoracic and abdominal viscera may be the seat of its migrations and give symptoms according to the particular location of the worm and the interference with organic and functional action which its presence creates. Another remarkable fact is its disposition to leave the body of the host as soon as he dies.

Treatment. - To remove the worm one begins by administering an active dose of calomel followed by another cathartic. The first may be given at night, the second on arising the next morning. These should be followed by a sufficient quantity of santo- 
nin, which stops short of producing the full physiological effects of the drug. The danger signal is the disturbance of vision that makes all objects seem yellow to the sight; if the drug is pushed yet farther, a yellow or reddish discoloration appears in the urine.

This course may have to be repeated on one or more days until the intestinal track is clear.

Oxyuris vermicularis (synonym : Ascaris vermicularis) is commonly called thread or seat worms. The females only are generally seen; they look like small shreds of white thread. Although they are only from eight to twelve $\mathrm{mm}$. long, and one-half $\mathrm{mm}$. broad, nevertheless, they are more than twice as large as the male. The tail of the female is long and pointed; that of the male is fairly blunt. The eggs are oval and very numerous. They are taken into the stomach with raw or unclean fruit and vegetables, or through the agency of dirty hands. In the stomach, the embryo rapidly develops, at times in little more than two weeks. In the small intestine, the male, after impregnating the female, dies, while she descends to the cæcum where her eggs develop. She is then ready to emigrate to the rectum, and out of the anus.

Symptoms. - The main effects which this parasite produces are local irritations of the rectum and the skin about the anal, perineal, and genital surfaces; various skin diseases, enuresis, abnormal sexual excitation, disturbances of the gastro-intestinal, nervous, and genito-urinary systems likewise show themselves.

On account of the itching, the child rubs or scratches the affected parts, contaminating his hands, fingers, and nails with the worms and their eggs, and then by bringing the hands to the nose or mouth, reinfects himself. In this way the vicious circle of contagion may be perpetuated for years.

The only sure way of diagnosticating the disorder is by seeing the worms in the fæces or about the anus and genitals.

Treatment. - The care of these cases, in order to be finally effective, should be continued for six weeks. The principal method of cure is the administration of enemata of quassia, salt water, garlic, vinegar, and diluted alcohol. Suppositories of quassia or garlic and cocoa butter accomplish the same result. Although it is impossible to reach the cæcum with the small enemata which we use in this disorder, nevertheless the repetition of this administration, by getting rid of the parasites in the rectum, will finally 
accomplish a cure. In order to throw the enema as high up into the bowel as is conveniently possible, the child's position during the operation should be on the back, with the hips somewhat higher than the shoulders.

The itching and irritation of the skin, especially at night, yield to bathing with carbolic solutions or the use of salicylic acid or mercurial ointment. During the whole course of treatment and until the parasites are finally eliminated, scrupulous care must be exercised to keep the hands and nails clean and the body thoroughly covered at night by such sleeping garments as prevent the child from touching the parts and the worms from escaping into the bed and about the patient. Where there are several children in a family, especial care must be exercised to prevent infection.

Ascaris mystax is a parasite that normally inhabits cats and dogs. By handling and playing with these animals, especially if they are not kept clean, children may become infected. The worm is small, the female not growing beyond twelve ctm. in length, and the male half that length. The eggs are covered with a reticular formation which is said to resemble the mace on a nutmeg.

The worm is rarely seen.

\section{Intestinal Colic in Infants}

This troublesome symptom occurs mostly in young infants. It depends upon fermentative changes in the intestinal contents by which gases are generated. The distention which thus arises, in conjunction with more or less muscular spasm, is the cause of the pain. These changes in the food are based upon the imperfect digestion, especially of proteids. All children are liable to this condition, but especially those who are fed upon cow's milk; for such milk possesses casein that in coagulation is heavy and difficult to digest. Too much sugar, especially cane sugar, in the food may cause similar symptoms; fats also may bring about, although more rarely, a like result.

The colic of congestion belongs in a different category.

The treatment consists in administering enemata that by stimulating peristalsis will expel the gas. One hundred and twenty to two hundred and forty grammes (four to eight ounces) of 
warm water and soap-suds will usually give relief, especially if aided by a hot bath and the application of a hot water bag to the abdomen. After the child has become quiet, the intestines should be emptied by a full dose of castor oil ; and thereafter such changes should be made in the food as will prevent a repetition of the pain and spasm.

A very severe case of colic may possibly be confused with volvulus, intussusception, with appendicitis, or intestinal obstruction ; but premonitory symptoms of slight intestinal disorders, the sudden attack of pain without prostration, and the relief which follows an effective enema usually show without delay the real nature of the disorder.

Only in the severest cases does one need to use opium.

\section{Habitual Constipation}

Young children are peculiarly liable to this disorder because their anatomical features, their necessities for a special diet, and their physical immaturity predispose them to it. The subject is an exceedingly important one and requires serious attention.

Causes. - The anatomical peculiarities should be well understood." "During infancy and childhood the intestines grow irregularly, by fits and starts, as it were; their position varies from that of the adult, and also they are less fixed. The constriction which may be found in adults at the junction of the first and second parts is commonly absent in infants; the transverse colon is relatively low. In the large intestine, up to four months of age, the length remains quite stationary. After that time a remarkable change takes place; the upper portion begins to grow at the expense of the sigmoid flexure, which at birth is nearly one-half of the whole large intestine, while at four months it already assumes its permanent proportions. The ascending colon in children, owing to the higher position of the cæcum and the greater size of the liver, is very short. This part of the colon has more often a mesentery than in the adult, and also a relatively larger portion above the cæcum is invested with peritonæum so that the gut is here absolutely free. The cæcum alone changes its position and relations so much in the course of development that the transitional nature of childhood is clearly made apparent.

1 Oppenheim: "The Development of the Child." Macmillan, 1898. 
"About the fourth month of fœtal life this part of the intestines is situated near the median plane; and at a higher level than in the adult. As it grows it passes to the right side, in front of the second part of the duodenum, and then descends into the iliac fossa. Even then it is apt to be placed high up near the anterior superior spine of the ilium. A corresponding position is held by the sigmoid flexure, hardly any of which is found in the pelvis until this bony basin is more widely spread out by later development."

"The rectum, as one would expect, shows conditions somewhat similar to the main part of the large intestine. In the adult it is situated entirely within the true pelvis and presents three curves : one in the lateral and two in the antero-posterior direction. On the other hand, in the infant a large part of the rectum is in the abdominal rather than the pelvic cavity; it is nearly straight and occupies a more or less vertical position. Its attachments do not extend as high in children, and the reflection of the peritonæum is placed lower down." Thus one can understand how with a narrow, undeveloped pelvis, with a great length of intestine much of which lies in the abdominal cavity, with comparatively few fixed points to act as points d'appui, and with sectional directions that give the least advantage in promoting peristalsis, one can understand, I think, how liable infants and young children are to constipation.

In addition, the muscular fibres in the walls of the intestine are often poorly developed. This may be characteristic of the whole or a part of the intestine. Moreover, since with growth and exercise every part of the child's body may develop favorably, such a condition may be transitory, not necessarily characteristic of the child's whole life.

Another important cause is a poorly constituted diet. Breast milk that is poor in fat, or too rich in proteids, or deficient in quantity may logically give rise to constipation; for not only are local necessities unsatisfied, but also there is apt to be a deficient nutrition. When the food is an artificial one, there is still more likelihood of constipation; for milk that differs much from mother's milk, that has too hard a curd, that is boiled or sterilized, has an undoubted tendency to bring about this condition. Irregularity of habits and lack of attention to the subject have also a share of responsibility.

Pathological conditions, either of the intestines or of the ner- 
vous system, - such as intestinal adhesions on the one hand, and tubercular meningitis on the other, - are purposely excluded from the ætiology ; for in them the constipation is merely a secondary symptom.

There are no characteristic lesions, excepting the local irritation and possible erosion of the mucous membrane which the passage of hard, scybalous masses might cause.

Symptoms. - Outside of the familiar phenomena of constipation, the possible symptoms are functional derangements of the gastro-intestinal track, subsequent nervous disorders, eruptive diseases of the skin and scalp, adenitis, prolapse of the rectum, intoxication from absorption of matters in the intestine, and pressure effects which may interfere with the action of the diaphragm, heart, and visceral circulation. One should especially keep in mind fretfulness, irritability, and seeming viciousness of conduct that may arise from some small degree of intoxication.

Treatment. - The first step in the successful handling of these cases is the determination of the cause or causes which lie at the root of the matter. If an artificial food is the diet, it must be made to simulate in its physical and chemical composition mother's milk. If breast milk is the food, and if on analysis it proves to be deficient in fat, then the requisite amount of cream may be given after each feeding. If the milk be too rich in proteids, the daily life, diet, and exercise of the mother or nurse must be so regulated that the secretion becomes normal. If the milk for any reason remains abnormal, it is much better in the long run to wean the child without too much delay.

In older children regulation of the food may be of considerable use in promoting a satisfactory intestinal condition. 'The meals must be regular and should include such articles as fruit, breakfast cereals, stale bread made from the whole wheat, green vegetables, unboiled creamy milk, and sufficient water. They must not contain dried fruits (with the exception of figs), spices, or pastry.

An earnest attempt should be made to promote regular habits. The child must go to the closet or vessel at regular times, even though he may not at first feel the impulse. The formation of a habit is highly desirable, especially since children are so easily confirmed in one custom or another. Also the attendant should see to it that the patient assumes the proper crouching position which 
will enable the muscles to exert all of their power in aiding peristalsis. In addition, massage and kneading of the intestinal walls and coils are of use. A further measure that I have found valuable is abdominal exercise. This is accomplished by teaching the child to relax and contract the abdominal muscles. The amount of motion which children are able to acquire, thus insuring a marked increase in the development of the intestinal muscular fibres, is surprising. Also, one may prescribe gymnastic or calisthenic exercises that tend to strengthen these muscles; and if they are followed out faithfully and long enough, they will undoubtedly be beneficial.

The medicinal remedies are simple. Small enemata of oil or glycerin and water are temporary helps. Gluten suppositories may be used for a short time. For longer use a combination of strychnine, belladonna, and aloin may be given by mouth or in rectal suppositories. The former method gives the better result and has as its only objection the disagreeable taste of the drugs. I have found it possible to give small pills, embedded in fig paste, to children of two and one-half years. Such a prescription would read : -

$\mathrm{B}$

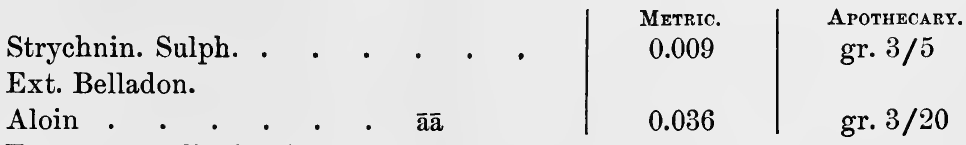

my Ft. massa et div. in pil. no. XV

Sig. Pil. no. i, t.i.d.

Each individual case will present peculiarities that a general survey of the subject can not and need not foresee. But if the physician will show the possession of logic, ingenuity, and patience, he will be fairly sure of gratifying results.

Prognosis. - The outlook, if the cases are well handled, is favorable. One must not expect good results too easily or quickly.

\section{APPENDicitis}

An inflammation of the appendix usually begins as a primary disorder which may spread in various directions; but the main fact to be remembered is that the appendix is the real seat of the disease even though neighboring parts may likewise become in- 
volved. For this reason, instead of speaking of typhlitis, perityphlitis, and paratyphlitis as distinct diseases, we now recognize them as being a few of the many manifestations of inflammation about the ileo-cæcal region. Since almost all of these cases hinge upon lesions in the vermiform appendix, we naturally group them under the generic term of appendicitis.

Causes. - The structural differences between very early life and adult life have a fairly important bearing upon the occurrence of appendicitis. In early childhood the appendix is shaped more like a funnel and less like the finger of a glove, than it is in later years. It is noticeably long, its mesentery is long, it is more freely movable than in later life, and also its location is higher. The former is said to lend itself less readily to receiving and containing concretions than the latter. Also, some stress may be laid upon the fact that the appendix is a functionless organ whose value was lost in primeval times. Following the nature of vestigial structures, it is more easily attacked by pathological processes than parts which have an active and useful life. This would in part account for its frequent disease, especially in adults. In infants, however, a modification of the rule would hold good. For they in some respects have, as normal features, peculiarities that were characteristic of less highly evolved forms. Thus, for instance, with the germs of the third molars, which develop as late as the fifth year, a fourth molar, a feature that generally, if not regularly, occurs in the platyrhine apes, is sometimes found. By an analogous force the appendix very possibly has more vitality in the first period of life than later on. Also, shortly after birth, it has a greater blood supply than in the mature person; for in the latter its mesentery and the included artery run only to the middle instead of, as in the former, nearly to the apex. An additional fact that must be kept in mind is that this artery runs along the free concave border of the appendix. Thus one can easily see that any twisting or pulling, due to the pressure of a large amount of gas, to colic or excessive peristalsis, is capable of causing sufficient interference with the local circulation to bring about congestive and catarrhal changes. When this happens, the bacterium coli commune, which appears to be harmless in health, attacks the injured tissues. When in addition there is a flourishing growth of streptococci in the affected region, the resulting intoxication is very serious and of a sort that 
tisese young patients cannot well withstand. Thus one can see that in children there are reasons for both the greater and less prevalence of appendicitis than in adults. And it is necessary to keep this in mind, because although these young patients are supposed to be more exempt from attacks than their elders, nevertheless I believe that more exact observation in the future will bring about a change of opinion.

Lesions. - The mucous membrane is swollen, the capillaries are injected, there are an emigration of red blood cells and a diapedesis of white. The course of the inflammation goes through the

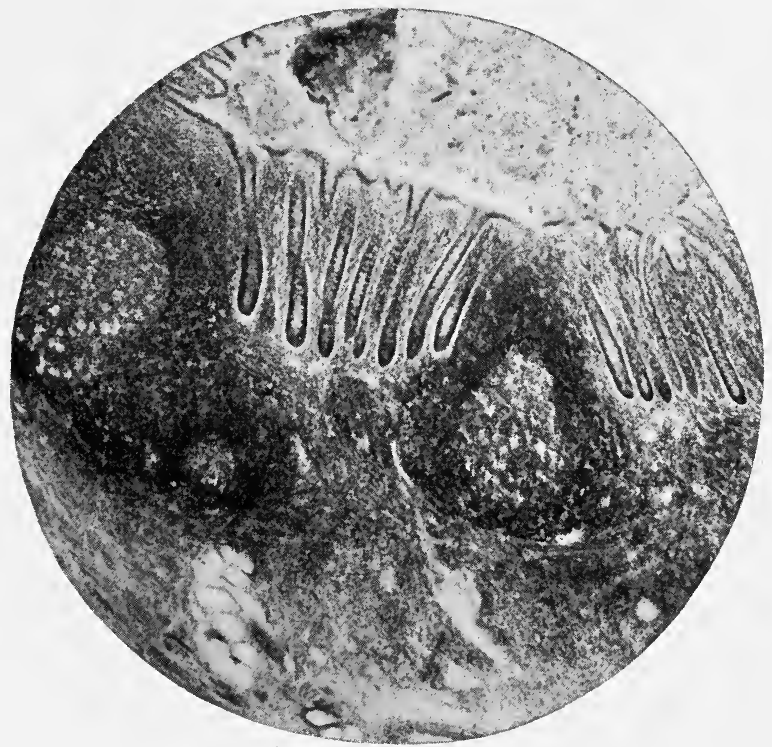

Frg. 27.- Acute Appendicitis. $\times 25$.

various stages to which all mucous membranes are subject. The congested condition of the capillaries fades into an acute catarrhal appendicitis. This may become resolved, or it may be followed by other similar attacks. The exacerbations give rise to a stricture at the base which is commonly attended by the accumulation of mucus, pus, fibrin, broken-down epithelium, bacteria, and fæcal material. The resulting irritation and pressure may start an ulcerative condition or empyæma of the appendix, which in its most virulent form may assume the nature of a gangrenous inflammation. The natural spread of a congestive and catarrhal appen- 
dicitis results in a localized peritonitis ; but in the ulcerative and gangrenous forms a pus process has formed which is located in the appendix as a focal point. From this focal point an abscess may start which later on will open into the peritonæum. If, on the other hand, the inflammation is so located that the appendix becomes fixed by an adhesive process to the parietal peritonæum, the general peritoneal cavity is not invaded. Instead, the inflamed part may become shut in by adhesions, or an abscess may be formed in the connective tissue back of the peritonæum, which may break through the lumbar region, along the psoas muscle, or into one of the abdominal viscera. If it extends above the liver and causes a subphrenic abscess, it may work its way through the diaphragm and start an empyæma, the pus from which may burrow through the lung tissue, and finally be coughed up. The various combinations of inflammatory extension and burrowing of pus are almost without limit.

Symptoms. - There is usually a previous history of derangement of the stomach or intestines, with the production of considerable amounts of gas. The characteristic beginning of the disease is a chill or an excessive attack of colic. The pain may be referred to the whole abdomen; in fact, it is rare for a child to localize it in the right iliac region. It is only when the physician carefully palpates the abdomen - a procedure which should never be omitted from the examination of a child with intestinal symptoms - that the tenderness is clearly located. This will be sometimes found on the line running from the anterior superior spine of the ilium to the umbilicus, about three or four ctm. from the former point. This localization, known as McBurney's point, which occurs almost always in adults, is less frequently seen in children. In them the higher position of the appendix, its greater length and longer mesentery, make a higher position of the tender area possible. For this reason the search for it must extend up to the free border of the ribs. In some cases, especially after the disease has had time to develop, a fairly large, doughy mass may be distinguished in this locality. At the same time, one should remember that exceptions may occur, and that the tumor in rare cases is otherwise situated.

The temperature does not run very high, usually ranging from $38^{\circ} \mathrm{C}$. to $39.5^{\circ} \mathrm{C}$. $\left(100.5^{\circ} \mathrm{F}\right.$. to $103^{\circ} \mathrm{F}$.). Generally, infants will suffer from a greater amount of fever than older children, and 
their prostration will be greater. In children of all ages the temperature, especially in the initial stage, is somewhat higher than in adults. The respiration and pulse are affected by the temperature, the former becoming shallower, and the latter thin and wiry as the fever ascends. The tongue is coated, and there may be much vomiting, first of food and later of clear liquid mixed with bile. The paralysis of the bowel which often precedes fæcal vomiting is rarely seen in appendicitis excepting where the conditions simulate an obstruction of the bowel. The child is apt to lie on his back, turned somewhat to the right and holding the right knee drawn up so that as little strain as possible be laid upon the corresponding rectus muscle. There may be an irregular constipation, varied occasionally by short attacks of diarrhœa. But this feature is not characteristic enough to deserve much importance. There is always marked thirst.

If the tenderness spreads farther and farther across the abdomen, with accompanying symptoms of suppuration and shock, a rupture of the appendix may be suspected.

Treatment. - The first step in the treatment formerly was the emptying of the intestines by repeated small doses of calomel followed by a saline. At the present time objection is made to this on the score of the danger that vigorous peristalsis might create. Therefore, in place of the calomel, we now give a dose of castor oil ; and some practitioners, believing that this likewise is dangerous, order no more than an enema. The patient should then be kept as quiet as possible, and if necessary opium by mouth or hypodermatic injection may be used. An ice bag should be kept on the affected region continuously; in using this, one should be careful not to make it too heavy, and in addition the skin should be protected by the interposition of a cloth between it and the bag. The food must be restricted to modified or peptonized milk; if vomiting is severe, one may have to resort to nutrient enemata. The administration of stimulants may at times be demanded; for this purpose good whiskey and small doses of nux vomica are of value.

If fluctuation is detected, or if the tenderness shows a disposition to spread, the opinion of a surgeon will be of value. A large percentage of these cases require operation, and a delay means greater immediate risk of death, or at any rate an almost certain possibility of future attacks. The more I see these cases, 
the firmer becomes my belief that the operation should be done as soon as the indications permit.

Convalescence should be carefully managed, and the patient must not be permitted to take liberties in the way of movement or diet until every question of danger is settled.

Differential Diagnosis. - In distinguishing appendicitis we must keep in mind intestinal obstruction and intussusception. The main signs of appendicitis are the hard, colicky pains, immediate rise of temperature, constipation, moderate vomiting, a tumor usually on the right side, and pronounced thirst. In obstruction, since there are at the beginning no inflammatory lesions, there will be no fever, no vomiting, and gradually appearing pain. In intussusception the tumor is commonly on the left side, pain appears only late in the sickness, the temperature rises no more than slightly or may be subnormal, the vomiting is apt to be stercoraceous, and there are mucous movements slightly stained with blood.

Prognosis. - The prognosis is, on the whole, better under surgical than medical treatment. Some surgeons report a mortality as low as two per cent. Children stand the operation well, and enjoy a fairly rapid convalescence.

\section{Proctitis}

The rectum, in the presence of inflammation in the intestine above, sometimes becomes affected in a like manner. In this way one may account for the large majority of the cases of proctitis. The extended disorder is of the same nature as the original complaint, and needs very little differentiation from it. In some few cases, however, a proctitis may start as a new disease, after a periproctitis, as the result of injury from a syringe-nozzle, irritating suppositories, pin worms, gonorrhœal infection from the vagina or criminal violence, and by means of foreign bodies of all sorts. In addition, wounds of the rectum may be attended by a variable amount of inflammation. Diphtheria may invade the rectum, and some of the other acute infectious diseases, as well as tuberculosis and syphilis, may also cause disturbance, although rarely in this locality alone.

The symptoms do not differ much from those of an ileo-colitis, with the exception of greater heaviness, pain, and tenesmus which 
are referred to the region of the rectum. There is a formation of mucus that is sometimes streaked with blood. The straining may be so great as to produce a prolapse of the mucous membrane of the rectum.

The membranous form is apt to be severer than the catarrhal, and almost always is characterized by the presence of streptococcic infection. The only method of sure differentiation is finding pieces of membrane in the stools.

As an extension of an ulcerative inflammation from above, or on account of an exaggeration of a catarrhal proctitis, the rectal mucous membrane may become riddled with superficial ulcers. In some cases these eat their way through the deeper layers of the bowel. Usually the attention is drawn to them by marked pain and variable amounts of hæmorrhage. Often the ulcers may be seen on ocular examination.

The treatment of proctitis includes the general care of the diet, rest in bed, and the application of such means as will keep the rectum approximately clean and bearably quiet. This can be accomplished by flushing out the bowel with a warm saturated solution of boric acid, followed when necessary by suppositories of cocaine hydrochlorate or opium. In the later stages astringent injections may be used, although in most cases one can get along quite well without them. The general scheme of treatment is, on the whole, the same as in ileo-colitis, with the possible exception of those cases where there is a local cause at fault, such as a foreign body or pin worms, in which case the offending cause must first of all be removed.

\section{Prolapse of the Anus and Rectum}

A sharply differentiated nomenclature will distinguish between a prolapse of the anus and one of the rectum. In the first named there is a protrusion of the anal mucous membrane; in the latter there is a descent of the whole wall of the rectum; either may project one or more centimetres. In the beginning the protrusion occurs only on defæcation, but later on it may happen at any time of strain or movement.

Causes. - The condition has two main rtiological factors : one is straining in having a movement, the other is physical debility. Such complaints as the various functional and organic derange- 
ments of the large intestine, phimosis, cystic calculi when they attack rachitic and poorly nourished children, especially those under three years of age, very easily bring about a protrusion of the rectal structures.

Treatment. - Each time the prolapse occurs it should immediately be replaced after being oiled. Where much swelling is present, the application of ice will often reduce the size enough to permit reposition. In a few cases it may be necessary to anæsthetize the patient and stretch the sphincter.

The most troublesome part of the treatment consists in trying to overcome the constant recurrence of the disorder. To meet the requirements the child should defæcate while lying on the back or side, so that he may not strain too much. The nurse or mother may aid him in this by holding the buttocks with a light but firm pressure. After the movement the child should continue in the recumbent position for a quarter or half an hour to allow the bowel to become quiet. At the same time the movements must not be constipated, and to accomplish this end the diet must be regulated and mild cathartics administered. After the movement, a $T$ bandage should be adjusted or the buttocks be held together with adhesive plaster. Astringent injections may in some cases be of use, but as a rule one can do without them. Most of all is this true if one recognizes the cause of the prolapse and abolishes it. In the majority of all cases there is marked general weakness which must be earnestly combated.

In exceedingly obstinate cases occurring in older children, it may be necessary to cauterize the prolapsed bowel with the Paquelin cautery or nitric acid. Before being replaced it should be well oiled, and in the lumen a small plug of gauze may be placed to keep opposing surfaces apart. Only in the very severest cases is a more radical operation required. And when the proper measures are employed, the prognosis is good.

\section{HeMORRHOIDS}

This condition is not often seen in the general practice among children; in hospitals and dispensaries it occurs somewhat more frequently. It is apt to follow chronic constipation or other disorders which entail much straining at stool; this factor combined with an atonic condition of the rectal mucous membrane is sufficient to cause local dilatation of the blood-vessels. In 
some cases there may be small hæmorrhages, especially if the tumors are internal.

The symptoms are protrusion of the hæmorrhoids while at stool or straining, some pain and occasional hæmorrhages.

The treatment consists in keeping the movements loose and in the use of astringent ointments. At the same time the diet should be regulated and tonics administered. In cases of neglect the disorder may be so firmly seated as to call for operation.

Hæmorrhoids have been confounded with rectal polypi, prolapse of the rectum, and even invagination of the intestine. A short time since I was called to see a girl of four and a half years of age in whom a diagnosis of hæmorrhoids had been made. Examination showed an internal pile and a rectal polypus; the straining produced by the latter was doubtless the cause of the hæmorrhoid. When the polypus was removed the hæmorrhoid disappeared without further treatment.

\section{Rectal Abscess}

Rectal abscesses are either ischio-rectal or marginal. They arise from traumatism of some sort, or from absorption of toxic matter through lymph channels. In addition, some few cases of extension from a tubercular area occur in this region. Intramural abscess of the rectum and perirectal abscess are local subdivisions of the general subject. All these special forms usually attack children who are in poor health.

The symptoms are the same as in adults, and consist in a feeling of heaviness and pain in the perineal and rectal region; frequently there is brawniness, followed by fluctuation, which may be detected on palpation. There may finally be an escape of pus.

The treatment is surgical ; free incision should be performed as soon as one detects signs of pus.

\section{Nevus of the Rectum}

No more than a mention of this condition is needed. It is of rare occurrence, and is characterized by hæmorrhage from an otherwise unimpeded bowel.

Its treatment consists in the applications of the Paquelin cautery. 


\section{Rectal Polypus}

A pedunculated tumor of the rectal mucous membrane is a not very uncommon complaint in children of all ages and conditions. In character it may be adenoid, cystic, or fibrous, the first being the usual form. Its pedicle is of varying length. Usually it is situated near the sphincter; cases, however, of much higher location have been reported. The child complains of straining and

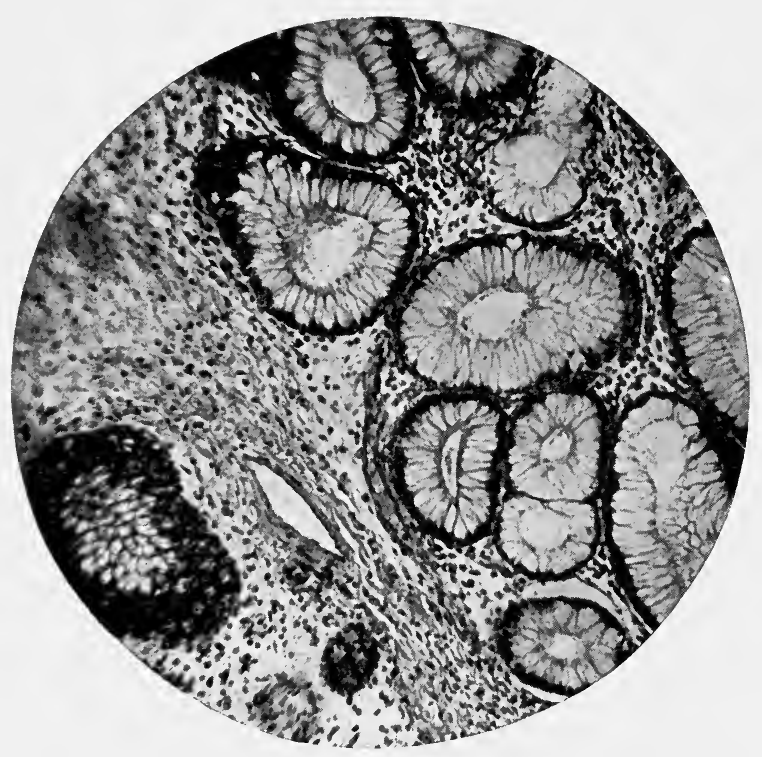

FIG. 28. - Rectal Polypus. $\times 100$.

tenesmus, occasionally of slight pain. Some blood and mucus may be passed with the stools.

One can be sure of the diagnosis only by seeing or feeling the polypus. It is cured by twisting off the pedicle and touching the stump with a Paquelin cautery or nitric acid. In some cases it may be possible to withdraw the tumor, tie off the pedicle near its base, and amputate above the ligature.

Rectal polypi must be differentiated from hæmorrhoids and prolapse of the rectum. The diagnosis is so easy that nothing more than careful attention is needed to confirm it. 


\section{Fissure of the Anus}

As a result of scratching the anus, of very hard stools, of traumatism produced by syringe nozzles and foreign bodies in general, the mucous membrane of the anus may be fissured in a vertical direction. The result is considerable pain on defæcation, straining, or manipulation.

The active cause should if possible be discovered and abolished. The movements should be kept loose, and a five per cent or ten per cent icthyol ointment should be applied, and kept in place by a pad and $T$ bandage. In severe cases it may be necessary to touch the fissure with nitrate of silver, and in the worst instances one may have to stretch the sphincter. 


\section{CHAPTER XII}

\section{DISEASES OF THE PERITONAUM}

\section{Ascites}

THE occurrence of this symptom may mean either local or general disease. A knowledge of the causes which produce it is the readiest means of referring the responsibility to its proper source. For the most part the effusion is caused by disease of the liver, or a condition which gives rise to pressure upon the portal circulation. In other cases it is due to cardiac disease pressure upon the inferior vena cava, to pulmonary, peritoneal, and occasionally nephritic disease. Marked anæmia may be at the root of the trouble, or tumors of the abdomen and the nearby viscera may be the cause. Finally, it may be a local symptom of a general anasarca caused by marked cachexia.

Chylous ascites is the name applied to the presence in the abdominal cavity of a milky-colored fluid containing a large percentage of fat. Its causation is obscure; and although we know that it may follow perforation of the thoracic duct or filariasis, nevertheless this knowledge does not explain all the cases that we meet.

The principal inconvenience which ascites gives is that due to pressure. As the primary disease which causes it disappears, it likewise is apt to diminish. Where it persists the usual treatment has consisted in the administration of salines, followed if necessary by tapping. A more satisfactory method would be the operation of laparotomy.

\section{Acute Peritonitis}

Causes. - An acute inflammation of the serous membrane lining the abdominal cavity and covering the viscera therein contained is not a rare condition in childhood. Sometimes it is seen at birth; such cases are generally thought to be syphilitic, although 
I have found seeming exceptions to the rule. It is possible that infectious disease in the mother before delivery, such as measles, may be the cause. In infants of a few days or weeks the cause is usually an indirect one, such as septic inflammation of the umbilical vein, or a disease, e.g. measles, that is able to produce a complicating affection of the serous membranes. The essential condition seems to be the active presence in the body of the streptococcus, staphylococcus, pneumococcus, or bacterium coli commune in such locations that it can find its way through lymphatic channels to the peritoneal cavity. Most cases occur in children of a greater age, of four or more years. Here the disease may be traced to falls, blows, and injuries of the abdomen, disease of the abdominal viscera, such as rupture, intussusception, volvulus or inflammation of any part of the intestine; pathological conditions of the stomach, vermiform appendix, liver, kidneys, spleen, pancreas, pleura, and lymphatic glands; hydatid cysts, pyogenic diseases of the bladder, uterus and its connections, and male genital track; inflammation of adjacent bony structures, and the acute infectious diseases, including septicæmia.

Lesions. - In the first stage there are in the peritoneal membrane an active congestion, a heightened red color, and a proliferation of endothelial cells. This is followed by the production of fibrin, serum, and pus in variable quantities. The milder cases have but little pus, and so have been termed fibrous or serous. Nevertheless there is practically always a formation of pus cells which collect on the surface and work their way in between the endothelial cells. The surface is likewise studded with small or large masses of fibrin. The peritoneal cavity contains serum in variable quantity in which there may be uncertain quantities of bacteria, pus cells, and endothelial cells. After the disease has been in existence for a week or more, the connective tissue cells may be increased to a marked degree. In addition, the fibrin may cause neighboring folds of intestines to adhere to each other by bands which may finally become permanent adhesions.

Symptoms. - The disease may or may not be ushered in with a chill. Vomiting is apt to show itself and to become worse when food is administered ; there may be diarrhœa or a decreased peristalsis - for one cannot formulate a hard and fast rule in regard to this detail. The urine is scanty, high-colored, and may burn in passing. It often contains considerable quantities of indican, 
and some acetone. The child is plainly in much pain, which he involuntarily tries to diminish by flexing the legs upon the abdomen, thereby reducing strain upon the abdominal structures. His attempts are evidently unsuccessful, and his suffering becomes progressively more intense. Any form of examination, pressure, movement, or peristalsis is scarcely bearable; even the act of approaching his bed frightens him. The pain is apt to come in waves and spasms, rather than in one continuous stream. There is much prostration, the face has an anxious, sunken, and drawn look, the pulse is small and rapid, the respiration is quick and shallow, and the temperature ranges from 1 or 2 degrees above normal to $41^{\circ} \mathrm{C}$. (about $106^{\circ} \mathrm{F}$.). The high temperatures are commonly present in cases of associated intestinal disorder, while those of the uncomplicated disease show moderate pyrexia. Occasionally a very toxic case will give a normal or a sub-normal temperature. The abdomen is swollen and typanitic over its whole surface, except where the peritonitis is distinctly localized; such an area is often recognized by the circumscribed tenderness and pain. The abdominal swelling varies according to the thinness of the walls and the amount of tympanites. The latter element may be distinguished not only by the exterior form, but also by the hyper-resonant percussion note; on the other hand, an overloaded state of the intestines or the presence of a considerable amount of fluid may make the note fairly dull. These remarks are most applicable to a general peritonitis, in which there is a moderate distribution of the symptoms. At the same time one must keep in mind that a localized peritonitis may at any time, and often does, become general. In some cases there is a successive involvement of one part after another.

When recovery takes place, it shows itself by gradual amelioration of all the symptoms. One must be prepared for a slow and tedious convalescence which is characterized by a train of intestinal derangements.

Treatment. - The first step is to empty the gastro-intestinal track, preferably by small and rapidly repeated amounts of calomel followed by a saline. Thus one may order 0.006 gramme ( $\frac{1}{1.0}$ grain) of calomel for six to ten doses at half hourly intervals to be followed by a sufficient amount of the sulphate or citrate of magnesium to flush out the intestines. Hot stupes may be continuously employed. As soon as the bowels have moved sufficiently, enough 
opium or morphine must be given to quiet pain and peristalsis. It may often be necessary to administer this drug hypodermatically in order to avoid disturbing the stomach. This object will be still further effected by a rigorous care in feeding the child; the diet must be confined to fluid's, and often it will be necessary to predigest all food until convalescence sets in. Since the patient obtains a certain amount of ease by flexing the knees, this position may be maintained by placing a folded pillow under them. A further degree of comfort may be obtained by allowing the child to lie with his hands above his head. If the signs show a noticeable increase of fluid in the peritonæum, especially if pus infection seems to be growing, the treatment becomes surgical.

Prognosis. - The outlook is always doubtful, and from day to day the chances for recovery may change in material degree, largely according to the amount of pyogenic intoxication. The chances of recovery vary according to the area involved, the child's vitality, and the amount of the septic element. One can easily see that each case must be judged by itself, and that no single opinion can be universally applicable.

Differential Diagnosis. - The main facts to keep in mind are the history, the gradually increasing pain and tendermess, the elevated temperature, the hard abdominal wall, and the flexed knees. From this group of symptoms we must differentiate colic, acute intestinal obstruction, enteritis, renal or biliary calculus, and neuralgia. Colic gives a sharp, acute pain, which doubles the patient up; there is no fever or prostration, and the patient is unable to lie quiet. An enema or a cathartic will promptly give relief, and clear up the diagnosis. In acute obstruction there is at first no fever, no abdominal tenderness, the belly wall is not tense; there will be copious vomiting at first, which later on becomes stercoraceous. In the beginning there may be a number of small movements; and when they have ceased, one may at times feel an abdominal tumor. Enteritis should rarely be a confusing factor, since its tenderness and pain are not so marked, nor is the abdominal wall so hard and rigid. Renal calculus and neuralgia give no material elevation of temperature, no general tenderness, no characteristic position of the knees, no regular history. In appendicitis the distinct localization and the tumor are usually sufficient to show the nature of the trouble. 


\section{Chronic Peritonitis}

A chronic inflammation may follow an acute process, may complicate chronic disease of the heart, lungs, spleen, or liver, or may occur as an original sickness. The tubercular form is described under the head of tuberculosis.

Lesions. - The changes are not always the same: in a few cases they consist for the most part of a proliferation of the endothelial cells; the connective tissue is increased at the same

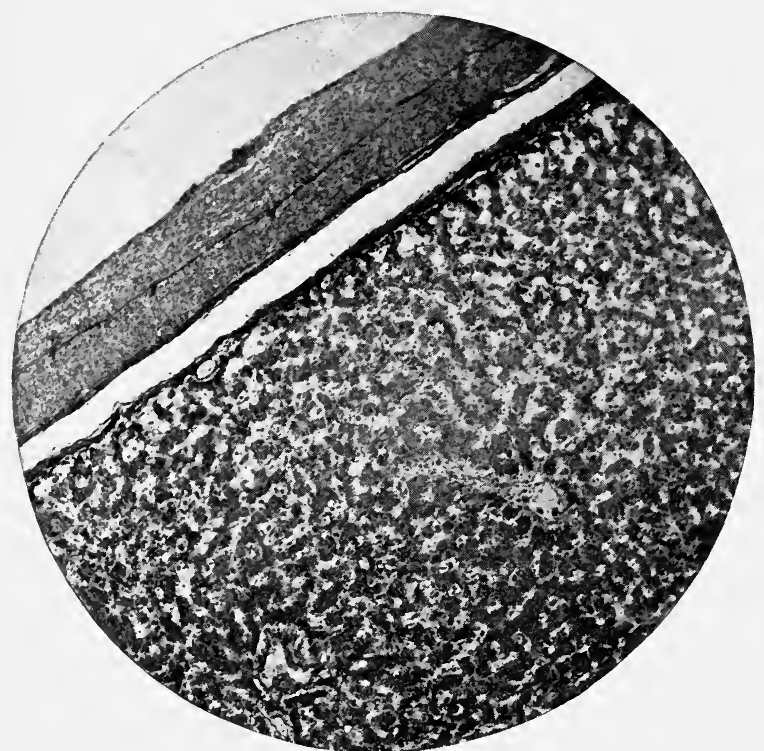

FIG. 29. - Perihepatitis. $\times 50$.

Occurring with Chronic Peritonitis.

time. In addition there may be more or less extensive adhesions, particularly in the neighborhood of a focus of pus. In other cases there is a thickening of the peritonæum with the production of much connective tissue, a deposition of fibrin, the formation of adhesions between opposing surfaces of peritonæum and intestines, and the presence in the cavity of an increasing quantity of clear or purulent serum. At times the capsules of the liver, spleen, and kidney may be involved and become very much thickened, especially when the process has been localized. Evidently in such cases the inflammation has gradually spread until it involved the whole peritoneum. 
Symptoms. - If the process follows an acute attack, the child after defervescence does not seem to recover. He is unable to exert himself and complains of tenderness and some pain in the abdomen. A variable quantity of fluid collects in the cavity, and the whole abdomen begins to be distended. At times there are areas which continue to be painful or tender, even when the main symptom is the ascites, which doubtless represent foci of severe inflammation or adhesions that have been put on the stretch.

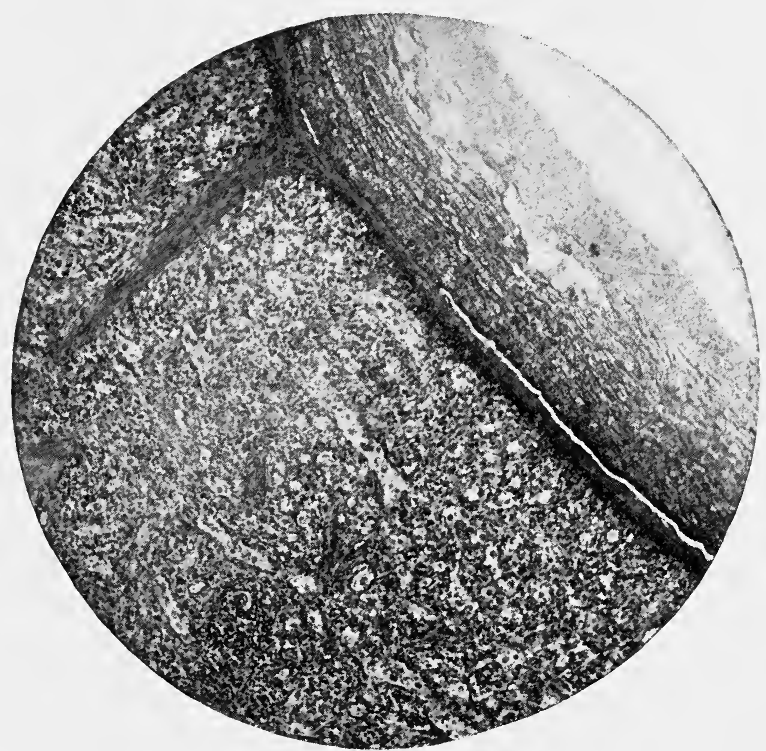

Fig. 30. - Perisplenitis. $\times 50$.

Occurring with Chronic Peritonitis.

There may be no fever or only a slight evening rise. The general nutrition is poor, and the child is liable to have many coincident symptoms in other organs as well as a proneness to contract intercurrent disease. The coincident symptoms are commonly the ones that attract most attention, and give most trouble in the successful treatment of the general condition.

Treatment. - If improvement does not follow the use of tonics, careful nursing, and a moderate amount of salines, and if the fluid does not increase in quantity, laparotomy is advisable. I believe that this measure has certain advantages over aspiration; by it one would avoid the tedious recurrences of fluid after tapping, and thereby avoid much loss of strength and 
vitality. In addition, it is wiser, surgically, to have a fairly large open wound, in the manipulation of which the operator can see what he is doing, rather than to make a puncture more or less blindly, and thus carry a possible infection in inserting or withdrawing the instrument.

After the fluid has been disposed of, the physician must for a long time keep the child under observation, carefully regulating tonics, food, exercises, and hygiene.

Prognosis. - The outlook is better than in the active acute form. The patients are generally in the second half of childhood, and so have greater vitality to draw upon than if they were younger. The main factor in the prognosis is the differentiation of the simple from the tuberculous form. 


\section{CHAPTER XIII}

\section{DISEASES OF MALNUTRITION}

\section{LARYNGISMus Stridulus}

THE penalties of malnutrition are so far reaching that it is almost impossible to set a limit to the potential effects of the disorder. We are apt to think of this condition as, for the most part, being confined to strongly marked and familiar pathological states such as gastro-intestinal disorders and poorly nourished bones, and there seems to be difficulty for most people to conceive that the influence of malnutrition extends beyond these limits. As a matter of fact the more closely one looks at the phenomena of development, the more one is impressed by the prevalence of the effects of defective nourishment.

Causes. - A striking instance of this generalization is the neurosis called laryngismus stridulus, spasm of the glottis or internal convulsions. The disorder occurs among imperfectly nourished children whose deficiency may result from the evils of luxury as well as those of poverty, from exposure or the confinement of too sedulous care. Thus the artificial life which is imposed upon so many babies in cities makes the condition more prevalent there than in the freer circumstances of the country. As a matter of experience we know that it occurs somewhat more often in boys than in girls; but the reason of this is hard to find. That it is confined to the first year or two of life may depend upon the unstable and easily disturbed nervous equilibrium of that time, in which the transitional character of the child's physical being is so plainly evident.

Some of the abnormalities, such as enlarged and irritated glands and a disturbed detention, which have so regularly been cited as causes, are no more than proofs of this. For cases have occurred time and time again without their possible influence; while, on the other hand, the debased physical condition which causes this neurosis is likewise able to have as some of its partial 
characteristics these and other similar disorders. The elements of heredity, so regularly mentioned as a more or less active cause, acts mostly in the way of providing a possible weakness of assimilation and excretion; but it is very apt to be helped out by unwise general care of the child, which commonly, in the absence of intelligent effort and instruction, blights one baby after another in the same family. Often enough the increase in maternal wisdom and knowledge is not in direct ratio with the number of births in the family.

Lesions. - There are no known local lesions in this disorder. In fact, the spasm of the glottis, or adductor spasm of the larynx, which is so startlingly evident as to draw the observer's attention to itself and away from other characteristics, is merely one of the symptoms. Thus, some clinicians speak of a diaphragmatic form, of a laryngeal form, of a mixed form, of a form which involved the pharyngeal as well as the laryngeal muscles, of cases in which the neurosis is so widely diffused that.it shows itself in a spasm of the carpo-pedal muscles. And, in addition, acute observation will convince one that other muscular tissues, such as those of the thorax, the abdomen, the eyes, and probably the heart, may be involved. Indeed, it is hard to draw a line at the possible convulsive effects, for, more or less directly, any portion of the body may be involved.

Symptoms. - The most prominent symptom is the spasm of the glottis, which in proportion to its completeness stops respiration until relaxation occurs. The attacks usually begin mildly, so that, as the low form of intoxication which comes from malnutrition increases, the spasms likewise increase. The more strongly marked paroxysms come at night with all degrees of rapidity of recurrence. In the milder forms of the disorder the glottis closes completely or nearly so for a few seconds, so that there is practically a total stoppage of respiration; then it opens slightly and the air rushes through with a convulsive, crowing sound. In severe attacks the apnœa is of longer duration, the muscles are convulsively fixed, the skin becomes cyanotic and slightly puffed, and finally the child drops back unconscious. In extreme cases, and where the intoxication is evidently extreme, plainly marked general convulsions supervene, in some instances followed by death. In these cases it is hard to say in what degree the fatal termination is due to asphyxia or to the general intoxication. 
Treatment. - The treatment looks to the breaking up of the spasm and the removal of the physical conditions which cause it. Hot or cold applications, used separately or alternately, or the various well-known expedients for restoring impeded respiration, will be of service in ending the convulsion. To quiet the irritated nervous system, antipyrin or bromoform or bromide of soda is efficient. The gastro-intestinal track should be thoroughly emptied, and then the main idea of treatment should be carried out. This consists in minutely examining every detail of the child's life, in carefully searching for faults in the methods of feeding, in the circumstances of sleeping, clothing, exercise, hygienic and moral control. Tonics, when needed, - and they usually are, - should be given until the child is in a normal, strong state of health. The organic preparations of iron or the compound syrup of hypophosphites, for such children as can digest it, are highly desirable. But the main thing is to regulate the child's general mode of life so as to diminish sources of weakness and improve the sources of strength.

Prognosis. - In most cases the attack passes off without any more serious effects than temporary exhaustion; and for this reason we commonly regard the disease as not especially dangerous. Nevertheless, one should not forget that the spasm may be severe and long enough to bring about a fatal result.

\section{Simple Atrophy}

This is a condition of extreme malnutrition which results from bad methods of feeding and unhygienic surroundings. In fortunately situated families it is rare, but in large institutions, as well as among the poor and ignorant frequenters of dispensaries, it is common. Such sources supply a continuous stream of these cases in some of the various stages of the process ; they are commonly unsatisfactory cases to treat, not because the condition is necessarily fatal; but rather because the original circumstances of ignorance and poverty are extremely difficult of amelioration.

Causes. - Simple atrophy is practically always acquired. The child at birth may seem healthy and well nourished. Then, as the result of the mother's milk being poor in quality or quantity or both, or on account of a substitute food which is too difficult to digest or absorb, a process of starvation begins. Unhygienic environment supplies the missing unfortunate element of decline 
until the child is ready to slip from this life to the next with the least show of resistance. In other cases the process of malassimilation may start from some exhausting sickness, such as gastrointestinal disease, syphilis, bone-disease, which so far depresses the child's vitality that the economy is unable to perform its functions, and assimilation practically stops. Thus, to cite examples, in a case of severe septic infection following circumcision, I saw a most impressive instance of this truth ; and again, after a most trying broncho-pneumonia and pleurisy, I saw the same experience repeated. As a rule, however, the disorder begins in the gastro-intestinal track where the functional disability is so long continued, with its attendant train of far reaching symptoms, that a sort of starvation ensues.

Symptoms. - While the different degrees of severity bring out appropriate signs, nevertheless the main tendency is in all alike. The child begins to lose flesh, in some cases so rapidly that it seems almost to melt from the bones. But whether the loss is rapid or slow, he finally comes to be markedly emaciated, the skin hangs in sagging folds, the face looks grotesquely old, furrowed, and puckered. The skin generally is dry, harsh, cool, and clanımy. Here and there, and especially on the face and head, it begins to assume a brownish yellow color and a thin, dry crust. At this time the patient is very apt to suffer from exacerbations of gastric or intestinal disorders which necessarily show their logical symptoms. In this way the number of fæcal movements may be increased or diminished, although the ordinary course of the disease gives a moderate number of large stools. For the same reason the color, which generally is light, may be changed to a greenish or a clayish hue. Frequently there is no marked vomiting except in the presence of intercurrent gastric disorder. The child is constantly hungry, and, until he becomes quiet from exhaustion, shows it by restlessness and crying.

The course of the disease is steady, and the symptoms from week to week increase in severity. The temperature is usually normal or subnormal ; nevertheless this rule will often be modified on account of the susceptibility of these children to intercurrent disorders, any of which may regularly bring an elevation of temperature. The fontanelle may be slightly depressed, anæmia be marked, and œdema progressive. The patients are liable to complicating disorders of the skin, mouth, congestive 
bronchitis, and hypostatic inflammation of the lungs, and occasionally to functional nervous complaints.

Treatment. - The preventive treatment is obvious: merely to avoid errors in feeding and hygiene. If this were done, there would be few cases of marasmus. In the presence of the disease drugs are of little use; for the disease is a mode of starvation. The main object is so to modify the milk that the patient can digest and assimilate it. Empirically we have found that the best combination is composed of a very small percentage of fat, a moderate percentage of proteids, and a high percentage of sugar. Beyond these details one cannot well go ; for one child cannot digest more than 0.75 per cent of fat, while another may thrive on 1.5 per cent. The same rule holds good for the proteids and sugar. The besc plan is to begin with very small percentages and increase them as the patient's condition permits. The quantity at each feeding should be small; and in order to ascertain whether it is nourishing the child, his weight should be carefully ascertained twice a week or oftener. At the same time it is advisable to examine the stools macroscopically and microscopically, and adapt the treatment to the indications thus ascertained. If the child does not gain, the food must be continuously modified to suit his needs.

The question of ventilation, exercise, and hygiene in general is almost as important as food. The child must be bathed daily, his sleeping room should be thoroughly ventilated and well lighted, and the care of his body should be scrupulously exact. Best of all, he should be kept in the country as much as possible.

The treatment of all temporary and intercurrent diseases must be as simple as possible.

Prognosis. - The forecast of these cases is purely relative. If the disease has not run too long, if the child is not too young and weak, and if the requirements of treatment are carefully and logically carried out, the child has a fair chance to live and attain a good development. The farther these suppositions are from fulfilment, the poorer is the result of the case apt to be.

Differential Diagnosis. - The main error into which one may fall is to confuse simple atrophy with tuberculosis. The latter is more apt to give appreciable pulmonary symptoms or other signs of metastatic invasion, and in addition is always attended by an evening rise and a morning fall of temperature. Observation for a few days will generally exclude one or the other of the diseases. 


\section{RACHITIS}

Causes. - Rickets is a condition of hypotrophy plus the effects of the deviations from the normal process of growth. These deviations, as well as the hypotrophy itself, are of various kinds and degrees, so that the resultants are likewise variable. In the presence of such imperfect and abnormal states of nutrition many inflammatory states - acute and chronic, active and passiveare both possible and probable. The statement of what the disease is bears with it an understanding, to some extent at least, of its ætiology. It is not hereditary, nor if one wishes to be exact can it be called congenital. The very rare cases of so-called fotal rickets, the achondroplasia of Parrot and the chondrodystrophia of Kaufmann, should be put in a different category. These instances of "dwarfism" or "infantilism" are really examples of physical deficiency, comparable to certain forms of mental deficiency; but the continued use of improper food, and a life passed in a badly arranged environment, especially in the earliest and most easily disturbed age, will usually bring on the rachitic condition. It takes a not extended experience in hospital and dispensary practice to form a good idea of the requisites for producing the disease. One very soon notices that almost all of these cases have been fed on artificial foods, and that only in the rarest instances has the patient been nourished at the breast. The usual diet thus includes a small quantity of fresh milk and a large quantity of dessicated substances that are rich in sugars and starches. The normal milk ingredients which commonly are lacking are fats and proteids, and sometimes mineral salts. Even where fresh cow's milk is used, the differences in chemical composition between it and mother's milk so often set up functional disturbances of the stomach and intestines that digestion and absorption are rendered impossible, and the infant is in the same position as he would be if fats and proteids were excluded from his food.

These errors in diet are most commonly found among the poor who may have difficulty in obtaining and modifying good milk. On the other hand, the complaint may be seen in the children of wealthier persons who, on account of indolence or ignorance, commit practically the same faults. These patients are fed on cereals, vegetables, sugars, and starches ; they are coddled 
and pampered and deprived of opportunities of obtaining sufficient light and air. This is the state of affairs that exists widely among the poor, especially in large cities, the cubic space of whose rooms is small, whose apartments are too often dark and poorly ventilated, and whose opportunities of breathing the air of country, seashore, and park are sharply limited by necessity, ignorance, or indolence. Such deprivations bear most heavily on children in temperate climates whose parents have emigrated from warmer lands. For the natural habitat of rickets is in these temperate zones where the changes in temperature are

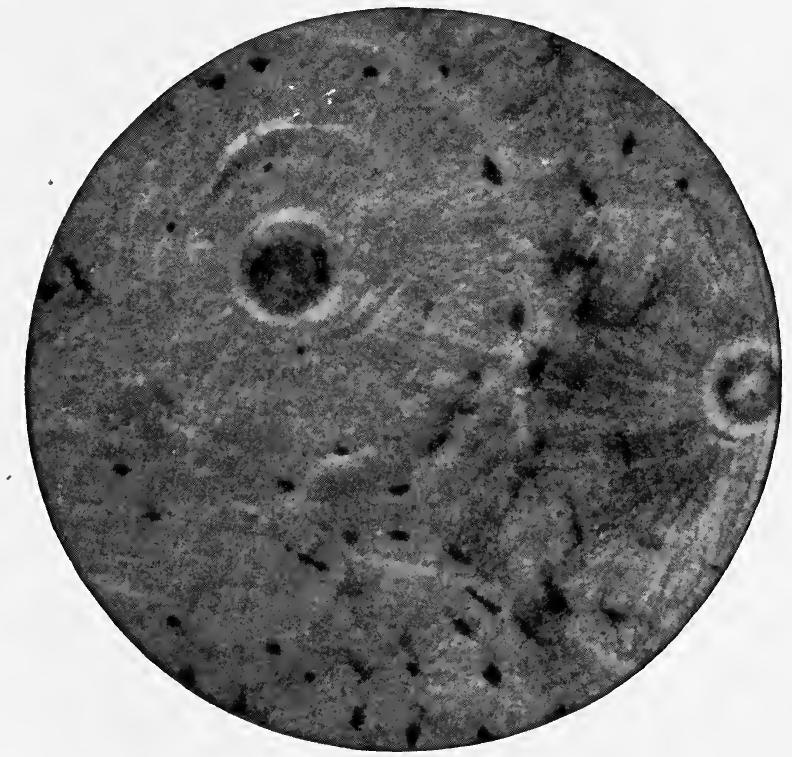

Fig. 31. - Normal Bone. $\times 220$.

great, where there are weeks and months of cold and dull weather which bring with them confinement and suffering. An additional danger comes from the weakened bodies which the conditions of life impose upon the parents; for such people, although they do not transmit the disease itself, do transmit to their offspring a deficient vitality which is easily influenced by unfortunate environmental conditions. In the same fashion, any other circumstances which diminish the patient's vital resistance will produce a similar result. In this way hereditary syphilis makes a child more easily subject to rachitis than he otherwise 
would be. Again, exhausting diseases, such as severe gastrointestinal disorders, may in the same way accomplish a similar end. In short, the only positive causes of rickets that we know are the errors in diet above mentioned, with or without unfortunate environmental conditions.

Lesions. - All through the body the changes exist in varying degrees, affecting the bones, joints, muscles, nerves, and viscera. Those of the bones are most prominent, and generally have been thought to be the characteristic ones of the disease. While all

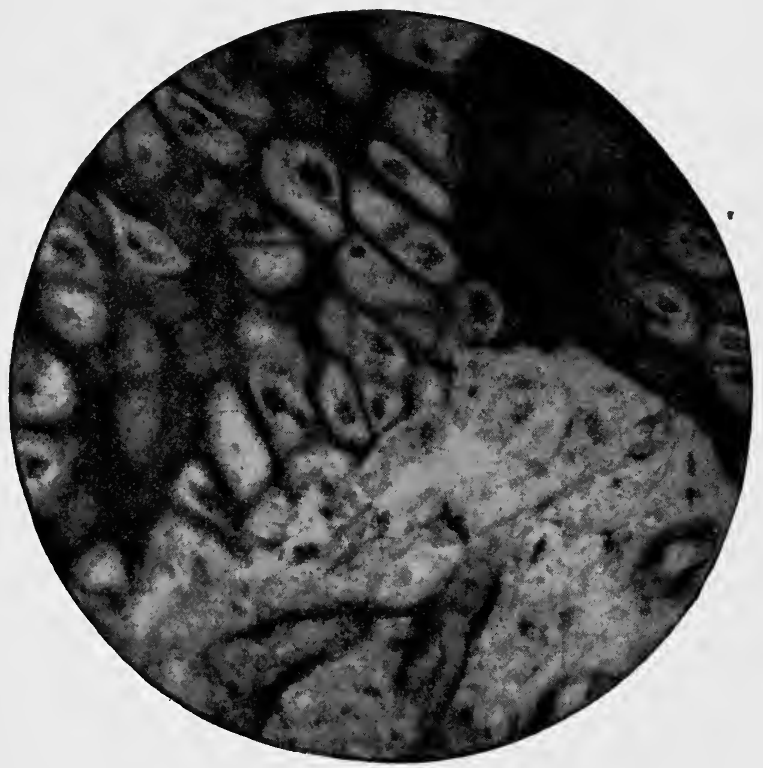

Fig. 32. - Rachitic Bone. $\times 220$.

the bones may be and usually are involved, nevertheless the long ones show the principal modifications in both structure and form. The process of growth is changed; the increase in true bone structure is slow, irregular, and partial; the increase in the cartilaginous cells and those on the periphery of the bone is, on the contrary, very marked in the epiphysis as well as to a less extent along the shaft. In normal growing bone the medullary cavity becomes enlarged through the length of the bone by the gradual and regular absorption of the medullary layers of bony cells. In rickets, the absorption and consequent dilatation occur unevenly 
both in regard to time and extent. In the part between the epiphysis and diaphysis the cartilage cells are not placed in regular rows ahead of the zone of ossification, but on the contrary are piled up in irregular formations in which there may be some calcific areas, or none at all. The blood-vessels of the whole bone are large and plentiful instead of being small and few. The area of ossification is likewise irregular, and cartilage cells may, by means of the microscope, be seen scattered through it. The sharp differentiation between the bony and cartilaginous areas is not always preserved, and the integrity of both is deficient. Under the periosteum a similarly asthenic condition exists. The central strata of cells are proliferated, and studded irregularly with varying areas of calcific deposition. Here, as well as in the midst of the bony structure, there is a spongy, irregular growth which takes away from the normal strength. This loss is accentuated on account of the increased size of the central cavity of the bone, and the fragile and unstable condition of its surrounding layers. The medullary substance is congested, swollen, and at times considerably inflamed.

As the result of these changes, the epiphyses are enlarged and irregular in form, the shafts or bodies are lacking in true bony tissue, and therefore are much softer than the normal. A graphic way to represent this is to remind the reader that such bones lack one-quarter or even one-half of their whole percentage of calcium salts. It is therefore easy to understand how they may be pulled out of shape by muscular traction and atmospheric or adventitious pressure. The ribs, on account of their long and narrow form, are the most easily affected; and after them, the long bones of the arms and legs. The epiphyses are clubbed and knobbed, the shaft may be thickened and bent out of shape, either forward or to the sides. Similar processes may attack the pelvic and cranial bones, which, on account of their softness, their irregular thinning and thickening, and the mechanical strain put upon them, become, the one narrower, the other flattened (rachitic pelvis and craniotabes).

The ligaments of the joints partake of this structural weakness, and by the effects of weight and pressure become stretched irregularly. In this way knock-knee results, or the foot turns out at the ankle, the arch of the instep weakens and sinks, and the vertebral column bends backward or to the sides. The 
muscles also are weak and flabby, and their bundles and markings look indistinct and blurred. The subcutaneous fat is commonly increased, so much so as to suggest the process of fatty degeneration ; this tissue is easily affected and easily dispelled, so that in the presence of any wasting condition it melts away and the patient rapidly becomes emaciated. This asthenic tendency pervades the whole body, so that specialized tissues show the effects of the strain in ways that are characteristic of their location and function. Thus the mucous membrane, all through the gastrointestinal and respiratory tracks, becomes readily irritated and inflamed, the effects of which vary according to the nature of existing excitant. The lungs are materially influenced by the beading and malformations of the ribs; the weakness of these bones, aided by atmospheric pressure and the pull of the diaphragm, causes a depression of the chest wall along the diaphragmatic line (Harrison's Groove). Under the enlarged costo-cartilaginous joints and this "rachitic girdle" the pulmonary tissue may be collapsed. In addition, the obstructive action of concomitant disease, such as pertussis or bronchitis, may enlarge the collapsed area to a dangerous degree. In the presence of this collapse there will be a compensatory emphysema, especially in the front of the lungs where the weak confining structures permit dilatation.

The changes in the lung accompany alterations in the structure and position of the heart. The muscle fibres are blurred and indistinct, with the natural result of weakened functional activity. Also the organ is pushed downward and outward, so that the apex beat is seen farther toward the mammillary line than is normal. The liver, spleen, and lymphatic glands may, one or all, be enlarged as the result of simple hyperplasia due to weak pulmonary and cardiac activity. But only in unusual cases do the changes involve a noticeable interstitial change. The spleen, especially, may be enlarged on account of the anæmia that is so commonly present. The red blood cells are then decreased in number, and their size, shape, and vitality are more or less impaired. Coincidently the hæmoglobin is decreased and the number of leucocytes is increased.

The nerve tissues are doubtless affected; but in what the changes consist has not been thoroughly ascertained.

Symptoms. - In mild cases all that one can see is a dispo- 
sition to sweating, especially about the head, during feeding and sleeping. The hair on the back of the head is worn off, so that this part of the scalp may be bald while others are fully covered. The child may be fat and round, but his flesh is flabby, his vitality is deficient, and he is liable to have a succession of sicknesses. He develops slowly, he walks and talks later than one expects. The epiphyses are somewhat enlarged, especially at the wrists, elbows, and ankles. On slight provocation he contracts diseases of the lungs and gastro-intestinal track, and whatever sicknesses visit him run a more serious and protracted course than in robust patients. Unless active disease is present, the temperature is commonly subnormal.

In a severe case of rickets the symptoms are very much more distinctive. The head is large, square, distorted by prominent bosses on the frontal, occipital, and occasionally the parietal bones. In other parts, especially along the margins, the bone is noticeably thinned, sufficiently so to bend under pressure. The craniotabes is on the inner, while the bosses are on the outer plate of the skull. The fontanelle remains open much longer than is usual, and may at the same time be larger than the ordinary size. The sutures remain unclosed for a comparatively long time, and have along their margins a series of roughnesses. The general appearance of the head is one of asymmetry, of incompleteness, of bad modelling. The teeth appear later than usual, or if they are not slow in their development they are commonly friable, weak, and easily decayed. The long bones are enlarged at the epiphysis, particularly the lower, and to a less extent along the shafts. The tibia, fibula, radius, and ulna are more frequently attacked than the femur and the humerus. On account of the enlarged epiphyses and the relaxation of the ligaments, the various deformities of the joints ensue. With these deformities there is commonly some twisting or bending of the shafts of the bones in one or other direction.

One of the earliest and most constant changes is the enlargement of the ribs at their junction with their cartilages. In marked cases the increase in size is very noticeable, and the protuberances, on account of their resemblance to a string of beads, have been called the rachitic rosary. Although they are plainly marked on the anterior aspect, they are regularly larger on the posterior surface of the ribs. Both the bones and joints are 
softer than the normal. Pressure exerted on these structures necessarily produces an alteration in their form. This is most noticeable in the furrow, produced in this way, which runs from the lower part of the sternum downwards and across the chest to the posterior axillary line, following the attachment of the diaphragm. Since the chest wall is held down at this line, the parts of the ribs below it are gradually forced outward by strong respiratory efforts. Another furrow is formed in a similar way along the costo-cartilaginous junctions, thus running parallel with the "rosary." Another deformity, the so-called chicken breast, is the narrowing of the anterior chest and projection of the sternum which is likewise due to atmospheric and intra-thoracic pressure and the strain of muscular activity. While these changes are going on in the ribs, the clavicles are likewise becoming enlarged, irregular, and weak; and in some cases the scapulæ follow suit and assume the curve of the rounded back. All the long bones mentioned above may on account of their softness and friability be subject to partial or complete fractures, most of which are of the green-stick variety.

The relaxation of the ligaments is plainly evident in the back, which thereby becomes rounded. In addition, the vertebral column may assume a lateral curvature, and at the same time a kyphosis of the lower half exists which throws the upper half into a compensatory forward curve. While the child is very young these accentuations may be temporarily abolished by traction, but if they continue until the bone softness disappears, they are apt to remain unchanged. The bones of the pelvis are thickened and softened; and when the child begins to walk, the weight of the body from above pressing down upon the resisting femora forces the sacrum and the related parts forwards and makes the pelvic space shallower than it normally would be. These and all other bones which are touched by the rachitic changes do not attain their full size.

Weakness is not confined to the bones, but pervades the whole body. Nowheres is it more noticeable than in the muscles. For this reason rachitic children are not able to sit up alone, to stand, walk, or obtain proper muscular coördination. This weakness varies widely in different cases : one child may on account of it be regarded merely as delicate, another may not have sufficient strength to move a limb, and lies in bed as if partly or wholly 
paralyzed. The asthenia may have far reaching effects, may diminish peristalsis of the stomach and intestines, may permit gastric and intestinal dilatation, and is the cause of the characteristic pot-belly of rachitis. The ordinary effects of gastric and intestinal fermentation, of functional disorders of the stomach, of constipation alternating at times with diarrhœa, may be expected. The mucous membranes in their irritated condition are prepared to act as a fertile culture ground for severe and organic diseases.

A somewhat similar condition exists in the lungs, which is exaggerated by the deficient pulmonary activity. There is constant danger of inflammation of the larynx, naso-pharynx, and bronchi. Upon these the most serious diseases of the lung may be built up, which in the debilitated condition of the patient have a gloomy outlook.

The nervous symptoms are not absolutely definite, but they are undoubted. The sweating of the head and body, which may be the starting point of many congestive attacks, is in all likelihood due to some unknown defect in nerve activity. The ease with which rachitic children have convulsions may be caused by abnormal reflex irritability and imperfect cerebral control; with disorders of the gastro-intestinal track as a beginning these factors could certainly produce the characteristic condition as one usually sees it. In the same obscure way one may trace the cause of laryngismus stridulus, so often seen in these patients. It is impossible to account for its frequent occurrence. A reasonable conjecture may include a series of effects starting with an atonic condition of the gastro-intestinal track, the fermentation of food, the absorption of products of fermentation and putrefaction, and a final stage of low intoxication, one of whose expressions is a spasmodic condition of the larynx. The tonic spasm of the hands and feet, so often associated with laryngismus stridulus in rickety children, may be caused in the same way. The tetanic condition is noteworthy: the thumbs begin to turn in toward the palm, and the nail presses into the surface of the third finger. The fingers are squeezed together in the form of a cone, and the whole hand is flexed from the wrist. The feet are similarly contracted, and in both hands-and feet, if the spasm is very marked, the violent contraction of the muscles may shut off the return circulation and make the dorsal surface œdematous and purplish. 
Some variations from the ordinary occurrence of rickets are handed on from one writer to another as a sort of literary bequest. One of these is the so-called congenital rickets which has been seen in England and Austria much oftener than in America. Another is late rickets, occurring in children of five, six, or more years of age. Although in my clinic I see a fairly large number of rachitic children, nevertheless I have never met a true case of either of these variations.

Treatment. - The preventive treatment should be an important matter of thought in families where a pregnant mother is in a debilitated condition, also where an older child has suffered from rachitis. By proper arrangement of the patient's food the disease will not be allowed to develop.

This same fact is at the bottom of the treatment after the disease has once obtained a foothold. Since sugars and starches in excessive quantities are, in large part, responsible for the rachitic condition, they should as far as possible be replaced by nitrogenous foods plus easily digested fats. Milk and cream properly combined, as described in the section on infant feeding, are to be the food for infants. Older children may in addition have eggs, beef and mutton, meat gravies, fresh fruits, and cod-liver oil. These foods must be given in their most easily digestible forms; and the various accidental disorders of the gastro-intestinal canal will have to be taken into account before a hard and fast dietary may be prescribed. This is especially true in regard to the administration of cod-liver oil. The value of a food depends not so much upon its intrinsic worth as upon the ability of the patient to digest and assimilate it. And although the oil may in itself be an excellent ingredient in the child's diet, nevertheless until he can absorb it there will be more harm than benefit obtained from its use. Therefore, if one is sure that the patient's intestines have a sufficiently vigorous action, small doses ( 1 gramme - 16 drops - for a child of one year) may be given two or three times a day. As the patient proves his ability to digest the oil, the size of the dose may be increased.

An equally important factor is the regulation of the general life. The child must be kept in the air as much as possible; if a trip to the country or seashore can be provided, the cure will be much more rapid and thorough. Even if the weather is not ideal, 
the child should nevertheless be kept out of doors for at least a part of every day; for there is less danger of coughs and colds in the open air than in overheated, underheated, or poorly ventilated rooms. The same general idea applies to the sleeping room. Care should be taken that its supply of fresh air is sufficient, and that the cubic area is enough for the child's needs.

Daily baths should be the rule. For infants the ordinary tepid bath is best, and may be followed by a brisk rubbing with alcohol. Children who are able to support themselves may be stood in a tub containing a few inches of warm water, sufficient to cover the feet. Cold water should then be rapidly thrown over the body; the child may be dried and rubbed smartly with alcohol. With these weak children one should take care to secure a good reaction after the bath, on penalty of harming more than helping them. The clothing should be light but warm; and all through the year woollen underwear may be used, the only difference required by change of seasons being one of weight.

Concurrent disorders must be treated according to their several symptoms. Deformities of bones in their very early stages require no treatment outside of massage, and if the general treatment is vigorously pursued, the affected parts will soon regain their normal form. Since the natural tendency of the disease is toward eventual recovery, special measures for the cure of minor deformities are not often necessary. In extreme cases of deformity of the extremities it may be necessary to keep the patient in bed in order to prevent use of the arms and legs. Curvatures of the spine may be helped by splints, plaster jackets, passive motion, and postural treatment. The child should lie upon a hard flat mattress with the whole body in one plane.

A number of drugs have been recommended for this disease, among which phosphorus occupies an important place. The value of this drug is doubtful, and on the whole, after a fair trial of it, I believe that one can get along as well without as with it. Its capability of irritating the stomach and intestines is large, and great care must be observed in its use. The ordinary dose is from 0.0003 to 0.0006 gramme $\left(\frac{1}{200}\right.$ to $\frac{1}{100} \mathrm{gr}$.) dissolved in olive oil. Other drugs that are much used are the lime salts, such as the hypophosphite or the lactophosphate.- Their use began with the theory that since calcium salts were deficient in the bones, a cure might be obtained by supplying them through the stomach. This 
theory is not necessarily founded upon truth; and while these salts may have a tonic value, it is no greater than and possibly not as great as that of iron and strychnine. These with proper food and general care will be sufficient for practically all cases. Accessory symptoms, such as nervous disorders or sweating, will, as the cure progresses, subside without additional treatment.

Prognosis. - As has been stated, the progress of the disease is toward recovery. In the presence of proper treatment the patients, unless some intercurrent disease sets in, should regularly recover. Even without treatment, if the character of the disorder is not especially severe, the changes in the bones gradually resolve themselves, and these parts may even become harder than they are in normal children. Marked deformities, such as knockknee and bow-legs, may require orthopædic treatment. Under such circumstances a radical operation is to be preferred to the slow treatment by pressure splints; the operation should not be performed until the child is old enough to bear it without dangerous shock.

The main danger of rickets lies in the general weakness which characterizes it, and in the presence of which diseases of all kinds are easily contracted and feebly resisted. Of these the gastrointestinal complaints are most to be feared in the summer and pulmonary in the winter.

Differential Diagnosis. - Rickets is not often confused with other disorders ; for the enlarged bones, the profuse sweating, the square head, large fontanelles, lax joints, pot-belly, delayed or poor teeth, the slow development, and general weakness are characteristic enough to point unerringly to the correct diagnosis. Occasionally a very marked case of muscular weakness may suggest from the utter helplessness an anterior poliomyelitis with paraplegia. The previous histories of the two diseases are quite dissimilar; the rickets would have its other symptoms, and the infantile paralysis would give its own electrical reactions. Cretinism may, on account of the partial growth, the open fontanelles, and delayed eruption of teeth, be thought of ; but the other symptoms of rickets are not present. Syphilis and scorbutus are mentioned as confusing agents; but the pictures which they present are not enough like that of rickets to suggest more than temporary doubt for the physician who is fairly familiar with all three. 


\section{InFANTILE Scurvy (Barlow's Disease)}

When adults are deprived for a considerable time of fresh food their liability to contract scurvy is well known. The same possibility exists in children. The one has been recognized for a long time; the other, strangely enough, a comparatively short time. In the light of present knowledge it seems strange that this important fact could so long have been overlooked. It is only since 1873, when Ingelev first suggested the possibility of infantile scurvy, that attention has been drawn to the disease; and since 1878, when Cheadle published in the Lancet the diagnosis and records of three cases, we have become comparatively familiar with such cases. In America these cases are now frequently recognized.

The cause is regularly seen to consist in the continued withholding of fresh food, principally milk. Since the diet of children, other than infants, is more or less general, the disease is practically confined to the first year or year and a half of life ; for during this time the exclusive use of dried, farinaceous, and proprietary foods is most common. Children who are brought up on condensed milk and possibly sterilized milk are liable to this disorder. Since the use of proprietary foods is widespread among the rich as well as the poor, scurvy is found in both classes. Indeed, a strict division of the cases will show a greater proportion among the rich, whose children are apt to suffer from too much care, or rather from too much unwise care. Such people are apt to believe that the expensive food is the best food, and therefore restrict their children's diet to what seems to them the best. Poverty produces rickets; wealth, unwisely used, makes scurvy.

Lesions. - The main changes consist in extravasations of blood into the periosteum, bones, skin, subcutaneous and muscular tissues. The cause of this is doubtless some deterioration in the blood, whereby the integrity of the vascular tissues is impaired. It has been supposed that a decreased alkalinity is at the bottom of this deterioration. Such a theory is good as far as it goes, but it is not by any means a final explanation of the phenomena of the disease. The extravasations are oftenest seen in the legs, the vascular periosteum of the femur and tibia being the most commonly affected part. Other bones, such as the ribs, scapula, hu- 
merus, the radius, the orbit, are similarly affected. Hæmorrhages may take place into the cavity of the long bones, and the medullary substance may be softened and degenerated.' The muscles and mucous membranes, the joints, kidneys, mesenteric glands, liver, spleen, lungs, and pleura may likewise be the seat of extravasations, and in the heart, liver, spleen, and kidneys there may be some degree of fatty degeneration. Thus we see, as a result, the swollen, spongy, and bleeding gums; also the ecchymotic eyelids and the sensitive swellings on the extremities and about the joints. In the last named structures the hæmorrhages may be considerable, and may be followed by a slow inflammatory process that finally destroys the tissue. The changes in the ribs may be followed by separation of the sternal ends from the cartilages.

Symptoms. - Scorbutic children are anæmic and poorly nourished, they may be thin or fat, and they are always weak. Attention may first be drawn to their condition on account of an increasing physical tenderness, which is noticed on washing, dressing, or handling the child. He not only becomes quiet when untouched, but also shows a decided disposition to avoid all movement, and lies unusually still without changing the position of legs and arms. The temperature is moderately elevated, not often above $39^{\circ}$ C. $\left(102.2^{\circ}\right.$ F.) Examination reveals the presence of subperiosteal swellings, caused by hæmorrhages, along one or more of the long bones above mentioned. If a hæmórrhage has taken place under the periosteum of the orbit, a correspondent degree of proptosis may be noticed. The ordinary swelling is fusiform, and gives on palpation the feeling of deep fluctuation. When it is near the joint, the swelling may to some degree extend on to the articular surface, but only in rare cases is the joint itself involved. The separation of the epiphysis from its shaft, as seen in the ribs, gives the sound and the deformity of fracture.

When the muscles are involved, they seem soft and boggy; the skin may also be involved, and become dusky and ecchymotic. In this way, especially when there is a subperiosteal hæmorrhage in the orbit, the eyelids may be dark and mottled, like the familiar "black eye." A fairly constant symptom is seen in the gums, which gradually become heavy, swollen, soft, and purplish. The surface may be eroded and superficial ulcers arise. If teeth are present, the tissue about them swells, bleeds, and is very tender. There is so little firmness left in the gums that the teeth become 
loose and finally fall out. The resulting cavities are apt to be partially filled with a purulent exudate which increases the offensive odor coming from the rest of the gums. Hæmorrhages from mucous membranes, such as from the nose, mouth, stomach, and intestines, may be seen at any part of the disease ; and occasionally hæmaturia may be the first symptom to attract attention. The general anæmia may at times grow more profound on account of this hæmorrhagic tendency. In all cases, however, the complexion is peculiarly anæmic and has a dull, earthy hue.

Treatment. - The prevention of scorbutus consists in avoiding the exclusive use of condensed milk, dried, proprietary, farinaceous, and peptonized foods. In the very rare case of a scorbutic nursing mother, the child should immediately be weaned. The active treatment does not necessarily call for the use of any drugs, but only for the substitution of fresh for dried foods. Fresh milk, modified according to the age and digestive ability of the child, must be the main diet for infants; in addition, he may daily have a small cup of strained beef tea in which potatoes and carrots have been cooked. One of the best means of treatment at our command is potato which has been steamed and rubbed up with milk to the consistency of cream. A teaspoonful of this mixture may be put in the patient's milk three or four times a day. Orange juice is also beneficial. After the scorbutic symptoms have passed away, the anæmia and asthenia will require appropriate treatment. In older children this plan of treatment holds equally good; and the diet must include as much fresh food, both vegetable and animal, as circumstances permit. Such children should be treated very much in the way that an adult in similar pathological circumstances would be.

Prognosis. - The outlook is usually good. Within a week or two after proper diet has been established, the symptoms have in large part disappeared. It is only in unrecognized cases or those in which recognition of the nature of the disease has come very late, that death must be expected. Some scorbutic children recover without any treatment whatever, but the majority of those untreated gradually become more and more anæmic, weak, and prostrated, until death takes place through hemorrhage, exhaustion, or intercurrent disease.

Differential Diagnosis. - The diseases with which scurvy may be confounded are rickets, rheumatism, osteitis, anterior polio- 
myelitis, and stomatitis. All of these have at least one symptom that occurs in scurvy; but none has a group that in its entirety closely resembles that of this condition. A child that exhibits subperiosteal swellings which are sensitive to the touch, spongy and swollen gums, hæmorrhages from the mucous membranes, and marked anæmia, is certainly scorbutic ; and no other disease presents a similar combination of signs. In rheumatism the lesions are in the joints; in scurvy they are almost always along the shafts of the bones. The pain on movement may keep one or more limbs abnormally still, and this has been mistaken for infantile paralysis. But as this is the only symptom in which the two diseases coincide, the resemblance should not be very misleading. Hæmaturia has been rapidly diagnosticated as nephritis, and the scorbutic gums have been treated as an isolated condition; but these mistakes are doubtless due to carelessness rather than lack of knowledge. In the very large majority of cases there should be no difficulty in naming the disease aright. 


\section{CHAPTER XIV}

\section{DISEASES OF THE NOSE AND THROAT}

\section{Acute Rhinitis}

ONE of the commonest ills of children is an acute rhinitis, commonly called "coryza," or head cold. In almost all cases it is regarded as a trivial matter. In the adult it is rarely important, but in children and especially in very young children its possibilities for harm are much greater. When one calls to mind the peculiarities and sensitiveness of the infant naso-pharynx, one can at a glance see how easily an inflammation of its mucous membrane may begin, and why it is able to entail serious results. In these very young patients one should look upon every rhinitis, no matter how slight it may be, as a probable starting-point of a bronchitis. Leaving out of consideration every other harmful contingency, this fact in itself is enough to dignify the disease to a place of some importance.

Causes. - The best known cause is exposure to unfavorable atmospheric conditions. Nevertheless, in all likelihood, there must be some antecedent cause, such as a micro-organism, or an atonic general condition, or both. Other things being equal, the more poorly nourished the child, the greater the liability to this disease. And the communicability of it is demonstrated every day in our ordinary lives.

Lesions. - The pathological changes are characteristic and plainly marked. The mucous membrane of the nose and nasopharynx is swollen and congested. There is a diapedesis of red blood-cells, an emigration of white blood-cells, and a transudation of serum. In the severer cases there may be an exfoliation of epithelium with possible erosions.

Symptoms. - At first the child is apt to sneeze frequently, the eyes are more or less suffused, and the nasal discharge is prominent. This discharge is thin and acrid, but as the disease develops it becomes more purulent. On account of its irritating 
character it is able to bring about an irritation of the skin of the upper lip and even the neighboring portions of the face. On account of the swelling of the mucous membrane and the presence of the discharge, the nose may become more or less stopped up, thus producing the natural sequence of mouth-breathing, impaired sleep, and, in infants, defective ability to nurse. There may be a slight elevation of temperature, loss of appetite, general depression, and irritability. In the same way that the lower portions of the respiratory system may, by an extension of the inflammation, be attacked, so may the lesions be communicated to the Eustachian tube and thence to the middle ear. The glands in the neighboring regions are irritated and swollen.

Treatment. - It is a good plan to institute treatment by clearing the intestinal track, preferably by divided doses $(0.006$ gm. $-\frac{1}{10}$ gr.) of calomel followed by a mild cathartic. Warm baths are useful, as they relieve the general depression and help to resolve the local congestion. The interior of the nose should be frequently sprayed with an alkaline solution, or a solution of boric acid, while a spray of an oily material to sooth the inflamed area may be next applied. The end of the nose and the whole upper lip should be covered with vaseline as a protective against the acrid discharge. The food should be carefully regulated, and as soon as possible the child should be sent into the air for exercise. To guard against a recurrence of the attack, the patient's general condition should be improved as much as possible by regular exercise, bracing baths, and tonics.

Prognosis. - The disease lasts for a few days only, and the outlook is uniformly good. It is necessary to keep in mind both the weakened condition which is apt to precede the rhinitis, and the further weakening which must follow it.

\section{Chronic Rhinitis}

Causes. - Repeated attacks of acute rhinitis are commonly sufficient to determine a chronic inflammatory condition. In the face of improper climatic conditions, weakened nutrition, and lack of remedial care, this condition may lead to effects which, if not quite permanent, are hard to remove. Also the presence and the continued irritation of adenoid fungations are equally capable of producing a like result; again, the rarer 
occurrence of nasal polypi may cause much the same state of affairs, although not so regularly as the other factors mentioned.

Lesions. - The pathological changes are the natural extension of those found in the acute form. But the mucous membrane is much thickened and has a tendency to the infiltration of connective tissue. In some rare cases the necrosis of tissue is marked, and the stroma is infiltrated with fibrin, which holds in its meshes many pus cells. As a result the discharge is purulent, and the surface is so vitally affected in small areas that ulcers of various size result. This constitutes the so-called purulent form of chronic rhinitis. In the course of time the ulcers heal and the space is filled with granulation tissue; here the growth of connective tissue may start, which, infiltrating the surface in all directions, produces contractions and deviations from the normal histology. The glands are encroached upon and gradually destroyed. The discharge, mixed with fibrin and pus, adheres to the surface, decomposes, and emits a most disagreeable odor. This is the so-called atrophic form of chronic rhinitis, formerly known by the general name of ozæna.

Symptoms. - The symptoms consist in a more or less constant discharge, especially on exacerbations, the formation of crusts, interference with the sense of smell, deviations from the normal shape of the nose internally or externally, depreciation of the general health, and a marked tendency toward the acquirement of laryngeal, bronchial, and pulmonary diseases. In the purulent form the fetid odor is plainly noticeable.

Treatment. - The cure of these conditions requires much care and time. One should, as a matter of the first importance, seek by every possible means, to improve the general health. Without this I doubt whether a cure is possible. Some obstinate cases require removal to a favorable climate. Where abnormal antecedent causes, such as new growths, exist, they must first of all be removed. The mucous membrane must be frequently douched and cleansed, and for this purpose alkaline and antiseptic solutions, such as Seiler's, are required. The thickened mucous membrane may require cauterization, deviations of the septum may need rectification, and, in short, one should endeavor to remove or abate the pathological conditions as one finds them in individual cases. Children differ so much, one from the other, in the manifestations of this disease, that it is undesirable in a 
gencral treatise to go into all the possible peculiarities of occurrence and treatment.

Prognosis. - The outlook is fairly good, certainly better than in adults, if parents are able and willing to respond to all the necessary demands upon them. They should be informed concerning the protracted nature of the disease and the necessity. of devoting to its cure considerable attention, for the gravity of the possible consequences is worthy of much respect.

\section{Epistaxis}

Causes. - A frequent and usually unimportant complaint of childhood is bleeding from the nose. It usually follows some trivial cause, such as picking the nose, insertion of foreign bodies, blows, or falls. A weakened general condition produces a greater liability to this disorder, and an abnormal state of the mucous membrane, as in a chronic rhinitis, is a further provocative. Bleeding from the nose happens with some acute fevers as well as constitutional diseases; but under such circumstances its occurrence is not necessarily regular. In atonic conditions, such as anæmia and scurvy, it is often seen, and its connection in a grave form with hæmophilia is rare but startling.

Symptoms. - Outside of the hæmorrhage, it has almost no symptoms. In the ordinary slight forms it is unimportant; in the hæmorrhagic diathesis and allied conditions it may be very serious, even fatal.

Treatment. - In most cases the hæmorrhage will cease spontaneously. In others it will be necessary to plug the nostril, and while one is about it one should make the plug fairly tight. This plug should be secured by a string for ease in removal. When the bleeding-point is far back, it may be necessary to place the plug in position by passing a soft rubber catheter, to the end of which the cotton is attached, through the nose into the mouth. If the plug is soaked with a four per cent solution of antipyrin, its styptic effects will be increased. In recurrent cases one should locate the bleeding-point and cauterize it.

In any but trivial cases it is advisable to improve the patient's general health by hygienic measures, diet, and tonics. 


\section{Nasal Polypi}

The presence of polypi in the nose is very apt to be overlooked, especially by the general practitioner who has not the opportunity of full acquaintance with them. Although infants are not exempt from them, the growths are most apt to occur in the middle and later periods of childhood. Outside of the symptoms of chronic rhinitis which they entail, there are not many diagnostic features which inevitably draw the observer's attention

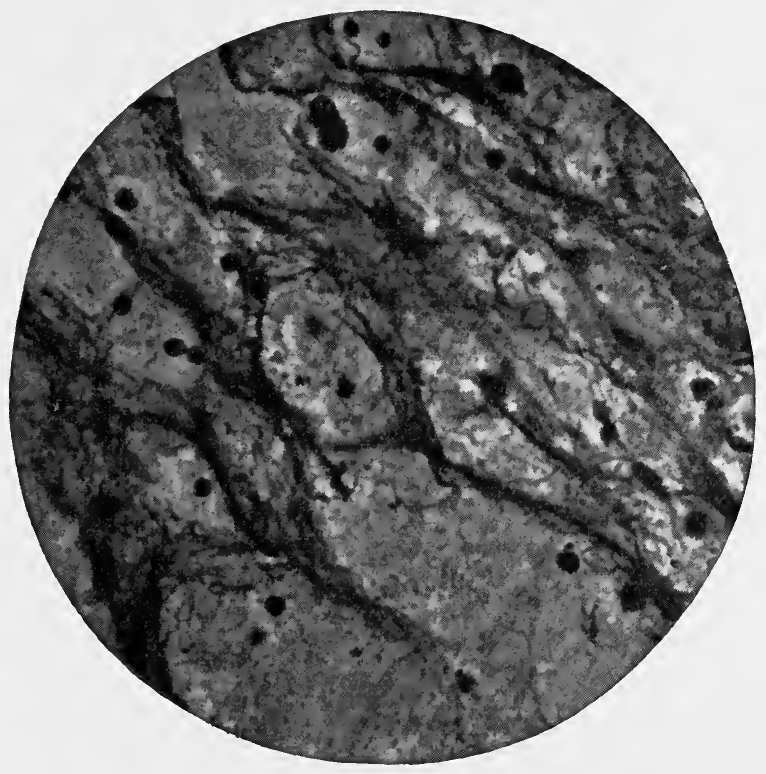

Fig. 33. - Polypus of Nasal Mucous Membrane. $\times 320$.

to them. In part this is due to the difficulty which children have in translating subjective sensations; for such sensations are a part of the symptomatology of this disorder.

The growth, either fibrous or mucous, hangs from the middle turbinate bone by a pedicle. It varies in size at different times, becoming larger with irritating atmospheric and local conditions and smaller when these irritants are removed. Attention is drawn to them in the examination which follows disorders of the nose, larynx, and ear, complaints of headache, partial lack of smell, restless sleep, headache, and malnutrition. The only way in 
which one can be sure of the presence of nasal polypi is to see them on examination.

The treatment consists in twisting them off by a forceps or removing them with a snare.

\section{Adenoid Vegetations of the Pharyngeal Vault}

In comparison to the disabilities entailed, there is none of the diseases of children outside of the fatal ones which is more important than hypertrophy of the pharyngeal tonsil. Moreover, there is none that originates more easily, has more widely distributed effects, and is more easily cured. All in all, it is a most interesting condition, especially in view of the many results, with the most diverse tendencies, which follow it.

Causes. - Although no child is free from the possibility of its attack, poorly nourished, rachitic, and lymphatic children have a predisposition to it. Such a patient should always be suspected, until cleared by examination, of being thus afflicted. Under such circumstances trivial adventitious causes, such as slight rhinitis, inclement weather, or unimportant injuries to the nasal mucous membrane, are sufficient to start it into existence. It often shows itself after the acute fevers, especially measles.

Lesions. - In the mucous membrane of the naso-pharynx one finds many characteristics of that of the pharynx. An example is the analogy between the two faucial tonsils and the pharyngeal tonsil, sometimes called the third, or Luschka's, tonsil. In health, this third tonsil is very small, but when hypertrophied it may attain so great a size that it fills up the vault. It is made up of lympho-mucoid follicles which are an exaggeration of simple normal histological elements. It may attain various shapes and sizes, may grow in separate masses, in grapelike branches, or merely as a heavy formation without definite outline.

With this growth certain other changes frequently occur. These may be the pathological features of an inflammation of such parts as the nose, pharynx, larynx, Eustachian tube, and middle ear. Also on account of the impeded respiration, there may be a deficient oxidation of the blood with consequent low forms of intoxication. These obscure changes are among the most interesting and protean features of the disease. 
Symptoms. - The first things of which the parents are apt to complain are restless sleep and some degree of impeded development. Questioning will usually bring out the fact that the child snores in his sleep, sleeps with open mouth, is easily subject to rhinitis, laryngitis, and bronchitis, is poorly nourished, and is apt to be peevish and finical. In marked cases, especially those of long standing, deformities of the face and chest may occur. The bridge of the nose may be high and thick, the palatal arch high and narrow, the upper jaw contracted so that the teeth project, crowd, and override one another.

After having seen a few characteristic cases, one ought to be able in many instances to make a diagnosis merely from the child's appearance and expression. The mouth remains open, the lips are thick and heavy, the eyes are dull and stupid. Examination of the chest will show a greater or less degree of flat-, barrel-, or pigeon-breast deformity. Whether one or the other of these conditions will follow depends upon the existence of such complications as bronchitis, rachitis, and the different degrees of malnutrition. Frequently one sees various forms of pharyngitis, rhinitis, and otitis, caused by adenoid vegetations, running a most obstinate and protracted course, defying all curative measures until the growths are removed. In the same way, a chronic bronchitis, with a similar ætiology, may give rise to an emphysema which in its turn may start an interstitial pneumonia. Often enough one may notice that certain children, who suffer from adenoid vegetations, frequently and easily fall into congestive pulmonary conditions which render them a fertile culture-ground for specific lung diseases.

Another interesting feature of the symptomatology is the subjective impressions induced by the abnormal conditions from which these children suffer. For instance, take the disorders which attack the ear, and their possibilities. These follow from the occlusions of the Eustachian tube by the growth, which thereby prevents the normal ventilation, and dams up the natural secretion. As a result, the tube becomes congested and inflamed; the middle ear becomes similarly influenced, with a natural accompaniment of subjective noises. These the child translates in various fantastic ways, thus unwittingly bewildering and deceiving his parents and attendants. "Such incidents can color his whole life with a hue of unreality, involving all his sense-impressions, 
and ways of thought. Attacks of asthma or hay fever may likewise follow in this obscure train of complicating symptoms.

Treatment. - There is one sure way of dealing with adenoid vegetations, and that is their radical removal. This should be done under chloroform anæsthesia by means of a post-nasal forceps or curette. The mouth may be held open by a gag, and the operation should be rapidly executed. While in most cases the hæmorrhage is trivial, it may occasionally be serious; and I have had two cases in which it was necessary to plug the posterior nares. In making the preliminary examination the physician can avoid having his finger bitten if he will press a fold of the child's cheek, with a finger of his free hand, between the open jaws. This is easily accomplished, can do no harm to the patient, and gives the examiner a full opportunity to acquire all necessary information.

For a few days after the operation it is advisable to spray or douche the cavity at least four times daily with an alkaline antiseptic solution. The child should be confined to bed for two days, and not permitted to go into the open air for two days more. The medical treatment usually consists in the administration of a tonic such as one of the organic preparations of iron.

Prognosis. - The outlook is uniformly favorable except in very marked cases of long standing where permanent deformities may exist before the operation. These cases, however, are exceptionable.

\section{Acute Simple Tonsillitis}

After infancy children often suffer from various degrees of inflammation of the tonsils; but during the early months of life the disorder is more rarely seen, possibly because the child has a less active life with fewer opportunities of infection from food and foreign bodies put into the mouth, as well as fewer occasions of exposure to unfavorable climatic conditions. In the presence of irritation of the superficial tissue, the tonsils easily become subject to congestive changes as well as invasion by bacteria. One attack makes the child more susceptible to the disorder than he originally was; and in the spring, more than at any other season, he is apt to contract this acute infection. Usually there is apt to be a generally lowered nutrition; and such a condition not only permits an easier entrance of the disease, but 
also makes the attack severer than it otherwise would be. Other diseases, such as scarlet fever, measles, diphtheria, and sometimes lobar pneumonia, bring about a general inflammation of the mucous membrane of the throat, in which the tonsils are included.

Lesions. - The changes which take place include congestion of the vessels, an exudation of serum with some fibrin, an emigration of red and white blood-cells. In this exudate large numbers of bacteria may be found.

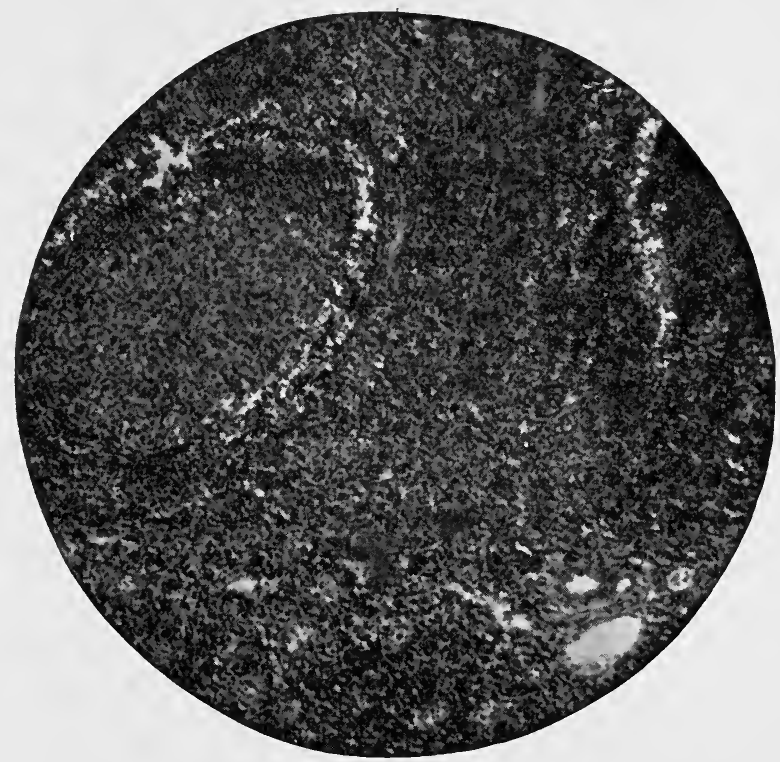

Frg. 34. - Acute Tonsillitis. $\times 80$.

Symptoms. - The symptoms are of all degrees, from barely noticeable pain to severe prostration. The tonsils are red and swollen, and the cervical glands may be somewhat enlarged. The temperature may range from normal to $39.5^{\circ} \mathrm{C}$. $\left(103.5^{\circ} \mathrm{F}\right.$.). There may be sufficient soreness in the neck to make deglutition unpleasant or difficult.

Treatment. - The treatment is simple; in fact, the simpler one makes it, the more comfortable will the child be. First of all, the gastro-intestinal track should be thoroughly emptied. The throat should be regularly and frequently sprayed with an 
alkaline, antiseptic solution. In marked cases the application of ice bags to the neck may give relief. For internal medication remedies which will promote excretion, such as the compound mixture of rhubarb and soda, with small doses of nux vomica, will help the patient make a quicker and better recovery.

Prognosis. - The outlook is good. One should, however, keep in mind that every attack of tonsillitis makes a chronic inflammation so much the more possible; and also that the inflamed mucous membrane easily becomes the seat of further disease.

\section{Acute Follicular Tonsillitis}

This is an acute, contagious, self-limited disease, whose symptoms are out of all proportion to its real danger. While the disease is not limited to children, it attacks them more frequently than adults; on the other hand, they generally seem to suffer less from the prostrating efforts than mature patients. This may be readily demonstrated in families where one person after another is attacked. Certain individuals seem to be predisposed to it, and those of a rheumatic tendency are included in such a category. Any simple acute or chronic inflammation of the tonsillar mucous membrane makes an attack of the follicular disease more easily possible than healthy tissue does. No specific germ has been found in the exudate on the tonsils, although large numbers of the ordinary streptococci and staphylococci of purulent processes may regularly be distinguished.

Lesions. - Although the whole tonsil is inflamed, nevertheless the process is primarily localized in the crypts. These crypts become blocked up with masses of serum, fibrin, pus, and blood cells, ephithelial detritus, bacteria, and particles of food. In first attacks the lymphoid tissue for the most part is involved and swollen. As the attacks are repeated, there is a progressively greater amount of fibrous tissue present and involved in the inflammation. According to the intensity of the pathological process and the weakened resistance of the patient, the inflammation spreads over the adjacent mucous membrane.

Symptoms. - The first sign that attracts the parents' attention is the marked prostration. The child seems to be seriously sick, the temperature runs high, often reaching $40.5^{\circ} \mathrm{C} .\left(105^{\circ} \mathrm{F}.\right)$ and occasionally higher. The pulse is rapid, the general pain is 
severe. Urinary analysis frequently shows the presence of febrile albuminuria, which, however, persists for no more than a short time. Inspection of the throat shows an inflammation of the tonsillar mucous membrane, which gradually fades off into the near-by parts. Scattered over the tonsil are the swollen follicles, and from some of them small plugs of exudate project. Some of these when close together may coalesce, forming a membranelike deposite of a yellow, thick, uneven, and dull appearance. The cervical glands are enlarged and tender, but do not go on to suppuration. The purely local symptoms may be bilateral, but the membrane does not often go beyond the tonsils. Occasionally one is apt to see a fine, erythematous rash that accompanies this disease. It fades away after the lapse of a few hours or a day, and requires no especial attention.

Treatment. - The treatment, while not specific, is satisfactory. Sufficient calomel or a saline cathartic should be given to empty the stomach and intestines. Ice bags should be placed on the sides of the neck opposite the tonsils, and the throat may be frequently and thoroughly sprayed with a solution of hydrogen peroxide and boracic acid. Relief can be given within a short time by the use of equal parts of phenacetine and salol, taken every two hours; the dose of each for a child of about five years is gm. 0.12 (gr. ij). The food should be small in quantity and of an easily digestible nature. In cases of a rheumatic tendency one may get relief from fairly large doses of salol, salophen, or similar preparations.

Prognosis. - The outlook is almost always good. In some few cases a peritonsillar abscess may follow the acute tonsillitis or one of the various other inflammations of the throat and mouth, which naturally find a good starting-point in the inflamed surface.

Differential Diagnosis. - The diagnosis rests between follicular tonsillitis and diphtheria. The former attacks the tonsils only; it appears in scattered areas, and if these coalesce, the membrane has a clear, whitish appearance. In diphtheria the membrane is on any part of the tonsils, pharynx, or larynx ; or if in the beginning it is confined to the tonsils, it soon spreads to the adjacent parts. In appearance it is yellowish, dull, and not clean looking. In follicular tonsillitis the fever is high, and the pain and discomfort marked. In diphtheria the fever is moderate or low, and the pain not so great. In the former the depression passes away in the 
course of a day or day and a half; but in the latter it lasts much longer, and may progressively increase until the disease ends in its usual manner or as the result of treatment. Finally, the matter is definitely settled when a culture has been made, which shows the presence or absence of the Klebs-Loeffler bacillus.

\section{Chronic Inflammation and Hypertrophy of the Tonsils}

Causes. - Hypertrophied tonsils at birth are rare; nevertheless they occur. Still oftener one sees children whose tonsils are predisposed to inflammation and consequent enlargement. With such patients slight causes of irritation are sufficient to set the process in motion. Thus, a harsh and trying climate, a rheumatic predisposition, or the lymphatic tendency is sufficient to produce tonsillar hypertrophy. In a few young cases, and in a yet larger proportion of older children, repeated attacks of acute inflammation of the tonsils, or even of the pharynx, make a progressive increase in all the various elements in the gland so that the throat gradually becomes more and more obstructed. Whether the glandular or connective tissue predominates is of but little practical importance, with the possible exception of a few rare cases where the interstitial tissue is so very much in excess as to render possible a greater hæmorrhage after amputation. This is not a serious danger, and should not be allowed to prevent the operation.

Lesions. - The pathology is not particularly interesting, and from it we derive no especial indications for differential treatment. Where the connective tissue is most involved, the tonsil is hard and gnarled; where the glandular tissue is principally the seat of inflammation and hypertrophy, the tonsil is larger, redder, and more prolific in secretion. One usually finds varying degrees of mixture of these elements.

Symptoms. - The child suffers, for the most part, from symptoms of obstruction. The voice may be thick and wooden, there may be a chronic associated pharyngitis, rhino-pharyngitis, and rhinitis. The two glands may be so large as to meet, or nearly meet, thus interfering with respiration and the consequent oxygenation of the blood. With the condition there may be an hypertrophy of the pharyngeal tonsil, but even if there is not, the symptoms may be much alike. Thus the bulging growth may 
press upon or occlude the pharyngeal entrance to the Eustachian tube, setting up an inflammation that extends into disease of the middle ear. 'The same results of restless sleep and breathing, of deformity of the chest, and impaired nutrition may be present in both. Where the faucial tonsils are hypertrophied, one should always suspect the pharyngeal tonsil of being similarly involved, and removal of the first should not be decided upon without a digital examination of the naso-pharynx in order to convince oneself of the potential necessity of likewise clearing it out.

Treatment. - The treatment is plain, and should not be obscured by palliative measures. ' If the tonsils are large enough to produce obstruction or marked irritation, they should be amputated. While one is about it, the patient will obtain most benefit by leaving very little stump; for the removal of no more than the cortex permits too frequently a return of the whole trouble. Rarely is the operation troubled by hæmorrhage. If it is, in the vast majority of cases, it can be checked by pressing the thumb, which has been covered with a bandage, against the bleeding surface while the fingers make counter pressure from the corresponding part of the outside of the neck. Another satisfactory way of obtaining the same result consists in having the patient suck fairly large pieces of ice for a few minutes. In very rare cases the galvano-cautery may be needed to stop the hæmorrhage ; but the usual experience is that the bleeding stops spontaneously. The throat should then be kept clean by frequent spraying or gargling with boracic acid.

When one desires to remove an hypertrophied pharyngeal tonsil in addition to the faucial tonsils, an anrsthetic should always be administered. The faucial tonsils should first be removed. After almost all cases of tonsillotomy it is advisable to give tonics, and in all ways sedulously to promote the general nutrition.

The outlook is good, and the operation should not be regarded as dangerous.

\section{Peritonsillar Abscess}

Children who are in poor general health, especially those who have been suffering with some form of throat disease, are liable to contract an inflammation of the cellular tissue around the tonsil. While the general practitioner may not have many occa- 
sions to treat such a condition, the specialist in charge of a large clinic or hospital sees a sufficient number of cases to keep fresh in his mind the possibility of this disease.

There may or may not be a chill; there is always a rise of temperature to about $40^{\circ} \mathrm{C} .\left(104^{\circ} \mathrm{F}\right.$.). The pain is mostly local and the general prostration is not in all cases great. As the swelling increases the head may be held in a cramped position, and in unusual cases of extreme degree the acts of eating and drinking may be impeded; in other cases the infiltration may be so great as to effect the larynx. When the abscess is fully formed, it may rupture spontaneously. If this happens during sleep, the pus may be inspired, with a fatal result. 'This, however, is unnecessary. If, after examining the throat, the peritonsillar tissue is seen to be swollen, and the application of an ice bag is without avail, one should be prepared to operate.

Treatment. - As soon as one can detect fluctuation, the abscess should straightway be opened freely; it is advisable to enlarge the incision by passing into it a pair of forceps which then should be opened, thereby materially enlarging the drainage area. The incision should be made outside of and above the tonsil, and downward.

The treatment is not entirely surgical. The first step should be the thorough cleansing of the gastro-intestinal track by means of calomel or a saline cathartic. The food should be as easily assimilable as possible. During convalescence one may have to administer tonics, and to supervise the diet and mode of life. The mouth must be kept as clean as possible.

Prognosis. - If the disease is promptly treated the outlook is good. 'The main danger arises from allowing the abscess to increase in size until the larynx is encroached upon, or until spontaneous rupture occurs with the attendant danger of inspiring the pus. Both of these conditions may result in death.

\section{Uvulitis and Elongated Uvula}

As a primary disorder an inflammation of the uvula is rare : so much so, that when it occurs one regards it as a curiosity. For the most part, it comes with acute pharyngitis and rhinitis. The uvula is swollen, the vessels are congested, and there is a hypersecretion of mucus. The child has a feeling of discomfort 
in the throat, as if an unnatural substance were there; he is tormented by an irritable cough, although the lungs are quite clear. Especially on lying down this symptom is annoying; and until the uvula resumes its normal size the patient will experience all the discomforts that come with irritating the surrounding parts. In this way attacks of laryngitis or laryngismus stridulus may be put in motion; and doubtless in some cases asthma may originate in the same way.

An astringent applied to the part will reduce the size; and a spray or gargle of an alkaline and antiseptic solution will promote a normal condition. In very severe cases it may be necessary to make many minute scarifications. In case the uvula remains too large, it will be necessary to remove its tip. This is easily clone with a forceps and scissors. One should be careful not to amputate too much.

The uvula is sometimes congenitally elongated. It then causes all the irritating effects mentioned above. The treatment is amputation of the tip. Some cases of bifid uvula seem to have a natural tendency to elongation.

\section{Acute Pharyngitis}

Causes. - An inflammation of the pharynx or any part of it is a frequent incident in a child's life. The conditions which predispose to and cause it are so easily contracted that the disorder is one of the most common with which we have to do. The existence of any pathological condition of the near-by parts or an hypertrophy of the tonsils, either faucial or pharyngeal, easily starts the inflammation in motion. Such a process is the more readily possible on account of the softness of the youthful mucous membrane; its structure is weak, its blood-vessels are loosely held and easily become dilated and congested, its glands readily become hypertrophied and their characteristic action abnormal. Thus, even harsh changes of weather can produce enough irritation to start an acute inflammation into active being. Most assuredly is the irritation sufficient to make the mucous membrane a good culture-ground for the micro-organisms which are responsible for some attacks of pharyngitis.

Lesions. - The pharynx is rarely alone in such an inflammation; one usually finds the process involving the mucous mem- 
brane of the nose or the throat as well. This membrane is swollen, the vessels are congested, the glands are hypertrophied. At first, secretion is diminished, but shortly it becomes profuse. It is composed of mucus, fibrin, red and white blood-cells, and epithelial débris; in follicular pharyngitis, the inflammation centres about individual follicles, which thereby become swollen and prominent.

Symptoms. - The child complains of local pain, a rise of temperature, loss of appetite, and general malaise. The lymphatic glands in the mouth, throat, and neck become swollen and often tender. In itself, acute pharyngitis is not a very important sickness ; its main interest lies in the fact that it may be one of the prodromata of some acute infectious diseases, such as diphtheria, scarlet fever, and measles. Scarlet fever even more than measles may thus be overlooked, for it gives a red hue to the pharynx that in typical cases differs materially from the dotted and irregular appearance seen in measles. Likewise in these two exanthems there is apt to be a precedent or accompanying rhinitis and conjunctivitis. Nevertheless, the diagnosis in acute pharyngitis must always be a guarded one until sufficient time has elapsed to settle the question of systemic involvement.

Treatment. - The care of pharyngitis is more worthy of full attention than is generally thought, mainly because if untreated it may extend in all directions or pursue a prolonged course. The gastro-intestinal canal should be emptied, the diet should be restricted, and the nose and throat should be sprayed with an alkaline antiseptic solution. If the attack is in any way severe, the patient should be confined to bed and in every way exposure must be avoided. For elevation of temperature, sponge baths are more valuable than the antipyretic drugs. When convalescence sets in, tonics may be needed.

The prognosis is good.

\section{Chronic Pharyngitis}

Causes. - The chronic form of pharyngitis is not so often seen in children as in adults. Nevertheless, occasionally one meets such a condition which may have resulted as an extension of an acute attack, or from the constant irritation of a long uvula, from enlarged tonsils, hypertrophy of the pharyngeal tonsil, nasal 
polypi, and similar causes, that keep the mucous membrane in a state of excitement. Living in a harsh climate not only predisposes to the disease, but also aggravates it.

Lesions. - The changes are much the same as in the acute form; but the mucous membrane is apt to be irregularly thickened and slightly infiltrated with new tissue. In the region of this new tissue the follicles are apt to be prominent. The secretion is often less copious than in the acute form, but it is thicker and more tenacious.

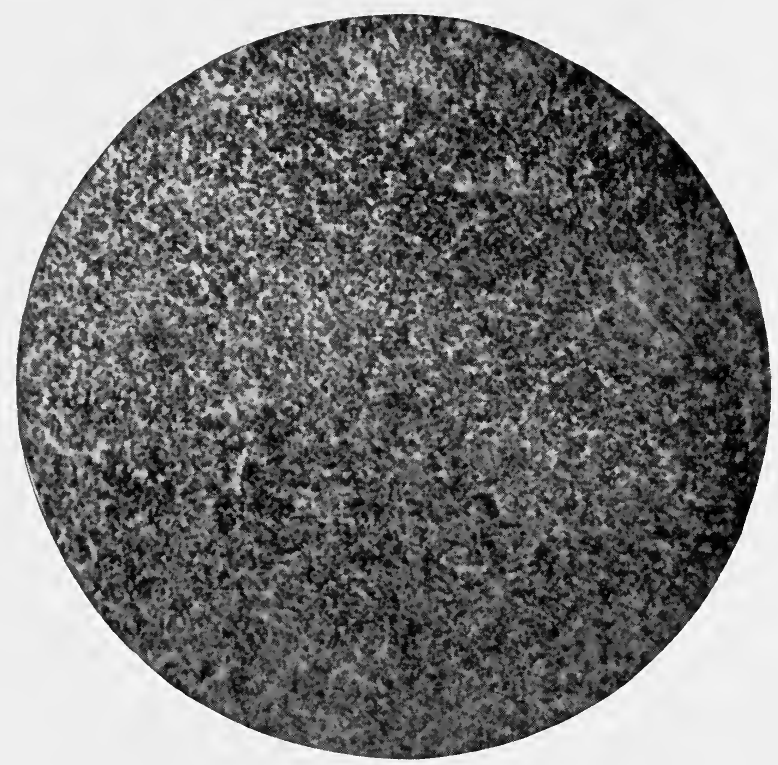

FIG. 35. - Normal Lymph Node. $\times 80$.

Symptoms. - The principal symptoms are cough, some degree of malnutrition, impaired appetite, and disturbed sleep. In addition there will naturally be the signs of the particular cause which is responsible for the pharyngitis, such as an enlarged bifid uvula or pharyngeal tonsil.

Treatment. - The treatment consists primarily in getting rid of the cause, whatever that may be. It will be necessary, then, to keep the nose and throat very clean by means of an alkaline antiseptic solution. This may be followed in some cases by the application to the pharyngeal mucous membrane of a weak astringent, such as a one per cent solution of nitrate of silver. It is 
highly desirable that the child be put into good condition by whatever means of tonics and regulation of daily life one may find necessary.

\section{Retro-Pharyagal LYMPH-Adenitis}

As a result of infection young children are apt to suffer from an inflammation of the lymph nodes in the neck. As these nodes are comparatively large at birth and gradually shripk during the first three years, the greatest liability to disorder exists in babies

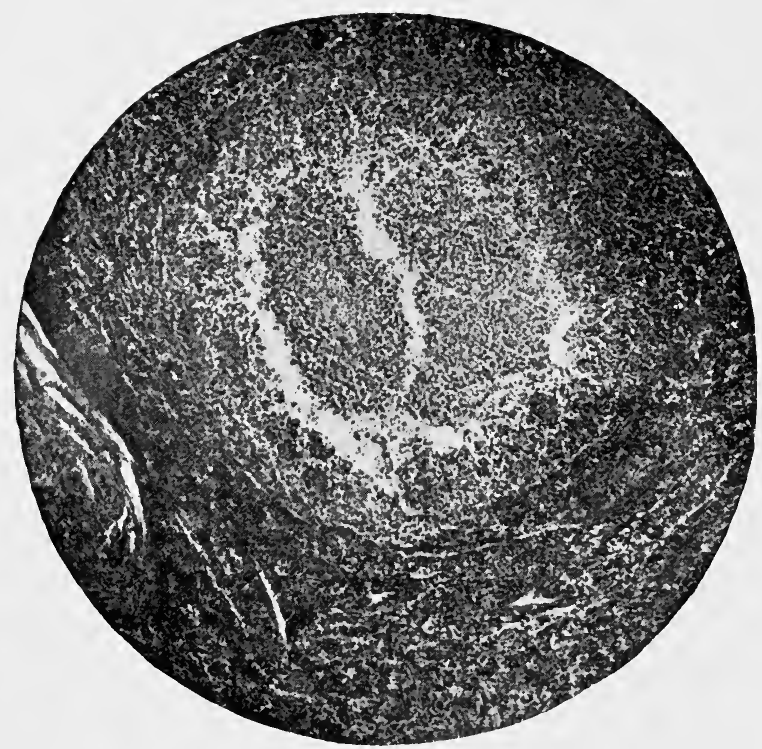

Fig. 36. - Suppurative Lymph Node. $\times 70$.

of this early age. Occasionally, however, the glands persist for some time later, during which there is a simultaneous proneness to such involvement; and I have had occasion to treat a retropharyngeal abscess in a child of nine years.

Causes. - In almost all cases the disease begins as an infection by the ordinary pyogenic bacteria, such as streptococci and staphylococci. These are most apt to find a lodgment when the child's nutrition is impaired and when the local condition in the throat is poor. Such a combination one finds after a severe rhinitis and pharyngitis, the acute infectious diseases and diseases of debility. Chil- 
dren whose mouths are not kept clean contract the disease much more readily than those who are well cared for. In a very small proportion of the cases one will find the tuberculosis bacillus. Rarely one finds a retro-pharyngeal adenitis following caries of the cervical vertebræ. Here the location and extent of the infiltration are somewhat different from the other cases.

Symptoms. - The first symptoms which the parents notice are unwillingness to eat, pain, restlessness, and fever. The child cries steadily and peevishly and shows progressive degrees of prostration. The cry is quite different in pitch and tone from what we hear in any other disease, so that after one has heard it a few times, one is able without difficulty to recognize it even without seeing the child. It is hard to describe this pitch and tone ; some call it a thin crowing sound, others describe it as quacking. The only way in which one can obtain a definite conception is by hearing it.

Another characteristic symptom is the position in which the child holds his head. It is inclined somewhat to the side away from the inflammation and backward. These things naturally direct the attention to the throat, and on examination one finds a hard, brawny swelling which may be so large as to cause dysphagia and even dyspnœa. In some cases the interference with the related parts is so great as to induce hoarseness, and sometimes a loss of voice. Where the swelling is very marked one.is often able to notice it externally on the side of the neck. In rare cases there may be as a final complicating symptom œdema of the glottis.

The common name for this disorder, retro-pharyngeal abscess, is misleading. Not every case goes on to suppuration, and a still larger number when first seen contain no pus. The same rules of development hold good here as in other cases of inflammations of glands. If there is sufficient infecting material with a deficient power of resistance in the patient, the ultimate outcome of the process will be an abscess. Thus a case that at first shows no more than some induration and swelling may a few days later show the characteristic signs of an abscess, which finally, if neglected, may open spontaneously and allow the child to inspire a fatal amount of pus.

In the retro-pharyngeal abscess which accompanies tubercular cervical spondylitis the course is somewhat different. The onset 
is less sudden, and usually, although not always, the bone disease precedes the adenitis by weeks or months. The tumor, instead of being almost on the side, gradually makes its way to the back of the neck, and may even partly encircle the neck in a heavy ringlike mass. If left untreated, it may open spontaneously through the exterior of the neck or into any of the structures of the throat, neck, or air passages. In the last-named case the result is death.

Treatment. - If the case is seen at its inception, one may apply ice bags externally with a view to checking the process. If this does not succeed directly, or if the case has been progressing for more than a day, heat should preferably be used in order to hasten the breaking down of the glandular tissue into an abscess. As soon as this takes place, there should be no delay in opening it. The patient's arms should be pinned to his sides by a large towel or blanket. An attendant, holding him on her lap, should grasp his body with one arm and with the other his forehead. The operator, depressing the patient's tongue, opens the abscess with a knife whose blade has been wrapped to near the point. If the incision has been properly made the pus will gush out. The patient's head should immediately be bent forward so that he may not swallow or inspire the fetid material. Another method, that may be advantageously used, consists in placing the child on his back, and allowing his head to hang over the edge of the operating table. One thus obtains the same immunity from inspiring pus. Then a closed forceps should be put into the wound, and gradually and steadily opened in order to make the aperture large enough. No further treatment, outside of keeping the mouth and nose clean, is needed. The after treatment consists in building up the child's general condition by all means of tonics, diet, and exercise.

While the operation for simple abscess can be performed by the general practitioner, that for the tubercular variety coming with cervical spondylitis should not be attempted by any one but a competent surgeon. Therefore the description is left to special works on surgery.

Prognosis. - The outlook where the operation is done promptly and thoroughly is good, and the patient recovers in a few days. If the matter is neglected, the child's general condition may be so seriously depressed as to endanger life. A still more imminent 
danger is the possibility of compression of the larynx, œdema of the glottis, or inspiration of the pus. These things, however, should not happen.

\section{Acute Laryngitis}

Causes. - A simple laryngitis is a very common occurrence, both as a primary and secondary complaint, in the diseases of children. It exists in the widest range of severity, from a slight hoarseness to the gravest organic and functional changes. It may result from exposure, from the corrosive effects of irritating vapors, as an extension of an affection of any other part of the respiratory system, and as a complication of the acute infectious diseases. In its mild form it may be due to violent erying and shouting.

The characteristic pathological changes are not striking, especially when one compares them with the severity of the symptoms, which in marked cases are often startling. The changes confine themselves for the most part to the mucous membrane; this becomes hyperæmic, swollen, and uneven. In proportion to the congestion the swelling and the dark hue of the tissue increase. The epithelial layer begins to desquamate, and may go on to such an extent that superficial ulcers of various size result. In the severer forms of the inflammation the deeper strata may be invaded; and the climax of progressive involvement is reached when cedema of the glottis supervenes. An injection of the neighboring mucous membrane is commonly seen. As a rule, not much mucus is formed and less pus.

Symptoms. - There are in this disease so many degrees of severity that one's conception of it must be somewhat elastic. Almost all cases begin lightly, commonly showing an acute rhinitis followed by hoarseness. The patient coughs in a dry, harsh, irritating manner. Disturbances of temperature, pulse, and respiration come with the severer attacks; in such cases these disturbances may be much or little. The child in all likelihood will show signs of tenderness and pain in the larynx, the cough becomos nearly continuous, and the gencral prostration is apt to be excessive. In the older children a laryngoscopic examination will show much the same picture that one is accustomed to see in adults, excepting that a certain definite amount of pathological change gives a severer set of symptoms in the former, than in the latter. Dyspnœa is likely to be present 
during part of the day and regularly so about midnight. As this increases cyanosis sets in, the attempts to breathe become more and more violent and shallow, until relief is obtained or the child becomes unconscious.

In all affections of the larynx there seems to be a neurotic element which makes the liability to spasmodic contraction of the muscular tissue ever present. The explanation of this fact is hard to find; we merely know that it exists, sometimes in such confusing forms as to render a distinction between the catarrhal and spasmodic varieties very difficult.

Treatment. - Even mild cases should be confined to bed and carefully nursed; if this is done, there will be fewer dangerous attacks. The intestinal track should be emptied by repeated doses of calomel or citrate of magnesia. Small and frequent amounts of antipyrin or bromoform with a stimulant, such as the aromatic spirits of ammonia, and dissolved in syrup of tolu, will give considerable ease. Inhalations of steam should be frequent; in artificially heated houses it is often wise to provide a continuous supply of steam in the sick-room. It is necessary at all times to maintain the patient's general strength as much as possible. When the hoarseness, cough, and harsh breathing become alarming, it may be necessary to administer emetics. On the other hand, mild cases do not require this measure; and not infrequently physicians see it adopted when unnecessary and are compelled as a result to treat a consequent gastritis. When the dyspnœa is extreme, it will be necessary to perform intubation or tracheotomy.

Prognosis. - The prognosis in the ordinary cases is uniformly good. As the attacks become more and more severe, the danger increases. But even in these skilful treatment can afford so much relief that the outlook is in almost all cases favorable.

\section{Chronic Laryngitis}

Causes. - Chronic laryngitis is rare in infants, but in somewhat older children - those of five or more years of age - it is fairly common. Especially is this true in the harsh climate of the north Atlantic coast. The disease may occur as an extension of an acute laryngitis that has had many remissions and exacerba- 
tions, or it originates in the irritation set up by adenoid fungations, new growths, and some specific diseases.

Lesions. - The lesions are somewhat of the same nature as those in the acute form, but more deeply seated. The epithelial layer is irregular and wavy in contour, for the most part thickened, but with scattered areas where it is thin and wasted. The underlying structures are thickened, inelastic, and infiltrated with small, round cells. The mucous glands are hypertrophied, and secrete a thick discharge which is found to be mixed with some pus-cells. The condition is especially favorable for the growth of the tubercle bacillus.

Symptoms. - The symptoms consist of hoarseness and some degree of aphonia, which are for passing reasons easily exaggerated. On slight exposure the patient is attacked with a cough which one finds difficulty in quieting. At night there may be attacks of dyspnœa, especially at a time of an acute exacerbation of the pathological condition. In the older children a laryngoscopical examination will show the thickened and reddened mucous membrane, and occasionally the presence of erosions of greater or less size. The general nutrition is apt to be affected so that the patient, even when his physical circumstances are fairly favorable, is weak and easily subject to acute sickness.

Treatment. - The treatment should be aimed at removing whatever causes may be present, such as papilloma and adenoid fungations, in building up the child's nutrition by properly selected foods, hygienic conditions, and tonics. In older children one may use the same direct intra-laryngeal applications, somewhat diluted, that are prescribed for adults. Thus weak solutions of silver, ethereal solutions of iodoform, or medicated vapors, may be employed; but they are of less importance than the general methods of treatment. When the little patient can be sent to a favorable and even climate, his comfort as well as health will have the best chance for improvement.

Prognosis. - The outlook is not very encouraging, excepting in the case of those who are able to select the climate in which they are to live. Even such patients are liable on exposure to a recrudescence of the disease.

Pseudo-membranous laryngitis is treated under the head of diphtheria. 


\section{SPASMOdIC LARYNGITIS}

It is a remarkable fact that very young children, who as a rule are not liable to noteworthy nervous impressions, are subject to special neuroses of the larynx. In general, we may divide these manifestations into the two forms called spasmodic laryngitis and laryngismus stridulus. They are characterized by spasms of the larynx which temporarily interfere with the act of breathing.

Causes. - The cause is unknown. Logically, there seems to be no more reason why the automatic respiratory function should be injuriously affected than other similar faculties. One can do no more than cite the fact that it is so modified, and then state certain concomitant facts. Thus we know that this neurosis occurs in a few years of early life, that some children have a congenital tendency to it, that various forms of malnutrition, among which faulty digestive functions take an important place, aid its development. This last factor doubtless has much to do in determining the presence of heredity in the equation. Outside of these indefinite grounds, it is easy to understand how the irritation of associated abnormalties in the throat should be capable of setting up a spasmodic contraction of the laryngeal muscles. It is more apt to occur in bad than in fine weather. In spasmodic laryngitis there is usually some local injury or irritation, with which the poor general condition unites, to act as the foundation of the disease. But in laryngismus stridulus there is usually no such injury, or at all events it is of an entirely subsidiary character.

Lesions. - The lesions are generally slight; in some children they are fairly noteworthy, while in others they are barely perceptible. There may be a mild inflammation of the laryngeal mucous membrane, with hyperæmia of the small vessels. The surface seems higher in color than in health, dry, and bright. Later on there is an increased supply of mucus, which contains small amounts of fibrin and pus-cells. In other cases there may be small erosions, the effects of injury, or the irritated condition brought on by disorders of neighboring parts. The direct lesions may not be very marked, but their influence becomes felt when it acts in conjunction with a poor state of the general health.

Symptoms. - The impression which the child during an attack makes upon the attendants is most disquieting. He' may have 
been fairly well, or have had no more than an ordinary cold, such as children commonly contract. In the middle of the night he begins suddenly to breathe with less ease. Gradually the difficulty increases, and he may wake in fright. In the severe cases his distress increases; he labors wildly and unrestrainedly to obtain a greater amount of air. As his difficulty increases his efforts are frantic, the air whistles hoarsely through the contracted larynx, he becomés somewhat cyanotic, and is plainly very weak. The attack in its fulness lasts only a short time and then rapidly leaves him. Before long he is again sleeping easily with nothing more than an occasional cough to testify to the past respiratory crisis. In these attacks there is often only a slight or even no disturbance of the temperature, the pulse is not markedly increased in action, and the respiration is slow. The greatest difficulty comes with each inspiratory effort, and at this time every accessory muscle of respiration is put on the utmost tension. After the attack is gone, the child may or may not be hoarse. Commonly he may have an attack on one or more succeeding nights, but this is not invariable. Also there are some cases where an acute catarrhal laryngitis or bronchitis may follow the original attack.

Treatment. - As soon as the spasm shows itself the child should be put into a warm bath; steam inhalations should be begun, and then if relief does not quickly follow, an emetic should be administered. Syrup of ipecac is useful in very young children; older patients may take small doses of apomorphine, which in suitable quantities may be administered in hypodermatic injection. To prevent further attacks antipyrin or bromoform, given in the evening, will be found of use. In some cases of marked severity it will be necessary to practise intubation. After the attack every effort must be directed to improving the patient's general condition.

Prognosis. - The outlook is good; for, although the condition seems most alarming, serious results need not be feared, especially if means for relief are promptly instituted.

\section{CEdema of the Glottis}

The mucous membrane of the glottis may, as the result of disease, and with or without inflammation, become infiltrated. 
The condition is called œdema of the glottis. In children it is rare.

Lesions. - The changes are generally situated throughout the upper part of the larynx and are most pronounced in the posterior aspect of the epiglottis and the ary-epiglottic ligaments. The tissue is sodden with a serous infiltration in all degrees up to complete closure of the larynx. When the causes are such as to induce an active inflammation, the appropriate changes are added to those mentioned above.

Symptoms. - On account of the swelling, the lumen of the glottis is decreased, with a consequent difficulty in drawing air through it. The condition comes on suddenly, and generally leaves but little time for action. If one is able to practise laryngoscopy, one can see the swollen and infiltrated condition of the parts; the examining finger will confirm the opinion.

Treatment. - The treatment consists in cutting and puncturing the mucous membrane in the hope of reducing the œdema. Its success is not likely to be brilliant on account of the patient's exhaustion. For a similar reason intubation, which is logically called for, is also disappointing. Tracheotomy, if performed without too much delay, may be of some use. As a rule, however, the child is in danger as soon as the disorder begins; any delay is then momentous. And the inability to obtain immediate medical aid, or the physician's possible hesitancy to use intubation or tracheotomy before the child is moribund, is often the cause of death.

The prognosis is bad. 


\section{CHAPTER XV}

\section{DISEASES OF THE BRONCHI, LUNGS, AND PLEURA}

\section{Acute Bronchitis}

THE inflammation of the mucous membrane of the bronchi and their ramifications, called bronchitis, is one of the commonest pathological conditions from which children suffer. There is hardly a child to be found who has not at some time felt the strain of this disorder. The physical peculiarities of a child's lungs invite congestion and inflammation, the conditions which aid in developing the disease are almost omnipresent, and as a result the child must at some time or other fall more or less sick with this disease.

Causes. - In settling upon the causes of bronchitis, one must keep in mind that the nose, throat, larynx, trachea, and lungs are closely related. An inflammation of any of these parts may extend in the easiest manner to the bronchi; and the degree of severity of the ultimate disorder need bear no direct proportion to that of the original trouble. A comparatively trifling rhinitis may extend into a severe or fatal bronchitis, most of all in children who are weak or exposed to unfortunate circumstances. Such circumstances may be atmospheric changes, rough weather, irritating gases. Indeed, all of these irritants may act independently and start an inflammation of the bronchi without any precedent change. Exposure of almost any sort can act as an exciting cause, even the "drooling" of young babies being at times sufficient. Systemic poisoning from acute infectious diseases, such as measles, scarlatina, pertussis, and typhoid fever, can act with much certainty. In addition one must take into account those cases which occur with intestinal diseases of an infective, putrefactive, and fermentative character. Sufficient attention has not been given to these diseases as near or remote causes of bronchitis. Not only do we commonly have an inflammation of the bronchial mucous membrane in such disorders, but also we often find Bac- 
terium coli commune and other pyogenic micro-organisms in the affected area and the exudate; in addition observers have many times associated the bacillus of Lumnitze with the so-called putrid form of bronchitis. Finally, one should emphasize the importance of all weakened general conditions as predisposing causes, such as in rachitis, in syphilis, in the wasting diseases of adenitis, and in the circulatory interferences of cardiac disease. Such things, even if they do not act as direct causes, nevertheless make so favorable a physical environment for the development of bronchitis that even a trivial circumstance will start the disease into vigorous action.

Lesions. - The lesions in this disease vary considerably according to the seriousness of the attack. They may on the one hand be trivial, but on the other may be so severe as to produce death. Also the disease varies considerably according to the age of the patient, for infants suffer much more acutely than other children. In the former the large proportion of capillary tubes to the rest of the lung-structure makes an extension of the inflammation to them comparatively easy. As a result, in this class of patients one often finds very severe forms of the disease closely approximating to or coinciding with broncho-pneumonia. However, in the majority of cases, the disease has its own individuality and appears distinct.

Usually parts of both lungs are involved; less often the inflammation limits itself to one lung, and in other cases whose seriousness is in direct proportion to their youth it may spread over the whole of both lungs. In the ordinary cases the mucous membrane of the middle and large-sized bronchi and even of the trachea is affected. This membrane becomes swollen and congested, the capillaries are engorged, the mucous glands cease to secrete. Then the columnar epithelium begins to desquamate, and when once destroyed it is not renewed during the process of the disease. On the other hand, the basement membrane is not to any noteworthy extent disturbed. Within a short time the congestion subsides somewhat and the muciparous glands begin to secrete vigorously: The secretion becomes mixed with exfoliated epithelium and small round cells, with many white cells and a smaller number of red cells. The deeper epithelial layers increase and remain attached to the basement membrane.

One should keep in mind that the inflammatory process is not 
in all cases sharply defined. On the contrary it may spread in any direction. Frequently enough it extends to the pleura so that all grades of this disease may result. Or instead of spreading widely, it may show an unusual virulence in small disseminated areas. In such cases one sees a bronchitis with associated small areas of pneumonitis, the whole condition being to all intents and purposes a form of broncho-pneumonia. This is especially apt to occur in infants. Likewise they show much greater enlargement of the bronchial and lymphatic glands than do older patients, and are more deeply affected by absorption of small amounts of the pus which may be formed here and there in the affected area.

In the cases that recover, no permanent changes in organic structure remain, although a variable time may elapse before the previous normal condition is thoroughly reproduced. But after death from the disease one sees on section a congestion of the vessels and mucous membrane, some pus-cells and broken-down epithelium, new epithelial growth, and a muco-purulent discharge in the bronchi.

On account of the disability of certain parts of the lungs and the strong respiratory efforts of coughing, more or less emphysema is apt to supervene. Indeed, I believe that this complication, to which young children are prone, happens more frequently than is usually accredited. Or the disabled parts are liable to fall into a state of atelectasis or partial collapse, the latter occurring generally in small areas. Other complications, such as bronchopneumonia and the various forms of pleurisy, are fairly frequent.

Symptoms. - The symptoms of bronchitis, while on the whole following a general course, change considerably according to the age of the patient and the intensity of the pathological invasion. In the majority of cases one often finds the attack beginning with coryza and slight conjunctivitis, a variable appetite, moderate or slight fever, and general malaise. The cough is at first hard and dry, the chest is evidently sore, and the child is plainly seen to suffer additional pain with each attack of cough. The sleep is disturbed and uneasy, and on waking he seems not to be rested. After two or three days the cough is looser and clearly less painful, and the patient-breathes much more easily. Since young children swallow the sputum, at no time need we look for expectoration. 
Inspection shows the breathing to be labored and hurried. Fremitus may be somewhat increased. One hears scattered râles all over the chest, but in the later stages these signs are most plainly heard in the posterior part and lower half. In infants one does not look for the classical sibilant and sonorous character as regularly as in adults or even in the older children; indeed one

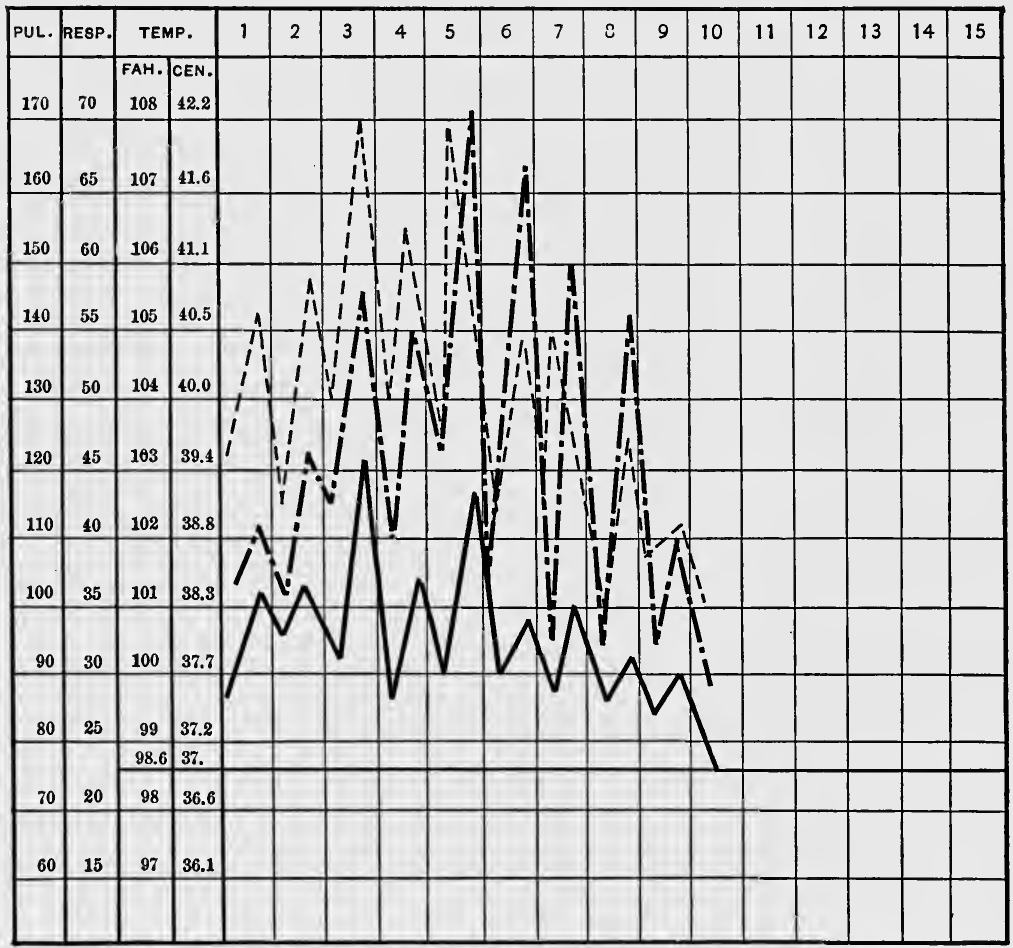

PULSE, RESPIRATION AND TEMPERATURE CHART OF ACUTE BRONCHITIS. AGE, 7 MONTHS.

PULBE. .REBPIRATION

TEMPERATURE

Fig. 37.

is apt commonly to find a wide variety of sounds, mostly mucous These are smaller in the congestive stage, becoming larger, looser, and less compact as resolution sets in.

Respiration is hurried and outstrips the pulse-rate. Especially in very young infants it may attain a startling rapidity, while at the same time the general condition may be fairly good. The ordinary cases, as a rule, do not suffer much from interfer- 
ence with the circulation. The temperature varies between normal and $38.5^{\circ}$ C. $\left(101.5^{\circ} \mathrm{F}.\right)$. When these averages are largely exceeded there is generally more or less severe systemic poisoning or a complication with a concurrent disease. There is apt to be some cyanosis, especially in severe attacks, which is in proportion to the dyspnœa.

Occasionally one sees cases that begin with great general disturbance, very rapid respiration- and pulse-rates, and high temperature. These attacks happen for the most part at night, and by the next day the child is in an almost normal condition. This state of things I have seen almost exclusively in infants. It is in all likelihood due to spasm of the muscular coat of the bronchioles, with or without the presence of toxæmia. All in all, one should regularly expect to see the severest effects of the disease in infants, rather than older children who are much more liable to dyspnœa, cyanosis, and suffocative invasions than adult patients. Their anatomical peculiarities make a rapid extension of the inflammation to the capillary bronchioles comparatively easy ; and with this go higher temperature, pulse, and respiration; the prostration is more pronounced, the anorexia or inability to nurse is plainly noticeable, and resolution is more delayed. The more fragile and delicate the child, the greater is the exaggeration of these symptoms.

Very often one sees disorders of the alimentary track brought on, in part, by the swallowed sputum with its irritating chemical characteristics, its difficultly digestible elements, and its bacteria. As a result one sees frequent attacks of vomiting, especially after coughing. The existence of a bronchitis in the presence of a tendency to gastritis, or vice versa, is highly favorable to the development of both diseases.

The nervous symptoms in some infants are marked, but rarely do they indicate a graver outcome of the disease.

Treatment. - In the treatment of acute bronchitis nothing is of more importance than the general care and nursing. Children should be kept in bed and well protected, although not too warmly covered. The intestinal track should be emptied, preferably by fractional doses of calomel, followed by an ordinary cathartic ; and the food should be as digestible as possible. Fever calls for thorough sponge baths or cool packs, and in addition the use of warm baths morning and night is to be praised. The extremities 
should be kept comfortably warm, and on the body there should be no undue burden of clothes. The mouth, throat, and nose should be thoroughly sprayed and cleansed with an alkaline or boric acid solution. The child should not be allowed, in order to prevent hypostatic congestion, to remain too long in one position.

Counter-irritation, produced by rubbing the chest, front and back, with camphorated oil, should not be neglected. Oilsilk jackets, plasters, and poultices I believe, after systematic trials, are unnecesssry or even harmful. We can easily do without the increased liability to colds and congestion, as well as the clogging up of the skin, which they are apt to produce. On the contrary, the use of serviceable wool underwear is safer, more rational, and more desirable.

Medicines do not necessarily, most of all in the light cases, play the most important part in the treatment. Inhalations of steam or lime-water vapor are often a means of quieting the cough. The use of the strong disinfectants in vapor is of doubtful additional benefit. With the older children creosote may, after the onset of the disease, prove valuable. But if the milder inhalations are at all used, they should be liberally administered. In the first part of the attack, where the cough is hard, wearing, and continuous, one can give much relief by small doses of ammonium chloride with fairly generous amounts of bromide of soda. When the disease has a clearly marked spasmodic character, the liberal use of antipyrin with a cardiac stimulant will give prompt and satisfactory results. Not often is it necessary to resort to opium in any form for very young patients, but older children do obtain relief from small doses of the deodorized tincture. In very nervous cases, where neurotic symptoms are plainly emphasized, small doses of codein will give much relief. Throughout the whole sickness there may be attacks of prostration or collapse that require the administration of stimulants such as whiskey or nux vomica. Later on in the attack, especially from the beginning of resolution and convalescence, small doses of terebene or creosote in some of the malt preparations may be used. The function of expectorants is commonly overrated, especially in young children. Their place is in the treatment of older children whose expectoration is thick and copious.

It is important to remember that this disease is apt to attack 
children who are in a debilitated condition. Also, children who are fairly strong at the beginning are apt before the sickness has disappeared to be much weakened. Therefore, a proper attention to tonic remedies is imperative; and especially during and after convalescence such methods as will do most to build up the drooping vitality are sure to give satisfaction. For this purpose small doses of strychnine or nux vomica in combination with the organic preparations of iron may be safely recommended. Other tonic preparations that may be profitably used, so long as the child's stomach is in fairly good working order, are cod liver oil in a good emulsion, and the compound syrup of hypophosphites.

Prognosis. - As there is so wide a difference between the very mild and the very severe forms of bronchitis, the prognosis varies accordingly. The younger the child, the severer is the disease apt to be. In infants it is a really serious disorder; but as the children approach the age of puberty its likelihood of producing serious results decreases progressively. With proper attention the outlook is not as a rule grave.

Differential Diagnosis. - In most cases there is very little doubt concerning the nature of the disorder; but occasionally, and especially in the severe cases, it may be possible to confuse this disease with a pneumonia. In infants there is always the danger of an extension of the inflammation to the very fine tubes, and the resulting formation of a broncho-pneumonia. In either of these events the presence of consolidation and its characteristic objective symptoms will point the way to the right opinion. Also, it is possible that pleurisy with effusion might cause some doubt. This is most apt to happen if the amount of fluid is small and the chest walls thick. But careful inspection, percussion, and auscultation should clear up any obscurity, at all events after the child has been seen more than once. It is likewise possible that enlarged mediastinal glands may by pressure cause a cough that simulates some of the appearances of bronchitis. But here the acuteness of the attack is absent, and there is in addition the diathetic disorder which precedes such enlargement. When the physician makes his first examination of a moderate case of bronchitis, he must always keep in mind the possibility that the disease is preceding or accompanying one of the acute fevers, such as measles, epidemic influenza, - or even whooping-cough. 


\section{Chronic Bronchitis}

The causes of this disease are such as would logically produce it. First of all, as a predisposing cause, one should consider a general debility, either congenital or produced by adventitious causes. The rachitic, the syphilitic, the tubercular, the poorly nourished child, the sufferer from the impeded circulation of cardiac disease, are much more apt to suffer from a tedious and destructive bronchitis of long standing than a healthy and well cared for patient. Or a child who has been straining and choking with a protracted whooping-cough may make favorable conditions for the development of this disorder. Deformities of the chest which give rise to abnormal positions of the viscera, with the accompanying tendency to faulty development and congestion, may act as a fertile cause. When a weak and badly nourished child has suffered from an acute bronchitis or broncho-pneumonia, or from any cause producing a long standing congestion and an obstinate cough, the chances of his being afflicted with a chronic bronchitis are very good. Extensions or repeated attacks of the acute form are naturally the most fertile causes.

Lesions. - The lesions differ but little from those in acute bronchitis. The bronchi and bronchioles become thickened and hard, the mucous membrane instead of regaining its normal condition is heavy and inelastic, lacking in tone. In such an unpliable state the liability to form cylindrical and sacculated dilatations or areas of bronchiectasis is, as one can imagine, comparatively great. The air-spaces and bronchioles are filled with a thick and viscid exudate, which contains a greater amount of fibrinous material than does the thin secretion of the acute form.

Symptoms. - The symptoms consist of the cough, of the physical deterioration, and the subsequent physical alterations which result from these factors. The cough is not apt to be so hard as in acute bronchitis. There is a variable amount of sputum, but more is expectorated in the morning on arising than at any other time. The child complains of general disability, of loss of flesh and strength. The chest may, on inspection, seem unnaturally or irregularly puffed out. Commonly there is no rise of temperature, although the respiration and pulse may be slightly faster than is normal. The other physical signs are much the same as in the acute form. One should keep in mind that associated with 
this disease one finds here and there emphysematous spots or a bronchiectasis; the peculiar hyper-resonance of the one and the muco-purulent or purulent discharge of the other will then naturally show itself.

The course of the disease is slow, with periods of remission and exacerbation. These changes follow quite closely the fluctuations in the child's general condition. This is often so poor and delicate that the patient succumbs to comparatively slight attacking causes. The right heart and the large veins are dilated. Bronchiectasis and emphysema in varying amounts are apt to result from a chronic bronchitis.

Treatment. - The treatment is mainly a matter of general care, climate, and tonics. The child should be clothed in wool, should have his diet carefully arranged, should take as much of the easily digested fats as possible, should be carefully controlled in the matters of bathing, sleep, and exercise. It is of the greatest value to take him to a warm and clear climate where the changes are not extreme. Such treatment will commonly do more than drugs can ever hope to accomplish. In the way of tonics, it is wise to adhere to the compound syrup of hypophosphites, to cod liver oil, or at times to such other particular remedies as each case may call for. Considerable temporary relief for the cough may be had from inhalations of the steam of water, lime-water, and, in older children, creosote. Codein may give relief to the nervous cough or general nervous symptoms that are commonly seen in this disease.

Any primary disease or antecedent condition which persists will call for its appropriate treatment before one may look for much betterment of the chronic bronchitis.

\section{Acute Broncho-Pneumonia}

Broncho-pneumonia, formerly called catarrhal, lobular, or capillary bronchitis, is a disorder combining many of the features of bronchitis and lobar pneumonia. Its frequency of occurrence in children is in inverse ratio with their age; and the serious danger of it follows a similar rule. In certain aspects it is hard to decide whether this disorder should be classified as a distinct and separate disease, as a combination of two or more diseases, or an extension of a primary disease. There is much to be said in 
favor of the view that it may be a "mixed infection," a massing of symptoms that vary, as one or another of the elementary lesions predominates. Any other view makes it hard to understand its manifold appearances, its remarkable changes, its lack of unity in typical form. One can make out an indefinite number of individual forms in which it may appear; and only by the exercise of a fair amount of scientific imagination may these be grouped in large classes. At all events we know that broncho-pneumonia attacks very young children, that weak, debilitated, and rachitic children are most easily affected and killed by it, and that it has

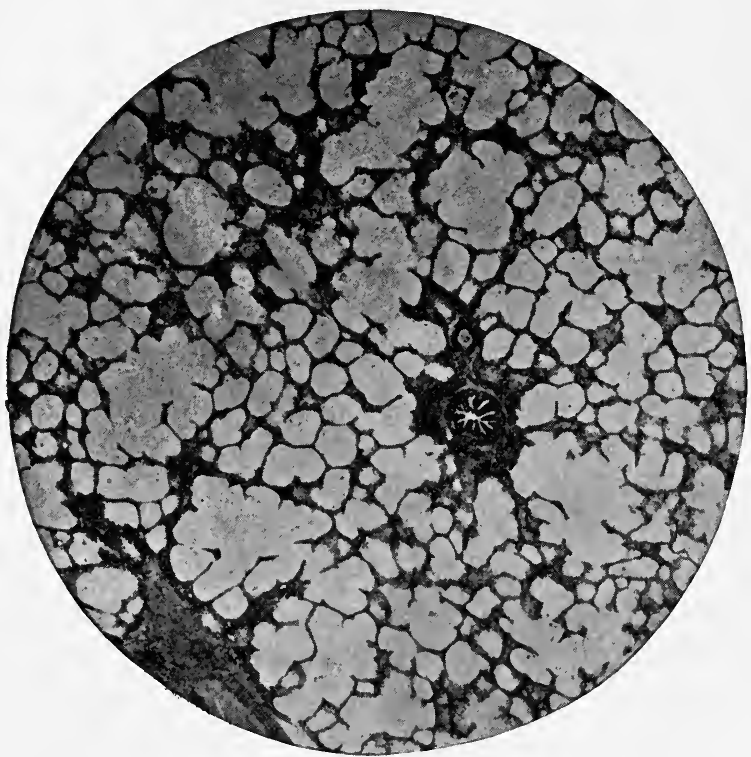

FIG. 38. - Normal Lung. $\times 25$.

no absolutely typical course, duration, or method of ending. The whole appearance of the disease impresses one as that of a sickness which is much influenced by the physical condition and environment of the patient. Herein it differs from lobar pneumonia, which seems to attack with equal virulence both the strong and the weak.

Where the child has been suffering from a bronchitis or any of the acute infectious fevers, broncho-pneumonia may frequently supervene as a complication. The specific toxines of these fevers seem to have an important influence in predisposing toward the 
disease, most of all when they are very severe or where the patient is unusually weak. In most cases the direct and acting cause of the sickness is some forms of pathogenic micro-organism such as streptococci, pneumococci, or staphylococci; in pure or mixed cultures. Subsidiary factors are the inhalation of irritating substances, hypostatic congestion, and unhygienic surroundings; and harsh weather and sudden changes of atmospheric conditions may probably have some influence as auxiliary circumstances.

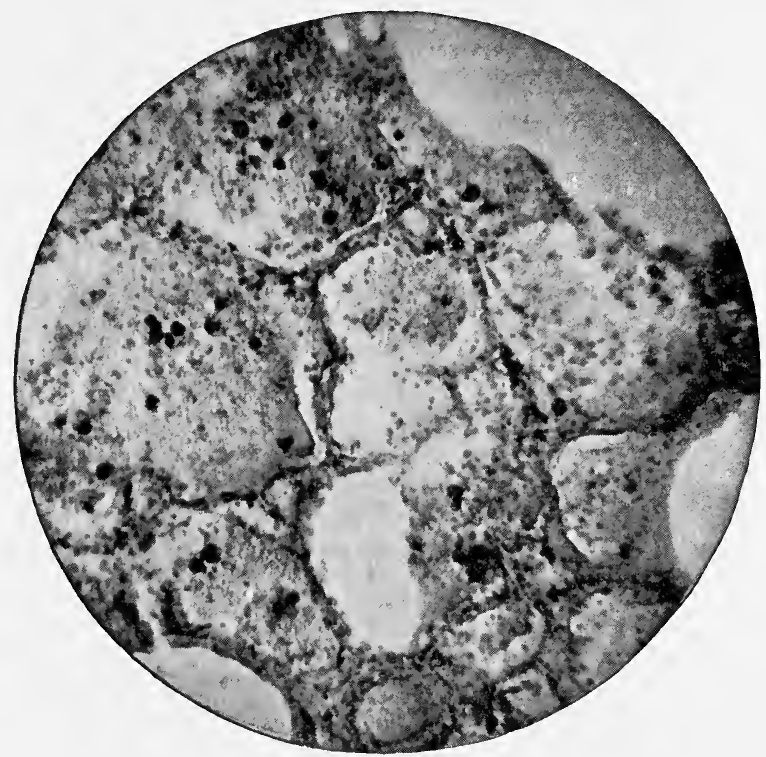

Fig. 39. - Broncho-Pneumonia. $\times 100$.

Lesions. - The main lesion is an inflammation of the walls of the bronchioles and air-cells. The inflammation of the mucous membrane does not play so important a rôle as in lobar pneumonia. The walls of the bronchioles and air-spaces are thickened and infiltrated. The spaces themselves are filled with the products of inflammation, with epithelial cells and shreds of epithelium, blood-cells, pus, and fibrin. In some cases the capillaries become so congested as to encroach upon and fill up the area of the air-spaces. In other cases the affected parts become thoroughly infiltrated with sclerotic tissue, with the natural result that their characteristic activity is lost. This process may take 
place in the walls of both air-spaces and bronchi, so that complete recovery is hard to obtain. This interstitial material may be so extensive that considerable parts of lung are thus rendered useless and become the starting-ground of a chronic process of tuberculosis. An additional aid to this result is atelectasis, which occurs in patches and mostly in the thinnest and most remote portions of lung tissue. This is brought about by the occlusion of the small bronchi, mostly by mucus or by a lack of the normal respiratory force. As a natural sequence, other parts, having to do more than their share of work, become, on account of the strain of coughing and over-exertion in breathing, emphysematous. These emphysematous spots are for the most part found in or near the apices, in compensation for the clogging of the lower portions of the lung which in this disease are more often affected. For analogous reasons the tubes dilate irregularly and mostly in a cylindrical shape.

As a general rule the larger bronchioles are not involved and the inflammation is irregularly disseminated. However, all cases do not adhere to this strict form, but have in addition inflammatory changes in allied and adjacent structures, such as the mucous membrane, pleura, and the surrounding lung tissue. This extension may proceed even as far as the trachea, although it progressively becomes fainter the farther it spreads from the centre of the disease. Where it is most intense there may be small collections of pus, of broken-down epithelium and cells, each of which areas has its small surrounding space of pneumonitis.

Excepting in cases where the characteristic changes are not marked, there are peribronchitic areas of true pneumonitis which spread with the extension of the original disease. Such cases may start with small and widely separated localities of inflammation, but the lapse of a few hours or days changes materially the condition of affairs. Occasionally these areas approximate so closely that they eventually give the appearance of a large original tract of pneumonia. There are some special types which should be mentioned :-

(1) In some very young infants these extensive changes do not occur; the affected portions are for the most part collapsed and œdematous, having neighboring areas of emphysema.

(2) Occasionally one meets with a broncho-pneumonia which is exceedingly rapid in progress. The occlusion of bronchioles 
and air-spaces is accomplished in a few hours, and the breaking down of both normal and abnormal tissue is marked.

(3) There is another irregular form, characterized by strong interference with the circulation, which is caused by an exceedingly rapid diffusion of the products of inflammation. Consequently the breathing is labored and the cyanosis is extreme.

On account of the interference with the circulation and the compressed state of the lung tissue, the right heart is enlarged without a commensurate thickening of its walls. Also the large veins are dilated.

In gross section the characteristic appearance consists of the involvement of scattered lobules. These project above the plane of the cut surface and are harder than the surrounding surface. There is a distinct contrast between them and the shrunken areas of atelectasis, especially as the latter are dark and cyanotic in color. The lower and posterior parts of the lungs show the greatest amount of congestion. The emphysematous spots are softer and more crepitant to the touch, especially as the most characteristic parts are most airless. Here and there in the peribronchitic zones of pneumonitis may be seen minute collections of pus. There is, besides, an exudate of clear or semipurulent mucus.

The pleura may have a coating of fibrin, or may be the seat of a marked inflammation. In the more protracted cases it may even be bound down by fairly strong adhesions. A purulent pleurisy is a common sequel, and is liable to run a very severe course on account of the exhaustion produced by the original disease. The larger bronchi and trachea may be congested and covered with fibrin. The involvement of these structures, and their lack of tone that is brought on by the strain and stress of coughing, may start a bronchiectasis of varying size and form.

Symptoms. - The most audible physical signs may be noticed at the back and sides, particularly in the lower parts, and usually in both lungs. Inspection shows the breathing to be labored, and instead of the abdominal it assumes the thoracic form. The pause in respiration comes before instead of after expiration. As one would expect, the expansion of the lungs is imperfect and irregular, the lower part of the chest and the intercostal spaces recede on inspiration, and the nostrils work convulsively with each respiratory effort. Dyspnœa is usually present, accom- 
panied at times by varying degrees of cyanosis. It is impossible in some very young infants to get anything more definite than this ; but in older patients vocal fremitus may be increased over the affected area excepting those parts where atalectasis exists.

The percussion note varies with the affected areas, so that the predominance of the pneumonic or bronchitic element in turn

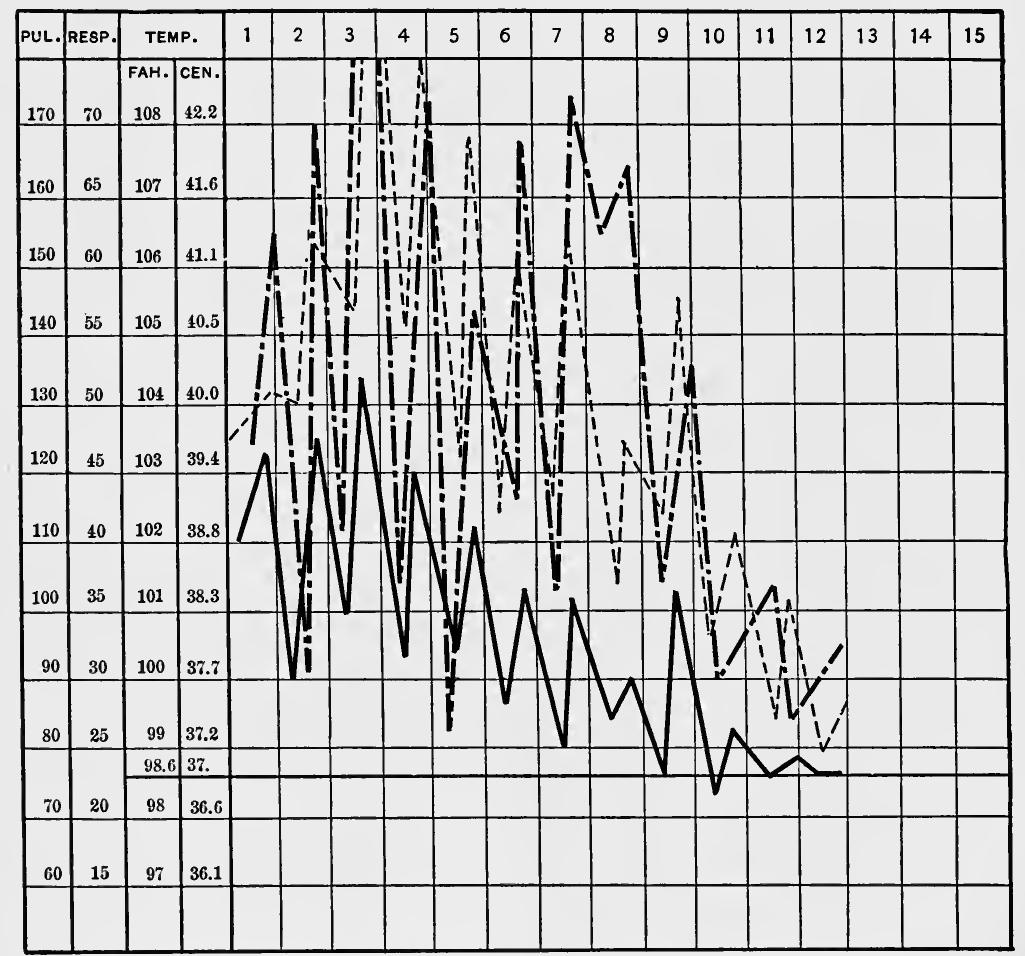

PULSE, RESPIRATION AND TEMPERATURE CHART OF BRONCHO-PNEUMONIA. AGE, 13 MONTHS.

PULBE_-- - RESPIRATION

TEMPERATURE

Fig. 40.

becomes apparent. Often one may notice a distinct dulness, especially where the affected area is sufficiently large. Again, this sign may be absolutely lacking, possibly on account of a central location of the inflammation or some peculiar position. Moist râles, both fine and coarse, may be heard scattered over the chest, while small dry crepitant râles sometimes occur at rarer intervals. In the older infants and children one may expect to 
hear a fairly distinct bronchial breathing, which in the younger may change and fade away to a sound that is merely harsh. Resonance is likewise variable; and where there is emphysema one may also find hyper-resonance.

The symptoms are not quite so characteristic as those of lobar pneumonia. Where the broncho-pneumonia complicates an infectious fever there may be at first no more than an exaggeration of the evidences of fever, dyspnœa, and malaise. Or the attack may be ushered in with a chill, with vomiting, or diarrhœa, cough, and pain. The fever rises irregularly and intermittently, usually having a maximum of $40^{\circ} \mathrm{C}$. to $41.1^{\circ} \mathrm{C}$. $\left(104^{\circ}\right.$ to $106^{\circ} \mathrm{F}$.), generally at night. In rare cases of profound toxic absorption the temperature may even be somewhat higher. The respiration and pulse are likewise irregular, but follow the general course of the thermometer, the former ranging from 50 to 80, the latter from 100 to 200. These figures are, of course, approximate. There is a class of unfavorable cases in which the fever line remains low or even drops to subnormal. Here there is, as a rule, marked weakness, the pulse and respiration vary considerable from time to time, and the whole picture is one of flagging vitality.

The cough at first is short and hacking, and, especially in children of a nervous disposition, may become continuous and very severe. In infants, and sometimes in older children, there is no expectoration because the sputum is swallowed. Where the child is being exhausted, the breathing becomes progressively more hurried and shallow, cyanosis is apt to be marked, and the little patient may become stupid or fall into convulsions. Occasionally in serious cases one meets with Cheyne-Stokes respiration. Such children are likewise apt, in case they recover, to have a delayed and tedious convalescence. On the other hand, if the disease assumes a milder type, the patient begins from the sixth to the ninth day to show a definite improvement. The breathing becomes easier, the color more natural, the cough looser, and the appetite somewhat more vigorous.

There are a few cases where one sees no symptoms except a small increase in the temperature and respiration, slightly harsh breathing, and some general malaise. The cough may be insignificant or absent. One becomes aware of such an attack after it has served as the basis of a chronic broncho-pneumonia or a 
tubercular process, of a dilated heart or a pleuritis. When death occurs, it is in infants mostly due to toxæmia or interference with respiration; in older children to heart failure.

The heart, stomach, spleen, and kidneys - in fact all the abdominal and thoracic viscera - may become the seat of an acute exudative inflammation which may be recognized by the characteristic symptoms.

The outlook is always a serious one, especially as the disease is apt to be a long and wearing ordeal. When it complicates the acute infectious fevers the prognosis is truly discouraging. In the cases that recover, convalescence as a rule comes gradually; in some rare cases the disease is limited by a distinct crisis.

Treatment. - Complications involving the heart, pleura, or meninges may result, and need scrupulously faithful attention. The treatment of broncho-pneumonia demands judgment, sympathy, and care. An exact and rigid over-sight of the details of nursing is of the highest importance. This is all the more to be observed because there is no specific treatment with drugs. At the beginning the bowels should be emptied by repeated small doses of calomel followed by a saline cathartic. Where the disease occurs as a complication or a secondary disorder, the treatment is in the main that of the primary sickness. It may be necessary to use a drug to sooth an irritable cough; and for this purpose one is safe in using the bromide of soda, which very young infants tolerate even in comparatively large doses. In older children one may use this same remedy, or, where it is insufficient, very small doses of opium. The temperature should be controlled, not by the synthetic antipyretics, but rather by cool sponging, cool and tepid packs, and the graduated bath. In cases of marked weakness, cold extremities, or cyanosis, one should employ warm packs and baths and eschew the use of cold.

It is of the very highest importance to keep up the patient's strength. One must be prepared for a long siege of sickness, and therefore must be ready to face the problem of constantly recurring attacks of weakness or a persistent condition of asthenia. The gradual unfolding of all of one's resources in the way of stimulants and tonics permits one to use much fine judgment and clear thought. On the whole, and excepting cases of sudden collapse, one can do without much whiskey and brandy. And when there are gastro-intestinal disturbances it may be advisable 
to give them by rectum rather than by mouth. One can, in many cases, get along better with strychnine or nux vomica, which should be reënforced by sufficient doses of digitalis. Occasionally, where the bronchitis is the predominant feature, there may be a need of steam inhalation; or the patient may require small doses of potassium iodide to liquify the thick mucus which he strains to expectorate. The use of oxygen in inhalations has been praised in the past; but at the present time the tendency of the opinion is much less favorable. Many observers believe that it gives no help at all.

Since broncho-pneumonia is so often the concomitant or the sequel of debility, one naturally expects a hypostatic congestion occurring in the course of the sickness. Conscientious care will minimize this lesion, which otherwise may act as the determining factor against the immediate or eventual recovery. It is wise to vary the position of the patient from time to time, at the same time consulting his ease.

Food should be given in small amounts and frequently. It is hardly necessary to say that it should be fluid and as easily digestible as possible. More harm than good can be accomplished by overloading the stomach.

The ideal treatment for this disease would be by means of an antitoxic serum. The experiments have up to this time been unsuccessful, partly on account of inability to ascertain before death what germ is at the basis of the sickness, and partly because the disease almost always represents a mixed infection.

Prognosis. - Broncho-pneumonia is always a dangerous disease, especially in infants, and when it complicates the acute infectious fevers. One of its worse features is the likelihood of delayed resolution, with the attendant physical debility and the opportunities for the growth of chronic processes. In ordinary private practice the mortality ranges from fifteen per cent to thirty per cent; but in hospitals and institutions for children it is twice as large. In giving an opinion about the probable outcome, one should be guided by the patient's age, his vitality, the presence of other diseases, and the virulence of the broncho-pneumonia as shown by the symptoms. The complicating symptoms of disorders in other organs must naturally have a place as factors in the equation.

Differential Diagnosis. - The main features of this disease are 
the gradual onset, the rapid and irregular respiration and pulse; the prostration, high temperature, - which frequently assumes a remittent type, - the physical signs of scattered areas of consolidation, and some degree of dyspnœa. One must be prepared for the development of a broncho-pneumonia from a severe bronchitis or a bronchitis that has been neglected. And in the acute eruptive fevers an exacerbation of the symptoms is commonly due to this cause. When the items mentioned above exist, the disease should be suspected even if the physical signs in the lungs are not clear. It is possible, but not probable, that some doubt may exist between a diagnosis of broncho-pneumonia and that of pleurisy with effusion. But if one keeps in mind the objective symptoms which the presence of fluid creates, the difficulty should promptly fade away.

The disease which is most readily confounded with bronchopneumonia is lobar pneumonia. The diagnostic points between them are given in the account of the latter disorder.

\section{Chronic Broncho-Pneumonia}

Causes. - In weak and debilitated children recovery from an acute broncho-pneumonia may be indefinitely delayed; or successive attacks of the acute form may follow each other closely enough to make each attack harder to throw off than the preceding one. In either of these cases a chronic broncho-pneumonia may result.

Lesions. - Outside of the regular inflammation with which we are acquainted in the acute form, the fact which will impress us as most noteworthy. in the microscopical examination of this disease is the large amount of connective tissue that is laid down around the bronchioles. In cases that are not too far advanced this shows on section as small, light fibrous nodules. Where the disease is farther advanced, these peribronchitic areas spread out and merge, so that finally one sees a section of a lobe or even an entire lobe reduced to solid connective tissue. The exaggeration of this process has sometimes been called by the faulty name of fibroid phthisis. In the chronic form of broncho-pneumonia not only does a portion of a lobe succumb to connective tissue changes, but also the alveolar and bronchial walls are likewise thickened, the pleura is bound down to the lung by adhesions, the alveolar 
spaces may become filled with exudate, and thus obliterated. Following these changes, the smaller and medium-sized bronchi may be distorted, dilated, and sacculated. But these parts are not the only ones that suffer; even the pulmonary pleura may likewise be involved, and become thicker and less elastic than in health, as well as bound down by adhesions. Such a condition favors the development of tuberculosis ; and many of these cases finally become tubercular.

Symptoms. - The symptoms are very disturbing. Even without the strain due to the cough, the child becomes very much exhausted and emaciated. He suffers from periods of fever, at which times the pulse and respiration are somewhat heightened. He now and then is markedly prostrated and cannot avoid being attacked by intercurrent illnesses. His appetite and sleep are poor, the loss of flesh and strength is progressive. Rarely the formation of a bronchiectasis shows itself in the profuse mucopurulent or purulent expectoration, which is commonly most profuse in the morning. The whole picture is that of a wasting disease, and with it may be associated a simple or tubercular inflammation of the bronchial and lymphatic glands. Also one may find areas of emphysema. The course of the disease is a wearisome succession of exacerbations and remissions in the pulmonary complaint, the causes of which are at times hard to locate.

Inspection may show the chest to be retracted on the injured side. Where the sclerotic tissue exists in large amounts one finds dulness on percussion, poor respiratory murmur, and increased vocal fremitus. One is immediately impressed by the idea that the characteristic structure of the lung has been changed and abolished.

The child is very apt to become tubercular in other parts of the body than the lungs.

Treatment. - The treatment consists for the most part in attention to the general care and the climate. The food, clothing, rest, bathing, and exercise must be most scrupulously regulated, and every attempt should be made to keep the patient's vitality as high as possible. He should be removed to a warm and dry climate where he will not be oppressed by great and harmful barometric and thermometric changes. The best tonics at our command should be given. The selection of these must be made to suit individual cases. 
Prognosis. - Some, but not many, of these cases recover. In general the outlook depends upon the patient's vitality, his environment, and the ability of his parents to provide all the various helps that intelligence and money can give. Even where death does not result, a complete return to health and strength is scarcely to be expected.

Differential Diagnosis. - The diagnosis lies between chronic broncho-pneumonia and a tubercular process. The question is decided against the latter by inability to find the characteristic signs of tuberculosis or the specific bacillus.

\section{Interstitial Pneumonia}

This is a disease that is not often seen in children. And when it does so occur it is always a noteworthy process, because the development of infantile tissues lies away from the great increase in the connective tissue elements. All their efforts in growth are directed to an increase and development of parenchyma; but a growth of sclerotic tissue is essentially senile in its nature.

Causes. - Given a weakened nutrition, or exhaustion brought on by diathetic disease, with repeated attacks of pleurisy, pneumonia, or bronchitis, and the possibility of an interstitial inflammation is always present. Not only do adhesions between the lung and pleura bind and distort the tissues, but also an analogous production of connective tissue may gradually follow. In a few other cases a scar of a healing cavity may act as the starting-point of an interstitial process.

Lesions. - The disease may start in any part of the lung, may extend in any direction, may involve any amount of tissue. The appearance on post mortem examination is very striking : adhesions bind the thickened pleura to the lung and the chest wall, squeezing and twisting the lung out of its normal shape and position. The tough adhesions may pierce the surface of the lung and make their way through the pulmonary substance. All over the affected area the laying down of connective tissue spreads slowly and gradually until every element is involved. The bronchioles, as the surrounding tissue contracts and recedes, become irregularly dilated and enlarged. The lung loses its characteristic structure and remains homogeneous and undifferentiated. 
Curiously enough, one lung only is involved. Then, the other lung being forced to do more than its share of the work, becomes emphysematous. Where only a part of a lung is attacked, the neighboring areas must in the same way become emphysematous until they in turn become absorbed.

In some few cases of marked adhesions the pericardium is involved along with the pleura and lung surface. In these especially does the general rule of glandular enlargement hold good. The whole condition is one which invites invasion by the tubercle bacillus.

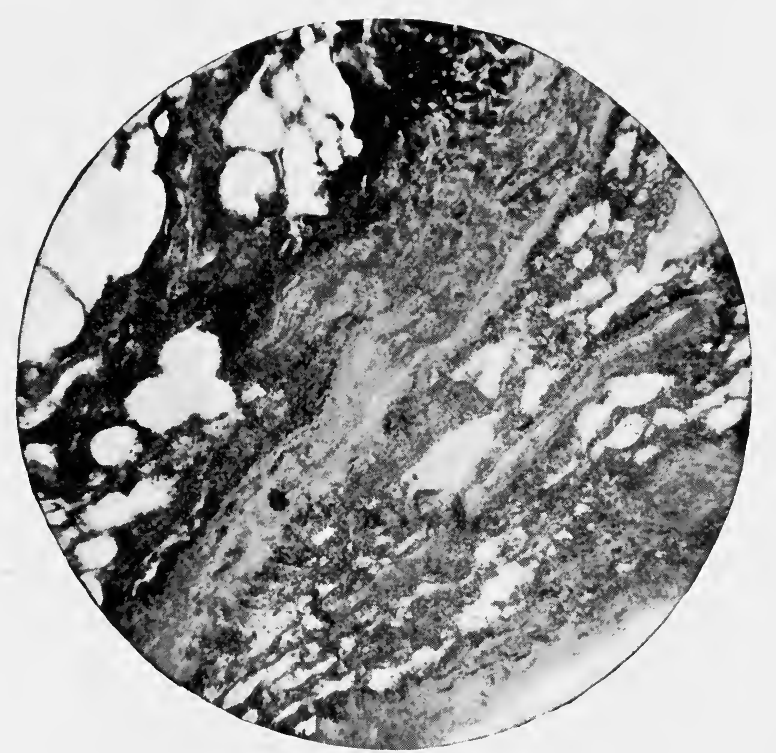

FIg. 41. - Chronic Interstitial Pneumonia. $\times 30$.

Symptoms. - The symptoms of this disease are often difficult to recognize in a clear fashion. One is constantly liable to be misled by a history of an ordinary sub-acute or chronic cough which started in some of the common pulmonary disorders. There is finally a more or less lengthy period of delicate health, lack of resistance to the attack of coughs and colds, flabby physical development. There may be no elevations of temperature, and interference with the regular ratès of respiration and pulse will depend upon the extent of the disease and the amount of distortion. In each case the observer will have to work out such factors himself. 
Over areas of interstitial tissue percussion gives a dull note, over emphysematous parts hyper-resonance, and over a bronchiectasis a cavernous note. There is very apt to be more or less bronchitis or a low grade of broncho-pneumonia which must give their characteristic symptoms. In advanced cases deformities of the chest wall and position of the heart may be striking. As the lung tissue becomes obliterated, the respiratory murmur becomes fainter and fainter until it finally may cease. At any time these symptoms may be increased by those of acute or subacute tuberculosis.

Treatment. - The only treatment consists in conserving and developing the patient's vitality by the strictest attention to diet, hygiene, tonies, and climatic changes. There is no drug that by itself is able to stop the disease; and often one can do little more than treat the symptoms. If the patient's environment is good, his chances for life may be bounded by a few years. If he is attacked by an intercurrent disease, the difficulties of successfully caring for him are very much increased.

Prognosis. - While life may be prolonged for months or years, there is little hope for permanent cure and a return to health and strength; moreover it is impossible to say how long these cases will live, for the most surprising changes are apt to occur.

Differential Diagnosis. - The diagnosis lies between the interstitial pneumonitis and pulmonary tuberculosis. Usually it is not difficult to exclude the latter, since the symptoms of fever, night sweats, the involvement of other parts of the body, the finding of the specific bacilli in the sputum, and the general impression of tuberculosis are absent.

\section{Secondary Pneumonia}

As the result of pronounced weakness, prostration, and engorgement of the pulmonary small vessels, a child may be affected with a low form of inflammation that may be called a secondary pneumonia.

Causes. - The recumbent position, most of all when the patient's posture is not from time to time changed, is in fact responsible for the condition. The severe wasting diseases are those most apt to debase the child's physical state to such a degree that the tone of the tissues is seriously impaired. If the 
antecedent disease is markedly microbic in nature, the germs are apt to find a fertile culture-ground in the weak and atonic pulmonary structure.

Lesions. - The posterior parts of the lungs are the ones most affected. A broad strip on each side may be involved from top to bottom, or the lesions may be scattered in small areas here and there, but without penetrating into the interior. Some of the cells may be aërated, others will be in a condition of red and gray hepatization. In the latter position the arterioles are congested, there are a diapedesis of red cells and an emigration of white cells. Also one may see mixed with them epithelial cells, pus, and fibrin.

In some few cases the inflammation seems to centre about the smallest bronchioles, each of which may have a small zone of peribronchitic pneumonitis. These bronchioles would then 'be subject to the usual degenerative changes and be filled with the products of inflammation.

Symptoms. - The symptoms are few and not especially characteristic. The child's condition is so poor that no definite rates of the respiration, temperature, and pulse can safely be given. Since the disorder comes only in the wake of some pathological condition, these three factors will assume the general character which belongs to the precedent disease. There may be a muffled sound on percussion and fine râles on auscultation. Here and there one may distinguish coarse râles. The breathing is apt to weak, shallow, and irregular.

Treatment. - In the course of any disease which brings with it marked prostration, the attendant must keep constantly before him the possible supervention of a secondary pneumonia. $\mathrm{He}$ should require the child's position to be changed from side to side, and thus avoid too long an exposure from lying on the back. The treatment of the original disease must look to the maintenance of as much vitality as possible, and should, if at all allowable, include the active administration of efficient tonics.

Prognosis. - The prognosis, on account of the patient's debility, is bad.

\section{BRoNCHIECTASIS}

By this term is meant a dilatation of parts of the bronchi which, in different cases, varies in size, location, and contour. 
These dilatations rarely occur in the largest tubes, and in children are more apt than in adults to affect the fine bronchioles. On account of their variations in size and shape they have been roughly divided into cylindrical and sacculated forms. The firstnamed variety consists of a fairly regular enlargement which gradually merges into the normal contour of the tube; the second consists of an abrupt enlargement which need not assume any regular form, but may vary all the way from a pouch-like dilatation to a practically closed cavity. It is doubtless true that the condition occurs more frequently in children than is commonly believed.

Causes. - The constant strain of coughing, the pressure of large quantities of mucoid secretion, and the weakened condition of the bronchial walls make dilatation a fairly common sequel to chronic bronchitis. Occasionally, and more especially in young children, it may follow the acute form of bronchitis as well as all forms of broncho-pneumonia. It may regularly be expected in some degree with emphysema and pertussis ; almost as regularly it occurs in interstitial pneumonia and pulmonary tuberculosis; while in a severe pleurisy with extensive adhesions the possible extension to the lung tissue of the newly formed bands and the distortion to which the lung is subjected are sufficient to weaken the bronchial walls and pull them out of shape. In short, any severe inflammation which affects the bronchi is capable of producing bronchiectasis.

Lesions. - In the cylindrical form the bronchial tissue may in the light cases be not altered. In the severer ones the mucous glands are flattened and wasted. In the dilated portions there will be large quantities of mucus with some pus. The lesions in the sacculated form are regularly severer than this. The whole wall is more or less intensely atrophied and infiltrated with connective tissue. In the extensive cases one dilatation may succeed another until the bronchus retains but little of its normal contour. At the same time its structural elasticity may vanish to such an extent that to limit the degree of the possible changes is difficult. Thus the tissue between dilatations may become so thinned as to allow them to communicate; or the walls of the enlargement may become so debased in vitality as to permit the growth of a pyogenic process.

Symptoms. - The symptoms are often obscure or inappre- 
ciable. However, the disorder occurs so frequently that weak children who suffer much from the pulmonary diseases which cause bronchial dilatation should be suspected of having it. In some cases there are some mucous râles, the respiratory sound is tubular, and over a large bronchiectasis one may discern the percussion note of a cavity. The expectoration, especially in the morning, is apt to be large, so large, in fact, that patients claim to vomit it. A peculiarity of it is that on standing it separates roughly into three layers. The bronchiectatic patient suffers from successive attacks of bronchitis, which often arise without sufficient ostensible cause.

Treatment. - There is no specific medical treatment; all that one can do is so to treat pulmonary cases that the severity of the cough may be diminished and the general strength and tissuetone as little impaired as possible.

The administration of tonics must be consistently carried out for a long time, and the general health must be promoted in all possible ways. Children who live in cities and in harsh climates should be kept in the country where the extremes of temperature and the degree of atmospheric moisture are not excessive. In addition, one should direct the daily practising of calisthenics which tend to promote the activity of the chest and lungs. While it is not possible to bring about an immediate cure, considerable improvement may be made in the child's health and strength. The latest and best method of relief is purely surgical. It consists of pneumotomy, with drainage. This is really the logical cure; for a bronchiectasis is to all intents and purposes a chronic abscess. And the regular treatment for abscess-formation must always be incision and removal of pus.

\section{Acute Pleurisy}

The more one is able to compare the conditions found after death in the bodies of children who succumbed to pulmonary disease with the symptoms which exist in the progress of sickness, the more will one believe in the widespread occurrence of the various forms of pleuritis. And when the frequency of this disorder is thoroughly appreciated, when its importance as a primary disease and its salient influence as a complication in exhausting a patient are put at their rightful value, an important step in the 
direction of efficient protection to the sick will be made. In adults the evil effects of any and all forms of pleuritis are bad enough; but in children they are both actually and potentially very much worse.

Causes. - The causes which bring about this disorder are unquestionably prolific. They are such that neither sex nor social condition can make much variation in its occurrence. Moreover, there is one important interpretation of this statement: a poor physical state, no matter what the cause, renders the child much more susceptible than he otherwise would be. "Whether this

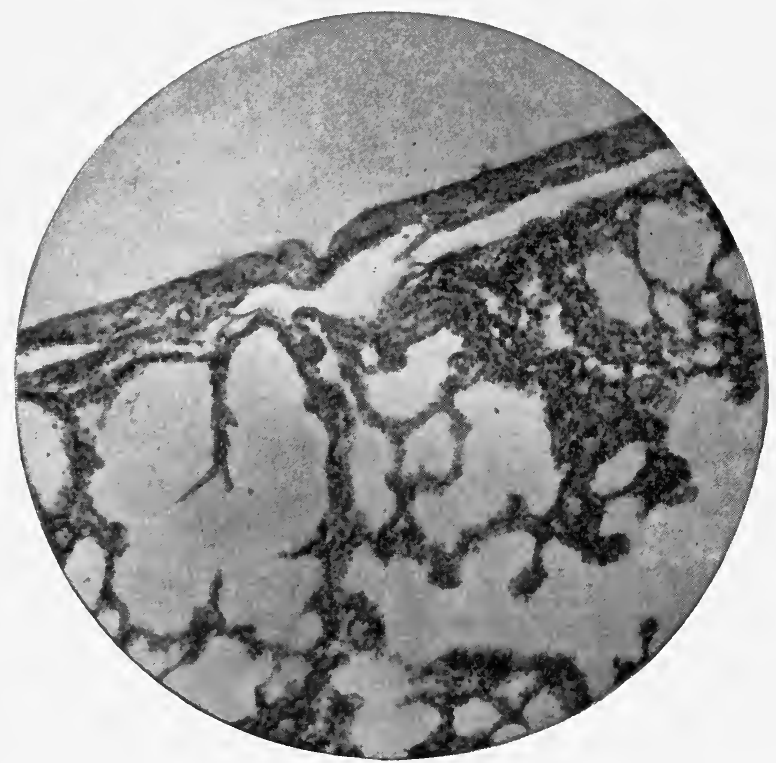

Fig. 42. - Normal Pleura. $\times 85$.

physical degeneration is due to underfeeding or overfeeding, to too little or to too much care, the final condition is to all intents and purposes the same. Robust health is not an absolute protection, but it certainly has some influence in limiting the course and severity of the disease. The main fact is that physical deterioration and deficient vitality lay a child open to attacks of pleuritis and likewise contribute to the likelihood of those attacks assuming the more serious forms.

In all likelihood this cause of malnutrition plus that of exposure should be regarded as for the most part contributory. In 
addition a very slight active agent, such as a blow, a fall, an intercurrent disease, may start the complaint in full force; and extensions of ordinary pulmonary inflammations are almost regularly to be expected. Therefore one often sees pleuritis occurring or following pneumonitis and bronchitis, the acute infectious fevers, especially those of a severe type, severe intestinal diseases, most of all in young infants, and even rheumatism. Moreover, whenever the pneumococcus, streptococcus pyogenes, staphylococcus pyogenes aureus, or the bacillus of tuberculosis can reach the pleura, there is danger of an acute inflammation of it. No age is free from it, for even before birth the infection may take place. In addition, inflammation, especially of a purulent type, of any part of the throat, the lungs, mediastinum, ribs, vertebræ, the serous envelope of the heart, as well as abscess or echinococcus of the liver, purulent disease of the pancreas, intestines, and peritoneum, and bone diseases have been known to act as irritants.

The disease, according to statistics, occurs somewhat more frequently in boys than in girls, and oftener on the left than the right side. However, these are facts of little importance and can easily enough be accounted for.

Lesions. - The different forms of pleuritis are commonly regarded or spoken of as different diseases. This can result only from an inadequate attention to the pathology and to experimental researches. As a matter of fact it is highly advisable to consider all the various types as phases of one disorder which may assume greater or less features of pathological development according to the presence of different factors. In one case we see a simple inflammation of the pleura with a rational formation of fibrin and fibrous adhesions. In another there is an effusion of serum added to the characteristic changes of the first form. In still another we find that the effused material has in some way become infected with pus-forming elements, and as a result the pleural cavity contains larger or smaller amounts of pus. Where for proper reasons this pus material is very intense and acrid in its nature, we see a gangrenous condition of the tissues. And where for special reasons, as in scurvy, there is a tendency toward the breaking down of blood-vessels, we have the rare so-called hæmorrhagic form of pleuritis. But all these processes have a common foundation, and vary merely in the superstructure which that foundation supports. 
At the first stage of the inflammation, the pleura becomes congested, its color deepens, the capillaries are plainly injected, and the endothelial cells swell up and are shed. Besides these small cells white cells also may be seen. Then on the surface of the pleura fibrin begins to appear in scattered bits, in threads, in spreading areas. One should keep in mind that these changes are confined to the surface, and that below the surface the tissue is scarcely, if at all, affected. This fibrin, holding in its meshes endothelial and pus cells, increases very rapidly in quantity. 'The opposed surfaces become simultaneously affected, and strips and strings of fibrin finally join and bind them. The superficial layers of connective tissue cells become swollen and enlarged, causing with the layers of fibrin a distinct increase in the thickness of the pleura. This process naturally goes on to the production of new connective tissue cells. When the growth of the new materials reaches its height they become organized, and the blood-vessels may easily be traced ramifying from the pleura.

When convalescence sets in the fibrin begins to be absorbed, and at the same time the connective tissue increases yet further in quantity. As a result the thickening of the pleura goes on, but unevenly and in patches. Then the bands of fibrin between the pleural surfaces become in the same way organized and strengthened, and thus form more or less rigid adhesions. These on recovery need not have the same shape and relations as while they were forming. On the contrary they may be partly absorbed and so produce eccentricities in outline with corresponding deformities.

This is what one sees in a simple pleuritis, called dry or fibrinous pleuritis. More frequently one sees a course of the disease which in addition to these changes has the characteristic of an effusion of serum. This serous fluid contains about five per cent of albumin, has a specific gravity of about 1.018, and varies in color all the way from a clear amber to the yellow and green of pus. It may on the one hand be quite sterile, or on the other hand be loaded with great quantities of divers micro-organisms. It may contain collections of fibrin and in rare cases, scurvy for example, it may hold varying amounts of blood. In quantity it may range from grammes to litres. When the amount is large, the pressure effects may be plainly visible, even so far as to produce the most alarming symptoms. At the same time one should 
remember that; on account of the pliability of the infant chest wall, these effects are not so marked as they would be in the adult. The effusion naturally forces the viscera in this direction and that, according to the line of least resistance. The lung on the affected side is pressed upward and back; or it may be hemmed in on both front and back and thus compressed, and at the same time the heart is forced away from this side. The diaphragm is pushed down; the stomach, spleen, and pancreas are in similar ways variously affected according to the controlling

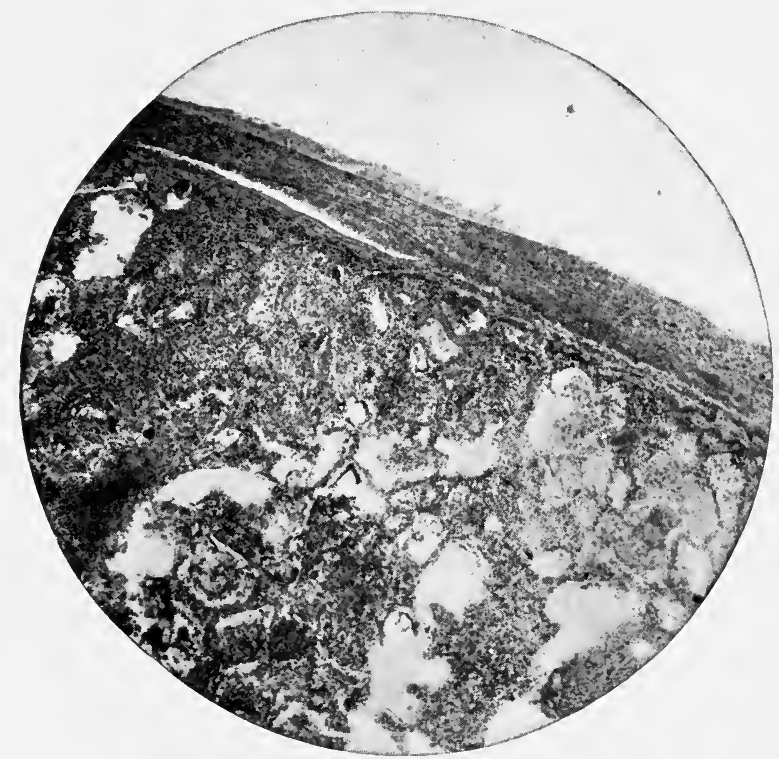

FIG. 43. - Fibrinous (Adhesive) Pleuritis. $\times 25$.

To the right the visceral and costal layers of the pleura are bound together; to the left the process is as yet incomplete.

forces. The chest wall is increased in circumference on the affected side, the intercostal spaces may be stretched apart and bulged out. As the result of these conflicting forces, most of all when the adhesions are strong and eccentrically disposed, the patient may become afflicted with distortions of the chest walls and spinal column. In other cases the fluid by the agency of the adhesions may be shut off in curiously shaped cavities. In a different class of cases, where there are no adhesions, the fluid in an upright chest has a regular location, being lower 
behind than on the sides or front. With changed position there naturally follows some change of contour.

This form of the disease may be designated pleuritis with effusion, or pleuritis with fibrin or serum.

The third form, commonly called empyema, consists in the addition to the second form of a purulent element which may begin with the inflammation or be secondarily acquired. It should be clearly understood that this division is to a certain extent conventional, and that the majority of cases have a greater or less mixture of characters. Thus the fibrinous form often has a small amount of fluid, although not enough to modify the symptoms in any considerable degree. Again, the serous type commonly has a few pus-cells in the effusion which may, under the circumstances of weakness and debility, increase to a great extent. Even the purulent form may begin with a comparatively small micro-organic element, which rapidly develops, so that in certain cases it comes to be the predominating feature of the disease.

Purulent pleuritis may be of a short or long duration; in infants, even when there is no high temperature, it may continue for a considerable period. Nevertheless, one is safe in saying that children under favorable conditions of treatment and vitality react more rapidly in convalescence than do adults. When protracted cases occur the organic changes may be as great as the pleural thickening is marked.

There are a few virulent cases where the purulent exudate is, or becomes, gangrenous. Such patients are always very dangerously sick, and the pathological changes rage with intense severity. The infected fluid is exceedingly offensive in appearance as well as odor, and is characterized by the formation of gases of putrefaction. In this last event the pressure effects are decidedly greater than where the fluid alone is the active cause. Such a condition may be one of the causes of a rupture from the lung or the chest wall. The consequent entrance of air would constitute the condition of pyo-pneumothorax.

Symptoms. - Palpation shows the lessened movement of the chest, and, when there is effusion, much diminished or absent vocal fremitus. A case rarely occurs where the fremitus is not much altered. As a rule, however, its absence is one of our most valuable signs. 
Percussion gives a dull note over thickened pleura and a flattened note over the effusion. This note may not be evident at the very onset of the disease; in fact, the child may be sick for hours or even days before we get a clearly defined change. The variations are not susceptible of intelligible and efficient description, since we seldom see two cases exactly alike. The comparison of a number of cases will demonstrate the differences better than words can do. Nevertheless, a few examples will show a peculiar heavy flatness which is not easily mistaken. The main source of error exists in cases where a thin stratum of fluid allows the subjacent lung tissue to modify the note. Likewise we must be prepared to find any curiosity in the distribution of the altered note, especially if the effusion is purulent; for on account of the encapsulating tendency of adhesions any sort of pockets or compartments may be formed. Therefore the percussion note should be most carefully practised.

The physical signs of the three forms change in character as effusion becomes added to the dry pleurisy, and then increases in quantity. When the disease is fresh, the two affected surfaces rub upon each other in an unconfined way, and their condition is plainly heard. When adhesions exist, such movements are much decreased. If fluid is added to a new pleurisy, the physical signs are at first not pronounced. With a small amount of fluid and an absence of adhesions, the lung is floated somewhat higher than its usual level. But if the fluid is large in amount, and especially if the pleural surfaces become bound down adhesions, the lung tissue becomes hard, airless, and twisted out of its natural shape. The physical signs then become of much greater diagnostic value.

Above the pleuritic area, and especially if there is a large or even a moderate effusion, the percussion note is clear, resonance is exaggerated, and often has the unchanging character that belongs to Skoda's resonance. As we approach the level of the pleuritic area or the surface of the fluid, the percussion note changes to dull, and over large effusions it becomes flat. With such amounts of fluid the respiratory murmur becomes low and harsh, and finally may disappear. In the older children it may assume an amphoric character. One regular sign which we expect to hear in this disease is the characteristic friction sound. It is a fine, sharp, grating noise, heard on inspiration and expiration, and is caused by the opposing surfaces rubbing against each other. Adhesions 
or large quantities of fluid may eliminate it, and thus produce a seeming exception to the rule. Its presence is diagnostic, but its absence is not. In other respects not too much reliance may safely be placed upon the results of auscultation. We may find bronchial or broncho-vesicular, diminished or puerile, breathing; also, curiously enough, all these variations may at times be heard above or below the level of the fluid. The voice may be normal or bronchophonic. The râles which regularly belong to the disease are fine crepitant ones; any others belong to coexisting disorders. In a word, the auscultatory signs in infantile pleurisy may be confirmatory, but outside of this have not much force. It is naturally understood that with increasing age children present signs that progressively approximate to those which the adult presents.

A rarely encountered sign is ægophony. When it is distinguishable, and where the lungs are not compressed by large quantities of fluid, it may be heard at the back on either side of the spine.

Pain and dyspnœea are most apparent at first. The pain may vary or disappear after two days. When present in infants it is shown by the wrinkled and puckered face and repressed breathing. Even the youngest patients try to minimize the respiratory effort, and the attempts are often so plain as to be truly impressive. Older children show their distress by unwillingness to be touched, by a cramped posture, by shrill cries on coughing, on voluntary or involuntary movements. The dyspnœa depends upon the amount of pleuritic inflammation as well as the amount of compression of lungs and heart by fluid. At times it is very alarming, owing to the fact that on the left side the quantity of fluid may be so extreme as to squeeze and compress the lung, and float the heart out of its normal place into a twisted position in the right mammary region. On the other hand, effusion into the right side causes much smaller symptoms. The rule in infants, however, is that remarkable quantities of effusion are not common. The greater the amount of surface involved, the greater will the patient's efforts to restrain respiration be, with a consequent decrease in oxygenation. Likewise the greatest compression and displacement of lungs, heart, and the near great vessels will produce corresponding effects upon the circulation.

The patient, as if fearing the pain that movement induces, is seen to lie unusually still. He prefers to rest upon the side 
which is diseased, because thereby he avoids a certain amount of movement. The affected side is apt to be fixed, and the diaphragm is allowed less movement than in normal conditions. In spite of the fact that the restrained breathing seeks to limit expansion, careful measurement will show a slight increase in the circumference of the chest due to the bulging out of the affected side. According to the degree of this bulging, there will develop a

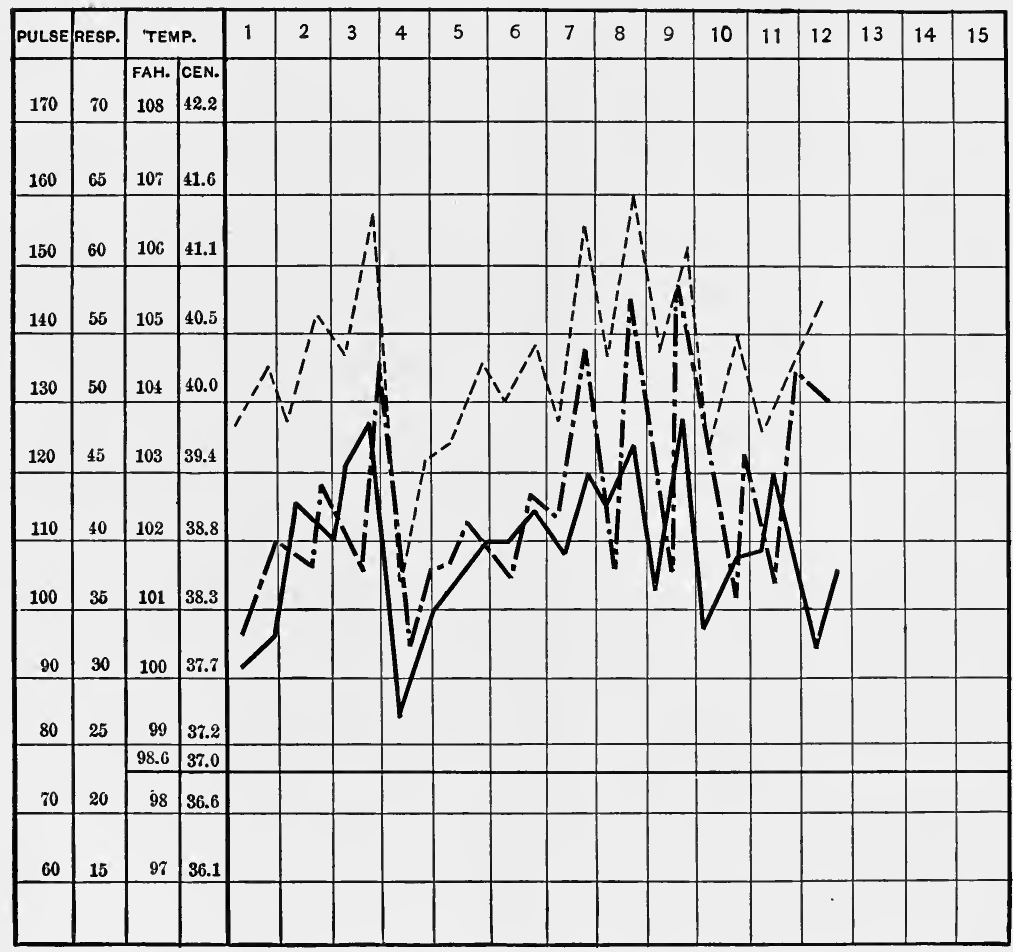

PULSE, RESPIRATION AND TEMPERATURE CHART OF PURULENT PLEURISY. OPERATION, DEATH. AGE, 8 MONTHS.

PULSE RESPIRATION

FIG. 44.

tendency, most of all in large purulent effusions, to alter the contour of the intercostal spaces.

The fever is not a constant one, and commonly has a distinct remittent form. As a rule it is high, especially when the pleuritis complicates another disease. The surface of the affected side has a higher temperature than any other part of the skin.

The pulse follows the general temperature fairly well. In 
young infants it may reach a maximum of 190 to 200 . Usually it is thin and hard, excepting in cases of complication with acute lobar pneumonitis, where it is distinctly soft. But this is due to the predominating effect of the pneumonitis upon the vaso-motor circulation.

The respiration is hurried and shallow, and, on account of the pain which each effort produces, does not follow the fever and pulse. Usually it is irregular; but there is no predominating form of irregularity which one can crystallize into a general statement. A noticeable observation, however, is that the breathing varies from the thoracic to the abdominal type. In addition to the usual prostration, anxious feverish look, coated tongue, and scanty urine, we may, in some severe acute cases of a purulent type, have the added symptoms of pyæmic infection, such as stupor or delirium, convulsions, unusual ranges of temperature, and finally collapse.

In the cases where effusion is suspected, the diagnosis is to be confirmed by means of a large hypodermatic needle. Under careful antiseptic precautions there are almost no objections to this step. The most favorable position to make the puncture is in the sixth or seventh interspace in the posterior axillary line. In rare cases, when this test shows no fluid, the failure is due to the fact that the needle enters an adhesion. In such a contingency the point of the needle is not to be moved about, but the instrument should be entirely withdrawn and inserted anew.

Complications. - The usual complications are those resulting from poor arterialization and hampered respiratory effort. Where the disease is of a purulent type we may encounter complications arising from a systemic infection. Also, where the purulent fluid is not liberated by operation, it sometimes burrows its way through the chest wall or through the lung tissue into a bronchus. The termination of this condition is generally favorable. Where pus forces its way through the chest wall there may supervene, as a result, a caries of the ribs with a possible extension to the vertebræ.

Treatment. - In the first form, the treatment consists in the nursing, the regulation and modification of the food so that it will be most easily assimilated, and the control of pain. Considerable discomfort can be relieved by counter irritants, such as liberal applications of iodine. The actual cautery, if judiciously used, 
may be of a great value. With this agent, however, one must be careful, keeping in mind the ease with which a young child's skin becomes irritated. At times it may become necessary to use small doses of opium. It is desirable to see to it that the patient's strength is wisely conserved; the use of stimulants may from time to time be indicated, and their quantity must be regulated to suit individual cases.

Where effusion exists one must be particularly careful of pressure effects and heart failure. Caution in allowing the patient to assume an erect position must be exercised. To facilitate absorption, tonics and diuretics are to be given, the latter in liberal doses. For this purpose, plain or alkaline waters will be found of value. At times it may be necessary to aspirate the chest, in order to diminish the amount of fluid. This measure is of temporary value and does not prevent the return of the fluid; if symptoms of pressure are very marked, the best plan of action is the same as in the purulent form : to open the chest wall with a free incision and secure sufficient drainage. The process of absorption by the economy is often so slow, that we are justified in operating and draining in a much greater number of cases than we now so treat. After the acute stage is past, the operation is even more desirable than before.

The treatment of purulent pleuritis should resemble that of any pus cavity. The chest wall should be opened and thoroughly drained. It may be necessary - I believe it is practically always desirable - to resect a rib. Thereby one obtains a more thorough drainage.

As the patient begins to convalesce, he should be most carefully treated and nursed so that every opportunity for the regain of health and strength may be improved. Even under favorable circumstances he is apt to suffer for a long time on account of compression of the lung and changes in pleural surface.

Prognosis. - In primary pleuritis, the prognosis is usually favorable; but when the disease arises by extension, the outlook is more serious. In almost all cases the original infecting disease is apt to be made severer by the lighting up of the pleuritis with its peculiar disabilities.

Differential Diagnosis. - The main signs are the evidences of pain, the dull note over the pleura, and the flat note over the fluid. When this much is distinguished and there are besides no râles or 
very few râles at the margin of the affected area, the diagnosis is more nearly certain. With fluid the character of the bronchial breathing is modified and friction sounds are absent. The distinction between a purulent and a non-purulent effusion may be made from the more virulent character of the symptoms in the former, but most of all by aspiration. From a pneumonitis one may distinguish pleurisy with effusion by the former's high temperature, greater resulting prostration, and more acute course; by the pleurisy's greater area of dulness or flatness, and the changing location of this flatness, as well as the absence of many reliable auscultatory signs.

\section{Chronic Pleurisy}

Causes. - A chronic form of inflammation of the pleura exists more often than is generally believed. When one considers the fertile causes that may produce it, this conclusion seems inevitable.

Lesions.-- After an acute pleuritis it is practically impossible to lose all trace of the changes in the involved tissue. The pleura remains thickened and heavy. Frequently the thickening exists in more or less scattered areas, but sometimes the process involves the whole pleura. In these latter cases a partially uniform condition exists; here and there the changes are especially marked, so that one is apt to find spots where the characteristic pliability and functions are quite absent. The hypertrophied portions are covered with large endothelial cells in great numbers. There is an increase in the connective tissue, which on account of the marked growth of the blood-vessels has no natural tendency to decrease. The pulmonary and costal membranes are bound together by strong, firm adhesions which are apt to be surprisingly large in number. These adhesions become thoroughly organized, contain blood-vessels, and are sheathed with endothelium.

Symptoms. -- The symptoms are such as commonly escape connection with the lesions and often are attributed to quite a different cause. On inspection the chest is seen to expand irregularly; and in marked cases the difference between the two sides is so great as by comparison to make the affected part seem stationary, while the rest of the chest exerts unnaturally intense efforts. Percussion gives a dulled note which in severe cases may become almost flat. The ear, on auscultation, hears the 
breathing as if from a distance; and a few moist râles are often present in scattered areas. When the lung is free, one is apt to make out all the signs of emphysema, which on account of the inactivity in certain parts is bound to develop in others. Sometimes there is a tenderness of the chest which is liable to become in the face of an irritant an acute pain. Where the chest walls are bound down, there may be considerable deformity. With this goes an imperfect aëration of the lung tissue with the consequent proneness to acute inflammations. For trifling causes the patient may have a rise of temperature that is apt to puzzle the attending physician. This may be unattended by a correspond-

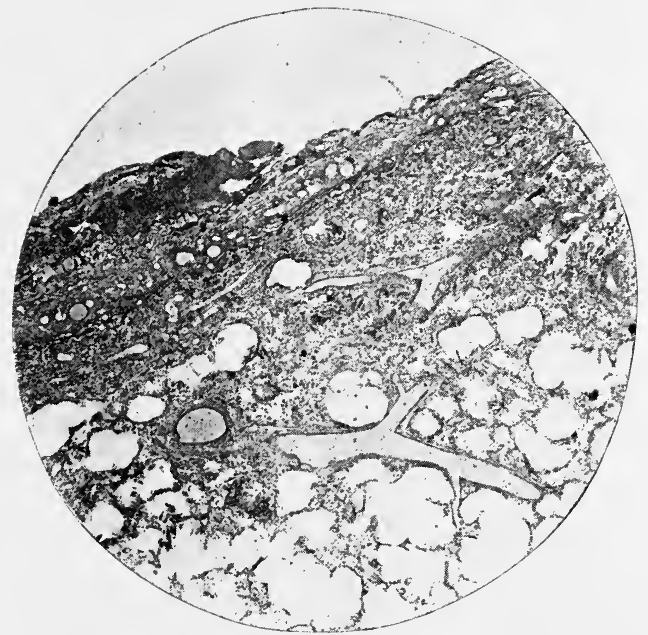

Fig. 45. - Chronic Pleurisy. $\times 25$.

ing rise in the respiration- and pulse-rate, excepting in those cases where an appreciable acute sickness supervenes. There is a great variation in the appearance of the patients, some showing at first sight hardly any physical deterioration, while others are clearly exhausted, ready to lapse into a serious decline. There is a constant disposition to respiratory irritations which may easily start into active disease. There is a chronic hacking cough that is hard to quiet, returning on slight provocation.

Treatment. - The treatment of building up the general health by exercise and tonics is of most use. Such tonics as the compound syrup of hypophosphites or cod liver oil in a malt prepara- 
tion may be recommended. Exercises which tend to expand the chest and stimulate the lung tissue should be carefully and vigorously employed. At the same time it is wise to counsel the continued practice of athletic sports and games as well as every means that can promote the general health and strength. Living in high latitudes, on account of the increased expansion of lungs which follows, is often of decided value. By such simple means a satisfactory improvement can be brought about which requires no more than a continued regulation of all the details of daily life for its perpetuation. Much patience may be required; but the result will justify the outlay of effort.

\section{Atelectasis}

By this name is meant a condition in which parts of the lung become entirely deprived of air ; as a result the unaërated portions collapse, and when once they are in this state their tendency is not to resume their function. Any part - much or little - of a lung may be involved, and at any age from birth up to adult life. As a rule, however, we meet this condition in infancy and most of all in weak babies.

Causes. - The existence of marked physical weakness is the main predisposing, and often the main active factor in the production of atelectasis. Whether this weakness is congenital or the result of disease makes little difference. The prematurely born child may equally with the syphilitic run the gauntlet of atelectasis; or the tubercular, the rachitic, the poorly nourished baby may likewise be afflicted. Following out this general idea we get a conception of the causes which work at any period; for even in the face of an active cause of atelectasis, there still must be the foundation of noteworthy exhaustion. Such active causes might be the occlusion of a bronchiole in the course of a local disease by a plug of mucus, the exhaustion of the residual air sometimes resulting from the prolonged spasm of pertussis, the viciously congestive action of severe cardiac and pulmonary disease, tumors of the chest and abdomen, deformities of the chest, and foreign bodies in the lung, all of which may exert so much pressure upon an area of the lung that the air is forced from it. One should keep in mind those rare and interesting cases of nervous disorders where, on account of paralysis of the pneumogastric nerve or dis- 
ease of the respiratory centre, the act of breathing becomes exceedingly difficult and progressively less efficient. Naturally such disability can act just as surely in the long run as the more acute obstructions mentioned above. In case the obstructions are quickly removed the lung naturally seeks to resume its form and function; but where the relief is too long delayed the harm is apt to be irreparable.

Lesions. - If the atelectasis occurs at or immediately after birth and before the lung has been well expanded, one fails to find any inflammation - merely rather hard, flesh-like tissue that has a

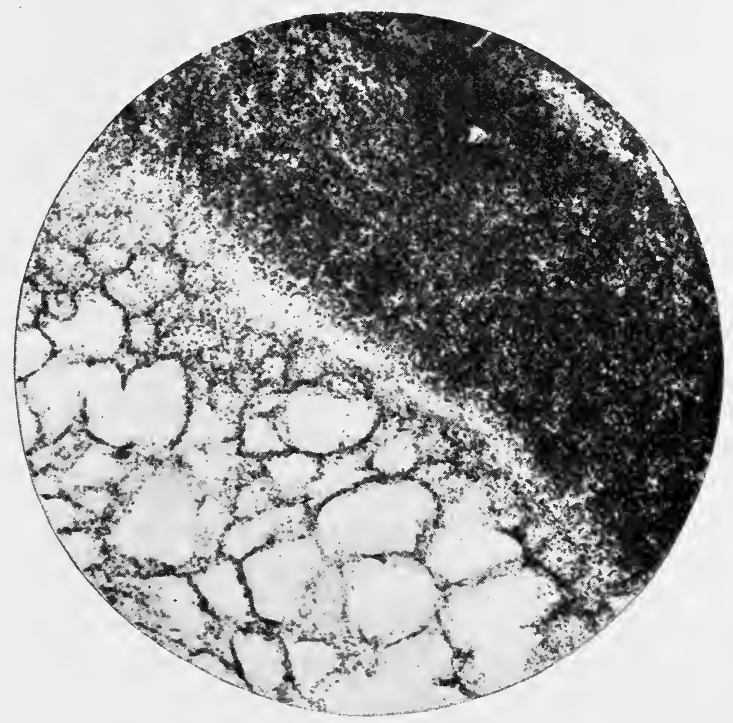

Fig. 46. - Atelectasis with Compensatory Emphysema. $\times 43$.

somewhat darker color than liver. The longer the tissue remains unaërated, the harder and less lung-like the affected part becomes. In children of greater age, where the collapse has been caused by obstruction, one is apt to find more or less bronchitis scattered here and there. The collapsed areas will be found to be connected with the inflamed tubes and to radiate in a fan-like fashion from them. These patches are, of course, much heavier than normal lung tissue - so much so, in fact, that they cannot float in water. On account of the uselessness of these portions, the neighboring areas of the lung must - or at least try to - make up the deficiency. In consequence they become plainly emphy- 
ematous. This lack of balance in the relation of parts is a fruitful source of congestive disorders, which in turn may be followed by any manner of lung disease.

Symptoms. - The symptoms in a fairly well-marked case are impressive. The child is restless, somewhat cyanosed, and suffers from dyspnœa. The face has a sunken appearance, the nostrils work convulsively, the breathing is shallow, and the chest wall under the load of atmospheric pressure is apt to be retracted. The rate of respiration is hurried and irregular, the temperature is very slightly elevated or normal, or in serious cases subnormal. The ordinary relation in rapidity between pulse, respiration, and temperature may be quite lost.

Over the atelectatic area the respiratory murmur is faint or imperceptible. If this area is not too deeply located, there is dulness on percussion. If emphysema, bronchitis, pneumonitis, cardiac or intestinal disease be present, the characteristic symptoms will naturally manifest themselves.

Treatment. - There is no especial treatment except the wisest possible regulation of all the details of food, bathing, clothing, and general management. As these cases are very weak and exhausted, one has opportunities for the exercise of much resource, ingenuity, and patience in the attempt to develop as far as possible the little patient's vitality. Stimulants and tonics must be employed, and their use will be directed and regulated by the varying necessities of individual cases.

Prognosis. - The prognosis is bad.

\section{Emphysema}

Causes. - This condition consists in an abnormal dilatation of the air-vesicles of the lung, with a consequent increase in the amount of contained air. Although this amount of air is often large, the lung does not get the benefit of a large amount of oxygen, for the walls of the air-vesicles are so constantly kept on the stretch that they lose their tone and resiliency. In consequence, their functional activity suffers. The marked pliability of the tissues in childhood makes the occurrence of an attack of emphysema very easy. Indeed, it is very probable that in all the sicknesses which involve severe spasms of coughing, there is more 
or less dilatation of the air-vesicles. In addition, the barrelshaped chest of the infant by its very form invites emphysema.

On the other hand, if it is easy for these little patients to acquire this disorder, it is likewise easy for them, excepting in the case of the so-called substantive form, to rid themselves of it. On the return of a fair amount of vitality, the lung tissue resumes its usual anatomical condition, and its function reëstablishes itself. In children one meets with three forms which, on account of their manner of occurrence, are generally designated as vesicular, interlobular, and substantive emphysema.

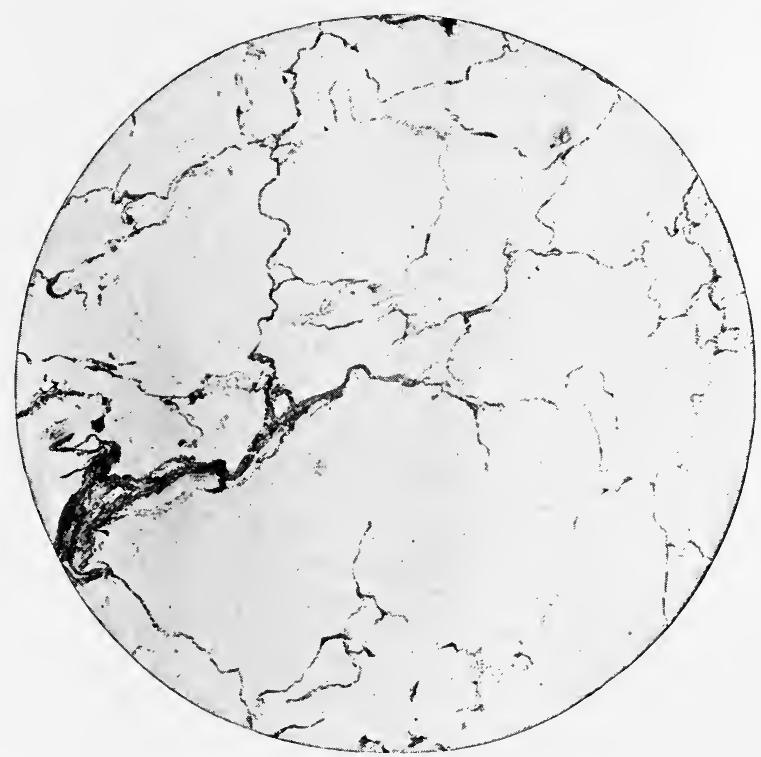

Fig. 47.-Emphysema. $\times 31$.

When a child, on account of the disability of some of the bronchi or bronchioles, or when a part of a lung has been rendered incapable of operation, attempts to perform the usual respiratory motions, the undisturbed portions of the lung are forced temporarily to do more than their share of work. As a result they become stretched out and dilated during the progress of the impeded breathing. This is called vesicular, or compensatory, emphysema.

When the expiratory efforts are really severe, when a great strain is put upon the air-spaces, their walls are apt to break. 
The air rushes in, making the cavities progressively larger with fresh exacerbations of the exciting cause. Thus the air may work its way in all directions through the interstitial tissue of the lungs. It may even find an outlet through the pulmonary pleura into the neck, mediastinum, face, or other parts of the body. This, the so-called interlobular form, may arise from a severe pneumonitis or broncho-pneumonitis, from whooping-cough, asthma, or laryngitis. The lesions are more firmly seated than in vesicular emphysema, and when the disabled parts are large in extent may persist for years.

In substantive emphysema one finds the most serious cases. Not only the form, but also the substance, of the space walls may be changed. Indeed, the alteration in the form may not be very marked, the amount of dilatation may not be very great, but there is a slow but sure laying down of connective tissue in the walls of the air-vesicles, which renders them practically immovable. This is not apt to occur excepting in older children, especially those of a vicious physical disposition.

Lesions. - In the mildest forms the lesions, outside of the changed shape of the air-spaces, are barely noticeable. In the severe forms the whole lung may be increased in size. The small bronchi may be thickened, and here and there along their course one may see spots of pneumonitis. There may be much mucus secreted, or mucus mixed with small quantities of pus. The air spaces are often affected in groups, so that the whole appearance of the lung is irregular. The diseased air-vesicles have ruptured, thickened walls which, when the chest is opened, do not collapse. In such cases disorders of circulation are apt to occur; but as there must be some fairly severe disease to cause the emphysema, one is not positively sure whether the original disorder or the emphysema was the cause of the imperfect circulation.

In the severest form of emphysema, one notices a tendency to the production of connective tissue in small, scattered areas. The walls of the air-vesicles, of the small bronchioles, and of the arterioles are thickened and slightly rigid, but irregularly so. Sometimes one can notice a slight proliferation of the endothelial cells.

Symptoms. - In the mildest cases practically no symptoms reveal themselves to the examiner. The infantile chest, being naturally barrel-shaped, is apt to assume the appearance of being 
afflicted with dilatation of the pulmonary elements. But in the more strongly marked cases there is a hyper-resonant note on percussion. Expiration is apt to be long drawn out. There may be some of the auscultatory signs of a sub-acute bronchitis, and, in the severest cases, of a slight pneumonitis. But even where these are absent, the respiratory murmur lacks the clear, even character of health. Where deformities occur, such as those of the spine or pleuritic adhesions, their presence will help to define and emphasize the symptoms. Moreover, one may expect congestion of any viscus or the skin. As the disease is apt to accompany conditions of defective strength, one is apt to see such signs of malnutrition as rounded shoulders and bent backs, depressed surfaces over the poorly aërated apices, lower ribs that are bent out, retracted upper abdominal region, and an indefinite number of characteristics that go with a run-down and worn-out physical state.

The patient on slight provocation complains of dyspnœea, less frequently of asthma and rarely of pain and soreness. He shows a notable liability to pulmonary diseases and, if they attack him, he resists their inroads with poor success.

Treatment. - The treatment, when there is an antecedent disease, consists in no additional measures until its acute symptoms have ceased. Then, and as the surest means of recovery, every attempt must be made to build up the patient's health in every possible direction. The diet, largely nitrogenous, should be as hearty as his digestion permits. If the climate in which he lives is trying, he should be removed to a locality which is not too high, where the air is clear, and where there are no extremes of temperature. Tonies, such as nux vomica, the organic preparations of iron, hypophosphites, and - where the digestion is good - small doses of cod liver oil, will be found of use. One must be careful not to permit violent exercise.

Prognosis. - Where the disease is mild and merely compensatory, there is a good likelihood of complete recovery. This is especially true when the pathological changes have not existed for long. However, when the disorder has persisted for long periods of time, especially in the serious cases, such as the substantive form, it is unreasonable to expect a complete recovery. 


\section{GANGRENe OF THE LuNGS}

Gangrene of the lungs, while seen quite often in adults, especially in exhausted patients, is rarely encountered in children ; in infants it is barely liable to occur. It is in its final aspect a senile condition rather than a disease. It means the quick sucking up of the very life of the affected parts, and their invasion, as so mach inert organic matter, by pus-forming bacteria. There is no physiological reason why children should be more exempt than adults from its attacks ; but in most cases of profound loss of vitality children will succumb before the stage of gangrene supervenes.

Causes. - In the progress of some primary disorder the lung tissue becomes broken down. This primary disease may be one of the inflammations of the lung coupled with a weakened cardiac condition. A rational reason for the starting up of a gangrene is a wound of the lung which admits putrefactive bacteria or the introduction by any means of a foreign body. In fact, any method of bringing pathogenic micro-organisms to a weakened lung area, especially in the presence of a general physical impoverishment, is sufficient to bring about the disease. In addition, any interference with the integrity of the arterioles, which is fortunately rare in infancy and childhood, can by withdrawing the local means of nourishment cause the area to degenerate and break down.

Lesions. - When the process has once begun, the affected portions become hard, dry, and discolored. The surrounding area takes on a low form of general inflammation, involving the parenchyma of the lung, bronchi, or pleura. A fatty infiltration makes its way through the margins of the gangrenous portions, the central parts of which begin then to soften and break down. The normal color fades and changes to yellow and green, and finally, when decomposition has fairly begun, an intensely offensive odor develops. At this time the various bacteria of putrefaction may be found in huge swarms. No one form seems to have an inevitable predominance, either in the production of the diseased condition, or as a disposing factor in influencing the course of the sickness.

The process may be limited to a single focus, may be scattered in many different parts, or may invade the whole lung. Which course it will take is decided by the question of general and local 
nutrition. As a result of these differences in location we are in the habit of classifying the forms as circumscribed and diffuse. In the first a single spot may be affected, while the rest of the lung remains fairly normal. It is possible that this spot after breaking down may be eliminated, with the result that the cavity becomes filled with connective tissue which contracts and leaves a healthy scar. In the second form the whole lung is disintegrated, it may even become fluid, and then the possibility of recovery is past. When the necrotic fluid, containing products of decomposition and bacteria, is able, either by its corrosiveness or by the weakness of the tissues, to make its way into different parts of the

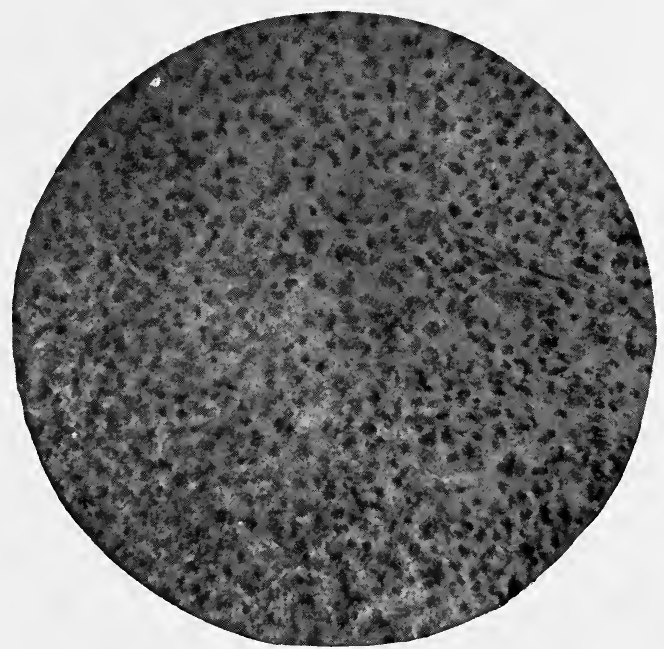

Fig. 48. - Gangrenous Abscess of Lung. $\times 235$.

lung, direct contamination follows. If this process extends to a blood-vessel it continues its course without interruption, the walls are destroyed, and a more or less serious hæmorrhage results. In the same way a bronchus may be perforated; in this case, however, the patient experiences some relief because the products of necrosis thus find a free exit. Such a situation is far preferable to the one in which the decomposition eats through the lung to the pleura, thereby starting a purulent pleuritis.

Symptoms. - Even before the gangrene fully begins, the symptoms of the primary disease become exaggerated. In addition the patient plainly suffers from extreme prostration. The 
loss of flesh and strength is rapid, the face has an anxious, worried look. The temperature is irregular and remittent, the pulse is rapid, thin, and irregular. The picture is such as to force upon one's mind the idea of purulent process. If the disease is far advanced, the destruction of tissue may be sufficiently extensive to give the signs of a pulmonary cavity. The frequency of this last condition is in inverse ratio to the youth of the patient; for the younger the child, the less can he resist the invasion of gangrene. Finally, one characteristic sign should be mentioned : there is a very foul odor about the patient, which emanates mostly from the breath, but also from the body as well. I well remember a case in a boy of ten years, where this odor could be discerned at a distance of from twelve to fifteen feet.

Treatment. - There is no specific and successful treatment. The regular course is to give inhalations of disinfectants, such as terebene, creosote, thymol. Their value is doubtful, for the process is too intense both in its retiology and its course to yield to such slight remedies. All that one can do is to give tonics and stimulants while in every way conserving the patient's physical resources.

Prognosis. - The prognosis is bad. 


\section{CHAPTER XVI}

\section{DISEASES OF THE HEART}

\section{Functional Cardiac Disorders}

Is infants and young children functional disorders of the heart are few and not very important. All those which we may designate as subjective are, from the very fact of youthful immaturity and imperfectly conscious mental conditions, ruled out. There would then be left variations of rhythm only, such as tachycardia (rapid heart action), brachycardia (slow heart action), and arrhythmia (irregular heart action).

The rtiological factors are common to all, and for the most part are grouped about the central fact of impaired nervous control. In very young babies this is a common condition, due largely to a naturally deficient coördination. Evidences of this one sees every day in the aimless spasmodic movements of arms and legs, in the temporary lack of control of the eyes. In a similar way, close observation will show temporary defective nervous control of the heart action.

In older children this state of things is naturally brought about by nervous shock and strain, by diseases of the brain and cord, by cardiac and pulmonary disease, by inherited weakness or sickness, during convalescence after the acute fevers, by diathetic and wasting diseases.

The neuroses have no characteristic physical signs which are common to all. Tachycardia is often associated with a strong apex impulse, as brachycardia — although less frequently - is with a weak one. As a rule, all that there is to be found out may be ascertained by inspection or palpation. Auscultation will occasionally and in very weak cases reveal a murmur which is often hæmic ; but it is possible that such a sign is coincident with the neurosis, not dependent upon it.

It is best to regard all these cases, unless there are concomitant reasons for alarm, as of comparatively little importance. 
Not only does organic heart disease not develop from them, but also there is no particular reason for believing that the neurosis will persist for a long time, most of all if the general condition and circumstances of the patient are good. If diet, exercise, and moral control are good, the outlook is excellent.

\section{MYOCARDITIS}

Myocarditis is an inflammation of the heart substance which begins with the vessels and extends to the muscular fibres. Then

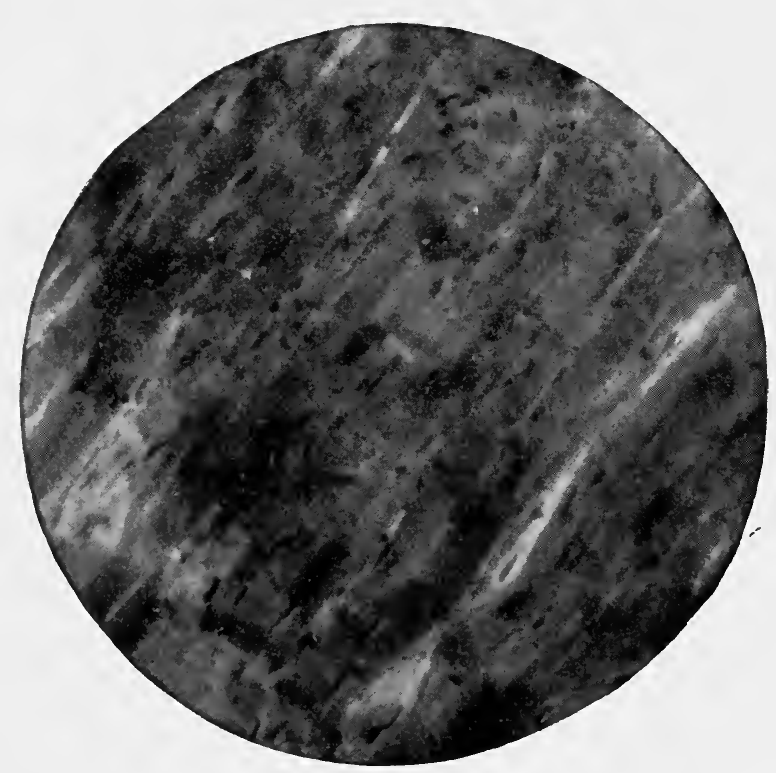

Fig. 49. - Normal Heart Muscle. $\times 220$.

it may proceed inward or outward, involving the endocardium, or pericardium. It may occur in intra-uterine life or early infant life, and may extend indefinitely into childhood.

Causes. - The disease usually appears as a complication or sequel of endocarditis, pericarditis, diphtheria, scarlatina, py:emia, septicæmia, phosphorus poisoning, pernicious anæmia, and other infectious or non-infectious diseases. There is reason for believing that severe wasting diseases may bring about the atrophic changes of the so-called parenchymatous form. 
Lesions. - According to the pathological changes one may divide the cases into parenchymatous, interstitial, and purulent forms. The first-named is characterized by a loss of the proper conformation of the muscular fibres, which degenerate and become infiltrated with granular matter. The full color fades and becomes dull and heavy. In the insterstitial form the process assumes a chronic character. The muscular fibres becomes irregularly infiltrated with small cells, and the normal tissue may gradually be replaced with new connective tissue which is apt to

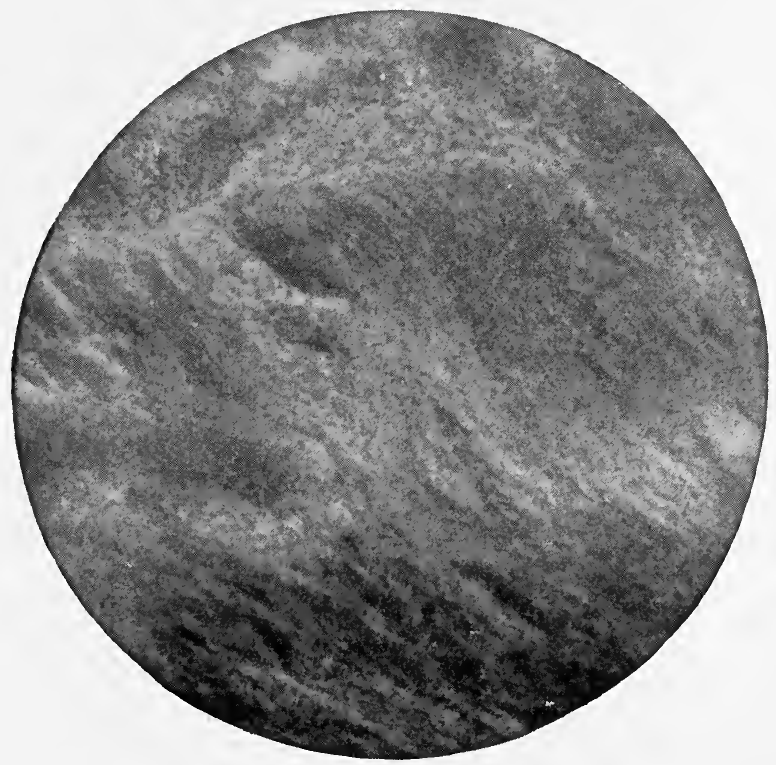

Fig. 50.-- Parenchymatous Myocarditis. $\times 80$

be unusually hard and inelastic. In the purulent form bacteria find a lodgment in the weakened spots. These areas then slowly break down, forming larger or smaller abscesses. They may extend and break in any direction, or they may slowly heal, the pus being absorbed while the empty space becomes filled with granulation and connective tissue.

In that form of degeneration which is commonly called "fatty," the muscular fibrillæ are infiltrated with droplets of fat. They may vary in quantity from a hardly appreciable amount to so great a number that the characteristic appearance of the tissues is clouded. In the fatty myocarditis that occurs in pernicious 
anæmia and phosphorus poisoning the color of the organ may be changed to light reddish yellow, and its consistency may be soft and yielding.

Symptoms. - In infants the symptoms are very obscure, especially as the disease almost never occurs alone. There is no murmur to be expected unless the spread of the lesions is very great, and even then there is apt to be some concomitant disorder which would likewise explain it. One should, however, expect a disturbed heart action, with weak and irregular pulse, fluctuating

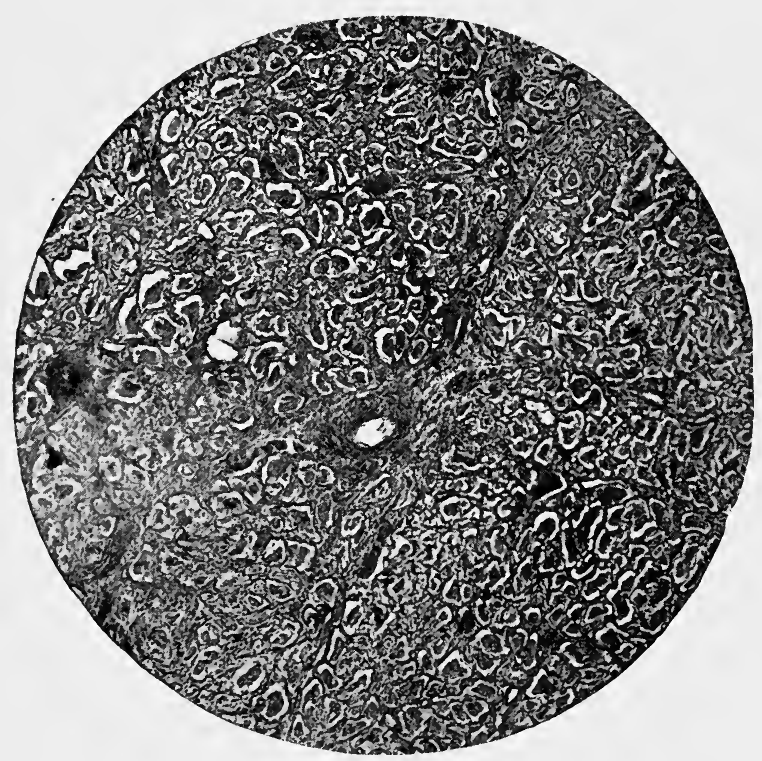

FIg. 51. - Interstitial Myocarditis.

temperature, dyspnœa, cyanosis, and prostration. The symptoms are all the more obscure because the disease may begin very early and in a very gradual and insidious way.

Full knowledge of the disease is usually obtained on postmortem section.

Treatment. - All that one can do for these cases is to husband and nourish the resources of the patient and answer the demands which the symptoms make. In doing this digitalis should be excluded, or at most should be used with great caution. The prognosis is bad, not only on account of the disease itself, but also 
because the conditions with which it occurs are so serious and exhausting.

\section{Acute Endocarditis}

An inflammation of the endocardium, the lining membrane of the heart, is in childhood a common and very disturbing disease. The least informed parent has heard and is deeply impressed by the dangers of it. On the whole, this fear is not misplaced, for even if the sickness does not result in death, nevertheless it so seriously affects the growing body that in many cases the most rigorous attention will often be unable to atone for the damage.

Causes. - One of the curiosities of endocarditis is its occurrence before birth. Naturally, this is a rare condition. When it so exists it is evidently caused by pressure on the pulmonary valves, and thus affects the right heart. It may likewise be due to maternal or inherited disease; but occasionally one will search in vain for the cause which has set the process in motion. A peculiarity of the disease at this time is the preference which it has for attacking the right heart. This is doubtless due to the peculiar state of development in which the heart and lungs find themselves. When the disease is not congenital, it is rarely seen during the first two years of life. Then it may accompany chorea, pulmonary, and septicæmic or pyæmic diseases. As instances one may cite cases of purulent inflammation of the umbilicus, the bones, and the kidneys. It may be due to diathetic diseases, syphilis, and tuberculosis. The commonest causes, however, are scarlet fever and rheumatism. One constantly sees the connection in patients suffering from the latter disorder, but infants, since they do not seem to be often attacked by rheumatism, form a partial exception to the rule on account of their age. However, when young children do have rheumatism, they are most likely to suffer from a complicating endocarditis.

The belief in the bacterial origin of endocarditis is obtaining a wider hold with successive attempts at forming a rational retiology. The proof of the theory is as yet incomplete, and therefore it is advisable to do no more than mention it.

Lesions. - In this disease the endothelial connective tissue becomes brighter in color, most markedly so at the edges of the valves where the inflammation oftenest occurs. The capillaries, with which the tissue is but poorly supplied, become enlarged and 
develop in every direction. The valve increases in size, and becomes succulent. The products of inflammation, as seen in a disorder of the mucous membranes, are scanty. On the other hand, the connective tissue cells and the basement membrane are swollen and hyperplastic.

In the severer cases the sub-endothelial connective tissue, besides being swollen, becomes infiltrated with new proliferating cells which extend to the membrane below. The surface may then be roughened and studded with nodules. Also the cells may proliferate, the nodules may become eroded, with the resulting

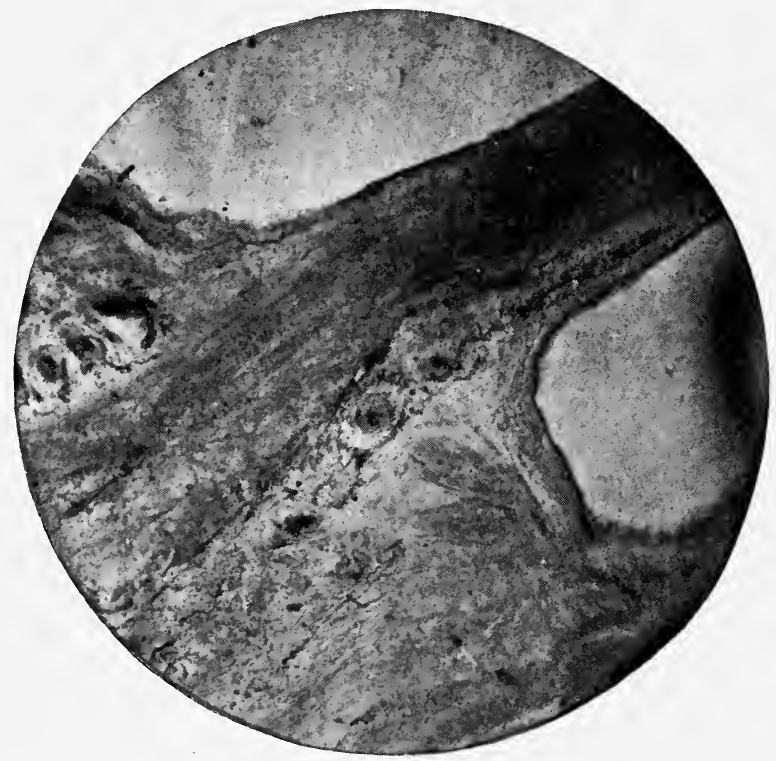

FIG. 52. - Valve in Acute Endocarditis. $\times 25$.

formation of ulcers. On these nodules and ulcers fibrin collects as on a foreign body ; the resulting formations, consisting of granulation tissue covered by fibrin, constitute vegetations. These vegetations may vary in shape from minute elevations to much larger growths having a broad base. While this process is going on, the newly formed vascularization tends to bring about an exudation of fibrin. Masses of this material, whatever their origin, may be rubbed off, and are then carried in the blood current to any part of the body to form thrombi and emboli. Under the microscope these masses show a mixture of proliferated cells. 
The inflammation may extend all over the endocardium, and even penetrate into the inner coating of the arteries.

Symptoms. - The heart action, as usually seen, is rapid and violent, especially in young children, whose thin and soft chest walls most rapidly lend themselves to transmission of the motion. For this reason, partly, the great veins are likewise easily seen to be engorged. At the same time one may notice that the apex beat is displaced. On palpation one detects the irregular heart action, as well as a thrill, which is often of much significance. One may recognize the failing force of the apex impulse as well as its diffusion.

On account of the conformation and relations of the chest walls, the soft, blowing mitral systolic murmur which occasionally comes with this disease is, as a rule, found to be transmitted to the left. Generally this, when present, is distinctly heard, but with this distinctness comes a liability of confusion with other murnurs, mainly hæmic. The second pulmonic sound is commonly exaggerated, on account of the inevitable pulmonary congestion; or as the result of the impeded circulation one may hear a secondary murmur at the tricuspid valve, and sometimes - but much more rarely - at the aortic. Murmurs so located are rarely anything but secondary. All these facts will naturally be modified by the intensity of the cardiac inflammation as well as by complicating signs in other organs.

The general symptoms are neither many nor always quite reliable. There may be plain evidence of pain in the chest, so that even younger children, by their unconscious motions, locate it with a fair amount of distinctness. Handling the patient in any but the gentlest way is apt to eall forth such signs of distress that the attention is necessarily called to the unusual manifestations. There may be coughing, especially if the disease is associated with a dilated heart. Dyspnœa is one of the most constant symptoms which is always disturbing and sometimes startling. It may begin at the outset, but is most apt to appear at the time of intensest inflammation. At the height of the attack, when it is most alarming, it may bring with it as a potent accompaniment varying degrees of cyanosis. Then, in very severe cases, coma may supervene, and, as a frequent result, death comes. On section the heart is found to have stopped in diastole.

The pulse at first is full and rapid; as the disease progresses 
and weakness becomes more pronounced, it grows smaller, irregular, and dicrotic. The variations in rapidity may be great; indeed, the whole character of the pulse may, under the many circumstances of the disease, change to an appreciable degree. The temperature is atypical, rarely running very high, and may be somewhat remittent. At serious stages of the disease it sometimes shows a tendency to fall below normal.

The respiration is rapid and irregular; often the child stops to catch his breath, as though oppressed. The difficulty in breathing is especially apt to follow very high and very low temperatures. At such times of strain the tendency toward congestion of any part of the body is unusually great; even under ordinary circumstances it is great enough to cause various symptoms, which are referable to the part involved. Thus where the peritonæum is concerned, there would be ascites; where the stomach is attacked, there would be nausea, vomiting, and disturbances of digestion ; in the kidneys there would be the symptoms of congestion, with a small amount of urine holding a little albumin and a minute quantity of casts. It is especially common to have an obstinate bronchitis, which at times persists in spite of almost everything that the attendant may do to check it. The vegetations on the valves, or fragments of them, may be swept away from their location, and thus form embolisms in the kidneys, lungs, brain, or other organs. Appropriate symptoms would, of course, then show themselves.

Treatment. - The treatment, after all is said, is mainly symptomatic. Naturally, if there is an antecedent or complicating disease, such as rheumatism or scarlet fever, that must be vigorously treated. For the endocarditis, the first thing to do is to secure absolute quiet and rest of body and mind. And one must remember that children are equally as, or even more, prone to have worries of mind than adults. The diet should be fluid, easily digestible, and not given in too large amounts. For the pain it will in most cases be necessary to give opium, excepting in infants, who may often be quieted with sodium bromide in fairly large doses. In either case the drug must be prescribed in large enough amount to produce its characteristic effect and the desired relief. For the weak heart action one may use with success repeated doses of digitalis, nux vomica, and sometimes alcohol.

All through the disease, lasting from a few days to a month, 
one should keep in mind its exhausting nature. Children very soon show the effects of it, and become markedly weak and anæmic. The attendant has the opportunity of showing, in his ability to cope with these changing circumstances, much judgment and fertility of resource.

Prognosis. - The prognosis for attacks of moderate severity is, on the whole, good. Those which are characterized by a marked intensity of the inflammatory process have little chance of life. Most children recover, but they remain for months weak, and liable to a recurrence of the disease. Repeated attacks become more and more serious, for with each one the organic changes in the heart become progressively more pronounced.

Differential Diagnosis. - Often the disease, especially at the beginning, may be hard to recognize. The heart murmur, which is so characteristic of the chronic disease, is commonly absent. Also, the wear and tear of the sickness bring on a rapid invasion of anæmia which commonly gives a hæmic murmur. This fact should constantly be kept in mind, so that the sound may not be diagnosticated as an organic murmur. The main elements of endocarditis to keep in mind are the cardiac pain and distress, the violent heart action with the frequent accompaniment of a thrill, the marked dyspnœa, and the possible cyanosis. The only disease that is apt to cause confusion is an acute pericarditis. Here the peculiar friction sound, the possible effusion, pericardial swelling, and likelihood of cardiac displacement are sufficient to show the true diagnosis.

\section{Chronic Endocarditis}

This disease is encountered in comparatively large numbers; and in many ways it is intensely interesting. As much as any other, and more than most other diseases, it has a widespread influence upon the child's entire life. It may produce any amount of disability, it may gather in its train an endless procession of direct symptoms and complicating disorders. More than most diseases it calls for a careful consideration of all its possible developments.

Causes. - The causes which produce it do not differ much from those of the acute form. In fact many cases are merely extensions of a previous acute attack. In the vast majority of 
cases the endocarditis accompanies or follows rheumatism; in other patients all that one can find is a history of chorea, or possibly of some bone or microbic disease. The varieties of fotal or congenital endocarditis have been described on pages 41-43.

Lesions. - The lesions are the natural development of those of the acute form. The endocardium of the cavities and the mitral valve is oftenest involved; next in frequency comes involvement of the aortic valves. The tricuspid or pulmonary valves are much less commonly attacked. The surface of the endocardium may have the little smooth beads or the large eroded vegetations that exist in the acute form. The main additional features are the marked production of connective tissue, the destruction of the endothelium, and some infiltration of calcium salts. The changes in the endocardium cause the valves to be twisted, puckered, shortened, and heavy. 'The milder cases show little interference with the smooth surface of the endocardium; the severe cases, on the other hand, are the ones in which roughening and distortion occur.

Symptoms. - The objective symptoms permit, in the attempt properly to interpret them, of the keenest exercise of observation and logical deduction. In fact there is no disease in the exact recognition and treatment of which these faculties are of greater use. For in chronic endocarditis the effects may show themselves in any part of the body and involve widely separated functions. Frequently the abnormal conditions come about in an indirect way, and it is only by tracing the pathological conditions to their logical causality that one reaches a fairly positive result. Many a time a child, with few or no direct objective symptoms of endocarditis, may begin to complain of a disorder which is seemingly far removed, and only after careful examination is the fact of a long continued inflammation of the endocardium firmly established. This, when one takes into consideration the widespread effects of such a disease, is not at all remarkable. For when the integrity of the valves is impaired, an abnormal strain is put upon certain parts of the heart muscle, with the result of a varying amount of dilatation or hypertrophy or both. Or the minute structure of the muscular elements may be affected so that some degree of myocarditis may exist. In other cases the lining of the arteries may be injured so that the child will show the symptoms of endarteritis. In still other cases the tone of the 
arterial vessels will be altered, the blood pressure will be abnormal, and then the step to a congestive inflammation of the kidneys is an easy one. In fact all the viscera may in an analogous fashion be influenced so that corresponding results will show themselves. Thus the lungs may deteriorate to a condition of chronic pneumonitis with patches of emphysema, the stomach may fall into a state of troublesome irritability, the liver, spleen, and even the brain, may give their respective signs of pathological disturbance. In short, there may be a general venous congestion as well as an irritation of the mucous membranes that is multiform, elusive, and wearying to both patient and physician.

The main thing, however, is the endocarditis itself. This may be of any degree of severity and may continue for a variable time, according to its intensity and the patient's power of resistance. Outside of this it shows itself principally in various murmurs, caused by stenosis or insufficiency of the valves. Such a narrowing of the orifices (stenosis) does not permit the requisite quantity of blood to pass, and naturally the chamber from which it flows is overcharged with its contents. Insufficiency results where the muscular tissue as well as the valves is in such a condition that when the blood has passed, the orifice is not entirely closed; therefore some of the blood regurgitates into the cavity from which it came. It is possible by hypertrophy of the heartwall or dilatations of the cavities, which are great enough to equalize conditions, that no effects of pathological changes may be apparent. Such a fact is spoken of as compensation. Again, this artificial equilibrium may, by an acute attack of rheumatism bringing with it an exacerbation of the endocarditis, or by such vicious agents as failing vitality, no matter what the cause may be, fail; then the patient will immediately show the symptoms of cardiac trouble, generally by one or more of the ordinary murmurs.

Mitral insufficiency is very frequently seen in childhood. It gives a systolic murmur, heard most plainly at the apex and transmitted to the left. Unlike some other murmurs, it is nearly always constant, especially if preceded or accompanied by rheumatism. Here, on account of the insufficiency, the blood regurgitates during systole from the left ventricle into the left auricle. As a result, the left ventricle is hypertrophied, or dilated and hypertrophied. The pulse is full. 
Mitral stenosis is indicated by a systolic or presystolic murmur. The presystolic murmur is heard over a small area about the location of the apex impulse. When there is a systolic murmur it is transmitted to the left, but not so distinctly as the murmur of mitral insufficiency. This is one of the murmurs that is not always constant; on the contrary, it may come and go, and sometimes does not appear at all. Here the impediment in the flow of blood lies between the left auricle and the left ventricle. As a result the left auricle and the right ventricle may be hypertrophied and dilated; in some cases the left ventricle alone is

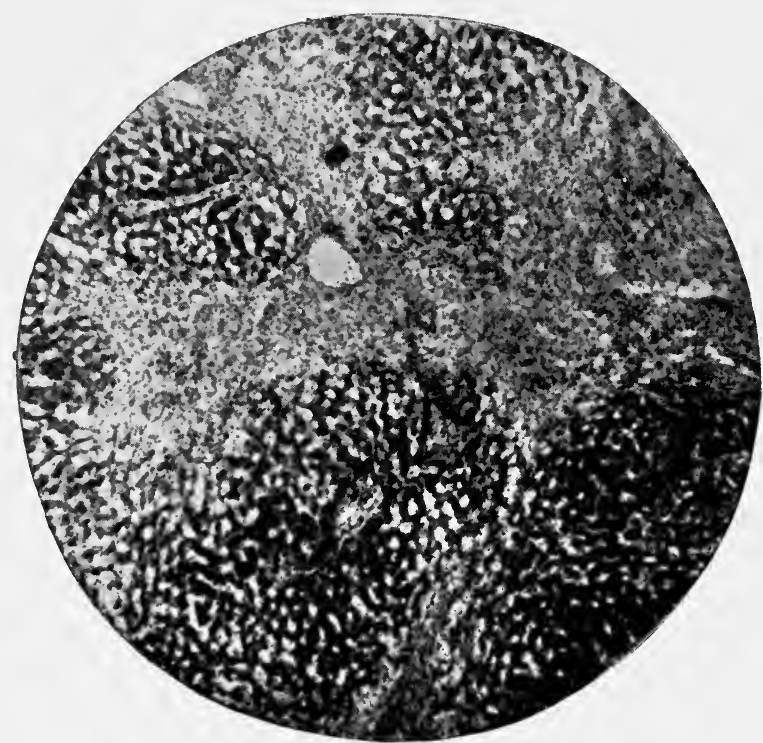

Fig. 53. - Cirrhosis of the Liver. Passive Congestion from Mitral Stenosis. $\times 50$.

thus influenced, or in some few cases the whole heart, and especially the left ventricle, is unusually small.

Aortic insufficiency is for the most part of merely theoretical interest to us, as it hardly ever occurs in childhood. Its murmur comes with the second sound, is most plainly heard at the sternum, especially on the right side, between the second and third interspace, and is transmitted upward to the right and also down the sternum. The lesion gains its activity in the diastole of the heart, and is the cause of regurgitation from the aorta to the left ventricle. Consequently with this lesion one may expect to find dilata- 
tion and hypertrophy of the left ventricle, or at times the ventricle is merely dilated. This condition more than the others is apt to give rise to cerebral symptoms.

Aortic stenosis is also rarely heard in children. Its murmur is systolic, heard at mid-sternum opposite the third interspace, and is carried over into the silence between the two sounds. It is usually transmitted up the sternum to the right and sometimes down the sternum. Sometimes it may be heard at the back to the left of the third dorsal vertebra. Occasionally on autopsy one finds an aortic stenosis in a case that presented no murmur at all. As a result of the lesion both left and right ventricles are dilated and hypertrophied. One can easily understand how this comes about, because the flow of blood from the left ventricle into the aorta is impeded. Both aortic stenosis and insufficiency are apt to accompany mitral disease rather than occur alone, although they sometimes happen alone in congenital heart disorders.

Tricuspid insufficiency is for the most part of theoretical interest. It originates in a rough ventricular surface, or may be due to the presence of thrombi in the ventricle. It is not constant, and when it can be plainly heard is fairly sure to accompany mitral disease. It is systolic, most plainly heard just to the right of the apex, and is circumscribed. Usually it is diagnosticated on autopsy.

The other symptoms do not occur in any fixed order, and may for considerable periods be absent, at any rate in an acute form. The child is apt to be weak and poorly nourished; but until compensation fails he is apt to cause no unusual worry. At the same time he is very liable to react unfavorably to exposure of any sort. Mental excitement is apt to affect him very unfavorably, and his frequently changing condition will perforce excite comment. He is subject to pain, which he refers to the heart, to malnutrition and anæmia, to headaches, dyspnœa, and, in the event of unusual strain, cyanosis.

In young children one of the symptoms first noticed is the unusual heart and pulse actions. We do not so constantly look for differences of tension, which when they exist at all are so slight, and so hard to distinguish as to possess little diagnostic value. The action may be wild, irregular, intermittent, or weak. When it is weak, one is often able to count a greater number of pulsations in the arteries than beats of the heart. The temperature is 
variable, depending upon associated conditions. The respiration often follows the pulse, and when the disease is at all severe the dyspnœa may be alarming. When it is very bad, cyanosis will naturally follow.

In some severe cases one may find a subacute nephritis in which the urine contains albumin and occasionally casts. A more frequent set of symptoms may be referred to the lungs, where the congestion may occasion a bronchitis. In other cases a true pneumonitis, also of a subacute type, may be distinguished. This pneumonia is of an obstinate character, for not only are the capil-

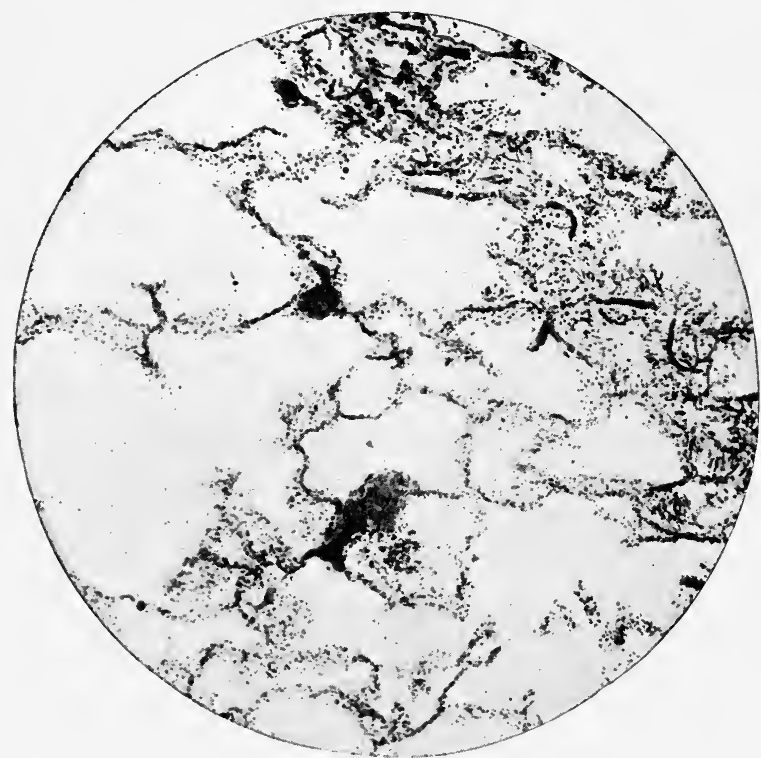

Fig. 54. - Pneumonia of Endocarditis. $\times 50$.

laries dilated and the lung tissue in a state of hepatization, but also the walls of the air spaces are materially thickened, and there is a considerable laying down of new connective tissue. In such an inflammation the color of the lung shows the effect of a pigmentation and is darker than in a primary inflammation.

The symptoms of congenital endocarditis are mentioned on page 43 .

Treatment. - The treatment is largely symptomatic. Heart stimulants and tonics may at various times be needed. At others it may be necessary to treat complicating symptoms. It is always 
desirable to correct the anæmia which springs up on slight provocation and persists with obstinacy. Even small attacks of physical deterioration require careful attention and tonics. When compensation is defective digitalis is beneficial, especially upon the left heart, while strophanthus has a good influence on the right side. In the flurry of an acute exacerbation, especially in the older children, the use of opium will often give valuable results. Strychnine or nux vomica is a very useful drug, and may be combined with iron or the simple bitters. Most of all it is of the highest importance to regulate every part of the child's daily life, every detail of rest, exercise, clothing, and study. Regular and systematic exercise should be prescribed, always excluding whatever involves much strain. Thus, walking, riding, driving, and similarly moderate exercises are of the greatest benefit. The child should be under constant supervision so that no detail may escape observation.

Prognosis. - The outcome of the disease is better than one would expect. Compensation comes quickly in children. And even in severe cases the patient will sometimes live and seemingly recover in the face of disabilities which at first seemed inevitably fatal. Nevertheless a guarded opinion must be given, even in mild attacks; this is especially true when the general circumstances of the patient are not of the best.

\section{Malignant or Mycotic Endocarditis}

This form of endocarditis in children has been regarded as a pathological curiosity. It doubtless occurs rarely; and therefore when it does, it may be so difficult of detection that one should not be too much blamed for failing to recognize it. Most of our information concerning it comes from the study of adult cases, but as there is practically no difference brought about by age, and since the degree of fatality in children is as severe as in adults, such knowledge efficiently serves our purpose.

Causes. - The disease does not occur as a primary disorder, but follows such sicknesses as are able to provide the infecting material. These are the acute infectious fevers, purulent diseases of the bones, septicæmia, and pyæmia. In these cases, no matter with what sickness they may be associated, cultures made from the vegetations show the presence of diplococcus lanceolatus, 
staphylococcus pyogenes aureus, gonococcus, or streptococcus pyogenes. A previous lesion of the endocardium favors the lodgment and development of the bacteria at the affected part.

Lesions. - The pathological process seems to start from the subendothelial layer. The involved parts become swollen, filled with white cells, and covered with fibrin and bacteria. Here and there the endocardium is covered with vegetations of different sizes and of varying consistency. The smaller ones as a rule gradually accumulate a fibrinous covering which checks further development

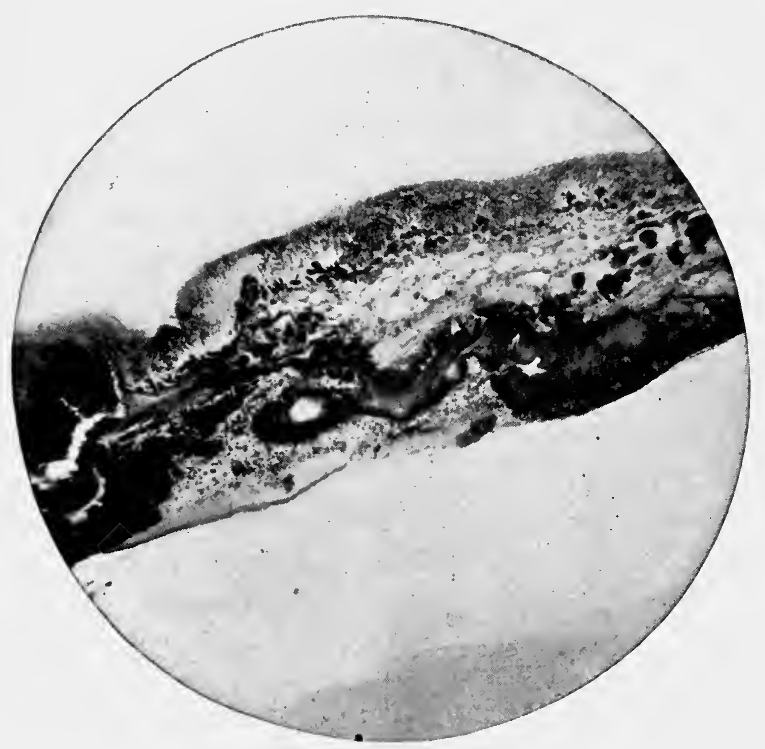

FIG. 55. - Aortic Valve: Acute Mycotic Endocarditis. $\times 30$.

and change. Or the process may not stop here; the vegetations may increase in size to a marked extent, and alter their comparatively benign character. They then seem to be made up of necrotic tissue, proliferated endothelium, and masses of bacteria. Coagulated blood is deposited on them and helps to swell their size. Such parts have a tendency to break down in necrosis, thereby forming ulcers; detached portions are torn away by the blood current, and find a lodgment either in the heart cavities or any of the viscera. Thus they may act as thrombi or emboli of an infectious nature. 
Symptoms. - The physical signs do not differ naturally from those of an acute simple endocarditis. The disease may be ushered in with a chill, or there may be a number of them ; nevertheless this is not invariably the case. One naturally expects to see ordinary symptoms of endocarditis plus those of a septicemic invasion. The temperature may mount up to $41^{\circ} \mathrm{C}$. $\left(106^{\circ} \mathrm{F}\right.$.)

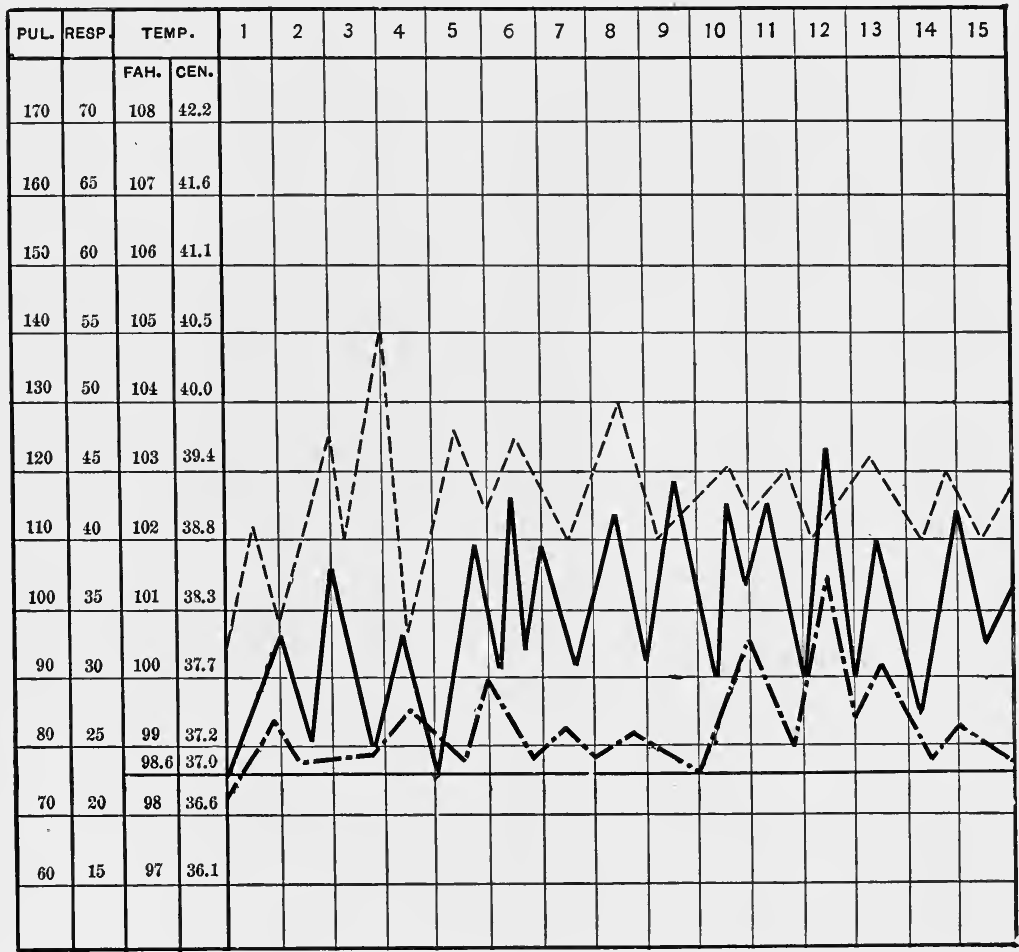

PULSE, RESPIRATION AND TEMPERATURE CHART OF MALIGNANT ENDOCARDITIS. AGE, 9 YEARS.

PULSE

TEMPERATURE

Fig. 56.

or more in the evening, and then in the morning may fall two or more degrees. It may be very irregular, or even distinctly remittent. The quality of the pulse and the character of the respiration follow the variations of temperature, the pulse especially showing a disposition to become irregular. There may be symptoms of embolism, more frequently referred to the cerebral arteries than to other vessels. The urine regularly contains albumin and 
casts, the spleen usually is enlarged, soft, and tender, the liver may be enlarged, the serous membranes may become subject to acute inflammation either simple or purulent, and the joints may be similarly affected. In the more mature children lodgment of emboli may cause paralysis, in infants more frequently convulsions. Any or all of the valves may be involved, so that one is apt to hear various murmurs. Some authors believe that the rarer heart murmurs should always suggest malignant endocarditis.

Treatment. - The treatment is purely symptomatic. One should do everything possible to keep up the patient's strength. Even although the prognosis is thoroughly bad, nevertheless one's efforts should never relax. It is especially important to use stimulants up to the point of toleration.

Prognosis. - The outlook is gloomy. One cannot give a reliable opinion which will cover all cases; in fact, each must be worked out by itself, and the separate factors of endocarditis and septicæmic infection must be regarded as complementary forces. Few children survive the disease.

Differential Diagnosis. - The diagnosis is often very hard to make. The most one can say is that an endocarditis which has an emphatically septicæmic character may be taken to be mycotic. The presence of a remittent temperature may cause the observer to make a diagnosis of malaria. But in this event there will be no characteristic history, no plasmodium in the blood, and no response to the administration of quinine. In addition, observation of the disease will soon correct the error. Very commonly the diagnosis is made on autopsy.

\section{Cardiac Hypertrophy and Dilatation}

There is a large class of cases in infants and children to which sufficient attention has not been given. The patient complains of symptoms which are referable to the heart, and yet one cannot hear heart murmurs nor distinguish the regular signs of endocarditis. Such cases are apt to worry the parents as well as the physician; for, although the children do not seem dangerously sick, nevertheless they have many disturbing symptoms, and their general development is substantially retarded. These cases come properly under the head of hypertrophy and dilatation, presenting the picture of an overacting heart. 
Cardiac hypertrophy is more frequent in infants and children than in adults, partly on account of the weaker muscular tissue, partly from the frequency of the responsible causes. In some instances it seems to occur as a congenital condition. The usual rule, however, involves the idea of a naturally weak and pliable tissue put to a severe test, and thus showing the effects of strain. The cases of muscular strain due to prolonged work and athletic exertions in adults are replaced in children with strain due to violent coughing (as in pertussis or severe bronchitis or asthma), or excessive crying; to the constant restlessness, the running, jumping, and continuous activity of early years. As far as precedent pathological disabilities go, one should mention, as predisposing causes, endocarditis, serious pulmonary disease, congenital atresia of the aorta, chronic nephritis, rheumatism, the acute infectious diseases, and prolonged disorders of malnutrition.

Cardiac dilatation, like hypertrophy, may be seen much oftener in children than in adults. It may happen as a fortunate accompaniment of hypertrophy, resulting in an equilibrium between these two abnormal conditions. In consequence, the heart may work in a regular and satisfactory way, and in the course of its normal development may use the increased size in place of the expected growth. Thus the child may in time lose all trace of his previous lesions. On the other hand, it may come less fortunately as an induced condition of -heart weakness after cardiac and pulmonary disease, in which the blood pressure has been greater than the heart muscle's power of resistance. Again, a thinning of the muscular structure, due to wasting diseases, may cause larger cavities than are normally found, although the size of the whole heart is not increased. Another common cause, in young children even more than in youths or adults, is acute inflammation of the lungs and pleura. This action comes about by obstruction of the pulmonary arterioles so that the blood is dammed back upon the right heart. In such ways one can account for the origin of many cases; on the other hand, one is apt to meet patients in whom no assignable cause can be discovered. In all likelihood the ætiology of these cases is obscure simply because of the habit of poor observation in regard to young children.

Symptoms. - An hypertrophied heart may often be diagnosticated on inspection. The chëst walls are so thin and pliable, that the increased size of the heart is seen by the bulged-out contour 
of the præcordium. The apex beat is likewise seen with greater distinctness than in normal cases, while its position is changed according to the line of greatest increase. If the left ventricle alone is hypertrophied, the length of the heart is increased. Naturally the beat would then be seen farther to the left as well as lower down. If the right ventricle alone is hypertrophied, the heart increases in size on its right side This would not necessarily change the apex beat at all, or it might be seen farther to the right than normal. When both are hypertrophied, the result would naturally be the sum of both.

Percussion will show an increased area of dulness, and when carefully practised will sometimes tell us what part of the heart is increased.

Auscultation will give a muffled and softened first sound which is in contrast to the sharp, hard, and definite character of the second.

In dilatation one looks for the greatest changes in the auricles sooner than in the ventricles. The apex beat may again be changed if the dilatation is accompanied by hypertrophy; otherwise it is in its normal position. In the latter case one does not look for a bulged-out chest wall; in fact, the conformation of the anterior thorax may not, unless in the presence of further pathological factors, be materially changed from the normal. By palpation one may notice the weak, weary, and undecided heart action. In other cases this weakness may be so marked that the impulse is very gently spread over a comparatively large surface; as a result one can often detect it with the eye even though the superimposed hand is unable to feel it.

From dilatation alone one commonly does not expect much of a change in the percussion note. On auscultation one hears a sharp, metallic accent to both sounds which gives the impression of strain or effort. On slight provocation such a heart loses its normal and regular action, taking on a functional character that may be eccentric and puzzling.

One of the most noticeable symptoms of hypertrophy and dilatation is a short, hard cough, occurring without adequate involvement of the lungs. This cough is worst at night and early morning, while during the day it may almost be, and possibly is, quite absent. In general the child's nutrition is apt to deteriorate, he looks pale, he lacks energy, his appetite is poor, 
and he is inclined to suffer from attacks of bronchitis, headache, and the effects of vicarious congestions. The mental condition may be somewhat similarly influenced so that it is noticeably dull and apathetic.

Treatment. - Attention to the regulation of the child's life, to the enforcement of quiet in action and habit, to strict hygienic and dietary control, and the administration of tonics will do much to eliminate the evil effects of these conditions, and allow the impeded heart to resume its normal size, contour, and functions. For the cough, large doses of bromide of soda, with or without nux vomica as the case demands, will be found of use. In older children codeine may prove of marked service. These drugs should be soon changed to tonics, such as the compound syrup of hypophosphites or the organic preparation of iron with small doses of strychnine. In the use of these means one should under no circumstances neglect the main factor of regulation of every detail of daily life.

Prognosis. - The outlook usually is excellent, so that with conscientious attention the patient should in a short time be completely cured.

\section{Anamic Murmurs}

Anæmic murmurs should in most cases be diagnosticated without much difficulty from those of organic heart lesions. They are not constant, there are no regular signs of heart disease, and there is no history of the antecedent conditions of endocarditis. As the functions of the heart are not altered, so its size and contour are not regularly changed. The child is plainly ancmic, may for a time have systolic murmur, which is heard over the heart and the large vessels as well, and recovers in a comparatively short time after appropriate treatment has been instituted. His improvement need not go very far before the murmur disappears. The treatment is that of the anæmia.

\section{Pericarditis}

The rare phenomenon of an inflammation of the pericardium is one of the most serious diseases from which infants suffer; older children stand the strain somewhat better, but even their greater vitality is poor when opposed to the wearing, exhausting, 
and distorting effects of the disease. Generally we recognize three forms of inflammation, - one characterized by the production of fibrin, another by that of fibrin and serum, and the third by that of fibrin, serum, and pus.

Causes. - The disease very rarely occurs as a primary disorder; often it accompanies or follows some sickness which has the power of general infection. In older children, as in adults, rheumatism is a common cause ; but infants, being less frequently rheumatic, do not so frequently exemplify this rule. Pericarditis,

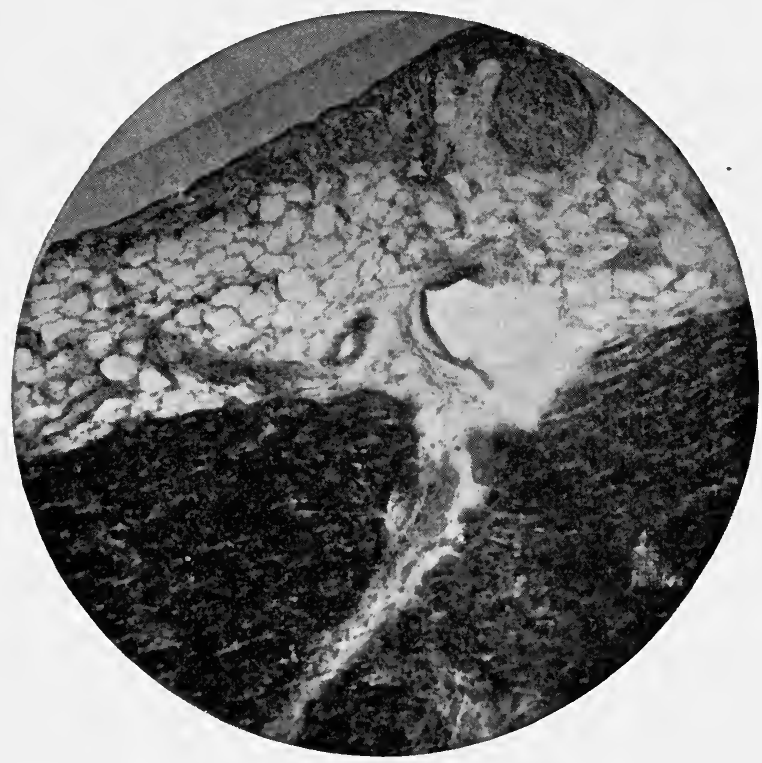

Fig. 57. - Normal Pericardium. $\times 50$.

in some very few cases, begins before birth as a so-called idiopathic affection, or as the result of septicæmic contagion from puerperal disease in the mother, or from inherited vice. Under these conditions the effusion may be either serous or purulent. After birth it may appear, seemingly without cause, as the concomitant of nephritis, of inflammation of neighboring organs, as the lung or pleura, with endocarditis, with septicæmic diseases either local or general, with bone diseases, and with the acute infectious fevers, notably scarlet fever and diphtheria. The micro-organisms commonly found in this disease are the usual streptococci 
and staphylococci of pus processes, the bacillus tuberculosis and bacillus lanceolatus.

Lesions. - The pericardial membrane becomes congested, the capillaries being dilated and visible. Fibrin in varying amounts is thrown out and covers the surface irregularly. If the amount is large, adhesions are formed at the points of greatest collection between the two pericardial surfaces. On recovery part of the exudate is absorbed; the rest, however, may remain and slowly give way to permanent connective tissue. A large number of

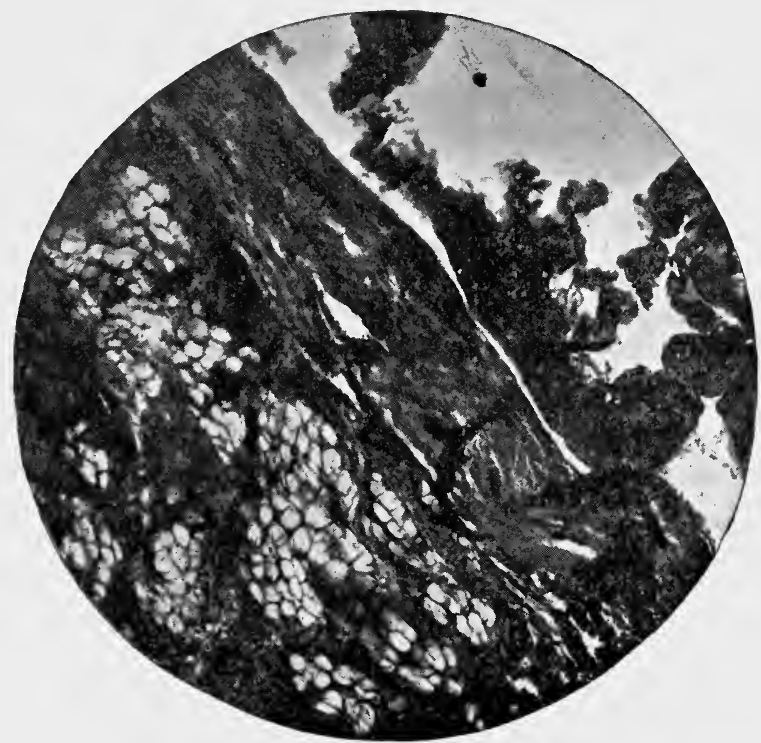

Fig. 58. - Simple or Fibrinous Pericarditis. $\times 50$.

cases may in addition have an exudation of serum, more or less tinged with blood. It collects in the lower and posterior part of the sac, forming a wedge-shaped body. The serum may be quickly or slowly absorbed, and then the fibrin remains as in dry pericarditis. This is what commonly happens in young children who may on post-mortem examination be found to have had a primary inflammation without fluid.

The purulent form is likewise more common, comparatively, in children than in adults. Here there is a distinctly purulent exudation tinged with blood. Allso one notices a fairly large infiltration of the membrane itself, with small cells. This class is natu- 
rally the most serious, because the exudate is both large in quantity and poisonous in character. Likewise does this class leave the largest number of permanent changes, such as adhesions between the pericardial surfaces, a tendency to septicæmic attacks, the formation of connective tissues which become thickened and toughened by calcific infiltration, a proneness to tuberculosis, usually pulmonary.

At times one sees a form of pericarditis that may roughly be called hæmorrhagic. The dilated and newly formed vessels, as

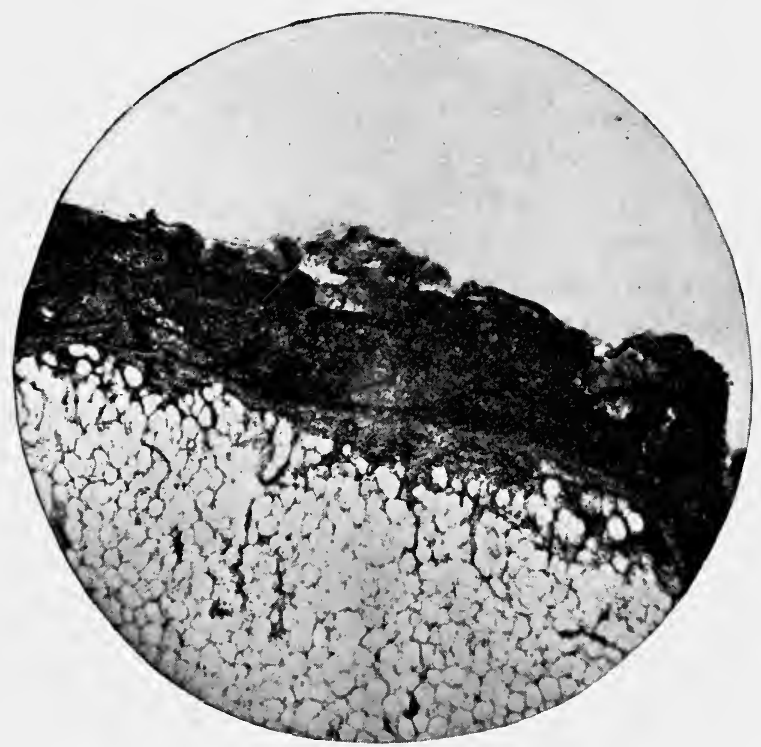

Fig. 59. - Acute Sero-Fibrinous Pericarditis. $\times 30$.

well as the surrounding tissue, are very delicate, and at the least provocation break, allowing hæmorrhage in various degrees. And even without a direct rupture there may be an effusion of blood through the wall of the vessels. This form is always serious, more on account of the physical depression which accompanies this condition than the mere fact of the hemorrhage.

Symptoms. - In the first form there may be a friction sound, which when present is characteristic. It can be heard at both first and second beats of the heart, but not exactly synchronous with them, and is independent of the breathing. It is apt to dis- 
appear quickly, especially if effusion is present, but on absorption may reappear. When present, it is best heard at about the nipple line, between the nipple and the sternum. It is usually harsh and rubbing, and when once heard should not be confused, as it sometimes is, with a congenital heart murmur.

With effusion of serum the area of dulness is increased, and assumes a roughly triangular shape having its base at the bottom. Formerly it was thought that this general form was easily confused with that of an enlarged heart, but that the latter was

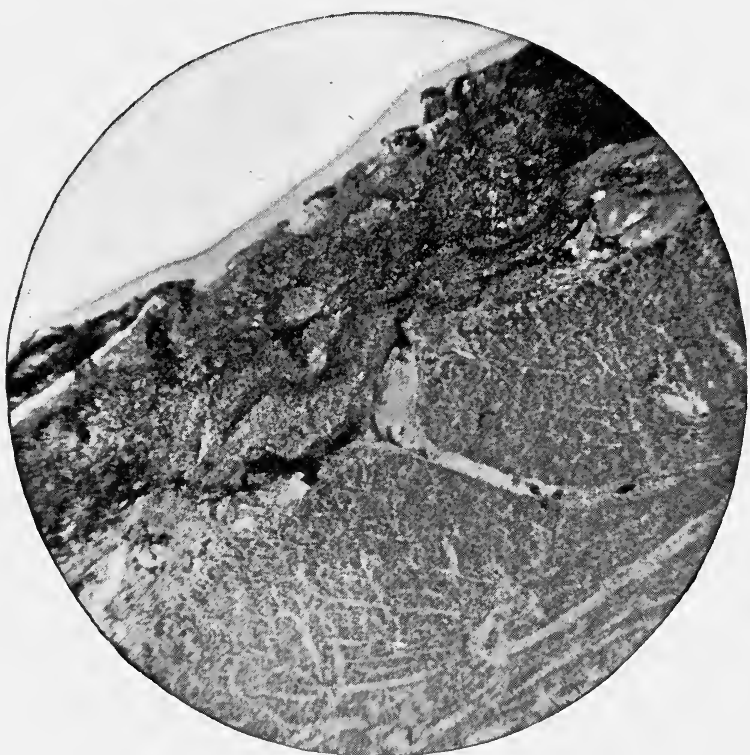

FIg. 60. - Acute Fibrino-Purulent Pericarditis. $\times 30$.

differentiated by a space of dulness to the right of the sternum. Since then observations and experiments, notably those of Rotch, have demonstrated that a pericardial effusion commonly gives an even greater area of dulness to the right of the parasternal line than the enlarged heart. The main point of distinction lies in the general form of the area of dulness : that of the enlarged heart resembles a truncated ovoid, while that of effusion is, as stated above, a triangle which rests like a saddle upon the diaphragm. As the fluid increases in quantity, the pericardium is apt to thicken, the heart sounds are muffled, and the pericardium, 
on account of the pliability of the chest walls, is apt to bulge out. In very marked cases the dulness may cause an alarming dyspnœa with cyanosis.

In the purulent form there is a tendency to a large exudation and a chronic course. The apex beat, owing to the effusion, may be absent, but in its place one often feels a wavelike impulse which may be transmitted to some distance from its source. In the newly born the only sign may be a wild heart beat; the older children have a greater liability to begin the disease with a chill or convulsions. The physical signs may be somewhat obscured or changed by adhesions, but these occur much less often in children than in older patients. This new tissue finds its natural and best opportunities for growth in the purulent exudation, and the alditional gravity which it entails is always considerable.

Precordial tenderness, especially in older children, is always apparent. The patient is easiest when lying with rigid body and elevated head, and change of position induces discomfort and oppression, which increase in proportion to the amount of displacement and compression of viscera. In the third or purulent form there are, in addition, the symptoms of septicæmic invasion, the lowered vitality, the worried, anxious look, the tendency to stupor and convulsions. The disease may be complicated with a myocarditis either circumscribed or diffuse, an endocarditis which is usually very dangerous, or an extension of the disease to some other serous membrane.

There may be an effusion of serum into the sac caused by heart, lung, or kidney disease, by malformations or tumors of the chest. Since there is no inflammation involved, the condition is called hydro-pericardium. A related phenomenon, hæmo-pericardium, consists of the presence in the sac of a fairly large quantity of blood which has escaped by rupture of the arterioles. Or by fermentation of putrefactive material there may be a production of gas. This is the so-called pneumo-pericardium.

Treatment. - The two main indications for treatment are the tendency to heart failure and the presence of pain. The first is combated by digitalis, strychnine, and tonics. Entire ease and undisturbed rest must be provided. For the pain bromide of soda in infants and opium in older children may be necessary. Some relief can almost always be obtained by the use of cold applications, such as light ice-bags. The diet must be as light 
and nutritious as possible, and every attempt to help excretion, especially by the kidneys, must be made. When the effusion collects much more rapidly than it can be eliminated, it may be necessary to practise paracentesic. This is accomplished by aspiration of the pericardial cavity in the fifth right or left intercostal space. In some few cases of purulent effusion, it may be necessary to resort to the dangerous operation of opening and draining the pericardium. Naturally, when there is an antecedent disease, its treatment must occupy a place of much importance.

Prognosis. - The outlook is generally unfavorable, and especially so when there are complications.

Differential Diagnosis. - The condition with which pericarditis may be confused is endocarditis. Between these two diseases the principal point of differentiation is the abnormal sound which is heard on auscultation. In pericarditis it is crisp and harsh, it accompanies both sounds of the heart, it may under certain circumstances disappear and reappear, and it commonly fades away as the disease subsides. This, as one can immediately see, constitutes quite a different impression from what one finds in endocarditis. If effusion is present, the added signs will make the diagnosis yet clearer.

\section{Chronic Pericarditis}

Lesions. - In the chronic form the lesions are the same as in the acute, with an accentuation of the tendency to form connective tissue and curiously shaped adhesions.

Symptoms. - The symptoms after the acute attack may be not especially prominent. The physical signs are much the same as in acute pericarditis, with the possible addition of distortions of the chest, an enlarged heart, myocarditis, and endocarditis. There may be recurring attacks of cardiac weakness, and a general malnutrition. Exact diagnostication of the condition calls for judgment and thoroughness of examination.

Treatment. - The treatment tries to build up the general health and strength by every aid which tonics, diet, and hygiene can afford. Each case will call for its own proper methods, for what may be needed for one child would for another be superfluous. The varying needs of the patient will, in all likelihood, 
call for the exercise of the full resources of the attending physician.

Prognosis. - The outlook is not good. The patient has a probable future of weakness, functional debility, recurring exacerbations, and complicating visceral congestions. His parents or attendants should regard him as living under a constant threat. 


\section{CHAPTER XVII}

\section{DISEASES OF THE BLOOD}

\section{Simple Secondary Anmemia}

As the result of unfavorable and exhausting conditions, children easily fall into a state of hæmic deterioration. The younger the child, the more easily does this happen, and the more serious are the results apt to be. On the other hand, they recover with comparative ease, so that such an anæmia bears the characteristics of a transitory phenomenon rather than a definite disease. Logically, it is a symptom whose appearance can be predicted, providing certain precelent causes, organic or functional, have been set in motion. These causes are descent from parents who, at the time of conception and pregnancy, are anæmic and exhausted, deficient blood supply or hæmorrhage from the cord, hereditary disease, and poor quality of food during the first few weeks of life. After this initial period every factor which makes for deficient nutrition, for impaired function, for waste of tissue, and for organic disease, may likewise be regarded, indirectly at least, as a cause of anæmia. This is so constant and significant a factor in the care and development of a child, that it must be reckoned with in every abnormal condition in which he may happen to be. In infants the most fertile causes of anæmia are provided by the various severe disorders of the stomach and intestines, which not only absorb vitality, but also diminish the possibility of assimilation and growth. As the child grows older, the number of diseases which affect him become larger; and, likewise, there is a correspondingly larger amount of toxines and tox-albumins absorbed, which are in large part responsible for the process of degeneration in the quality of the blood. Under this heading come such diseases as tuberculosis, diphtheria, scarlet fever, and the other infectious diseases. Closely allied to these, as far as their effect goes, 'are unhealthy environment, uncleanliness, bad food, intestinal parasites, and pathological conditions like rickets 
and scurvy. In fact, in practically all diseases it is very likely true that anæmia results not so much from what is taken from the system as what is added, in the form of poisonous products, to it. Even in malaria, in which we know that the plasmodium attacks the red blood corpuscles themselves, it is not at all certain that the anæmia is due entirely or mostly to this struggle, but rather to the formation of toxines which are simultaneously formed. The action of certain well-known drugs, such as most salts of mercury, the iodides, and potash salts in large doses, is often fully as sure as that of rheumatism or nephritis.

The changes are confined, for the most part, to the blood. There is a variable decrease in the number of red blood corpuscles, of hæmoglobin, and the specific gravity. In addition, many of these cells may be altered or injured in form and possibly in function. The leucocytes are often increased both relatively and actually.

Symptoms. - The most prominent sign of the sickness is the paleness of the skin, mucous membranes, and ocular sclerotic. The ears and the tips of the fingers and toes have a particularly blanched appearance. The child is listless and weak, irritable, not easily interested. The appetite is poor and fickle, and digestive disturbances of the gastro-intestinal track are common. The cardiac action is weak and sometimes irregular. Anæmic murmurs may be heard over the precordial region, and a loud buzzing sound may often be distinguished over the great veins of the neck. Children who are able to run about may complain of dyspnœea or a short, dry cough that is at its worst at night and morning. The weakened circulation renders congestive disturbances of the lungs probable; and with this is associated a proneness to inflammations of the nose and throat. At such times the hypertrophy of the pharyngeal, as well as the faucial, tonsils is easily contracted, and may give so distinctive a mark to the symptoms as to stand out as the primary cause of the general condition, while the anæmia may be taken as one of the results. Hæmorrhage from the nose and throat is not infrequent, but is rarely serious. The spleen and lymphatic nodes may be enlarged, although not always enough to be plainly perceptible. On the other hand, the glands of the mediastinum may be sufficiently hypertrophied to give pressure symptoms.

These poorly nourished children are liable to subjective 
impressions that may have a far-reaching effect. During sleep they may have alarming dreams that make serious impressions on the mind, and turn it toward abnormal manifestations. Such impressions may persist in their waking hours, and may color the whole child life with a false hue. They may have pains in the head, over the heart, in the abdomen and extremities, that are apt to mislead one into conjecturing some local disease of the part affected. Some children become troubled with a weakness of sphincter control, which is oftener seen in the bladder than the rectum. Anæmic girls of any age often have some leucorrhœal discharge that cannot be entirely cured until the anæmia has disappeared.

The treatment consists in removing, as far as possible, the causes, and following the directions concerning hygiene and drugs which are recommended near the close of the section on primary anæmias.

The outlook is good in almost all cases. Where doubt should be felt is in those somewhat unusual instances of prolonged neglect which appear in hospital and dispensary practice. This does not include the extreme anæmias which occur in the course of long drawn out diseases. In these children it is hard to apportion to each phase of the sickness its suitable responsibility.

In ordinary attacks there is little chance for confusion. It is only in the very severe cases where one may be in doubt whether the complaint is not chlorosis or pernicious anæmia. In simple secondary anæmia there is a simultaneous reduction in both hæmoglobin and red blood-cells, in chlorosis the greater reduction is in the hæmoglobin, while in pernicious anæmia the decrease is the most marked in the red blood-cells.

\section{Chlorosis}

This disease in past times was called morbus virgineus, since it occurs mostly among young girls. From ten or twelve up to seventeen or eighteen years is the usual time for the disease to appear. It is not, however, confined to this period of life, but may occur in young and even middle-aged women. Although it is largely a disease of girls, nevertheless one occasionally sees instances of it in growing boys which correspond to all the ordinary tests. This item has for a long time been one of the 
grounds of contentions which have flourished so richly in the various phases of this disease, - one party contending that chlorosis is exclusively a complaint of young girls, the other that it appears in both sexes. But there is so much uncertainty in our ideas covering all the parts of chlorosis that no special stress need be laid on this point.

Causes. - The origin of this disease is unknown. Many theories have been enunciated to account for it, but one after the other has been overthrown with greater ease than is required in most doubtful diseases. Poor food and unhygienic surroundings have been cited as causes; but they are plainly not responsible, for many of these patients are in these respects fortunately situated, while as a counter fact every one knows that only a small percentage of the vast number of young people in poor circumstances have chlorosis. The disease occurs alike among the rich and poor, in the city and the country. Emotional excitement is just as unreliable as a sufficient cause; and the same may be said of losses of blood by hæmorrhage. Heredity seems to exert some influence, especially in those families where a large number of children is the rule. Even this influence is partial and does not explain the large number of cases in which there is no history of parental chlorosis. Some writers have laid much stress upon auto-intoxication from the intestinal canal, others upon uric acid poisoning, and still others upon an unknown microbic agent as the effective ætiology. But one and all are not proven. It is scarcely necessary to refer to the theory of hypotrophy of the arterial system, although it has been a favorite theory in Germany. In short, we are as far from a solution of the problem as we can be, especially in view of the centuries of familiarity with the clinical aspects of the disease.

Lesions. - Changes occur in the blood and viscera; and the more thoroughly one examines these cases, the more widespread are the effects of the disease found to be. The blood.is thinned, is brighter in color than normal, tending to assume an orange hue. Its hæmoglobin is much diminished in amount, being two-thirds or even one-half as much as normal. The red blood-cells are less reduced in number, and irregular forms may be seen; the number of poikilocytes, microcytes, and megalocytes varies from case to case. The number of blood-plaques is greater than normal, but that of leucocytes is practically unchanged. The specific gravity 
of the blood as a whole is somewhat lowered, while that of the serum is unchanged or raised. The reaction of both serum and the whole blood is in many cases more strongly alkaline than usual. Although the amount of fibrinogen is decreased, the tendency to the formation of thrombi is increased.

The heart and the great vessels have been found in some subjects to be under-developed. At the same time the cardiac fibres may be sufficiently weakened to produce a dilated right side with a compensatory hypertrophy of the left ventricle. There is a tendency all through the viscera to fatty degeneration, which is not easily distinguished excepting in severe and prolonged cases. In the heart it seems to make most headway in the left ventricle, and may extend to the vessels. It may gradually involve parts of the liver, the spleen, the stomach, and possibly the intestines. In the stomach the circulation may be sufficiently imperfect to allow the breaking down of small areas of mucous membrane. Most frequently the lack of tone in the gastric muscular tissue is great enough to render temporary dilatation from the piled-up food or the gas of fermentation inevitable. In some cases a lack of development in the uterus and appendages has been observed.

Symptoms. - One of the most characteristic signs is the peculiar yellow or yellow-greenish pallor of the skin. Most of these patients have a waxy uniformity of complexion; but there are exceptions in whom there is a bright patch on the cheek-bones. The inucous membranes are blanched, the sclerotic has a bluish tinge, the ear is waxy. The pupil is commonly dilated, and helps to give vivacity to the rather expressionless face. For the muscles may become so atonic as to be partially inefficient in depicting fine changes of emotion. The pulse is apt to be irregular and weak, the area of cardiac dulness is increased to the right; and on the least exertion the patient complains of dyspnœea. Hæmic murmurs may be heard over the region of the pulmonary artery, the aorta, or the apex, which are doubtless due to muscular flaccidity. These are often increased when the girl lies down, and under all circumstances are apt to vary in intensity. In the large veins of the neck a humming, buzzing sound may be heard, which is often greater on the right than on the left side. This likewise is variable, and from time to time may change considerably. There may be œdema of the feet and under the eyes which has no relation to a nephritis. 
In the digestive system almost all of the changes are based on atony. There may be constipation, fermentation of gastric and intestinal contents, and a temporary dilatation of stomach and often of the colon. The tongue is pale, fairly clean, and thick. The appetite is poor and capricious, sometimes perverted. Sudden nervous impulses may turn it in one direction or another. Not only in this respect but in the various functions and capabilities of the whole body these diverse influences play an important rôle. They may give rise to headaches, nausea, divers neuralgias, choreic symptoms, unreliable caprices, and outbreaks of multiform hysteria. There may be amenorrhœa, dysmenorrhœe, or menorrhagia. Rarely, in older girls, a thrombosis of the cerebral sinuses or of the veins of the legs has occurred. Many observers have remarked a "chlorotic fever," which seems to me to be caused by some intercurrent condition rather than by the chlorosis itself.

The treatment is given near the end of the section on anæmias.

Prognosis. - The outcome of this disease, although it may be delayed by various fluctuations, is almost always favorable. One of the greatest obstacles resides in the fickleness and unreliability of the patients, which render strict obedience to orders difficult. Thus the course of the disease may extend over months or even years. But so long as the condition has been accurately diagnosticated and the treatment faithfully carried out, a cure should result within a time measured by weeks rather than months.

Differential Diagnosis. - First of all one should ascertain whether the anæmia is primary or dependent on some precedent complaint, such as malaria, rheumatism, lead poisoning, nephritis, and other exhausting diseases. If these factors have been excluded, an examination of the blood, combined with the objective signs of the disease, will clear up any doubt.

\section{Pernicious Anfemia}

Chlorosis is mysterious in its origin; and if one may make a comparison, pernicious anæmia is much more so - it is baffling. While no one pretends to know how it arises, very many writers follow the classic examples of suggesting as possible causes all of the ordinary difficult and exhausting conditions in life. Thus, over-work, over-worry, intestinal parasites, severe sicknesses, profound anæmia, exhausting hæmorrhages, congenital syphilis, rachi- 
tis, auto-intoxication from the intestines, severe diseases of the gastric and intestinal mucous membranes, and micro-organisms have been mentioned. 'The connection between any or all of these diseases and pernicious anæmia has not been proven; on the other hand, it partakes largely of the nature of coincidence. As a counterfact one may note the occurrence of all these conditions without the sequence of pernicious anæmia, and the appearance of the anæmia without any visitation of the preliminary disorders. The true cause is yet to be discovered.

Lesions. - The blood is paler than normal, and its specific gravity is low. The striking feature is the great reduction in the number of the red blood-cells, which at the worst stages of the disease may be only 500,000 or even less to the cubic millimetre. Not only are the cells decreased in number, but also they are altered in form. The large proportion of poikilocytes is very striking, and some of them have been said to exhibit active movements. In addition there are fairly large amounts of microcytes and macrocytes. Nucleated corpuscles occur in their various phases of gigantoblasts, normoblasts, poikiloblasts, and microblasts. The nuclei as well as the blood-cells vary in different cases in their disposition to take up stains. The number of leucocytes may be slightly decreased and the cells themselves may be small in size. The blood-plaques are reduced in number.

Hæmoglobin is reduced in quantity, but not as much as the red blood-cells. It may be found in the plasma, as may methæmoglobin, and in some red blood corpuscles is present in unusually large amounts. The reaction of the whole blood is often markedly alkaline, on account of the increase in the alkaline chlorides, while at the same time the phosphates are diminished. The total quantity of blood seems to be diminished.

Throughout the thoracic and abdominal viscera there seems to be a noteworthy tendency toward the production of fatty degeneration; even the diaphragm may be involved. The heart may show this change in any part of its substance, and as the degeneration occurs there will also be a simultaneous growth of dilatations in the weakened tissue. The liver, kidneys, spleen, and pancreas show areas of fatty degeneration and softening, as well as deposits of iron and pigment. The size of these organs may be increased, and they-may be studded with patches of parenchymatous degeneration. The stomach shows some atrophy 
of its epithelium, its muscular layer is thin and easily dilated; similar lesions are present in the small intestines, and the mucous layer holds a noticeable excess of iron. The urine is markedly alkaline in reaction, contains some albumin and casts, especially hyaline, large amounts of normal and pathological urobilin, urea, and uric acid. After the disease has been actively set in motion, a sclerotic process may begin in the posterior cord which gradually works its way around to the lateral and anterior columns, principally in the cervical portion. All through the body there may be small ecchymotic hremorrhages, in the cord, the mucous

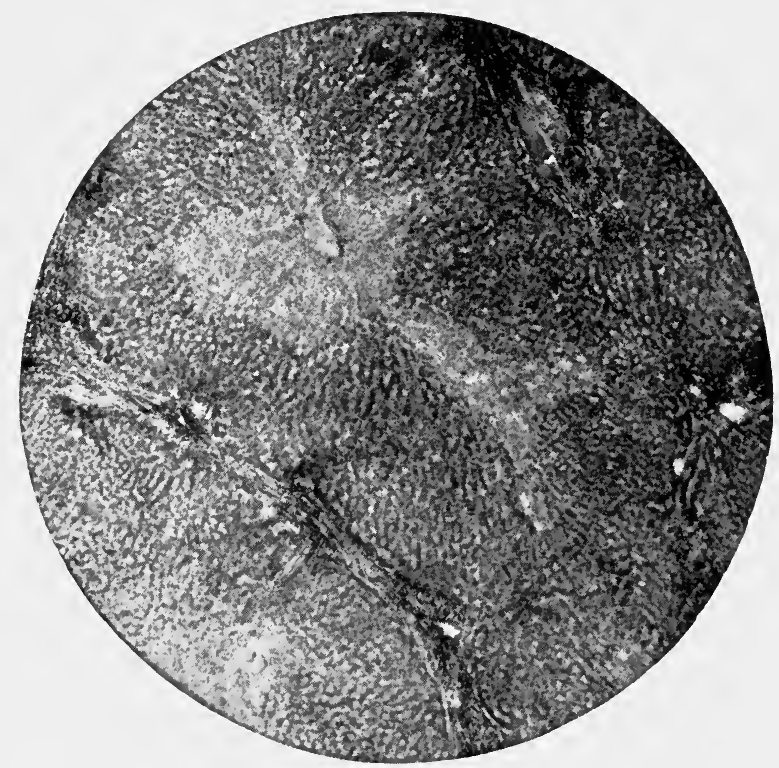

FIG. 61. - Liver in Pernicious Anæmia. $\times 25$.

membranes, the eyes, ears, and parenchymatous and subcutaneous tissues. Even the bone marrow may be degenerated and studded with minute hæmorrhages and deposits of iron.

Symptoms. - The symptoms begin mildly but develop and expand with inevitable sureness. The child at first is listless and without energy; he loses appetite, and his complexion becomes pale and then dull, sickly yellow. Functional disorders of the gastro-intestinal track are common, and may assume many forms. Dyspnœea is regularly present and is increased on slight exertion. The heart action is weak, the pulse is soft and com- 
pressible. The apex beat is apt to be low and displaced to the left. Hæmic murmurs may be heard at the aortic area and at the apex, but these may be in part caused by deficient coaptation of the valves due to cardiac dilatation. Over the great vessels in the neck one may hear a venous hum. In most cases there is a moderate, irregular fever, which may be due to the disease or to its complications. There may be some dropsy under the eyes, and later about the feet and ankles; more rarely there may be effusion into the serous cavities. Hæmorrhages, usually small in amount, may start from any of the mucous membranes, of which the nasal are the most fertile. Nervous symptoms mark the last stages and may be of varying severity. In some cases they are no worse than headaches, temporary lack of coördination, restlessness, and insomnia ; in others they may go as far as the effects caused by hæmorrhage into the brain or cord.

Prognosis. - The outlook is bad; there may be temporary improvements, and the fatal outcome may be delayed for months or even years. But the disease, so far as we know, is not curable.

Differential Diagnosis. - We make sure of the diagnosis of pernicious anæmia by the slow, steady, and progressive course; the evidence of hæmorrhages and fatty degeneration. The principal guide is the examination of the blood, the changes in which have been described among the lesions.

The treatment is given near the end of the section on anæmias.

\section{Infantile Pseudo-Leuchemic Anamia}

A condition of marked leucocytosis with oligocythæmia has been described by Von Jaksch which as yet has not secured full recognition as a distinct disease. Instances of it have not as yet been so common, and studies of it have not been so thorough that an exact and distinct picture is commonly recognized. Some observers have claimed that it is merely a profound anæmia with a very large percentage of leucocytes. On the other hand, Von Jaksch, Luzet, Hayem, Monti, and Berggrün believe that it is an independent disease. Its cause is unknown, but it has followed severe anæmias and the exhausted conditions of rickets and syphilis. Practically all cases reported were under five years of age. 
The red blood-cells may be very much reduced in number, so that only one-half or less of the normal amount is present. These may be variously altered in form, and in different cases there may be varying proportions of poikilocytes, microcytes, macrocytes, and polychromatophilic cells. The leucocytes are very much increased in number, so that in marked cases they form from eight to ten per cent of the whole number of cells. These leucocytes may be seen in their different varieties. Löwit reports a case in which he discerned amœboid bodies in the blood and spleen, the larger number being found in the latter. The spleen is much enlarged, but the lesions seem to be no more than a simple hyperplasia which may include all parts of the viscus. The liver may be unchanged or slightly increased in size, and the hepatic cells are usually normal. The statement has been made that in this condition the liver has " reverted to the embryonic state." And this is still another instance of the lack of agreement in the matter. -The glands may be enlarged by a simple hyperplasia, but they are neither very great in size nor tender.

The symptoms are those of an anæmic condition of much severity plus an enlarged spleen. The course is chronic and may be marked off by the variations in the size of the spleen. There may be exacerbations and remissions; and there is a noticeable weakness in resisting intercurrent diseases.

Prognosis. - The outlook in this complaint is supposed to be fairly good. But the extreme youth of the patients, as well as their necessarily exhausted condition, makes any serious impoverishment of the blood a matter of danger. A mortality of twenty per cent has been reported.

Differential Diagnosis. - The diagnosis is made from the greatly enlarged spleen and the equally great leucocytosis.

\section{LEUCOCYTH AMIA}

Leucocythæmia, or leucæmia, is a disease that one occasionally sees in childhood. At the time of the first report of this disease, in 1845 , the general conception of it was simpler than ours at present is. Greater experience with it has shown the existence of two varieties: the one; which is called the spleno-medullary type, consists in the most part in an enlargement of the spleen and the presence in the blood of certain large mononucleated cells. 
The other variety, called the lymphatic form, is characterized by an excessive quantity of small mononucleated cells, while the enlargement of the spleen is not so marked as in the first case. This classification is not a final one; and increased knowledge, as in most degenerative conditions of the blood, may overthrow the present beliefs.

Causes. - We are ignorant of what causes leucocythæmia. Although the greatest proportion of the cases occurs among adults, nevertheless it does appear in children of the youngest age. In these instances it may be associated with or precedod by exhausting diseases, malaria, rickets, syphilis, profound anæmia, and unfavorable conditions of life. Attempts have been made to trace an hereditary connection, but they are not promisingly successful.

Lesions. - The main change consists in the large number of leucocytes in the blood. This may be so great that the color of the fluid is noticeably light. If there is a coexistence of anæmia, the blood is thin and watery, and its hue has a yellowish cast. Although the amount of its fibrinogen is somewhat increased, it coagulates outside of the body with some slowness. The lymphatic variety is somewhat commoner in children than in adults. It shows an increased number of mononuclear leucocytes. Of these, the larger forms contain considerable amounts of protoplasm and some basophile granules, but the smaller ones have only a small quantity of protoplasm. As a rule, both of these forms are non-amœboid. On the other hand, the polynuclear leucocytes and eosinophiles remain stationary in number. If there is a marked anæmia, one may notice a few nucleated red corpuscles. In the spleno-medullary form the leucocytes in general are increased in size; most are finely granular, while only a few may be called coarsely granular, e.g. the mast-zellen of Ehrlich. The principal varieties are the large mononuclear corpuscles or myelocytes, the eosinophile corpuscles, the polynuclear corpuscles or cells with multipartite nuclei, and the coarsely granular leucocytes. In addition there may be many nucleated red blood corpuscles. In and about the leucocytes, Löwit reports the finding of sporozoa, and suggests from observation that these parasites go through a process of multiplication within the cells. He does not believe in the entire separation of the two varieties mentioned above, and speaks of transitional forms between them. 
The red blood-cells are diminished in numbers; they may be so much decreased and the leucocytes so much increased that the two become equal to each other. The hæmoglobin may be reduced in quantity in about the same proportion as the red blood-cells.

In the viscera the changes are not confined to the spleen, as formerly so often thought, but may extend all through the body. Thus, there may be a leucocytic infiltration, a leucocytic thrombosis, and hæmorrhages. The spleen is much enlarged and is infiltrated with leucocytes like those found in the blood. It may contain infarctions or be subject to hyperplasia. In chronic cases there may be a fibrosis which involves the pulpy network and the trabeculæ. The liver may be somewhat increased in size, but not so much so as the spleen. It is slightly pale in color, which may be due to anæmia, or leucocytic infiltration. This infiltration is most apt to occur about the portal track, from which it may work its way into the lobules. There may be some fibrosis followed by atrophy of hepatic cells. Where there is much anæmia there may be a fatty degeneration. The lymphatic glands, in the lymphatic form, are enlarged in practically all cases; in the spleno-medullary form they are rarely enlarged, and whatever enlargement exists is not at all great. In each variety the leucocytes are the same as those which are found in the blood. Bone marrow becomes altered by cell change and degeneration. It may be soft and have a resemblance to the appearance of a light-colored pus, or, if it is fluid and of a deeper pink hue, it may suggest lymphoid tissue. It replaces the fatty marrow in the shafts of the long bones, and may even bring about some absorption of particles of osseous tissue. The hyperplastic process is as consistent as in other parts of the body. 'The same general change takes place in the thymus gland, in the heart, where it is associated with fatty degeneration, in the lungs, where it brings with it thickening of the connective tissue, in the kidneys, in which the leucocytic infiltrations into the connective tissue may be noticeably large. In the alimentary canal similar hyperplastic processes in the glands of the mouth, stomach, and especially the intestines, may be expected, most of all in the lymphatic form. In the various viscera hæmorrhages may take place, as well as on serous membranes, periosteal membranes, skin, and mucous surfaces. These seem to rise from a leucsemic infiltration of the walls of the arteries, followed by local degeneration and rupture. In this way hæmorrhage may take 
place in the retina and inner ear. The amounts of excreted urea, uric acids, and xanthin bases in general has been found to be increased, but not regularly so. The phosphorus excretion is also said to be enlarged.

Symptoms. - The appearance of leucocythæmia is alike in children and adults. The beginning is slow and barely perceptible. The patient shows lassitude, weakness, anorexia, dyspnœa, disorders of the gastro-intestinal track, and often some pain in the left hypochondriac region. Examination shows an enlarged spleen, which may become so large that it approaches the superior

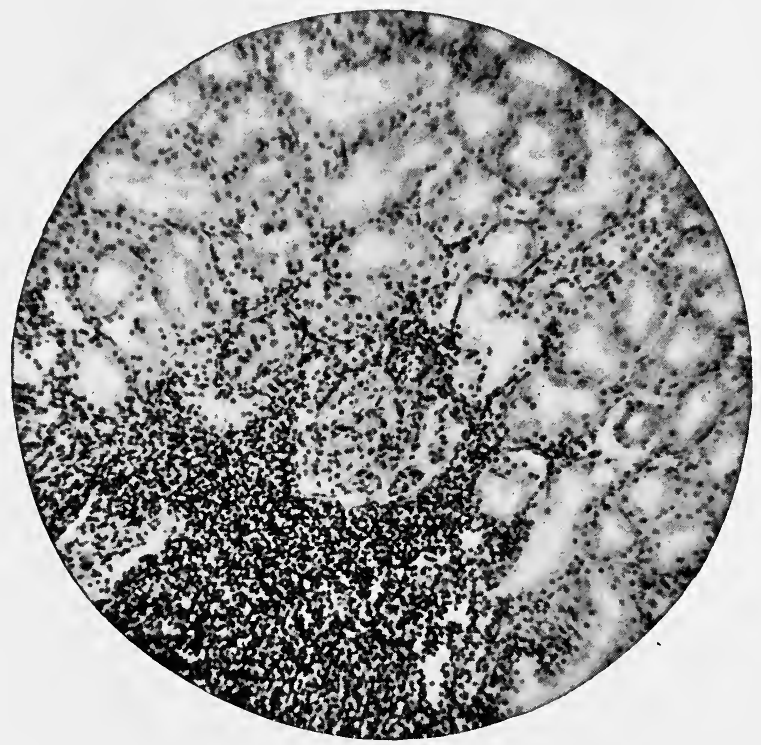

Frg. 62. - Kidney : in Leucocythæmia. $\times 100$.

margin of the ileum. Hæmorrhages from the stomach, intestines, nose, throat, eye, or other regions may occur. There may be an irregular fever which does not rise to a great height. There may be an irregular adenitis which occasionally is painful. Anæmia is often noteworthy, and gives its regular symptoms, including weakness, headaches, soft pulse, hæmic murmur at the apex, and changes in the composition of the blood. The complexion is dull, and especially at nights there may be some sweating of the head and chest. There is an acute form in which the progress of the disease is very rapid, so that days or weeks instead 
of months mark the limits of the disease. From the very beginning the symptoms are startling, the prostration is profound, the hæmorrhages may be frequent and copious, and œedema of the lungs or an intra-cranial hæmorrhage may suddenly end the child's life. From the very outset of the sickness the symptoms rage with so much intensity that very little hope can logically be entertained.

Prognosis. - The outlook is, on the whole, bad. There may be periods of improvement, and death may be delayed for some months or even years. The lymphatic form, which is doubtless the more common in children, ends fatally sooner than the spleno-medullary variety. The younger the child, the shorter is the course and the less is the chance of recovery.

Differential Diagnosis. - The significant facts are the enlargement of the spleen, possible hæmorrhages, and the examination of the blood. The main part of this examination is not merely finding a greatly increased leucocytosis, but rather the especial forms of leucocytes and myelocytes mentioned under the heading of lesions.

\section{Pseudo-Leucamia (Hodgkin's Disease)}

This is a disorder which is not often seen in children. It is characterized by a more or less general enlargement of the lymphatic glands, hypertrophy of the spleen, the growth of lymphomata in the abdominal and thoracic viscera, and anæmia. In recent times observers have shown a tendency to regard the disease as infective, but the matter is as yet not definitely decided. At any rate, no other ætiology is able to account for all the cases ; the connection between this disease and tuberculosis, syphilis, and malnutrition seems to be somewhat fortuitous. It occurs more often in boys than in girls.

Lesions. - The main changes may be found in the glands and the viscera. The glands may undergo a simple hypertrophy and retain their normal consistence, or they may assume a fibrous character. In the former the various elements exist in their normal relationship, but throughout the reticulum there is present a large number of lymphocytes. In the latter form there is, in addition to the increase of cells, a notable increase in the fibrous tissue of both the gland and its capsule. In the spleen there may be a simple hypertrophy or a number of lymphoid growths. As an 
additional factor, there may be one or more infarcts. There may also be a varying number of adenoid growths in the pharynx, nasopharynx, œsophagus, gastro-intestinal canal, the liver, the kidneys, heart, lungs, the testicles, ovaries, the thymus, thyreoid gland, supra-renal capsules, the dura mater, and the skin. The bone marrow may be altered by the growth of adenoid tissue, the heart muscle may fall into a condition of fatty degeneration, and there may be an effusion into the pleural cavity.

Symptoms. - The attack may come in an acute or chronic form, but we do not know the deciding factor which predisposes to one course or the other. In the majority of cases there is pyrexia, which may remain uniformly high, may from day to day describe an irregular curve, or alternately appear and disappear. The child becomes progressively pale and more anæmic, and the changes in the blood are among the first symptoms. The number of red blood corpuscles is decreased, and their form may be altered. In most cases there is no increase of leucocytes. The general effects of anæmia may be present.

Commonly, the first sign which the parents notice is a general adenitis. The superficial glands are involved before the deep ones, and the growth generally begins on one side only. The cervical group is generally the starting-point; and later on the submaxillary, subcervical, the mediastinal, the axillary, the mesenteric, the inguinal, and even the popliteal glands may become attacked. The glands may be hard or soft, and may retain their separate form until, by the action of a peri-adenitis, a mass of them may be matted together. As soon as the increase in size becomes considerable, pressure effects of corresponding intensity may result. This is the main source of the serious symptoms, since the glands themselves very rarely give pain. Thus in the throat, the calibre of the œsophagus may be lessened enough to interfere with swallowing, or the larynx or trachea may be pressed upon, so that articulation and respiration are impeded. Hypertrophy of the submaxillary and axillary glands prevents free motion of the respective parts. The deep glands of the chest may by their enlargement produce nervous congestion, cough, and dyspnoea. All through the body the symptoms may be explained by ascertaining what organs are involved, and how serious the functional interference is."

There is a moderate enlargement of the spleen, but no pain. 
The functions of the stomach and intestines may be impeded by lymphoid growths and the increasing weakness. The heart may become weak in its action, and its pulse may be irregular if the vagus is pressed upon. In the lungs there may be recurrent attacks of bronchitis, as well as an accompanying pleurisy. There may be a localized neuritis, following pressure, or cerebral symptoms from a similar cause may arise. There is always some anæmia, which in the later stages may become profound. In the prolonged form of the disease the symptoms may extend over months or years, until the patient dies from exhaustion or intercurrent disease.

Prognosis. - The outlook is bad. Some cases have recovered; but how and why they did we cannot say.

Differential Diagnosis. - The enlargement of the glands may suggest an acute adenitis. In this condition there would be considerable local disorder, pain, inflammation, sometimes suppuration. Also, there will commonly be a history of a sufficient cause. These signs would not be present in pseudo-leucæmia. Usually it is not hard to distinguish this disease from the spleno-medullary form of leucæmia, since in the latter there is no general adenitis, and the splenic enlargement is very pronounced. The enlargement of the abdominal glands and the intestinal symptoms may suggest typhoid fever. But the lack of eruption, characteristic temperature curve, and Widal's reaction would clear the doubt.

Treatment of Anæmic and Leucocythæmic Condition. - Although there may be the greatest differences between these various complaints, nevertheless the differences between their respective treatments are quite the opposite. Our lack of knowledge concerning even the most successful of them is undoubtedly great. At the same time we have a good therapeusis for most of the cases, even although the theory of it is not thoroughly understood. In the secondary anæmia of children, the first step is to remove, as far as possible, the primary cause. With this out of the way, the most important step toward health has been taken. The diet should be made as light and nutritious as possible (see section on Feeding), and the hygienic and sanitary conditions of the patients must be rendered thoroughly good. In the way of medicines one may give an organic preparation of iron, such as the peptonate, the albuminate, the malate, or the manganate. Some of these, notably the peptonate and the manganate, have been 
put up in a proprietary form that is really' excellent. These given alone are usually efficient; when they are combined with strychnine or the tincture of nux vomica, their value is enhanced.

In chlorosis the same directions concerning diet, hygiene, and sanitation, must be carried out. The same salts of iron must be administered, and the parents must be informed of the necessity of continuing treatment for several weeks, as well as of the fact that the disease has a tendency to recur. For this reason the treatment must be continued until after every vestige of a pathological condition has vanished. It is a useful thing, in the second half of the treatment, to combine arsenic, usually in the form of Fowler's solution. Especially is arsenic indicated in any hæmic disorder, where there is an enlargement of the spleen, or the lymphatic glands, or both. In pernicious anæmia our main reliance rests upon arsenic, which may be given in increasing doses, from three or four drops up to fifteen or twenty, according to the patient's age, three times a day. In infantile pseudo-leucæmic anæmia arsenic is again the main drug to be used. In leucocythæmia and pseudo-leucæmia we have recourse once more to arsenic, aided by iron and strychnine. In pseudo-leucæmia, as soon as the glands become enlarged, they should be removed by operation. Some observers believe that the disease in the beginning is a purely local infection, and that in this way its progress may be checked. At all events, there is a diminished possibility of pressure effects. As far as medicinal treatment goes, one can do no better than to give arsenic, to regulate the diet, exercise, and general mode of life, and treat the symptomatic needs. The iodides and mercury have also been used, but without success. In all these conditions there may be a wide necessity for a certain amount of symptomatic treatment. In those complaints which are characterized by marked leucocytosis and a tendency to hæmorrhages, sufficient realization of the dangers involved in doing surgical operations must be had.

\section{HAMOPHILIA}

This is a congenital disease which is always transmitted from mother to son; the daughters usually escape the disorder, although through them it is perpetuated. It is the same method of transmission that is seen in gout, polydipsia, and color-blindness. 
The sons of a bleeder are unable to hand on the disease to their children; but his sisters and daughters are potent to transmit it to their sons. Summarized, the case stands thus: the boys in such a family bear the effects of the disease, but do not transmit it; the girls bear no effects of the disease, but do transmit it. The compiled statistics of the disease gives a proportion of eleven occurrences in boys to one in girls. This hereditary factor constitutes the rtiology of the disease. There are no known lesions.

There are but two symptoms: hæmorrhages and arthritis. The hæmorrhage may appear at any time of early life, even intrauterine. Sometimes it shows itself at circumcision, cutting of the frenum of the tongue, lancing the gums, or opening an abscess. Usually it is not seen until the child is able to run about, for then he becomes liable to injuries that may cause hæmorrhage. Practically any trauma may start the bleeding; in some cases it may be a scratch or a slight cut, in others the drawing of a tooth is the opportunity. In some cases the bleeding may be spontaneous, or at least have no ascertainable cause. Such cases are probably at the bottom of the joint affections. These children suddenly have pain in the knee, ankle, elbow, shoulder, or hip joint ; very rarely in the fingers, toes, or wrists. There are signs of effusion (doubtless hæmorrhage), there may be slight fever. After a time the effusion becomes absorbed, and the joint returns to its former useful condition, or may be slightly disabled. Repeated attacks may spoil its integrity. In early childhood the most frequent seat of the bleeding is in the nose, and the second place in order is taken by the mucous membrane of the mouth. As the child grows older, the intestines and then the stomach furnish a large number of the cases. But any part of the skin, mucous membranes, or viscera may, on sufficient provocation, be the ailing part. The hæmorrhage varies in severity and in the time of its activity. It may be a mere oozing or a rapid flow; it may last for a few hours or for days. It has been said that the tendency may be outgrown, but the unfortunate possessor of a well-marked habit does not often live long enough to prove it.

Treatment. - The girls in a hæmophilic family should have the nature of the disease explained to them, and at the same time be advised against contracting marriage. The advice is almost always neglected, but should, nevertheless, be given. Hæmophilic 
boys should not be allowed to undergo any surgical operation, even so slight a one as drawing a tooth. However, in the case of opening an abscess, an exception may be made, because the hæmorrhage which is apt to ensue on spontaneous rupture seems to be equally great with that caused by incision. Still another exception is vaccination, which involves little or no danger. If hæmorrhage exists, the best means of help lies in the use of ice and pressure. Styptics and the actual cautery are not to be advised. In the intervals between the attacks the general health should be fortified as much as possible. Exercise should be regulated, and all violent games and amusements involving exertion should be proscribed.

The chloride of calcium has of late been much recommended, on account of its supposed faculty of causing increased coagulation of the blood. Its efficiency has not as yet been sufficiently demonstrated to warrant much reliance on it. Recently, attempts to control hæmorrhage have been made by allowing normal blood to coagulate upon the bleeding area; but the results are not encouraging. Another recent method proposed by Lancereaux, which gives somewhat better results, consists in the injection into the cellular tissue of sterile gelatine solutions $\left(\frac{1}{2}-2 \%\right)$. The ultimate fact remains that there is no really efficient treatment for a marked case of "bleeder's hæmorrhage."

\section{Purpura}

The appearance of hæmorrhages into the skin and mucous membranes is a sign of many diverse conditions. Some of these are of but little importance and fairly familiar; others occur in connection with practically unknown pathological states which are so mysterious and obscure that various observers differ considerably in their classification of them. As research becomes more thorough and voluminous, purpura is seen to be merely a symptom which in some cases may not be the head and front of the disease, which moreover may eventually be relegated into a place of much less importance than it now holds. The classification given below may be regarded as provisional; but until the sum of positive information is considerably greater than it now is, a better one is difficult to give. The cases divide themselves into two groups: one may be called secondary or symptomatic; the 
other - so far as we now know - is primary. Under secondary purpura are included the instances following infectious diseases, toxic conditions, mechanical strain, cachexia, and nervous lesions. The changes are generally superficial, but the element of severity is a large one and controls to some extent the phenomena.

The infective group follows severe attacks of infectious diseases. It is difficult to say whether they represent no more than intensely acute attacks, or the ordinary form of the disease plus some foreign element. At all events we know that these hæmorrhages occur with grave examples of these infectious diseases, such as measles, scarlet fever, diphtheria, malignant endocarditis, epidemic cerebro-spinal meningitis, pyæmia, septicæmia, and other like complaints.

The toxic group includes the effects of large doses of drugs, chemicals, snake venom, the absorption of poisonous matter from the alimentary track. The drugs which most readily produce this condition are the iodide and chlorate of potassium, quinine, phosphorus, mercury, salicylic and mineral acids. The matters which are absorbed from within the body include urinary and biliary constituents, and doubtless some unknown products of metabolism.

The mechanical group consists of cases in which unusual strain is put upon the blood-vessels, as in cardiac disease, convulsive seizures, pertussis, deficient physical support of the vessels such as occurs in the new-born, after removing splints from limbs, and the like.

The cachexia of exhausting diseases, notably the severe and prolonged disorders of the gastro-intestinal track, pulmonary system, and blood, may cause some deterioration of blood, or vessels, or both, that permits of superficial hæmorrhages.

The nervous group is rare in children, and appears with or after disease of the cord or of special nerves.

Primary purpura may be divided into the simple form, which when it becomes very severe is called purpura hæmorrhagica, the arthritic form or peliosis rheumatica, and the abdominal form, called Henoch's purpura. In these various conditions there are no constant known lesions with which we can account for the disease and its symptoms. Various theories have been enunciated, founded upon the pathology of one case or group of cases; but the theories are not of general application and cannot be regarded 
as having passed far beyond the limits of conjecture. Thus some writers advance the idea that inflammatory, hyaline, lardaceous, or unknown changes take place within the coats of the vessels, and are followed by oozing through them or distinct rupture; others suggest a chemical agent elaborated in the body by degenerative processes; others again offer bacterial organisms as the causes. Each of these factors has been urged with industry and ingenuity, and each has failed to prove its case. At all events, we know that hæmorrhages may occur in any part of the skin, mucous membranes, the solid and hollow viscera, and that in children the gastro-intestinal track, followed by the urinary system, are the oftenest affected. The frequency of occurrence in the other parts of the body is not by any means a matter of importance, since the disease is not frequent enough to allow compiling large numbers of cases and thereby providing a trustworthy guide.

Simple purpura occurs with or without preliminary indisposition, with or without general sickness. It appears in the form of petechial spots whose location is commonly seen on the legs. The size and shape are irregular; the eruption may come in one or more crops. Its character is that of an ordinary ecchymosis, and when disappearing it goes through the ordinary changes that one sees in a bruise. 'The condition is usually not serious.

There is a severe form that was formerly called purpura hæmorrhagica, or Werlhof's disease. The hæmorrhages are located in both skin and mucous membrane, and often are larger in size than in the simple form. The constitutional disturbances may be great, commonly taking the form of fever, disorders of the gastro-intestinal track, and systemic prostration. The mucous membrane oftenest affected is that of the nose; but those of the mouth, gastro-intestinal canal, lungs, and genito-urinary system are also attacked; the accompanying symptom of blood in the vomitus, fæces, pulmonary expectoration, or urine would naturally be present. Added to them would be severe prostration, anæmia, hæmorrhagic œdema, and fever. Ulcerations of the mucous membranes may occur, which under the fit conditions of exhaustion may become gangrenous. In some very acute cases, called purpura fulminans, the temperature is very high, the hæmorrhages extensive; prostration is great and is associated with serious cerebral symptoms. There may be an enlarged spleen and evi- 
dences of nephritic involvement. It has been suggested that this variety of purpura is doubtless of infectious origin. It is almost always fatal.

Purpura rheumatica, or peliosis rheumatica of Schœnlein, signifies those cases which have a joint involvement. There is commonly a small-sized eruption on the legs and sometimes the arms, and the temperature may be somewhat elevated. There is pain in the lower extremities, which increases at night and grows less in the morning. The joints, usually the knee and ankle, become tender, painful, often somewhat swollen, and occasionally the skin is reddened. This affection of the joints, added to the fact that among adult patients many give a history of having had rheumatism, has led to the beliefs that rheumatic purpura means a combined attack of purpura and rheumatism. Nevertheless, it is very probable that it should rather be regarded as purpura, characterized by hæmorrhage into the joint. The fact of a former rheumatism may be no more than a coincidence; and it is certainly noteworthy that aspiration of the affected joint shows the presence of serum mixed with larger or smaller quantities of blood. The constitutional disturbance is not very great, nor is endocarditis a common complication. The disorder is not very dangerous, but is apt to be repeated.

In Henoch's purpura the characteristic element is the occurrence of hæmorrhage into or from the abdominal organs, with tenesmus and pain and vomiting. There are apt to be tenderness, pain and swelling of the joints; the fever is irregular, and the general depression may be great. Besides the severe intestinal symptoms there may also be hæmorrhages from the stomach, kidney's, lungs, nose, and throat. The eruption generally appears on the legs, and may be complicated by an outbreak of urticaria, or nodular, or exudative erythema. This form of purpura, unlike the rheumatic, occurs principally in children.

Treatment. - The patient, even in mild attacks, should be confined to bed, and his environment should be made as quiet, as restful, and as healthy as possible. In almost all cases a tonic plan of treatment is indicated, with the additional means for relieving symptoms. Thus, an affected joint should be immobilized, and, as soon as tenderness and pain permit, may be compressed. Hæmorrhage from the bowel, stomach, and lung may require the application of ice. The use of drugs has not given 
very much satisfaction. The latest one to be recommended is the chloride of calcium, which is supposed to check hæmorrhage by its faculty for increasing the coagulability of the blood. It has not been sufficiently used to allow of an authoritative opinion concerning its efficacy. Arsenic in full doses is often recommended, and some cases are said to have improved materially under its continued use. Oil of turpentine, ergot, hamamelis, hydrastis, and the vegetable acids have all been used with various degrees of failure. Fresh fruits and vegetables have been suggested on account of a supposed relationship between purpura and scurvy. After the hæmorrhage has ceased, iron may be prescribed.

Prognosis. - In the milder cases, the outlook is good. In the severe cases the course of the disease is very trying and often fatal. It is very probable that more than one, possibly many, pathological conditions are included under the general term of purpura, and that with fuller knowledge a more exact idea of course and termination will come. At present one must be guided by the previous history and the character of the symptoms.

Differential Diagnosis. - The main fact to be kept in mind is that the eruption does not disappear upon pressure, and that in the large majority of cases the precedent cause, or rather condition, may be one of those mentioned in the classification. There may be some doubt for a time between scurvy and purpura; but a little comparison of the syndromes of the two complaints ought without difficulty to dispel it. 


\section{CHAPTER XVIII}

\section{DISEASES OF THE GENITO-URINARY SYSTEM}

\section{Functional Derangement of the Bladder}

THE delicate equilibrium of the urinary bladder in young children is often disturbed by disorders of the stomach and intestines involving faulty assimilation and excretion, by conditions of high fever, by exposure, or by nervous weakness and excitation. It is at times nothing more than a sympathetic irritation brought on by disease in a neighboring part. The symptoms consist of spasmodic pain, of difficult and frequent urination, of nervous irritation, especially when the child's attention is drawn to the idea of urinating.

In treating these cases, one should be careful to inquire concerning disease in any adjacent parts. such as the rectum, the genitals, or pelvic peritoneum, which might act as a cause. If such disease is present, it must of course receive immediate attention. Likewise if the child's stomach or intestines are disordered, they must be treated actively and promptly. For the derangement of the bladder one may prescribe hot baths and the local application of heat, sedatives - bromide of soda for infants and tincture of hyoscyamus for older children - and the drinking of much water. It must be kept in mind that in children, even possibly more than adults, water is one of the best diuretics at our command.

\section{Acute Cystitis}

This disease is somewhat rare in children, much more so than in youths or adults. The smaller amount of exposure of all kinds to which children are subject is the cause. When it does occur it generally is due to irritating drugs, to vesical calculus or other foreign bodies, to infected urine, or gonorrhœal urethritis or vulvovaginitis. At times one is unable to ascertain the cause.

The mucous membrane is swollen, congested, and bathed in a 
mucoid fluid containing pus and blood-cells. The epithelial cells are swollen and in places disintegrated, at times so much so as to produce ulcerations.

The symptoms consist of prostration, a moderate febrile movement, difficult, painful, and frequent urination, and vesical tenesmus. The examination of the urine is very important, and in order to exclude adventitious elements of confusion the urine should be drawn off by catheter. When the urine is allowed to stand, a heavy precipitate is thrown down, and some albumin may be held in solution. The color is reddish, the reaction is acid, but with a tendency to a rapid change to alkaline. The microscope shows pus-cells, blood-cells, squamous epithelial cells, epithelial débris, and bacteria.

The treatment consists in rest in bed, the administration of divided doses of calomel, the application of hot stupes or compresses over the bladder, the restriction of the food to milk, and the use of sufficient doses of sedatives to still the pain. In infants the bromide of soda may be used, but in older children hyoscyamus may be substituted. Warm baths and local heat are useful. If there is any antecedent disease, it must naturally be carefully treated.

\section{Chronic Cystitis}

A chronic inflammation of the bladder may be caused by an extension of an acute process, by vesical calculi, foreign bodies, and rarely by tumors. The mucous membrane is heavy, congested, and mottled in color. There is a constant formation of pus, and pus-cells may infiltrate the superficial tissues. The mucous membrane is changed variously in different parts, being hypertrophied, atrophied, or eroded. With a long continued course, more or less connective tissue may be formed, and the whole bladder becomes irregularly dilated.

The symptoms are apt to be bound up with the cause or antecedent disease. Thus vesical calculi will superimpose its own signs very prominently upon those of the cystitis. Ordinarily there are loss of flesh and strength, a querulous and irritated mind, frequent urination that at times may be painful. Some urine is always apt to remain in the dilated bladder, and there undergces ammoniacal change:

The treatment is much the same as in the acute form, with 
the exception of frequent irrigation of the bladder with boric acid solutions. As soon as possible the diet must gradually be enlarged and the patient's strength fortified by tonics. It is of paramount importance that the child be examined most carefully in order to ascertain the existence of any pathological conditions which can possibly perpetuate the abnormal condition in the bladder. When all such factors are thoroughly known, one is able to judge in a fairly rational way concerning the prognosis.

\section{INCONTINENCE OF URINE}

This disorder is apt to be one of the most troublesome with which the physician has to contend. Very often, in most cases in fact, it is difficult to describe a cause for it, except the very general phrase of deficient development, or general weakness with a local manifestation. This naturally does not include the cases of deformity or malformation of the genito-urinary organs, nor the functional lesions of the central nervous system of which incontinence of urine may be a symptom of long standing. In such cases, the weakness may involve the whole economy and be plainly visible in various phases of disordered secretion, excretion, and assimilation ; or, on the other hand, it may be more particular and local, while the rest of the body is fairly well nourished. This we often see well illustrated by deficiency in function following an exhausting sickness. For instance, after an attack of scarlet fever, the patient who formerly had had exemplary habits may be quite unable to control the bladder sphincter. Again, in cases of anæmia, a like condition may come into existence, not because there is any connection between enuresis and the disease in question, but merely because the sphincter control is one of the vulnerable points in childhood.

This is reasonable and easily understood from the common experience of teaching a child habits of self-control. In the ordinary course of growth, he usually learns this before he is two years of age ; if carefully and properly taught, he may gain this restraint when he is less than a year old. On the other hand, many a child is markedly deficient in this respect for months or years afterward, and the best that one can do in the way of diagnosis is to claim the persistence of infantile traits, of deficient development. Such, of course, would not be the case where a rationally sufficient irritant is present: such an irritant might. 
exist in an unusually acid urine, in a contracted or atonic bladder, or a bladder containing calculi, in pathological conditions of the uretha, in a small meatus, in abnormalities of the foreskin, in vulvo-vaginitis, adherent clitoris, in parasites and diseases of the rectum. There is one more factor, that of inheritance, which seems to exist in some families. Frequently enough parents, in stating the disability of their children, will give the additional information that they or their sisters or brothers had had a like failing. How far this is a question of true heredity or of habit, is always hard to say.

Treatment. - If an antecedent removable cause exists, it must of course be treated vigorously: thus an adherent prepuce or clitoris should be freed, a narrow meatus enlarged, a long prepuce with an insufficient orifice amputated, a calculus in the bladder removed, a highly acid urine neutralized and diluted by the administration of alkalies and large quantities of water. A principal part of the treatment comes after the abolition of these precedent conditions; for in the majority of cases the disorder is apt to continue in much the same way as before. In the first place, the general health must be carefully looked to ; the normal functions should be strengthened at every weak point, and the habits and environment of the patient regulated most scrupulously. The amount of water ingested in the afternoon and evening, except where the urine is highly acid, should be restricted, and the child must be ordered to empty the bladder at regular intervals which should not be too far apart; likewise it is generally advisable for the parents, before retiring, to arouse the child and make him urinate. This is especially useful if his sleep is unusually heavy. If the bladder is contracted, so that frequent urination is a physical necessity, the organ may be dilated daily by warm boric acid or normal saline solution.

As far as drugs are concerned, the best that one can do is to prescribe a combination of nux vomica and belladonna; the latter drug should be given in larger doses than the former and steadily increased to the point of toleration. One may use either the tinctures or the alkaloids (strychnine and atropine) of the drugs. The size of the dose varies with different patients, according to size, weight, and idiosyncrasy. The doses may be distributed through the day or confined to the afternoon and evening. I have found no advantage in one over the other method. 
These patients should not as a rule be punished for their lack of cleanliness. They should be regarded as sick, not disobedient, and punishment will be apt to diminish their confidence and selfrespect; they should instead be encouraged whenever praise is possible. For in a neurosis, such as most cases of enuresis, the mental condition is of great importance.

Prognosis. - At the beginning of treatment the parents should be informed that this disorder is apt to be tedious, that it has an obstinate tendency to recur, and that the therapeutic care must be continued for weeks or months after the patient has gained a good control over the bladder sphincter.

\section{Vesical Calculi}

There is no doubt that vesical calculi may occur in the earliest as well as the latest period in life. Since the time when Langenbeck reported the finding of a stone in the bladder of a six months' fœtus, a surprising number of cases of vesical calculi in young children have been diagnosticated. The cause is often hard to discover, and many factors have been regarded as the starting-point. Heredity, locality, climate, and diet have all been discussed in this connection. Much stress has been laid upon a possible lack of milk in a young child's food as being the frequent cause of the growth of a stone.

These formations are generally composed of uric acid; in some cases there may be an admixture of sodium oxalate and the urates of sodium and ammonium. Less frequently one sees calculi composed of sodium oxalate, ammonium urate, triple phosphates, and other rarer salts. Usually they originate in the kidney, from which they are washed into the bladder, where, by mixing with a colloid substance, such as mucus, they are capable of indefinite increase.

The symptoms may at first, especially in young children, be somewhat indefinite; but careful observation will generally without much delay show the truth of the matter, Summarized in a general statement, they are frequent urination, exaggerated on brisk motion, and at times incontinence of urine, generally more marked during the day than at night; the occurrence of pain, especially at the end of urination, referred to the under aspect and the end of the penis; sudden and unexpected stoppages of 
the flow of urine when the stone is drawn to the cystic end of the urethra; rectal irritation and possible prolapse of the rectum from straining; the presence in the urine of mucus and pus with the addition at times of blood. Besides these symptoms there may be nervous manifestations and reflex pains in almost any part of the body. Thus the effects may be very widespread, and may even go so far as to cause mental or moral degeneration. This will account for some of the cases of altered character and disposition, for the readiness with which some of these children fall into habits of masturbation and precocious sexual activity. The examiner must not expect to obtain as typical a picture as in adults. Not only are the symptoms, excepting in the older children, not so characteristic, but also they are less continuous and reliable in their manner of occurrence. Alnost always it will be necessary to observe the case for a short time before a reliable diagnosis can be made.

The treatment is surgical and consists in the removal of the calculus either by crushing or cystotomy. In performing the latter operation the supra-pubic method is generally preferred. After the stone has been removed, careful attention should be given to the diet to diminish the possibility of new formations. 'The patient should drink liberal quantities of water, and highly seasoned foods, fats, and sugars must be avoided. The food must be easily digestible, and not large in amount.

\section{Balanitis}

The prepuce may become inflamed as the result of injury, excessive masturbation, collection of smegma, decomposition of urine held under the foreskin, and other forms of uncleanliness. It usually occurs in presence of a phimosis which prevents the retraction of the foreskin and the thorough cleansing of the head of the penis and the preputial mucous membrane. In dispensary practice one frequently sees an inflammation of the meatus which follows such causes, or even disorders of the stomach and intestines. As a result, the prepuce and the glans penis may become swollen, inflamed, œdematous, and sometimes purulent. The process may be cut short, or go on to sloughing and gangrene.

The condition is treated by the application of cold or heat, and the injection under the foreskin of considerable quantities of a 
solution of bichloride of mercury, 1:8000, or permanganate of potassium, $1: 500$. If improvement is not rapid, the foreskin should be opened along the dorsum or sides, and the parts should then be thoroughly cleansed and treated antiseptically. After recovery circumeision should be done.

\section{Phimosis}

An unusually small preputial orifice is occasionally seen in young boys. The younger the child, the more apt is one to find this condition; and with advancing age there is a natural tendency toward a normal evolution. In some cases, however, the opening of the foreskin remains inadequate; as a result, and especially when the foreskin is long, urine may decompose about the glans, smegma may collect about the corona, and the prepuce may in some degree become adherent. A long train of widely divergent symptoms may follow, which show themselves in the way of nervous and hysterical manifestations that may attain the dignity of seeming perversions of character, defective growth and nourishment, enuresis, difficult or painful micturition, irritation of the sexual sense with excitement to masturbation. The range of effects is very inconstant as well as wide; in one patient the bladder will be seriously incommoded, while in another the complaints are referred to the rectum; in both cases the harm may finally become organic as well as functional.

These cases should be taken in hand early; where the orifice is not too small and the foreskin too long, dilatation may be accomplished by repeated and gradual attempts to retract the foreskin back of the corona. When this is successful, the parts should immediately be put in their normal relations to avoid the danger of paraphimosis. Retraction should then be practised daily for the double reason of keeping the orifice large and the parts clean.

In the severer cases it will be necessary to do circumcision. Of the two methods of treatment, excepting in trivial cases, I prefer the latter as being radical, rapid, and involving no tendency toward forming the habit of masturbation.

\section{Paraphimosis}

Paraphimosis is a stricture of a narrow prepuce drawn back of the glans penis. As a result there may be swelling, inflammation, 
œdema, sometimes a purulent discharge, followed in extreme cases by sloughing or gangrene. As soon as one sees a case of paraphimosis, one should immediately attempt to draw the prepuce into its proper position. If the swelling is too great to allow this, the application of cold or the use of multiple punctures of the œedematous tissue may give relief. If this is not promptly obtained, the prepuce should be divided; on recovery, circumcision should be performed.

\section{Urethritis in Male Children}

By far the largest number of cases of this disorder occurs among the children of the poor and ignorant classes, that make up a large part of hospital and dispensary practice; among people of favorable circumstances it is, on account of their superior habits, much rarer. The disease appears in two varieties: a catarrhal and a specific. The former is due to neglect, dirt, adherent smegma under a long and tight prepuce, and injuries; in some cases of marked malnutrition, such as rachitis, I have seen a simple urethritis that was subacute in its course and very obstinate in its resistance to treatment. Usually the general health required much careful treatment before the urethral inflammation entirely subsided.

In the cases of specific urethritis, the cause is always infection with the gonococcus (Neisser). The diagnosis must regularly be confirmed by the microscope and Gram's method of decolorization. It is only in this way, as various observers have demonstrated, that the gonococcus can be positively differentiated from the diplococci commonly found the urethra.

Gonorrhœa may occur in boys of any age ; even the new-born child may become infected in the process of delivery. At any later time of life he may contract the disease from soiled clothes or household utensils, from being handled or cared for by persons whose hands were infected, or by real or attempted sexual intercourse. Even very young boys may thus be exposed; and as the matter may at times be of considerable medico-legal importance, a diagnosis of specific urethritis should be made as stated above, only after microscopical examination and the use of Gram's method.

The lesions are no different from what we find in the adult, consisting of congestion and swelling of the mucous membrane, the 
production of mucus and pus, and (in the specific form) the presence of the gonococcus, lying free in pus-cells. The inflammation may extend to the deeper tissues of the penis, the bladder, the testicles, and spermatic cord. The prostate, partly on account of its rudimentary form and function, is rarely attacked in children. 'This immunity is relative, not absolute; and occasionally in hospital practice one may meet a specific prostatitis. In the specific urethritis there may be a clearly defined gonorrhœal arthritis, the favorite seats of which are the ankle, knee, fingers, and wrist.

Symptoms. - The symptoms correspond closely to those of the adult; and in many cases I have noticed that young boys stood the strain of the disease without marked effect. In spite even of severe manifestations of the disease, these children have showed remarkable activity. The ordinary signs of discharge, of burning and frequent urination, of partial physical depression, of chordee, may be regularly seen in the child as well as his older brother. There is always a possibility of the ordinary complications, except phimosis and balano-posthitis, which on account of the shape and form of the youthful prepuce are somewhat more liable to be probabilities in children than in adults.

Treatment. - The boy should be confined to bed, and his food must be limited to fluids. The bowels should be kept open and the urine rendered alkaline; the latter may be obtained by such simple means as bicarbonate of soda. He should have much water to drink, and any tendency to chordee may be counteracted by sufficient doses of the bromicle of soda, or, in the case of older children with unusually great excitation, suppositories of opium. The penis must be kept as clean as possible, and every attempt must be made to prevent phimosis. If this complication occurs, the foreskin must be drawn back, the glans penis cleaned, the foreskin replaced, and the whole organ covered with hot or cold applications. Where the phimosis is too severe to be treated in this manner, the foreskin must be slit, preferably on the dorsum, and treated like an infected wound. On recovery of such an operation circumcision should be performed. Injections may be used early in the disease, in fact as soon as the passage of a small soft rubber catheter can be accomplished without too much pain. A number of organic preparations of silver have recently been manufactured for this purpose, and have been highly praised by 
many observers. The most useful of these, according to my experience, is protargol. 'However, in spite of one fluid or another, the treatment of a large number of cases is apt to convince the physician that the course of the disease is not thereby materially shortened; and it is very possible that the old remedy of sulphate of zinc in a one-half per cent solution may be found worthy of use in almost as great degree as the newer preparations. Protargol need not be used in stronger solutions than a one-quarter to one per cent. At all events, toward the end of the disease, when the full discharge has been replaced by a small mucoid flow, a local astringent may with advantage be exhibited. The general health must then be protected by sufficient rest, food, and tonics.

Complications must be foreseen as in adults. Especially should especial care be taken to prevent infecting the eyes. For this purpose the child at night should be so clothed as to prevent his touching the genitals. Urethral stricture may be contracted in the very young as well as in adults; but it is a rare occurrence.

\section{Vulvo-VAGINITIS}

This condition in the female is in many ways the analogue of urethritis in the male. It occurs in a catarrhal and a specific form, the latter due to infection with the gonococcus (Neisser). The simple variety may be caused by poor nutrition, such as follows the acute infectious fevers, by lack of cleanliness, irritants, such as inflammation of the skin with the resulting scratching, scabies, thread worms, injuries, and direct or indirect contact with other cases of this same disorder. In the specific cases, where the presence of the gonococcus has been demonstrated by the usual methods of culture, Gram's method, and microscopial examination, the one way of contracting the disease is by direct or indirect infection from a person who has a specific urethritis or vulvo-vaginitis.

The changes include an inflammation of the mucous membrane, which may extend more deeply, accompanied by the production of mucus and pus. The inflammation may attack the urethra, bladder, vagina, cervix uteri, uterus, and appendages. It may in unusual cases involve the glands of Bartholini and the peritonæum.

The symptoms vary according to the severity of the attack, and both mild and severe cases may be seen in the specific as well 
as the catarrhal variety. In the mild cases the discharge may be thin, watery, light yellow in color, mild in odor, and not necessarily accompanied by severe physical depression. As the severity increases all the symptoms become exaggerated: the prostration is considerable, the discharge is thick, profuse, greenish yellow, and foul in odor. There are burning and frequency of urine, the inguinal glands may be enlarged, and as adjacent parts become infected appropriate symptoms appear. There are considerable tenderness and pain, and the general health suffers in various ways. The course of the disease is much longer than that of urethritis in the male, largely on account of the anatomical conformation which makes contamination of adjacent parts easy or inevitable.

The treatment is analogous to that of urethritis. The urine should be rendered alkaline, especially if micturition is painful. The vulvo-vagina should be washed and irrigated two, three, or more times a day, according to necessity, with a solution of permanganate of potassium $1: 1000$, or bichloride of mercury $1: 8000$, or similarly weak solutions of sulphate of zinc, nitrate of silver, or protargol. The choice of solution is largely a matter of individual preference: Usually the potassium or mercury salt, or protargol is used first, and then is later on followed by the zinc or nitrate of silver. Exacerbations or reinfections are very apt to recur.

It is of especial importance that the child from the very onset of the disease should be so clothed and controlled that auto-infection or contamination be prevented. The communicability of the disease must be forcibly impressed upon the parents, so that the child's eyes and mouth are protected and that other children are not endangered.

In this disease, in making a prognosis, the possibility of purulent inflammation of the uterine appendages or the peritonæum must always be kept in mind. It is of the greatest importance that the parents should appreciate the serious nature of the sickness and the widespread evils which it may entail. In all cases the long course of the disease should be predicted.

\section{Stricture of the URethra in Boys}

As the result of injuries, urethritis, or balano-posthitis, the urethra, in boys of any age, may in one or more locations become 
ringed with strictures. In addition to these acquired cases, the same disorder may occur congenitally.

It is merely necessary to mention this subject and to state that the symptoms and treatment are the same as in adults.

\section{Orchitis AND EpididyMitis}

Inflammation of the testicle or of epididymus is a rare disease in childhood, and presents no especial feature that is characteristic of the patient's age. It may result from injury, from operations in the immediate neighborhood, such as lithotomy or an operation for hernia, and diseases of the urethra, as gonorrhœa. A remarkable case that I saw a short time ago was one of specific urethritis in a child two weeks of age at the time of examination. He had evidently contracted the disease from his mother during parturition. When I saw him, the testicle and epididymis had been inflamed for twenty-four hours. Orchitis may occur in inherited syphilis; but it is a rare manifestation.

The treatment is simple, consisting of prolonged rest, elevation of the testicle, hot applications, and the use of sedatives.

The chronic form is extremely rare.

\section{Hydrocele}

Hydrocele is not an uncommon complaint in children; what is more, if the disorder is promptly recognized, it is more easily cured than in adults. The main point in diagnosis is to differentiate clearly between hydrocele and hernia. This may be done by observing that in the former the tumor is translucent, that to the touch it feels elastic, gives a flat note on percussion, and that when it is reducible the tumor disappears slowly, gradually, and without noise.

There are four varieties commonly recognized in males : -

(a) Where the vaginal process is open throughout its whole extent, so that serum derived from it or the peritonæum may pass from one cavity to the other. This form is congenital.

(b) Although serum is in the vaginal process, it cannot flow into the peritonæum, since in the inguinal canal there is a complete shutting off of the lumen. In the first form the tumor is long and regular in shape; in the second, however, the circumference of the swelling is greater below than above. 
(c) Or the fluid may be in the upper part of the vaginal process, for here the closure occurs about the testicle, while the canal remains open above it and up to the peritonæum.

(d) The vaginal process is closed at the top and bottom. The serum is held in sac-like pouches.

There may be a hydrocele of the spermatic cord, in cysts, or in one mass. Another, but still rarer, form of hydrocele occurs in the pouch of an inguinal hernia from which the knuckle of gut has receded.

In girls a hydrocele of the canal of Nuck may occur, which is similar to hydrocele of the cord in boys. It is a somewhat rare complaint; but it has no unusual features which call for special mention.

The treatment of hydrocele is simple; when the tumor is reducible, a truss may be worn. If this is unsuccessful, the mechanical irritation of repeated aspirations will generally effect a cure. It is rare that one needs to perform the so-called open operation.

When the hydrocele is irreducible, the fluid should be drawn off by an aspirator. If it recurs, one or more repetitions of the operation generally cure the disorder. At the same time, one should remember that there may be some danger of entering the peritoneal cavity while attempting to do no more than empty a hydrocele ; nevertheless this may usually be avoided if the point of puncture is made sufficiently low down on the tumor. In a number of cases in infants the injection of peroxide of hydrogen, after the serum has been aspirated, has given me good results. 


\section{CHAPTER XIX}

\section{DISEASES OF THE GENITO-URINARY SYSTEM (continued). DISEASES OF THE KIDNEYS}

\section{Acute Congestion of the Kidneys}

Causes. - Acute congestion, or a hyperæmia of the kidney, may result from exposure, wounds and injuries, irritant or poisonous drugs, the acute infectious diseases, the loss of one kidney, and certain more indefinite causes, an example of which is vasomotor paralysis.

Lesions. - The blood-vessels are dilated and engorged with red blood-cells, so that in certain places the walls permit of minute extravasations into the uriniferous tubules. 'The color of the renal substance is dark, and its consistence is soft. The gland may be increased in size.

Symptoms. - Outside of changes in the urine there are few symptoms excepting those of the exciting disease. The urine is diminished in quantity, its color is high, its specific gravity is unchanged or increased, and it may contain albumin, blood-casts, and an increased quantity of urates. Sometimes there are no albumin, blood, or casts. The condition exaggerates the gravity of the previous sickness, and makes its symptoms stand out more clearly than they otherwise would. The renal congestion does not, however, project itself as a sharply defined disease.

Treatment. - The treatment is that of the original disease with the addition of local and general heat and the exhibition of diuretics, especially water. Excretion by all the emunctories must be thoroughly promoted, and at the same time the diet must be made as easily digestible as possible in order to put no unnecessary strain upon the kidneys. The care of the patient must be scrupulous and exact, for the possibilities of the condition are always serious. Neglect of this precaution can make a change from an acute renal congestion to an acute nephritis very easy; and at all events a tendency tó subacute or chronic renal disorders is in this way commonly developed. 


\section{Acute Degeneration of the Kidneys}

(Synonyms: parenchymatous inflammation, parenchymatous degeneration of the kidneys.)

This process is usually due in children to the acute infectious diseases, although almost any severe sickness with its varying amounts of toxines and irritating by-products of partial digestion may likewise act as a cause. It may occur in connection with the administration of poisonous doses of phosphorus, arsenic, or mercury, especially if the doses are large. It is one of the commonest renal troubles among children.

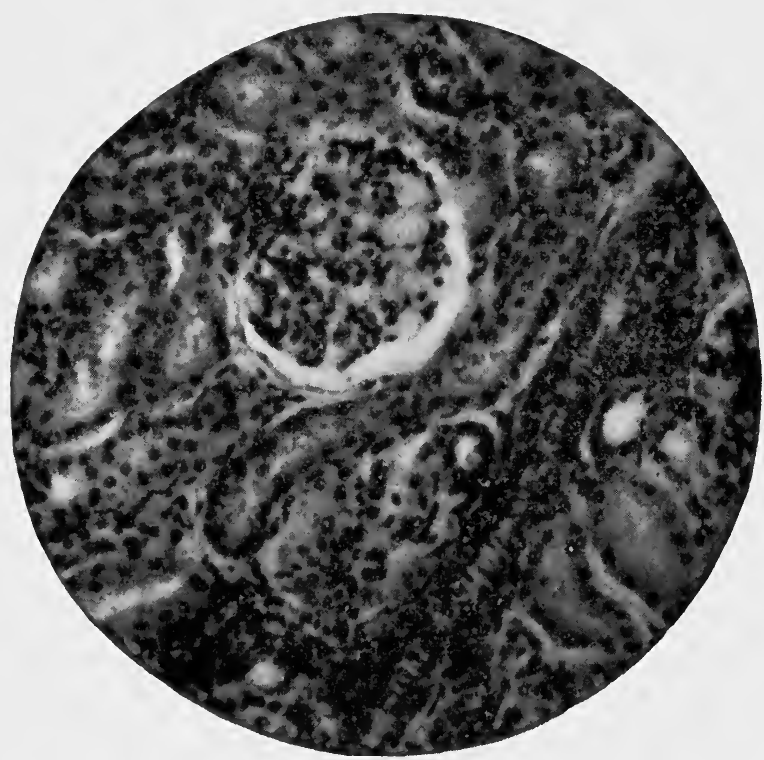

FIG. 63. - Normal Kidney. $\times 220$.

Lesions. - The changes are for the most part confined to the renal epithelium, especially of the convoluted tubes. The cells are swollen and congested, and may contain particles of fat, or the products of disintegration. In severe cases this process may go on so far as coagulation necrosis. The dead cells then desquamate, and on recovery are replaced by new cells.

The whole kidney may be enlarged, and exceptionally may be congested.

Symptoms. - Outside of changes in the urine there are not 
many symptoms more than the original disease produces. The urine is diminished and may temporarily be suppressed. Its specific gravity is unchanged, its color may be high, and in some cases there may be variable amounts of albumin and casts. The child may complain of headaches, of general malaise, of anorexia, or possibly nausea and vomiting. The course of the primary sickness is prolonged, and its severity is emphasized.

Treatment. - The treatment is that of the original disease with the addition of diuretics, especially water, and the application of heat. This last is conveniently accomplished by hot baths.

Excretion by means of the skin and rectum as well as the kidneys should be made active, and the diet must be inoffensive and easily digested.

\section{Acute Exudative Nephritis}

(Synonyms: parenchymatous nephritis, catarrhal nephritis, croupous nephritis, desquamative nephritis, glomerulo-nephritis.)

This is one of the commonest forms of nephritis that we find in children, being frequently set in motion by the acute infectious diseases, such as scarlet fever, diphtheria, acute lobar pneumonia, measles, the severe forms of enteritis, typhoid fever, erysipelas, septic inflammations of serous cavities, meningitis, endocarditis, and similarly acute and toxic disorders. It is worth while to lay especial stress upon the possibility of this inflammation's arising from serious disorders of the gastro-intestinal track; and this fact will explain why an acute exudative nephritis should follow an eczema, since the latter complaint is in the majority of cases preceded by disease of the stomach or intestines, or both. In fact some absorption of toxic matter is at the basis of this renal disease, either primarily or secondarily. Thus it may begin as the original disease, or may occur as a sequel or complication that is at times more weighty than the first disorder. It has been known to spread so rapidly among the children of a distinct locality that some observers believe it to be epidemic.

Lesions. - There are, first of all, the ordinary features of an acute or exudative inflammation : an exudation of serum, a diapedesis of red cells, and an emigration of white cells. In addition there may be various degrees of inflammation of the epithelium, mostly in the cortex of the organ. In children there is rarely, if ever, any serious structural change, so that when the process has spent itself before or at death, the kidney is found practically 
unchanged. On the other hand, on careful examination one will be surprised at the great number of white cells found in the stroma, the tubes, and even in the capillary veins.

In the capillary tufts of the glomeruli the cells increase in size and become cloudy; in marked cases these swollen cells have the appearance of considerable masses between the glomerulus and its capsule. The cavities of the capsules may contain white and red cells, and many coagulated masses of various sizes. The capsular epithelium may be much swollen. The tubules may be swollen; their epithelium may be swollen and cloudy, in places ragged and torn from the wall. Coagulated matter chokes up the convoluted tubes in certain areas, which may also contain hyaline plugs, and white and red cells. The stroma may be infiltrated with serum, in which case the kidney will be larger than otherwise. In some severe cases there may be minute collections of pus in the stroma.

Symptoms. - The primary cases are rarely seen and easily overlooked. And whether the cases are primary or secondary, the course of the disease is about the same. They are ushered in with a febrile movement, loss of appetite, disturbances of the stomach and intestines, headache, and some prostration. In the mild cases there may be nothing beyond this; but the severe attacks steadily get worse. As the disease progresses the patient's weakness increases, there may be some dropsy, vomiting may be obstinate, the cardiac action becomes excited or exhausted, and anæmia increases progressively. The left ventricle may be dilated, and the arterial tension heightened. There may be sleeplessness, restlessness, and twitching which may become exaggerated into convulsions; in other cases stupor and delirium may mark the late stages.

In mild cases the urine is slightly diminished in quantity, its specific gravity is unchanged, it may contain small quantities of albumin, casts, and possibly blood. The severer cases have much less urine, with larger quantities of albumin, casts of various sorts, and larger quantities of pus and blood. Such cases may go on to suppression of urine.

Some of the cases which follow severe attacks of the specific infectious fevers run a serious course. Usually the child suddenly becomes acutely affected, complaining of chills, headaches, and general prostration. He refuses food, is unable to sleep, and passes 
very little urine. There may be some dropsy, but in a fair number of cases it may be so small in amount that it is regarded as unimportant. The cerebral symptoms become so much exaggerated that they stand out as the principal signs. There may be pains throughout the body, the restlessness may change to delirium, and finally may give way to stupor. At any stage of the attack convulsions may set in, the outcome of which is commonly death. The urine is somewhat diminished in quantity, its color is high, its specific gravity is not materially changed, and it may contain albumin, casts, blood, and pus.

The treatment is the same as in acute diffuse nephritis.

\section{Acute Diffuse Nephritis}

Causes. - As far as serious and permanent results go, this form of nephritis is the most important inflammation of the kidney which one is called upon to treat. Usually it is secondary, following severe infectious diseases, notably scarlet fever and diphtheria. I have known typhoid fever and acute lobar pneumonia to be complicated or followed by this inflammation, and doubtless any sufficiently toxic condition could bring about the same result. The primary cases are said to be caused by exposure and congestion; at times the ætiology is very obscure.

Lesions. - These are, first of all, the same as one sees in acute exuclative nephritis; in addition there is the characteristic feature of diffuse inflammation, a growth of new connective tissue, which is located in the stroma. Besides this, we must reckon with a growth and proliferation of the capsule cells of the Malpighian bodies. The connective tissue is scattered here and there in the cortex in strips and patches which follow the direction of the arteries. In the Malpighian bodies, the growth of the capsule cells may be so great as to squeeze or even obliterate the tufts of vessels.

The whole kidney is apt to be large, white or mottled, and congested. The changes in it have a natural tendency to permanency.

Symptoms. - The disease may begin suddenly and the symptoms may be very acute; in other cases the onset is more insidious and may not be recognized-until it has existed for a week or more. Also there are cases where the symptoms may not show themselves in full force until months after the disease begins. In 
any case, the natural tendency of the inflammation is to continue and not to subside. It is commonly ushered in with a moderate febrile movement, disturbances of the gastro-intestinal track, supplemented at times by disorders of the circulatory system, loss of flesh and strength, and anæmia. Dropsy is very apt to occur in varying degrees in the face, legs, feet, scrotum, and serous cavities, in the order given. The urine is scanty, of unchanged specific gravity, of a dark color (due in part to the presence of blood), and contains albumin, epithelial, hyaline, granular, or blood casts, and may hold in solution a smaller quantity of urea

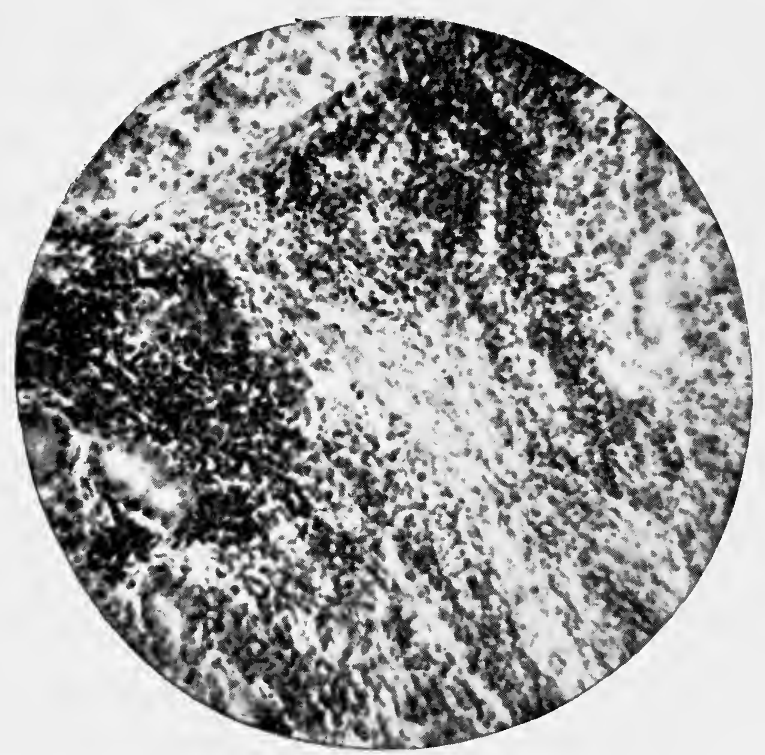

FIg. 64. - Acute Diffuse Nephritis, showing Plasma Cell Infiltration. $\times 135$.

than is normal. Occasionally the casts are not present; and as the disease progresses the specific gravity falls lower than it was.

The disease may last for weeks without a great deal of change.

Some of these cases are exceedingly severe. They may begin with or without a chill, but the general prostration becomes very marked. The child has no appetite, complains of nausea, vomiting, and disorders of the intestines. The anæmia may have a rapid course, the heart may be hypertrophied and dilated and give a hæmic murmur, and there is almost regularly a moderate or large amount of dropsy. The nervous symptoms are always prominent 
and sometimes painful; they progress from irritability to restlessness, then to involuntary twitchings of the extremities and face, and finally to convulsions. In other cases the child becomes stupid, falls into mild delirium, becomes comatose, and finally dies from uræmic poisoning. Very often there is some interference with vision, accompanied by lesions in the retina and optic nerve. The progress of the disease is fairly steady, and many of the seeming variations of the child's condition are due to the involvement of one organ after another in the process of exhausting inefficiency.

Treatment. - In both exudative and diffuse nephritis, the treatment is general and symptomatic. The patient is to be kept in bed, the diet should be restricted to fluids, calomel may be administered in small and repeated doses. Counter irritation may be applied to the surface of the lumbar region. The urine should be made as bland as possible by the use of diuretics, the best of which is pure water. The skin should be kept active by means of hot baths, and the bowels should never be allowed to become constipated. The ventilation of the room should be as nearly perfect as possible, and the patient's mind must be kept unruffled and fairly well occupied.

If dropsy is present, it is advisable to restrict the amount of fluids consumed to less than that excreted. This may require the substitution of an alkaline diuretic for the water that otherwise would be used. For the general weakness, nux vomica may be used with advantage. If the arteries are much contracted, nitroglycerin may be given; in case the heart is weak, one may prescribe digitalis, strophantus, or caffeine. There are some severe cases where the tension of the arteries is very high, the urine very scanty or suppressed, and the dropsy marked; these may require active sweating by means of hot packs, hot baths, and hypodermatic injection of pilocarpine.

As soon as the acute symptoms have passed, an organic preparation of iron, preferably combined with nux vomica, should be prescribed. The diet must be carefully restricted for a considerable time - in some cases for weeks. And above all, a rigid supervision of the hygiene and general manner of life must be sedulously maintained until every sign of disease or weakness has disappeared. Often it will be necessary to continue the use of tonics for weeks or months. 
Prognosis. - In acute exudative nephritis, the outlook, so long as the condition is not too seriously complicated by other diseases, is, on the whole, good. The natural tendency of the lesions is to resolve and leave an unchanged kidney. In the diffuse form, the result of the sickness is much more doubtful. The course of the disease is longer, its nature is much more insidious, its lesions have a greater likelihood of becoming permanent. After weeks or months it may show its worst features. Nevertheless, scrupulous care, combined with childhood's marked recuperative powers, can do much to bring about such an equilibrium and compensation in the kidneys, that to all intents and purposes the child may fully recover.

\section{Chronic Congestion and Degeneration of the Kidneys}

It is not easy to classify the chronic diseases of the kidneys. The causes are similar in all, and one condition is apt to be merged in another without giving very clear clinical evidence of the change. In chronic congestion and degeneration the differential diagnosis is largely pathological. And the main benefit in making the distinction lies in the scientific appreciation of the processes which affect the patient in the form of complications and sequels.

Causes. - In both the origin of the disorders lies in chronic disease of the heart and lungs - in short, whatever interferes with an unimpeded circulation of the blood. Thus the various forms of endocarditis, dilatations of the cardiac ventricles, pulmonary emphysema, and septic or non-septic effusions into the pleural cavity are all able to act as the exciting factor.

Lesions. - In chronic congestion the kidney at first is of ordinary size, but later on becomes large. Its surface is hard, sometimes called "stony," smooth, and congested. The veins of the pyramids are congested and in places dilated. The epithelium of the cortex tubules is cloudy, and in places swollen and, seemingly, packed tightly together. The capillaries of the glomeruli are dilated, their walls are irregularly thickened, and the cells which cover them are swollen.

In chronic degeneration there is apt to be more exaggeration in the alteration of size - either in the way of decrease or increase. The surface is smooth, the cortex is thick and of a light hue. The 
color of the pyramids is redder than usual. The epithelium of the cortex tubules is swollen, infiltrated with droplets of fat, granular, and occasionally broken down. The capillaries of the Malpighian bodies may be dilated, if the origin of the degeneration rests on cardiac disease. And in all cases both stroma and arteries are normal.

Symptoms. - The development of these disorders is a very gradual one. There is a progressive loss of flesh and strength, the muscles become weak and flabby, the child becomes more and more anæmic. Disturbances of other organs are common, and

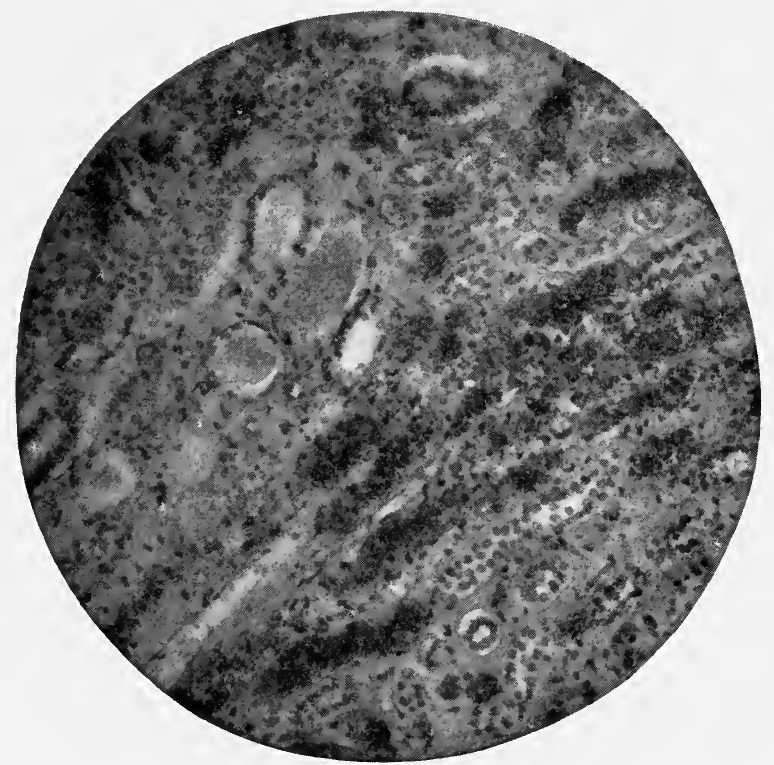

FIg. 65. - Chronic Passive Congestion of Kidney (Casts in situ). $\quad \times 135$.

may pursue an obstinate course. The stomach and intestines are very apt to be affected, so that the patient complains of anorexia, nausea or vomiting, headaches, constipation or diarrhøa. In the lungs there is a constant liability to bronchitis, which is the more persistent on account of the common irritation of the nasal and faucial mucous membranes. The heart easily falls into an overacting condition, with exaggerated second sound, and hard pulse.

In both conditions the main change in the urine is a diminution of the amount excreted." Only at certain times of exacerbations it is increased; and in chronic degeneration it occasionally 
may be suppressed. In congestion albumin and casts may be found. In degeneration the same casts and albumin may be found, but in smaller amounts.

Treatment. - In both conditions there is no specific treatment. The care of the original disease is the main indication, and as that improves, the condition of the kidneys is apt to follow. Every detail of nursing must be carefully ordered, and the child's mind be kept as quiet as possible. Digitalis must be used with caution, and at times may be really harmful, as it is apt to increase the pressure on the weakened capillaries of the Malpighian bodies.

Prognosis. - This depends, to a large extent, upon the progress of the original disease. If the congestion or degeneration continues for too long a time, it will naturally develop into a chronic diffuse nephritis.

\section{Chronic Diffuse Nephritis without Exudation}

In children the cause of this disease is always somewhat obscure, unless there has been an antecedent acute process. It is a rare disorder, and occurs, for the most part, in the older children. Like the other forms of nephritis, it may be rapid or gradual in its onset or course.

Lesions. - The kidneys are generally normal in size, until the later stages of the disease, when the organs may become shrunken; with the alteration in size the capsules grow onto the kidney walls in small areas, and finally are quite adherent. This form of nephritis is characterized by the greatest production of connective tissue, which invades not only the stroma but also the pyramids. As this process advances, more and more of the renal tissue is replaced, so that the remaining parts have an undue amount of work to do.

The tubules, partly from the strain put upon them, are irregularly dilated, and their epithelium may be congested or degenerated. The Malpighian bodies are in various stages of congestion and atrophy, and their capillaries may fall into amyloid degeneration. At the same time the larger arteries may be attacked by a slow sclerotic process that renders them rigid or occluded.

Symptoms. - The first thing that strikes the observer is the child's poor condition. He is thoroughly anæmic, exhausted, 
subject to many complicating disorders. He lacks appetite, complains of headaches, and shows the loss of flesh and strength. In addition to a hæmic murmur which may follow the anæmia, there is a strong likelihood of other murmurs being present that are symptomatic of a chronic endocarditis. The left side of the heart is oftener attacked than the right, and remains feeble. As a result of the endocarditis, emboli may become located in the meninges, the cerebral vessels, or the viscera; also the various organs may become congested, inflamed, and then give their regular symptoms of the respective disturbances. Thus there may be bronchitis,

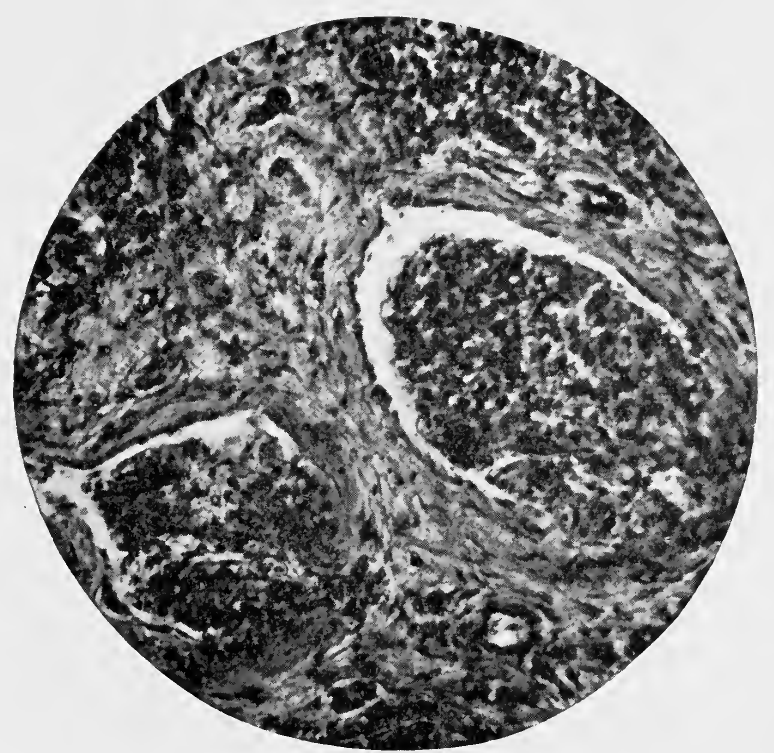

Fig. 66. - Chronic Diffuse Nephritis without Exudation; Connective Tissue, and Atrophied Glomerulus. $\times 180$.

pleurisy, gastritis, hepatitis, and perihepatitis, and even various forms of neuritis. Acute attacks of uræmia may occur in minor or major degrees of severity, producing irritability, muscular twitchings, or convulsions. There is little or no dropsy; and even if the pleura becomes inflamed there is less danger of a complicating effusion than in chronic diffuse nephritis with exudation.

The urine is usually large in quantity, unless there is an acute exacerbation of the disease, when the amount may become very small. After the acute attack has passed away, the copious flow is 
reëstablished. The specific gravity is low, the color is not high, and there is a diminished excretion of urea. Occasionally there may be some albumin and casts, but not as a general rule.

Treatment. - The principal line of treatment is to care for any precedent and concomitant disease, and to follow the needs which are demonstrated by symptoms. There is no specific drug to be recommended, but all cases derive benefit from scrupulous care in regulating the diet, hygiene, and general mode of life. Commonly we send these cases to a mild and pleasant climate,

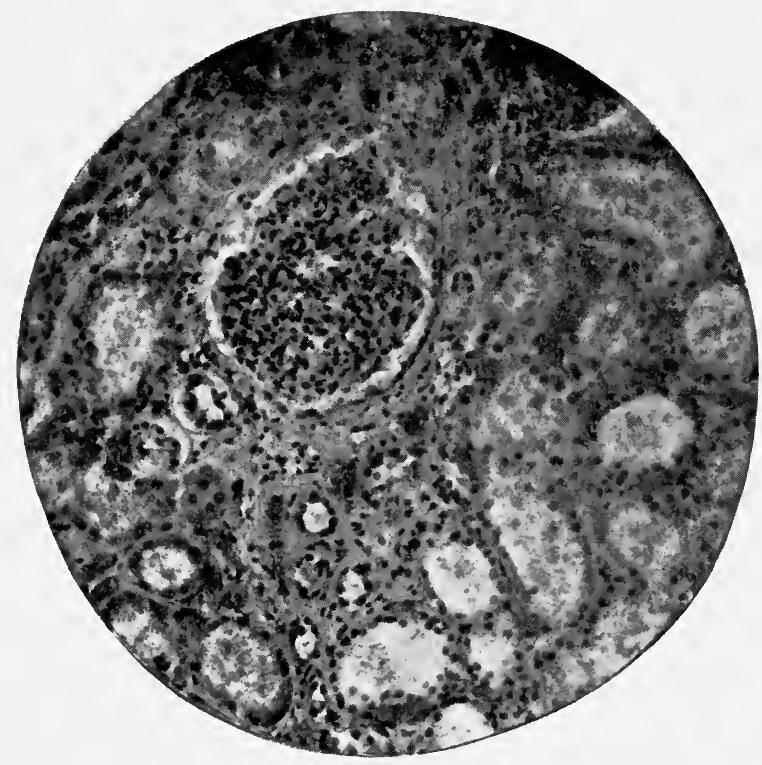

Fig. 67. - Chronic Diffuse Nephritis without Exudation. $\times 135$.

where there are no startling changes of heat and cold, dryness and humidity.

Prognosis. - The outlook is not encouraging; if the patient is in circumstances which allow the fullest attention to all his needs, his chances of obtaining a reasonable degree of health are much better than if he is cramped in means. Under all circumstances the course of the disease is long and tedious, subject to many exacerbations and remissions, while the general tendency is in the direction of deterioration.

In spite of the organic disabilities some children seemingly throw off the disease, and continue to live for years. 


\section{Chronic Diffuse Nephritis with Exudation}

This form of nephritis may occasionally be seen in well-grown children and youths as a primary disorder. In such cases it may be difficult or impossible to detect the exciting cause. In the majority of cases, however, the disease is a continuation of an acute nephritis, or an exaggeration of a chronic degeneration or congestion of the kidney. In a few instances it may follow long continued disease of the bones and joints, or chronic suppurative processes.

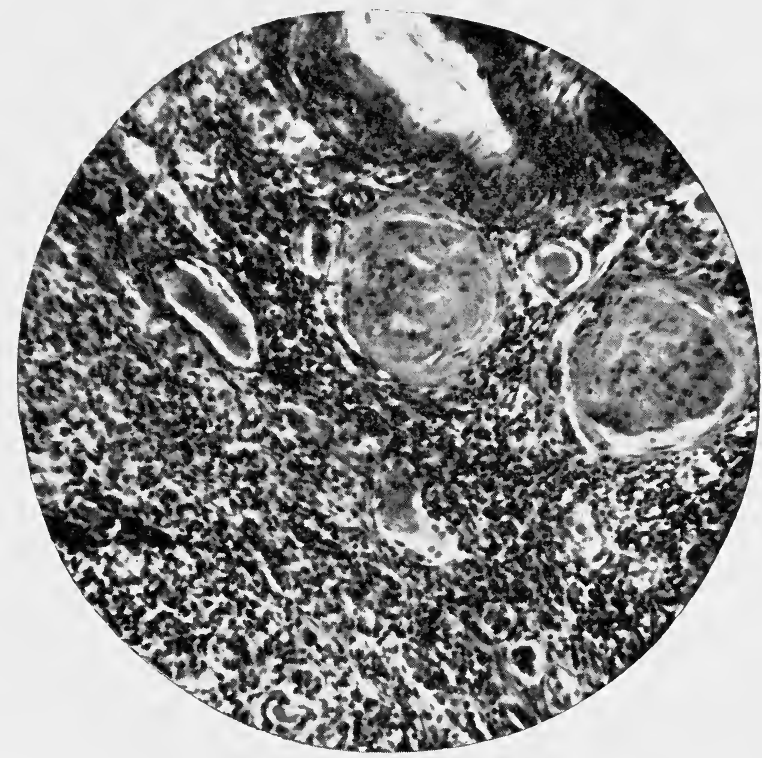

FIG. 68. - Chronic Diffuse Nephritis with Exudation. Hyaline Glomeruli. $\times 125$.

Lesions. - Generally the kidneys are large and their surface is rough in contour. The most important change is a deposition of new connective tissue in the stroma in gradually enlarging strips and wedges. At the same time the arteries may become thicker and less pliable than is normal, or, in advanced cases, the capillaries fall into a condition of amyloid degeneration. The epithelium of the tubules may degenerate, and the tufts and capsule cells of the Malpighian bodies may become hypertrophied. The nutrition of the structures may be cut off by the atrophy of the capillaries connected with them. 
Symptoms. - The symptoms which first draw attention to the case are, as a rule, apt to be the general poor condition of the patient, or the evidence of uræmia. He is noticeably anæmic, and on estimating the percentage of hæmoglobin and the number of red blood-cells the quality of the blood will be ascertained to be markedly deficient. The patient's general condition is poor, showing loss of flesh and strength, lack of appetite, headache, neuralgic pains, sleeplessness, disturbances of the gastro-intestinal track, and at times disorders of the heart. In almost all cases dropsy of the face, legs, and serous cavities is more or less evident.

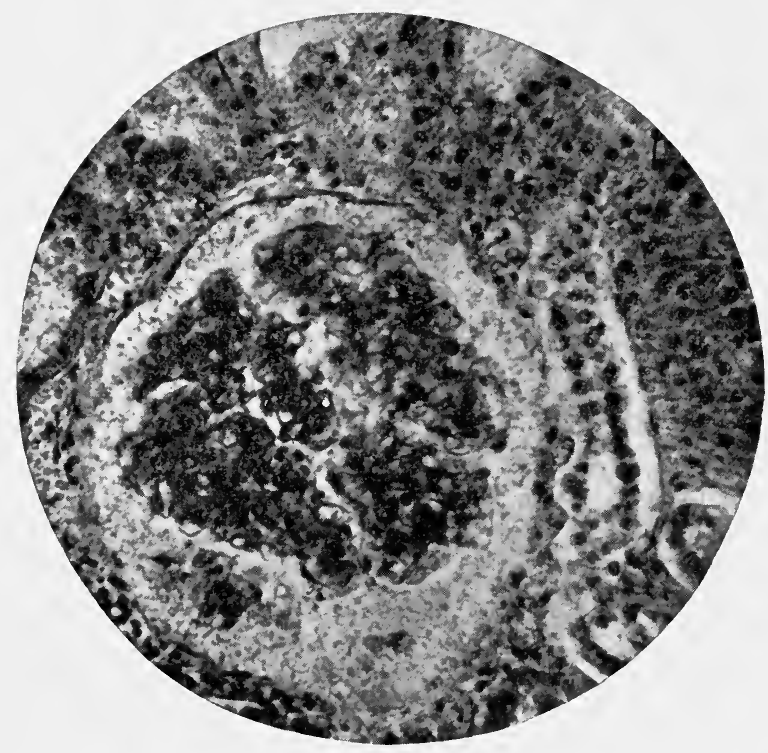

Frg. 69. - Chronic Diffuse Nephritis with Exudation. $\times 420$.

Thus a pleuritis which is contracted under such circumstances commonly has a complicating effusion that may or may not become purulent. Or the lungs on slight provocation become more or less œedematous. Examination of the eyes will at times demonstrate a neuritis or a retinitis; and in marked cases there may be asthenic diseases of the lungs, especially those dependent upon an impeded circulation. The urine varies widely from time to time in its quantitative composition. As a rule the specific gravity is low and the amount of excreted urea is small; but the daily quantity of urine changes within wide limits; as a rule, but not 
invariably, albumin and the various casts may be present in fairly large amounts, while in the acute exacerbations blood may be found in varying amounts.

The symptoms rarely continue in a steady course, but increase and decrease quite irregularly. In this way they may go on for months or years with discouraging remissions and intermissions. Thus, some cases fall into a condition of amyloid degeneration which affects, not only the kidneys, but often the liver, spleen, and intestines as well. Here there would be a long continued course of sickness with many and diverse complaints that try the vitality of

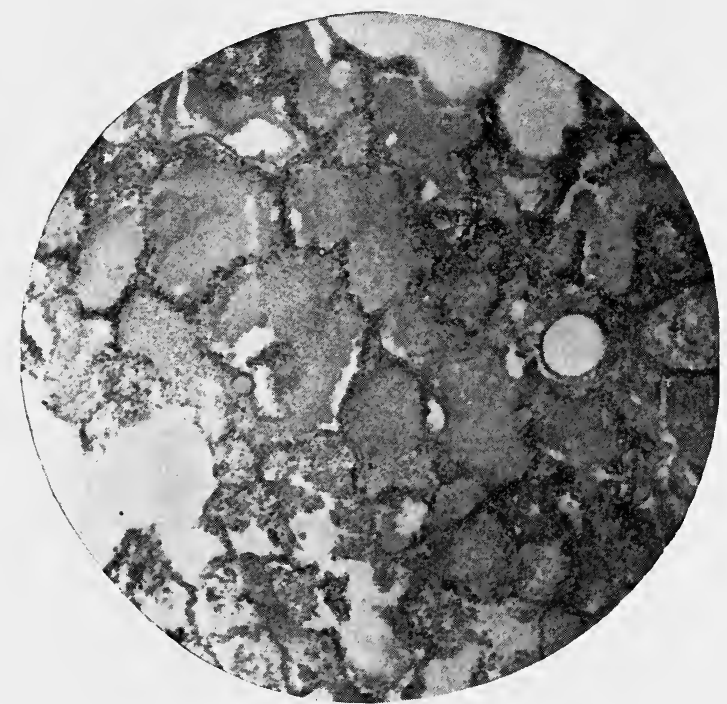

FIg. 70. - CEdema of Lung; in Chronic Diffuse Nephritis with Exudation. $\times 50$.

the patient and the resources of the physician to the utmost. The only limits to the complications seem to be the limits of the body.

The treatment is the same as in chronic diffuse nephritis without exudation. And what may be said of the prognosis in the one variety is equally true of the other.

\section{Suppurative Nephritis}

Causes. - A suppurative inflammation of the kidney is a rare disease in children. In some few cases it has occurred without discoverable cause, although that is no reason why one should 
put faith in the possibility of spontaneous creation. Ordinarily it has followed falls, blows, or wounds, the use of infected catheters or instruments, from cystitis, or embolic infection.

Lesions. - When due to trauma the disease varies in severity according to the extent of the injury. When death is not the immediate result, a pyogenic process may begin. Various amounts of tissue may break down into pus, with which necrotic kidney-substance and blood are mixed. The same result may be reached, in the course of a malignant disease, by minute emboli which the blood current carries to the kidneys. The abscess which follows may be of any size. The portion of the kidney outside of such a focus will be congested and inflamed, while inside of it one or more varieties of the pyogenic bacteria may be present. In the few cases of cystitis that one meets in children a rare complication is an extension of the inflammatory process to the kidneys. When the mucous membrane of the pelvis of the kidney, as well as the rest of the organ, is involved, the resulting condition is called pyelo-nephritis. In some cases the ureter is likewise attacked by congestion and inflammation, and varying quantities of pus may clog up its lumen.

In all these cases there are ordinary features of catarrhal inflammation plus the production of pus. Where the quantity of pus is noticeably large, we speak of the condition as pyonephrosis.

Symptoms. - Before the disease shows itself plainly, the child is plainly sick, has chills, and a hectic fever. His loss of flesh and strength is rapid, and he is subject to disorders of the stomach and the abdominal viscera, as well as diseases of exhaustion and malnutrition, to anæmia, to malassimilation, to complications that are both wide and remote. There are usually tenderness and pain in the lumbar region, and in some marked cases a tumor. The main help to diagnosis rests on the composition of the urine. The quantity is apt to be decreased, the reaction usually acid, and the presence of albumin is assured by both the inflammation of the kidney and the varying amount of pus held in suspension. This amount may be large or small, in some cases merely perceptible by means of the microscope, in others so abundant as to make the urine opaque and turbid. In addition, one is fairly sure to find red and white blood-cells, broken-down epithelium, and epithelial cells, as well as casts and micrococci. 
If there are calculi present, as they rarely are, the particular symptoms which they produce will show themselves. In the same way, if the pyogenic process extends into or behind the peritonæum, into the intestines or diaphragm, there will be corresponding signs.

Treatment. - The medical treatment consists of rest in bed, neutralization of the urine by giving alkalies when it is acid and benzoic acid when it is alkaline, the restriction of the diet to fluids, and the administration of diuretics. The general care of the case requires much attention. If the patient does not soon improve, surgical help must be summoned.

Prognosis. - The outlook is not good, but each case must be judged upon its merits. Children, except in the severest cases, recover more easily than adults. An important factor in deciding on the outcome of the disease is its retiology.

\section{Chronic Pyelo-nephritis}

A chronic inflammation of the mucous membrane of the kidney may in rare instances occur as the result of renal calculi or a chronic eystitis. The mucous membrane of the pelvis and calyces are in a condition of chronic inflammation, there is an irregular production of granulations under the epithelial layer, and the epithelium itself is ragged and hypertrophied. There may be a production of serum and pus. There will regularly be a formation of some connective tissue in the kidney.

The symptoms show themselves in a series of exacerbations and remissions. When the pus and serum collect in large quantities there is more pain than otherwise, the fever rises, and a tumor becomes evident. The urine shows these changes in the quantity of pus, and there may be a history of calculi, renal colic, and the passing of gravel.

The treatment is general and symptomatic unless surgical interference becomes necessary.

\section{Amyloid Degeneration of the Kidney}

This condition hardly ever occurs alone, but usually with similar processes in other viscera. One can scarcely regard it as a clearly defined disease; on the contrary it is no more than a stage in gradual dissolution, a state where the characteristic 
structure of the organs gradually fades away, and is replaced by a lardaceous formation that makes the kidney appear waxy and almost homogeneous. Its origin lies in the action of protracted exhaustion, such as occurs in the course of syphilis, tuberculosis, diseases of malnutrition, and long continued pyogenic processes. Thus, not only the kidneys, but also the spleen and liver, are commonly involved; and the effects of the degeneration show themselves in a slow but steady ebbing of the vitality as well as the functional activity.

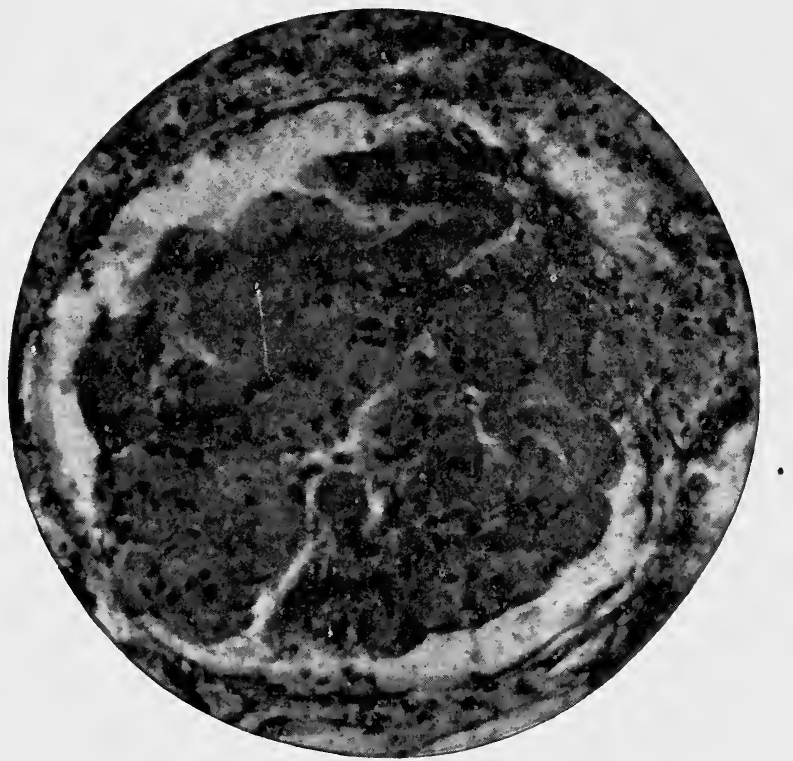

FIG. 71. - Amyloid Degeneration of Kidney, showing Glomerulus. $\times 220$.

The ordinary changes in the kidney have a very gradual evolution. The size of the organ steadily increases at first, but in the later stages it becomes smaller, and loses its regular contour. The capsule in the beginning is not adherent; but this fact in the advanced condition becomes reversed. The characteristic tissue gradually fades away, becomes pale, waxy, structureless, and gives a satisfactory reaction to the iodine test.

There are no distinct symptoms which belong to this condition. Its effects are merged into those broad disabilities of the general complaint, in which the signs that one viscus or another contributes may have a part. The whole picture is one of progressive 
wasting, of an almost uninterrupted loss of functional ability, and of steadily approaching death.

There is no especial treatment. All that one can hope to do lies in the direction of general care, answering the symptomatic indications, and most of all prosecuting the treatment of the original disease. Living in a favorable climate, the use of tonics, the employment of massage and other mild forms of exercise, and the regulation of the diet, all have some value. But, no matter what one may do, the patient as a rule dies.

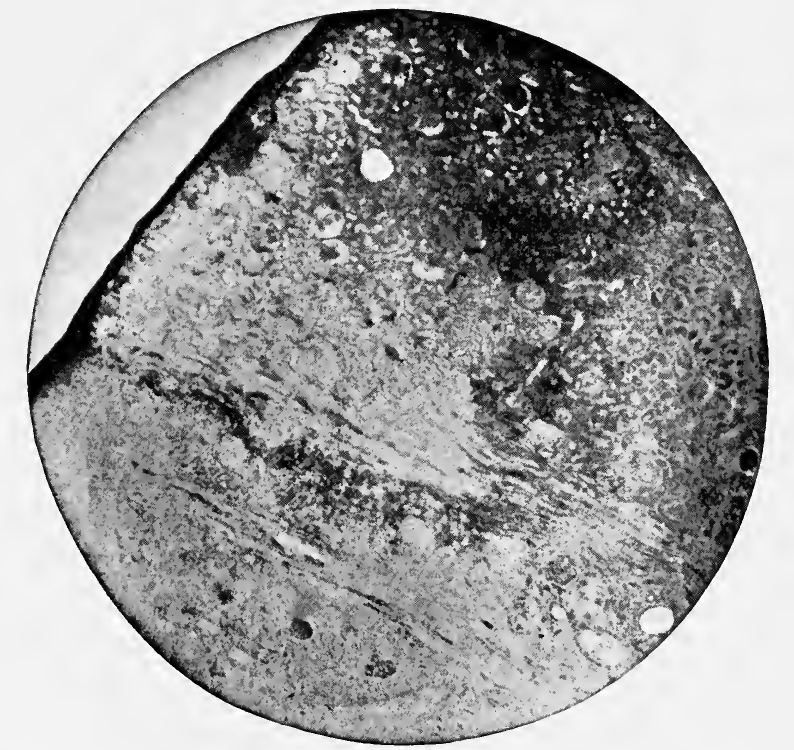

Fig. 72. - Infarction of the Kidney. $\times 10$.

(Endocarditis and Broncho-Pneumonia.)

\section{INFARCTION OF THE KIDNEY}

The terminal result of renal embolism, which we call infarction, has a keen pathological and academic interest, although its practical importance is not very great. When in the course of some grave disturbance of the circulation, such as may occur in endocarditis, a renal artery becomes plugged up and the area which it supplies is thus cut off, the effects are easily seen in the fan-shaped space which is thereby defined. The epithelial tissue thereby loses its source of nourishment, it disintegrates, its nuclei 
fade and disappear, and it goes through more or less of the process of coagulation necrosis. The broad end of the wedge is at the surface, while the apex projects into the body of the organ. The infarction may have a light red or yellow color, or in very recent cases a deep red hue. As the process of repair progresses the epithelial débris becomes absorbed, cicatricial tissue forms with the accompaniment of diminution of substance and color, and a limited establishment of collateral circulation.

It has been said that the production of an infarction in adults is indicated by a sudden pain in the region of the kidney. In children, however, the diagnosis is practically never made during life, for these patients are generally not capable of exact and faithful description of their subjective symptoms.

\section{Perinephritis}

For various reasons an acute exudative inflammation may attack the loose connective and fatty tissue about the kidney. The process may be resolved, or go on to the formation of an abscess. This is called perinephritis or perinephritic abscess.

Causes. - The inflammation may start from traumatism, exposure, or from some unknown cause. In the so-called secondary cases it may follow disease of the spine, suppurative disease of the kidney, renal calculi, abscess of the large intestine, appendicitis, disease of the ureters, bladder, or genital organs, and typhoid fever, smallpox, and other acute infectious fevers.

Lesions. - There is at first an exudative inflammation followed by a breaking down of the tissue into pus. The location of the parts admirably favors the burrowing and draining of this pus in various directions. Thus the abscess may finally point in the lumbar muscles, or on the inner aspect of the thigh as if it were a psoas abscess, or by means of the sacro-sciatic notch it may appear in the buttock; following another direction it may make its way through the urinary bladder or the peritonæum, intestines, or stomach; it has also been known to perforate the diaphragm or appear through some part of the pleural cavity or lungs.

The kidney itself is at first not seriously affected, and the urine shows few if any changes. As the abscess grows it compresses the neighboring tissues in all directions, and the kidneys themselves are naturally much affected. From congestion they 
pass on to inflammation, until they may finally succumb to an acute suppurative process.

Symptoms. - Generally there ought to be but little difficulty in making a diagnosis of perinephritic abscess because the symptoms are fairly characteristic. At first the child complains of chills, and has a varying but generally high fever; there may or may not be disturbances of the stomach and intestine. There are tenderness and pain in the lumbar region, sometimes followed by a red, swollen, and heated condition of the surface. As the abscess grows, there is a proportional inability to use the leg of the affected side; the thigh and knees are kept flexed, and the main trouble seems to be in extending them. The patient, on standing up, is apt to rest his hand upon the knee of the affected side, and so bends his torso that the spine describes a curve, the concavity of which lies toward the affected side. If the abscess points in other parts of the body there will be corresponding symptoms.

The urine shows no special changes except in some cases of injury, or where a suppurative nephritis comes to exist. Thus at last one may find some pus, blood, or albumin in the urine. But very often it is clear.

Treatment. - Rest in bed, the administration of divided doses of calomel followed by a saline, and the local use of heat or cold, are routine practices. If there is not a subsidence of the symptoms and if signs of pus formation appear, a surgeon should be allowed to operate. Delay is much more dangerous than the operation.

Prognosis. - The outlook is surprisingly favorable, especially if the process is not allowed to run on so long that it involves a fatal perforation.

Differential Diagnosis. - There seems to be a likelihood of confusing perinephritis with hip-joint disease. In most cases the doubt should not exist, for in perinephritis there is no joint tenderness, motion of the hip is impeded only in the direction of extension, and the leg is rotated outward. In hip disease motion in all directions is impeded, there is considerable tenderness in the joint, and the muscles of the thigh are wasted. Hip disease is much more chronic both in its onset and its course than perinephritis.

From cysts, tumors, and other diseases of the kidney, from dis- 
ease of the spine, psoas abscess, and growths of the abdomen, this disease may be differentiated by a careful consideration of the course and character of the various affections. For none of them imitates very closely the course and symptomatology of perinephritis. Finally the diagnosis may be confirmed by passing an aspirating needle into the abscess, and withdrawing some pus or pus-stained fluid. This must be done cautiously and with strict antiseptic precautions.

\section{HYDRONEPHROSIS}

As a result of an impediment to the flow of urine, the kidney may become dilated. This obstruction may be congenital or acquired. In the congenital variety the cause may be an imperforate ureter or urethra, a twisted or compressed ureter, or any malformation of the bladder which would interfere with its normal function. These congenital cases are often associated with other deformities, as harelip or club-foot.

The acquired cases of hydronephrosis may be due to calculi in the kidney or bladder, any stricture of the ureter or urethra by disease, injury, or new growth. Nephroptosis has been known to act as a cause.

The pelvis and calyces of the kidney are dilated to a small or great degree. The fluid may be a normal or altered urine, with or without an admixture of pus. The fluid distends the pelvis of the kidney, then the calyces, and finally may so wear down the kidney tissue that nothing more than a mere shell is left. As the tumor increases in size it may give pressure symptoms in any direction, and may be felt on palpation through the abdominal wall or even in the back. If one kidney alone is affected, the excretion of urine may be successfully carried on by the other; when both are attacked, the patient will show the signs of uræmia.

It is possible to confuse such a tumor with a cyst of the spleen, liver, ovary, or kidney. Generally a careful examination will suffice to clear up the diagnosis. Perinephritic abscess, pyonephrosis, and hydatid cysts may be diagnosticated in part by urinary examination.

There is no specific treatment for this condition except that of an antecedent causal disease, unless the tumor is very large or both kidneys are involved. Such cases call for surgical interference. 


\section{Parasites of the Kidney}

Echinococcus. - In a general invasion of this parasite, mother-, daughter-, and grand-daughter cysts may be formed in the kidney. A tumor may form, attended by pain and the passage of a small quantity of blood in the urine. The diagnosis is confirmed by finding the hooklets in the urine. The treatment is surgical.

Other parasites that have been seen, but rarely, in the kidney are Strongylus gigas, Filaria sanguinis hominis, Trichina cystica, and Bilharzia homatobia. They lie so far from the usual experience and practice in children that one should properly look for them in special works on parasites.

\section{New Growths of the Kidney}

In early childhood the kidney may be the seat of tumors which in most cases are of serious import. A very large proportion are

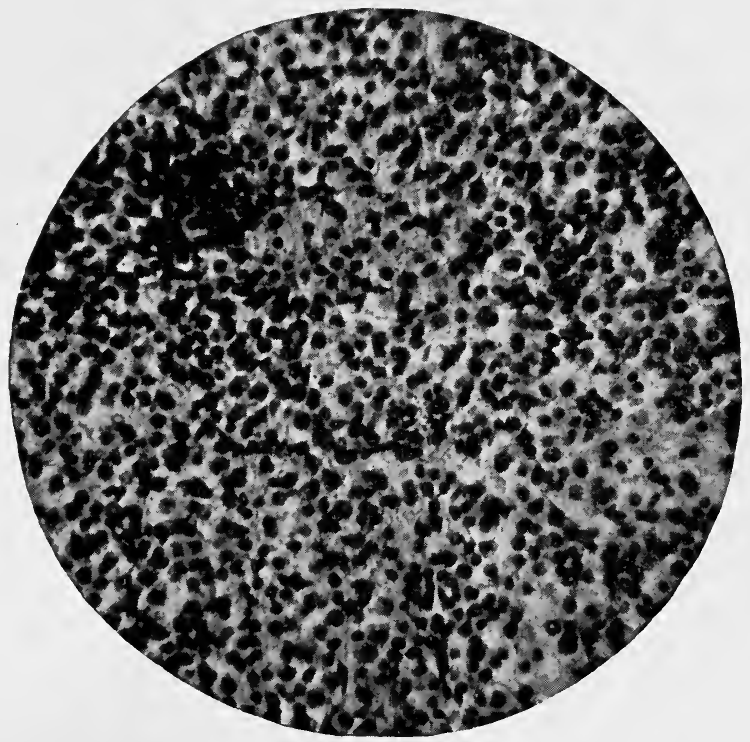

FIG. 73. - Large Round-Cell Sarcoma of Kidney. $\quad \times 180$.

malignant, and most of these are sarcomatous. The different varieties of round-celled, spindle-celled, and myo-sarcoma are represented; in a much smaller percentage one may find carci- 
noma or a mixture of sarcoma and carcinoma. Most of these tumors are congenital, and in the others the rtiology is obscure. A very few cases of benign growth have been recorded which were fibromata or adenomata.

The symptoms are not intelligibly discernible until the growth has assumed a sufficient size to render discovery by palpation possible. In some instances its bulk becomes enormous, especially in the later stages of the disease. Usually, however, the doubt is felt only in the earliest periods. The only exception to this rule is the fact that in some of the cases an early microscopic examination of the urine may show small amounts of blood-cells. The other symptoms are the deformity, pressure effects with their corresponding signs, gradually increasing malnutrition, cachexia, and a small amount of pain.

The diagnosis is usually not difficult; but the tumor of the kidney must be distinguished from growths of the liver, spleen, abdominal organs, uterus, ovaries, and from hydronephrosis.

These cases are surgical, and their treatment is therefore surgical. The sooner operation is performed, the better are the chances of recovery.

\section{Renal Calculi}

Renal calculi are concretions of various sizes formed in the pelvis or tubules of the kidney by the precipitation of the solid constituents of the urine or of matters held in suspension in the urine. They may occur in the form of small gravel-like bodies, or in masses so large that they block up the ureters. They are found at all ages of extra- and intra-uterine life. They vary in composition, being composed of uric acid, of oxalate, phosphate, or carbonate of lime, cystin, or xanthin. Often their composition is complex, being made up of two or more of these ingredients.

The cause of this precipitation is hard to state; and although many explanations have been given, nevertheless none has been entirely satisfactory.

Symptoms. - The small calculi may pass away in the urine without giving any signs. As soon as the concretions become so large as to pass into and through the ureters with difficulty, renal colic shows itself by tenderness and pain in the region of the kidneys; this pain may spread in the direction of the testicles (which from spasm of the cremaster muscle are then tender and 
retracted), the bladder, the end of the penis, the perinæum, the hypogastrium, and the inner aspect of the thigh, following, in short, the branches of the lumbar plexus. In some cases it may even be referred to the opposite kidney.

The attacks may last a short or long time; the latter cases are especially able to give general prostration, nausea, a rise of temperature, or even syncope. In a moderate as well as a severe attack the respiration is quick and shallow, the skin may be cold and clammy, and the face has an anxious look.

In the urine one may find crystals, small quantities of pus, blood, and epithelial cells. It is passed in small amounts and with spasmodic frequency.

If the ureter is occluded, hydronephrosis may result; or from irritation and pressure a pyelo-nephritis or atrophy of the renal substance may result.

Treatment. - For the relief of immediate pain, we may give hot baths and in older children hypodermatic injection of morphine. In addition, especially during the intervals between the attacks, large quantities of alkaline waters should be consumed, and the diet must be as largly fluid as possible.

If the symptoms point to the presence of a large calculus, one that is too large to be passed, an operation for its removal should be urged. 


\section{CHAPTER XX}

\section{THE SPECIFIC INFECTIOUS DISEASES}

Is the following two chapters there must necessarily be so many references to the lymplatic glands and their drainage areas, that it has seemed best to insert here a partial but satisfactory table of them. The adenitis that occurs in so many of the specific infectious diseases is by no means a matter of laphazard; on the contrary there is the closest connection between the swelling and inflammation of the glands with diseases of the associated part. It is advisable to work out this connection in every case ; for not only do we thereby obtain additional information about the disease in question, but also we get new aids in differential diagnosis. And most of all we are saved from the error of supposing that the child is sick with yet another disease.

\section{Lymphatic Glands, their Location and Drainage Areas ${ }^{1}$}

\section{HEAD AND NECK}

Glands

Drainage Area

Suboccipital

Mastoid . . . . . . . $\}$ Posterior half of head.

Parotid . . . . . Anterior half of head, orbits, nose, upper jaw, upper part of the pharynx.

Submaxillary . . . . Lower gums, lower part of face, front of mouth and tongue.

Suprahyoid . . . . . Anterior part of tongue, chin, and lower lip. Superficial Cervical . . . Exterior ear, side of head, and neck and face.

Retropharyngeal . . . . . Nasal fossæ and upper part of pharynx.

Deep Cervical . . . . . Mouth, tonsils, palate, lower part of pharynx, larynx, posterior part of tongue, nasal fossæ, parotid and submaxillary glands, interior of skull, and deep.parts of head and neck, upper set of lymph glands, lower part of neck, and joining axillary and mediastinal glands. 
UPPER EXTREMITY

Supracondyloid . . . . Three inner fingers.

Axillary . . . . . . Upper extremity, dorsal and scapular regions, front and sides of trunk and breast.

\section{LOWER EXTREMITY}

Anterior Tibial and Popliteal . Deep lymphatics of the leg, and receive some vessels from the skin of the leg and foot, chiefly the outer side.

Inguinal :

Femoral set, superficial . . Superficial vessels of lower limb, and partly of buttock and genitals, also perinæum.

Horizontal set, superficial . Abdomen below umbilicus, buttock, and genitals. The deep vessels of the lower limb go to the deep glands along the femoral vein.

Iliac . . . . . . The pelvic viscera and the deep vessels of the genitals partly.

Lumbar . . . . . . All the lower glands, uterus, testes, ovaries, kidneys.

Sacral . . . . . . The rectum. 


\section{ScARlet Fever}

Scarlet fever, or scarlatina, is an acute, eruptive, and infectious fever that is worthy of most serious consideration and study. Under favorable conditions its course and treatment may be very simple; but on the other hand it may be difficult to recognize, refractory to treatment, and serious or even fatal in its outcome.

Cause. - It is undoubtedly the result of infection by a microorganism; but what that micro-organism is and what its life history is, are still matters of investigation. Many attempts at isolating and cultivating it have been made; and in this connection one should refer to the outbreak of scarlatina in Marylebone, London, when the infection was traced through the use of milk obtained from a herd of cows in Hendon. The epidemic was practically confined to the users of this milk; and when the cows that supplied it were examined they showed evidences of a certain pyogenic disease of the teats and udders, as well as pathological changes in the viscera. From these animals Dr. Klein obtained a streptococcus that was similar to one found in the affected consumers of the milk. The validity of this claim has been strenuously attacked on the ground that the streptococcus involved was not the cause but the accompaniment of the scarlet fever, and that it was no other than the well-known streptococcus pyogenes. Confirmation of this view is given by the occurrence of various forms of the fever, some of which are clearly complications of streptococcic invasion. It is undoubtedly possible that the so-called septic and malignant forms are merely ordinary cases of scarlatina plus a varying but always severe invasion of one or more varieties of streptococcus. The more one sees of these cases and the more one observes their course and complications, the more one is convinced that they make a picture, not merely of a severe eruptive fever, but also of a septicæmic or pyæmic disease grafted thereon. Examples of this may be seen in the trying and dangerous cases of tonsillitis and laryngitis, of cellulitis, adenitis, and nephritis that complicate attacks of scarlatina which otherwise would be comparatively simple. This view is made stronger by the fact that the occurrence of such complications acts in the way of a second infection, that they are not necessarily a part of the original disease, and that when they get a hold upon a hospital or locality a large proportion of the cases therein become affected by 
them. Moreover, additional evidence from another side is provided in the good results obtained in some cases by the use of anti-streptococcus serum where these severe complications were thereby simplified or nullified. The point wherein we still are weak is our ignorance of streptococcus, its many possible varieties, the differentiation of one from another, and the consequent difficulty in the production of specific serums.

Lesions. - In the ordinary case the pathological changes are simple and not many. The eruption is evidently the result of the absorption of toxines. There is congestion of the capillary vessels in the cutis vera, with a consequent swelling in the surrounding area. There are an emigration of white cells and a proliferation of round cells into the Malpighian layer. The inflammatory process continues in and about the sweat glands and ducts, by which the epithelial layer breaks down and exfoliates. When desquamation takes place a minute quantity of serum makes its way between the superficial layers of the epidermis, whereby small scales are forced off. When the amount of fluid is somewhat greater than ordinary, the pressure may become great enough to cause the papule to rupture. From this point the scaling spreads out on all sides.

The only other changes that belong to uncomplicated scarlatina are those in the throat. Here the pharynx goes through the ordinary changes of a catarrhal inflammation characterized by congestion, swelling, emigration of white cells and diapedesis of red cells, proliferation of epithelium, and the production of varying amounts of mucus and fibrin. According to the severity of the process, superficial ulcers may occur. The same changes occur substantially in the tonsils; but here the glandular tissue may increase markedly in size and the follicular secretion be much exaggerated.

In the ordinary simple cases the viscera are very slightly affected; there is doubtless some degree of acute degeneration or acute inflammation which expresses itself with greater or less force in this organ or that, according to the circumstances of the case. Even without a septic element the kidney, liver, intestines, and glands may be adversely influenced; but the changes have a natural tendency to resolve themselves, so long as the fever is uncomplicated.

Of all the viscera the kidneys are the most easily affected. 
Even in the mild attacks and when the general disturbance is comparatively small, there is some degree of congestion and cortical cloudiness. Under the impulse of an asthenic condition, unhygienic surroundings, unwise treatment, or disorders of assimilation and excretion, this congestion easily develops into an acute exudative nephritis (q.v.). The lesions may be of all grades of severity; they may be so slight that the symptoms and the urinary analysis can scarcely suggest their presence, or if there has been a marked absorption of toxines they may become so grave as to assume a hæmorrhagic character, give the manifes-

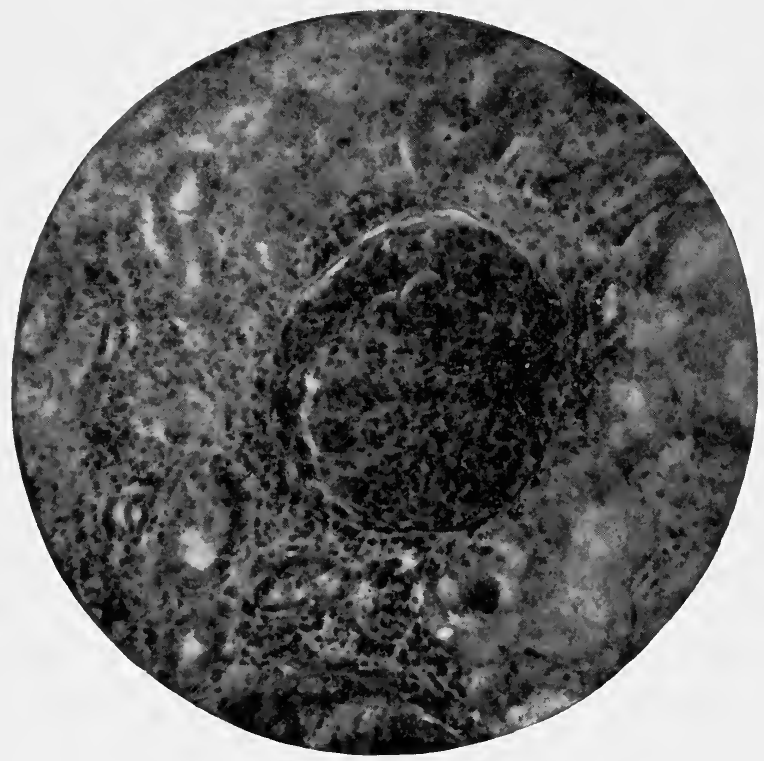

Fig. 74. - Acute Scarlatinal Nephritis. $\times 135$.

tations of acute uræmia, and soon result in death. In other cases the progress of the disease takes on a sub-acute, and finally a chronic, form which is characterized by the various changes of a diffuse inflammation.

Symptoms. - A patient who contracts scarlet fever may have been infected in various ways : directly from another patient, indirectly from some person who has been near the patient, or even through a third person who obtains the infection from such mediate agency, from clothing, furniture, domestic utensils, books, toys, household animals, - in fact, practically any person or thing 
that has been in the patient's vicinity, - and food or drink. The patient is the main source of contagion, by means of contact after the period of incubation, by the desquamating scales, excreted matter, such as fæces, urine, perspiration, discharges from the ear, and possibly the breath. A member of the household, the physician, or nurse may likewise be instrumental, directly or indirectly, in spreading the disease; and so volatile is the poison that it may be wafted from contaminated articles through considerable spaces of air. Moreover, this poison is very tenacious, so that rooms, furniture, books, and clothing may retain their virulence for weeks

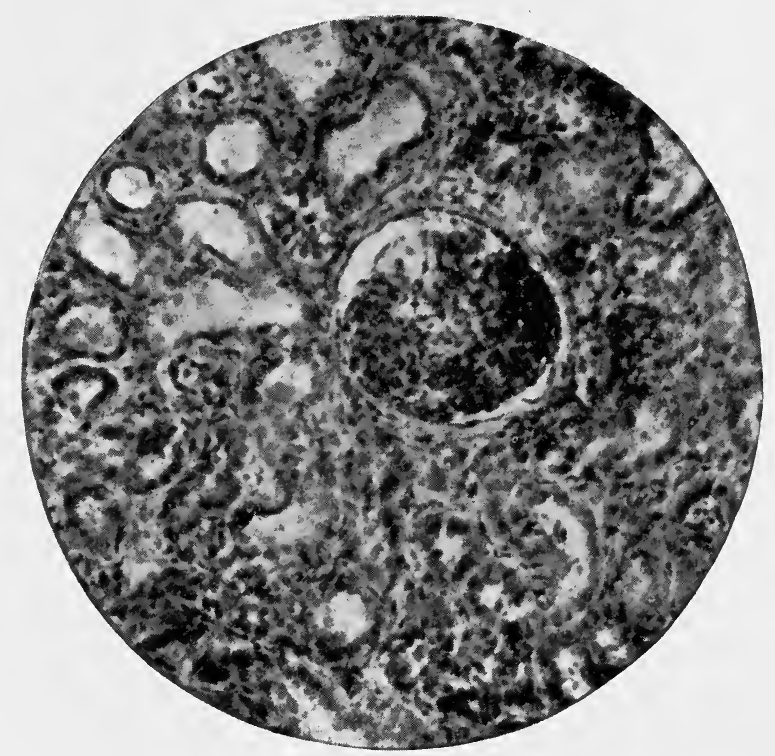

Fig. 75. - Acute Hæmorrhagic Scarlatinal Nephritis: Glomerular Thrombosis. $\times 135$.

or months. The possibility of infection by means of such articles as water, milk, bread, and fruit, is well known and occasionally very fertile.

When the patient has become infected, he goes through a period of incubation that varies in different cases. In the majority of cases the time is from two to four or five days, but the extreme limits are from a few hours to about two weeks. Remarkably short or long periods should always be scrutinized with suspicion.

The onset of scarlatina is almost always sharp and abrupt. 
In most cases soreness of the throat is the first symptom shown, followed by vomiting, - often without nausea, - malaise, and rise of temperature. So constant are these symptoms apt to be that any combination of them, even if another rtiology seems evident, should command respectful consideration. Their intensity varies in different cases : the vomiting may be trivial or severe, occasionally accompanied by other digestive disturbances; the flush on the faucial mucous membrane may be light or deep, and the hard palate may be studded with few or many small red spots, all of which appear from twelve to twenty-four hours before the exanthem and continue into the second week of the disease; simultaneously the associated glands may be slightly tender; the temperature is in no way characteristic, and may be trivial or severe, slightly more than normal, or as high as $40.5^{\circ}$ or $41^{\circ} \mathrm{C}$. $\left(105^{\circ}\right.$ or $\left.106^{\circ} \mathrm{F}.\right)$. The amount of malaise or prostration varies with the severity of the attack and the vital resistance of the patient; in one case the child is scarcely sick, in another he is thoroughly prostrated, has chills, convulsions, and toxic depression. These differences may characterize the whole course of the disease.

At the expiration of a few hours or a day, rarely longer, the scarlet rash begins to appear, and persists for a variable period, which is usually about a week, but which may vary from one day to two weeks. One must be cautious in announcing the end of the eruption, for occasionally the red flush may disappear only to appear again. This is especially apt to happen at times of markedly deficient elimination or weak cardiac action.

The favorite seat of the eruption is on the chest and neck, whence it spreads to the face and the rest of the body. Close inspection shows it to be made up of a great number of small red points, the clustering together of which gives the characteristic scarlet flush. There are many possible variations, both in its course of development and appearance: it may develop from the legs as a starting-point, or the arms, or any other portion of the body; it may be heavy or sparse, deep or light colored, continuous or circumscribed, petechial, macular, or hæmorrhagic. Nevertheless, in practically all cases the characteristic quality is so clearly evident that little or no doubt concerning it need be felt. Especially is this true, if one keeps in mind that with the appearance of the eruption the other symptoms should develop with an equal step. The throat 
becomes more tender and painful, and occasionally is covered in part by a thin membrane; the glands become plainly enlarged and tender - so much so at times that they render movement of the parts painful; the tongue may be coated, the temperature rises, the pulse becomes correspondingly rapid, followed somewhat laggingly by the respiration, and the skin, on account of itching, becomes uncomfortable. The tongue, after being coated

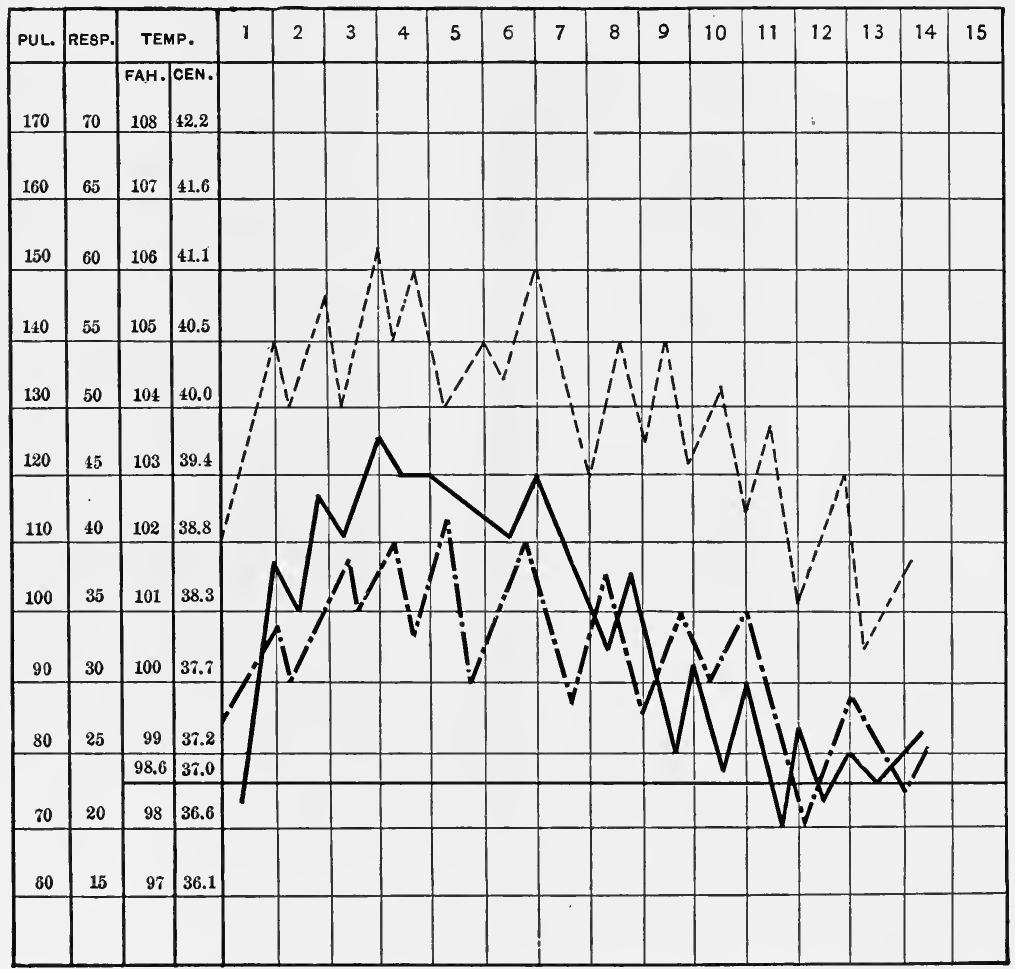

PULSE, RESPIRATION AND TEMPERATURE CHART OF SCARLET FEVER. AGE, 5 YEARS.

PULSE RESPIRATION TEMPERATURE

FIG. 76.

on the average for a half week, clears up and assumes the wellknown "strawberry" appearance; the temperature, pulse, and respiration begin to travel toward their normal limits; the throat gradually becomes painless; the sense of malaise steadily and appreciably fades away, and the child goes on to convalescence. 
At this time desquamation sets in, beginning on the portions of the skin first affected by the rash, and being most abundant where the skin is thickest. It continues on these parts longest-occasionaliy as long as two months, while other portions of the body, as, for instance, the face, may be quite clear in a week. It occurs in the form of fine flakes that are shed in copious amounts; on the fingers and toes, in other words where the skin is thick, it may change its form, and instead of fine flakes the skin may come off in patches and strips that are usually compared to sections of a glove-finger. In a few cases the hair and nails have been shed.

This is a description of a mild, or, at the most, a moderate case; frequently the variations in intensity are noteworthy. On the one hand, the cases may be very mild, so much so that carelessness in observation will pass over them entirely until attention may be drawn to the desquamation, possibly to ear disease, or to complaints that are founded upon a nephritis. On the other hand, the children may from the very first be exceedingly sick, the symptoms may in every way be much exaggerated, and the danger of the disease becomes great. These cases, according to their severity and the judgment of individual observers, have been called severe, or septic, or malignant forms. The distinctions are arbitrary and misleading, and subserve no good end. Rather it would be much clearer and much more in accord with our knowledge - even although our knowledge is small - to call these cases scarlet fever with bacterial complications. Upon this secondary infection depends, to a large extent, the severity, course, and outcome of the sickness. Unfortunately, our information of the bacteriology involved does not allow us to speak finally of the varieties and the possible differentiation of one from another. But, nevertheless, their septic character is not hard to discern in the prostration, the temperature, pulse, respiration, and the complicating features presented. In one form the throat is deeply involved: after the disease has well begun, an intense inflammation attacks the tonsils. They are much congested and swollen, small areas of membrane appear on them, and thence gradually spread over the pharynx and rhino-pharynx, the soft palate and uvula, sometimes the epiglottis and larynx, and into the Eustachian tube. The affected tissue becomes puffy, friable, and may break down into superficial ulcers. At the same time the fever rises to greater heights $\left(40^{\circ}\right.$ to $40.5^{\circ} \mathrm{C} .-104^{\circ}$ to $105^{\circ} \mathrm{F}$.), about 
which it describes an irregular curve, the breathing may be difficult, and the portion of the head and neck stiff and constrained on account of the swelling of the affected tissues; at the same time and as a predominant symptom, the signs of intoxication may be indefinitely great. According to the character of the changes in the tonsils and throat, one may speak of the inflammation as membranous, ulcerative, or gangrenous, although frequently the types are not sharply distinguished.

In these severe cases one is apt frequently to see the involvement of other parts of the body. Thus the submaxillary, cervical, and post-cervical glands become swollen and tender, and may later on break down into abscesses. The inflammatory process may extend to the cellular tissue of the neck, and the resulting cellulitis may be of serious import, most of all if septic absorption, followed by destruction of the walls of the large vessels or thrombosis, be not prevented by surgical means. Following the inflammation in the throat, one frequently sees the symptoms of an otitis media. The younger the child, the more apt, on account of the anatomy of the Eustachian tube, is this to happen, especially when the weather is bad. In such cases there is an exacerbation of the fever and constitutional prostration, the child throws his head from side to side, places the hand from time to time on the affected side, and cries in a sharp, piercing voice. If the pressure is not relieved by paracentesis of the drum membrane, the discharge may break its way through, thereby relieving the pressure symptoms. The otitis is subject to the same course and the same terminations that occur outside of scarlet fever, excepting that, in very malignant cases, the outlook is more discouraging, not only in regard to the safety of the ear itself, but also as far as life itself is concerned. Sometimes a septic inflammation of the bronchi, lungs, and pleura may arise, especially in the severe cases, attended by the features that mark such disorders.

In almost all cases, there is some disturbance of the kidneys. In the mild cases, this is barely recognizable; in the severe attacks, especially those which are characterized by deep intoxication, the lesions range from acute degeneration through exudative nephritis to a definite interstitial change whereby a greater or less amount of parenchyma is replaced by connective tissue. These serious lesions commonly do not occur until the second or third week of the sickness. The symptoms are described 
under the heading of diseases of the kidneys. In these cases the nervous symptoms are generally marked. Even in the mild attacks, some disturbance of the nervous centres is frequently encountered. Thus one can account for the chills, the pains in head and body, the tendency to convulsions, and the restlessness, especially at night. When the fever is very high or

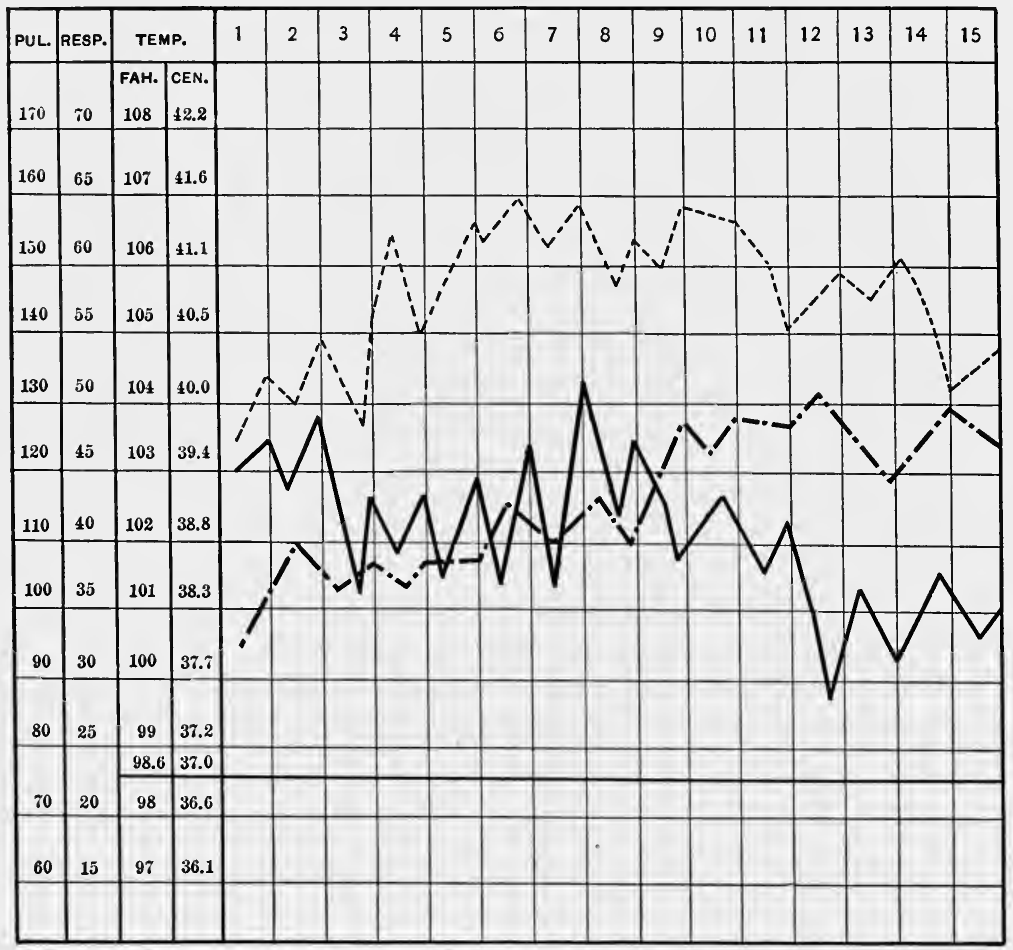

PULSE, RESPIRATION AND TEMPERATURE CHART OF SCARLATINAL NEPHRITIS.

THIRD AND FOURTH WEEK OF SCARLET FEVER.

AGE, 31/2 YEARS.

PUL8E

REBPIRATION

TEMPERATURE

Fig. 77.

toxæmia emphatic, these manifestations may develop into dulness, stupor, delirium, mania, or coma. In some instances peripheral neuritis with consequent paralysis, or chorea, or epilepsy have followed. There is a rare set of scarlatinas, sometimes called cerebral, in which a muttering delirium, stupor, and coma are present from the early hours of the disease. They are characterized 
by very high temperatures, unusual or deficient eruptions and a short course of two or three days. For the most part the result is death.

Another complication that occurs in from 6 per cent to 10 per cent of the cases of scarlet fever is an inflammation of the joints. This is commonly diagnosticated as rheumatism, although in most cases such a judgment is false. While it is quite possible for a scarlet-fever patient to contract rheumatism, nevertheless such a complication is not often seen. As a rule such attacks are synovitides, slight in mild cases, severe in strongly marked or septic fevers. In fact the degree of sepsis controls to a large extent the amount of disturbance in the joint, which may range from a simple synovitis to pyæmic abscess formation. Any articulation may be affected, although the small joints are oftener involved than the large ones. The history of the patient, the general tendency to spontaneous recovery in the light attacks, and the ease with which severe ones become purulent, the absence of the thick creamy coating of the tongue that is so generally present in rheumatism, and the small likelihood of a resulting endocarditis, are all apt to mark off these disorders as doubtfully rheumatic.

A more frequent accompaniment of this fever is some disorder of the gastro-intestinal track. This is generally functional, rarely organic. Outside of the vomiting which occurs in the beginning of the sickness, there may later on be vomiting as well as diarrhoea due to improper food, to sepsis, to uræmia, and to the inception of some complicating disease. Another series of attendant affections, but not so common, is that of the heart. Here again functional disorders rather than organic are the rule. In this connection, variations from the normal heart sounds do not necessarily signify endocarditis. In fact this condition as well as pericarditis is seen infrequently, excepting in the so-called malignant or septic attacks. In scarlet fever these inflammations have the same course as in their primary condition. In giving an account of the possible contingent affections, one should not forget to include the liability to contract eczema, which generally starts from the irritation produced by an acrid discharge, such as may flow from the ears, the nose, or even the mouth. There is nothing peculiar about these disorders of the skin, nor is their treatment difficult.

Operative cases, when brought within the range of scarlet. 
fever, very early become infected. Many of them illustrate the connection of the fever with pathogenic germs, such as streptococci ; for often the children show symptoms of sepsis, but have no rash, and possibly no sore throat. For such reasons these cases have been sometimes called "atypical," although there is a serious doubt whether they should be at all included under the head of scarlet fever. On the other hand, there have been some unquestionable instances of this manner of scarlatinal transmission, but they are not apt to be as severe as the atypical ones.

Treatment. - Prophylaxis is comprised in the phrase thorough disinfection. Since both patient and his apartment with its contents are capable of spreading the contagion, it follows that both these factors should be cut off from the outside world as carefully as may be. The child should be rigorously isolated until all traces of desquamation have passed; in case of doubt he should not be allowed to live with other children for upward of a month and a half, or two months. All clothes and washable fabrics in the sick-room should be boiled for a few hours; and to make this item as simple as possible, the sick-room in the beginning should be stripped of all superfluous carpets, curtains, hangings, pictures, furniture, and clothing. The nurse should be isolated from the family, and whenever she leaves the house should disinfect her hands and face, and, if possible, her hair ; she should in addition wear other garments than those used indoors. The physician on entering the sick room should don a gown, that is easily made from a sheet pinned or sewed into shape, and on leaving should disinfect his face, hands, beard, and hair. The furnishing's of the room should be thoroughly disinfected by steam, and whatever articles are of little value, especially if their nature or construction renders them hard to cleanse, should be burnt. Most of all, the pillows and mattresses should be treated with scrupulous severity. The walls, ceiling, and woodwork must be scrubbed and washed down with a strong bichloride solution, the wall-paper removed, and the painting and papering renewed. The old-time method of disinfection by means of sulphur fumes is useless, and gives a false sense of security.

The general care of the patient is simple. At the beginning of the fever the child should be placed in a bare, empty room, where good ventilation, directly from the window or by means of grate fires, can be easily obtained. The more efficient the prepa- 
rations for isolation at the beginning, the less trouble and regret does one have later. An important item is the disinfection of the fæces and urine, preferably by means of mixing with a strong solution (1:500) of corrosive sublimate, and the providing of napkins and handkerchiefs made of cheese-cloth or other cheap material, which may be burnt after being used.

The patient should take a cathartic, such as a saline or small and repeated doses of calomel followed by a saline; after this it is advisable to keep the intestines moving gently but regularly. A hot bath may be given at the beginning of the treatment whereby the rash will appear more fully, the skin will be less uncomfortable, and the patient much more at ease. On each succeeding day the whole body should be bathed or sponged with lukewarm water once or more times, and in case of high fever one may prescribe warm or cool packs or the graduated bath. The higher the fever, the oftener should these baths be given, accompanied by cold affusions to the head and friction to the body. One should endeavor to forfend the depression which follows too long an exposure to cold, and to see to it that the child does not remain in a shivering condition and with cold extremities after being returned to bed. In the cases of hyperpyrexia, especially those with cerebral symptoms, much comfort may be obtained by the continued application of ice bags to the head.

The nose and throat should be copiously irrigated with antiseptic and alkaline solutions administered frequently. There is no drug that acts as a specific in scarlatina, and all that one can do in this direction is to promote elimination by some such means as the compound mixture of rhubarb and soda plus nux vomica. The diet should be simple and fluid, and liberal quantities of distilled or boiled water may be allowed. The complications should receive the particular treatment that each calls for.

As soon as desquamation sets in, the body should be anointed with vaseline after each sponge bath. When the normal temperature is restored and the patient is free from serious complications, the diet should be progressively enlarged and a tonic prescribed. For this latter purpose an organic preparation of iron is useful and readily borne. When the means of the family permit, the child should be sent into the country for a prolonged vacation.

Prognosis. - The danger of scarlet fever is controlled by the 
youth of the patient, his environment, the severity of the epidemic, and the existing complications. Infants bear the disease poorly, and some statistics give a mortality of fifty per cent. As a rule, the older the patient, the smaller the chance of death. Up to the age of puberty the general average of all cases may be reckoned as ranging from eight to twelve per cent. A child who was previously sick or weak makes a poorer resistance than one who is healthy and vigorous. With the presence of marked sepsis the danger becomes greatly exaggerated, most of all if the truth of the matter is not promptly recognized and considered in the treatment. Nothing can be more unfortunate than the view that these cases are scarlet fever pure and simple, but merely a more severe type than the ordinary. The complications carry their share of danger, especially the more serious ones, such as cerebral disease, nephritis, lung and laryngeal disease, and joint infection.

A child suffering from scarlet fever may suffer from a relapse or a simultaneous attack of measles, diphtheria, chicken-pox, pertussis, erysipelas, typhoid, and typhus fevers. All that one can say is that with the severity of the new attack and the weakness of the patient, the prognosis becomes proportionately worse. The occurrence of typhoid and typhus fevers in this connection is very rare.

Differential Diagnosis. - A difficulty in diagnosis for the most part depends upon an imperfect or irregular eruption. This difficulty exists because a somewhat similar irritation of the skin may be seen at times in diphtheria, the early stage in smallpox, in typhoid fever, in gastric disturbances, associated with urticaria, heat erythema, and following the use of quinine, belladonna, and antipyrine. The observation and history of these cases soon make the nature of the disorder plain, and especially if the physician keeps in mind the characteristics and location of the scarlatinous eruption, the throat lesions, the glandular involvement, the sharp onset of the attack, and the usual prostration. More serious confusion may exist when the question of differentiating scarlet fever from measles and rubella arises. The points of difference are made plain in the table facing page 424 . Tonsillitis and diphtheria by their temperature, characteristic appearance of the throat, their history, and the occurrence of disease in the neighborhood and adjacent school usually are sufficient to remove doubt within a short time. 


\section{MeAsles}

This disease, otherwise called morbilli, is the commonest of the acute infectious fevers. It has been known so long and so intimately by all classes of people, that one of its dangers lies in the familiarity that brings contempt. By a large part of the laity it is regarded as of very little importance, although the most serious conditions and even death itself may result from it. This is especially true in regard to peoples to whom it is a comparatively new disease, and to infants under eight months of age.

While measles is, on the whole, a disease of early childhood, nevertheless older children, and even adults, may become infected. There is no age so young or so old as to possess immunity; even a babe at birth may bear the contagion, and in proportion to the youth of the patient the disease is dangerous. Everybody is susceptible to the contagion of measles, which herein has a point of difference from that of scarlet fever; for the latter undoubtedly seems to possess a liability to attack certain persons. In addition there are adventitious circumstances which modify the course and fix the character of the measles. Thus what may in one child be a simple disorder, easily and quickly thrown off, may in another be a matter of the most serious import. Measles should never, $\grave{a}$ priori, be regarded as a trivial disease ; on the contrary it should be regarded as a fertile means of physical deterioration, of opening the way for a large number of possible pathological effects.

Causes. - It is generally agreed that this disease is caused by an infective micro-organism which as yet has not been isolated and described. Several investigators have at various times believed that they had found the bacillus in question, but time did not verify their claims. It is unnecessary to go into the details of these investigations, since their interest is in the main historical.

Whatever the germ may be, it is at all events very active in its diffusion and certain in infection. It is believed to distribute its contagion from the body surface, the bronchi, mouth, nose, and tears. The disease is generally transmitted from the patient directly to the prospective victim, and rarely through the intermediary of another person, às for instance the attending physician. In the same way the apartments of the patient, and the clothes and utensils which he used, do not remain contaminated 
in an obstinate manner. On the contrary, on full exposure to light and air and with sufficient cleanliness, they easily become innocuous.

Lesions. - The changes that occur in measles are located in the eruptive areas of the skin and in various degrees of acute exudative inflammations of the viscera of the abdomen, thorax, and head. The inflammation in the skin is located, for the most part, in the corium and rete ; there are considerable swelling, congestion, and infiltration of white cells. These round cells are seen in greatest number around the small vessels, along the hair ducts, the follicles and sweat glands, and in the papillary layer.

The other lesions are of much more importance. They consist of inflammations of the mucous membrane of the eyes, nose, pharynx, mouth, larynx, trachea, and bronchi, and the intestinal track. The kidneys may fall into some degree of acute degeneration or acute exudative inflammation, and the lymphatic glands may likewise be congested and inflamed. The severity and often the character of these changes depend upon the predominant bacteria present, and the power of their toxines.

Incubation. - The average incubation period is said to be about ten days, but the variations in both directions are great. A series of cases, in one epidemic, or in one neighborhood, is very apt to give an individual and separate number of days for each child. Thus the limits extend on the one side to about four days, and on the other to two weeks. The eruption, as a rule, does not appear until from three to five days after the period of incubation.

Symptoms. - The first signs which the parents notice are generally described as a "cold." There is a catarrhal inflammation of the mucous membrane of the nose, eyes, mouth, throat, and bronchi, all of which give their characteristic symptoms. On the palate and mucous membrane of the cheeks one may notice a few small, dark red spots, and Koplik has described some scattered bluish macules, in the centre of which is a white point, that appear on the inner aspect of the lips and the opposing surface of the gums as much as three days before the exanthem. The general appearance of the child now begins to be indicative of the disease; the cough becomes harsh, the face looks peculiarly puffed up and doughy, the fever rises, and the child complains of malaise and irritability. As the nose and throat become more and more involved, the glands in the submaxillary and post-cervi- 
cal regions begin to swell and be tender. There may be one or more chills, and the child evinces some slight prostration.

About three or four days after the period of incubation, an eruption appears on the face and body. Usually its first location is behind the ears or on the forehead; thence it spreads to the face, and so makes its way to the body and extremities. This course is not always alike; it may be rapid and uniform, or slow and uniform, or broken up by intervals of various degree. Likewise the eruption may be light or heavy, according to the severity of the attack. The rash at first is indistinct, more like an irregular blush with a few spots here and there; in a short time it ought to give way to small, reddish, flat papules, that gradually become macular in form. These spots have a tendency to coalesce into a rough crescentic shape with a somewhat irregular outline. A profuse eruption may fully cover the body surface, while a mild attack may leave many areas untouched. The amount of rash usually gives rise to a corresponding amount of swelling of the face. In those cases where the congestion about the small blood-vessels is very great, minute hæmorrhages into the skin occur which go to form the hæmorrhagic rash. Such an eruption may likewise be variable in extent. In very weak children the petechial points on the back and thighs may be so large as to merit the name of ecchymoses. A steady development of the eruption is much to be desired; and any interference or retardation must be regarded with apprehension as an indication of unusual intoxication and consequent exhaustion. On the other hand, the rash may appear, run its course, and reappear.

When the eruption has been in existence for about a day, it begins to fade away in the order in which it came, taking from less than a day to a week to disappear. Most cases clear up fairly well in from two and a half to four days. Until it has vanished the child is noticeably troubled with the swelling, tenderness, and itching of the skin. These symptoms are in proportion to the profuseness of the outbreak. When the eruption has faded away, a fine desquamation takes place, and continues for from a few days to about two weeks. As the rash develops, the general symptoms maintain an even pace. The temperature, reckoning from the beginning of the attack, generally assumes a double maximum curve ; by the end of a day and a half or two days it may amount to $38.5^{\circ}$ or $39^{\circ}$ C. $\left(101.2^{\circ}\right.$ or 
$102.2^{\circ} \mathrm{F}$.), it then drops a variable amount only to ascend again at the height of the eruption to $39.5^{\circ}, 40^{\circ}$ or $40.5^{\circ} \mathrm{C} .\left(103^{\circ}, 104^{\circ}\right.$, or $105^{\circ} \mathrm{F}$.) Therefore one speaks of the initial and the eruptive fever. Various elements, such as complications, surroundings, and the patient's vitality, may to some extent modify these curves, but even under such disturbing factors one can often dis-

\begin{tabular}{|c|c|c|c|c|c|c|c|c|c|c|c|c|c|c|}
\hline PUL. & RESP. & TEI & MP. & 1 & 2 & & & & 10 & 11 & 12 & 13 & 14 & 15 \\
\hline 170 & 70 & $\begin{array}{c}\text { FAH. } \\
108 \\
\end{array}$ & $\begin{array}{l}\text { CEN. } \\
42.2\end{array}$ & & & & & & & & & & & \\
\hline 160 & 65 & 107 & 41.6 & & & & & & & & & & & \\
\hline 150 & 60 & 106 & 41.1 & & & & & & & & & & & \\
\hline 140 & 55 & 105 & 40.5 & & & & & & & & & & & \\
\hline 130 & 50 & 104 & 40.0 & & & & & & & & & & & \\
\hline 120 & 45 & 103 & 39.4 & & & & & & & & & & & \\
\hline 110 & 40 & 102 & 38.8 & & & & & & & & & & & \\
\hline 100 & 35 & 101 & 38.3 & & & & & & & & & & & \\
\hline 90 & 30 & 100 & 37.7 & & & & & & & & & & & \\
\hline 80 & 25 & 99 & 37.2 & & & & & & & & & & & \\
\hline & & 98.6 & 37.0 & & & & & & & & & & & \\
\hline 70 & 20 & 98 & 36.6 & & & & & & & & & & & \\
\hline 60 & 15 & 97 & 36.1 & & & & & & & & & & & \\
\hline & & & & & & & & & & & & & & \\
\hline
\end{tabular}

PULSE; RESPIRATION AND TEMPERATURE CHART OF MEASLES.

$A G E, ~ 4$ YEARS.

PUL8E.

TEMPERATURE

REBPIRATION

FIG. 78.

tinguish the general plan. After this the fever as a rule gradually subsides to the end of the disease. Defervesence may come suddenly, as by crisis, when there may be some degree of collapse ; as a rule, however, it occurs slowly and more or less regularly. The pulse and respiration generally follow the variations in temperature. The progress of the other symptoms depends very 
much upon the degree of toxicity, the vitality of the patient, and the character of his environment and care. The question of toxicity is not, as a rule, sufficiently considered. To it the worst effects of the disease are due, and, what is of still greater importance, by it the liability of contracting complications is controlled. A person in a state of disease is peculiarly liable to the attack of related sicknesses, and in measles the various affected organs very easily fall into a more serious condition. Thus the mouth, pharynx, and larynx, that in the beginning suffer from a mere catarrhal inflammation, may easily be invaded by specific bacteria, and membranous or true diphtheritic inflammation is the result. The case would then, in addition to its original symptoms, have the modifying influences of the newly added element.

Under the stress of the disease, the mouth likewise may contract a more or less serious infection, ranging all the way from a parasitic stomatitis to noma. Such a stomatitis is particularly liable to take place in the sulcus between the lips and the teeth. Small pieces of white membrane may dot the mucous surface, giving way in time to small erosions, having a tendency to flow together. From these ulcers the Staphylococcus aureus may be isolated. The trachea in its turn may be attacked, and provide a path for the downward spread of infection. This organ has a marked danger in the diphtheria that complicates measles, since a common location of the false membrane is on the larynx.

In the lungs we find the commonest and the most serious of these changes. The original condition is one of bronchitis, not necessarily of a serious type, excepting in very young infants whose anatomical configuration of the lungs tends to the involvement of the finer capillary tubes. From the mouth, nose, or inspired air are taken various pathogenic germs, such as streptococcus, staphylococcus, and the pneumococcus, which find a fertile culture-ground in the affected tissue; and some degree of true pneumonic inflammation is very apt to result. This may take the form of a serious bronchitis, or a broncho-pneumonia of variable extent and severity, or a true lobar pneumonia. The bronchitis may be ordinary in character, or may have a spasmodic, suffocative element that lessens -as soon as the eruption has fully developed. The broncho-pneumonia bears even more the general appearance of a secondary infection, and its frequency of 
occurrence is in proportion to the obstacles in the way of wise treatment and hygienic care.

The stomach and intestines are regularly affected, but the intestines seem the more liable to serious disorder. The condition assumes the type of a sub-acute derangement, with mucoid, offensive movements. When this condition occurs before the

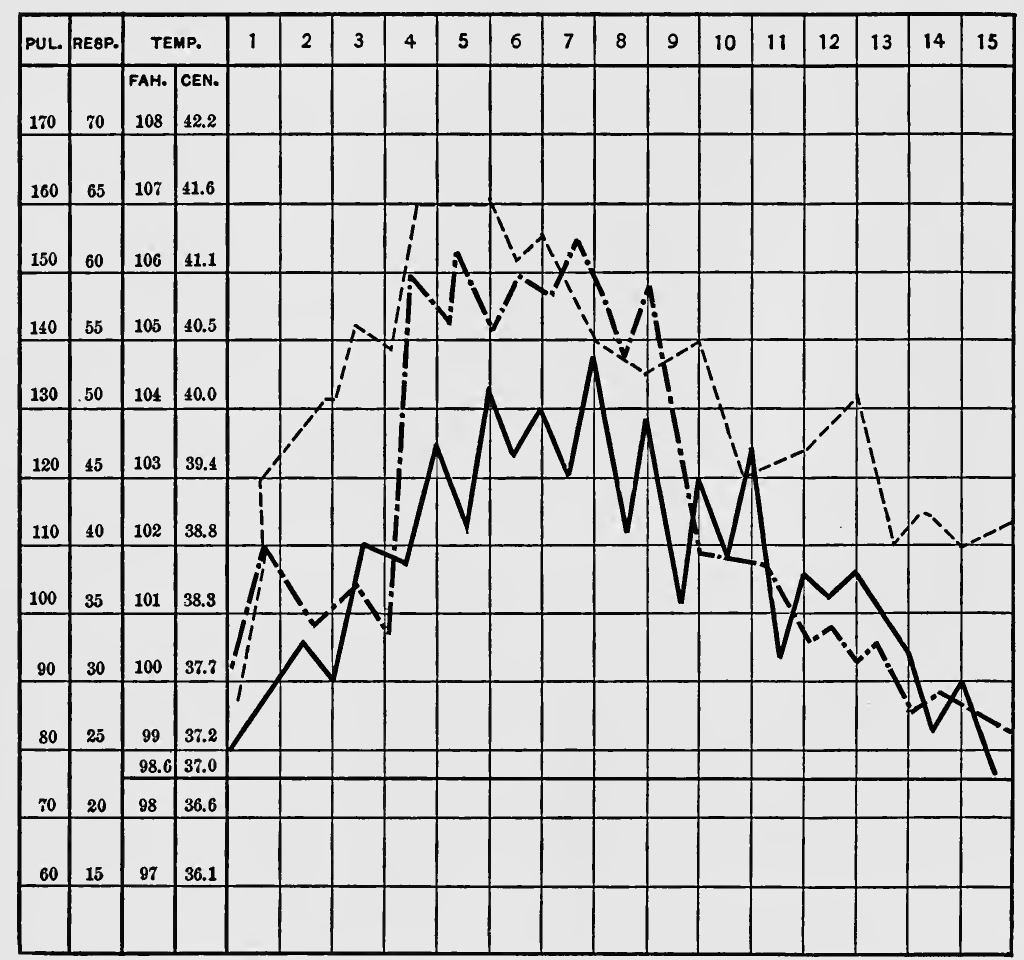

PULSE, RESPIRATION AND TEMPERATURE CHART OF MEASLES; ACUTE BRONCHO-PNEU.MONIA. $A G E, 2$ YEARS.

PULBE, RESPIRATION TEMPERATURE

FIG. 79.

eruption is fully developed, especial attention must be paid to it with the view of preventing the more serious organic lesions of the ileum and colon with an accompaniment of prostration.

Among the rarer conditions is an inflammation of the vulva; in badly nourished children who are not kept clean, a vulvo-vagi- 
nitis may follow. In its worst form, the inflammation may become gangrenous. The result is then, usually fatal.

An otitis, from infection through the Eustachian tube, happens more frequently in children than in adults. The main danger is the possibility of an extension to the mastoid cells. If the inflammation and effusion in the middle ear are great, paracentesis may be necessary. The symptoms are the usual ones of middle ear disease. In the inflammation of the eyes, there are no features that are peculiar to measles.

The kidneys are usually affected in no serious degree, and the graver forms of nephritis need not be apprehended where the child's environment is fairly good. The heart and pericardium are also rarely involved, and in the same connection one may mention severe skin diseases, mental, and nervous disorders. Still less frequent are sudden hæmorrhages from mucous membranes.

Especial importance should be laid upon the possibility of tuberculosis as a complication or sequel, most of all in children who are weak or of the tubercular predisposition. In measles, glandular enlargement is a common condition ; when this enlargement persists after the other symptoms of the disease have subsided, there is always cause for apprehension. But the attacks of tuberculosis may be widespread, so that any part of the body may be involved. Either these complicating attacks may come during the immediate convalescence, or they may be deferred until weeks or months afterward. At all events the utmost care should be exercised to forestall or ward off this danger.

Treatment. - The preventive measures consist in isolating the patient for at least three weeks, and in the thorough cleansing and disinfection of his apartments. The room and all its furniture should then be exposed for two weeks to the fullest light and air. After this, danger may be supposed to have been outlived. One should keep in mind that the patient's person, even in the earliest stages of the disease, is a more fruitful source of contagion than his apartment, and that the germ of the disease is not so tenacious of life as that of other fevers, for instance, scarlet fever and diphtheria.

The treatment of the disease itself is symptomatic and general rather than specific. While nothing can be done to check or diminish the course of measles, still we may accomplish much in the way of conserving strength, warding off complications, and 
making convalescence easy and comfortable. As soon as a diagnosis - or even a probable diagnosis - is made, the gastro-intestinal track must be emptied and the patient confined to bed. The room should be kept cool and dark, and the eyes, if their irritation is great, should be irrigated with a boric acid solution, and treated with ice cloths. The diet should be restricted to fluids, and water should be given in liberal quantities. An item that I consider of great importance is the thorough cleansing of the nose, mouth, and throat, preferably with an antiseptic alkaline fluid, such as made from Seiler's tablets. In place of this, one may use a saturated solution of boric acid with peroxide of hydrogen in the proportion of two to one. These fluids may be used in an ordinary atomizer; and in order to be of definite use, they must be administered with a liberal hand. Their value in preventing complications of the ears, nose, throat, mouth, larynx, trachea, and lungs, and even the gastro-intestinal track, is not easily overestimated; also at the same time they contribute materially to the patient's comfort.

If the rash is tardy or halting in appearing, a hot bath will materially hasten its eruption. The patient should then be bathed one or more times daily in lukewarm water. This alone will make him feel lighter, fresher, and his skin less irritable than otherwise; in addition, after the bath, he may be anointed with a small quantity of vaseline.

The control of fever and irritable cerebral symptoms may be left to hydro-therapeutics. The graduated warm bath and cool packs applied to the body are, in almost every case, efficient; especially is this so if they are thoroughly and liberally employed. It is wise practice to use cold affusions to the head and heat to the feet. The latter precaution should be observed after every bath or pack, most of all if there is any depression or deficient circulation.

The medicinal treatment is simple; if there are no especial indications, one's attention may be directed towards promoting glandular activity by the administration of such means as the compound mixture of rhubarb and soda, with or without nux vomica. Individual symptoms will call for special prescriptions : the cough may need a sedative mixture, as bromide of soda for infants, or the deodorated tincture of opium for older children; the diarrhœa may call for liberal doses of bismuth subgallate. 
The sick-room should be kept cool and dark, and when possible one room should be used in the day and another at night. The physician should see to it that the temperatures of both rooms are about the same. The freest possible ventilation should always be maintained. The child's bedclothes and nightgown should be changed night and morning, and under ordinary circumstances he should not be overburdened with blankets. Mentally and physically he should be kept as comfortable as possible.

Prognosis. - In robust children, over two or two and a half years of age, whose surroundings are favorable, measles is generally not a serious disease. On the other hand, weak children, those with a tubercular history or predisposition, children who live in asylums or in circumstances of ignorance, uncleanliness, and stupidity, find in it a frequent cause of prolonged sickness or even death. According to some statistics more than fifty per cent of children under one year of age, who suffered from measles, died during or shortly after the attack; while those from five to ten years of age lost but little more than two per cent. The same rule may, in a general way, be distinguished in almost all mortality tables, and variations in one direction or another are the result of adventitious circumstances. In a fair grade of private practice the deaths should not average more than one and one-half per cent. In asylum practice it may easily amount to six or seven per cent.

The more carefully we try to prevent and diminish complications, the smaller will the death-rate be; this holds good with especial force to the danger of tuberculosis. The physician should endeavor to impress upon his patients the necessity of looking upon the potentialities of measles with respect, that the disease may prove to be more than a passing inconvenience, and that only by rigorous care are they able to minimize its dangers.

Differential Diagnosis. - A typical case of measles is hard to mistake; but all cases are not typical, and so the disease has been confused with acute eczema, rubella, scarlatina, varioloid, and copaiba eruption. The catarrhal symptoms in the throat, nose, and eyes, the enanthem or eruption in the mouth, the history of infection, and the development of the disease, which a few hours will bring, are entirely sufficient in almost every case to clear up any doubt. 


\section{RUBELLA}

This disease, sometimes spoken of as German measles or rötheln, has as its main symptoms a rose-red rash of short duration, long incubation, slight prodromal signs, mild course, and almost no complications. It may occur sporadically or in epidemics, and is often mistaken for slight attacks of scarlet fever or measles.

Cause. - It is commonly believed to be due to infection by a specific micro-organism, which as yet has not been isolated and described. This germ attacks infants and adults rarely; nevertheless one must keep in mind that these persons are not exempt. About five years ago I saw a well-marked case in a young man of twenty-two years of age.

Symptoms. - The main signs of the disease appear after an incubation period of from one to three weeks or more. There is often considerable difficulty in fixing the beginning of this period on account of the slight symptoms. Different observers give varying accounts of this item. However, the incubation period toward its end is characterized by slight fever that rarely rises above $38.5^{\circ} \mathrm{C} .\left(101.3^{\circ} \mathrm{F}\right.$.) and a slight or moderate enlargement of the post-cervical glands, some malaise, and in a rare case a perceptible irritation of the mucous membrane of the throat and eyes.

A few hours later the eruption appears in the form of a small or moderate number of pink or rose-red papules that at first are confined to the face, or to the forehead, or behind the ears. The intensity of the color varies in different cases, disappears on pressure, and on fading leaves a light brown pigmentation for a short time. The papules may be almost flat or, in well-marked cases, somewhat raised. They are usually well scattered, but in their developed form may flow together and form red patches. In exceptional cases the eruption appears first on the body or in the mouth. Later on a part or the whole of the surface may be covered with a fine, blush-like redness. There is no subjective irritation of the skin connected with the rash.

When these maculo-papules are at the height of the efflorescence, the temperature reaches its climax. Not often does it pass $38.5^{\circ}$ or $38.8^{\circ} \mathrm{C}$. $\left(101.3^{\circ}\right.$ or $102^{\circ} \mathrm{F}$.). At the same time whatever additional symptoms belong to the case reach the fullest develop- 
ment. These may be some bronchitis, some suffusion of the eyes - commonly called pink eye-without photophobia, possibly a slight soreness in the throat, or some glandular enlargement along the sides of the neck, under the angle of the jaw, and in rare cases in the groin. There may or may not be noticeable depression, and often the patient can scarcely be called sick.

The eruption, after lasting one, two, or three days, begins to fade rapidly, and the child immediately feels almost well; whatever disability remains is for the most part a slight weakness. In some cases a fine desquamation, usually small in quantity, begins as soon as the eruption fades, and may continue for a week or less. Often there is no recognizable scaling at all.

The gastro-intestinal track seems not at all to be affected, nor are any sequels or complications usual. The occurrence of disease of the eyes, throat, and ears may be regarded as much in the way of a coincidence as a result. The only children that are endangered by rubella are those who are very weak or sick at the time with some serious disorder, such as tuberculosis.

Treatment. - All that we need to do for these cases is to clear out the gastro-intestinal track, restrict the diet to fluids, keep the patient in bed for about a week, and give daily baths. It is rare that one meets indications for further treatment.

The patients should be isolated as soon as the disease is recognized or suspected. They should be kept in seclusion for at least three weeks, and even those who have come in contact with them before the diagnosis was fully made should be sequestered for a like period. The bodies of the patients, as well as their apartments and clothing, should then be disinfected.

After recovery the patient may be benefited by a tonic.

Differential Diagnosis. - The main points of distinction between rubella on the one hand, and measles and scarlet fever on the other, are the history of infection, the mild symptoms, the lack of marked catarrhal inflammations, the eruption, the time when the enanthem appears, and the absence of high fever and complications.

\section{VARICELLA}

Chicken-pox is a highly infectious disease, commonly seen in young children, and caused by a specific micro-organism which as yet is not known with certainty. Bareggi reported investigations in the course of which he isolated an ovoid coccus from the white 
blood corpuscles of patients who had gone as far as the fifth day of the disease. He stated that by means of cultures of this micrococcus he inoculated children with varicella. Before we can regard the matter as definitely settled, further information is needed.

The disease may be communicated by contact with the patient, indirectly through persons who have been in communication with the patient, with infected articles of clothing or furniture, and possibly through the air near the patient. After exposure a period of about two weeks is required before the disease shows itself, the limits of incubation being from eleven to seventeen days.

Symptoms. - In the majority of cases there are no prodromata. The child may not at all feel indisposed until the rash appears. On the other hand, in unusually heavy attacks there may be some little malaise, nausea, and possibly a slight rise of temperature. In very rare cases severe prodromal symptoms have been reported. The usual experience, however, is that the child is not considered sick until the eruption appears and thus heralds the fact. The rash consists of several crops of small rose-colored macules usually scattered over the chest, back, and arms, and the mucous membrane of the mouth and throat. Thence they may spread to the face, scalp, and legs. The spots on the body are apt to be irregularly oval, while on the extremities they approach the more nearly round form. In some instances the order of appearances is changed, the face or other part being first affected. The macules, the number of which is rarely large, have often a pink or reddish areola, and become decolorized on pressure. A few hours after appearing they become hard, change into papules, and then into vesicles. These changes take place so rapidly that a patient on almost any day of the eruption shows all three stages. The vesicles are smaller than the macules, have a very thin covering, are unilocular, and are filled with a very clear, alkaline serum. Their appearance is so characteristic as to remind one of an old and good comparison to small white blisters raised by scattering drops of boiling water on a tender skin. The covering of these vesicles may rupture, and a part or a whole of the contained fluid may escape, leaving a depression or umbilication in the centre of the structure. Some of these formations become covered with a crust, while underneath them a small pyogenetic process may exist. This leads to a variable amount of ulceration with a resulting scar. 
In the well-marked cases there is doubtless an element of local infection with pus-forming germs such as might attack a similar small wound. Reports of hæmorrhagic and gangrenous varicella, especially in tuberculous children, have been made, but they are exceedingly rare. Their names sufficiently describe them.

The eruption lasts from two days to two weeks, according to the virulence of the infection and the number of crops of the rash. The disease is communicable from the first outbreak of the spots until desquamation has been completed.

The complications are not dangerous so long as the child is well cared for. The open wounds produced by the rupture of the vesicles gives an opportunity, as stated above, for infection. In this way erysipelas has been known to supervene. Diseases of the kidneys, lungs, and nervous system may occur, but they are rare.

Treatment. - All that one need do consists in making a diagnosis and supervising the general care of the patient. The gastrointestinal track should be emptied, the patient confined to bed, the diet restricted to fluids, and the body bathed or sponged daily. In case the fever mounts to an uncomfortable height, cold packs or the graduated cold baths may be ordered. Any restlessness or delirium may be relieved by the use of ice bags to the head. If the skin itches to an uncomfortable extent, it may be sponged frequently, and then anointed with vaseline or lightly covered with a dusting powder.

Complications should be treated according to their needs.

Differential Diagnosis. - Confusion of varicella with skin diseases is not apt to occur after the physician's first visit, for the latter give no fever and have no enanthem. The history, the manner of eruption, the simultaneous existence of macules, papules, vesicles, and crusts, the occasional pitting of scattered vesicles, the appearance of the enanthem, all these mark off varicella from the other eruptive fevers. See table facing p. 424 .

\section{VARIOLA}

Smallpox, one of the most venerable diseases, is an acute epidemic fever, with a high degree of infectivity, and an eruption that quickly passes through the stages of macules, papules, vesicles, and pustules. In all-likelihood it has had more of an effect upon the history of the ancient and mediæval world than any other similar sickness. 
Causes. - The works of Klein and Hart have done much to fix the responsibility of the disease upon a specific bacillus. But since the investigations are as yet incomplete, we may at any rate conclude on the grounds of analogy that the active cause of smallpox is a micro-organism that finds its way into the economy through the mucous membianes of the nose, mouth, throat, lungs, and possibly stomach. The contagion is carried by the air, in contaminated articles, such as furniture, clothing, rags, books, and domestic utensils, from the patient during life or after death, from fæces, or by a healthy person who has been in communication with the contaminated person or things. The virulence of this infection is strongly marked, but most so during the stages of the vesicles and pustules, and the formation of crusts ; however, the virulence of the poison is not so important a factor in the spread of smallpox as is the susceptibility of the prospective patient. A virulent degree of infection may be followed by a light attack, while a mild degree may give rise to a severe, protracted, or fatal sickness. The personal equation is after all the deciding consideration, and even in the very young ages it has an important influence. While from certain tables of statistics one would draw the conclusion that older children and youths are the greatest sufferers from this disease, nevertheless no age, not even the unborn child, is free from danger. The main fact is that infants and young children are less exposed, and therefore seem to be less often attacked. In similar ways one may get the impression that males are more susceptible than females; but here again the question of exposure is a weighty one, and in all probability may be sufficiently so to bring about this apparent conclusion.

Symptoms. - From the time of exposure to that of invasion is a variable period of from one to three weeks. Exceptional cases of still greater extremes have been reported, but are so rare as to have no practical importance. Even these limits are greater than the large majority of cases possess, for usually incubation takes from ten to twelve days. At the end of this time the characteristic signs of the disease begin to show themselves. The child complains of headache and backache, the latter being especially severe. The pain is located in the small of the back, may radiate into the legs, and generally continues until or after the eruption appears. The general impression which the child makes is one of being thoroughly prostrated and seriously sick. The tongue is covered 
with a grayish fur, the breath is offensive, and nausea, vomiting, and epigastric pain are apt to be pronounced. Constipation is much more common in children than diarrhœa, and chills followed by convulsions are more often seen than in adults. Other nervous symptoms, such as drowsiness which may develop into coma, restlessness, lack of muscular control, teeth grinding, and delirium, are often seen. The temperature from the beginning of the symptoms mounts rapidly until within a few hours it may reach $40^{\circ}$ C. $\left(104^{\circ} \mathrm{F}\right.$. $)$, and then may rise still higher. During these first symptoms, which last for two or three days, the throat shows nothing abnormal except an enlargement of the tonsils.

A noteworthy part of the preliminary symptoms is the appearance of the so-called initial rash. It may come out during the first few days of the disease, and remain for a variable time up to four or five days. Its usual location is on the body and extremities, rather than the face, and marks the places where the true eruption does not appear, especially in copious amounts. The space that it covers is variable, so that no rule concerning this detail can be formulated. In character it may be erythematous, hæmorrhagic, or a combination of both. The erythematous variety may resemble the rash of scarlet fever, of measles, or of erysipelas. The resemblance is general, for the color is apt to be less bright, it fades on pressure, leaves no stain, does not show the same local predilections, and is not accompanied by catarrhal symptoms. 'The hæmorrhagic variety usually appears on the body during the first three or four days, and for the most part is confined to the lower part of the torso. It may be light or dark in color, petechial in form, and in heavy crops may by convalescence produce large confluent spots. The light-colored rash disappears on pressure, while the dark does not entirely fade. In many cases the rash is a combination of erythema and petechiæ, so that neither term may be used exclusively to designate it. A sub-variety, seen in children rather than in adults, is the so-called purpuric rash, which receives. the name from its close resemblance to ordinary purpura. It is dark in color, fairly circular in form, and irregular in distribution.

Within a short time, usually one, two, or three days, after the outbreak of the first signs, the ordinary eruption of smallpox makes its appearance. Its primary location is on the face, scalp, hands, and arms, then on the rest of the body. The enanthem breaks out at the same time on the mucous membrane of the 
mouth and throat, and in a few rare cases on the conjunctivæ, vagina, vulva, and rectum. Usually two or three days are required before the external eruption is fully out; it may be of use to call attention to its tendency to appear most luxuriantly on the parts most affected by pressure or friction from clothes. Its character and conformation in different children may vary considerably, and these different forms have been called discrete, confluent, and hæmorrhagic eruptions.

Its first appearance is in the form of small red spots which fade on pressure. They rapidly grow in size and hardness until they are distinctly papular. During the next day they increase still further in size, and show at the summit a metamorphosis into vesicles; this change in the course of four or five days converts the whole papule into a vesicle which is about $0.5 \mathrm{~cm}$. ( $\left(\frac{1}{5}\right.$ to $\frac{1}{4}$ inch) in diameter, dirty white or pearl-gray in color, multilocular, translucent, filled with serum, often pitted in the centre, and surrounded by a small reddish areola. At about the sixth day, the vesicle begins to change to a pustule. The umbilication begins to disappear, and its place is taken in almost all the vesicles by a small, dull-looking spot which gradually spreads out in all directions. At the same time the areola increases in size and brightness, the vesicles grow in extent and height, and within two, three, or four days have become a pustule. 'This formation persists for about a day, when it spontaneously breaks. The liberated pus and serum dry, and form an offensive yellow or greenish yellow crust. After the lapse of a few days the crusts fall off, leaving the new, tender covering which gradually becomes like the rest of the skin surface. Where the ulceration has descended deeply into the skin, a permanent depression or pitting may remain; but in most cases where proper care is exercised the process does not extend so far. In fact in some cases the pustule never breaks at all, but dries up and then is shed, leaving a clean, fresh surface.

As the exanthem appears and develops, the enanthem keeps an an equal pace. From the beginning, however, its changes are not so radical and startling. It starts in the form of minute red spots which slowly enlarge somewhat, but never reach the size or elevation of the spots upon the skin. Simultaneously the mucous membrane becomes swollen and congested, so that at times it is not easy to make out all the characters of the eruption upon it. Here and there one can see an areola about a 
somewhat flattened papule, whose apex has a whitish hue. As the exanthem becomes pustular, the papules in the mouth and throat break down, leaving in its place a small ulcer. Juxtaposition may cause several of the ulcers to coalesce, thus producing a suppurating area that is more or less thickly covered with necrotic tissue, pus, mucus, and bacteria.

Such is a description of the discrete eruption. In the confluent rash the vesicles are close together, and the intervening space is covered by an erythema, so that the affected area, whether large or small, is entirely covered by the outbreak. The essential elements of the main type are, however, present. In hæmorrhagic smallpox there are subcutaneous or cutaneous hæmorrhages with or without the ordinary features of a variolous eruption. This variety when well marked is sometimes called black smallpox. It is often accompanied by hæmorrhages from the mucous membranes and kidneys. A sub-variety, called peri- and sub-vesicular, is occasionally seen in which the vesicles, whether the eruption is discrete or confluent, become injected with a dull purplish color, while the areola is of a garnet rather than a red hue.

The other symptoms have a close connection with the character and development of the eruption. The high temperature, which with pains in the head and back accompanies the initial symptoms, abates as soon as the eruption develops. In the course of a few hours, or a day or two, the fever largely or wholly disappears until pus begins to form in the vesicles, when it mounts to about $39.5^{\circ}$ or $40^{\circ}$ C. $\left(103.3^{\circ}\right.$ or $104^{\circ} \mathrm{F}$.). The degree of fever depends upon the comparative severity of the disease and the amount of pus. As the case progresses toward recovery the temperature gradually falls, the morning remissions becoming more marked, while the evening exacerbations mount less and less high. In very mild cases the fever may never run very high, but in severe attacks, especially in confluent smallpox, it may in the pustular stage reach $41.5^{\circ}$ C. $\left(106.7^{\circ}\right.$ F. $)$, or higher. The respiration and pulse follow the temperature fairly well, except during unusual pus production, when the pulse-rate becomes very rapid, and its character at times is weak and irregular.

One of the most painful and troublesome features of smallpox is the œdema and swelling of the body surface, especially where the eruption is located, during the pustular and the second half of the vesicular stages. This swelling is irregular, so that the 
contour of the face, neck, and even the body is quite changed. The eyelids are puffed out so that they are beyond control, the nose is huge, the ears large, thick, and bent forward, the lips are strangely out of their regular shape, project, cannot coapt, nor restrain the flow of saliva. The whole body surface is excessively irritable, sensitive, and painful, so that the ordinary friction of the bedclothes and necessary manipulations are hard to endure. Thus one finds much difficulty in keeping the eyes, mouth, ears, and body clean, so that there is additional discomfort as well as danger of any complicating condition which may be brought about by septic or irritating accumulations and deposits. Salivation, dryness of the throat, and laryngeal congestion may be particularly troublesome, although in most cases not dangerous. The main disorder of the gastro-intestinal track is constipation, which is not necessarily serious. The kidneys in ordinary cases may be in a state of acute degeneration, while in serious attacks there may be a distinct exudative nephritis. The inflammation of the skin may be of various degrees, and on account of the abscesses may leave an opening for the production of more extensive ulceration, erysipelas, and cellulitis. The nervous symptoms of irritability, delirium, convulsions, and mania vary according to the patient's weakness and sepsis. Partial or total paralyses of the face, neck, or extremities sometimes occur, but usually are not permanent.

Treatment. - The prophylaxis is plainly of fundamental importance. This is to a certain extent simplified in most houses because the patient as soon as a diagnosis is made is taken to a special hospital whose situation renders the spread of the disease almost impossible. Smallpox cases should not be treated in a city, but should always be removed to some remote locality. The necessity for this is very plain, since the virulence of the poison is such that aërial contamination is possible for the residents within a radius of three-quarters of a mile. When a patient has been removed, his apartment and every article of clothing, furniture, or domestic use must be disinfected by washing, boiling, or steaming, according to the nature of the thing cleansed.

The medical treatment at present does not include the use of a specific. Doubtless there will be in the near future an anti-toxine; but now we restrict our efforts to symptomatic and hygienic measures. The patient must be confined to bed, the diet restricted to fluids, and the gastro-intestinal track emptied by means of small and 
repeated doses of calomel followed by a saline. The eyes should be thoroughly washed as often as may be necessary, and if the lids swell very much they may be treated with ice cloths. The nose and mouth must be thoroughly and frequently irrigated, and the whole body may be washed daily once or more times for the double purpose of cleanliness and the regulation of fever. The importance of keeping the skin as clean as possible in all stages is unquestionably great. Not only is the patient made more comfortable, but also the likelihood of extensive abscess formation with the resulting disfigurement of deep pitting is lessened. As soon as pus begins to form, the continuous use of moist dressings (bichloride of mercury in weak solutions) will give considerable relief, and hold in check the septic element of the skin lesions.

As far as drugs are concerned, little need be done. One of the principal indications is the relief of pain. In almost all cases the most direct method for this purpose is the use of opium in sufficient doses to keep the patient quiet. In young infants or in older children for whom opium may not be employed, the bromide of soda, with or without a small quantity of chloral, may be used. An additional need of treatment is the cardiac and general weakness which is apt to occur about the middle or end of the sickness. To combat this we may use alcoholic stimulation and also fairly large doses of strychnine. The complications should be treated according to their various indications.

Prognosis. - The outlook depends upon the patient's vitality, age, and the question of previous vaccination. The younger the child, the greater the mortality. Children under four years of age seem to be only half as resistant to the disease as those of twelve or thirteen, and the difference in the death-rate between vaccinated and unvaccinated children is about as one is to ten. The difference in sex seems to have a very slight bearing on the question, since statistics covering large numbers of cases show a greater mortality in males of about one per cent. The difference between discrete cases and confluent or hæmorrhagic cases is, in regard to mortality, very great. In the former the prognosis is good, but in the latter the question of recovery is always a serious one. The degree of gravity seems to be. in direct ratio to the amount of confluence or hæmorrhagic involvement.

The presence of serious complications, such as organic disease 
of the lungs, kidneys, heart, or nervous system, provides an additional element of danger, the exact importance of which varies with individual cases.

Differential Diagnosis. - Smallpox is so rarely seen now, at least by the general practitioner, that delay in making a diagnosis, most of all in the initial stage, is very apt to occur. The main facts to be kept in mind are the presence of marked pains in the back, head, and epigastrium, chills, nausea and vomiting, high temperature-, respiration-, and pulse-rate, prostration, and the appearance from the first to the third day of an erythematous or petechial eruption or both on the abdomen, while no enanthem may as yet be seen. If after the lapse of the usual time, as stated before, a macular eruption appears on the face and scalp, accompanied by the formation of smaller macules on the mucous membrane of the mouth and throat, the conclusion that the case is variolous is almost certain. The contrasting points between this sickness and the other exanthemata may be seen in the table facing this page.

\section{VACCINIA AND VARIOLOID}

\section{VACCINIA}

The practice of vaccination produces a local eruption of a papule, which changes into a vesicle, and then into a pustule. Commonly there are some general symptoms of adenitis, fever, and malaise. To induce this protective disease we use a lymph obtained at the present time from inoculated calves. The former method of employing humanized virus, the so-called arm-to-arm method, has wisely been done away with on account of the pos: sible danger of a simultaneous communication of syphilis, tuberculosis, erysipelas, cellulitis, septicæmia, and other suppurative processes. With this objection out of the way, there is very little danger left. It is held that absolutely sterile lymph is never found. Over against this we may set the fact that the microorganisms which are regularly found, Staphylococcus albus epidermidis, S. pyogenes aureus, and S. cereus flavus, are not, unless present in relatively large quantities, necessarily dangerous; and we have the additional fact that even these organisms we can remove from the fluid. 
With all extraneous infections eliminated, the result in the vast majority of cases ought to be productive of very little inconvenience or sickness, especially if the patient is properly prepared, and is in a fit condition to receive the inoculation. In the first place the child should be in fairly good health; unless there is immediate danger of infection new-born children should not be vaccinated, and the operation should be delayed until the patient has shown his ability to live and thrive. When this matter is settled, the part that is to receive the inoculation, usually the outer aspect of the left calf or upper part of the left arm, should be prepared as if for an operation. The skin should be thoroughly scrubbed with hot water and soap, then washed with alcohol, with corrosive sublimate solution, and finally rinsed with sterile water. There are various ways of accomplishing the vaccination: a space about three-quarters of a $\mathrm{cm}$. square is scarified until the blood flows, or is covered at fairly wide intervals by a few lines which intersect at right angles. The dried vaccine is then moistened and thoroughly rubbed into the wound; the liquid vaccine which is now put up in sealed capillary tubes is a distinct improvement over the quills and ivory points, since it is incapable of becoming contaminated by adventitious influence. Another method of using the liquid virus is to put a drop of it on the selected spot and then with a sterile needle or knife to scarify through the lymph. Still another method, and one that has given me much satisfaction, is to scrape off the upper skin with a scalpel until one produces a fine serous oozing on the denuded surface. The virus is then applied and allowed to dry. I have used this last method in many hundred cases, and I feel safe in heartily recommending it. One should try in a general way at least to regulate the amount of lymph to the age and weight of the child.

The general practice of leaving the wound uncovered, or at best protected by some sort of wire shield is not, I believe, a good one. Here again the general principles of surgery should guide us. No one would think of leaving any other kind of wound quite unprotected, unless of course he were perfectly willing to invite infection. For the same reason a vaccination wound should be covered with an aseptic dressing held in place by a roller bandage. The result will be a very much smaller number of serious, or at least troublesome, inflammations and deep abscesses. The 
course of this disorder will in most cases be uneventful, there will be comparatively little fever, prostration, and adenitis. The bandage may be allowed to remain for seven or eight days, when the wound should be inspected.

Usually the first four days pass uneventfully. Then one or more papules appear which quickly change into vesicles surrounded by a light areola. The vesicle steadily increases in size, and the contained lymph gradually becomes turbid. As the growth nears completion the summit becomes foveated and progressively dessicated; about the eleventh, twelfth, or thirteenth day the vesicle dries up or ruptures, leaving a crust of variable thickness. Within a short time this scab should fall off, and nothing but a reddish or purplish scar remains.

When the symptoms and course of the disease are markedly severer, the cause resides in extraneous infection. This does not refer to variations in the development of the pock, such as supernumerary and confluent vesicles, or hæmorrhagic development, or keloid, or the appearance of a harmless urticarial or erythematous rash. These are unforeseen variations, and cannot be guarded against. But such complications as noteworthy suppuration, gangrene, auto-inoculations, the serious skin and systemic diseases, should not occur. Likewise one cannot foresee or prevent the few rare cases of delayed incubation. In this as well as in other problems of the subject are involved the elements of age, idiosyncrasy, season, and strength of vaccine. A weak virus may lead the physician to believe that the child is not susceptible or only faintly so, or two weeks, or more may elapse before the vaccination "takes." Almost everybody has noticed that a pock develops more quickly in warm than in cold weather. The question of susceptibility is hard to understand; occasionally one meets a child who seems proof against successive attempts, although success is almost bound to come if the inoculations are repeated often enough. Equally inexplicable are such susceptible cases as the remarkable one of Allbutt, where there were three successful vaccinations, each one of which was followed within a comparatively short time by an attack of variola.

The treatment of the various conditions that call for attention should be conducted upon surgical principles. 


\section{VARIOLOID}

The practice of vaccination is so widespread that some cases of variolous infection which run a modified course must naturally be seen. The disease is then of a mild type, so much so at times that a mistake in diagnosis is readily excused. The same phe-

\begin{tabular}{|c|c|c|c|c|c|c|c|c|c|c|c|c|c|c|c|c|c|c|}
\hline PUL. & RESP. & TEM & & 1 & 2 & 3 & 4 & 5 & 6 & 7 & 8 & 9 & 10 & 11 & 12 & 13 & 14 & 15 \\
\hline 170 & 70 & $\begin{array}{r}\mathrm{FAH} . \\
\mathbf{F a}\end{array}$ & $\begin{array}{l}\text { CeN. } \\
42.2 \\
\end{array}$ & & & & & & & & & & & & & & & \\
\hline 160 & 65 & 107 & 41.6 & & & & & & & & & & & & & & & \\
\hline 150 & 60 & 106 & 41.1 & & & & & & & & & & & & & & & \\
\hline 140 & 55 & 105 & 40.5 & & & & & & & & & & & & & & & \\
\hline 130 & 50 & 104 & 40.0 & & & & & & & & & & & & & & & \\
\hline 120 & 45 & 103 & 39.4 & & & & & & & & & & & & & & & \\
\hline 110 & 40 & 102 & 38.8 & & & & & & & & & & & & & & & \\
\hline 100 & 35 & 101. & 38.3 & & & & & & & & & & & & & & & \\
\hline 90 & 30 & 100 & 37.7 & 4 & & & & & & & & & & & & & & \\
\hline 80 & 25 & 99 & \begin{tabular}{|l|}
37.2 \\
\end{tabular} & & & & & & & & & & & & & & & \\
\hline & & 98.6 & 37.0 & & & & & & & & & & & & & & & \\
\hline 70 & 20 & 98 & 36.6 & & & & & & & & 1 & & & & & & & \\
\hline 60 & 15 & 97 & 36.1 & & & & & & & & & & & & & & & \\
\hline & & & & & & & & & & & & & & & & & & \\
\hline
\end{tabular}

PULSE, RESPIRATION AND TEMPERATURE CHART OF VARIOLOID, $A G E, 4$ YEARS.

PULBE_-_- RE-O RESPIRATION_-__ TEMPCRATURE

FIG. 80 .

nomena may be seen in a patient who has had variola or who for some puzzling reason is only partially susceptible to the poison.

Usually the disease begins like a mild variola, but the regular course is not followed: the attack may suddenly wither away, or the development of the eruption ceases before pustulation sets in, 
or there are very few and scattered pocks. The initial symptoms are short, and the papules may change into vesicles within a few hours or a day. The appearance of the eruption is not quite like that of variola: the papules and vesicles are smaller, more irregular in form and outline, less likely to have a well-marked areola. After two, three, or four days many vesicles dry up, and only a few develop into pustules. These latter are short-lived, reach their maturity within a day or two, and they break open or dry up. Finally there may be a slight desquamation about the new epidermis. The general symptoms are likewise abortive or mild. The temperature is moderate or scarcely above normal, there is little pain, tenderness, or swelling, and the nervous symptoms are mild.

The complications are rare, and if any occur they are generally the result of infection of the surface wounds.

The treatment is purely general and symptomatic. The important precaution of isolation must be kept in mind, no matter how trivial the attack may be; for a trifling case in one person may be the starting-point for a violent variola in another. Prophylaxis, isolation, and disinfection should be as rigorous as in an ordinary case of smallpox.

\section{Diphtheria}

Within the last few years our ideas concerning this disease have changed so radically that at one stroke all our statistics of mortality as well as methods of treatment have become antiquated and useless. Diphtheria, instead of being a frightful scourge, the very name of which made parents tremble, may now be looked upon as a curable disease in most children who receive reasonably good care.

Cause. - The discovery of the specific germ by Klebs in 1883, and its isolation and description by Loeffler in 1884, have dwarfed every other element of rtiology into total insignificance. Thus we are assured that this bacillus, when once lodged on a surface, usually mucous, which is in a fit condition of asthenia or irritation, will develop in its characteristic fashion, will give rise to the formation of a false membrane in the locality in question, and the elaboration of soluble toxins and tox-albumins throughout the whole body. These latter elements are the really serious factors of the disease, and should receive the greatest part of our atten- 
tion when we attempt to treat it. In the diffusion of the infection the bacillus itself is the responsible agent, and to exclude it is to stamp out the dangers of diphtheria. This is no easy matter on account of the tenacity with which this micro-organism resists extinction. But in addition to this we commonly find, especially in severe cases, one or more other forms of bacterial life, usually streptococci and staphylococci. These may complicate the specific condition to such an extent that the strict idea of diphtheria no longer holds good, and we should then speak and think of a mixed infection of various degrees of complexity and severity.

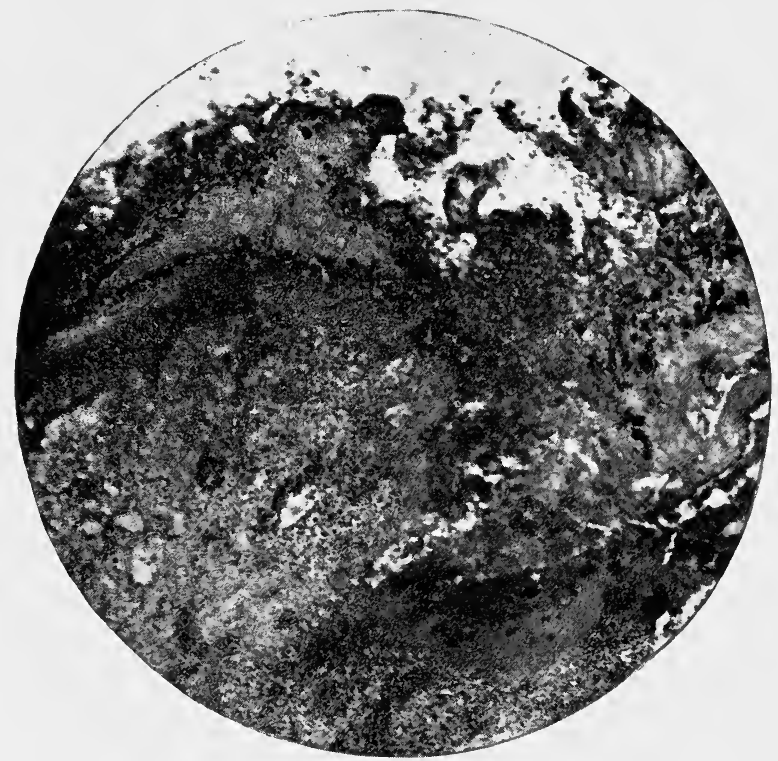

Fig. 81. - Diphtheritic Inflammation of Pharynx. $\times 25$.

Lesions. - The parts which most strikingly attract attention are such as are covered wholly or in part with the local inflammation and membrans. These are the tonsils, pharynx, uvula and soft palate, nose, lar ax, trachea, and bronchi; in addition the process may, ir rare cases, extend through the lungs, into the œsophagus, stomach, and intestines, may invade the conjunctivæ, the ears, the vulva, glans penis, anus, and recent wounds. In the more remote situations, such as the eyes and genitals, the membrane in all likelihood is caused by tactual infection as often as by anatomical extension. In similar ways fresh wounds, such 
as tracheotomy wounds, may become the seat of local inflammation. The farther away from the mouth and nose, the rarer is the spread of the diphtheritic membrane. In this structure the KlebsLœffler bacillus is found in great abundance; in some cases, usually those of great virulence, it may be encountered in the spleen, in the blood, in leucocytes, possibly in other organs.

When the mucous membrane is attacked, it falls into a state of catarrhal inflammation and the epithelium degenerates. In some rare cases the process seems to stop here; but in the majority the degenerated epithelial layer disappears, and its place is taken by

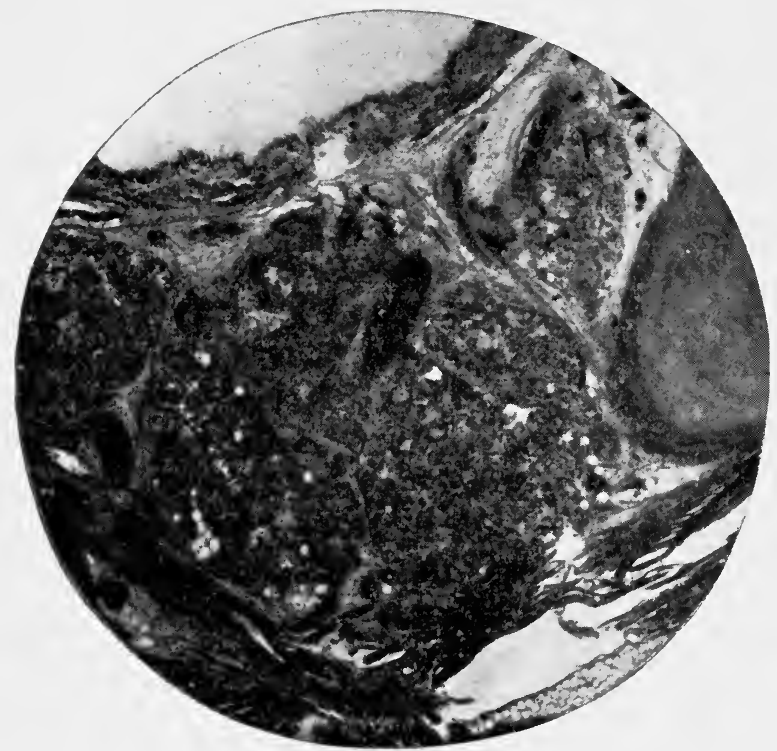

Fig. 82. - Diphtheritic Inflammation of Trachea. $\times 25$.

a meshed stratum of fibrin which holds epithelial débris, pus-cells, blood-cells, cocci, and the diphtheria bacillus. The membrane grows from its under surface, or the mucous membrane, up; and therefore, near this mucous membrane, the Kleos-Lœffler is most commonly found, sometimes in almost pure cultures, while in the upper layers one generally finds the various kinds of streptococci and staphylococci that readily find access to the mouth. The diphtheria bacillus usually, on disappearing, leaves no permanent marks of its invasion; in some cases, however, more or less extensive ulceration or necrosis occurs, which may be attributed to 
the other pyogenic cocci, that constitute the ordinary mixed infection.

Outside of this local process, changes of considerable importance occur in other parts of the body, such as the cervical, bronchial, and mesenteric glands, which become swollen, softened, and possibly broken down; in the kidneys, which are cloudy, anæmic, degenerated, and acutely inflamed; in the liver, which shows areas of cell-necrosis and sub-capsular hæmorrhage ; in the heart muscle, which often has areas of fatty degeneration; in the spleen, which is swollen, congested, and degenerated; in the lungs, which show

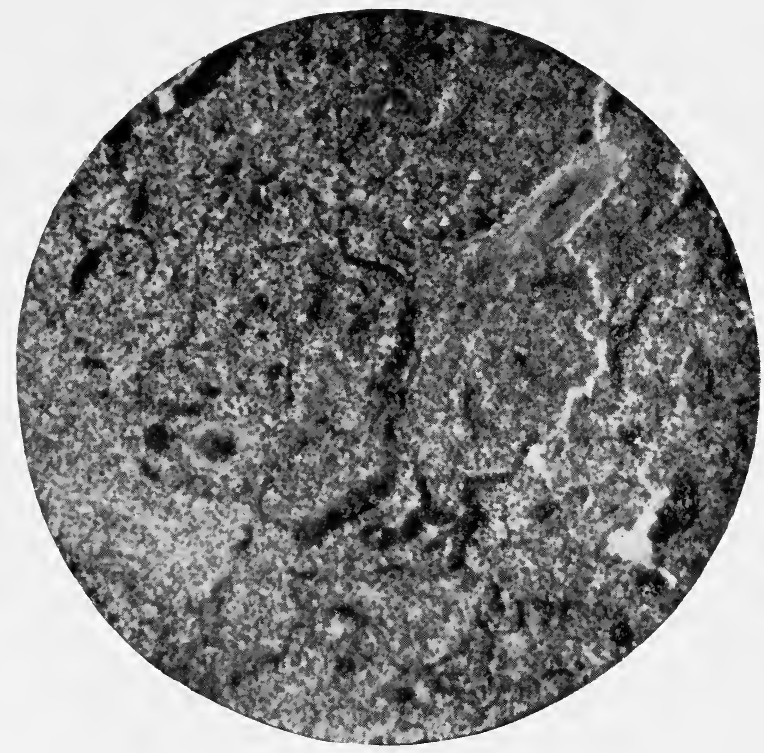

Fig. 83. - Lymphadenitis of Diphtheria. $\times 80$.

the signs of a bronchitis or a profuse growth of the diphtheria bacillus with varying amounts of membrane and a resulting true diphtheria pneumonitis; or on account of the presence of the pneumococcus or varieties of streptococcus, a lobar pneumonia or broncho-pneumonia. In the blood there is a decrease of red blood-cells and hæmoglobin, and in the severe cases an increased leucocytosis. One of the most constant and important changes is the parenchymatous degeneration that occurs in the peripheral nerves. The toxines of diphtheria seems most of all to affect nerve tissue, and thus, even in comparatively mild cases, we find 
the white substance of the medullated fibres as well as the axis cylinder degenerated and broken down in irregular areas. So long as parts and branches of the affected nerves are left unharmed, the function is not entirely destroyed, and in ordinary cases the impression which is thus conveyed is one of asthenia rather than a degree of paralysis.

Symptoms. - From the time when the child receives the infection until the first symptoms appear is a period which may vary within wide limits. This has been known to constitute no more than half a day; usually the symptoms manifest themselves before a week has elapsed; in rare instances the first signs may be put off for two or even three weeks. The deciding factors seem to be the condition of the rnucous membrane upon which the bacillus seeks to grow, and the vitality of the patient. A mucous membrane which is irritated or inflamed acts as a fertile culture-ground for this as well as other micro-organisms. On the other hand, a healthy mucous surface resists the attack so successfully that unless some form of irritation or degeneration supervenes, the triumph of the germ may be indefinitely postponed. The hypertrophic tissue of adenoid vegetations is a good resting or storage ground for this organism until some disorder of the surrounding or adjacent tissue lights up the disease, and permits it to pursue its course.

Commonly, careful observation will discern prodromal symptoms before membrane appears. There are moderate fever, a gradually increasing sense of physical depression, possibly a chill. There may be disorders of the digestive system, followed sooner or later by a sore throat. Rarely there may be no membrane at all, but the constitutional symptoms go on in the ordinary way. In the vast majority of cases, however, the faucial mucous membrane becomes swollen, reddish, and glistening. There may be disinclination to swallow, and the glands at the angle of the jaw are swollen and tender. In some few cases the parotid gland is likewise enlarged and sensitive, and often the whole neck may be sensitive. This is followed at an uncertain interval by the appearance of one or more small areas of membrane which, starting commonly on the tonsils, uvula, or pharynx, grow or coalesce so that from hour to hour the increase in size may be noticed. The membrane varies in character in different cases: it is gray or dull white in color, only occasionally taking on a darker hue; it may be thick or thin, closely or loosely adherent, tough or friable. 
The pure diphtheritic membrane is often thinner and less easily removed than that due to mixed infection, which also has the greater tendency to assume a dark and peculiarly unwholesome color. The unmixed infection has a mucous membrane which, when freed from its peculiar covering, is not badly abraded or torn, while the opposite is often the case in the so-called mixed infection. The last-named membrane more easily decomposes than the first, thus giving rise to the so-called putrid sore throat. In proportion to the severity of these inflammations there will be a swelling of the underlying parts, so that the usual relations are disturbed, and the characteristic form is temporarily destroyed. In rare cases of marked severity the cellular tissue of the neck may likewise be involved and very much swollen. On the other hand, the disease when found in the nose frequently has much less swelling of the tissues; there is a concomitant nasal discharge that is thin and sharp at first, gradually becoming puriform and blood-streaked.

While these local phenomena are developing, the general symptoms progress with an equal step. Their seriousness varies not so much with the extent of the membrane as with the intensity of the intoxication, which is the principal factor in the disease. The younger the child, the harsher is the onset of the disease apt to be. The temperature is atypical, rarely exceeds $30.5^{\circ}-40^{\circ} \mathrm{C}$. $\left(103.1^{\circ}-104^{\circ} \mathrm{F}\right.$.) and in markedly asthenic cases may be subnormal. The respiration is fairly rapid, excepting in laryngeal and nasal cases, where it becomes rapid, strenuous, and labored. The pulse is quick, shallow, and sometimes irregular, most of all in severe attacks and with weak patients. The weak heart is one of the characteristics of diphtheria, and may exist in a noteworthy degree when muscular degeneration is not noticeably present in other parts of the body. The peculiar toxines have an especial affinity for the cardiac structure, and may bring about a fatal result, either by an acute myocarditis, or by a paralysis of the par vagum; in rare cases the same result is obtained by cardiac thrombosis.

The wearing effects of the sickness are regularly shown in an anæmia, whose tendency is rather in the way of excess than triviality. The sense of prostration continues throughout the disease; when the fever is high there may be marked restlessness and possibly delirium, while in the asthenic cases the child may be 
dull, stupid, or even comatose. After the disease has well begun, the kidneys fall under the action of the toxæmia, and the urine shows the presence of albumin and casts in varying amounts.

In young children there is always an acute danger of an involvement of the larynx, to a greater extent than in those of more advanced age. This may occur as an extension of the pharyngeal membrane, or the disease may begin in the larynx,

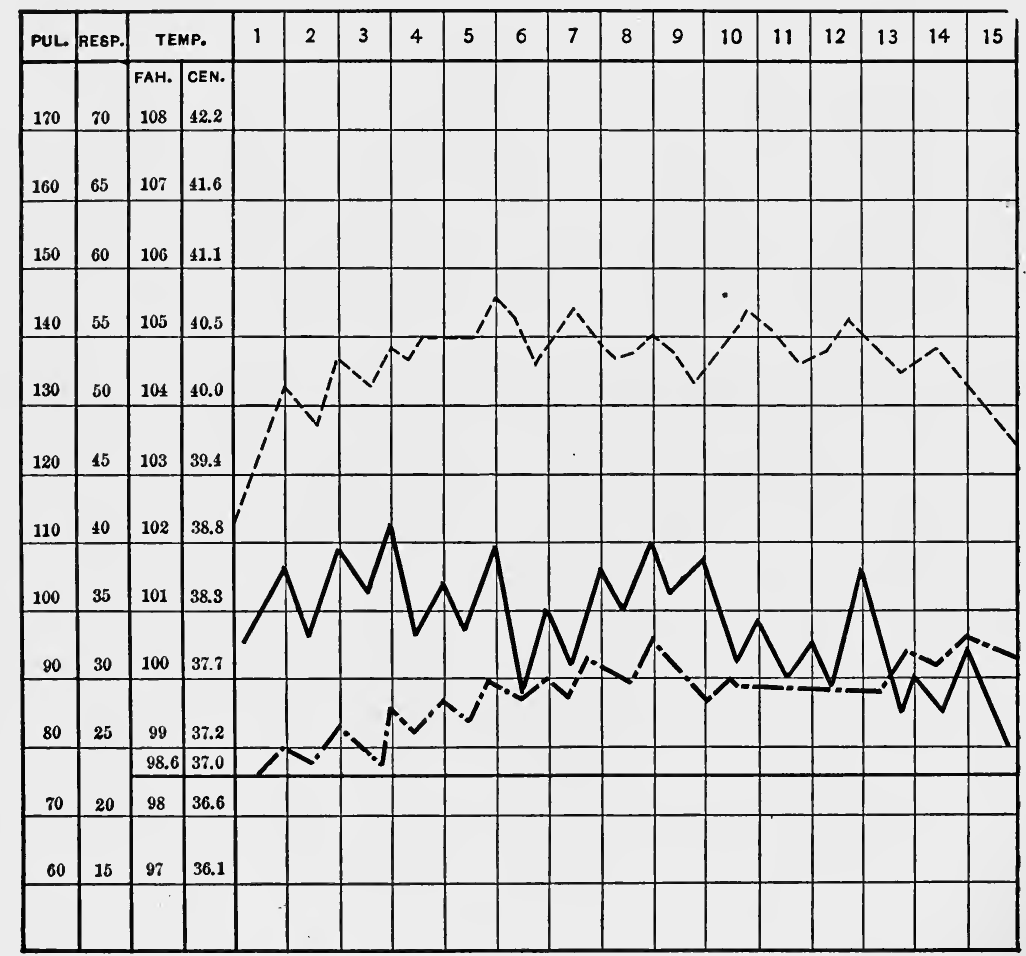

PULSE, RESPIRATION AND TEMPERATURE CHART OF DIPHTHERIA.

AGE, 5 YEARS.

PUL8E

REBPIRATION

FIG. 84.

and thence spread up or down. In some cases there may be one or more patches in the throat, an area in the larynx, and between them a clear space. The laryngeal symptoms of hoarseness, difficult breathing, and spasmodic cough are so characteristic that they cannot be mistaken. When the larynx is invaded, not only may the diphtheritic germ and membrane make their way into the 
trachea and lungs, but also there is imminent danger of the aspiration of other pathogenic germs, such as pneumococcus and streptococci, with the resulting development of various degrees of pneumonia and broncho-pneumonia. There is great danger of such complications when the larynx and throat have become partially paralyzed.

Another extension of the disease involves the nose. This location may in a few cases be primary, but usually it is secondary to pharyngeal diphtheria. The nose is occluded, and the patient is forced to breathe through his mouth. Occasionally in the thin muco-purulent discharge there may be traces of blood. This condition, which is easily confused with rhinitis fibrinosa, is especially liable to persist for some considerable time, even when the membrane seems to have vanished. It is commonly capable of producing hypertrophy of the pharyngeal tonsil, in the recesses of which the bacillus lies concealed for weeks after the end of the disease. In other rare cases the membrane covers more or less of the buccal mucous membrane - the cheeks, tongue, gums, even the lips. Outside of the results occasioned by so great a spread of the disease and the consequent swelling and loss of local muscular control, this condition gives no especial symptoms. In some of these very rare cases the membrane may extend into the œsophagus, and thence into the stomach. The resulting inflammation is accompanied by such persistent vomiting that the outcome of the disease is seriously influenced. This, however, is not the only occasion for this symptom, which may occur as the result of the toxæmia, or the nephritis.

One of the commonest and most dreaded effects of diphtheria is the paralysis that comes during or after the main course of the disease. Commonly it occurs during the second week, but it has been known to appear as early as the third day, or as late as two months after the main symptoms have vanished. Its commonest seat is in the fauces. It is manifested by a strange wooden tone of the voice and some degree of difficulty in swallowing. The degree of palsy varies in each case, and the accompanying symptoms of functional disability will demonstrate both the location and the extent of the lesion. In some places this complication immediately becomes of the gravest importance; thus, if the glottis is affected, the epiglottis and larynx cannot react to their usual stimuli; as a result, particles of mucus, membrane, and food may, 
enter the lungs, and start a fatal septic pneumonitis. Any other part of the body may be likewise affected so that deformity of the trunk or extremities, or death from respiratory or cardiac paralysis, may occur. Lesions of the spinal cord are very rare in children.

One ought to call attention to the possibility, which, by the way, is not common, of an arthritis, that is apt to involve the smaller joints. This complication is characteristic, I believe, of mixed infections, and should be regarded as a clear case of pyæmic infection.

Treatment. - Since the brilliant success of the diphtheria antitoxine has been demonstrated, the former methods of treatment have been entirely overthrown. At the outbreak of the sickness the patient should immediately be isolated, and the remaining members of the family, especially the children, be injected with an immunizing dose (150 to 300 units, Behring standard, according to age) of the serum. The sick-room should be as remote from the other apartments as possible; it should have as much air and light as the situation of the house permits, and must be stripped of its carpet, pictures, curtains, and all unnecessary furniture. Those who enter or attend on the sick-room must not mingle with the family, and every effort should be made to divorce the two establishments to the last detail. The routine practice of clearing the patient's gastro-intestinal track by small and repeated doses of calomel must not be omitted. Without delay the child should receive a full dose of the anti-toxic serum (from 1000 to 3000 units, Behring standard); within a few hours the temperature will fall, the respiration become easy, the nervous symptoms and depression markedly decrease, and the spread of the membrane be checked. If these results do not appear, the injection may be repeated on the following day. In some cases a discrete maculo-papular rash may appear about one week after the injection. Some practitioners have claimed that this is due to deficient purity of the serum. At all events, it is not dangerous, and requires no more than symptomatic treatment.

Since cases of mixed infection are so common, we must be prepared to treat other bacteria besides the specific bacillus. Wanting an efficient anti-streptococcus serum, our efforts are directed to keeping the nose and throat as clean as possible, by free irrigations of the former and sprayings of the latter. Saturated solutions of boric acid, with or without the admixture of peroxide of hydro. 
gen, are a useful means for the throat, as is likewise (for older children) a weak solution (1:10,000) of bichloride of mercury. For nasal irrigation one may employ warm normal saline solution, or Seiler's solution. From the first it may be necessary to use stimulants, the best of which are alcohol and strychnine. The diet should be fluid, and administered in small and repeated amounts.

Complications should receive the treatment which belongs to them. Laryngitis, when combated by the anti-toxine, rarely calls for tracheotomy, and not nearly so often as formerly for intubation. After the fever and the local evidences of the disease have disappeared, the child should yet be kept in bed for from one to two weeks, to guard against the danger of heart failure due to exertion. After he has left the sick-room all its furniture, bedding, utensils, books, and toys should be most thoroughly disinfected or burnt. The paper should be taken from the walls, and the woodwork most thoroughly scrubbed and cleaned. After the room has been exposed to the air and sunlight for at least a week, it may again be occupied.

Although the need of intubation is not as frequent as it formerly was, nevertheless there are occasional cases where it must be practised, especially in infants. It is indicated where the laryngeal obstruction is marked; such obstruction may be due to diphtheritic laryngitis or to œdema of the larynx, resulting from mixed infection, burns, scalds, and wounds, especially when there is no great swelling of the fauces and tonsils. The instruments which we use are those devised by the late Dr. O'Dwyer of New York. The set consists of seven tubes, each of which has an obturator, an introducer, an extractor, a mouth-gag, and a scale with which to select the proper tube according to the age of each child. In the right shoulder of each tube is a small hole, through which a silk thread, designed to prevent the swallowing of the tube, may be passed. All the instruments before being used should be sterilized, and then placed in hot water in order to be properly warmed.

The patient should be securely wrapped in a blanket, so that his arms, body, and legs may be comfortably and securely held. The nurse supports him on her lap, grasping his arms above the elbows with her two hands; his head may be held rigid by another assistant, who simultaneously may keep the mouth-gag from slipping. The head, neck, and body of the child must be held in a 
right line, and one must not allow the head to be strained backward and the throat to be contracted and protruded, as the patient in his struggles is bound to attempt. The introducer is then attached to the obturator in the tube, the thread passed through the aperture in the shoulder and held by the fingers of the operator's right hand. The index finger of his left hand, after being protected by a few layers of gauze or rubber protective, is passed into the patient's throat until it touches the epiglottis. Using this finger as a guide, the operator passes the tube until its free end enters the larynx, when a slight pressure will place it in its proper position. While the obturator is being removed the tube may be kept in place by the guiding left finger. The operation is not a difficult one if the tube in passing through the mouth is kept in the median line, and is not allowed to rise in the throat above the base of the tongue. If the tube enters with difficulty, the cause is either that it is too large, or that a plug of mucus and membrane has been forced down in front of it. In either case no force should be used; a smaller tube should then be substituted, or the child be allowed to expel the obstacle. As soon as the tube is properly located the child will cough more or less violently for a short time to clear the passage, and then should breathe with a reasonable amount of ease, while the cyanosis and other signs of respiratory obstruction should pass away. After the child has become quiet, the thread which secures the tube may be passed between two of the teeth or along the gums, out at the angle of the mouth, and secured around the ear or fastened to the cheek by a strip of adhesive plaster. If its presence in the mouth prove too uncomfortable, relief may be obtained by passing it through the nares, and then making it fast to the cheek. Some children persist in pulling at the string as soon as they are freed from the blanket; but this is easily obviated by covering their hands with stockings, which are to be fastened above the wrists.

If the tube, while it is in process of insertion, is not kept in the median line and sufficiently close to the root of the tongue, it is apt to pass into the pharynx or the cesophagus. The error is usually recognized without trouble, not only because the shoulders are not felt in their usual position, but also because the patient experiences no relief. Sometimes, but rarely, a mass of membrane may be pushed before the tube into the trachea; in this case the respiratory obstruction increases, and if, after the tube is removed, 
the patient is unable to expel the mass, tracheotomy should be performed without delay. A very rare accident has been known to follow the use of too small a tube or the employment of too much force: here the shoulders of the tube slip within the false vocal cords instead of resting upon them. In such a contingency the instrument must be withdrawn, and replaced by one of a proper size, or greater gentleness must be observed. In the few cases where the tube has become freed from the thread and swallowed, it has been passed through the intestines without serious trouble.

The feeding of these cases is always a matter of importance, on account of the danger of inspiring liquid food through the lumen of the tube. Some children soon acquire a remarkable proficiency in directing the food away from the partly inefficient epiglottis. In those patients who are less capable one may obtain safety by placing them, while feeding, on their backs, and allowing the head to hang lower than the chest. Older children who experience this trouble may be fed upon semi-solids, such as strained gruels, softboiled eggs, mush, and milk-toast. In both infants and older children the amount of fluids that usually would be swallowed may be decreased if enemata of water, each containing about thirty grammes (one ounce), are administered. In all cases the throat must be thoroughly sprayed after feeding, as well as in the intervals, with a mixture of peroxide of hydrogen and a saturated solution of boric acid.

There is no fixed time at which the tube may be permanently dispensed with, but it may vary from two days to two weeks. Before the use of the diphtheria anti-toxine the longer periods were more frequently encountered than at present. As a rule, an attempt at dispensing with the instrument may be made at the end of two or three days; if the child is yet unable to breathe with sufficient ease, the tube should be replaced, and allowed to remain for two days more. The operation of intubation need not be looked upon as an especially difficult one, but it demands skill and judgment on the part of the physician, and ceaseless vigilance on the part of the nurse.

Prognosis. - The former opinions concerning the chances of life in diphtheria must be put aside. For we know quite certainly that in the ordinary pure infection which has been promptly treated with the anti-toxic serum the danger to life is comparatively little, nor are the nervous complications to be feared as much as in former 
times. In the mixed forms of infection, most of all where there is a broncho-pneumonia due to the invasion of streptococci, the case becomes quite different, and a fatal outcome is not unusual. In institutions these latter cases have a mortality of fifty per cent or more ; but the experience of institutions is not that of a favorable class of private patients. All in all, one may say that diphtheria is dangerous in proportion to the youth of the patient, to the delay in recognizing the disease and administering the anti-toxine, and finally, to the presence of mixed infections, especially when such infections cause a broncho-pneumonia. In institutions, and according to municipal statistics, with the unfavorable class of patients, the mixed infections, and the many inevitable inaccuracies, the mortality has been lowered to about fourteen per cent or fifteen per cent. In private practice it should fall to one-half or one-third of these numbers.

Differential Diagnosis. - The main sources of confusion are follicular tonsillitis and allied disorders of the mouth, the membranous sore throat of scarlet fever, and the mucous eruption of syphilis. The history, the surroundings, the occurrence of diphtheria in the house or neighborhood, and the presence of albuminuria will do much to clear up the diagnosis. Usually the difficulty is to distinguish between diphtheria and follicular tonsillitis: the former has a moderate fever, usually a gradual onset, and a membrane that has a tendency to spread and maintain its continuity. The latter has a high temperature, a sudden onset, and its membrane appears in isolated dots that later may coalesce. The supreme test, however, is the bacteriological examination, which may, in doubtful cases, need one or two repetitions. These doubtful cases should, until certain information is obtained, be regarded as diphtheria, and treated accordingly.

\section{Epidemic Infectious Parotitis}

Mumps is a somewhat uncommon specific epidemic disease, characterized by its high degree of infectivity, and a swelling of the parotid glands and the adjacent tissue. The micro-organism which causes it is, as yet, not positively known, although Michaels has recently described a small diplococcus which he believes to be the germ in question. It was taken from Steno's duct and a wound of the gland. Whatever the poison is, it is communicated 
by direct contact or by indirect means, such as an otherwise unaffected person, by clothing, or furniture. Infants are rarely attacked, and most of our cases occur between three and a half years and the end of childhood.

The process, it is believed, usually begins in the salivary ducts, which become congested, swollen, and more or less thoroughly occluded. The gland itself then partakes of the same inflammation, which spreads and infiltrates the surrounding area. Sometimes there are infiltration and œedema of the buccal and pharyngeal mucous membrane.

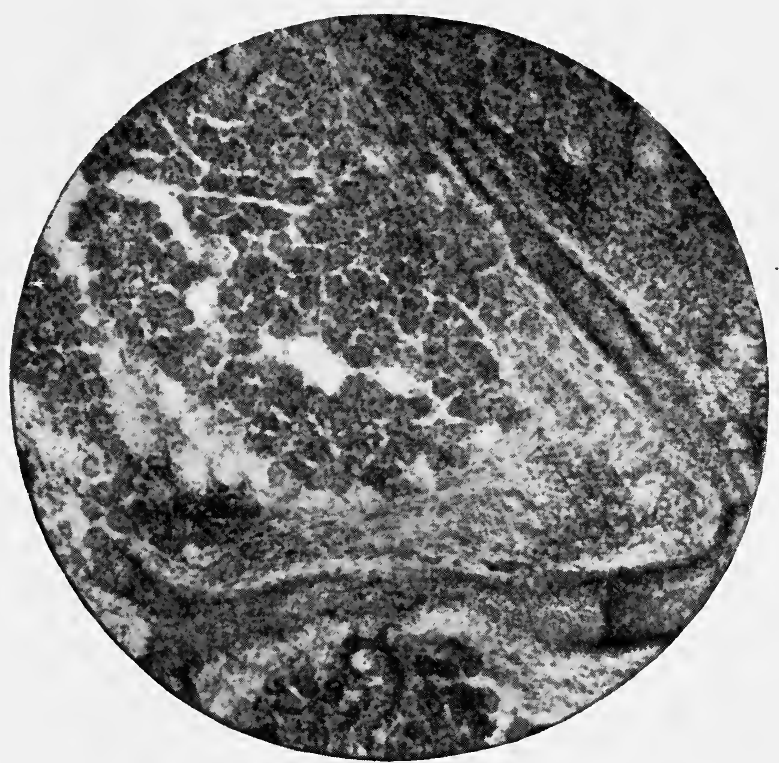

FIG. 85. - Epidemic Infectious Parotitis (Mumps). $\quad \times 80$.

Symptoms. - From the first sign of sickness, after an incubation period of from two to three or three and a half weeks, the possibility of contagion exists, and is supposed to continue for upward of three weeks. The first signs are apt to consist of a slight amount of fever, restlessness and malaise, nausea, and tenderness about the affected glands. This prodromal period lasts, roughly, for a day or two, after which the hollow under the ear begins to fill out. The swelling continues to increase for three or four days, the circumjacent area is markedly enlarged, the submaxillary glands are also swollen, and the whole throat feels stiff 
and sore. At this time the temperature may mount as high as $40^{\circ} \mathrm{C} .\left(104^{\circ} \mathrm{F}\right.$.) ; the swelling extends from behind the ear well on to the face, so that the lobe of the ear marks the approximate centre of the enlarged area; the pain and discomfort may be extreme, and the child looks worried and prostrated. The fever, if left to itself, would now subside; but in most cases the gland on the opposite side begins to swell, and goes through the same course as the first. After the swelling has reached its height in the later or second gland, the temperature quickly returns to normal. As the contour of the face falls back into its natural lines the salivary secretion becomes smaller, and may even go so far as to leave the mouth in an exceedingly uncomfortably dry condition. After the lapse of a week and a half or two weeks the parts usually return to their normal shape.

The order of occurrence of the swelling is not always the same. While it is true that in three-quarters of all the cases one gland closely follows the other, nevertheless one occasionally sees both sides simultaneously involved, or one side following the other at an interval of several days or weeks, or one side may alone be affected. Other unusual features are a complicating orchitis in older boys, in girls a swelling of the external genitals and breasts, enlargement of the inguinal glands, and a tenderness of the ovaries. Other rare complications are meningitis, meningo-encephalitis, and functional nervous disorders, deafness caused by disease of the middle ear or labyrinth, nephritis, functional disorders of the gastro-intestinal track, and adventitious suppuration of the affected parotid gland.

Treatment. - From the first the child should be confined to bed, where he must remain until the temperature is normal. He should then stop indoors until three weeks from the beginning of the disease have elapsed. The gastro-intestinal track should be emptied by a saline cathartic, or small and repeated doses of calomel followed by a simple cathartic. The mouth and nose should be kept clean by frequent sprayings and douchings with an alkaline antiseptic solution, and the diet should be restricted to fluids. The patient should receive one or two lukewarm sponge baths daily, and the ventilation of the sick-room must be carefully provided for. Very little treatment need be given to the enlarged glands. In ordinary cases they may be left alone; but very large swellings give somewhat less pain if hot applications be prescribed for them. A pad of aseptic gauze, wrung out of a hot two per cent 
carbolic solution, and covered by gutta percha tissue, answers the purpose much better than the old-fashioned and unclean poultices.

The complications, whatever they may be, are to receive their ordinary treatment. It is wise, during and after convalescence, to promote excretory action; for this purpose one may use small doses of the citrate of magnesia or the compound mixture of rhubarb and soda.

Differential Diagnosis. - The one point of confusion is the possibility of mistaking mumps for an acute swelling of the lymphglands of the neck. If one keeps in mind the history of contagion, the usual involvement of both sides, the locations of the swelling from behind the ear on to the face, the rapid rise and fall of the tumor accompanied by a similar movement of the fever, and on the other hand, the fact that swollen lymph-glands usually have a discoverable cause, it ought not in the large majority of cases to be difficult to get at the truth of the matter.

\section{Pertussis}

This disease is one of the most infectious disorders that attack children. It is characterized by respiratory spasm and the convulsive cough that are exceedingly trying to the patient, and almost equally so to the relatives and attendants who are present. This is especially true of infants or very young children who form by far the greatest number of patients, for fully fifty per cent of all cases occur before the third year of life is passed. Most children by the time that they are ten years old have passed through the ordeal, and thus become reasonably well protected. Nevertheless, one occasionally meets the disease in youths, early manhood, and rarely in old age.

Causes. - Many attempts have been made to decide upon the exact cause of whooping-cough. That it is due to the invasion of a micro-organism is certain, and Afanassief, Burger, Cohn, Neumann, and Koplik have done much to give us a good idea of what this germ is. While not absolutely certain, nevertheless one may reasonably believe it to be a streptococcus which is communicated through the expired breath and saliva. Thus the contagion is easily spread, especially as the patient is not necessarily confined to his room during the infective period.

Lesions. - The pathological changes which pertussis entails are few and unimportant. All that one can find is a moderate de- 
gree of congestion in the mucous membrane of the pharynx, larynx, trachea, and the larger bronchi. There is likewise a tendency to the production of acute degeneration in the kidneys, exaggerated in rare cases to an acute exudative nephritis. The stomach and intestines very easily become congested, and are copiously bathed in a mucoid secretion. Any further changes that occur are the effect of extraneous circumstances, or of inability on the part of the patient to withstand the shock of the convulsive cough. Thus, the congested mucous membrane may, as the result of constant irritation, become a fertile culture-ground for bacteria of various sorts, so that various inflammatory conditions may result; or the weakened chest-wall, most of all in asthenic and rachitic children, may be unable to withstand pressure, so that pulmonary emphysema occurs. In similar ways collapse of lung tissue, surgical emphysema, thrombosis of the intercranial sinuses, prolapse of the rectum, or hernia may readily result. It is no uncommon thing to find considerable enlargement of the bronchial glands, which may make the cough still worse than it otherwise would be.

Symptoms. - The infectivity of this disease is supposed to begin as soon as the catarrhal symptoms appear, and to last as long as the characteristic cough persists. The exact limits are, however, hard to set. The whole early history of whooping-cough is more or less indistinct. As an example of this, one may cite the usual statements that the incubation period is a fortnight at the most and two to four days at the least, and that no symptoms before the catarrhal ones are present. Nevertheless, Illoway has noticed a short, hacking cough that shows itself without noticeable changes or symptoms in the throat and lungs a month or less before the onset of the well-known symptoms, and this he considers pathognomonic. I have been able to verify his observation, but only in a few cases; the small number may possibly be due to the deficient faculty of observation in mothers and nurses.

With the so-called catarrhal stage, the general picture is that of an ordinary cold. The child is listless, easily fatigued, poor in appetite. There is a cough of ordinary severity that gradually shows a disposition to become worse at night; there may be a rise of temperature of one or one and a half degrees, principally in the evening. At this time auscultation discovers almost no signs of disease in the lungs, or at most a little harshness in the breathing, and an occasional râle. 
The length of the catarrhal stage is not fixed; in some it is a few and in others it is many days. But the change in the main symptom soon becomes clear and striking. A spasmodic element then makes itself felt; the cough comes in a series of quick, hard hacks until the air in the chest is exhausted, when with a strong effort the air is sucked through the narrowed and rigid larynx, making the well-known crowing sound. The patient when this begins fixes himself as strongly as he can to withstand the shock; he bends over and grasps a chair, or his knees, or a friendly arm or hand; his face becomes puffed out, dark red or purplish; his eyes become suffused and bulged out, and from mouth and nose a rough flow of saliva and rnucus bursts forth. Such a paroxysm may be repeated time and again, until the child falls to the ground, or lies exhausted in the attendant's arms.

These attacks may come with varying frequency; as the disease progresses they occur oftenest at night, and one of the first signs of the approaching end of the sickness is the decrease in frequency of these night paroxysms. After the cough the child commonly vomits, especially if he has recently eaten. Particular attention should be called to the fact that adenoid vegetations will increase the severity and prolong the course of the attacks. Occasionally one meets a case in which the vomiting comes before the cough. The two facts point to a central as well as a local gastric irritation. When the paroxysms are severe, there may be an involuntary passage of urine and fæces. Regularly during the crisis the heart action becomes progressively more rapid until at times one is unable to count the pulsations.

There are many secondary conditions which may supervene: hæmorrhage, usually unimportant, may occur in any mucous membrane, especially that of the nose ; in one case I saw the conjunctivæ completely colored by a thin layer of blood from a small ruptured vessel. The lids may be heavy and discolored from ecchymoses; even the skin of the face may be puffed out and blotched. During dentition a small ulcer may be produced on the sublingual frenum by the scraping of the tongue, during the spasms, on the two lower central incisors. In the lungs there is in typical cases some degree of emphysema ; in addition the thinned walls of the air vesicles may break under pressure, allowing the air to make its way into the cellular tissue of the face and body. In other cases varying degrees of bronchitis, or broncho-pneumonia, or lobar pneumo- 
nia may supervene. Serious cases of laryngitis are not common, nor are well-marked examples of collapse of the lung. The stomach and intestines easily become deranged, and may even occasionally fall into really serious conditions of gastritis and entero-colitis.

The nervous symptonis in some degree are commonly seen. There is always some restlessness and irritability; there may in addition be a tendency to spasm of the glottis and to convulsions. Usually after such a seizure the patient quickly regains his ordinary appearance; if, however, this recovery is delayed, if stupor persists, one should suspect cerebral, usually meningeal, hæmorrhage. Paraplegias and hemiplegias of all sorts may come with or without such a prelude, the particular form depending upon the location and extent of the hæmorrhage.

Finally, great stress should be laid upon the exhaustion which falls upon pertussis patients : the long, tiring sickness, the deficient nourishment, made so by gastric irritability, the disturbed rest, and the presence of complications, - all these are factors in wearing out the child. $\mathrm{He}$ is in a favorable condition to contract disease, and especially is he liable to contract tuberculosis.

Treatment. - The first care is to provide against the infection of other children. There may be considerable trouble in doing this, for the patient in the intervals between paroxysms resents being too closely confined. In the inclement weather of winter and spring, when the disease is most prevalent, it may be possible to keep him in one or two rooms; but in summer and autumn this is out of the question, especially as the period of infectivity extends over the whole active range of the disease and until the convulsive element in the cough has vanished. Since it is impracticable to confine the patient to his room, the only other plan is to remove the healthy children to a safe locality. At all events the patient should not be allowed to associate with other children, even in the open air, until he is practically well. His apartments do not require other disinfection than what thorough cleansing and air give. The towels, handkerchiefs, and personal linen should be well boiled before being put where they can spread the infection.

The medicinal care is simple. Almost every drug within the range of the anti-spasmodics has been used and recommended by some one, and many claims have been made that common experience could not endorse. On the other hand, some observers 
believe that no drug is of any real use, and that the disease must wear itself out. Both views are extreme, and the truth lies between them. While there is no drug that acts as a specific, nevertheless, in antipyrine we have a remedy that will materially mitigate the violence of the paroxysms, and, as I believe, in many cases, shorten the attack. But it must be given fearlessly, in comparatively larger doses than an adult would take. For the past three and a half years I have been so prescribing it without bad effects, the sole precaution being to add to each dose a stimulant, usually aromatic spirits of ammonia. The dose which I commonly use is 0.06 gramme (one grain) for each year of life up to 0.45 gramme (seven grains) every four, three, or even two hours. In exceptional cases of tolerance, where necessity demands, the dose may even be slightly increased. The next best remedy is bromoform, in the same dose in minims, and used with the same precautions. Another useful measure is to spray out the nose and throat with an alkaline antiseptic solution several times a day. This, I believe, is superior to the making of topical applications by means of swabs or brushes, which so commonly have the effect of exciting new paroxysms of coughing. This can be demonstrated by touching the back of the throat, and especially the arytenoid cartilages, with any instrument, with the almost constant result of a fresh spasmodic attack. In fact, a practical method of diagnosis is, while examining the mouth, to touch the back of the throat with the tongue depressor, whereupon the characteristic cough is commonly elicited. The practice of providing medicated vapors seems of doubtful utility. With very young children such means can be used only when the atmosphere of the whole room is well charged with the remedy; if this is thoroughly done the harm to be derived would in all likelihood be greater than the possible good. Just as much benefit will be derived from moistening the air, if it is too dry, by steam without medicinal admixture.

The vomiting in some cases is so severe as to call for careful consideration. In most cases, as the violence of the paroxysms falls under the control of antipyrin, the stomach becomes less irritable. In a few cases it may be necessary to regulate the food very carefully, or to predigest it, giving it in small quantities. Eustace Smith recommends cocaine in doses of 0.01 gramme ( $\frac{1}{6}$ grain) twice a day for a child one year old. A few extreme cases will be benefited by giving no food at all by the mouth, the 
child being supported for a few days by means of nutrient enemata.

The general care and nursing of the child should be as good as possible. So long as he has no fever and the weather is good, he should be carried into the air every day. Daily sponge baths, so long as precautions against draughts are observed, are beneficial; and everything possible to soothe the patient's irritated nerves, and keep him contentedly occupied should be done. Toward the end of the sickness a tonic should be administered to combat the inevitable weakness and anæmia.

Complications should be treated according to their various indications.

Prognosis. - In infants, especially those who are weak or rachitic, pertussis is a very serious disease. Their weakness necessarily restricts within small limits the amount of medicine which we may prescribe, they are quickly exhausted by the convulsive strain of the cough and the interference with retaining food, and they are most susceptible to the various complications. Among such patients the mortality may easily be from fifteen per cent to forty per cent. With each succeeding half year the danger rapidly decreases, and at four or five years of age a death from this disease is rare. The complications, such as broncho-pneumonia, tuberculosis, and entero-colitis are to be feared according to their several characters.

The time length of the disease is very uncertain, ranging from two weeks to two months, and in unusual cases possibly longer Scrupulous attention and good management will, I believe, make these very long cases exceptional.

Differential Diagnosis. - The early diagnosis of pertussis is not often a matter of certainty. If there is a history of prevalence of the disease, of a short, hacking cough for days or weeks, unattended by physical signs in the throat or lungs, of a later cough which steadily becomes worse, especially at night, without being controlled by the ordinary means, if the physical signs continue to be insignificant in proportion to the cough, and if this cough finally becomes convulsive, the diagnosis of whooping-cough can be made with a reasonable degree of certainty. One source of serious confusion is the enlargement of bronchial glands to a sufficient degree to produce an irritable cough; but in this case pressure symptoms would likewise be present. The ordinary throat and lung diseases, 
among whose symptoms would be a cough, have physical signs that make them easily distinguishable from pertussis.

\section{Epidemic Influenza}

La grippe is in many ways one of the most remarkable diseases which we are called upon to treat: it has been known for centuries, but, nevertheless, unless it comes in epidemics it frequently is not recognized; it attacks all ages, but in some epidemics adults are more affected, while in others the disease shows a greater affinity for children ; the direct mortality is small, but the depression which the disease produces and the liability to long continued or fatal after-sickness is great; the cause is single and specific, but its manifestations are manifold and diverse.

Cause. - The active cause of the disease is a minute bacillus $(0.4 \mathrm{~m} . \times 0.8 \mathrm{~m}$.) which Pfeiffer was the first to describe accurately. Its culture-ground is on the mucous membrane of the nose, throat, and lungs, where it occurs in large numbers. Its habitat suggests immediately its manner of infection as well as the most direct method of disinfection.

Lesions. - The pathological changes are confined for the most part to the mucous membranes primarily, from which they may spread in various directions. As far as one can see, they are no different from ordinary catarrhal processes of the bronchi, trachea, larynx, pharynx, nose, ears, eyes, stomach, and intestines. The intensity of the inflammation is irregular, and varies not only between different epidemics, but also between individuals. The main and most serious change seems not to be due to the direct effect of the poison, but rather to the ease thereby produced for the entrance and development of other pathogenic micro-organisms, such as streptococci, the specific bacilli of lobar pneumonia and diphtheria.

Symptoms. - The period of incubation before the symptoms appear is a short one, probably not more than five or six days, and generally about half that time. Then the signs break out suddenly, and often with violence. The violent symptoms usually last for less than a week, although one occasionally sees a case that lingers on for a week or ten days longer. The child complains of pain in the head, frontal, and orbital, and in the back. These may be so severe as to make the patient cry out, and to 
frustrate any attempts to obtain an easy position. There will be some fever which rarely exceeds $39.5^{\circ}$ or $40^{\circ} \mathrm{C}$. $\left(103.1^{\circ}\right.$ or $104^{\circ}$ F.) in ordinary cases. While there is nothing characteristic about the fever curve, one may notice that its variations, especially its rises, do not necessarily carry with them a proportional increase in the pulse-rate. The heart does not work very well, and its beating

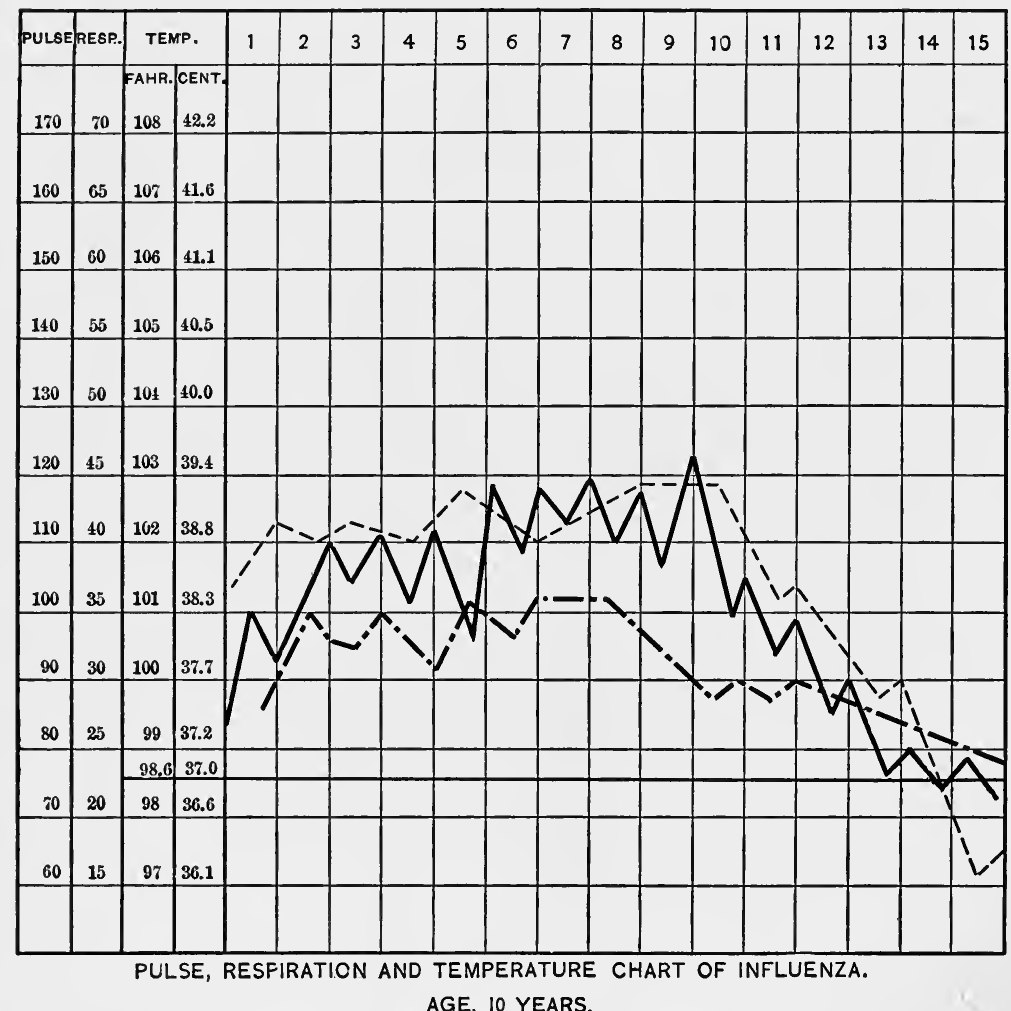

AGE, 10 YEARS.

Fig. 86.

gives the observer the idea of weariness. There may be one or more chills, followed by profuse sweats. The tongue is coated with a creamy fur, the breath has a disagreeable musty odor, and the teeth may be covered with sordes.

In most cases the source of the attack spends itself upon the mucous membrane of some part of the body, the selection being 
doubtless due to an existing weakness of the locality in question, or to the presence of some particular organism that finds in the irritated surface its proper culture-ground. Thus there is a class of cases in which the lungs bear the brunt of the attack. There may be a bronchitis of any degree of severity, in infants involving the smaller tubes; in older children the lung tissue itself is involved so that there is a broncho-pneumonia or an acute lobar pneumonia. In these cases, especially the latter, there is apt to be a complicating pleuritis. One should keep in mind that such a pleuritis, whether it occurs immediately, or only as an extension of a pneumonitis, has a strong tendency to become purulent. In all these sicknesses one of the most striking features is the weakness of the heart, and the consequent general depression. In close relation stand the cases in which the heart seems deeply affected by the poison of the disease. The beat is at times alarmingly weak, irregular, or even dicrotic. In some children there may be cyanosis, oppressed breathing, or even syncope; the pulse-rate may be unnaturally fast or slow, and even after convalescence may take long to regain its natural quality. It is possible in rare cases to find a thrombosis as a result of the circulatory insufficiency.

In other children the main symptoms show themselves in the nose and throat. There may be a follicular tonsillitis, or a stomatitis, or a laryngitis of one or other variety, or a rhinitis with an acrid, muco-purulent discharge ; under these circumstances there would naturally be an enlargement of the corresponding glands. These disorders of the throat are oftener in children than in adults complicated by disease of the middle ear, which may occasionally involve mastoid disease. In two cases during the past winter I saw empyema of the antrum of Highmore.

The nervous manifestations are multiform. The pain in the head, back, and extremities, convulsions, the stupor or mild delirium, are phenomena that one must be prepared to treat. These latter symptoms may at times remind one of a meningitis; while in other cases the patient is so indifferent and dull as to suggest the typhoid condition. Occasionally in older children one may see neuralgias of various distribution, which may later develop into a distinct neuritis.

Frequently there may be derangements of the stomach and intestines; these in children are apt to be very often present in some degree even when they are not among the main characteristics 
of the disease. The vomiting commonly occurs earlier than the diarrhœa, while the latter is more apt to be persistent and at times severe. In serious cases, especially where the type of the disease is more septic and malignant than the ordinary, there may be an acute degeneration of the kidneys which in rare cases is exaggerated into an acute nephritis.

In epidemic influenza there is always a wide range of possibility through which the severity of the case may pass. And at any time the symptoms may assume so threatening a form that doubt as to the true nature of the disease may logically exist. Thus it is impossible to set hard and fast limits to the various phenomena that may show themselves in this case or that. The convalescence is apt to be long and trying.

Treatment. - In the absence of a specific drug, the care of these cases is symptomatic and general. The precaution should be taken to disinfect or destroy the handkerchiefs which the patient uses, and also to place in the cuspidores that are in the room, a solution of carbolic acid, or some other efficient disinfectant. The sick-room on being vacated should be thoroughiy cleansed and aired. The patient's nose and throat should be carefully, thoroughly, and frequently sprayed with an alkaline antiseptic solution. The child should be kept in bed and the diet restricted to fluids. $\mathrm{He}$ may receive sponge baths daily, and if his temperature runs above $39.5^{\circ} \mathrm{C}$. $\left(103.1^{\circ} \mathrm{F}\right.$.) he may be treated with the cold pack or the graduated cold bath. The pains which are so formidable a part of the early symptoms may be kept in check by frequent doses of phenacetin and salol ( 0.12 gramme - gr. ij of each, every two hours for a child of five years). The heart weakness may be combated by liberal doses of strychnine, and throughout the whole disease alcholic stimulation may be indicated. The various complications must be treated according to their various needs.

The treatment of the convalescence is of the utmost importance. The diet, bathing, exercise, and general mode of life should be carefully regulated. For weeks and even months the child should be kept upon tonics, and if possible should be sent into the country for an extended vacation.

Prognosis. - The main danger for children, as for adults, resides not so much in the disease itself as in the various complications and sequels which are so apt to occur. The worst of these are the acute lobar pneumonia, broncho-pneumonia, and pulmonary 
tuberculosis. Not often does a child die from influenza alone, but frequently enough he may be left in a weak, irritable, and debilitated state for many months. At times it seems almost more than we can accomplish to get him back to rugged and blooming health, and in some cases we are quite unsuccessful. One discouraging feature about these complications is that they commonly rage with a greater fierceness than when they occur as primary diseases. Throughout the whole sickness the main object is to conserve the patient's strength and to ward off as well as possible any complications.

Differential Diagnosis. - The crucial point in diagnosis is the existence of much prostration, fever, and pain without correspondingly marked physical signs. This, and the course of the disease, will ultimately, even if at first there is doubt, clear up the matter. Meningitis, on account of its peculiar course, would within a day or two stand out clearly; pneumonia would give its characteristic signs and rapid pulse; typhoid and malaria would be differentiated by their especial temperature curves. In many instances the diagnosis cannot be made immediately. The simultaneous existence of an epidemic naturally makes the case much simpler, for the greatest confusion exists in the sporadic attacks.

\section{Lobar Pneumonia}

Lobar pneumonia, sometimes called croupous or fibrinous pneumonia, is an acute inflammation of micro-organic origin. While it generally locates itself in one lobe of a lung, it may on the one hand be confined to a mere spot, and on the other may cover a whole lung. Its infectious character is so plainly marked that it sometimes occurs in epidemics; often a house or a room containing the germ will infect persons living therein, so that one member of a family after another will suffer from it. Although young adults fall victims to it frequently enough, nevertheless children of all ages are not susceptible in quite the same degree. Indeed, it does not often occur before the age of three years; but from that time until the tenth year it is most frequent. After this time the danger of its occurrence lessens progressively. Boys seem to be slightly more liable to it than girls; it is more prevalent in spring than in other seasons; it attacks robust as well as weak children. For all these facts it is hard to find a reason; and 
similarly it is hard to account for the greater frequency of invasion in certain parts of the lungs. Some observers, after having collected many cases, believe that the base of the left lung is most easy of attack, that the apex of the right lung comes next, and that the base of the right and the apex of the left follow after. Others believe that both lungs at their bases are about equally liable, and that the apices are less commonly the seat of the sickness. The gist of the matter seems to be that, whatever part of the lung tissue is attacked, one may feel fairly sure of the fault's

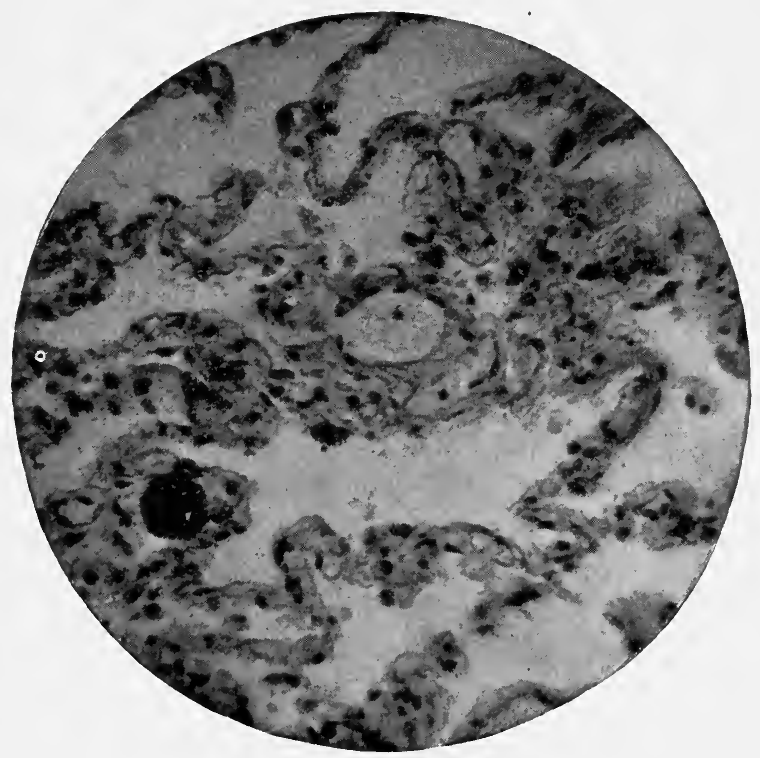

Fig. 87. - Lobar Pneumonia : First Stage. $\times 250$.

lying in the weakness of the diseased area, not in a predilection of the germ for any definite part.

Causes. - The cause may be almost regularly attributed to the germ called, on account of its shape, Micrococcus lanceolatus. In a few cases the micro-organism has not been found; and especially is this apt to be true in those cases where the pneumonia complicates or directly follows another microbic disease. This germ occurs not only in disease, but is also found in the sputum of healthy persons. While acting as the causative factor of lobar pneumonia, it sometimes occurs in other disorders, such as bronchopneumonia and acute exudative inflammations of serous mem- 
branes. Thus it has been observed in meningitis, empyema, peritonitis, endocarditis, and pericarditis. It has been seen in acute inflammations of joints, as well as in purulent inflammations of the tonsils and middle ear. Its development is attended by the production of an albuminous poison called pneumo-toxine. This is the direct toxine of the disease, and shows its power in the symptoms of a general toxæmia. Some experimenters hold that the physical economy in the course of the sickness elaborates a certain substance which acts as an antidote to pneumo-toxine. When

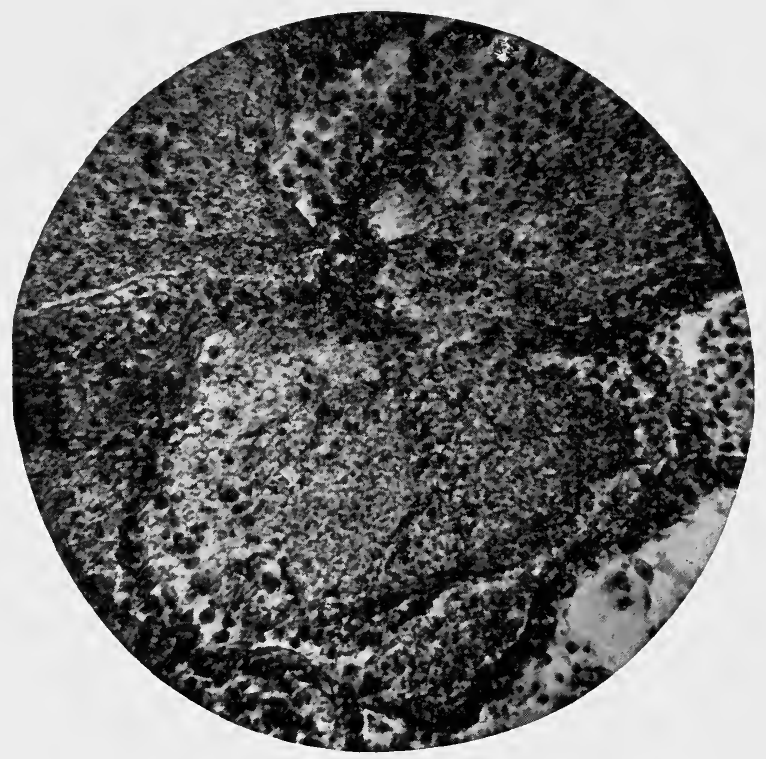

Frg. 88. - Lobar Pneumonia: Second Stage. $\times 180$.

(Red Hepatization.)

these two forces meet and offset each other, the approximate balance of health is restored, and shows itself in the so-called crisis.

Lesions. - The morbid changes which arise are quite characteristic. At first the affected parts are heavy, congested, and slightly increased in size. The air-spaces become choked with the products of inflammation: red and white cells, broken-down epithelium, and fibrin. The capillaries in the walls of the air-spaces are dilated and hold a larger percentage of white cells than usual. 
The tissue, on account of its engorgement, takes on a dark red color, and may become so choked up as to contain no air.

When this process of filling up is thoroughly developed, the lobe is solid, the air-spaces are plugged with small masses of inflammation products, the alveoli are full of a serous fluid holding many epithelial and white blood-cells, bacteria, and much fibrin. The color is somewhat lighter than in the preceding or congestive stage. The lung may be then so much swollen as to show, like a cast, the shape of the ribs. On section one sees a multitude of small elevations, representing the filled-up alveoli. The whole

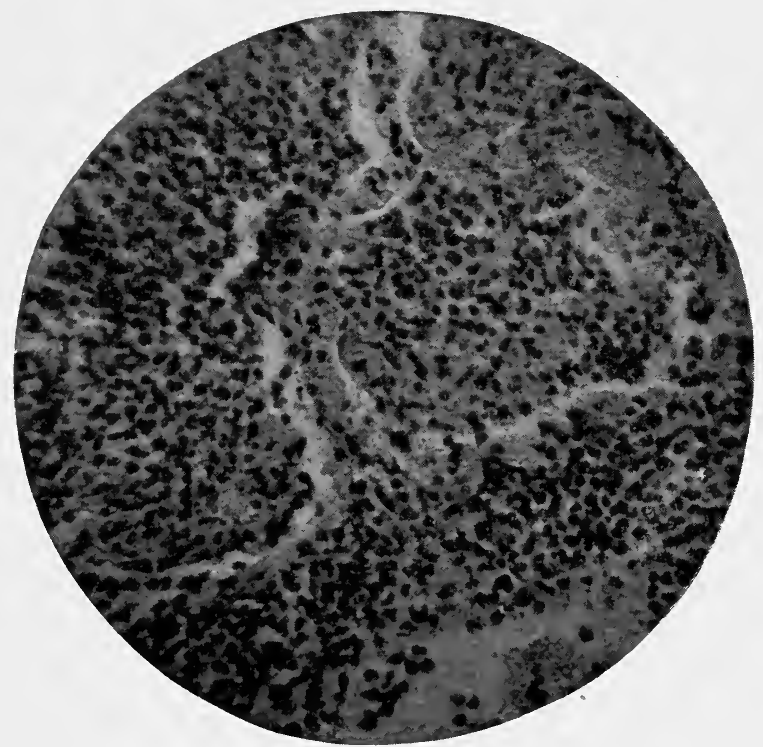

Fig. 89. - Lobar Pneumonia: Third Stage. $\times 250$.

appearance resembles so much that of the liver that we call this stage the period of red hepatization. The inflammation is so intense that it overflows the parenchyma of the lung and extends to the smaller bronchi or the pleura.

After the stage of red hepatization is fully developed, the color begins to change gradually to yellow and gray. The exudate then on account of fatty degeneration begins to soften, and should be absorbed. The time of absorption or resolution naturally varies according to the amount of material to be consumed and the eliminating powers of the patient. 
In some cases the exudate with the surrounding lung tissue breaks down and an abscess results. This may become encapsuled or absorbed, or go on to a fatal gangrene.

Symptoms. - The physical signs are occasionally not very distinct on the first, second, or even the third day; after that they are clear enough. 'The child is seen to lie for the most part on the affected side, thus preventing free expansion, while the unaffected lung is left free to do the work of both. Fremitus is increased and is distinguishable when other signs are not marked. Resonance is im-

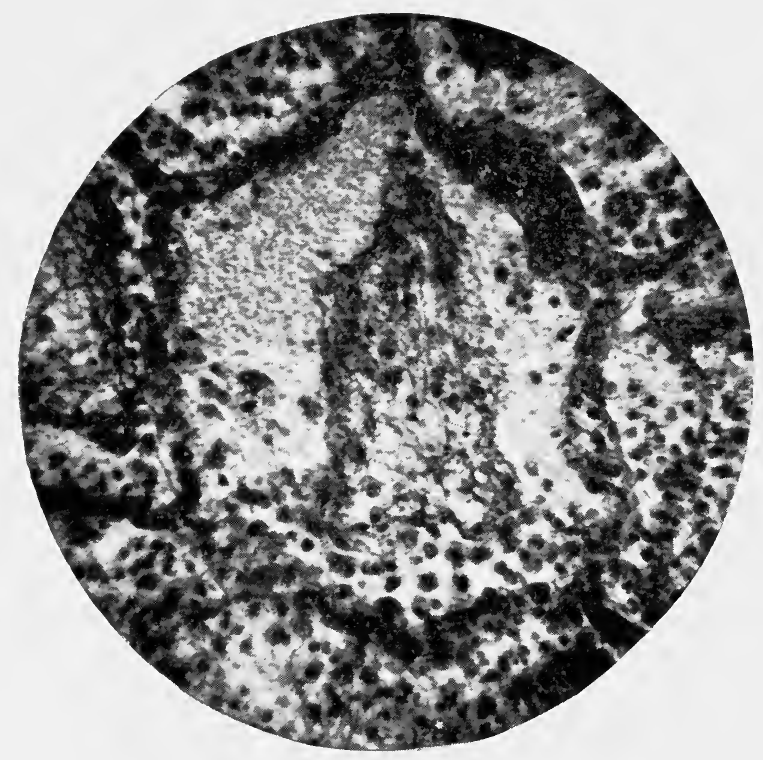

Fig. 90. - Lobar Pneumonia: Third Stage. $\times 250$.

(Fibrin Threads.)

paired; breathing is at first rough, and later on bronchial. In the full development of the disease one generally finds fine crepitant râles which are heard most plainly on the borders of the inflamed area; in addition there may be some coarser râles, moist or dry, scattered here and there, which are due to a natural extension of the inflammatory process. Also, the unaffected lung, on account of its increased work, becomes somewhat dilated in a part of its air-spaces, and thus-gives harsh breathing and an increased resonance.

It is commonly supposed that the physical signs in acute lobar 
pneumonia may sometimes be absent. This is due to the fact that at times the area involved is so small as easily to be overlooked, most of all if the inflammation is located deep in the centre of the lung. One should also keep in mind that the inflammation may extend in such a manner that one hears the signs of a bronchitis or a pleurisy. Or the exudate may be so profuse as to overflow its original limits and fill up adjacent alveoli and bronchioles. Thus again one may hear physical signs that are outside of the classical form. Also, when resolution sets in the exudate, preparatory to being absorbed, begins to soften. This may frequently give rise to confusion, because various parts of the affected tissue may at one time be in different degrees of consolidation and resolution. This is a more common condition in infants than in older children, and much more than in adults.

The disease may or may not begin with a chill, vomiting, or convulsions; on the whole the first does not so regularly occur in young children as in older patients, while the others are almost confined to children. In addition there may be beginning attacks of diarrhœa in a fair number of children. It is common to have the picture of a healthy child suddenly attacked with high fever, rapid and labored respiration, hurried heart action, short, dry cough, flushed cheeks, pain in the chest near the affected area, or somewhat removed from it, and the additional symptoms dependent on a disordered alimentary track, or the involvement of other viscera. Especially is the spleen apt to be enlarged.

Since the germ of lobar pneumonia makes its way to regions outside of the lungs, one may find symptoms which are characteristic of the invaded part. Thus if the meninges are affected, stupor or delirium may result; in a like manner the peritonæum may become the seat of inflammation. In this way arose the unfortunate terms "cerebral pneumonia," and "abdominal pneumonia." Nevertheless, the truth of the matter is that the pneumonia remains always the same, excepting that in the cases mentioned there are added to it symptoms of another disorder. Of course one must keep in mind that the stupor may in fact be due to the systemic intoxication by the specific poison.

The usual temperature varies between $39.4^{\circ} \mathrm{C}$. and $40.5^{\circ} \mathrm{C}$. $\left(103^{\circ}-105^{\circ} \mathrm{F}.\right)$ and is characteristic. At times it mounts still higher, but in the morning, at least, there are apt to be small remissions. Nevertheless, the course is in general fairly regular 
until the crisis, when it suddenly drops to normal or subnormal. In the beginning the pulse is small, thin, soft, and rapid, at times running as high as 160 , or even more to the minute; it may even become dicrotic. Later on it is full and bounding, but not hard. The heart is seen to work with difficulty, and the two sounds become uneven, the second being plainly accentuated. The respi-

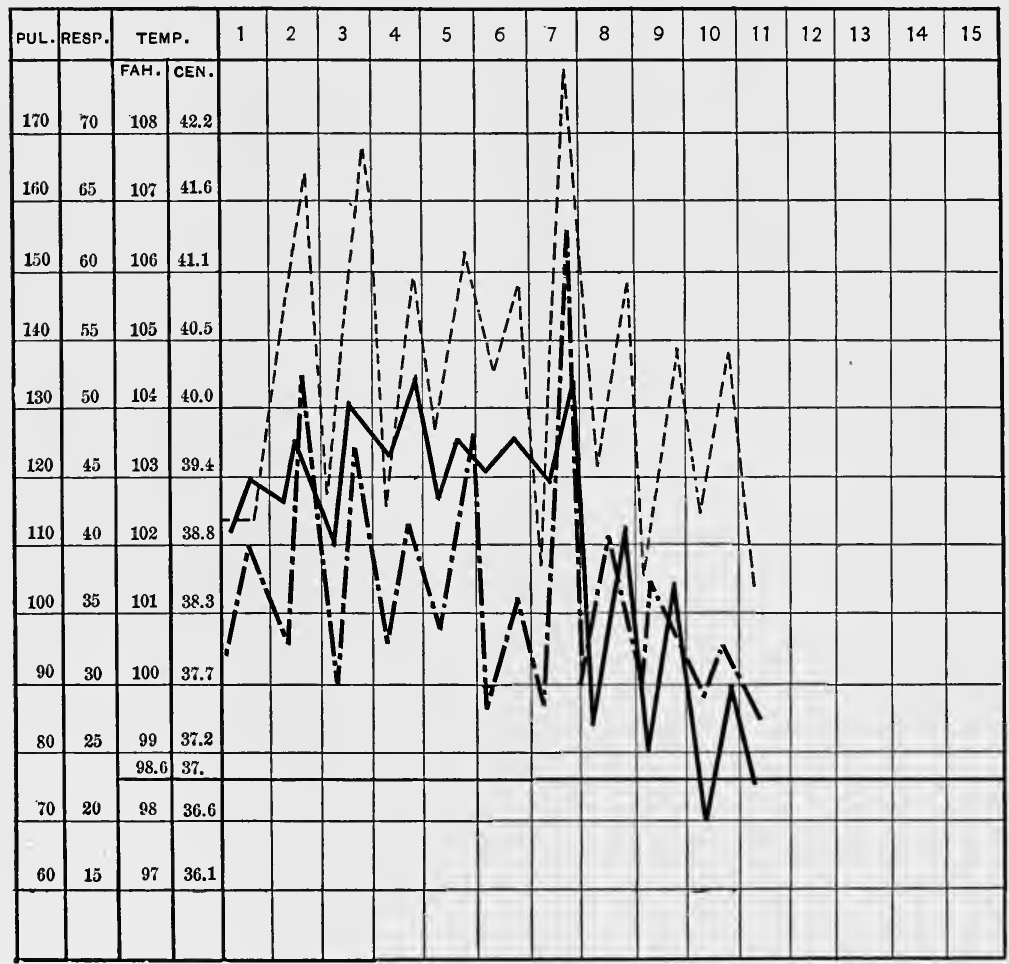

PULSE, RESPIRATION AND TEMPERATURE CHART OF LOBAR-PNEUMONIA.

AGE. 11 MONTHS.

PULSE

RESPIRATION

TEMPERATURE

Fig. 91.

ration is very fast, so much so that it outstrips the ratio with the pulse. Thus one often sees this ratio as one to two and a half instead of one to four. During the first day or two the child may pause after every few expirations, and give a short, catchy moan. One should keep in mind that infants are more subject to high pulse- and respiration-rates than adults, and that any of the ordi- 
nary complications may give symptoms which may seem far more startling than the true state of the case warrants. It is necessary to distinguish between such a condition and one where there is a large amount of lung tissue involved, or an unusually severe systemic poisoning. A want of harmony between the temperature, pulse, and respiration curves is unfavorable. And irregularity in any of these factors is likewise unfortunate, pointing as it does to added disease or failing strength.

In this disease the patients are apt, partly on account of the pain which effort of any sort brings on, to be much quieter than one would expect from so much general and local irritation. This does not, of course, apply to cases of unusually high temperatures or unusually severe infections; for here one is apt to find delirium or stupor, varying in severity according to the comparative resistance of the child. Such symptoms have helped to bring about a belief that meningitis, tubercular or non-tubercular, very often complicates lobar pneumonia. This is far from the truth; although it is a fact that cerebral symptoms are easily developed in children who are suffering from a toxic disease, and that a meningitis may be caused by the pneumococcus, a complicating disease is not by any means a common occurrence.

The crisis is characterized by a sudden dropping of the temperature to normal or subnormal, by free, quiet respiration, and a full transpiration. The urine, which had been scanty, high colored, and sometimes charged with albumin, becomes light colored, clearer, and more nearly normal. Occasionally the patient will be fortunate enough to have also a free, thorough catharsis, after which he will be found to be materially more comfortable. In the cases that end suddenly, the crisis may come between the third and fourth days, on the fifth, or between the seventh and eighth, even as late as between the eighth and ninth. There are some exceptions to this - cases, on the one hand, of a slow and gradual dropping of temperature, the so-called resolution by lysis; on the other, those rare cases of delayed resolution where the children's symptoms may persist for from ten day to three weeks. There are occasional examples of a very rapid course that seem to end a few hours before the third day.

This disease is not commonly liable to many complications. There is, as has before been stated, a likelihood of more or less bronchitis, so that at times one finds the picture of an acute lobar 
pneumonia associated with scattered areas of broncho-pneumonia. Then again, an extension of the inflammation to the pleura, with or without effusion, often enough occurs. As has been stated above, there may be a complicating meningitis, which is somewhat unusual. Also, one should bear in mind the possibility of an associated inflammation of the serous membranes as well as the kidneys. And where resolution is delayed, where the pulmonary tissue breaks down, one occasionally — or rather rarely — sees a gangrene of the affected area. It may be mentioned that infants are not able to expectorate, and that therefore one should not look for the characteristic sputum; however, in patients over five or six years the adult appearance is gradually assumed.

Treatment. - The treatment of lobar pueumonia requires judgment and thoroughness, rather than the strict adherence to a routine. To begin with, it is advisable to discard the poultices, pneumonia jackets, and breast pads that for so many years have been in almost universal use. Their value is more than doubtful; employment of them is attended by plain dangers. The surface of the body should be kept as active as possible, not hindered and clogged up. Antipyretics, likewise, are with advantage omitted. The mere lowering of temperature is not necessarily of the first importance; where the temperature is lowered with a consequent decrease in vitality, one is apt to do more harm than good. Where the commonly employed antipyretics are freely given, the temperature chart may, it is true, be decidedly altered; but there is very little comfort in having a baby die, even though his temperature be normal.

Moreover, these agents are really not at all required because we have safer and more efficient means in baths, spongings, and packs. A robust child will bear a graduated bath (down to $60^{\circ} \mathrm{F}$. $-15^{\circ} \mathrm{C}$.) with comfort and benefit. Or one may refresh as well as cool him by sponging the body for a period of from ten to thirty minutes with lukewarm or cool water mixed with alcohol. Or, again, one may, where the other methods are not advisable, use hot or cold packs. The main rule is that a robust child in comparatively good condition may be treated with cold water; but a little one who is very weak, whose circulation is poor, whose extremities are cold, should by all means have warm applications. And when, on account of the application of cold, the extremities become cyanosed or chilled, one should directly resort to methods 
of heating the surface. All in all, one must keep in mind that practices for lowering the temperature should be kept as clear as possible from the suspicion of exaggerating the patient's weakness.

On the other hand, every effort should from the first be made to sustain the patient's strength. One must keep in mind that he is suffering, not only from the toxic effects of the disease, but also from a defective circulation, and that one of his gravest dangers lies in the strain put upon the heart. The stimulation will in all likelibood be in continuous demand through the whole sickness, and therefore should be accordingly arranged; one should proceed cautiously, always remembering that it is wise to keep a reserve of stimulation for occasions of sudden weakness or collapse. One of our most useful drugs is strychnine in doses of $\mathrm{gm} .0 .0005$ to $0.001\left(\frac{1}{120}\right.$ to $\frac{1}{60}$ of a grain) three, four, or five times a day. Nitroglycerin in very small amounts combined with large doses of digitalis is also of value, as is likewise strophantus or sparteine. In the whole matter of treatment one must pay most attention, not merely to the heart as a whole, but rather to the right side of the heart. And wisely directed efforts will ameliorate such symptoms as nephritis, œdema of the lungs, distressing dyspnœa, and the concomitant effects of impeded circulation.

When the cough is very distressing, it must be quieted; and for this purpose bromide of soda, which the youngest babies tolerate well even in large doses, will be found of use. In older children it may be necessary to use small doses of opium. But so long as this can be avoided, it is better so to do. When resolution sets in, expectorants are plainly indicated; and if by the use of alkalis one can render the mucous secretion thinner and more easily movable some advantage will have been gained. Even at this time the danger of extreme weakness or sudden collapse must always be kept in sight and carefully provided for. The use of oxygen has not as many adherents as in past times, for various competent observers have declared that the mortality of their cases is not at all lessered, nor the comfort of the patients improved by it. In spite of this opinion, which is doubtless correct, the inhalations of the gas continue to be used.

A suggestion, that has some value, has of late been receiving renewed emphasis: it is that the amount of food should be restricted. It is urged that a full stomach diminishes the amount of space which the swollen lung ought to have, and that the gas- 
tric enlargement is easily increased if fermentation of the contents occurs. It is held that for the short period of the pneumonia the child can easily be maintained on a reduced diet, especially if he is receiving alcoholic stimulants.

Some very encouraging experiments with a pneumonia antitoxine have been made; but since they are as yet confined to the limits of research, they should not receive further mention.

Prognosis. - The prognosis is, on the whole, fairly good, since no more than from two per cent to four per cent should die; in very weak children or where there are complications which diminish the amount of available lung tissue, or increase the inroads upon the general strength, there is naturally an important element of added danger. When death occurs, one can see the strain which the impeded circulation put upon the heart by its relaxed, slightly dilated condition. And when it stops it is in diastole.

Differential Diagnosis. - The main signs of high fever, rapid respiration, cyanosis, and cough, plus the physical signs in one lobe or in one lung, make the diagnosis of this disease fairly plain. Nevertheless it has been overlooked or mistaken for other sicknesses, some of whose symptoms are more or less related. Thus the sudden prostration with cerebral symptoms has suggested meningitis; but in this disorder the respiration and pulse are slow and irregular, while in pneumonia they are rapid; in meningitis the cerebral symptoms increase as the case progresses, but in pneumonia they decrease; in meningitis physical examination does not implicate the lungs at all. Lobar pneumonia has been confused with malaria, gastritis or gastro-enteritis, measles and scarlet fever, tonsillitis, and, most of all, broncho-pneumonia. Malaria has its history of exposure, its characteristic fever and chill, and freedom from pulmonary disease. Scarlet fever and measles present their eruptions and frequently a history of exposure. Tonsillitis is distinguished by the appearance in the throat, and although there may be a cough, the signs of consolidation are absent. Broncho-pneamonia commonly attacks infants, while lobar pneumonia is rarely seen under three or four years of age; in the former the onset is commonly gradual, in the latter it is abrupt. In the first the process is diffuse, in the second it is circumscribed. In the former the disease is commonly secondary; while in the latter it is almost always primary. In the one the signs of consolidation are combined with those of a marked bronchitis, but in the other the con- 
solidation gives the main or only signs, and if a bronchitis is present it is easily recognized as of subsidiary importance. In broncho-pneumonia there is no typical course, the disease may last for weeks, and commonly ends by lysis; but in pneumonia the course is typical, rarely extends over eight or nine days, and ends by crisis. The prognosis in the former is serious, in the latter it is comparatively good ; and when the disease is past, the bronchopneumonia is often enough followed by chronic pulmonary disease, while lobar pneumonia is rarely so attended. The resolution in the one is slow and halting, but in the other it is rapid and complete.

Lobar pneumonia is distinguished from purulent pleuritis by the latter's fluid, and the signs which fluid necessarily produces. The opinion can be confirmed by aspiration, even if the examiner is unable to satisfy himself concerning the usually plain evidences of consolidation.

\section{ENteric Fever}

Typhoid fever, as it is usually called, is an acute infectious disease that is more commonly seen in adults than in children. Nevertheless it occurs at all ages, from fœtal life to old age; in children under two years of age, doubtless on account of their comparatively sterile food, the disease is not often seen. After that infection becomes more and more common, until it reaches its fullest occurrence in youths and young adults.

Cause. - The specific cause of enteric fever is Eberth's bacillus, a short, thick organism whose ends are rounded, and from which eight to twelve flagella project. The vitality of this bacillus is noteworthy; freezing does not necessarily kill it, but continued exposure to temperature over $100^{\circ}$ C. $\left(212^{\circ} \mathrm{F}\right.$.) is fatal. When dried it has been known to live over two months. On food, milk, water, dirt, refuse, and ordinary household utensils, it is able to exist for periods varying from one week to two years, the best environment predicating the absence of sunlight and air. The manner of transmitting it is thus easily understood: it is carried from the body in the frees, and doubtless the urine and sputum as well. It then finds its way into refuse, sewage, cesspools, and rivers. Thence it contaminates articles of food and drink, and thus is taken once more into the gastro-intestinal track. In other cases it may be carried by hands which have become infected to the mouth, and thus equally well finds an entrance to the body. 
Lesions. - The characteristic changes occur in the intestines and spleen; but, in addition, there are various others that may be seen in different parts of the body according to the severity of the disease. As a rule, typhoid fever runs a mild course in children, and the pathological changes are, therefore, not extreme. In the intestines they are, for the most part, grouped in the lower ileum near the ileo-cæcal valve; this does not preclude the involvement in seattered patches of other portions of the large intestine or of the small intestine. The process occurs most fully in the follicular elements. There is first an inflammation of the mucous mem-

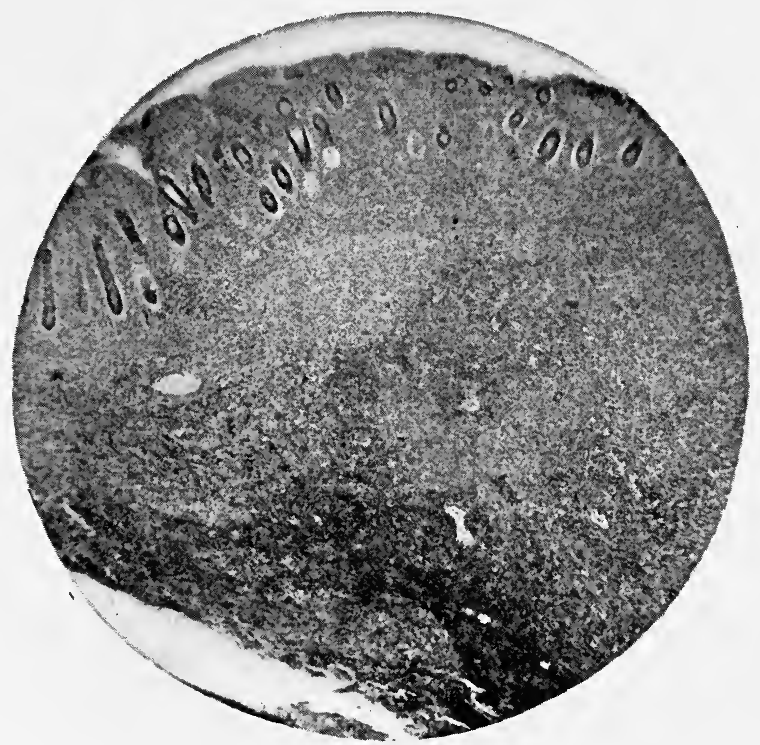

Fıg. 92. - Typhoidal Swelling of Lymph-Node. $\times 30$.

brane which spreads to the underlying follicles and lymphatic glands. The different elements which go to constitute a Peyer's patch may not be equally involved, and the resulting appearance is one of unevenness. With this there is a considerable infiltration of both solitary and agminated glands; the enlargement may be so great as to cause a noticeable projection into the lumen of the gut. These affected follicles then soften and break down, leaving an ulcerated surface behind them. The portion of the bowel which remains intact may be exceedingly thin. The bacilli from the intestine make their way through its walls into 
the lymphatic tissue, and thence into the mesenteric glands and the blood. The mesenteric glands undergo similar changes as in the gut, and may go on to suppuration. Although the typhoid bacillus may cause suppuration, nevertheless such a process is usually one of mixed infection; in either case, however, the result is a purulent peritonitis. The spleen is affected in somewhat similar ways as the intestines. One of its most marked changes is its increase in size. This begins at the first stage of the disease, and continues for about two and a half weeks in young children and a somewhat longer time in the older ones. The swelling may be

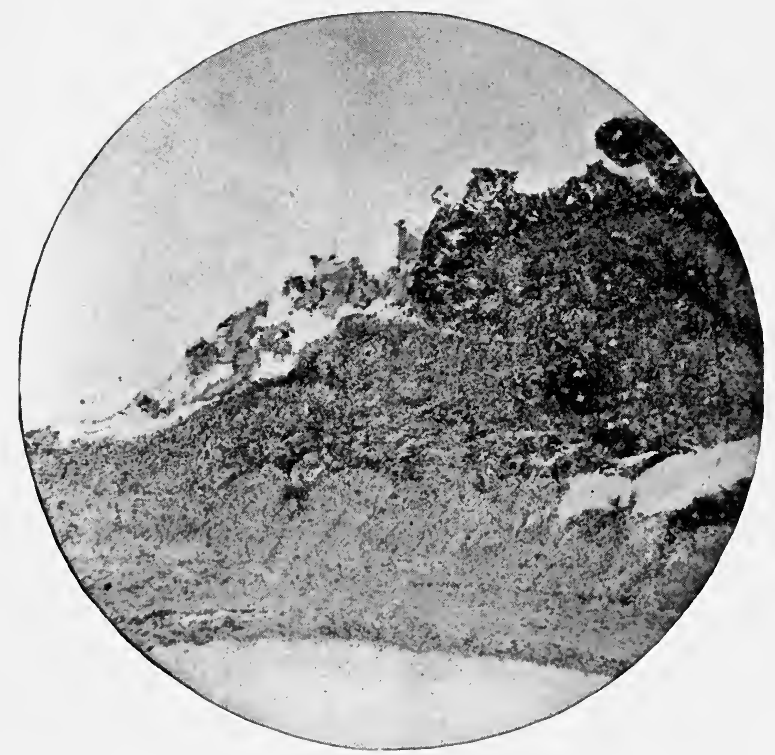

Fig. 93. - Typhoidal Ulceration of Intestine. Healing Stage. $\times 30$.

so considerable as to stretch the capsule. At the same time the stroma becomes indistinct, the parenchyma soft and pulpy, and the whole organ infiltrated with leucocytes. Infarctions may occur after the first stages of growth and may result in peritonitis. This last-named complication may likewise occur from an extension of the inflammation through the serous coat of the gut even when the ulcer has not caused perforation. When perforation does occur the amount of peritonitis is naturally much greater.

The whole gastro-intestinal track may become, to a greater or less extent, involved. From the mouth to the rectum there may 
be various degrees of inflammation, varying in different cases in severity as well as location. The pharynx may be congested and inflamed, the tonsils may be enlarged, and the follicles may be involved in the attack; at the same time the associated submaxillary glands, and even the parotid gland, may be swollen and tender. In well-marked cases there may be an acute degeneration in the pancreas and liver, but the latter viscus does not appreciably increase in size. The heart shows a tendency to atonicity, is pale, and the muscular fibres may be degenerated. The larynx is apt to fall into a catarrhal inflammation which only rarely in children assumes a serious form. The lungs easily become irritated, showing the changes of acute bronchitis. When the child is weak, and in the presence of the proper micro-organic life, the transition to a broncho-pneumonia or an acute lobar pneumonia is easy. The kidneys, as in all acute infectious fevers of much severity, are commonly irritated. There is often, even when the clinical symptoms do not draw attention to these organs, a cloudy swelling of the epithelium, an acute degeneration, or even an acute nephritis. In children the toxines of the typhoid bacillus have a disproportionately marked effect upon nerve tissue, as far as the symptoms go. In severe cases there may be a hyperæmia of the cerebral meninges, and some effusion into the ventricles of the brain. Nerve fibres are flaccid, and here and there may show minute areas of fatty degeneration.

Symptoms. - From the time when the patient contracts the disease up to the appearance of the ordinary symptoms is a variable period, the mean of which is about a fortnight, while the extremes are three days and three weeks. During this period the child is commonly irritable or depressed, he tires of his ordinary pursuits and games, complains of headache, and possibly intestinal or gastro-intestinal disorders. These manifestations are often so trivial in their appearance that they escape any especial notice. Sometimes the disease begins its usual course, and on account of its mild character is not diagnosticated for several days. The typical picture of the onset is the occurrence of depression, pains in the limbs, headache, anorexia, epistaxis, and gastric disorders. There is a rise of temperature, and the child is plainly unfit to be about. Usually he asks to be put to bed, and instead of feeling better continues to get worse. This is usually considered the first day of the disease. 
One of the main features of the disease is the temperature curve. This does not show as high a range of fever as in adults, and often the period between its beginning and its end is shorter as well. For the first half-week the temperature rises progressively every evening until it reaches a maximum of from $39.2^{\circ}$ to $40^{\circ} \mathrm{C}$. $\left(102.5^{\circ}\right.$ to $104^{\circ} \mathrm{F}$.). The morning curve shows a fall of

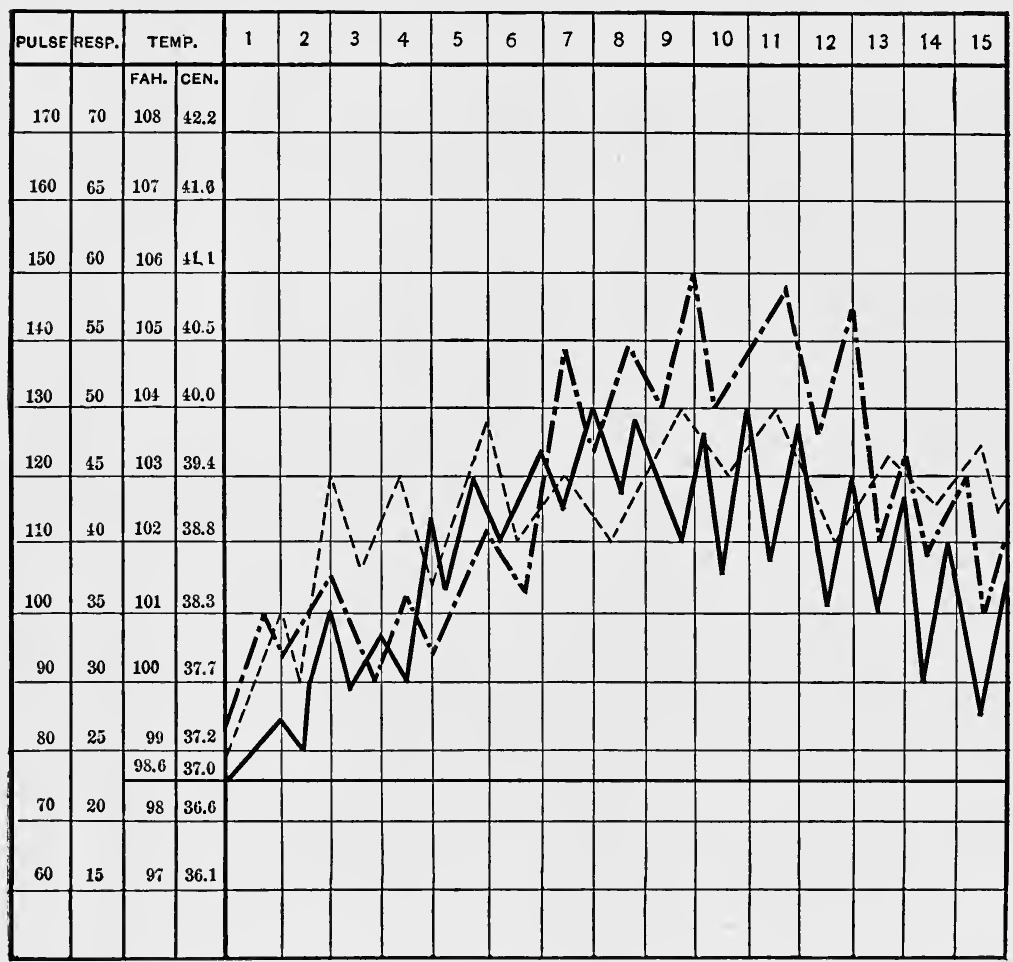

PULSE, RESPIRATION AND TEMPERATURE CHART OF MILD TYPHOID. AGE, 4 YEARS.

PULBE. RESPIRATION TEMPERATURE

Fig. 94.

from $\frac{3}{4}$ to $1 \frac{1}{4}$ degrees C. Generally the respiration and pulse follow the fever in a fairly approximate way. The tongue is coated, there may be diarrhœa or constipation, the abdomen may be slightly swollen and tender, especially in the right iliac region. At the end of this period the temperature remains at its approximate hcight, and the difference between the morning and evening 
figures is less than before. At the end of the first week, in the majority of cases, a roseola appears in the form of small rose-pink spots, disappearing on pressure and usually few in number, on the abdomen. There may be one or more crops of them, each crop lasting about three days. During this time the tongue is dryer and browner than before, the abdomen more distended and tender; there may be borborygmi, and sometimes a diarrhœa that is commonly likened in appearance to pea-soup. The patient may be semi-stupid or irritable, and in some cases there may be some delirium.

At the end of the second week the severity of the disease begins to moderate, but at the same time the increased weakness shows that the child has passed through a severe trial. The morning temperature shows greater approaches toward the normal, followed gradually by the evening fever. The pulse may be weak, irregular, and dicrotic, the heart may be noticeably weak, and the patient's resistance against attacks of pulmonary disease is poor. If there is delirium, it is apt to be at its worst in this period of weakness. Likewise this is the most likely time for intestinal hæmorrhage, and the evolution of nervous symptoms. Within a few days, however, a gradual return to health is made, and in children convalescence is commonly uneventful. After the disease has ended, one, two, or even three relapses are possible; usually they are of a mild type.

The main forms of deviation from the normal adult's type which we see in children are irregularity in onset, mildness of symptoms, markedly so in young children, sparseness or absence of eruption, and short course which in some cases may be called abortive. Occasionally infants show a temperature that one may call remittent. As has been said more than once, typhoid fever in children seems to have as many nervous as abdominal characteristics; in some cases the meningeal and spinal symptoms are so marked as to make confusion with meningitis easy. Likewise the possibility of complicating lung diseases is great, most of all hypostatic congestion and bacterial attacks which may result in bronchitis, broncho-pneumonia, and acute lobar pneumonia. The usual course, extensions, and outcome of these diseases may be expected. In addition the larynx may be involved, and occasionally, with or without a similar infection of the rest of the throat, becomes the seat of diphtheritic inflammation. This is undoubt- 
edly one of the sources of post-typhoidal paralyses. When any disease of the throat prevails, the possibility of middle-ear disease should always be kept in mind.

As there is a wide spread of intoxication throughout the body, it is possible that one or other of the viscera may show the effects. The kidneys may present the phenomena of degeneration or distinct inflammation, and the heart or its serous envelope those of inflammation or effusion. Frequently one may notice that children during typhoid fever increase in height to a noteworthy degree; the change occurs rather in the epiphyses than in the diaphyses of the bones, and sometimes is connected with an irritation or inflammation of the joints or their cartilages. Lastly, the debilitated condition of the economy invites, in the presence of the appropriate predisposition, attacks of tuberculosis, especially pulmonary tuberculosis.

Treatment. - Prophylaxis is an important element in the care of typhoid cases. All excretions and discharges should be mixed with strong antiseptic solutions, such as $1: 1000$ corrosive sublimate, 1: 20 carbolic acid, or slaked lime $1: 200$. The patient's bed linen, napkins, and towels should be boiled very thoroughly, and not with those of the family. His utensils of all sorts should be kept separate from the household's; his hands and mouth should be carefully washed before meals, and the nurse should be warned of the danger of infection. Where the patient has recovered, the sick-room and its contents should be cleansed and aired in the most thorough fashion.

The care of the patient, in the absence of an efficient serum therapy, is symptomatic. At the outset of the disease the intestines should be thoroughly emptied by means of calomel; the child should be confined to bed, and his diet restricted to milk. Attempts to abort the disease by means of intestinal antiseptics have been shown to be fruitless, for the simple reason that unless the drugs are administered in doses which are dangerous to the patient they are likewise harmless to the micro-organisms. If there is diarrhœa it may be treated by large doses of subgallate of bismuth, with or without starch enemata in which are mixed a small amount of opium. Constipation may be relieved by occasional doses of calomel followed by small amounts of salines; this may be varied by the use of enemata of soap-suds and water, or water with a little glycerin dissolved in it. Tympanites 
calls for the passage of a rectal tube, or the administration of enemata with a few drops of turpentine or a small amount of inspissated ox-gall mixed in them. The use of hot stupes may relieve the abdominal tension and tenderness. The nervous symptoms may call for the use of an ice bag, with or without fairly large doses of the bromide of soda. The use of stimulants should be begun as soon as the first signs of flagging vitality show themselves, and it is better to begin somewhat too early than late. For this purpose nux vomica, aided by a good whiskey well diluted with water, is useful. The main part of the treatment at the present time consists in cold or graduated baths given whenever the rectal temperature is $39.5^{\circ} \mathrm{C}$. $\left(103.1^{\circ} \mathrm{F}\right.$.) or more. For robust children the former may be selected, at a temperature of about $21^{\circ} \mathrm{C} .\left(70^{\circ}\right.$ F.) ; for very young or feeble patients it is better to start with a temperature of $37^{\circ}$ or $37.5^{\circ} \mathrm{C}$. $\left(98.6^{\circ}\right.$ or $99.5^{\circ} \mathrm{F}$.), at the same time paying the closest attention to the reaction of the patient. During all these baths the surface of the chest, abdomen, and extremities should be rubbed briskly with the hand, and the child, as soon as he shows depression, shivers, and turns blue, should be taken from the water, put in bed, and his feet warmed with hotwater bags. All complications should receive their approximate treatment.

As soon as convalescence is established, the child must be put upon a sufficiently prolonged course of tonics. Sometimes marked anæmia and asthenia may persist for months. Such cases most of all require constant attention and up-building until a normal state of health returns. When the circumstances of the patient permit, a protracted vacation in the country is of great value.

Prognosis. - The chances of recovery are very much better in children than in adults. Although the usual statistics give a mortality of from five per cent to eight per cent, nevertheless one may see that, since these are the results of hospital experience with doubtless the worst class of cases, the estimates in favorably situated families in private practice could safely be put at one half of the above figures. In estimating the probable length of the sickness, one should remember the shorter course in children, and that the usual period of from three to four weeks in adults has its analogue in two to three weeks in younger patients.

Differential Diagnosis. - The main difficulty in diagnosis occurs in the first week. In that time such symptoms as headache, epis- 
taxis, pains in the body, abdominal tenderness, and a continued and nightly mounting of fever should turn our thoughts to typhoid. At the end of the first and the beginning of the second week there are the additional symptoms of the eruption, enlarged spleen, tympanites, continuance of the characteristic temperature, and the added severity of the general symptoms. Ehrlich's diazo reaction is not of much practical importance, since it is present in other pyrexial and apyrexial conditions. Widal's reaction is of much more value, especially after the first week. As pointed out by Elsberg (New York), more than one trial may be necessary on account of the late development in some cases of the "agglutinizing power." Another method of differential diagnosis has recently been published by Piorkowski. He claims that cultures which are made on urine agar from typhoidal freces will show the typhoid bacillus within thirty hours.

The one disease that is most likely to cause confusion is tubercular meningitis. But in this disorder the slow, hard, and irregular pulse, the normal-sized spleen, retracted abdomen, persistent vomiting, coma, localized paralyses, absence of typhoid temperature, and Widal's reaction will make the matter clear. In other diseases there are plainly marked features that lead us aright: in malaria we see the peculiar temperature, the plasmodium in the blood, and the prompt reply to the administration of quinine. In pneumonia there are the rapid respiration and the physical signs in the lungs. In acute general miliary tuberculosis there is a plain involvement, usually of various parts of the body and commonly of the lungs, no eruption, no typhoid temperature or Widal's reaction, and often the tubercle bacilli appear in the sputum or the stools.

\section{Erysipelas}

This is an infectious inflammation caused by a specific microorganism, Streptococcus erysipelatis, that finds its way under the skin through small or large wounds. The micro-organism is medium-sized, occupying a mid-place in dimensions between the staphylococcus and the Streptococcus pyogenes. In former times it occurred in epidemics, and regularly in hospitals in infected operation wounds. At the present time it occurs in the new-born, in accidental wounds and abrasions of the skin, and mucous membranes of the face and body. It does not necessarily produce its 
characteristic eruption at the point of inoculation, and in some cases these two localities may be far separated. An attack evidently confers immunity for a short time, as Tehleisen has demonstrated; after this immunity has worn off, however, the patient may be repeatedly subject to new invasions.

Lesions. - The principal changes consist in inflammation and serous infiltration of the skin and subjacent connective tissue. In the lymphatic spaces and vessels of the corium the micro-organisms group themselves singly and in chains, clustering for the most part about the margin of the inflamed zone. As this zone clears up the streptococci die, and are replaced by varying amounts of leucocytes. The skin or mucous surface is congested, heavy, and filled with serum. In areas, on account of local lack of resistance or intense intoxication, the tissue breaks down and may become gangrenous. As in other infectious diseases, there may be an acute degeneration or inflammation of the abdominal and thoracic viscera, which in the presence of specific micro-organisms may take on the especial features that belong to their development. In this way we may find disorders of the nose and throat, lungs, kidneys, spleen, heart, peritonæum; there may also be inflammations of the meninges, or thromboses of the intercranial veins.

Symptoms. - According to Fehleisen's inoculation experiments the period of incubation varies from fifteen to sixty-one hours; clinically we speak in general terms of its being from one to three days. In the newly born the common locality of the disease is about the umbilicus, in children on the body and extremities, while adults have a larger percentage of involvements of the face. The invasion usually begins with headache, prostration, often a chill and vomiting. The temperature rises rapidly, usually attaining the maximum about four o'clock in the afternoon ; and at the same time the pulse rate keeps in equal step. The throat is often dry, hot, and congested. Simultaneously with these general symptoms the eruption appears on the skin or mucous membrane. Thus an area of inflammation - red, glistening, slightly raised, hard, fan-shaped, and sharply marked off - breaks out on the abdomen, the extremities, or the face. In the face the usual location is at the lower border of the nose, about the mouth, on the cheeks, or near the hairy scalp. The tendency of the inflammation is to spread irregularly in various directions; and as it-spreads, the previously affected part after a period of three, 
four, or five days, gradually returns to its normal condition and color. Thus, in one subject, various degrees of irruption may simultaneously be seen. As the main area loses its inflammation, the temperature falls, the whole skin surface loses its hotness and dryness, and may be bathed in sweat. At times, when one area is relieved, another, possibly at some distance, becomes involved with the natural accompaniment of recrudescence of fever and a return of the general symptoms. Such an attack has received the name of erysipelas ambulans in distinction from the first-described variety, where the eruption, creeping continuously from one part to its neighbor, is denominated erysipelas migrans. Occasionally the skin may have one or more blebs upon it, filled with clear or partially clear serum. At these localities, or near them, the skin may break down into a superficial gangrene.

In different patients the severity of the disease varies within wide limits. In the mild cases there is comparatively little prostration, pain, or after effects; in the severe attacks, however, the prostration is very marked, the intoxication very serious, showing itself in gastro-intestinal disorders, and serious cerebral symptoms, such as delirium, stupor, or mania. In children, even more than in adults, the spread of the disease to the throat is particularly to be feared. The mucous membrane throughout the mouth and throat may be dark red, much swollen, and infiltrated with serum, and here and there small blebs may be seen. These shortly break down, leaving a ragged, necrotic background. It is in the larynx that the greatest danger lies; for the tissues may become so much swollen that a dangerous laryngeal stenosis may rapidly spring up. When the mouth or throat is affected the connected glands are enlarged and tender.

A serious complicating feature is the occurrence of cellulitis. When this happens the danger of meningitis, pericarditis, pleuritis, and broncho-pneumonia is much increased, General visceral complications are not uncommon, and in well-marked cases some degree of acute renal degeneration is to be expected.

Treatment. - Prophylaxis in erysipelas is of the utmost importance; not only must the patient be rigorously isolated, but also must both nurses and physicians be careful not to act as carriers of the disease to other persons, sick or well. Neither nurse nor physician should, while attending the disease, treat a wound or 
conduct an obstetrical case. When convalescence sets in and the inflammation of the skin has subsided, the child may be removed to another apartment, and the sick-room must be thoroughly cleansed and disinfected. At the onset of the sickness the child should be put to bed, his bowels moved by small and repeated doses of calomel or a saline, and his food restricted to the simplest and most easily digestible articles. The body should be sponged daily, and graduated cold baths may be used for the control of temperature. Nursing children should either be taken from the breast or should suckle through a breast shield. The danger of infection must be clearly explained to the mother, as well as the best means of warding off the contagion by means of strict antisepsis. One of the main indications in the care of the patient is the necessary active stimulation with strychnine and alcoholics. These should be given liberally until all danger is past. The skin in mild cases may be covered with moist boric acid dressings or very weak carbolic vaseline spread on lint. Severer cases may be treated with moist corrosive sublimate dressings covered with rubber tissue. Various methods of treatment, looking to an attempt at limiting the spread of the disease, have been devised. For this purpose the affected area has been covered with a five or ten per cent icthyol ointment, or with iodoform collodion; or the skin outside of the inflammation has been painted with strong solutions of nitrate of silver, or with tincture of iodine; or the extra marginal tissue has been thoroughly scarified and covered with wet bichloride of mercury or carbolic acid dressings; in some cases hypodermatic injections of carbolic acid solutions have been made, but the method carries with it a certain amount of danger, especially for young children, and therefore should be used with great caution. The success of the attempts has not been fully demonstrated. Pain must be controlled by opium in older, and antipyrine or bromide of soda and chloral for young children.

Complications should be treated according to their several indications.

Prognosis. - Ordinarily an attack lasts for a week or less, but in erysipelas ambulans the disease may extend its course for two or more weeks. In strong children in whom the disease is not very severe the chances of recovery are very good. In nurslings the contrary is the fact; these patients seem particularly unable to stand the sepsis of the disease, and easily fall into collapse. 
Likewise, facial erysipelas is commonly worse in its prognosis than that of the body, and likewise gives severer symptoms. All in all, an equation must be made between the apparent virulence of the attack and the vitality of the patient.

The differential diagnosis is usually easy. If one keep in mind the peculiar eruption, the marked general symptoms, and the picture of intoxication, there is no other disease with which erysipelas can be confused.

\section{Tetanus}

Tetanus is an acute, infectious disease caused by the bacillus discovered in 1884 by Nicolaïer, and more fully investigated and described by Kitasato five years later. It occurs in children of all ages by inoculation; and on account of the large number of deaths due to it in the first few days of life, it was formerly included in the diseases of the new-born. But there is no especial reason for so classifying it, since the active cause is alike in all ages; nor is there any real difference in the symptoms between one time of life and another. It is doubtful whether the germ in its purity produces the characteristic manifestations as we commonly see them; on the contrary, there is good cause to believe that the presence of other pathogenic micro-organisms is necessary to its fruitful development. It occurs in the soil, dust, dirt, and has even been found in the atmosphere. 'The disease is by no means a frequent one, and is very rarely found in any but the environment of dirty and ignorant people, or in institutions.

There are no known characteristic lesions, although spinal hyperæmia has been found in a number of post-mortem examinations. Death seems to occur from the action of a poison or poisons which do not create organic changes. These poisons may be albuminous or non-albuminous. Martin believes that the main one is a proteolytic ferment, and that the albumoses are derived from its action. The various poisons so far obtained, such as tetanin, tetanotoxine, spasmodin, and spasmotoxine, are at present matters of further investigation.

The symptoms begin within an uncertain period after inoculation. The time, as usually stated, is a few hours or days. But experiments demonstrate that the bacillus requires several days to grow, and therefore to cause symptoms. Sometimes the effects show themselves only after the lapse of one or two weeks. Mus- 
cular rigidity is first noticed, especially when the patient is an infant and attempts to nurse. The spasms at first are transitory, but gradually they become severer and more frequent, until the intervals between them are comparatively few and small. When the disorder is at its height the jaws are set in a state of trismus, the back is hard and rigid, the arms and legs are extended, the thumbs turned in, and the hands closed. The face is set and has a pouting expression, the throat muscles are in such a spasm that deglutition and even breathing are difficult; in some cases the respiratory disability is increased by spasm of the diaphragm and the muscles of the thorax. The child is able to give no more than a faint, peevish cry, and rapidly becomes exhausted. The pulse is weak, the temperature irregular, the respiration shallow. At intervals he becomes bathed in a profuse sweat; and all through the disease there may be gastro-intestinal disorders that are very obstinate.

Treatment. - The most valuable factor is avoidance of infection by strict asepsis in the care of the new-born, and in older children of wounds and denuded surfaces. As soon as a diagnosis is made, the specific antitoxine should be injected in large quantities, and often enough to give relief. The drugs which were formerly employed are depressants and antispasmodics, such as chloral, bromides, and calabar bean. To them we may advantageously add antipyrine. All of them must be administered in large amounts and at frequent intervals. If the wound through which the inoculation took place is known and easily accessible, it should be freely opened, and thoroughly irrigated with a strong bichloride of mercury solution. Under all conditions absolute rest and quiet are essential. These are required not only for the child's comfort, but also for his safety. A comparatively slight amount of handling, motion, or even noise may be the immediate cause of a convulsion, or at any rate of a spastic condition that is not easily resolved. Stimulation should be liberal and unceasing.

On account of the spasm of jaws and throat, the child may be unable to drink; we can obviate this difficulty to some extent by passing a catheter through the nose into the stomach by which gavage may be practised.

Prognosis. - The disease occurs less frequently than in former times, and its mortality has been reduced. Some observers report a saving of about twenty-five per cent of the cases, in place of 
five per cent that formerly survived. The statistics are, however, unreliable on account of the comparatively small number of cases that come under our notice.

\section{Epidemic Cerebro-spinal Meningitis}

This is an acute infectious fever, which has been called spotted fever, epidemic meningitis, malignant purpuric fever, and cold plague. It attacks both human beings and animals in epidemics and sporadic cases. The epidemics have appeared more definitely or have been better recognized in the United States than in Europe; at all events we seem to meet it in America more widely than abroad.

Causes. - Although the causative micro-organism was described by Weichselbaum in 1887, the scientific world paid very little attention to his communication. Authors continued to speak of an unknown ætiology, or, doubting the specificity of the disease, mentioned it as an acute meningitis. In 1895 Jaeger, followed by Councilman and others, confirmed the findings of Weichselbaum, and in 1899 Osler, in his Cavendish Lecture, added still further proof that the disease was dependent upon infection by the Diplococcus intracellularis meningitidis. It is found in the cerebrospinal fluid, the blood, and sometimes in various parts of the body, and may be recognized on cover-slips and in cultures. It may occur alone, but frequently is associated with other microorganisms, such as streptococci and pneumococcus. In other cases although Diplococcus intracellularis alone was found in the meninges, the pneumococcus, staphylococci, streptococci, Bacillus coli commune, and Bacillus lactis ærogenes may be found in other situations.

Lesions. - The pia mater of the spinal cord and the convexity and base of the brain is the part directly affected. The tissue is congested, the vessels are dilated, and about them there is an infiltration of leucocytes. The pia mater of both brain and cord are infiltrated with a variable amount of serum, fibrin, and pus whose location is determined in part by the location of the affected area, and in part by the patient's position. The amount of the exudate varies within wide limits: it may be so small that the unassisted eye is unable to discover it, or, on the other hand, may be so large that it distends the ventricles. A similar fluid may be 
found in the central canal of the cord, in some cases being more or less deeply stained by small hæmorrhages. Such hæmorrhages may likewise take place in various parts of the pia mater and subjacent tissue. Long continued cases may present a lardaceous or amyloid degeneration of the pia mater. In some instances there may be purulent arthritides, multiple abscesses, hypostatic congestion of the lungs, parenchymatous degeneration of the kidneys, spleen, liver, and heart, subserous hæmorrhages of the endocar-

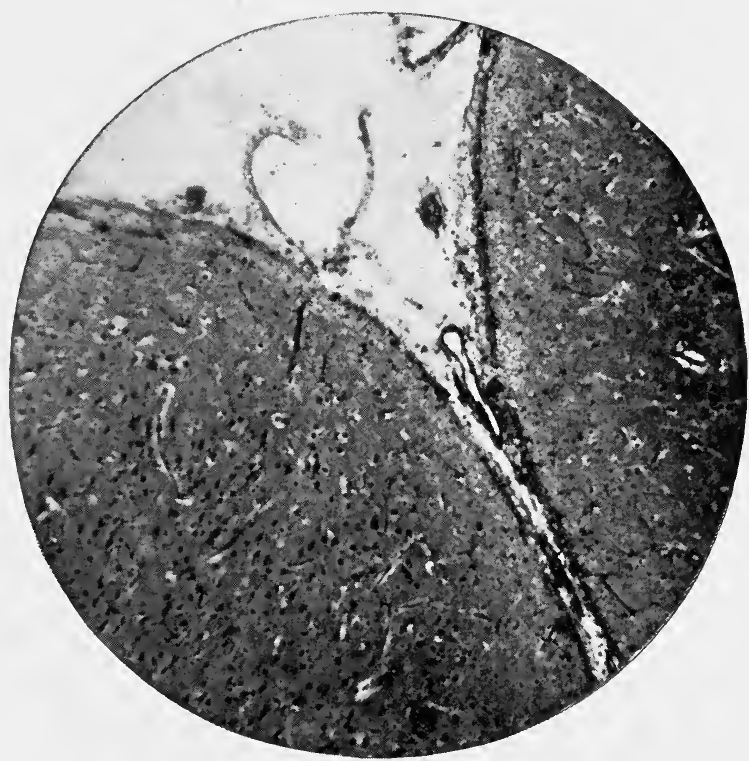

Fig. 95. - Normal Cerebral Meninges. $\times 60$.

dium, and hyperplasia of the intestinal lymph-nodes. A petechial eruption is one of the common features of the disease.

Symptoms. - As a rule the disease sets in suddenly, and very commonly with a chill. There may have been no prodromal signs at all, or the child may have complained of feeling depressed and weak. The first sign that is characteristic is a very intense sort of headache, located in any part of the head, which produces a prostrating effect. If the child tries to walk, he is dizzy and staggers about. From the head the pain extends to the neck and down the spine; on account of it the head may be held stiffly or be retracted. The fever sets in with a sudden impulse and may run high; the eyes are dull, the pupils small, and the irritability is 
marked. These symptoms may, in the mild form, constitute the whole picture; occasionally the abortive form occurs, in which there is no more than a beginning of the symptomatic course. These instances are somewhat rare, and are apt to be confused with cases of gastritis which are characterized by marked nervous and cerebral symptoms. In the severer cases the ordinary symptoms progress to a more radical development. In such an event, there will, in all likelihood, be vomiting; in some cases this is both violent and obstinate; the restlessness gives way to delirium

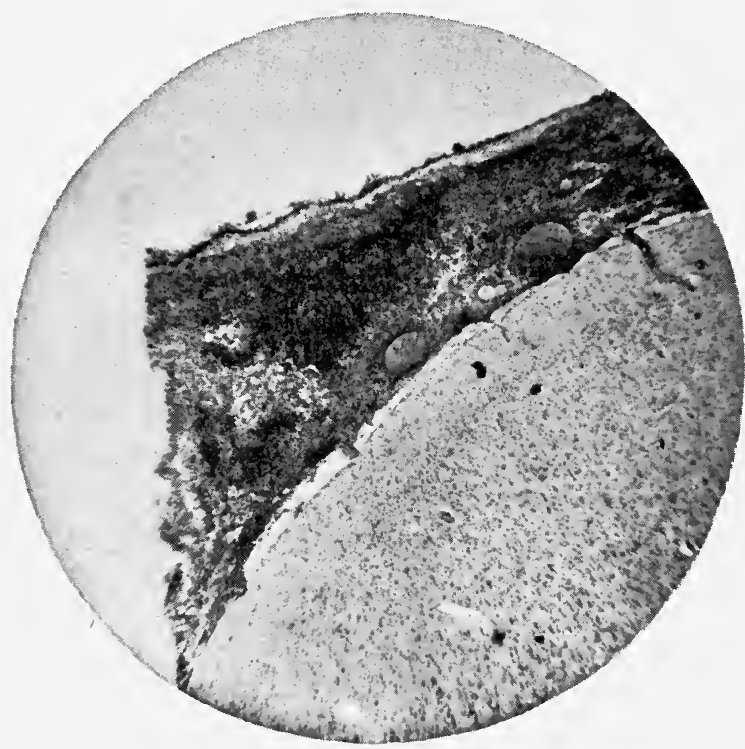

FIG. 96. - Epidemic Cerebro-spinal Meningitis. $\times 30$.

which may at any time change into coma. There may be a series of alternations between restlessness and delirium, delirium and mania, finally resolving into a comatose state; or the rigidity of the neck may change to opisthotonos varied by convulsions. The eyes are apt to have a dull and fixed expression, and if there is much effusion strabismus is common. The rapid development of such nervous symptoms is generally of grave import.

The pain in the head, coupled with the fairly constant photophobia, causes a puckering of the eyebrows and forehead, which often assumes the appearance of muscular spasm. It is frequently 
associated with twitching of the extremities. Kernig's sign may almost regularly be found, if it is carefully sought. To obtain this, the patient must be put in the sitting position, and one should try to extend the leg upon the thigh. One will then find that the flexors contract, and thus prevent the attempt to straighten the leg. Another method can be used while the patient is lying down: the thigh should be flexed upon the abdomen; if one then attempts to extend the leg, the flexors again contract, and leave the knee at a right angle. The tendon reflexes may be increased, decreased, or absent. Constipation is a fairly regular symptom, and occasionally it resists the action of really strong cathartics. If it is neglected, one may expect a gradual development of gastric and intestinal disorders. In some cases one of various rashes may appear: they may be petechiæ, herpes of the face and lips, erythema, urticaria, and less frequently eruptions that resemble those of measles, scarlet or enteric fever. In some severe cases there may be cutaneous hæmorrhages of small extent, which may occur on mucous membranes, and in the viscera as well as on the skin.

With these regular symptoms there may be complicating disorders of the lungs, heart, pericardium, stomach, intestines, liver, and kidneys. These inflammations vary in kind and degree according to the circumstances of each case. In addition there may be diseases of the eye, such as iritis, keratitis, and choroiditis, of the ear, and of the joints. After some exhausting attacks a chronic hydrocephalus may remain.

Treatment. - At the first symptoms, the patient should be put to bed in a quiet, dark, and well-ventilated room. The gastrointestinal canal should be emptied by means of small and repeated doses of calomel followed by a saline. Ice bags should be applied to the head, and, if possible, to the spine as well. It has been customary to prescribe ergot, with the idea of diminishing the cerebral hyperæmia; but at most the value of the drug is extremely doubtful. The pain, twitchings, and rigidity may be fairly well controlled by antipyrine in doses of 0.06 gramme ( 1 grain) for each year of age, every four, three, or two hours, in conjunction with a cardiac stimulant. This remedy will be found much more effective than the bromides, phenacetine, or the salicylates. Where it is insufficient, one may use opium to good advantage.

A comparatively recent procedure, that is destined to be widely 
used in this and other cerebro-spinal disorders, is Quincke's lumbar puncture. The operation, which has for the most part been used for diagnostic purposes, has been said to have therapeutic possibilities as well. If strict antiseptic precautions are taken, there is very little danger incurred in performing it. The puncture may be made between the third and fourth, or fourth and fifth lumbar vertebræ, and an amount of fluid may be removed which varies according to the pressure in the central canal; as much as sixty grammes or even more has been abstracted, with amelioration of fever and symptoms of pressure. Another surgical method, which will, as some writers believe, be used with advantage, is the removal of the spinous process of a lumbar vertebra, followed by a thorough irrigation and washing of the spinal canal. But such treatment would be so radical, would expose the patient to such great dangers, and necessarily presents so many objections, that its value is problematical.

In other respects the treatment is symptomatic, and the various complications call for their respective measures.

Prognosis. - It is hard to judge of the outcome of any case of cerebro-spinal meningitis, and the mortality in the various epidemics varies within wide limits. All that one can state is a few general observations: as a rule, the younger the child, the greater is the likelihood of death. The severe cases are most apt to die at the outset, the mild ones toward the end of the disease. Cases which begin with a brilliantly aggressive set of symptoms are more to be dreaded than those that start in a gradual fashion, and with a steady development of prodromata.

Differential Diagnosis. - The main points for diagnosis are the existence of an epidemic, the development of cerebral symptoms, and most important of all, the finding of the specific germ in the cerebro-spinal fluid. One must keep in mind that the repeated examination by both microscope and cover-slip may be necessary. Confusion with the acute exanthemata may be avoided by excluding their characteristic signs, At times it may be difficult to exclude pneumonia and typhoid fever, but here again not only the physical signs, but also a bacteriological examination, will decide the matter with certainty. Kernig's sign may be of considerable help in showing the existence of disease of the spinal meninges. There may be considerable difficulty in diagnosticating epidemic cerebro-spinal meningitis from tubercular meningitis ; in the former 
the attack is apt to be more acute, more startling in its manifestations, and more painful than in the latter. In addition, in tubercular meningitis one commonly may obtain a history of tuberculosis, tubercular involvement of other areas, and actual or recent physical deterioration from exhausting disease. In some cases the diagnosis can be made only after extended observation. 


\section{CHAP'TER XXI}

\section{THE SPECIFIC INFECTIOUS DISEASES (continued)}

\section{Tuberculosis}

Tuberculosis is an infective fever characterized by a specific ætiology, commonly a sub-acute or chronic course, the production of toxines whose results are an irregular febrile movement, and the formation of new and easily degenerating tissue called tubercle which may become so much further weakened as to deserve the name caseous. The disease may attack any part of the body, although some are frequently and others rarely involved. Wherever the characteristic changes occur, there one usually is able to find the specific micro-organism, the bacillus of Koch (1882).

The disease may occur at any age, and has even been found in the fœtus. Nevertheless, one may not say that it is hereditary. Tuberculosis of the placenta is very apt to result in infection of the unborn child, since the physiology of the circulation makes immunity improbable or impossible. On the other hand, a localized tubercular inflammation in any other part of the body does not by any means necessarily result in specific contamination of the fœtus, so that finally one must put the heredity of this disease in the same class as that of various acute infectious diseases, such as measles and scarlet fever, which may occur in intra-uterine life, but which are certainly not hereditary. 'The idea of heredity involves a change in germ-plasm according to some definite character in the body, and the inevitable transmission to the offspring of this changed germ-plasm and definite character. In tuberculosis this does not necessarily result. It is not at all hard to find cases of tuberculous parents whose children, especially if they were removed from the parental environments, developed into the ordinary degree of health and strength. And on the other hand every one is familiar with instances of children, whose heredity is clear, who under circumstances of poor local or general nutrition and in unfavorable surroundings quickly succumbed to the disease. The most that 
one may say in regard to the transmissibility of tuberculosis is that its presence so vitiates the patient's power of resistance, faculty of assimilation, and potentiality of development that his body becomes debased below its normal standard, and that his possibility of procreating fully vigorous offspring is thereby reduced. In addition, this weakness in his progeny seems to take the form of an especial susceptibility to attacks of the bacillus of tuberculosis. Farther than this one caunot logically go. Nor indeed may this accessibility be derived only from such a specific heredity; on the contrary, this susceptibility to invasion follows almost any condition of depression, such as what often follows measles, epidemic influenza, whooping-cough, enteric fever, and pyogenic processes. In other cases a similar result may be brought about by unwise or unfavorable methods of life, such as improper food, improper clothes, exercise, and general environmental conditions. This receptivity may be not only general but also local, for the same principle of lessened resistance is present in both. Thus an injured joint, a diseased lung, an overburdened and exhausted lymphatic system are all capable of acting as the starting-point for a tubercular process whose activity in spreading depends again upon the degree of vitality in the related parts. This idea of deficient resistance is at the base of processes attached to tubercular inflammations, but different from them. In this way other micro-organisms, such as streptococci, may be found in an active connection with the tubercle bacillus, and in all likelihood the relation in time between them is merely fortuitious, so that either may precede or follow the other. At any rate, a tubercular inflammation complicated by the action of additional micro-organisms must naturally give severer symptoms than otherwise would be felt.

The location of the primary tubercular lesion depends upon local conditions, although not all parts of the body are equally open to it. Wounds and abrasions of the skin, which one would suppose to be frequently affected, are very rarely involved. Occasionally in hospital practice such an inoculation is seen in circumcisions performed by a tuberculous mohel; but it should be regarded as something of a curiosity. The commonest method is by way of an injured, irritated, or inflamed mucous membrane. The converse idea is likewise true: that normal mucous membranes are one of the best defences against the disease. This membrane throughout the respiratory track is the favorite site of a beginning lesion, 
partly because of the ease of infection through the germ-laden inspired air, partly on account of the mucus in which the bacilli may thrive, and partly because there is no distinctly acid secretion or other sufficient agent to stop their growth. The frequency with which disorders of the respiratory track occur is another favorable condition for their flourishing, and in this light, attacks of rhinitis, hypertrophy of the pharyngeal tonsil, and inflammations of the throat and bronchi assume a greater importance than their immediate action would otherwise warrant. Poor ventilation, impure air, and a lack of cleanliness of the nose and mouth have a certain share in inviting these attacks.

The mucous membrane and glands of the gastro-intestinal track are less subject to invasion; especially is the stomach rarely involved, doubtless on account of the acid character of its secretions, although milk which forms so large a part of the child's dietary is an excellent vehicle for carrying the tubercular bacillus. The intestines are affected for the most part by bacilli in the food, or by extension of the disease through the lymphatic channels. In this region one may at times find the phenomenon of a transmigration of the bacilli through a normal mucous membrane into the mesenteric glands, thus producing the condition called tabes mesenterica. In all likelihood a similar process is responsible for some cases of lupus of the face, where the microorganisms pass through the mucous membrane of the mouth and nose, and finally produce the characteristic lesions in the skin.

The main highway along which the infection passes is the chains of lymphatic glands; and these are involved in proportion to the frequency of infection in the parts with which they are connected. This is the reason why the tubercular adenitis in a child has such an important bearing upon our prognosis of his disease, excepting in those cases where the process is plainly confined to the glands alone. Of the superficial glands, those of the neck are the oftenest involved; with much less frequency those of the axilla and the groin are implicated. Of the deeper glands, those about the trachea and bronchi are the ones commonly enlarged, although clinically we are able to recognize their existence only when they give rise to pressure effects. All these various phases of adenitis are more commonly encountered in young children than in adults.

Lesions. - The changes which occur in tuberculosis are the de- 
struction of fixed tissue, the production of miliary tubercles, of diffuse inflammation, caseation, and calcification of the products of inflammation. When other pathogenic bacteria are present, the ordinary degenerative processes attending their activity may be expected. Tubercle tissue may occur in small, miliary forms which remain discrete, or coalesce into larger masses. It consists of gray granulation tissue, with small or giant cells held in a fine network of basement substance. Around the affected area a large number of leucocytes cluster, which are most plentiful where the specific lesions follow the infliction of an injury. The tubercle tissue is poorly supplied with vessels, and as separate areas unite, a correspondingly large section becomes cut off from the circulation. This weak and vicious tissue is then said to become cheesy, and finally as the last step in this retrogressive action breaks down. At and beyond the margin a variable amount of fibrin-infiltration may take place; and as it tends to limit the spread of the local lesions, it really is a step in the direction of health. In other cases, where the disease is decidedly chronic in its course, the bacilli become coated with a material which becomes infiltrated with calcareous matter composed in the main of calcium phosphate. This process of calcification may spread until it replaces the tubercular tissue, kills off the bacilli, and puts an end to the disease. While these phenomena are characteristic of the disease, nevertheless the various parts of the body have individual peculiarities of involvement that are important enough to be known separately. In children the lungs, after the glands, bones, and joints, are the parts most frequently diseased, both primarily and secondarily, and the younger the child the more likely are they to be infected. The disease attacks by preference the upper rather than the lower parts of the lungs. After them, the brain and the meninges are the parts which are implicated in the next degree of frequency, while tuberculosis of other portions of the body is considerably more rare.

In the lungs the disease may occur as part of a general tuberculosis or as a distinct localization. In the first division the lungs, as well as the other viscera, are the seat of a miliary infection. These deposits may be found in large or small quantities, although in children the tendency is more in the direction of large masses than in adults; also a part or the whole of the lungs may be involved. The miliary tubercles may occur in various forms and locations: 
the walls of an air-space may be infiltrated and the cavity filled with tubercle or fibrin and pus; or the tubercle tissue may entirely replace the lung tissue; or the tubercles may infiltrate the walls of the small bronchioles on one or more sides and for a variable extent. The inflammation may extend to the near-by airspaces, the walls of which may be infiltrated and the cavities filled with fibrin and pus; or large masses may occur, formed by the infiltration of the walls of larger bronchioles, each of which has its surrounding area of infiltrated air-vesicles.

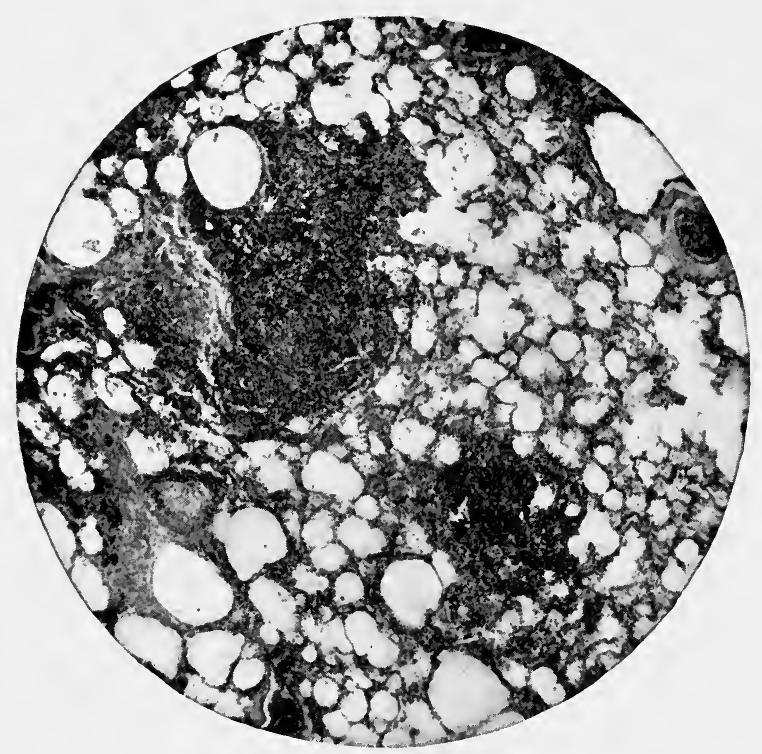

FIG. 97. - Acute Miliary Tuberculosis of the Lung. $\times 30$.

Instead of the acute course, the lesions may assume a sub-acute type. The disease is generally localized in the upper lobes, from which it may or may not extend. The walls of the small bronchioles and the adjacent air-spaces may be infiltrated, and in the surrounding area there may be the ordinary lesions of simple inflammation of the bronchi. In some cases the process may become chronic: then the tubercle tissue is harder than in the preliminary forms, and continues so until it breaks down into caseation. Its location is much the same as in the sub-acute course, although the duration of the process permits of indefinitely great extensions through the lungs or the rest of the body. Thus the 
pleura, the larynx, the glands of the mediastinum and mesentery, may become involved to any extent. In connection with this there is commonly a chronic inflammation of the bronchi and the lung tissue, with the accompaniment of conditions that characterize long continued pulmonary changes, such as the formation of connective tissue, emphysema, and bronchiectasis.

Local tubercular disease (acute or chronic pulmonary tuberculosis) is one of the commonest forms that the disorder assumes in children. In the acute form, besides the tubercular inflamma-

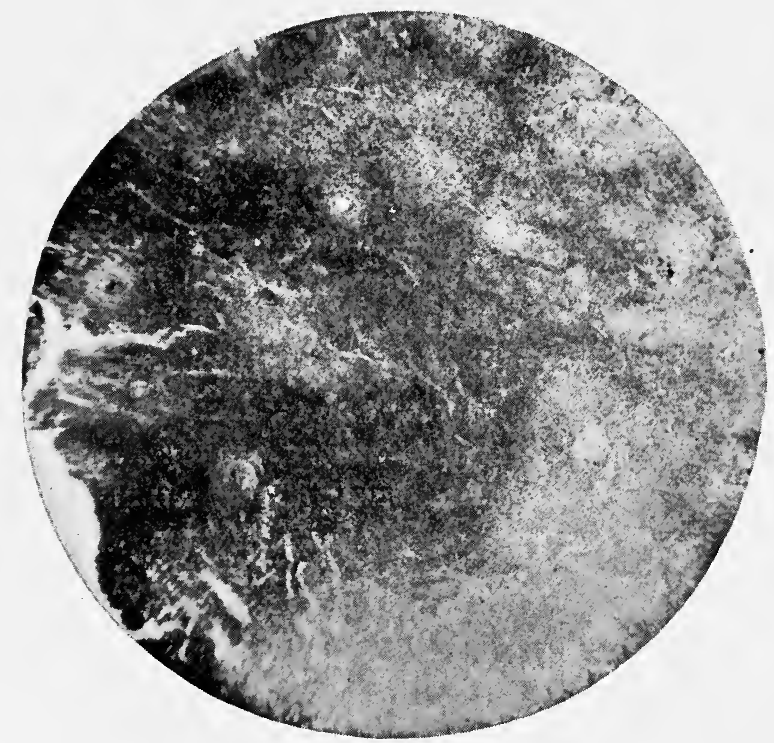

Fig. 98. - Chronic Tubercular Pneumonia with Cheesy Degeneration. $\times 30$.

tion, there are the regular changes of an ordinary broncho-pneumonia in one or more spots or lobes. This inflammation may be irregular in its distribution, the bronchitis being especially marked. In other cases there is besides the tubercular lesions a distinct pneumonia characterized by diffuse consolidation, with the ordinary phenomena of fibrin, pus, and broken-down epithelium filling the air-spaces. Where the pyogenic processes are far advanced, there may be areas of coagulation necrosis in all degrees of degeneration. In addition the pulmonary tissues may fall into the same pathological complications and sequels that attend a non-tubercular inflammation of them. For these conditions must be regarded as mixed 
infections in which the tubercular elements may develop side by side and with the several peculiarities that belong to each.

An acute pulmonary tuberculosis may gradually take on a chronic form, or the inflammation may from the first be of a chronic nature. The essential character of the disease is that of a tubercular broncho-pneumonia with choking up of the air-spaces by means of degenerated epithelium, or fibrin and pus, which have a tendency to caseous degeneration; in some cases there is a marked disposition to the formation of connective tissue in the

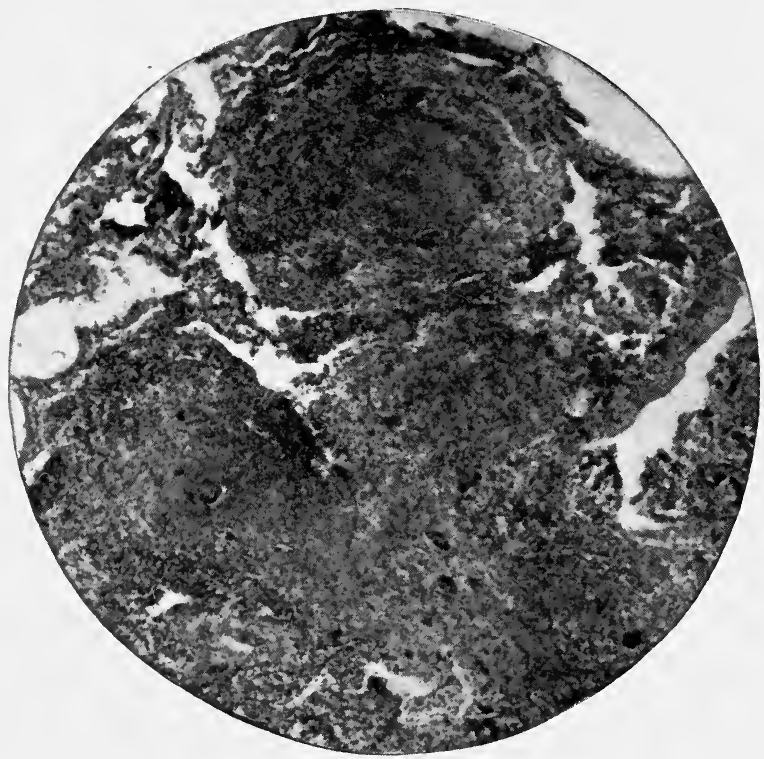

Fig. 99. - Chronic Pulmonary Tuberculosis with Conservative Fibrosis. $\times 30$.

air-vesicles, in their walls, in the bronchioles, and the septa. The sclerotic process is irregular, and consequently gives rise to various deformities. In the bronchi, in conjunction with the chronic catarrhal inflammation, there may be a cellular infiltration that weakens the bronchial walls, and thus causes dilatation; the walls may be riddled with tubercle tissue that inclines to caseation. As a result of the two processes cavities of various sizes may be formed.

The pleura is not often affected primarily, but merely as an extension of the pulmonary lesions. The first changes are apt to be a miliary tuberculosis on the pulmonary aspect of the mem- 
brane, followed by the development of adhesions and the deposition of connective tissue. Fluid may then be formed in the cavities, which by contamination with tubercle bacilli, streptococci, or staphylococci, becomes purulent.

The glands are among the first parts to show the effects of infection, not necessarily because the bacilli have a preference for them, but rather on account of their function of filtering out waste and foreign matter. Usually the tissues with which they are connected receive the infection, and then pass it on to them;

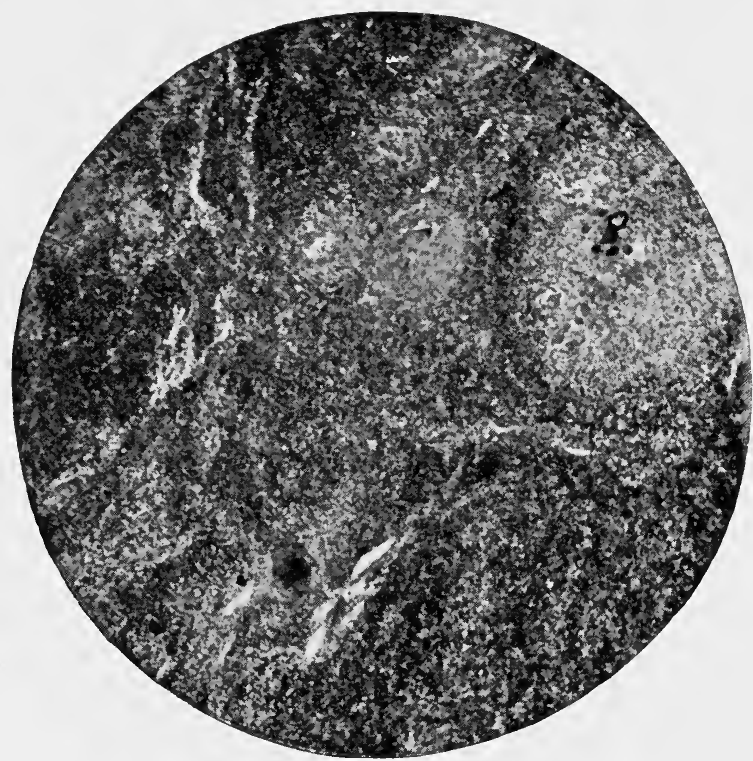

Fig. 100. - Tuberculosis of Lymph-Node. $\times 30$.

it is possible, however, for the bacillus to pass through uninjured mucous membrane, and find a lodgement in adjacent glandular structure. As an instance of the first process one may mention the adenitis in the neck following invasion through the pharynx, or about the roots of carious teeth, or the mediastinal adenitis following pulmonary infection; an example of the second process, as noticed above, is occasionally seen in the mesenteric glands, or in the occurrence of some cases of lupus on the face and about or near the nose.

The cervical glands are the ones most commonly involved; 
those about the trachea and bronchi are not so often plainly implicated through marked symptoms, but nevertheless pulmonary lesions regularly affect them, if only slightly. Many times they alone are attacked and remain the only seat of infection, even though they give no symptoms. Among the last named structures are the anterior and posterior mediastinal, the cardiac, the intercostal, and sternal groups. The first influence causes them to enlarge, and small miliary spots or areas appear which tend to unite, forming the location of caseation. This latter process

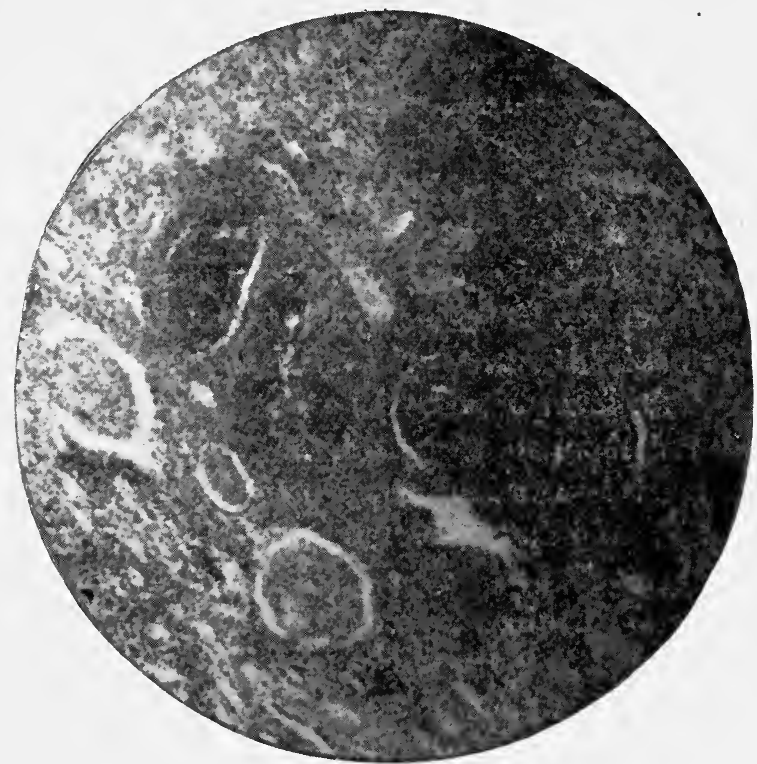

FIG. 101. - Tuberculosis of the Kidney. $\times 115$.

may be slow or rapid. In the first case the individual glands are small, although the union of many of them may produce a large mass; their capsules are thickened, hard, and fibrous, and do not readily become attached to the surrounding tissue or the skin. In some cases fibrosis may extend so far as to change the whole structure of the gland, with the attendant result of decreasing the number of bacilli present. In other cases the cheesy degeneration may undergo a process of calcification that is essentially conservative. Where the pathological changes are more rapid, as is common, they spread from the centre of the gland in 
all directions; all the surrounding and adjacent tissues are influenced accordingly, and consequently become matted together. The skin in turn may finally become involved, and at the point of attachment the subsequent abscess will break.

In the bones similar processes occur, having their location in and near the epiphyses of the long bones and in the centre of the short bones. The cancellous tissue is the part first to be affected, and the process goes through the stages of congestion, cell-infiltration, and caseation; frequently the new and debased tissue breaks

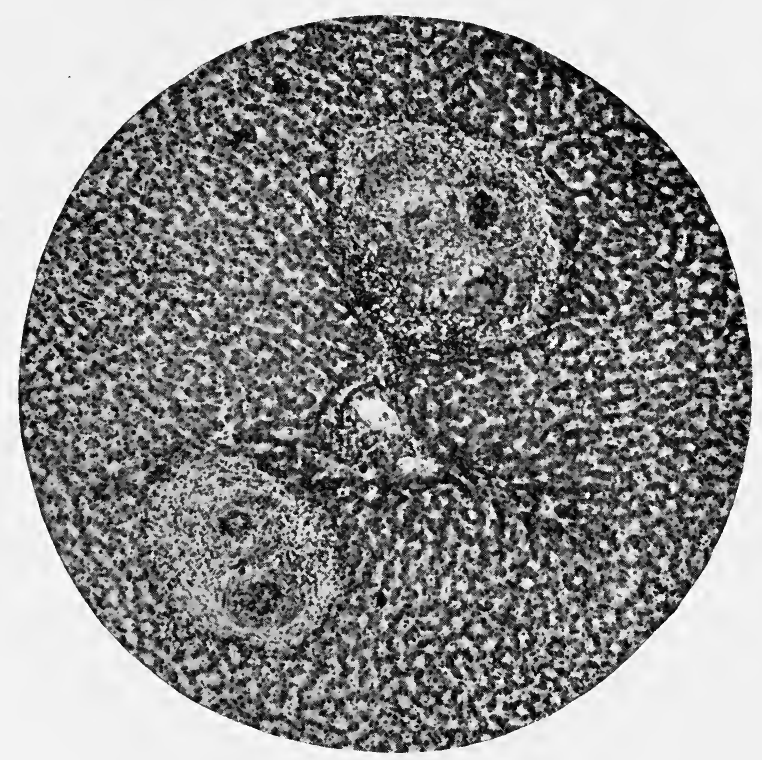

FIG. 102. - Miliary Tuberculosis of Liver. $\times 60$.

down, and is followed by suppuration. This process may stop here and encapsulation of the broken-down tissue may ensue, or it may go on and involve both bone and joint. In this way complete destruction of the part may take place, until the abscess finds an outlet either spontaneously or by operation. In either case, if the patient's vitality is good enough, a conservative and reparative growth of bone begins, which may to a fair extent replace the destroyed parts by new osseous tissue even in the joint. Anchylosis naturally results.

The kidneys are not often affected in childhood; but when infection does occur, it may be in the form of miliary tubercles, or 
larger cheesy areas. Either form may be multiple, and the separate patches may coalesce so that large masses of the organs become changed. Where the inflammation has not extended to a marked extent, the lesions may be shut off by a gradual fibrosis and calcification. This result may occur in tubercular disease of the suprarenal capsules, as was demonstrated to me in the autopsy on a boy of nine years. The whole organ, including the capsule, was shrunken and somewhat irregular in form. There were small areas of fibrous degeneration, and evidences of tubercular infiltration.

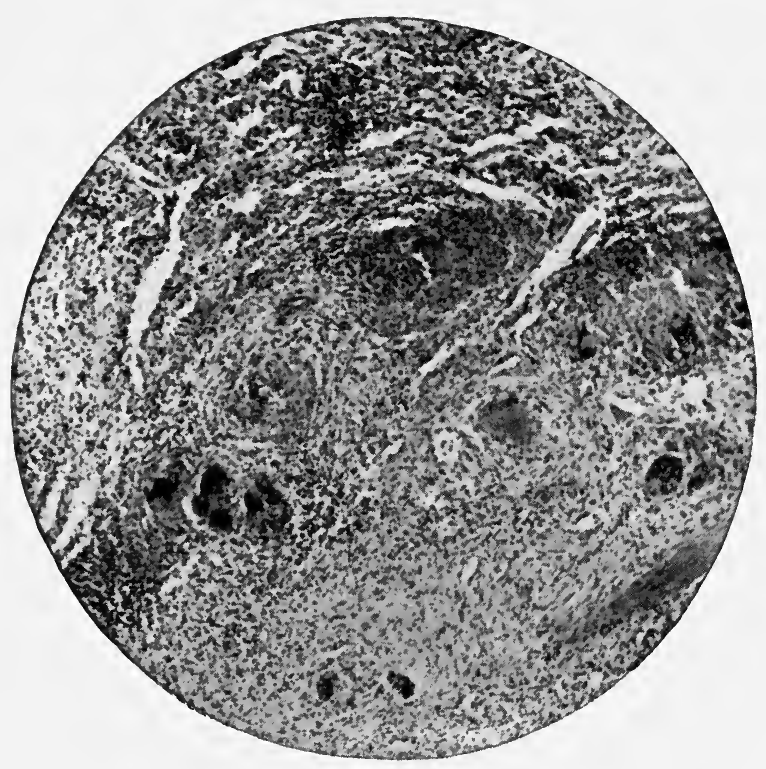

Fig. 103. - Acute Miliary 'Tuberculosis of Spleen. $\times 60$.

In the liver and spleen the infection occurs in miliary granulations. It is only in unusual cases that fairly large caseous areas are seen, although in advanced cases several miliary tubercles may coalesce, and thus form a larger growth. The disease appears in these organs as the result of extension rather than primary location. The size of the viscera may be increased, if there is a mixed infection with the production of pus, or if there is sufficient development of tubercles to increase by their bulk the ordinary dimensions. Such growth may oftener be looked for in children than in adults. In chronic cases amyloid degeneration of both organs may be expected. 
The stomach is involved in the rarest cases only, on account, as stated before, of the acid reaction of its secretions. When such involvement does occur, it is in the last stages of a practically universal infection. The intestines, on the other hand, are much oftener contaminated; here, again, the infection is generally secondary to that of the lungs or brain. The lesions are oftenest located in the small intestine, but in cases of very general distribution they may be in the large as well as the small gut. The disease usually begins with the formation of miliary nodules, which coalesce and

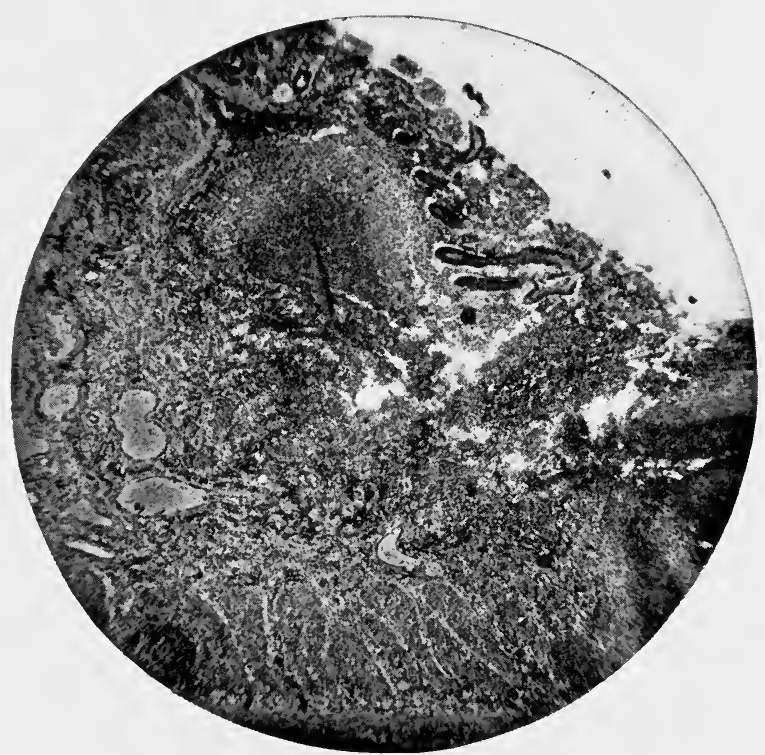

Fig. 104. - Tubercular Ulcer of Ileum. $\times 30$.

so form a much larger one. This finally becomes caseous, breaks down, and forms a superficial ulcer which extends around a part or even the whole circumference of the intestine. Its surface is rough, its edges are undermined, its base is infiltrated. It may be of any depth, and so involve only the mucous coat, or the others as well, even the serous. In such cases perforation may result; but it does not necessarily predicate the development of a general peritonitis, since the ulcer in its formation gives rise to a certain amount of plastic inflammation, which mats together adjacent parts and so prevents infection of the peritoneal cavity. The connected mesen- 
teric glands are always affected, and the amount of disorder is proportional to the extent and severity of the intestinal lesions. They undergo the same changes described above in the account of other glands throughout the body. Like them, also, they may become subject to fibrous or calcareous degeneration, or finally break down into a purulent condition. Extreme enlargement may cause pressure symptoms; but this is rarely seen.

The spread of the disease from the intestines to the peritonæum is always possible, especially in older children. In infants, tuber-

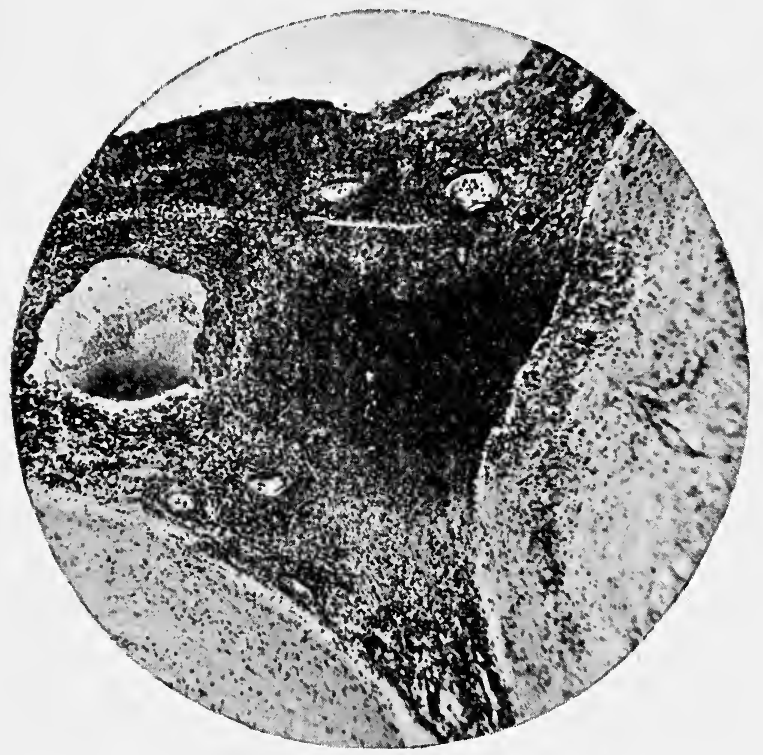

Fig. 105. - Tubercular Meningitis. $\times 60$.

cular peritonitis is not frequent by any means, nor is it common to see a primary tubercular peritonitis at any age. When the disorder does occur, it resembles the process in the gut. It usually begins as miliary granulations, which as they increase in number and size may coalesce and form larger masses. There may then be a cheesy softening, which on the one hand may be cut off and repaired by a more or less active fibrosis, or on the other may soften and break down into ulcers whose characteristics strongly resemble those of the intestines. As in other serous cavities, there may be large quantities of serum, with or without the addition of pus. 
In the brain tuberculosis occurs fairly often in children, especially between the ages of two and eight years. There are miliary granulations and small nodules which rapidly become soft and caseous. Around each is a small area of encephalitis; the older ones, however, may have a zone of fibrous tissue about them. These bodies may occur in any part of the brain, but in children the favorite seat is at the base. In the meninges the pia mater may be studded with miliary nodules, as in the brain. They may be seen strung out along the vessels which enter the brain, especially those which

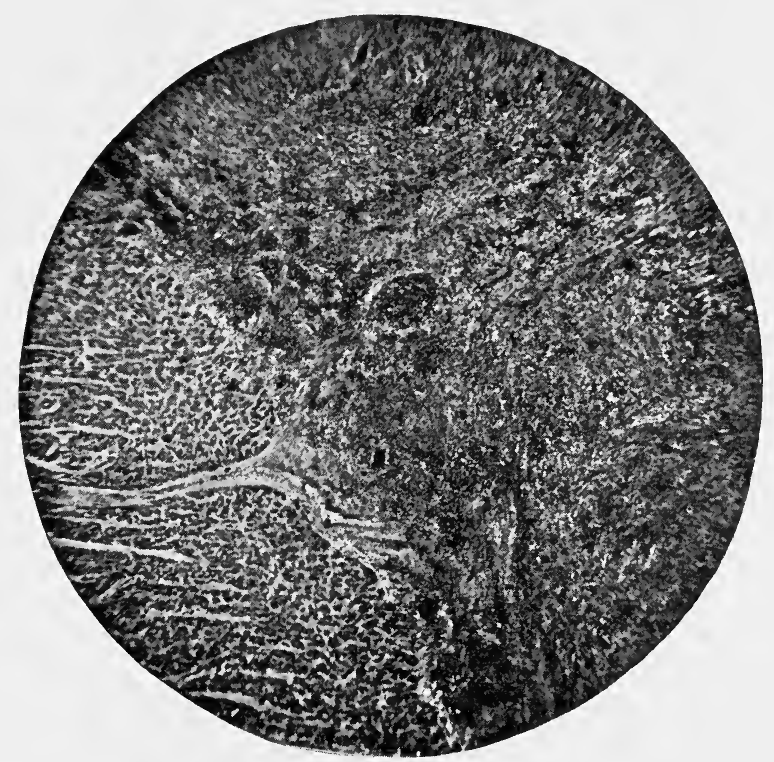

Frg. 106. - Tubercular Pericarditis. $\times 30$.

lead into the fissure of Sylvius. Likewise one may see them in the interpeduncular space, and thence in the membrane of the transverse fissure. The lateral ventricles are generally filled with serum, or serum and pus, the quantity of which increases with the duration of the sickness. The pressure may be great enough to weaken and flatten the ventricular walls, and in infants to make the fontanelles project beyond their usual plane. These infections of the brain and meninges, and among them may be included similar disorders of the cord, occur as extensions from tuberculous disease of the lungs, bones, joints, or intestines.

In cases where the lesions are widely disseminated other organs, 
such as the heart, pericardium, the choroid of the eye, the testicle, the Fallopian tubes, ovaries, and uterus may become involved. The conditions are not only rare, but have nothing peculiar to children in them. Even in the skin, where a chronic tuberculosis in the form of lupus sometimes affects children, there are no new factors outside of tissue necrosis and superficial ulceration. A noteworthy feature is the lack of fibrosis and calcification, excepting the amount of fibrous tissue which is produced in the scars.

Symptoms. - Tuberculosis has an incubation period, as we know from inoculation experiments; but in actual practice we are unable to fix and define it. The disease has certain peculiarities that mark it off from the other infectious fevers, such as its common tendency to chronicity, and its faculty of producing organic lesions of a peculiar kind in any part or organ of the body. The temperature is atypical, and varies with the severity and location of the infection; as a rule, however, the fever is not very great, and may at times be so slight as to escape notice. The disease is characterized by progressive loss of flesh and strength that is most marked in the last stages, by night sweats that begin toward the end of the night when the temperature falls, by anæmia, intestinal disorders, and occasionally by amyloid degeneration of the liver, spleen, and intestines. In the acute general infection, often seen in infants, the disease appears in its purest type. Every organ may be involved, and nevertheless no one organ may give predominant symptoms. The cough is often not impressive, the gastro-intestinal disorders may be transitory, the spleen may not be enlarged, or at most it is only slightly enlarged, and the cerebral symptoms are commonly of secondary importance. At the same time there is a marked and rapid loss of health which has a wide range of objective symptoms. These vary considerably as one organ or another feels the attack most acutely. Lack of appetite, vomiting, and constipation or diarrhœa are common, even if not impressive. In most cases the child is unable to digest and assimilate his food, and in spite of the most careful regulation and modification of his diet his condition does not improve. He is apt to be peevish and fretful, his sleep is disturbed, and as day after day passes by he looks more and more emaciated. Gradually a general adenitis may become evident, in some cases causing pressure symptoms. There may be sudden exacerbations of local disabilities, which progressively become 
more serious. Every organ may be attacked, even before the symptoms indicate the invasion. The whole course of the disease represents a steady and comparatively rapid deterioration of the body and its functions; and day after day the patient becomes weaker, sicker, and less capable of efficient, physiological activity. An acute course gives the most plainly marked signs, doubtless on account of the rapidity of absorbing the toxic products of the disease; a chronic course may give comparatively fewer signs, because the lesions are very gradual in their development and finally become shut in by the processes of fibrosis and calcification so that absorption is prevented.

Such an acute course characterizes the miliary tuberculosis of infants; and at times one may have difficulty in distinguishing it from marasmus, or any other wasting disease. Especially may it be hard to classify the symptoms properly if an added infection or an added disorder occurs. Under such circumstances one may need the observation of a few days to eliminate conflicting factors from the picture. The same idea occurs again in older children in whom, on account of their greater vitality, the disease takes a longer time to cause death. Here the long drawn out malaise, the increasing weakness, the possibility of an enlarged spleen, the possible disturbances of the intestines, and finally the supervention of a typhoidal condition, all go to suggest enteric fever.

When tuberculosis becomes localized in the lungs we have a combination of the general features of the disease plus the special form of the pulmonary disorder. Thus, a child who has tuberculosis in any other part or parts of the body, who has been suffering with other pulmonary or exhausting complaints, easily falls into a tubercular broncho-pneumonia, which nevertheless is not markedly different in its main signs from the simple bronchopneumonia. The length of the sickness is a point of some diagnostic importance, since instead of the ordinary recovery after a few days, the child continues very sick for two, three, or four weeks, or even more. Whether the case is rapid or protracted makes no great difference in the general symptoms. The temperature may not be as high as in the simple form, but as the disease progresses and the patient's strength fails, its variations become more marked. In the morning it may be near normal or subnormal, in the evening it may mount as high as $40^{\circ} \mathrm{C}$. (104 ${ }^{\circ} \mathrm{F}$.). Toward morning, when the fever abates, a cool, clammy sweat breaks out 
on the patient's chest, legs, or over the whole body. His appetite is poor, his power of assimilation defective, the loss of strength and flesh progressive. The cough cannot be said to have distinctive characteristics nor is the localization of the disease, which is often in the upper lobes and apices, regular enough to be of use in defining the disease. The physical signs are those of the ordinary broncho-pneumonia, but there may be more dyspnœa and

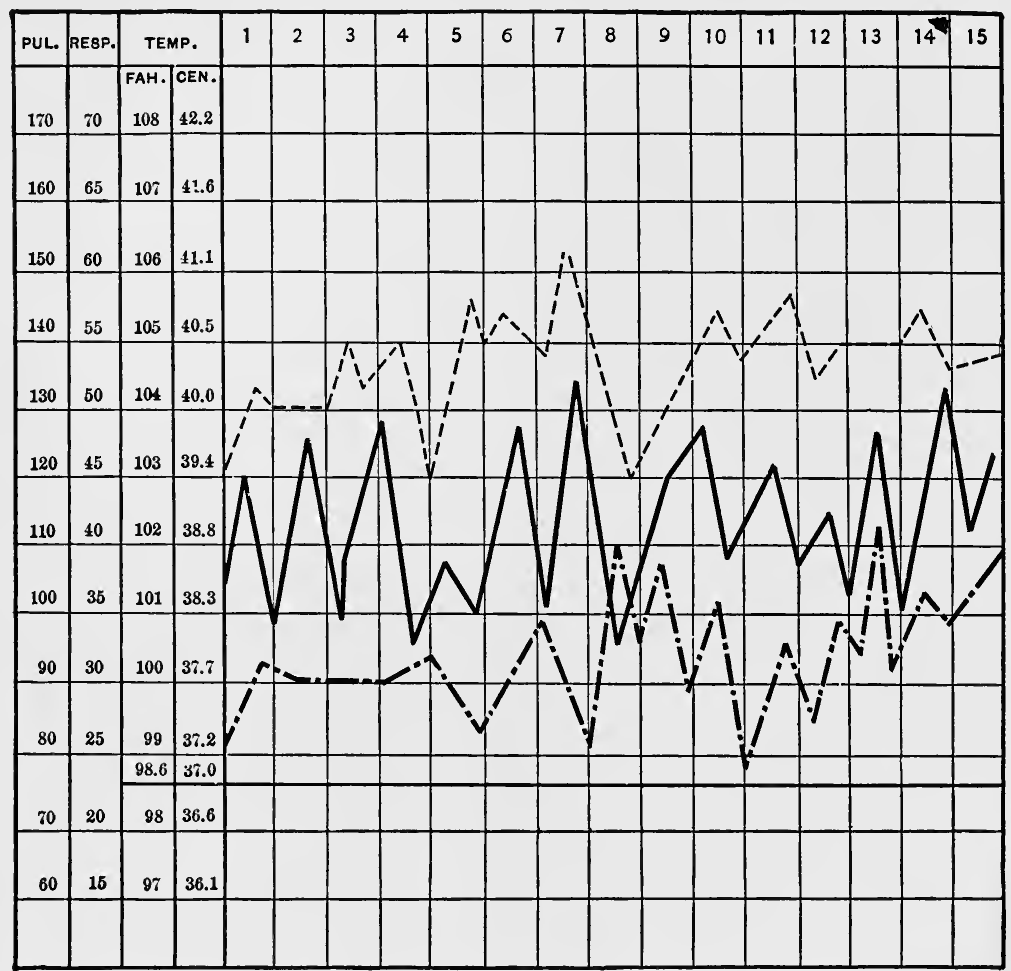

PULSE, RESPIRATION AND TEMPERATURE CHART OF GENERAL. MILIARY TUBERCULOSIS AGE, 7 YEARS.

PULBE

RESPIRATION

TEMPERATURE

FIg. 107.

cyanosis than one sees in non-tubercular complaints. In very rapid cases symptoms of breaking down of caseous areas may possibly appear, but they are not regularly to be expected; and it is yet more exceptional to find hæmoptysis. The main reliance in diagnosis rests on the general aspect of the case, its history, its 
development and course, the involvement of other parts of the body, such as the glands, joints, brain, and intestines. The rapid cases are apt to be the most obscure; the prolonged cases, on the other hand, involve such large areas of lung tissue, and in all likelihood bring out so many signs in the body, that any doubt is before long dispelled.

A chronic pulmonary tuberculosis is not often seen in very young children - largely because they have not the vital resistance to allow the process to become chronic. Whether the disease begins in a slow, chronic fashion, or is merely an extension of an acute or sub-acute attack, makes very little difference in the ultimate result. In the first case there may be a greater amount of interstitial tissues in the lungs than in the second; but the distinction is a pathological rather than a clinical one. Every part of the lung may be involved in the tubercular process, the caseous nodules may break down, and cavities of various size result. These commonly have a central location, doubtless because the extension of the disease takes place so often through the bronchial and peritracheal glands at the base of the lungs. This situation of the lesions may postpone an exact diagnosis for some time; the patient will have repeated attacks of acute or sub-acute pulmonary disorders, which by their frequency and continued course help to show the tubercular element. The general picture is that of a lung which steadily becomes more and more diseased, passing through successive stages of bronchitis, broncho-pneumonia, and abscess formation. The usual course is a succession of acute exacerbations each of which, although followed by a period of improvement, leaves the lungs in a poorer condition than before it began. As the disease progresses, the patient becomes weaker and more emaciated, the red blood cells and hæmoglobin are decreased, there is a marked leucocytosis, his appetite is poor, night sweats increase, and concomitant disorders - tubercular or non-tubercular - are very apt to appear. Especially are derangements of the gastrointestinal track likely to show themselves, bringing with them an exaggeration of the child's weakness. The temperature varies according to the changing conditions and complications, the pulse likewise varies but has a general tendency to thinness and weakness, the respiration is rapid and shallow, and as the disease becomes worse there may be dyspnœa and some cyanosis. The sputum in the older children - for very young children do not 
expectorate - is not abundant and does not become purulent until the late stages. On coughing or straining, the patient may complain of shooting and starting pains in the sides which are in part due to exacerbations of pleurisy.

The physical signs are often unsatisfactory; and while in many respects they resemble those of the adult, nevertheless in others they are deceiving. Some advanced cases show little disturbances in respiration, and the respiratory movement may be less marked on the side which is the more affected than on the other. Consolidated tissue gives a dull but rarely a flat percussion sound. The signs of cavity formation are especially deceptive; where a cavity is present we may, possibly on account of its small size and central location, not find the ordinary diagnostic symptoms; while on the other hand the most deceiving indications of cavity may be distinguished over a comparatively healthy part near a superficial bronchus or bronchiole.

Tubercular pleuritis gives no special symptoms by which one can readily distinguish it from the non-tubercular. Only in a small minority of cases is it empyæmic, unless it occurs as a mixed infection. It may, however, give signs of an irregularly thickened pleura and of few or many adhesions. A pleuritis occurring in a tuberculous patient is commonly regarded as tubercular.

The bronchial and peritracheal glands are frequently affected in children, occasionally as a primary disorder, but commoniy in conjunction with pulmonary tuberculosis. One may take for granted that a case of this lung disease involves changes in the associated lymph-nodes, even though no symptoms may appear. The younger the child, the easier does this involvement occur. When symptoms appear, they are in the form of pressure effects which may arise from one part or another according to the location of the enlarged glands. Thus the recurrent laryngeal or pneumogastric nerves give hoarseness, dyspnœa, and a dry, hacking, persistent cough in repeated attacks. When the trachea or a bronchus is affected, the attacks of dyspnœa come intermittently, the head is thrown back in a tonic spasm, and the effort to accomplish inspiration is at times pitiably great. In other instances the superior vena cava, the pulmonary artery, or the aorta may be impinged upon, giving symptoms of cyanosis, dyspnœa, and persistent hacking cough. It is also possible for ulceration to take place from these glands into the bronchi, trachea, œsophagus, the 
thoracic duct, the vessels mentioned, and the epicardium. When the bronchi or trachea are perforated death usually occurs, although rarely the pus may be coughed up and the patient thereby relieved. Rupture of the vessels is fatal.

The large size of glandular swellings may complicate the signs in the chest to an unfortunate degree. It may give dulness along the median line, both front and back; it may transmit the sounds of cavernous and bronchial breathing as well as large and small moist râles. The passage of air through a part of the lung may be shut off, and a condition of partial collapse be the result. These various symptoms are not to be expected unless the swelling of the glandular tissue is unusually great.

As far as tubercular disease of the bones is concerned we have to do with the first symptoms; for when the location of the disease is clearly defined the care of the case should be given to a surgeon. These children usually have a tubercular history, and often a previous tubercular lesion. The bones of the spine are most frequently attacked, and after them in the order of frequency are the hip, knee, ankle, elbow, wrist, and shoulder. In tuberculosis of the spine we may find the disease in any of the vertebræ. We determine the location by the signs of deformity, tenderness, and pain, by the muscular spasm of the part, by pains and pressure effects due to irritation of the spinal nerve roots and their distributions, and the manner in which the child mechanically disposes his body in order to obtain the greatest ease. Caries in the cervical region causes pain and, possibly, deformity in the neck; although this part of the spine is often the latest in showing full symptoms, nevertheless from the beginning there may be tenderness on pressure and some pain on movement. Attention to the disease may be first attracted by some degree of peritonsillar abscess. In the lower cervical vertebræ the deformity occurs as a kyphosis, while in the upper bones it projects forward so that one may palpate it through the pharynx.

In the dorsal vertebræ the pain is generally located below the injured part. The child tries to protect himself as much as possible against the shock of movement, and therefore walks and carries himself in an awkward fashion. The affected bones, following the line of least resistance, are pushed backward, forming a more or less pronounced kyphosis. In the lumbar bones the disease is often the hardest to diagnosticate. The patient keeps 
his spine in a posture of exaggerated rigidity; he complains of pains which radiate in the abdomen, the thighs, and the buttocks. After these signs appear, one should be prepared to find at any time a psoas abscess; in fact, tuberculosis of any part of the spine is apt to give rise to pus formation, the discharges from which in burrowing through the surrounding tissues follow the line of least resistance.

In the hip joint the disease begins in the head of the femur or the acetabulum. The child complains of some tenderness on movement, especially if the motion is at all violent. Little by little the tenderness may change to pain; the joint, on arising in the morning, or after being seated for an hour or more, is sore and stiff. Gradually this soreness and stiffness become fixed, the patient may complain of long continued pain, which, even in the early stages, radiates into the knee or the inner aspect of the thigh, although the hip joint itself does not seem really painful. At night, in the later stages of the disease, he suffers from sudden attacks of sharp, spasmodic pains. Inspection will show some atrophy of thigh and calf, the gluteal protuberance has but a single fold, and is flatter than the normal. Careful examination will show the tenderness on pressure and forced movements; the care of the case then becomes surgical.

In the other joints, such as the knee, the ankle, the wrist, and fingers, the same general course prevails. There is usually a history of tuberculosis, or one or more tubercular foci in other parts of the body. Either with or without injury, the joint in question becomes stiff, especially after disuse. The stiffness becomes less and less transitory, the tenderness gradually turns to pain. The joint gradually takes on a fusiform swelling which, on account of its lack of heat and redness, has been called a "cold abscess" or "white swelling." The associated muscles become atrophied, the inflammation passes from the joint to the soft parts about it, and pus may work its way in any direction in or about the joint. The diagnosis has by this time become clear, and surgical aid should be called to the case.

In the kidneys and suprarenal capsules tuberculosis gives no specific symptoms. There may be pain in the neighborhood, and micturition may give a sensation of burning, although the bladder and urethra are not affected. If an abscess forms in or about the kidney, one may distinguish the tumor on palpation, or pressure 
effects may gradually show themselves. The main diagnostic points are the presence of tuberculosis in other parts of the body, and the finding of tubercle bacilli in the urine. Likewise in the liver and spleen there are no distinguishing marks of tuberculosis beyond what one finds in non-tubercular disorders. The liver may occasionally be enlarged, and if there are sufficiently prominent nodules in the superficial parts of the organs, or in the capsules, one may possibly distinguish them on palpation. Otherwise one depends upon the conjunction of known tuberculosis in the body and the symptoms of hepatic and splenic disease to make a diagnosis.

The stomach is so very rarely tuberculous, and the few cases which have occurred have been so far advanced, that there has been little opportunity and seemingly no particular value in searching for special signs. In the intestines, however, infection is no rare occurrence. Outside of the general symptoms of the disease and its presence in other parts of the body, there are disorders of the gut, confined, for the most part, to the small intestine. The principal of these is a persistent and irregular diarrhœe that at times is exceedingly hard to influence. As ulcers are formed there may be hæmorrhage from them that in unusual cases is so great as to be serious. There may be tenderness on pressure, especially when the mesenteric glands are much affected. These glands, on deep palpation or manipulation, may be felt as single or fused lumps along the spine. They, likewise, may be tender, especially when the examining hand seeks to move them from their fixed attachment.

Tubercular peritonitis, in at least one of its forms, gives practically no symptoms. Where a miliary infection is present the disease is often not suspected unless it is sharply localized. In the latter instance there are, in some, long continued cases, localized swellings and thickenings of both peritonæum and omentum; at the same time there are irregular pains in the abdomen, and enlargements of the mesenteric glands may manifest themselves. Where the miliary tubercles are widely scattered over the whole peritoneal membrane, there is a strong likelihood of the transudation of a large amount of serum into the cavity. This may set in abruptly or in a sub-acute manner; but in either case the main symptom is the ascites. The abdominal wall may be so much distended that it is hard, tense, thin, and coursed on its surface 
with dilated veins. A well-marked wave-impulse is easily elicited by manipulation. Most cases exhibit irregular abdominal pains, irregular alternations of diarrhœa, and vomiting. The systemic effects of tuberculosis are coincidently present, and with the known existence of the disease in other parts of the body give the surest method of diagnosis. In those cases where the tubercular deposits, instead of being in the form of miliary granules, are caseous masses, degeneration of the new tissue may take place, and a diffuse purulent peritonitis results. Naturally this process is slow, and the symptoms develop slowly and irregularly. There are abdominal pain, functional derangements of the intestines and sometimes of the stomach, and noteworthy loss of flesh and strength. On palpating the swollen and sensitive abdomen, one distinguishes a heavy, thick consistency, swollen and tender glands, and matted masses of intestine and peritonæum. The purulent fluid may be diffused over the whole abdomen, or, if there are many adhesions, it may be shut off in pockets. One of the favorite locations for such encystments is about the umbilicus. In others of the cases of caseous deposits the process does not go on to purulent degeneration, and, instead, the cheesy areas have undergone a conservative change into fibrous tissue. The disease then becomes long drawn out, and its course alternates between improvements and retrogressions. There are many adhesions, encystments, and matting together of intestines and peritonæum. The mesenteric glands are enlarged and tender; and from them and the adhesions various pressure effects upon adjacent structures may gradually come into action. The amount of effusion is variable, tympanites is marked, and pain is considerable. The temperature is irregular, being often near normal, but rising easily on șlight provocation. There may, at various times, be derangements of the intestines, and alterations in the general condition of health, according to the fluctuations of the disease.

Tuberculosis in the brain is almost always a secondary condition, so that its general nature is understood as soon as symptoms appear. The tubercles vary from a minute size to that of a large nut or even a small egg. The amount of tissue thus replaced and the amount of pressure produced may, on account of the varying lesions, not be alike in any two cases. If the neoplasm is large, it may produce some effect even upon the exterior shape of the skull, or when it projects into the orbit it may cause the eyeball to project. 
The general symptoms are those of cerebral irritation, such as restlessness, pain, stupor or delirium, disorders of the cranial nerves, motor or sensory paralysis, incoördination, disturbances of vision, nausea, and vomiting. By ordinary methods of localization the tumor is generally located without difficulty. In the meninges tubercular deposits constitute the largest part of the diseases there found. In some cases preliminary symptoms, in the way of

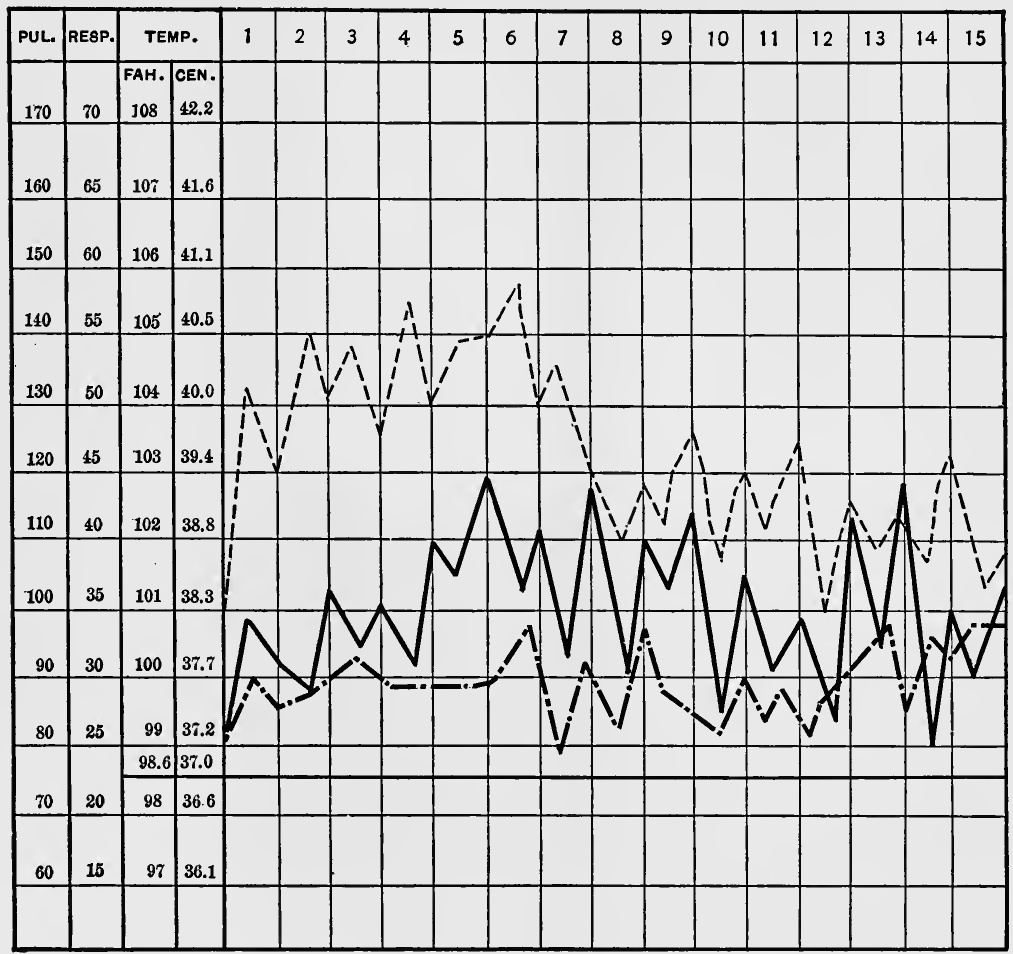

PULSE, RESPIRATION AND TEMPERATURE CHART OF TUBERCULAR MENINGITIS, FIRST AND SECOND WEEKS.

AGE, 5 YEARS.

PULSE___- _- RESPIRATION_- TEMPERATURE,

Fra. 108.

malaise, irritability, general weakness, gastro-intestinal disturbances, headache, and disturbed sleep, may be present. The appearance of gastric disorders as prodromata is both common and deceiving; and one may treat the symptoms for a week or two without being convinced of one's mistake. The first thing that 
appears suspicious is the comparatively mild nature of the attack and its failure to subside. On the contrary, the child gradually becomes worse, the pains in the head grow greater, the temperature is irregular, and has a tendency to mount, so that instead of oscillating from $37.5^{\circ}$ to $38.5^{\circ} \mathrm{C} .\left(99.5^{\circ}\right.$ to $101.3^{\circ} \mathrm{F}$.) it may attain to $39^{\circ}$ or $39.5^{\circ} \mathrm{C}$. $\left(102.2^{\circ}\right.$ to $103.1^{\circ} \mathrm{F}$.). A noteworthy fact is that the pulse rate, unless some complicating disorder interferes, does not increase in the same ratio as the temperature; the respiration shows more of a tendency to follow the pulse than the temperature. As the disease shows its character more plainly, the child loses his brightness and desire to be amused. He becomes more and more stupid, and shows an increasing desire to sleep, and in some cases it is almost impossible to wake him. The condition now becomes steadily worse. The respiration becomes irregular, the eyes dilate irregularly and respond poorly to light, and the skin is so sensitive that the non-pathognomonic tache cérébrale and the mark of Trousseau - redness of the skin caused by simple pressure - are commonly seen. The neck becomes rigid, opisthotonos may occur, the abdomen may be retracted, and ability to swallow is impaired. Bilateral or irregular paralyses may show themselves and remain temporarily or permanently. The superficial reflexes are diminished, and the deep ones are increased. The eyes show congestion of the papillæ and often an optic neuritis. The respiration assumes the Cheyne-Stokes type, the general prostration increases, the temperature shows a disposition to rise, convulsions supervene, and the child dies in a condition of respiratory and cardiac paralysis. The disease may last for a period varying from a few days to several weeks, the shorter periods being characteristic of very young children. In some infantile cases the disease runs a chronic course with symptoms which point to miliary formation at the base of the brain. There are opisthotonos, hydrocephalus, convulsions, gastric disturbances, and exaggerated reflexes. The pressure effects are naturally in proportion to the extent of the lesions, and the degree of ossification in the skull.

In the other parts of the body the occurrence of tuberculosis is almost always secondary; the involvement of these organs is not at all mysterious nor is the course of the sickness especially peculiar. As an example one may quote tubercular pericarditis, a disorder which most often occurs as a part of a general miliary tuberculosis. Since it commonly follows disease of the bronchial 
and mediastinal glands, it is very apt to be preceded or accompanied by pulmonary tuberculosis. Moreover, it occurs in a fairly late stage in the process when one or more glands have broken down, or ruptured into the pericardium. We consequently find the general features of the systemic process plus those of a purulent or sero-fibrinous pericarditis ; or where there is no effusion, the disease takes the form of a sub-acute or chronic inflammation with the production of a large amount of adhesions. The same general idea is true of tuberculosis of the testis, the Fallopian tubes, ovaries, and uterus. In the skin the disease is known as lupus vulgaris, although a more rational name would be tubercular dermatitis. Its favorite location is about the nose, cheeks, and mouth; but it may appear on any part of the skin surface with the exception of the palms of the hands, soles of the feet, penis, forehead, and chin. It may even extend to the mucous membrane. In the beginning one may notice small light brown spots raised above or depressed below the surrounding plane of the skin. These spots increase in number and size until they form a good-sized patch; there is an infiltration of the skin which helps to create the final picture of a raised and irregular periphery and a depressed centre, the color being an irregular mixture of red and brown. Following the common evolution of tuberculous tissue, there may be a conservative or destructive termination to the process. In the one case a gradual fibrosis begins which eventually covers the affected tissue with a depressed, shining scar. In the other the surface breaks down, leaving a superficial ulcer whose edges are rough, elevated, and infiltrated, and covered in parts by crusts which extend from the centre. Variations in form have been given appropriate names, so that if the affected area has a number of warts on it one calls the variety lupus verrucosus; if the outlines of the ulcer are wavy, lupus serpiginosus; if there is a rich and luxuriant growth of granulations, lupus hypertrophicus. The course of the disease is irregular, and on the whole chronic.

Treatment. - There is no doubt that many cases of tuberculosis are curable, and the clearly defined evidence which we possess of the healing of tuberculous process before a lesion has been known to exist, as demonstrated by the finding of scars after death, is enough in itself to encourage the physician never to cease in his efforts in any degree until the patient is dead. The dogged and steady persistence in carrying out every detail, no 
matter how small, of a wise method of care will unquestionably tend to produce ease and prolong life. In many respects the successful care of a tuberculous patient is a matter of minutiæ, of constant watchfulness, of unremitting activity. And the physician must try with all his power to prevent a spirit of pessimism from dominating himself or his patient. Psychological treatment has its place as well as physical.

Our first care, of course, must be in the way of prevention. Affected persons, those of a bad heredity who are in poor health, and those who have recently recovered from the disease, must not be allowed to marry until they are clearly beyond present danger. As a general rule, marriage should be forbidden until not only their general health but also their domestic, industrial, and climatic environment has been arranged with a view toward adequately protecting them from the probable likelihood of another attack. Following the same idea, such persons as are threatened should be placed in the most favorable circumstances to prevent the development of the disease, and best of all they should be removed from the family until a normal degree of health returns. This is especially true of young children who are naturally in a condition of unstable equilibrium, and for whom every circumstance of ordinary life may go far to produce health or sickness. Their food should be as untainted as possible, of the best quality, wisely selected, and properly administered. They should be made to live as much as possible on a nitrogenous diet, with sufficient admixture of fats, sugars, and starches to give variety. These children should be encouraged to eat much, to eat often, and to allow sufficient time after any violent exercise to permit undisturbed digestion. The ventilation of their sleeping rooms must be excellent, and whenever possible they should sleep in the open air. Their clothing should be warm and not too heavy; it should be sufficiently absorbent, and must be frequently. changed. They should bathe often, and should always have the benefit of cool or cold morning douches. Exercise must be carefully regulated, must not be omitted for a single day, unless they are confined to bed, and should be taken in the open air.

An infected child should be rigidly isolated, he should be removed to the country, and should live in the open air as much as possible. The climate should be moderate, dry, and not often subject to extreme changes. It should be such that the child may 
spend the greatest part of the day out of doors, eating there, and frequently sleeping there. No bed is quite as good as a hammock for such a patient; for it is most easily aired and transported from one place to another on the grounds as occasion demands. All discharges - pus, sputum, fæces, urine, nasal mucus - must be burned or disinfected before being thrown in the common receptacles, and all clothing and household utensils should be boiled and cleansed by themselves, and kept from contact with those used by the rest of the family. Books and toys should be kept apart from other children, and so disposed as to be free from the responsibility of carrying infection.

With these patients the recommendations concerning food, clothing, exercise, bathing, and ventilation must be carried out in the most consistent manner possible ; one must keep in mind that drugs, while valuable enough, have no specific action, that the surest method of help is to develop every possibility of vital resistance and recuperation. A corollary to this proposition is to remove every possible weakness or obstruction to perfect function. For example, hypertrophy of the pharyngeal tonsil, chronic intestinal derangement, or any other obstruction to normal physiological activity, calls more urgently than in ordinary cases for correction. Above all, one must regard the localization of the disease not merely as disability of the part involved, but rather as a partial manifestation of an infection that is doubtless wider than the objective symptoms denote, and in addition may be, or may shortly become, general. Potentially tuberculosis of any part means tuberculosis of every part, for the whole includes every element into which it may be divided.

The special methods of treatment are few : pulmonary tuberculosis calls for the same treatment that similar non-tubercular inflammations of the lung necessitate. In addition to the care of the symptoms, stimulants and tonics must be liberally and wisely used. Among these, alcohol holds an important place, and strychnine or nux vomica must be often and liberally employed. The organic preparations of iron may be needed to combat the anæmia that so often appears. Beechwood creosote is of value when it can be tolerated; it may be administered with three times the quantity of tincture of gentian, the mixture then being added to a small cup of milk. Another unobjectionable method is to suspend it in a good malt preparation; no matter how given, the doses may range 
from 0.03 to 0.3 gramme ( $\frac{1}{2}$ to 5 drops) three to four times a day according to the child's age. Cod liver oil may be used, but only where the child's power of digestion and assimilation are good enough to change and absorb it. In these pulmonary cases the factor of climatic treatment is of radical importance. The child should, with the least possible delay, be removed to a locality where the air is clear, dry, and sufficiently rarefied. The place should be so chosen that extremes of temperature are not common, nor should there be many obstacles in the way of living in the open.

The treatment of tuberculous glands is one of tonics and stimulants, of exercise, and regulation of the general mode of life. Thus iron, strychnine, arsenic, the compound syrup of hypophosphites, and alcohol are of considerable use. Where the glands are within easy reach, especially if marked improvement does not follow the use of the designated remedies, the ordinary surgical procedures are in place. Disease of the bones calls for similar care - rest, nourishment, stimulation, and finally operation. A tuberculous kidney, when its fellow is not involved, may require removal; and tubercular peritonitis, which is characterized by the presence of fluid, calls for laparotomy. When the testis, ovaries, tubes, or uterus are involved, operation may be useless because the rest of the body is doubtless also infected; but if these organs constitute the main seat of the disease, they likewise should receive radical treatment. Disease of the brain and meninges can receive no more than symptomatic care, and in this way resemble most cases of tubercular livers, spleens, intestines, and other viscera. Tuberculosis of the skin, lupus, calls for destruction of the pathological tissue by excision or caustics, and the covering of the freshly granulating surface by skin grafting.

Prognosis. - The outlook varies with the age of the patient, the location of the disease, and the possibility of commanding all the various means of treatment. An infant with general miliary tuberculosis has very little chance of life; if the disease takes the form of acute involvement of the lungs, most of all when there are characteristics of a rapid course, the termination is usually fatal. The chronic processes have a much better chance of life, or a fair measure of recovery. When the glands alone are involved, whether in the neck, chest, axilla, or elsewhere, with proper care the child ought in most cases to have a good chance to live and be comfortably healthy. The same remark is true of other parts where the diseased tissue 
may be reached by operation. Thus, the patient who suffers from the non-ulcerative form of tubercular peritonitis is generally restored to health, and almost regularly we expect a tuberculous bone to improve or heal after a well-timed and well-conducted operation. On the other hand, one cannot be so sanguine in speaking of the abdominal and thoracic viscera; for the disease generally spreads so rapidly that tuberculosis of one means tuberculosis of many organs, and the penalty of a resulting general infection is death. Even in tuberculosis of the kidney, although the organ may be removed, the doubt of uncertainty, of the possibility of hidden infection in the fellow-viscus or some other structure, must remain for years in the mind. There is always the fact to terrify us that in most cases the bacilli are not entirely eliminated. The outlook in all such cases is uncertain; one child may live, the next may die. In cerebral and meningeal infection the result is practically always death. In lupus the treatment may be long continued, but in most cases recovery results.

Differential Diagnosis. - The main guide in any local or general manifestation of tuberculosis is the characteristic symptomatology, and if one keeps this distinctly in mind most of the so-called doubtful cases after a compartively short period of observation become clear. A careful examination of the patient's previous history, of his heredity and environments, and whether he has recently had those exhausting diseases which predispose a child to tubercular infection, are important items in distinguishing this disorder. In the typhoidal type of the infection in infants, the absence of Widal's reaction, of characteristic temperature and eruption, and the long course of the symptoms are enough to clear any doubt. In pulmonary disease, confusion will occur only in the first stages, and then the doubt rests between a tubercular process and non-tubercular broncho-pneumonia. Here we need to keep in mind that the latter has an acute onset and usually a limited course, that resolution takes place more or less promptly, and usually leaves the lung clear. In tuberculosis there are generally prodromal symptoms, systemic signs, a long course, involvement of other parts, and a delayed and imperfect recovery. In addition we may often be able to find in the vomitus sufficient mucus from the throat and bronchi to allow a microscopical examination for the bacilli. The location may give a hint - not always trustworthy, however. Speaking in general terms one may say 
that the non-tubercular broncho-pneumonia more generally attacks the lower than the upper half of the lungs; the tubercular inflammation, on the other hand, more frequently attacks the upper than the lower half. The physical signs in both are too much alike to be distinguished.

Diagnosis of disease of the urinary track may be rendered more certain by microscopical and bacteriological examination of the urine. In the glands we have to distinguish tuberculosis from the enlargements caused by ordinary, pulmonary, and gastro-intestinal disease, by syphilis, malignant disease of the chest, and Hodgkin's disease. 'The mere statement of the case is almost sufficient to clear up the difficulty, especially when one remembers that the general picture of tuberculosis is different from that of the other complaints, and that they in turn have their own peculiar characteristics. It is only in the beginning stages that doubt should exist, and here a short period of observation should effectually clear up the difficulty. In tuberculosis of the intestinal and mesenteric glands, one may occasionally have much difficulty in distinguishing the disease from lympho-sarcoma. The main points of differentiation are the rarity of the latter disease, the absence of tubercular involvement of other parts, lack of tubercular history and general tubercular symptoms, and the very gradual growth of tubercular deposits. Sometimes the diagnosis can be made only after extended observation.

Tubercular peritonitis is to be distinguished from the nontubercular variety and cirrhosis of the liver. The last-named disease is very rare in children, and when it does occur it presents a hard, small liver, more or.less jaundice, and less temperature and gastro-intestinal symptoms than in tubercular peritonitis. In nontubercular peritonitis there would not be the history of tuberculosis; moreover, it is a rarer affection except in cases of wounds or similar means of pyogenic infection. Finally, the ascitic fluid will supply material for microscopical and bacterial decision.

Diagnosis in meningitis is not always possible in the early stages. Usually the confusing and doubtful diseases are gastric and intestinal disorders, and as these are much more frequent than tubercular meningitis, cases which involve rational doubt should not be decided upon until definite grounds for final judgment have been obtained. These grounds are discovery of past or present tuberculosis in any part of the body, continued disturb- 
ances of the gastro-intestinal track, increasing stupor, irregular pulse and respiration, convulsions, and in some cases opisthotonos. A further means of certainty is sometimes furnished by finding bacilli in the cerebro-spinal fluid obtained by tapping the lumbar portion of the spinal canal. One of the main objections is the difficulty of finding the micro-organisms in every case.

\section{INHERITED SYPHILIS}

Syphilis is an infectious disease that is peculiar in the widely spread range of its lesions, their durability, and the great length of time over which it may extend. It may be communicated to the ovum in the act of fertilization, it may appear in children, and may infect old age. But with the characteristics of the ordinary acquired disease we have very little to do, since they are about the same at all periods of life. On the other hand, inherited syphilis is a thing apart whose features and results are important enough to call for careful consideration.

Causes. - The disease is probably caused by a micro-organism ; in default of positive information writers have commonly referred to Lustgarten's bacillus as the likely cause. The lack of a suitable medium in which this bacillus may be cultivated has hitherto been a bar to the acquirement of certain knowledge. But recently the consensus of opinion has decided that this bacillus in all likelihood is identical with the smegma bacillus. That the nature of the disease is microbic is testified to by our appreciation, in the light of our experience with antitoxines, of the law enunciated by Colles in 1837, that a new-born syphilitic child does not produce on the breast of his mother, who may seemingly be free from specific taint, any ulcerations as the direct result of contagion from suckling. We naturally put this phenomenon in the same class with those of immunity against diphtheria or smallpox. Moreover, we may add to it the complementary law of Profeta which enunciates the immunity of children, one or both of whose parents are syphilitic. Some of these children continue immune against the disease.

It is always necessary to distinguish sharply between hereditary syphilis and those cases of acquired disease which occur early in life. Thus it is possible, although the occurrence is rare, for a child to be infected during parturition. Also, he may contract the disease from being suckled by a syphilitic nurse, by being handled 
and kissed by syphilitic persons, through the agency of infected foods, domestic utensils, toilet articles, linen and clothes, by sleeping with contaminated persons, through exposure in the rite of circumcision, and by sexual contact. These various means are to a certain extent accidental, and their likelihood changes according to the circumstances of the child.

The exact share of responsibility that should be attributed to the father or the mother in hereditary syphilis is rather an academic than a practical question. We know quite conclusively that an infected woman almost always passes on the disease to her child; that an infected father commonly, although not quite so regularly, brings about the same result; that the certainty of infection varies with the shortness of time between the contracting of the disease by the parent or parents and the date of conception. The question of infection of the fotus is quite a different one. The view held in past times that there might be a direct transmission to the child is untenable; it is true that the ovule may be infected at the time of conception by a diseased spermatozoön, but afterward infection must occur through the indirect means of the mother who acts as a primary agent. Much discussion has centred about the length of time during which the possibility of intra-uterine infection existed; in answer to the implied question one may state that reliable observers have considered a mother capable of infecting her fœtus even when she has become contaminated as late as the seventh month. On the other hand, Diday believes that a woman who becomes infected during the first four weeks of gestation may by promptly obtaining vigorous treatment ward off the danger from the infant.

Lesions. - The pathology of hereditary syphilis may properly be preceded by a glance at the changes which occur in the syphilitic placenta, on account of which abortion so frequently occurs. 'The villi, which at no time are lavishly supplied with blood, become vitiated by cell proliferation until their vitality is materially reduced; this change continues until the epithelium and connective tissue stroma are likewise affected to such a degree that the circulation is more or less shut off. A like process goes on in the pouches into which the villi project, so that finally the placental nutrition is cut off either throughout its whole extent or in scattered areas. Accordingly abortion occurs, or the nourishment of the fotus is abridged. 
While it is true that some syphilitic children present no characteristic lesions after death, nevertheless they are more liable than others to inflammations of many parts of the body. Indeed, there is no portion that is necessarily exempt; but, on the other hand, some are especially liable to attack, notably the osseous, visceral, and serous structures. The long bones of syphilitic children are especially liable to be involved, and mostly at the junction of the diaphysis and epiphysis. The temporary formation which constitutes the line of junction is broader and more irregular than normal, and consists in fairly large part of cartilage cells. This zone, instead of decreasing, may increase, and is bordered by a soft, rather vascular cartilage that is irregular in its composition. Finally the growth is so poorly controlled that as the areas of calcification and ossification increase, and the intermediary spots of soft tissue enlarge, the stability of the whole structure becomes seriously impaired. The perichondrium and periosteum become loose, thick, and irregular in contour, and the shape as well as the stability of the bone becomes altered. Thus the results may show themselves in the form of an acute epiphysitis with a possible cleavage at the line of junction, or of the more chronic osteochondritis. In the latter the cartilage is first affected, its cells proliferate, and become soft and loose. Simultaneously there is a wasting of the intercellular substance. In extreme cases the inflammation may extend through the muscular tissue and result in an abscess, or it may involve a near-by joint. These changes are apt to occur very early in life, and should regularly be looked for, even if the parents of the child do not include the symptoms of swelling and tenderness in their history of the case. In older children there may be a periostitis of the long bones of the arms and legs which has nothing peculiar in its pathology. In other children the changes may be located in the fingers and toes, the proximate phalanges being attacked in preference to the distal. Although abscess formation is here likewise liable to result, nevertheless the ultimate integrity of the part is apt to be restored as soon as proper treatment is vigorously instituted. Such involvement is characteristic of very young rather than older children. Another favorite seat for bone deterioration is in the skull, where the softened tissue may by pressure exerted from both sides be thinned to a marked extent. The form of the skull makes craniotabes of the occipital bone the most common variety. 
In the lungs of still-born and young children the condition, known on account of its color as white pneumonia, is commonly seen. The process involves a part or the whole of a lobe, and consists of a diffuse fibroid infiltration. The epithelium is in a condition of fatty degeneration, the septa are thickened, and the alveoli are compressed by fibroid tissue. The process is plainly marked in the peribronchial areas, and the pleura is often involved. The formation of gummata in young children is at times apt to be replaced in older patients by areas of pus production. As the

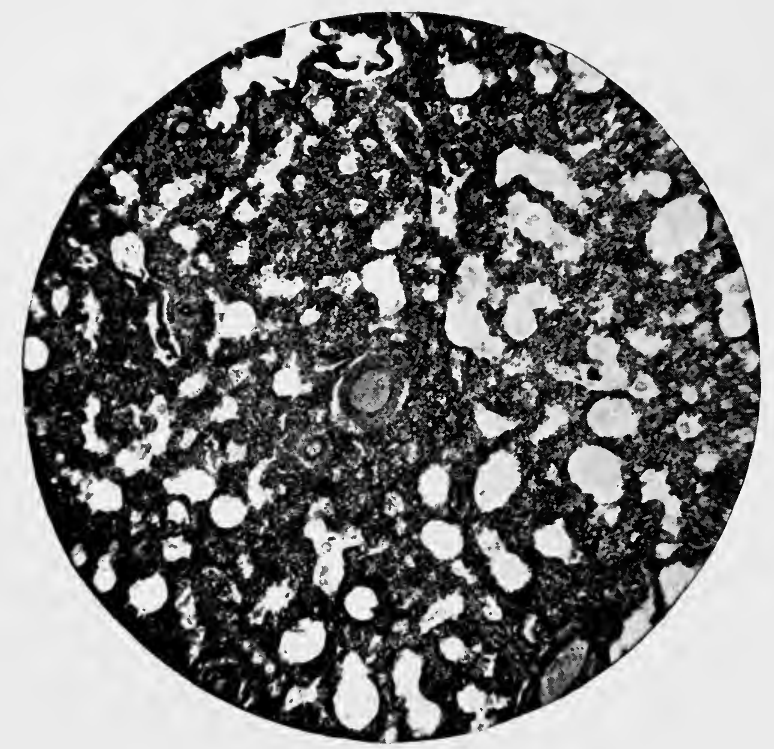

FIG. 109. - Infantile Hereditary Syphilis of Lung. $\times 30$.

result of these sclerotic changes, one rationally expects to find regions of emphysema, bronchiectasis, and atelectasis. In addition one occasionally sees a case of stenosis or ulceration of the bronchioles, trachea, or larynx.

The spleen is almost always enlarged, and the severer the grade of inflammation the more extensive are the changes in this organ. In infants there is a simple enlargement and hyperplasia of the tissue, and the capsule likewise may be thickened. In older children there may be a deposition of connective tissue that preserves the increase in size even after other pathological conditions may have become resolved. In the liver some what similar changes 
may be seen, with the exception that the interstitial inflammation occurs in the youngest children. The size of the organ is very much increased; the liver cells as well as the arterioles are squeezed and encroached upon by the new tissue, which gives a much lighter hue to the organ than it naturally possesses. The connective tissue may extend through a large part or the whole of the organ, or in other cases may be disposed in separated areas which at times may coalesce. In older children gummata of small size may be scattered here and there. In the pancreas a sclerotic involvement

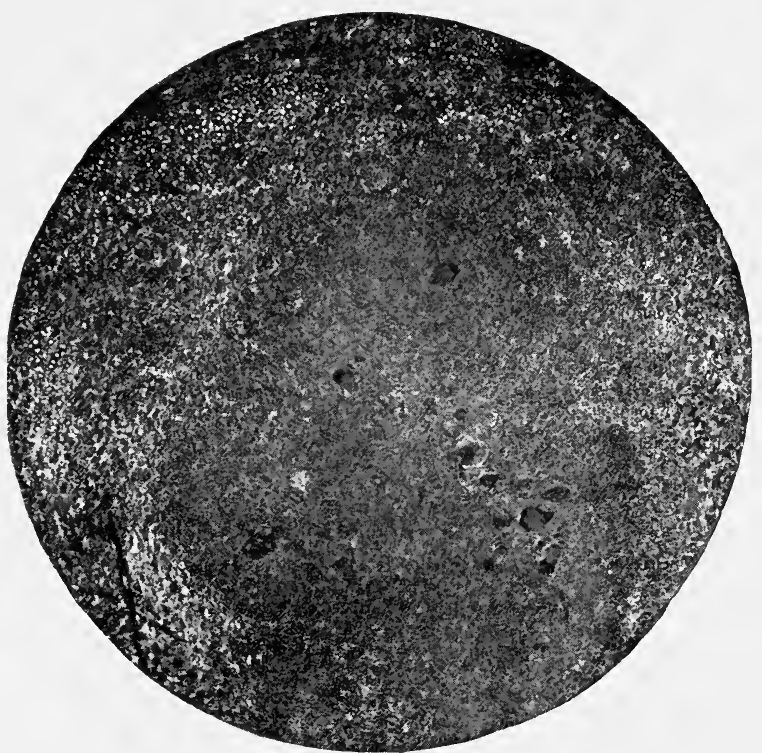

FIG. 110. - Gumma of Spleen. $\times 50$.

may occasionally be found; a similar course regularly affects the kidneys, where the inflammation assumes the form of a chronic interstitial nephritis, complicated in some cases by the presence of gummata. The suprarenal capsules go through a like development, as may any part of the genito-urinary system. Thus the urethra, or, more commonly, the body of the testicle, may be affected; and some years ago I saw a similar condition in the rudimentary prostate of a boy־only two and a half years of age. There is no viscus, in fact, which is free from the danger.

Among the parts most frequently attacked are the mucous membranes of the nose and throat. The inflammation is catarhal, 
with the addition at times of the so-called mucous patches. In the nose especially there is a likelihood of this tissue's breaking down, and of the ulceration's being followed by slight or severe degrees of necrosis. A specific otitis with consequent deafness is sometimes seen, but not quite so often as an interstitial keratitis which is seen in infants of the youngest age, or even before birth. The associated lymph-nodes are regularly involved,.but do not present any noteworthy features that are distinct from those seen in adults, with the possible exception of a greater readiness to

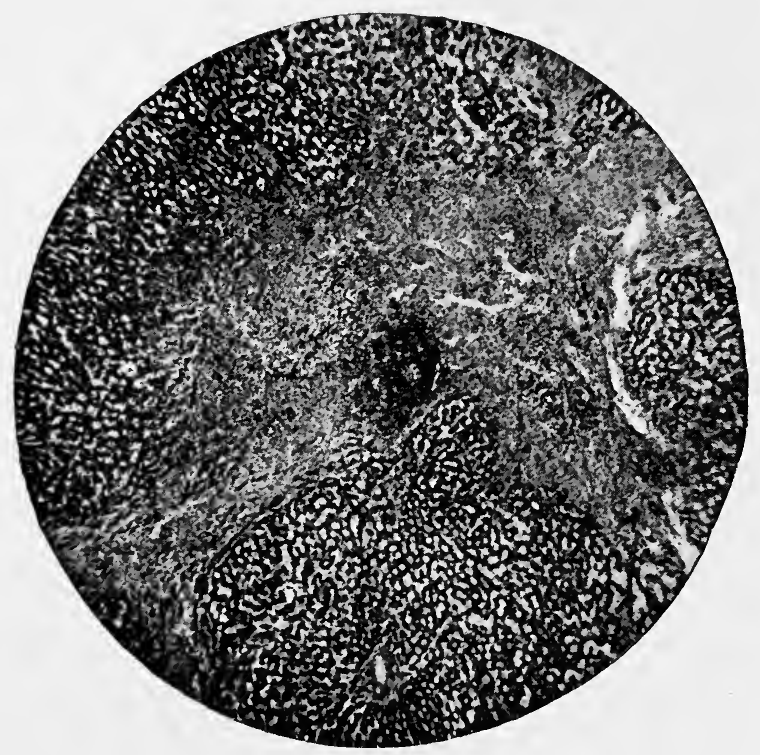

Fig. 111. - Gumma of Liver. $\times 30$.

break down in suppuration. In the brain and spinal cord syphilitic disease is rare. Hydrocephalus from this cause is sometimes seen; outside of this, the process may attack any part of the cerebrospinal system, but so infrequently that the cases are to a certain extent regarded as curiosities.

Symptoms. - In true hereditary syphilis there are no primary symptoms; the secondary signs may appear a short or a considerable time after birth, and only rarely are they present when the child is born. The various compilations of Diday, of Roger, of Miller, agree in placing the ordinary period when the symptoms 
appear as in the second half of the first month, although variations in both directions may naturally occur. These children may be born in good or poor condition, the decision resting upon the virulence of their infection and the mother's nutrition; the younger the child, the severer is the disease apt to be. Those who have symptoms at birth rarely live more than a few days or weeks. These characteristics are emaciation, prostration, asthenia; the skin is wrinkled, the face looks old, the temperature may be subnormal, the spleen and liver may be enlarged. Sometimes

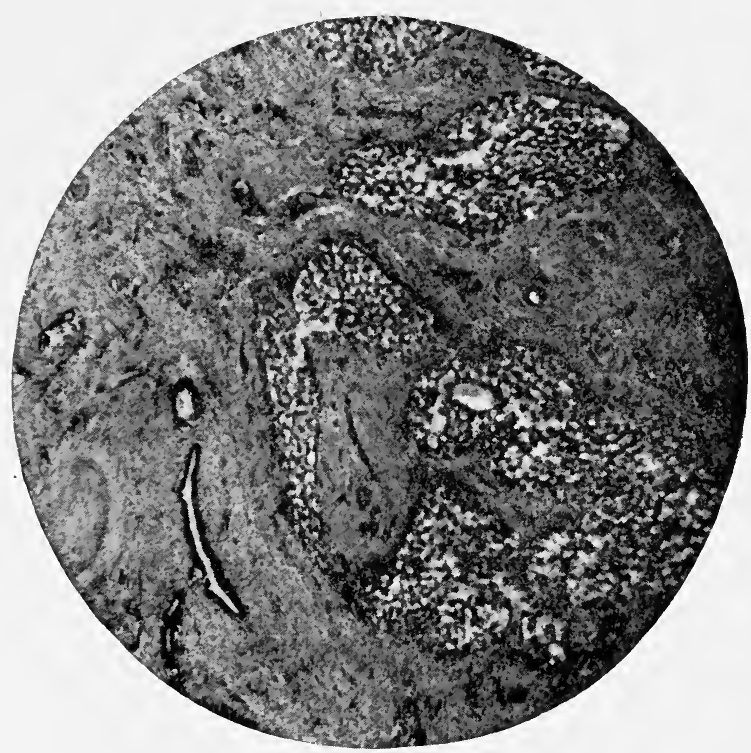

Fig. 112. - Syphilitic Cirrhosis of the Liver. $\times 30$.

there is an eruption of macules, papules, pustules, or blebs on the hands and feet. The cry is hoarse, and the nose is partly occluded by swelling of its mucous membrane and the resulting discharge. The children die because they have not the strength to live.

Those children who are born in better condition retain their health for a month or less, in rare cases for a possible period of three and a half months. Then their strength begins to fail, they become fretful and assume a weazened expression. They regularly become troubled with a persistent coryza, and the voice may be muffled and hoarse. There may be mucous patches on the throat and anus, the lips may be fissured, and condylomata may appear. 
Bone symptoms may break forth, and visceral diseases of various locations are to be expected.

The eruption appears in various forms ; its first location is generally on the abdomen, face, and nates, from which it spreads to the rest of the body. Its mildest form is the macular variety; its color is a dull red, which disappears on pressure. Later on, the hue is coppery and permanent. Papules are more serious in their import than macules; they are somewhat small in size and irregular in contour. The pustular syphilide may be seen in all stages from

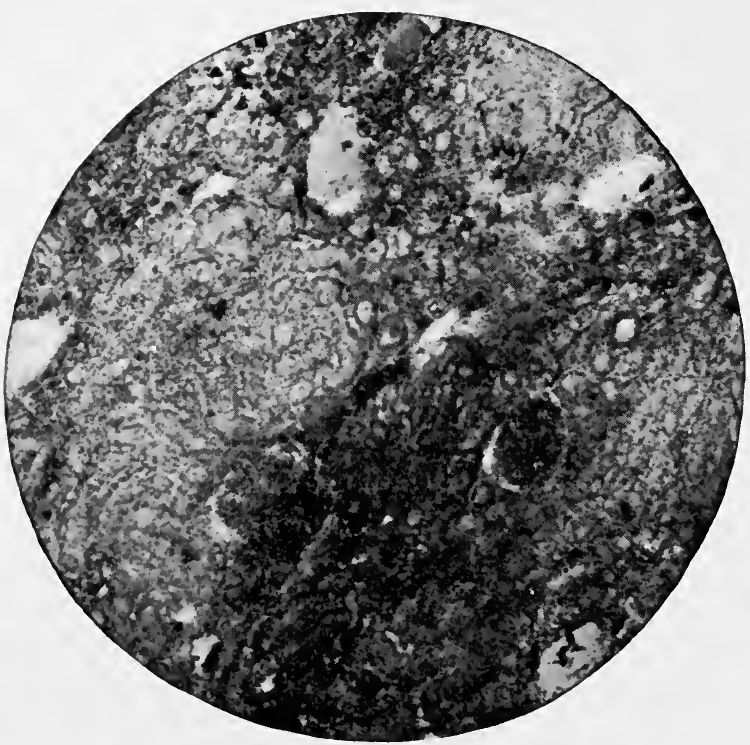

FIG. 113. - Induration of Kidney: Hereditary Syphilis. $\times 30$.

a changing vesicle to a completed pus formation. The worst of all is that made up of blebs or bullæ, filled with serum or pus, that has for its favorite location the plantar surfaces of the hands and feet, and sometimes the legs and arms. At the same time, when the eruption is developing, one's attention is commonly attracted to the mucous membranes. All over the body these may according to their conformation be attacked by fissures and patches. In the angles of the mouth such thin clefts, which are tender and easily made to bleed, leave scars that always point to syphilitic disease; they may also appear on the margin of the nose, and even more 
commonly about the anus. The patches are oftenest found on mucous membranes or their margins; but where the skin is thin and tender, or is irritated by friction, injuries, or any acrid discharge, they may likewise appear. At times they are hard to distinguish, but in general may be recognized as dull white in color, thin, slightly raised, and having a reddish border.

One of the characteristic facts about these children is their liability to succumb to inroads upon their vitality. A faulty milk supply, which may mean no more than a temporary indisposition

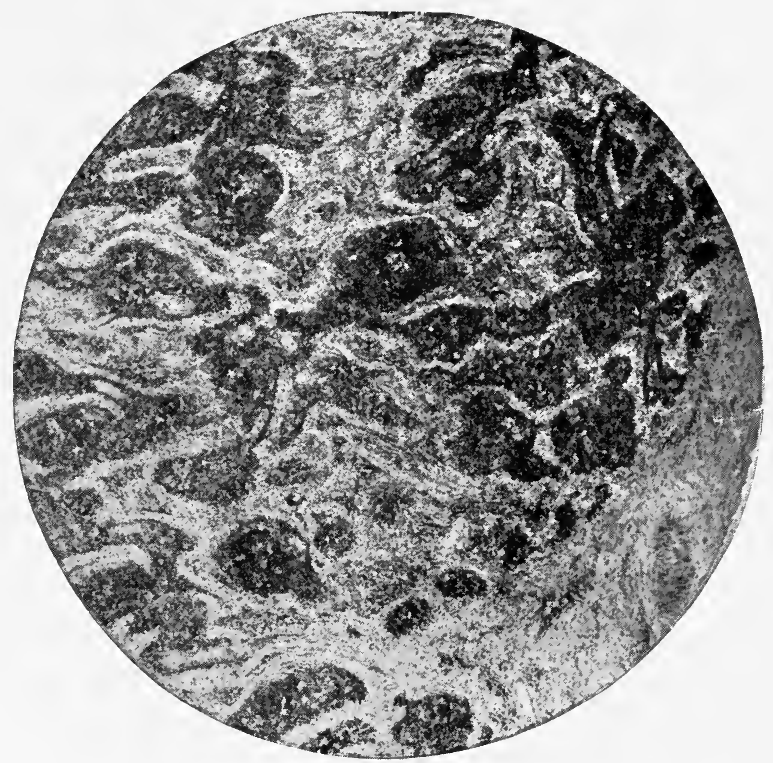

Fig. 114. - Syphilitic Lymphadenitis. $\times 25$.

in a healthy child, may in a congenitally syphilitic baby threaten life itself. For this reason the ones that are artificially fed, especially if their circumstances are not good, are poorly nourished, are commonly ailing, and are often unable to withstand the shock of ordinary physical disability. In some cases disorders of the gastro-intestinal track begin a series of sicknesses which successively attack one portion of the body after another. In this connection I am reminded of a syphilitic child who began to suffer with laryngitis and rhinitis, followed at varying intervals by peritonitis, gastro-enteritis, iritis, otitis with consequent deafness, peri- 
ostitis of the left femur, and then a similar process on the right side. Finally she died during an attack of broncho-pneumonia. These sicknesses extended over months, and during all this time the child was laboring under the pitiable disadvantage of inability to recuperate in sufficient degree from any one of the specific attacks, very much as a spendthrift continually takes more from his resources than he can add thereto. As a result of the steady physical deterioration an intense anæmia frequently ensues, characterized by a diminution of the number of red blood-cells, alterations in their form, decrease of hæmoglobin, and a noticeable leucocytosis. This condition of the blood, if it is not very marked, may be overcome; but severe cases are very dangerous. Especially so are the rare instances of hæmorrhagic syphilis in babies in which the bleeding may occur from the mucous membranes or under the skin, either from no known cause or slight injuries. Under this heading various writers have put hæmorrhage from the umbilicus which occurs within a few days after birth.

The symptoms of disease of the bones may easily be deduced from the pathological changes plus the ordinary course due to swelling, pain, and loss of function. It is sufficient to emphasize the necessity of carefully examining the long bones of every suspected or known case of inherited syphilis, and of regarding the first sign of tenderness or deformity as the probable precursor of serious changes. One should likewise watch for disease of the nails, and phalanges of the fingers and toes. There may be suppuration of the matrix around the upper margin of the nail, or the nails themselves may crack, break, chip off, or become wholly exfoliated. In either case the nails are apt to be furrowed, creased, irregularly thickened and discolored; at times they may be so much distorted as to suggest the picture of a claw. With or without these changes there may be disease of the phalanges, which more commonly occurs in the proximal than the distal segments. 'The inflammation is primarily one of the bone and periosteum; the swelling is hard and fusiform, and only rarely involves suppuration of the muscles and skin.

The false paralyses of early hereditary syphilis are not matters of serious moment, nor are they permanent. Very commonly the loss of function is not due to organic lesions of the nerve tissue, but rather to pathogenic conditions of the bones and muscles, which render action, movement, and even handling extremely 
painful. The existence of such disabilities, if no other sign is present, should always suggest the possibility, and often the probability, of inherited syphilis.

An exceedingly interesting class of cases is what is called late hereditary syphilis. Whether one will regard these cases as the delayed expression of inherited disease or the tertiary symptoms of acquired syphilis whose earlier stages have not been noticed, depends upon one's mental equation, training, and experience. Their characteristics closely resemble those of tertiary syphilis, and there are good reasons for believing them to be nothing more than tertiary symptoms. Nevertheless, as both views have able supporters, the matter cannot and should not be here treated as other than an open question. The principal instances of these cases are seen in the bones; there may be disease of the long bones and their periosteum, and also of the skull. The deformity may be considerable, but the pain and disability are often much less than in the early form of the disease. The skull is apt to have a heavy, square appearance, and the frontal prominence may be quite noticeable. The bones of the nose may be weakened, thickened, distorted, or necrosed. The hard palate is sometimes high and arched, and a necrotic process similar to that seen in the nasal structures may attack it in the median line. Many of these changes are not only due to the poison of the disease, but must also be in part attributed to a deficient and vicious nutrition. This is especially well seen in the second set of teeth. While all may be affected, the central upper incisors are the ones most plainly marked. They are so poorly developed that they lack their usual length and breadth, they are set irregularly in the gums, and according to their general deformities have been called screw-driver teeth, peg teeth, and the like. The classical form known as Hutchinson's teeth designates those upper incisors whose lower margin are grooved or notched in the centre. This is the result of softening and attrition of the enamel, and may occur in other diseases of malnutrition. Throughout the body there may be visceral changes which follow the general plan of tertiary disease in adults, the most frequently affected parts being the excretory and assimilative systems. The diseases of sense organs that are most often observed are interstitial keratitis with or without iritis, and a chronic otitis media whose ordinary end is loss of hearing. The skin is, in weak and poorly cared-for children, easily broken down, 
and a characteristic ulceration ensues that differs in no way from syphilitic ulcers in adults.

Treatment. - Prophylaxis in syphilis is one, if not the most important, part of treatment with which the physician must concern himself. The mother must be subjected to vigorous treatment, if she or her husband had the disease before conception, or if she or her husband acquired the disease after conception and before the birth of the child. A child born of such parents, so long as his heredity is known, and even if at birth he presents no symptoms, should be put under treatment until his normal development is fairly well assured. If the disease is not known or suspected at first, the treatment must be instituted with thoroughness as soon as the first symptoms appear. If a wet-nurse is to be employed, every applicant for the position must be rigorously examined and proved to be free from the disease before she is allowed to touch the child. And on the other hand, a syphilitic infant should never be suckled by a healthy wet-nurse. The physician's responsibility is as great in one case as in the other.

As in adults, our main reliance is placed upon the administration of mercury. This may be given in inunctions, in fumigation, or internally. The inunctions are deservedly the favorite mode of prescription, since we thereby avoid all injury to the sensitive gastro-intestinal track. The ointment of the oleate of mercury, either alone or mixed with lanolin or vaseline, or the officinal mercurial ointment mixed with an equal quantity of cold cream, may be rubbed into the inner surfaces of the arms and legs, one limb after the other being employed in regular rotation on successive days. Another way, and a very good one, is to spread about 0.75 to 1.0 gramme (gr. xij to $\mathrm{xv}$ ) of the ointment on a piece of lint which is to be firmly held by a roller bandage to the part selected. The warmth and movement of the body cause absorption, while the disadvantages of rubbing the ointment into the skin are done away with. If for any reason, such as erythema of the skin, it is necessary to discontinue this means, the internal administration of one of the soluble salts of mercury should be employed. The bichloride is commonly used in doses of 0.0003 to 0.0006 gramme (gr. $\frac{1}{2} \frac{1}{00}$ to $\frac{1}{100}$ ). The disadvantage in this is its disposition to irritate the gastro-intestinal track. In its place I have had satisfaction in using the protiodide in doses of 0.003 to 0.006 gramme (gr. $\frac{1}{20}$ to $\frac{1}{10}$ ) three times daily. In 
markedly severe cases where it is imperative to make an immediate impression on the disease, hypodermatic injections of the chloride in doses of 0.0003 gramme (gr. $\frac{1}{200}$ ) for a few times only may be given. In the late attacks one may use the iodide of potassium or the ordinary mixed treatment for the same indications as in the tertiary stage of adults, the quantity having been made suitable to the age and condition of the patient.

The administration of mercury is not the only matter of importance; for the child's general condition is often so poor, and the degree of anæmia may be so severe, that much attention should rightly be given to them. The food, if artificial, should be most carefully regulated and modified, and such details as bathing, rest, clothing, and exercise should be scrupulously supervised. In many instances it will be necessary to administer tonics early in the disease, and to continue their use until the patient is in good condition. As much judgment and resourcefulness may be shown in this direction as in the search for the most favorable method of exhibiting mercury.

Prognosis. - The outlook is not very encouraging; even if children do not die, they nevertheless may have such poorly nourished bodies that some form of disease is constantly threatening; and in such cases intercurrent acute sicknesses are apt to take a severer course than in children of a better heredity. The proportion of deaths depends upon the youth of the patient, the malignancy of the disease, the promptness and thoroughness of treatment, and the circumstances of the patient's home. These factors are so variable that statistics are not of much use. If we find in a public hospital, whose patients come from the poorest, the most ignorant, the most unfavorable classes in the community, a mortality of from fifty per cent to sixty per cent, we may on the other hand learn in private practice that eighty-five per cent to ninety per cent of syphilitic children live. Under the most favorable conditions the mortality may be even further reduced.

Differential Diagnosis. - The picture of hereditary syphilis is usually so clear, and the confusing elements are generally so easily eliminated, that a mistake in diagnosis should not often be made. The history of this specific disease in the parents, and the acute symptoms in the young child, such as snuffles, hoarseness, the skin eruption, the mucous patches, and anal condylomata, the tenderness in the bones, and the general malnutrition, all go to make convic- 
tion in one's mind. In the late form, the advanced bone disease, the small, poorly nourished, and irregularly developed teeth, the flat head and sunken nose, the rhagades, the marks and ulcers on the skin surface - these are sufficient to lead one to the truth of the matter. In case one finds symptoms of visceral involvement or the presence of gummata, the characteristic treatment will almost always relieve the patient without delay, and confirm the diagnosis.

\section{Malaria}

Malaria is an acute infectious disease caused by the hæmatozoön described by Laveran in 1880, and later named Plasmodium malarice by Marchiafava and Celli. It flourishes in greatest activity and luxuriance in hot climates, especially in the basins of the large rivers, and in swampy ground. In temperate zones it is not nearly so frequent, nor is its virulence nearly so great. In these climates, low, undrained ground, most of all where an impervious layer of clay is near the surface, and holds stagnating water within reach of the air, is the usual malarial locality. In cities large and deep excavations, tearing up of streets, residence near undrained parts, river fronts, and dumping grounds, are the usual means of infection. The plasmodium finds its way into the body through the inspired air, and Ross claims to have produced the disease in persons who drank water in which the bodies of mosquitoes that carried the hæmatozoön in their bodies had died. The disease is most common in the spring and early summer, somewhat less so in the autumn, and least abundant in the winter. Children are frequently attacked, more often than adults; moreover, they are liable to show irregular forms, even more so than mature patients.

Lesions. - Most of the changes which occur in malaria are confined to the blood, and to a smaller degree to the liver and spleen. The plasmodium enters the red corpuscles where it undergoes a series of changes in form; it increases in size, becomes pigmented, and then segments. Finally it presents the picture of a many-armed rosette about whose centre the pigment granules cluster. The process of division produces from twelve to twenty units, which finally become free by the rupture of the enclosing capsule, and prepare to enter other corpuscles. The evolution takes forty-eight hours, and constitutes the so-called tertian form of the disease. It is possible that there may be two sets of plas- 
modia which mature on successive days, thus producing the double tertian or quotidian form.

It is probable that there is more than one form of plasmodium; at any rate in the so-called quartan fever the appearance and evolution of the hæmatozoön are somewhat different. In the simple form it matures at the expiration of seventy-two hours. Likewise there may be a double quartan fever with two groups which may mature on successive days with an interval of one free day, or a still more complex combination of three groups, the triple quartan,

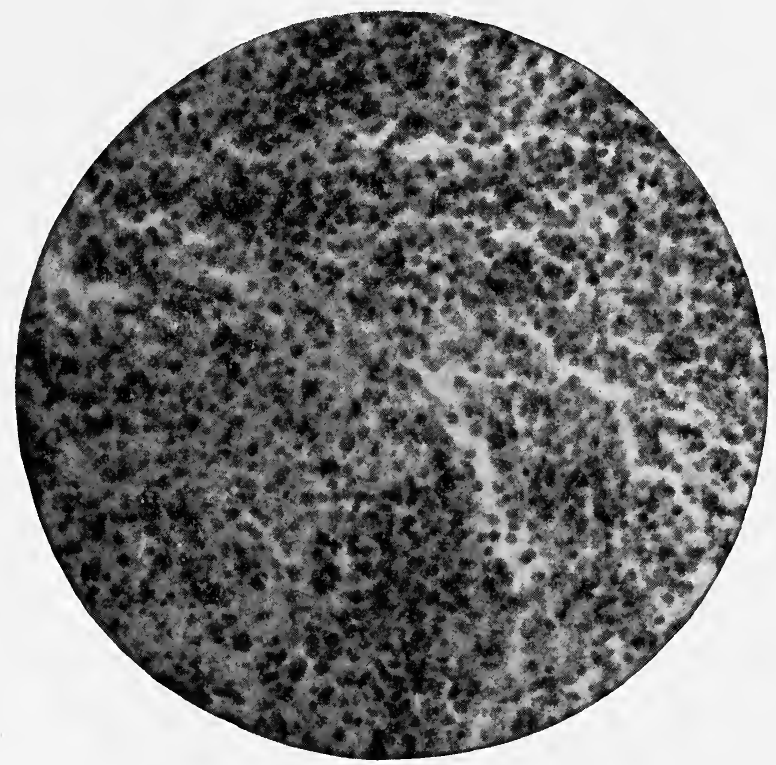

Fig. 115. - Spleen in Acute Malaria. $\times 220$.

one of which matures on each successive day. When the maturing organism segments the process is marked by the characteristic phenomena of chill and fever in the patient.

Still other forms of plasmodium have been noted whose segmentation takes place in the bone marrow, the liver, spleen, and other viscera. Not enough is at present known about them to classify them properly as far as concerns their evolutionary changes and the symptoms which they produce. All that one may say is that they are irregular, and the symptoms are likewise different from the ordinary type in irregularity of seizures and 
continuance of fever. Among these we may recognize a ringlike, highly refractile, and imperfectly pigmented form, a flagellate form, and the crescent form of Laveran. This last-named variety is especially connected with the æstivo-autumnal fever, and produces a very troublesome attack. But no matter what the form may be, all are attacked by leucocytes which keep up a. militant phagocytosis. As a result of pigment production, of

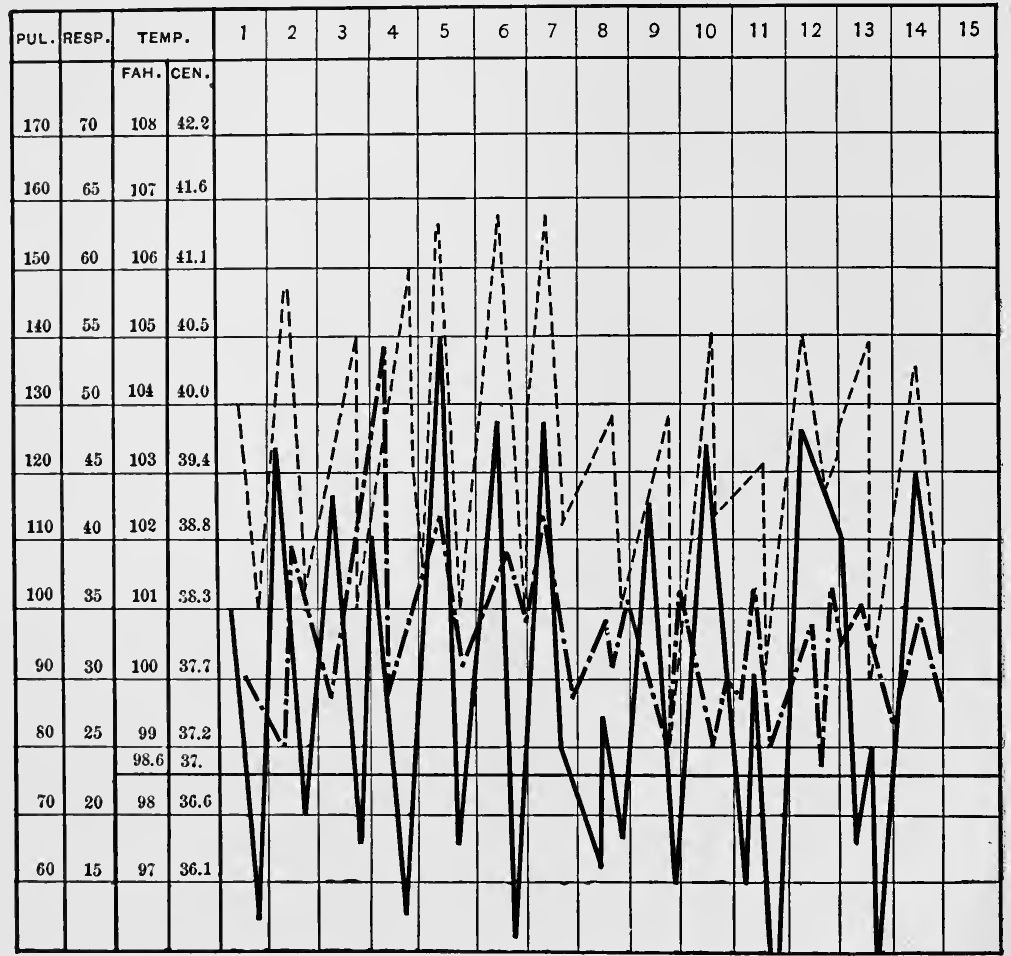

PULSE, RESPIRATION AND TEMPERATURE CHART OF INTERMITTENT FEVER. AGE, 6 YEARS.

PULBE, RESPIRATION

FIG. 116.

breaking up of red corpuscles, and the formation of free melanin, a more or less active melanæmia may exist; and in severe cases marked by a large mortality of red corpuscles there will be a corresponding anæmia and hæmoglobinæmia. The spleen and liver undergo interstitial changes, the mucous membrane of the gastrointestinal track and the kidneys show evidences of acute degener- 
ation, and a possible thrombosis of the cerebral capillaries may occur.

Symptoms. - Inoculation experiments show an incubation period of ten, eleven, or twelve days ; in the unclassified irregular forms the period ranges from three to five days. The doubletertian and tertian types are often seen, but the quartan, the double- and triple-quartan are rare. The paroxysm is divided

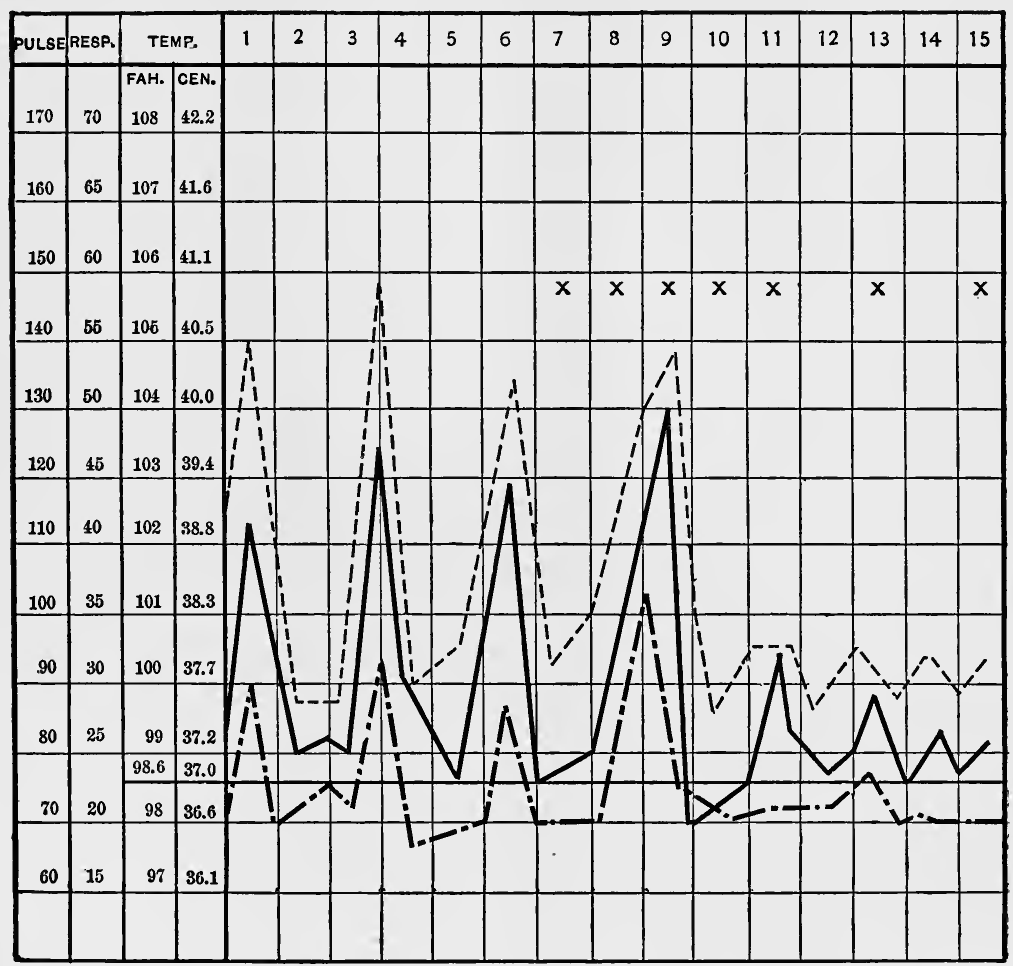

PULSE, RESPIRATION AND TEMPERATURE CHART OF TERTIAN INTERMITTENT FEVER. AGE, 9 YEARS.

$X=$ QUININE' PULBE TEMPERATURE

Frg. 117.

into a cold and hot stage. The onset of the first is marked by weariness, lassitude, disturbances of the gastro-intestinal track, general pain, and finally a chill. The child feels cold and depressed; the surface feels cold, but the temperature of the rectum begins to rise. This stage lasts from a few minutes to 
one and a half or two hours, and is followed by a fall of temperature and a return of warmth which gradually changes to a striking heat. The skin is then hot and dry, the eyes are shining, the face seems congested, the heart action is hard, forcible, and rapid. After an interval of from one to three hours, the skin is bathed in sweat, the temperature falls to about the normal, and the child drops into a quiet sleep.

There is usually an enlargement of the spleen and less often of the liver. There may be in addition disorders of the stomach and intestines, irritations of the skin, bronchial or pulmonary congestion. These symptoms are more apt to appear in children than in adults. They likewise are liable to severe nervous and circulatory depressions; the attack may be ushered in with convulsions, or by a chill followed by convulsions. In other cases there will be marked cyanosis and general prostration. In other cases periodic frontal headaches constitute one of the main features of the disease; occasionally the child complains mostly of indefinite depression, lack of energy, general massive pains. Occasionally one sees distinct nervous manifestations that stand out prominently in the mixture of indefinite symptoms: in one child there may be a localized neuralgia, oftenest in the face, arms, or chest; in another a multiple neuritis, in another a diarrhœa of a purely nervous type. The pernicious form is very rarely seen, and when it does occur it is usually in a child who has recently come from a warm or tropical climate. Some mention should be made of those very irregular cases, which generally occur in the autumn, characterized by continuous fever, or gastric disorders and general prostration which persist for several days at a time.

Among the cases occurring in the autumn and the latter part of the summer - the astivo-autumnal type - there is a notable percentage which run not only an irregular but also a severe course. The attacks may be long drawn out, so that they occasionally coalesce and give a continuous fever; or they may be irregularly intermittent; in other cases the paroxysms may at first be not distinctive, but may represent a combination of small chills, gastric disturbances, anæmia, and marked depression. Some of these irregular attacks may be acutely dangerous to life, and on account of their destructive course have been called pernicious malaria. This term includes the so-called algid, comatose, and hæmorrhagic forms. 
In the large cities, where sanitation is steadily improving, the type of the disease seems to be milder and less characteristic than in former times. There is more and more variation from the classical, intermittent form, from the ordinary manner of onset. In place of the regular picture, one sees a periodic recurrence of signs that usually belong to some common visceral or functional disturbance. In such cases diagnosis is often very difficult until the blood has been examined and quinine prescribed. In these children, as well as those who have too soon abandoned treatment, the sub-acute and chronic forms of malaria occur and present the signs of marked anæmia, various and indefinite functional disorders, nervous symptoms, enlarged spleen and liver, and visceral disturbances. All the phases of pronounced secondary anæmia are present, including diminution of red and white blood-cells, dyspnœa, hæmic murmur, and great general depression. The patient's resistance to disease is much reduced, so that he easily contracts intercurrent disorders. The picture of this malarial cachexia bears a notable resemblance to that of pernicious anæmia, although it more readily responds to appropriate treatment.

Treatment. - The preventive care includes living in a welldrained locality and the drinking of pure water. If the ground is wet or marshy, it should be reclaimed; but often the quickest and surest preventive is removal to a healthier place. When the disease has found a lodgement, it must be combated with quinine. The least objectionable salts for internal administration are the bisulphate and hydrochlorate, dissolved in water, or, for older children, enclosed in capsules. The method which I have found most useful is to give three doses per day, the one before the paroxysm being twice as large as the others. Thus, for a child of five years whose paroxysm occurs at noon every day, the early morning dose would be 0.2 gramme (gr. iij ss), the dose before noon 0.4 gramme (gr. vij), and the evening quantity the same as the morning. The large dose will kill the newly formed spores produced by segmentation, and the expected chill of forty-eight hours in advance will not occur. If the child is unable to take the drug by the mouth, it may be administered in double quantity by the rectum. In a few urgent cases where the depression and prostration are very urgent, the drug may be given hypodermatically; for this purpose the bimuriate may be used in doses of $0.1 \mathrm{gm}$. (gr. ij). During the chill and the fever the care of the patient is purely symptomatic; for 
the first one may use heat in various forms - hot-water bags to the feet, sufficient blankets, hot drinks. Some children find relief from a very hot bath, followed immediately by being wrapped in warmed blankets. For the fever, one may use cool baths, sponging with alcohol and water, and the application of an ice bag to the head. The sub-acute and chronic cases improve best under the administration of arsenic with small doses of quinine, or such a mixture as arsenic and strychnine. If the spleen and liver continue large in spite of these drugs, some improvement may be obtained by massage and systematic exercise. All cases, when convalescence sets in, require the use of iron, with which arsenic may often be advantageously combined, until the anæmia has passed away. Finally, obstinate cases and those that are left much weakened are benefited by removal to a healthy neighborhood where the conditions of soil, climate, and general mode of life are favorable.

Prognosis. - The outlook is almost always good. In temperate climates the disease is very rarely fatal. The main question is one of diagnosis in irregular and doubtful cases, which an examination of the blood will deternine. Unless treatment is very thorough and long continued, the likelihood of recurrence is great, especially in the late spring. In some cases the disease, after the lapse of a variable time, is very apt to assume one of the nervous forms with a sub-acute or chronic course. These in turn demand patient treatment and untiring watchfulness for their successful handling.

Differential Diagnosis. - The fact that malaria is so multiform in children permits of some confusion; nevertheless, this disease is too often used as the most convenient term for a congeries of symptoms that is not thoroughly understood. A malarial attack with cerebral symptoms has been mistaken for meningitis, or the stupor that one occasionally sees has been diagnosed as heat stroke. Attacks accompanied by vomiting and diarrhœa have often been called gastro-enteritis or ileo-colitis. Malaria has been diagnosed as typhoid fever and vice versâ, but the Widal reaction in the one and the presence of the plasmodium in the other are characteristic tests. The instances of confusing malaria with tuberculosis, broncho-pneumonia, septicæmia, pyæmia, endocarditis, and pyelitis are classical. The best way to guard against these errors is to keep as close as possible to the few tests of which we are certain. A thorough examination of the blood is the best of all ; in addition, 
one should try to obtain a history of residence in malarial locality, to distinguish the element of periodicity and an enlarged spleen. The diagnosis can be clinched by noting the effect of quinine. If there is any delay in effecting improvement, the case is either not malaria, or a complication with some other disease. In some irregular cases, which by the way are apt to occur in the autumn, the blood-examination may be doubtful; it is then advisable to puncture the spleen with a fine needle to obtain a sample of blood that commonly gives a large amount of plasmodia. Before taking this step, one must assure oneself that the swelling of the spleen is not due to syphilis, to rachitis, anæmia, leucæmia, or Hodgkin's disease.

\section{Weil's Disease}

This disease was first described by Weil in 1886 . It occurs as the result of infection by bacillus proteus fluorescens, which was isolated and named in 1891 by Jaeger. The patient becomes contaminated by eating decomposing meat and drinking infected water. The bacilli are found in the urine, the internal organs, and pus cavities.

Lesions. - The lesions consist of a fatty degeneration of the kidneys and a cloudy swelling of the renal epithelium; the liver shows a fatty degeneration, and a similar appearance may be seen in the spleen. In these and other viscera there is a variable degree of small-celled infiltration, and in one or more organs various amounts of hæmorrhage may occur. In general terms, the changes may be said to represent an acute parenchymatous inflammation.

Symptoms. - The symptoms cannnot be readily interpreted; in their simplest form they consist of headache, moderate fever, prostration, and abdominal pain. The liver and spleen are slightly enlarged, and possibly tender; there is almost always some degree of jaundice, and commonly the patient complains of articular pain. There may be nausea and vomiting, and possibly constipation. The stools on account of the obstructive hepatitis are dry and light colored. Albumin may commonly be found in the urine, at times accompanied by casts, and rarely by blood. The patient has a liability to pus-formations.

Treatment. - The treatment is largely symptomatic. The patient should be confined to bed, and the bowels must be emptied 
by small and repeated doses of calomel followed by a saline. The body should be sponged two, three, or more times a day; according to the range of fever. The food should be fluid and easily digested, and stimulants may be needed in liberal doses. The main drugs that are indicated are a few doses of bromide of soda to relieve pain and irritability, followed, when these symptoms have subsided, by strychnine or nux vomica, with or without the compound mixture of rhubarb and soda. The course of the disease is rarely longer than a week or ten days, and in most cases the patients recover.

Differential Diagnosis. - The disease may possibly be confused with acute hepatitis, typhoid fever, relapsing fever, and acute arthritis. The main diagnostic features are the enlargement of liver and spleen, jaundice, abdominal and articular pain, and the short course. If Jaeger's bacillus proteus fluorescens is found in the urine or a pus cavity, the diagnosis is at once confirmed. 


\section{CHAPTER XXII}

\section{NERVOUS DISEASES}

\section{Acute Leptomeningitis}

ThIs disease is known also as purulent meningitis, acute internal meningitis, acute simple meningitis. It is an inflammation of the pia mater, which commonly involves the arachnoid and often the inner layer of the dura mater.

Causes. - The disorder is generally due to bacterial invasion. It most frequently follows the acute infectious diseases, such as acute lobar pneumonia, erysipelas, ulcerative endocarditis, septicæmic processes. Or it may begin with an injury which is not great enough necessarily to produce fracture of the skull or spinal bones. In some cases it may not be possible to find any cause, and one may hear such cases referred to an indefinite over-exertion, heat stroke, exposure, possibly a fall.

Lesions. - In addition to the congestion of the vessels, there is an exudation of serum, fibrin, and pus in varying quantities. The three factors may be evenly mixed, or any one may predominate over the others. The serum, pus, and fibrin are held in the meshes of the pia mater so that the membrane looks thick and œdematous. Fibrin and pus may be collected in thick, heavy lines along the blood-vessels, or several of these lines may coalesce and cover considerable areas. When the exudate is very plentiful it may fill up the spaces between the convolutions, and form a cast of the convexity. It may make its way from the pia mater along the vessels into the cortical substance of the brain, which thereupon becomes infiltrated, œdematous, and degenerated. There may be a large number of red and white blood-cells in the exudate which get there by hæmorrhage, or diapedesis, and emigration. Cultures will show the presence of one or more varieties of bacteria according to sort of infection present. These, with the exudate, may extend to the ventricles and along the cord. The changes which are thus produced may persist for weeks after the acute inflamma- 
tion has subsided. There is a strong possibility of the sequence of chronic meningitis.

Symptoms. - The disease usually begins in a quiet, gradual fashion. The child complains of weariness, dizziness, and nausea. He is stupid, cross, and complains of indefinite pains. These symptoms may improve, remain stationary, or increase. Occasionally they may so far improve that they ultimately pass away. As a rule, they very soon become more serious; the nausea may be followed by vomiting, or vomiting may come without warning, before or after meals. It is projectile, more or less violent, and often is not associated with any gastric disturbance. Weakness increases very rapidly, the temperature rises irregularly, often attains a height of $40^{\circ}$ or $40.5^{\circ} \mathrm{C}$. $\left(104^{\circ}\right.$ or $105^{\circ} \mathrm{F}$.), and the pulse changes from quick to slow and irregular. There is much pain in the head which the child commonly refers to the forehead. Gradually the restlessness gives way to apathy; photophobia becomes marked and convulsions may now appear. Constipation is marked, the abdomen is retracted, the pupils lose their contraction, and become dilated on one or both sides. Following the convulsions there may be a spastic rigidity of the neck or limbs, and in some cases opisthotonos sets in. This condition lasts only a short time; without much delay the disease begins to resolve or hurries on to a fatal end. On the one hand, the symptoms may gradually become milder, in some instances falling into a sub-acute or chronic state. On the other, partial paralysis may set in, there is loss of sphincter control, and vaso-motor disturbances develop progressively. The respiration assumes the Cheyne-Stokes type, the child becomes blind from optic neuritis, falls into unconsciousness, and finally dies.

Treatment. - The care of these cases is symptomatic. The sick-room is to be kept dark and quiet. The diet is to be fluid and easily digestible. The bowels are to be moved as often as necessary by means of calomel and a saline, or by enemata. An ice bag should be kept on the head, and the pain and restlessness may be overcome by antipyrin given in solution with a cardiac stimulant. If the stomach is unable to retain the food and medicine, they should be given by rectum.

Prognosis. - Complete recovery is not common. Of course there are cases that seem to bear no traces of the past disease ; but, as a rule, those children who survive are left more or less incompetent in mind or body, or both. The extent of the lesions and the 
degree of severity have much to say in the probable outcome of the sickness.

Differential Diagnosis. - There is much difficulty, in the general run of cases, in promptly arriving at a diagnosis. Usually the mistake of too rapid recognition is made, so that the cerebral symptoms which attend some attacks of the acute infectious fevers and otitis media are wrongfully interpreted as signs of leptomeningitis. One should keep in mind the main characteristics of the disease, - the gradual onset, pain in the head, projectile vomiting, unequal pupils, convulsions, and stupor.

\section{Chronic Leptomeningitis}

Chronic inflammation of the pia mater usually has a history of a preceding acute attack. In some few cases its course from the outset may have a chronic character. In the latter case syphilis is the only known cause, although there are cases which arise from a different ætiology. Occasionally we see a case which has an apparent tubercular connection; but as a rule we are not justified in making a more definite assertion concerning this cause.

The lesions are the natural outcome of the acute changes. The pia mater is thickened in areas of various size, it has deposits of connective tissue, and is bound down by adhesions to the brain or dura mater, or both. The fourth ventricle may be shut off, and some distension of the lateral ventricles usually results. In the exudate there may or may not be a small amount of pus. The Pachionian bodies may be increased in size.

The symptoms are of the same nature as those of the acute form, but instead of running a steady course they come in remissions and exacerbations. There are periods of stupidity and pain in the head; there may be nervous disorders and irregularities of the pupils as well as of visual function. During the exacerbations there may be spastic rigidity, exaggerated reflexes, convulsions, vomiting of a projectile type, and even opisthotonos. If the amount of fluid in the closed ventricles is sufficiently large, the child will suffer from pressure effects, such as epileptic attacks and paralysis involving a large or small area.

Treatment. - Since the only direct, known cause of this condition is syphilis, iodide of potassium should be given whenever there is any reason to suspect the existence of this specific disease. Otherwise the treatment is general and symptomatic. Thus, in 
those cases which give a tubercular history, one can prescribe no one drug which has an inevitable value for all.

Prognosis. - The disease may continue for two, three, or even many more months. In all excepting the syphilitic cases the chances of recovery are very small. But even if, as a very unusual occurrence, recovery should take place, it will be under such conditions of mental and physical disability that little would thereby be gained.

Differential Diagnosis. - The main diagnostic points are involved in the chronic course, apathy, vomiting, headache, convulsions, and opisthotonos. It will not be difficult to distinguish it from tubercular meningitis, for the evidences of tubercular history and infection are wanting. Marasmus may infrequently give a certain amount of opisthotonos, but this one factor is not enough to confuse the two diseases. The chronic inflammations of the ear may in their acute exacerbations provoke some cerebral symptoms, but if from this cause a meningeal inflammation resulted, the course of the disease would not be very long.

\section{Pachymeningitis}

Inflammations of the dura mater may be confined to the external layer or the internal layer, and are accordingly named. Generally, however, the inflammatory process is not so sharply marked, and may penetrate from one layer into the substance of the membrane, and even into the other layer. It may be acute or chronic.

Acute external pachymeningitis generally follows injuries of the skull and disease of the mastoid cells, the inner and middle ear. The inflammation produces congestion, thickening, and softening of the dura mater. The affected area is generally small; on it pus collects and thence may spread in all directions. In other cases the process may be confined to the original space, and finally be absorbed.

Acute internal pachymeningitis may follow inflammation of the outer layer, the acute infectious diseases, pyæmia, syphilis, or syphilitic bone disease, or may occur without any known cause. The membrane is congested, thickened, and covered with pus.

The symptoms of the external form are clearly distinguished only when the inflammation is so confined that local pressure effects are produced. In children the disease generally compli- 
cates mastoid or middle-ear suppuration, and gives no especial signs. The internal form practically never gives separate symptoms, for in its inflammation it involves the pia mater, whose symptoms are so impressive that they alone can be seen.

Chronic pachymeningitis is almost always internal. There is a chronic thickening of the membrane, with the formation of connective tissue and adhesions. When the process involves the outer layer as well, the whole membrane may become bound down to the skull. In children the most important form of chronic pachymeningitis is the so-called hæmorrhagic internal meningitis. Its causes are unknown, and in some cases it is not distinctly recognized until autopsy. Examination shows the presence of fine connective tissue which supports in its meshes a large number of fine vessels, the outgrowth of the dural vessels. From these capillaries numerous extravasations of blood as well as hæmorrhages may take place. The amount of blood varies from a minute to a fairly large amount. In the intervascular spaces are variously shaped cells and blood-cells, pigment, and chalky concretions. The new tissue becomes gradually thicker and denser, and finally its vessels are unable to withstand the increasing fibrosis. Under this fibrous covering large and small hæmorrhages occur through the delicate vessel walls, and with the newly formed tissue produce varying amounts of pressure upon the brain. These pressure effects may be thus created under any part of the dura mater, although their usual site is on the convexity.

Symptoms. - The less severe forms of pachymeningitis may, and commonly do, give no symptoms during life. When, however, a hæmorrhage occurs, the effect is directly seen in nausea, vomiting, convulsions, unconsciousness, followed by paralysis in commensurate degree of the parts of the body which are controlled by the compressed brain. The respiration is slow, irregular, and sometimes of the Cheyne-Stokes variety. The pulse is likewise slow, irregular, and soft. There is little rise of temperature, and in mild cases there may be none. In such cases, after a period of active cortical irritation, the symptoms subside, and the child recovers, bearing usually some partial paralysis. Death may take place immediately, or after repetitions of the attack, or may be delayed indefinitely.

Treatment. - The care of these cases is purely symptomatic. The child is to be kept very quiet; ice bags must be applied to the 
head, and the bromide of soda or antipyrin should be given in sufficient doses to still the signs of cortical irritation.

The prognosis depends upon the size of the hæmorrhage; small ones are usually not fatal, large ones are commonly so. At the beginning of the attack one can never tell how extensive the process is going to be, nor whether it will recur. While we are acquainted with this tendency to repeated hæmorrhages, nevertheless we not very rarely see traces of these changes in children who died from other causes, and in whom the existence of an old pachymeningitis was never suspected until after death.

It is not always possible to make an exact and prompt diagnosis. When the pachymeningitis occurs in the course of some other disease, or without marked pressure symptoms, it is often very difficult to differentiate or even to recognize its existence. Progressive muscular atrophy and myelitis can usually be barred out with no great difficulty. From simple acute meningitis it may be distinguished by its more rapid development, quick growth of pressure, and little fever.

\section{Spinal Meningitis}

Inflammation of the spinal meninges generally occurs as an extension of a similar process in the cerebral membranes. While a direct inflammation of the spinal meninges may possibly be caused by trauma, and infection which follows trauma and operations, nevertheless, such cases are rare; and our usual experience leads us to expect an involvement of the spinal membranes in a fair proportion of the cerebral cases of all kinds. The attacks may be acute or chronic; when the latter is the case, the disease is usually located in the dura.

The symptoms of internal pachymeningitis of the spine are not easily dissociated from those of cerebral meningitis. In addition to them there will be pain and tenderness along the back, spasmodic rigidity in the back, extremities, or chest. If the spinal nerves are compressed, there will be corresponding symptoms along their distribution; and if the cord itself feels the pressure of the swollen membranes and their exudate, the necessary results of interference with movement and feeling may ensue. When the pressure is long enough continued it leads to atrophy of the substance of the cord. 
In the acute spinal leptomeningitis the cause is almost always dependent upon injury or infection. Here also the possibility of extension from a cerebral leptomeningitis is great. The symptoms are preceded by those of the active cause. Then there may be vomiting or convulsions followed by tenderness along the spine, rigidity of the back, flexure of the limbs, and retraction of the head. As the medullary substance becomes involved, there may be cardiac disability, the respiration may be difficult and often assumes the Cheyne-Stokes type. Spastic contractions of the muscles, of the rectal and vesical sphincters, will give their characteristic signs. As the condition is prolonged, paralysis, anæsthesia, and contracture may follow the distribution of the nerves whose roots are involved.

In the rare cases that recover, a chronic process is very apt to ensue. The symptoms are the natural extension of those in the acute form.

The treatment of all these varieties is symptomatic, excepting in the few cases where operation is attempted.

\section{SYRINGOMYELIA}

Syringomyelia, or as it has lately been called, myelosyringosis, is a chronic disorder of the spinal cord which is marked by the growth of gliomatous or glio-sarcomatous tissue near the central canal; this newly formed tissue breaks down, thereby forming cavities. The disease is seen in boys oftener than in girls. While any part of the cord or even the adjacent part of the brain may be involved, the lesions are generally situated in the upper dorsal and lower cervical regions. The cavities vary in number, size, and shape, and are filled with fluid; there is a delicate lining membrane, outside of which are the gliomatous or glio-sarcomatous cells. From the central portion of the cord the new tissue may invade the anterior and posterior horns, and the posterior and lateral columns.

The symptoms are focal, and thus follow closely the involvement of various parts of the cord. The destruction of the anterior horns produces muscular paralysis and atrophy, and loss of reflexes; and the parts affected will vary with the location of the new tissue. The central gray matter when attacked will give vaso-motor symptoms and trophic disorders. When the posterior 
columns are invaded, there will be analgesia and sensory disturbances. The lateral columns as soon as they are attacked produce exaggerated reflexes and stiffness of the legs; while the posterior columns cause incoördinated movements, deficient reflexes, and hyperalgesia. In each case the symptoms can easily be deduced from the known functions of any segment of the cord, and, vice versâ, the segment involved can be readily diagnosticated by following up the several symptoms to their necessary source.

Myelosyringosis, which may occur in late childhood, must be separated from hydromyelia, which is a congenital dilatation of the central canal of the cord. Also it must be distinguished from hæmorrhage, whose onset is sudden, and tumors. If the new growth is syphilitic or tubercular, there will be symptoms of constitutional disease. In other neoplasms there will rarely be the symptoms of more than one diseased locality, the opposite of which may exist in myelosyringosis.

The disease progresses in a series of exacerbations and remissions which are irregular in intensity and length. The final prognosis is bad, but there is no known method of influencing the disease, outside of symptomatic and general care.

\section{Myelitis}

The fact that inflammation of the cord is very rare in patients under the age of puberty, and that there is a noticeable sameness of the disease as it occurs in children and adults, make an extended account of it unnecessary. It may follow the severe infectious diseases, Pott's disease, or injury, and doubtless has in some cases an infectious nature. Its course may be acute, sub-acute, or chronic.

Lesions. - The dorsal region is most often attacked, and the process may be transverse, disseminated, or diffuse. The affected part is congested and swollen, and one cannot plainly see the distinguishing characteristics between the gray and white parts. The consistency of the cord has changed and become soft; in such areas one may see dilated vessels, leucocytes, granules of myelin, and corpora amylacea. When a recent hæmorrhage has taken place, the color of the softened part is red, but with the passage of days the red changes into yellow. The softness varies in different cases from a slight degree to one of fluidity. As the 
result of secondary changes, the tracts in the cord will be involved upward and downward, according to the direction in which their impulses are directed.

Symptoms. - The beginning of the disease is usually steady and gradual. As the lesions develop, the symptoms naturally follow their location. Complete transverse myelitis causes impairment of motion and sensation, disorders of rectal and vesical functions, and disturbed reflexes. If the cervical part of the cord is attacked, there will be paralysis of the upper and lower extremities, the first stage of which is flaccid, the second spastic. Anæsthesia exists in all four limbs as well as in the body as far down as the plane of the lesion. In addition there may be disorders of the pupil and unilateral vaso-motor disturbances. In dorsal lesions the arms are not affected, the lumbar region is attacked by secondary degeneration, and the eyes are not involved. With an affection of the lumbar region, the arms and legs are paraplegic and anæsthetic; rectal and vesical sphincter control is impaired, and knee reflex is lost. An indication of the diseased locality is the distribution of anæsthesia; these areas must correspond to the parts that are supplied by nerves which lead off below the level of the lesion. At the superior margin of the anæsthetic area a zone of hyperæsthesia exists. In some cases there may be a distinct girdle sensation which defines the boundary line between the healthy and abnormal sections.

The affected muscles show the reaction of degeneration; involuntary spasmodic twitchings are almost universal, and trophic disturbances, in the form of bed-sores, are common. There may be disturbances of temperature, but they are quite atypical, varying according to the pathological processes which are present in each case.

When the myelitis is disseminated or diffuse, there will be a mixture of the localizing symptoms according to the parts involved. The process in practically all cases is slowly progressive, and as the acute form changes into the sub-acute and chronic the temperature falls, complications of various sorts ensue, and the patient may finally die from their action or that of the original inflammation.

Treatment. - The care of these cases is largely general and symptomatic. Where there is a known preliminary disease it naturally must receive its full share of attention. 
The inflammation of the spinal cord calls for complete rest in bed, the clearing of the intestinal canal by means of calomel and a saline, the administration of nutritious and easily digested food, and the careful catherization of the bladder. The various symptoms as they appear must be wisely attended. Ice bags may be applied to the back, and mild counter-irritation may be used, but always with the thought that it may easily start an obstinate irritation of the skin and trophic disturbances of serious import. Such drugs as potassium iodide and ergot have in past times been recommended, but no good need be expected from them. It is possible that surgical means may be found to open the vertebral canal, wash it out, and so reduce in part the inflammation as well as the amount of exudate.

The prognosis in these cases is bad; a few mild cases recover, but usually leave behind them permanent traces of their former presence. The sub-acute and chronic cases usually die from exhaustion or intercurrent disease.

Differential Diagnosis. - The diagnosis may be made from the slow and steady unfolding of the symptoms, the permanent nature of the anæsthesia, disturbed rectal and vesical control, and the atrophy and flaccidity of the muscles at the level of the lesion, combined with spastic contraction of those below that level. Usually it is not difficult to distinguish myelitis from spinal meningitis, for the latter is very rare unless it occurs with cerebral meningitis or trauma, and is marked by pain in the affected region as well as some degree of neuralgia in the nerves of its distribution. Hiemorrhage may be marked off by its very sudden onset, its great and immediate pain, possible lack of fever, and the gradual abatement of symptoms as the effused blood is absorbed.

\section{Compression of the Spinal Cord}

This condition, synonymously called compression myelitis and Pott's paralysis, is the result of pressure upon the cord and its membrane by caries of the vertebræ, injuries, tumors, and aneurism. In the large majority of cases tuberculosis of the vertebræ is the active cause.

Lesions. - The bones enter upon a course of tubercular degeneration which may involve the intervertebral cartilages. As the result of the weight contributed by the parts above, the spinal 
curve is broken, and a displacement to the rear is produced. The cord is caught in the narrowed channel, which is made still narrower by the accumulation of the products of inflammation in front of the cord. 'The cord is somewhat œdematous, its color becomes pink or reddish gray, and the differentiation between the white and gray matter is gradually lost. The dura mater is commonly involved, so that a synchronous pachymeningitis takes place. In the cord there is an increase in the interstitial tissue,

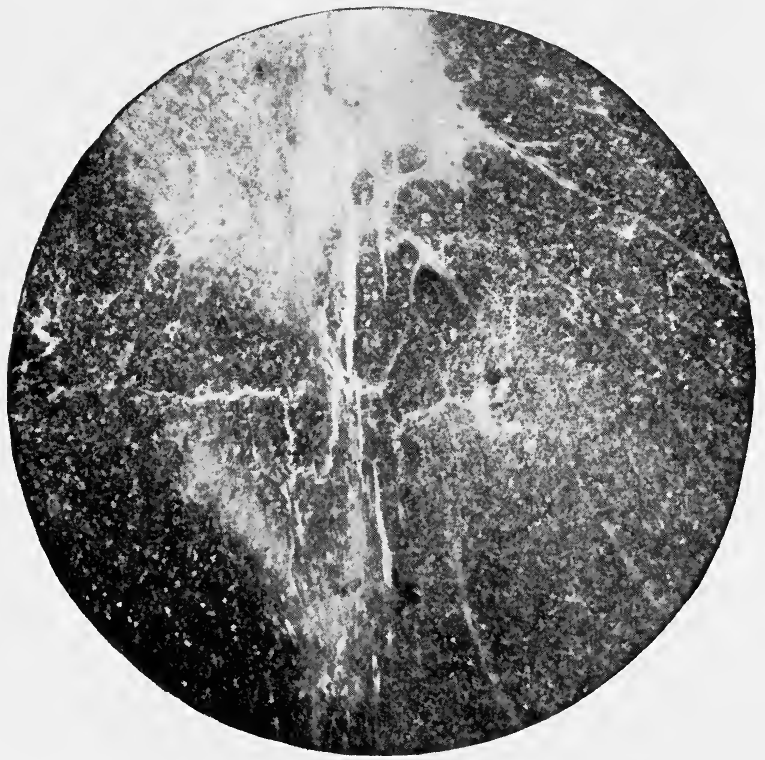

FIG. 118. - Compression of the Spinal Cord : Degeneration of Descending Columns. $\times 25$. (The light portions represent the spreading degeneration.)

a cellular proliferation, and a progressive degeneration of nerve elements. Outside of the tissue directly affected, there will be ascending and descending degeneration, and the peripheral nerve roots will naturally respond to the degree of strain and irritation that is put upon them.

Symptoms. - The signs of the disease may not show themselves until some time after caries begins; and on post mortem examination one may be surprised at the comparatively small amount of disturbance which the pressure has caused. The symptoms are generally referred to the cervical and dorsal regions, where tuberculosis of the vertebræ is usually found. From the 
parts involved pains will start, following the distribution of the various nerves. Hyperæsthesia of the surface is often the precursor of anæsthesia; and with this condition there is a gradually increasing spastic paralysis of the legs, or of the legs and arms. Contractures are not common. The inflammation may now spread from the point of pressure, and gradually take on the character of a myelitis; in fact this is the usual course of development, whether the lesion is originally unilateral or bilateral. In this event the picture will finally merge into that of a myelitis of the ordinary type with atrophy, contractures, loss of rectal and vesical control, and bed-sores. Among the rare manifestations of the disease are paralysis of the diaphragm, irritation of the phrenic nerve, gastric and pupillary disorders.

Treatment. - The general care of the child is very important. Diet, hygiene, and sanitation must be of the best. The child should be confined to bed, and as soon as the acute symptoms have passed, he should wear a plaster of Paris jacket. As this remedy indicates, relief from the pressure is one of the main objects desired. Tonics should be administered in order to help nature in the conservative effort to throw off the disease. Other means, such as counter-irritation, ergot, and potassium iodide, have been used; but their value is doubtful.

A logical method consists in surgical measures that are devised to take away the diseased bone. While the attempts have heretofore not been successful, nevertheless they should not be abandoned; and although children bear operations upon the skull and spine poorly, the only real hope of permanent improvement lies in such radical measures. At the same time it is only fair to state that surgeons do not regard the operation favorably, because it is apt to be so difficult and dangerous.

Prognosis. - Each case must be judged by itself. If treatment is promptly begun and pressure removed, a favorable outlook is not unusual. The farther the symptoms have developed, the poorer are the chances of recovery. Nevertheless, at any stage of the disease hopes for betterment should always be entertained. Death may occur from exhaustion, from medullary irritations of an extreme degree, from dislocation of the odontoid processes, or from intercurrent disease.

Differential Diagnosis. - The existence of no other cause than the fact of or the predisposition to tuberculosis, the gradual course 
of the disease, the weak spot in the spine with or without kyphosis, the vertebral stiffness, radiating pains, and increased reflexes, mark off compression of the cord from myelitis. The fact that the latter disease may be a sequel to the symptoms mentioned above will render confusion not unusual.

\section{Infantile Spinal Paralysis}

This disease is known as infantile spinal paralysis, acute anterior poliomyelitis, and the essential paralysis of children. It occurs more frequently in warm than in cold weather, and attacks boys oftener than girls. In the majority of cases the patients are under five years of age, the largest number being in the second year. Its causation has in the past been attributed to a wide variety of unfavorable conditions, which in the last analysis we may regard as coincidental rather than ætiological. On the other hand, observation of a large number of cases scattered over several years tends to convince one that the origin of the disease is purely infectious, that it may occur in epidemics, and that the true ætiology must be sought in some specific micro-organism or poison.

Lesions. - The cord is commonly attacked in the lumbar or cervical regions, and the changes may be confined to these parts, or may be scattered irregularly over a considerable extent of tissue. The anterior gray horns bear the brunt of the attack, and in them the ganglion cells undergo shrinkage, pigmentation, degeneration, and atrophy. The same processes may by extension involve the gray and the white matter, the anterior and lateral columns. In these parts, as well as in the anterior horns, there may be a deposition of connective tissue that helps to change the normal form and cause depressions and pittings. The peripheral nerves, which lead off from the involved cornua, show similar degenerative changes, but their destruction is usually incomplete. Likewise the muscles that are supplied by the affected cornual cells undergo a process of atrophy by which they lose their structure, and in extreme cases are turned to fibrous bands in which there may be a fat-infiltration. Even the bones to which the paralyzed muscles are attached show the effect of the paralysis. They are smaller, thinner, and weaker than they normally would be; but this change comes, doubtless, from the inability of the muscles to provide exercise. 
Symptoms. - Very often the disease begins as any other acute infectious disorder, but without recognized prodromata. In two cases I obtained a history of an initial chill; then one may be told of a sudden rise of temperature, projectile vomiting, sometimes of convulsions and coma. The parts that are to be paralyzed are generally painful, but the amount of pain varies within considerable limits. The variations in severity of all these symptoms are considerable: the convulsions may or may not be repeated, the vomiting may be trivial or alarming, the temperature may rise only a little above normal or may reach $40^{\circ} \mathrm{C}$. $\left(104^{\circ} \mathrm{F}\right.$.). These acute symptoms may last for as short a time as a day, or may be prolonged until a week has passed. A dysarthria may be present that may easily be mistaken for a partial aphasia. From the first hours of the sickness one may recognize the paralysis in limbs or body; generally, however, the strain and stress of the acute onset draw the attention from the muscles, and the paralysis is not noticed until these acute symptoms have passed.

The paralysis now remains as the principal feature of the disease. Often it bursts out in a stormy manner which after a few days spends its force, leaving certain parts more or less permanently affected. This paralysis may be denominated flaccid; the parts become atrophied, their reflexes fade away to a greater or less extent, and the electrical reactions are those of degeneration. The final paralyses may be situated in various parts of the body, but certain ones are more commonly affected than others. The left leg is the favorite seat of the disorder, followed in frequency by the right leg; a leg and the opposite arm are a fairly common combination; while all four extremities, both legs and both arms, are much more rarely affected together. Involvement of the trunk muscles is not often seen. The extensor muscles of the leg are oftener attacked than the flexor, and in the hand and forearm a similar rule holds good. As a result, the deformities which we usually see are of the type of drop-foot, talipes equinus, equinovarus, or valgus, flexed knee, and flexed thigh. Flexion of the wrist and fingers is seen, and occasionally a subluxation at the shoulder joint follows deltoid atrophy. Deformities of the back and spinal cord may occur, particularly lateral curvature.

Treatment. - The treatment of the acute stage is that of all acute infectious diseases; rest in bed, free catharsis, regulation of food, promotion of hygiene and sanitary conditions. Seda- 
tives may be needed to control vomiting, convulsions, and pain. As soon as this stage has passed, the principal care relates to the prevention and cure of deformities. For this purpose one obtains the best results from passive motion and massage, while the benefits to be derived from the commonly employed electricity are doubtful. The main object is to develop whatever muscles or muscle fibres that are left to their best degree of utility. As soon as the child is able to be about, systematic exercise with chest weights, dumb-bells, and the bicycle must be used under skilled supervision. Tonics are generally indicated, such as iron and strychnine; but the latter must be prescribed in small doses to avoid the danger of over-stimulation. Orthopædic splints may be of much use in the presence of deformities. In some cases tendon grafting has been done with good results.

Prognosis. - During the acute attack no reliable judgment of the ultimate outcome can be regularly formed. One should remember that after the first flurry there is a period of rest, followed by some improvement before the permanent paralysis becomes established. In this way one is fairly safe in believing that the final condition will be better than the initial appearance would warrant. The appearance, extent, and duration of the reaction of degeneration - a failure to respond to faradic stimulation and a changed galvanic reaction, so that anodal closure contraction equals or exceeds the cathodal closure contraction will give the best idea of the child's future condition. One must remember that the condition may gradually improve, especially with proper developmental care, during the first six months or year. Danger of death is so small that it need not be seriously considered.

Differential Diagnosis. - The main facts to be kept in mind are the acute onset, the periods of rest and improvement, followed by permanent paralysis, the lack of sensory disturbances, reaction of degeneration, and atrophy of the paralyzed muscles. These facts will mark off the disease from any form of meningitis with its marked cerebral symptoms ; transverse myelitis, with its anæsthesia, exaggerated reflexes, deficient sphincter control, normal electrical reaction, and trophic disturbances, will likewise be excluded; cerebral paralysis gives a picture of spastic instead of flaccid paralysis, contractures, normal electrical reaction, disturbed intellectual faculties, epileptic tendency, and a disposition to hemi- 
plegia; multiple neuritis seldom simulates anterior poliomyelitis, since it gives sensory symptoms, a history of slow growth and previous disease, pain along nerve branches, and shows a tendency to disappear after a few weeks or months. Occasionally confusion has been caused by the muscular disabilities of rickets and scurvy; but here the systemic condition alone, even if we omit the important factor of normal electrical reaction, is sufficient to define the disease.

\section{Multiple Neuritis}

Causes. - Multiple neuritis, also called polyneuritis, designates those pathological conditions in which disease of various nerves, related or unrelated, cause the various symptoms. This disease is the result of some form of poisoning, such as the toxines, or tox-albumins generated in the acute infectious fevers (of which the most prominent is diphtheria, followed by such others as enteric fever, smallpox, scarlet fever, measles, malaria, tuberculosis, syphilis), by various micro-organisms, beri-beri, exposure to cold, alcohol, and the metallic poisons, arsenic, lead, mercury, and phosphorus.

Lesions. - The process is a degeneration of the nerve fibres; there are congestion and swelling of the nerves, and an exudation of serum and fibrin. The fibrillar nuclei are enlarged, the connective tissue between the nerve elements proliferates, and round or spindle-shaped granular cells may be seen about the sheath of the nerve. The process is not alike in all areas; in some the inflammation is interstitial, in others it is parenchymatous. Only in rare cases is the perineurium involved. While the changes are confined to the peripheral nerves, not all parts of them are affected with equal facility, and the farther removed a part is from the mother cell the more readily does it become affected. The fact that there may be associated degeneration of ganglion cells in the cord does not necessarily bear any rigid relation to the changes in the nerves.

Almost any part of the body may be affected, but in children we most commonly meet disorders of the anterior tibial, musculospiral, and peroneal nerves, or stated more broadly - the extensors of the wrist and feet.

Symptoms. - The disease may begin in an acute flurry, but usually the onset is gradual, so that one, two, or three weeks 
elapse before a full development of the signs. The main symptoms consist of motor and sensory paralyses, both being symmetrical and having the same distribution. The affected muscles are flaccid, begin soon to atrophy, and in proportion to the degree of wasting show the reaction of degeneration. Although any part of the body may be affected, nevertheless the disease so often settles in the extensors of the wrist and foot that the resulting drop-wrist and drop-foot are almost characteristic of the disease. With the motor paralysis there is a decrease of sensation accompanied in many cases by pain along the course of the nerve. 'The parts which are farthest removed from the centre are the first to suffer, and one of the initial signs is the inability while standing with the flat of the foot on the ground to raise the toes. From the extremities the paralysis works its way upwards until almost all the muscles may be disabled and atrophied. In unusually severe cases the trunk and neck may likewise be involved, so that finally the child is absolutely helpless. Even in the milder cases there will be diminished reflexes, tremor, incoördination, and the presence of Romberg's symptom (inability to stand erect and firm when the eyes are closed). The development of these symptoms is not absolutely fixed in time, and they may be among the first or the later manifestations of the disease.

In the first part of multiple neuritis normal sensation may change to hyperæsthesia, which in turn gives way to anæsthesia. This sensory disturbance may be complete, including the temperature, touch, muscular, and pain senses. On the other hand this impairment may, like the motor paralysis, vary in degree. Vasomotor changes are commonly seen, producing a shiny and puffy condition of the skin. A characteristic sign when the hand is affected is the gradual tapering of the fingers to a noteworthy extent. Another important diagnostic point is the normal condition in which the rectal and vesical sphincters are allowed to remain.

The form of multiple neuritis which follows diphtheria is common and important enough to require special mention. It may follow any degree of infection, from the slightest to the profoundest, although the latter is the more frequently seen in this connection. It rarely occurs in infants; and when it does break out two or three weeks usually intervene between it and the diphtheritic infection. In most cases the palate is the first part 
to be attacked, and its involvement produces regurgitation of liquids through the nose, and nasal speech. Inspection of the throat will enable one to notice the loss of the normal curve of the soft palate, and the flaccid manner in which it hangs from its attachment. Sometimes the paralysis is not confined to this part, but may invade the throat, face, the extremities, and thorax. Affection of the third nerve produces ptosis, of the sixth produces inability of the rectus externus ; in addition there may be strabismus and dilatation of the pupils. The pharynx and larynx may also be attacked, the latter giving the symptoms of severe, choking cough and patent glottis during the act of swallowing. When the laryngeal muscles and the vocal cords are partly disabled, there will be imperfect tone production and hoarseness. When the paralysis spreads to the thorax, the muscles of respiration, the diaphragm, and the heart may be affected. The resulting respiratory symptoms are progressive attacks of subjective and objective dyspnœea, and thoracic breathing. The cardiac signs are weak and disturbed beat and pulse, which may precede paralysis of the heart muscle.

In other respects the paralysis of diphtheria is the same as the general type of multiple neuritis.

A third form, that, on account of its isolation, is worthy of special mention, is the so-called facial or Bell's paralysis. It may occur after exposure, with acute or chronic ear disease or neighboring disorders, and as a symptom of disease at the base of the brain; the facial nerve will thus have been respectively affected after it emerges from the skull, in the bony canal, and within the skull. The muscles about the forehead, the cheeks, eyes, nose, and mouth will be more or less influenced. As a result there is lagophthalmos which cannot entirely be overcome, inability to elevate the affected side of the nose, flaccid condition of the cheek, and imperfect success in puckering or raising the lips. Attempts to close the involved lids are not successful, and in addition cause a straining of the corrugator supercilii and frontal muscles of the other side; if the child tries to display the teeth, the whole mouth is pulled away from the paralyzed side, and if the tongue is protruded it inclines in the same direction. There are no sensory disorders, and the electrical reactions are changed in correspondence to the degree of paralysis.

Treatment. - The care of these cases is, on the whole, general; 
there is no specific remedy at hand, and, indeed, in most cases none is needed. Whenever the cause or precedent pathological condition can be distinguished, it should if possible be removed. Since the tendency of the disease is toward recovery, our attention should be directed toward the general care of the child, his hygiene and sanitation, the proper regulation of his food, the proper control of his excretory organs. Special symptoms, such as pain and restlessness, may be controlled by heat and sedatives ; and in most cases general tonics are indicated. As a general rule the use of passive motion and massage is of decided benefit; and occasionally some sort of orthopædic splint may be required to give support to exhausted parts of the body. In the heart failure of diphtheritic neuritis the patient is soonest relieved by hypodermatic injections of small doses of morphine with or without strychnine. And for treatment of the respiratory paralysis the faradic current has been much commended. In laryngeal paralysis the danger of inspiring food requires that the child be fed by means of nutrient enemata, or through a tube which is put into the stomach by way of the nostrils and œsophagus. If the laryngeal paralysis is so slight as not to interfere with the ordinary manner of eating, the food should be solid rather than fluid. It goes without saying that all complications should receive their requisite treatment. When improvement has once set in, the continued use of strychnine or nux vomica combined with an organic preparation of iron is likely to give good results.

Prognosis. - In most cases the outlook is good. 'The paralysis begins, becomes worse, and then improves - all within a few weeks. Most cases recover completely, although there are a few where a part or the whole of the original paralysis remains. In those forms where the laryngeal and throat muscles are involved, particles of food may be inspired into the lung with a fatal result. Involvement of the respiratory and cardiac muscles usually signifies a severe form of the disorder with a comparatively large mortality. In all these conditions the general health is more or less undermined, and intercurrent diseases, which are sometimes fatal, are easily contracted. The amount and persistence of the reaction of degeneration is a good index by which to judge of the probable permanence of the disabilities.

Differential Diagnosis. - The history of the cause or antecedent pathological condition may turn one's attention to the possibility 
of multiple neuritis. When the characteristic features of the disease appear, the diagnosis is usually not difficult. These features are the gradual beginning of the neuritis, the great area over which the lesions may be distributed, the coincident occurrence of motor and sensory disturbances in time and locality, the development of wasting in the paralyzed muscles, and the partial or complete electrical reaction of degeneration.

\section{Progressive Muscular Atrophies}

This heading includes a group of disorders the rationale of which is not as yet entirely known. They all have a common feature of steadily increasing weakness and final atrophy of certain muscles. Some of them have their origin in abnormal conditions of the cord, and are called amyotrophies; others begin with disease of the muscular system and are called myopathies. Of the amyotrophies the main variety is known as progressive muscular atrophy, or the Aran-Duchenne type, so called after the men who first described it. The principal variety of myopathies is called muscular pseudo-hypertrophy, and its subdivisions are called after the anatomical distribution of the hypertrophy and atrophy. The causes of these diseases are not known, and how much importance should be laid upon their occurrence in family lines is hard to say. So little is positively known about the lesions that the best course until fuller information is obtained is to leave the matter untouched.

The Aran-Duchenne type of progressive muscular atrophy attacks the hand. The thenar and hypothenar muscles are first attacked, followed by the interossei, the flexors, and extensors of the forearm. Contractions naturally result, and form what is known as the claw-hand. If the process continues, it slowly involves the flexors of the upper arm, the triceps, the deltoid, the muscles of the shoulder, back, and trunk. According to Duchenne the disease may sometimes begin in the inverse order, or even in the legs. One of the first symptoms is a fine fibrillary contraction in the muscles affected; added to this is a moderate degree of change in the electrical reactions. Progressively the atrophy becomes more and more pronounced until the patient is quite disabled. The disease is incurable, but may allow the patient to live for years, until exhaustion, intercurrent disorders, or even an 
unusual atrophy of the muscles of the neck, which interferes with breathing and swallowing, puts an end to life.

The peroneal form of progressive muscular atrophy, known also as the Charcot-Marie-Tooth type, and called by Sachs progressive neural muscular atrophy, usually attacks the extensor muscles of the toes, spreads to the small muscles of the feet, and then works its way upward from one to another, after the fashion of the disease in the arm, until the whole leg may become involved. A later extension may in a few cases finally involve the hand and forearm. In this peroneal form contractures occur, and form pes equinus, pes equino-varus, and club-foot; sometimes the disease is bilateral, and thus produces a double club-foot. Sensory changes may occur, and the reflexes are lessened or abolished. In most cases the reaction of degeneration is present in the affected muscles in proportion to the degree of atrophy. On the whole, the wasting is not so marked as in the hand type.

Muscular pseudo-hypertrophy is usually found in early childhood. Boys are more frequently attacked than girls, but the inheritance is almost always from the mother. At the beginning an increasing weakness is seen in the legs, followed by an increased size of the calf ; in exceptional cases the increase may start in the muscles of the thigh. The child has a clumsy, waddling walk, and on account of his weakness is partially disabled from climbing stairs, or accomplishing similar acts. In some late cases there may be an associated pseudo-hypertrophy of parts of the upper extremity. The weakness of the hypertrophied muscles finally becomes so extreme that the child becomes absolutely helpless. Finally there may be an atrophy of the thigh, arm, and shoulder muscles. There will generally be signs of degeneration: reflexes are diminished, there is a lessened response to faradic stimulation, there may be nystagmus and some feeble-mindedness. The course of the disease is chronic, irregular, and usually ends in death.

Erb's type - the so-called juvenile form of progressive muscular atrophy - attacks children or youths in the manner of a continued atrophy of some of the shoulder, upper arm, pelvic, thigh, and back muscles. After the lapse of a considerable period the muscles of the leg and forearm may likewise become involved. In conjunction with the atrophic process there may in some cases be a real or pseudo-hypertrophy of certain muscles, such as the deltoids, supraspinati, and infraspinati. These stand out very 
plainly in contrast to the withered pectorals, latissimi dorsi, trapezii, serrati, and rhomboids. The condition is sharply marked off from the previous types by the absence of the reaction of degeneration, fibrillary contractions, sensory and visceral disorders. The type is rare and its course is long.

The Landouzy-Dejerine type - the facio-scapulo-humeral form - is seen in very young children. The face is first attacked, and on account of the atrophy in all the muscles excepting those about the mouth the lips project, may become hypertrophied, and thus make the deformity of "tapir-mouth." From the face the atrophic process may spread to some of the muscles of the shoulder and arm; but the flexors of the hands and fingers, the subscapulares, the supraspinati, and infraspinati are not involved.

The treatment for all these conditions is indefinite. The general care and nutrition should be made as good as possible; in most cases the patient should be confined to bed, and given the benefit of massage and passive motion. If he improve enough to warrant greater exertion, carefully regulated exercise may be prescribed. General tonic mixtures may be indicated. Sachs believes in the early and thorough use of orthopædic appliances. Under any circumstances the outlook is not very encouraging.

\section{Hereditary Ataxias}

Under this head are included hereditary spinal ataxia, commonly called Friedreich's disease, hereditary cerebellar ataxia, or Marie's disease, and ataxic paraplegia. These were originally included in locomotor ataxia; but careful observation has demonstrated the separate identity of each. One of the main characteristics of all, is the fact that they occur in families. Any other cause is at all events doubtful. The occurrence of previous injury or acute disease cannot be regarded as more than coincidence.

Friedreich's Disease. - In hereditary spinal ataxia there is a degeneration or lack of development in the columns of Goll, the columns of Burdach, and the pyramidal tracts. In the columns of Goll the whole area is affected; in the columns of Burdach all except the column of Lissauer may be irregularly involved ; and the pyramidal tracts may be also entirely changed. The lesion consists of a diffuse sclerosis. The first symptom is an incoördination of mus- 
cles joined to weakness of the extremities. In the legs this produces an ataxic gait; in the arms it causes inability to perform finely adjusted movements, such as writing, drawing, and carving. In addition there may be involuntary choreic movements that result from efforts to balance the body. There will also be some incoordination of the muscles which are concerned in speech and articulation, so that the former is arhythmic, unchanging in pitch, often nasal, while the latter is jerky and syllabic. Gradually the reflexes become diminished, and finally are altogether absent. As the patient tries to balance himself, the extensor tendons of the toes may be seen to relax and contract. This is all the more noticeable when the disease is so far advanced that the characteristic deformity of the foot has developed. This deformity consists of an over-extension of the first and second toes, and a cupping of the arch, the result of which is very much like pes cavus. Simultaneously there will be a lateral or rotatory curvature of the spine. When the lesions have advanced far enough to attack the medulla oblongata there will be a gradual, development of dizziness, fainting, tachycardia, and sharp, neuralgic pains. In some cases there may be lateral or rotatory nystagmus.

The course of the disease progresses steadily toward death, and may last from ten to fifteen years. There is no special treatment excepting massage, passive movements, and exercise.

Marie's Disease. - In hereditary cerebellar ataxia there are hypoplasia and degeneration in the cerebellum, which may decrease so much in size that its bulk is reduced one-half, or so little that the change is barely noticeable. The cells of Purkinji are much reduced in size and number, while there may be a simultaneous increase in the neuroglia. The symptoms begin with incoördination of the upper and lower extremities, on account of which the arms become more and more useless, while the legs acquire the ordinary cerebellar gait. While the progress of the muscular inability is steady, it may nevertheless be slow. The patient in walking assumes a characteristic attitude : the upper part of the body is bent, the chin is held out and up, the head is thrown back, the widely planted feet and the staggering gait go to make a striking picture. A noteworthy and logical fact becomes evident when the patient lies down; then the incoördination of arms and legs practically vanishes, and he is able to make movements that were quite impossible in the erect posture. As 
the disease progresses, the muscles which are concerned in speech and articulation become affected, so that the pitch is monotonous, the rhythm faulty, the tone nasal, and the pronunciation jerky and syllabic. In many cases the movements of the eyes show a weakness of the external recti muscles, but there is no nystagmus. Ophthalmological examination sometimes shows an optic atrophy and progressive choroiditis. The reflexes are regularly increased, particularly in the knees. One of the main characteristics is the gradual loss of mental power, especially the faculties of association and memory. A remarkable fact that is not infrequently seen is a flattening of the skull over the cerebellum, which doubtless follows the decrease in size of the hind brain.

Hereditary cerebellar ataxia may continue for several years before exhaustion or intercurrent disease puts an end to it. The treatment is symptomatic and general; attention should be paid to massage and passive movements.

Hereditary ataxic paraplegia, described by Dana, is a condition that attacks children at about the age of puberty. There are, evidently, a hypoplasia and degeneration of the lateral columns and some of the peripheral and central neurons. The changes affect consecutive levels of the cord until the medulla oblongata is attacked. The course of the disease is progressive with remissions. The first symptom is a clumsy, awkward manner of walking that gradually becomes exaggerated into a typically ataxic, paretic gait. Later on there is a similar affection of the upper extremities which ends in thorough incoördination. A noteworthy fact that differentiates this disorder from hereditary cerebellar ataxia is that the incoördination persists in the reclining as well as the erect posture. The reflexes gradually become exaggerated, most of all in the lower extremities. After a variable time the feet assume the cuplike deformity that is regularly seen in hereditary spinal ataxia, and the hands become crooked; this latter deformity consists of a flexure of the first and second phalanges, and a slight extension of the terminal phalanx. The eyes are gradually involved: early in the disease there is a beginning optic atrophy, and later on there may be nystagmus, paralysis of the external recti muscles, and partial paralysis of the lids. The throat muscles likewise become involved, and give rise to difficulty in swallowing, phonation, and articulation. Sphincter control is deficient in the later stages, the muscular sense is lost, 
complete paraplegia supervenes, and a fatal result is then merely a question of time, accident, or intercurrent disease.

The treatment is general and symptomatic.

\section{Acute Ascending Paralysis}

Acute ascending paralysis, called Landry's paralysis, is so rarely seen in children that no more than a mention of it is required. Its cause is unknown, and its lesions are extremely obscure. Various inflammations of the cord have been found after death, but they are so irregular and so inconstant that no reliable connection between them and the disease can be made. The symptoms may begin with disturbances of pyrexia; then, more or less rapidly, the paralysis sets in. It begins in the feet, and gradually works its way through the legs, body, neck, and face. Each part as it becomes involved gives its particular symptoms. The muscles are flabby and weak, and as those in the abdomen become affected there will be constipation; as those of the diaphragm become attacked, there will be a corresponding difficulty in breathing; as those of the larynx and throat become involved, there will be impaired power of phonating, articulating, and swallowing; as those of the eyes become disabled, there will be paralysis of the lids. Sensation is progressively lost, sphincter control impaired, and electrical reactions changed.

The course of the disease is short, and usually ends in death. Some cases have, however, recovered; in that event the arms are the first to recover their function.

The treatment is symptomatic and general.

\section{Infantime Cerebral Paralysis}

This group of cases represents, in varying degrees of paralysis, some injurious impress on the brain. They occur at any time of life - before, during, or after birth. According to the part of the brain which is affected, there may be a hemiplegia, a double hemiplegia or diplegia, a monoplegia, or a paraplegia. The disease occurs somewhat oftener in boys than in girls, although it is impossible to state a reasonable cause for the preference. The paralysis is the most prominent, but not necessarily the only or most important, symptom of the disease, and thus gives a name to the whole group. 
Causes. - Intra-uterine cases may be due to injuries, infective and serious diseases from which the mother suffers, and the many obscure conditions in which the equilibrium of nutrition is seriously disturbed. Among the active causes of such disturbance may be systemic or constitutional disease, marked weakness, disturbed functional activity, and psychical shock and strain. The arrests of development which result are frequently very obscure in their retiology. The cases that occur during labor are the result of slow and difficult expulsion of the fœtus, due to its large

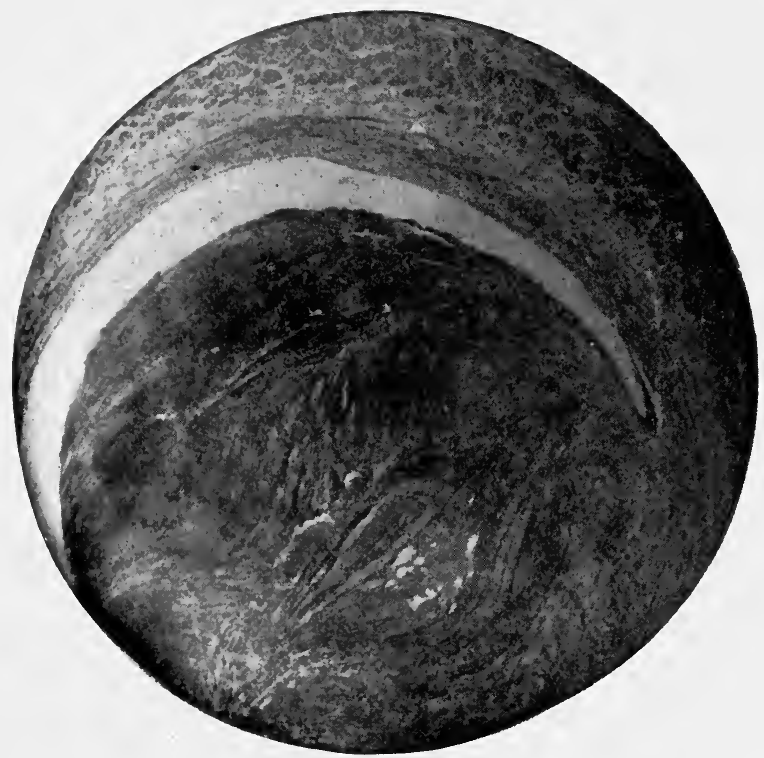

Fig. 119. - Organizing Thrombus in Vein. $\times 10$.

size, absolute or comparative, to malposition, to premature rupturing of the membrane, or to atonicity or pathological inability of the mother to complete the labor. The extra-uterine or acquired paralyses are due to injury, the infectious diseases, constitutional disease, psychical shock, convulsions, apoplexy, and epilepsy.

Lesions. - We know more about the results than the process of the pathological changes. Thus in the intra-uterine cases the process is one of arrested development, with a resulting absence of certain elements of normal brain equilibrium. In the paralysis of parturition the main process is meningeal and cortical hæmor- 
rhage, or bleeding from a venous sinus, with resulting pressure, atrophy, cyst formation, sclerosis, or porencephalia. In acquired paralysis there may be apoplexy, embolism, thrombosis, and fatty degeneration, with their consequent loss of tissue and function.

Hæmorrhage is usually the beginning, and is commonly followed by convulsions, with or without coma. Since the lesions are for the most part located in the cortex, the effect may be expected to produce a prompt effect upon the body. According to the localities involved there may be a hemiplegia, a diplegia, a paraplegia, and rarely a monoplegia. With any of these conditions there may be strabismus. In the majority of cases there is a variable amount of rigidity and contraction in the paralyzed muscles. As a result there may be a large degree of deformity. Thus, in the face the affected side may seem pulled down and flattened, and the normal folds are diminished or absent; or the arm may be drawn to an angle, and the fingers more or less flexed; in the thighs spasms of the adductor muscles may cause cross-legged progression, and in the feet talipes equinus and talipes equino-valgus may occur. Frequently the muscles are flabby and weak instead of rigid.

The affected part loses not only in function, but also in its growth in all dimensions. It is undersized, and seems rightfully to belong to a younger child. While it undoubtedly does grow, nevertheless the degree is much smaller than that of the uninvolved parts. But even these, and in fact the whole body, are restrained in their development. This weakness is shared to some extent by the mental capacity, so that these children are crippled in both body and mind. This intellectual infirmity is of all grades, from slightly impaired vigor to idiocy. In addition, a large percentage of these cases (estimated at about fifty per cent) is afflicted with epilepsy, which in most instances is general, but occasionally may be Jacksonian. Another comparatively common feature of the disease is the occurrence of athetoid or associated movements. The former occur as involuntary, slow, and uniform movements, usually of the fingers and toes; the latter as involuntary movements by the paralyzed extremity in general imitation of voluntary movements made by the healthy extremity. In some hemiplegias choreiform movements are seen, and nystagmus in some diplegias. In other cases tetanoid movements, rhythmical contractions, tremors, and ataxia may at times be observed. Still 
another somewhat rare condition in very young cases is hemianopsia. And in those children who are able to talk aphasia may result from a lesion of the motor speech centre. In this connection one should mention the occasional occurrence of the posthemiplegic polymyo-clonus of Peterson, a steady clonic contraction of some of the affected muscles, which happens at the approximate rate of five to a second.

In most cases the superficial and deep reflexes are exaggerated in the affected parts, although a marked rigidity of the muscles may render their detection somewhat difficult. It is unusual to find them normal or decreased, although this does now and then happen. It is no uncommon thing to find one or more of the stigmata of degeneration, such as deformities of the skull, face, or any of its parts, of the body, genital organs, extremities, and perversions of senses and functions.

Treatment. - There is no cure for these cases; and all that one can hope to do is to relieve the symptoms, and then to develop to the utmost whatever powers of mind and body are left uninjured. The acute symptoms require rest in bed, applications of ice to the head, and, in the case of convulsions, the proper doses of antipyrine, chloral, bromide of soda, or inhalations of chloroform. The general care of the patients should be of the best; the bowels should be kept in a free condition, the food should be nutritious and easily digestible, the skin must be made vigorous. Massage, passive motion, and electricity, aided by tonics, may help to strengthen the weakened muscles. These cases, in order to attain a useful position in life, demand special education which is designed to develop individual deficiencies. They gain little or nothing at the ordinary school, where they are discouraged; disheartened, and made to recognize their shortcomings in the keenest possible manner. Under skilled direction, which is able to locate the mental as well as the physical weaknesses, considerable improvement may be looked for. Much patience, application, and considerable expense may have to be incurred, but, as I have repeatedly seen, the result will commonly justify the outlay. Surgery has not as yet been able to give any radical help.

Prognosis. - The outlook in almost all of these cases is not good. Where the lesions are large, as in the paraplegias and diplegias, one need not look for recovery. In the other paralyses the disability is commonly confined to comparatively few muscles, 
and the steady development of others may atone in part for the loss. Thus an arm or a leg may be made somewhat useful, defective speech may slowly be improved, and by prolonged efforts, rightly directed, the mental condition may be somewhat bettered. One should take care to foretell the possible supervention of epilepsy, and especial stress should be laid upon the liability of these patients to contract intercurrent diseases, and the weak resistance which they are apt to offer to the attacks.

Differential Diagnosis. - The disease is not hard to recognize, and the only disorder that is at all commonly apt to create confusion is infantile spinal paralysis. In cerebral paralysis, however, the existence of the history of the case, the hemiplegia, paraplegia, diplegia, or monoplegia, with rigidity and contractures, the natural electrical reaction of the muscles, the morbid movements, epilepsy, and stigmata of degeneration, all are able to fix the nature of the disease.

\section{Thrombosis of the Intracranial Sinuses}

Thrombosis of the intracranial sinuses may result from two general causes : one, called primary or cachectic, follows conditions of exhaustion, such as those caused by simple atrophy, prolonged intestinal disorders; the other, called secondary or septic, follows some form of pyogenic infection. Primary thrombosis, which is rather rare in childhood, usually involves the longitudinal sinus, and may also extend to the cavernous and lateral sinuses. Secondary thrombosis is more apt to be loeated in the lateral or transverse sinuses. The clot may arise either from a coagulation in a near-by vein, or directly from an inflammation of the walls of the sinus. It finds its origin, in almost all cases, in the development of some infective disease that is in active existence before the thrombosis begins to be formed.

The symptoms of primary thrombosis are not easily distinguished, for the patient's condition is so poor, and there are apt to be so many serious signs of physical incompetency that in the general breakdown the diagnosis is easily overlooked. In the secondary form the symptoms resemble in a general way those of meningitis, which, by the way, often complicates the process. The general symptoms would be prostration, headache, stupor, spastic rigidity of the neck or extremities, convulsions, thin and 
irregular pulse, and interference with ocular functions. The temperature varies according to the circumstances of the complaint.

Thrombosis of the lateral and petrosal sinuses happens with less rarity than the other forms, since it is often secondary to some of the aural diseases which are so common in childhood. In involvement of the lateral sinus there is œdema over the mastoid region, and swelling of the veins. There may or may not be some tenderness on palpation, which is confined to the affected side. When the superior longitudinal sinus is attacked, which commonly occurs in severe aniemia, there is odema of the scalp and swelling of the frontal, occipital, and parietal veins. Thrombosis of the cavernous sinus is often connected with that of the petrosal. There are œedema of the eyelids, ptosis, paralysis of the external rectus muscle, supraorbital pain, and exophthalmos. In some cases there are practically no characteristic symptoms, and the diagnosis can be made only on post mortem section.

The treatment of the secondary form is purely surgical; and the sooner the operation is performed, the better is the chance of recovery. Under all circumstances the prognosis is very doubtful, not only on account of the severity of the measures, but also because the child's condition is regularly so poor.

\section{Abscess of the Brain}

This rare condition is much less frequently seen in children than in adults. The known causes which produce it are nevertheless comparatively common in these young patients, such as otitis media, purulent disease of the nose, infected wounds of the scalp and skull, and fractures and caries of the cranial bones, cerebral tumors, and metastatic abscesses from any pyogenic disease. In some cases the cause cannot be traced.

Lesions. - The abscess may be encapsulated or non-encapsulated. In the former there is an enclosing shell of connective tissue which is filled with creamy or greenish pus, broken-down cerebral tissue, and bacteria. In the latter the abscess cavity is irregular, the walls are necrotic and infiltrated with pus. For a variable distance the zone about the abscess is soft, degenerated, and œedematous. There may be extensions of the process in various directions, which thereby involve increasing amounts of tissue. Multiple abscesses are usually small, but may increase to a large size by 
coalescence. The usual locations are the temporo-sphenoidal lobe, the cerebellum, the frontal, occipital, and parietal lobes.

Symptoms. - The abscess may give symptoms immediately, or may be latent for weeks or months. Its first manifestations are those of pus formation - fever, chills, malaise, prostration, and disorders of nutrition. There may be nausea or vomiting, constipation, and lowered pulse rate. Headache is a constant symptom, and may in a general way correspond to the location of the lesions; if the abscess is superficial, there may be a corresponding tenderness of the scalp. Cerebral irritation may be severe, and, especially. as the pus formation enlarges, may be merged into convulsions, which in time may be followed by coma. Optic neuritis commonly ensues, and with it a resulting blindness which may be confined to one side. If by extension of the process a meningitis begins, it will give its characteristic symptoms. The same may be said of involvement of the special localizations of the cerebrum and cerebellum; but these focal manifestations are often not seen because the disease is confined to the frontal and temporo-sphenoidal lobes. The abscess may in some instances fall into quiescence for months or years, and then, for some known or unknown reason, undergo an acute exacerbation.

The treatment is purely surgical; and the sooner the abscess can be opened and drained, the better is the prognosis. Under all circumstances the chances of recovery are not great, and when the lesions are situated far from the surface the prognosis is absolutely bad.

The diagnosis from meningitis may at times be difficult; but if one keeps in mind the tendency to spastic rigidity, convulsions, opisthotonos, and superficial location of the meningitis, the difficulty may be lessened. In addition its course is more rapid, uninterrupted, and violent. Commonly there is a history of injury or preceding disease, from which an abscess may start, to turn our attention to the right opinion of the case. Between abscess and tumor a diagnosis may be obtained by noting the apyrexial course, the steady growth, and the equally steady increase of focal symptoms in the latter condition. An occasional or rare case may occur, as an exception to this rule, in which a cerebral abscess may give no fever, and in which the symptoms are practically identical with those of cerebral tumor. Here the diagnosis can be made only on post mortem section. 


\section{TUMIOR OF THE BRAIN}

New growths of the brain must include those of the cerebral tissue proper, its meninges, and those neoplasms of the scalp that by wearing a way through the bone finally produce pressure symptoms upon the cortex. About fifty per cent of these tumors are tubercular, about twelve per cent gliomata, eleven per cent are sarcomata, ten per cent are cysts, about three per cent are carcinomata. and the remainder is divided among glio-sarcoma,

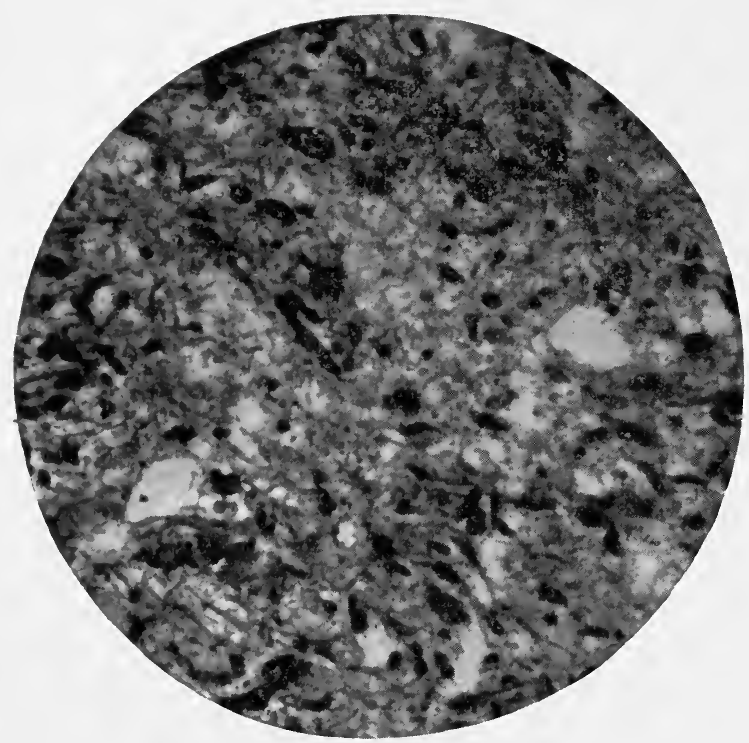

FIG. 120. - Neuro-glioma of Brain. $\times 110$.

angio-sarcoma, myxo-sarcoma, papillary epithelioma, gummatous, and unclassified tumors. In such a work as the present, an extended description would be out of place. For an exhaustive account the reader should consult special works on nervous diseases.

The tubercular tumors are almost always secondary to tubercular disease in other parts of the body. They may vary in size from a pinhead to an egg; they may be single or multiple, encapsulated or non-capsulated. Generally the interior of the tumor is cheesy, while the periphery is made up of giant cells, granulation tissue, and epithelioid cells. 
Gliomata are caused by some mysterious degenerative hyperplasia of the neuroglia. It is generally found in the ependyma ventriculorum and the retina. A common characteristic is their great vascularity, on account of which hæmorrhages may easily occur. When the tissue takes on a mucous character the tumor is called a myxo-glioma, and a growth of round and spindle cells is designated glio-sarcoma.

Sarcoma has a rapid growth, and therein differs from glioma, from which it is also distinguished by the fact that it is sometimes multiple. Variations are called myxo, fibro, lympho, glio, cysto, melano, angio, endothelial, round-celled, and spindle-celled sarcomata. Its course is malignant.

Cysts may result from degenerative changes in organic lesions, when they are called secondary. Metastatic cysts are caused by echinococcus or Cysticercus cellulosæ. They may be single or multiple, and may grow to a great size.

Symptoms. - There are certain signs that belong to all new growths, such as headache, nausea and vomiting, constipation, sleeplessness, mental disorders, optic neuritis, dizziness, convulsions, and in some cases enlargement of the skull, erosion of the cranial bones, and exophthalmos. In individual cases there may be special and characteristic symptoms due to local pressure and distension. The growth of the symptoms is slow and gradual, and when a sudden outburst of disturbances occurs it is due to hæmorrhage or meningitis. New growths located in the axis at the base of the brain give symmetrical symptoms; other locations produce unilateral signs.

In the frontal lobe the growths commonly give disturbances and decrease of the intellectual faculties, and sometimes their manifestations are slight or latent. 'There is apt to be an ataxic, staggering gait, and as the tumors grow they may exert pressure in various directions, and thus produce symptoms which do not properly belong to their location.

Those growths which directly or indirectly impinge upon the third frontal convolution (in a right-handed person) pro- . duce motor aphasia, and in older children a partial degree of agraphia.

Growths in the motor area (fissure of Rolando) produce spasm (Jacksonian epilepsy), convulsions, or some degree of paralysis of the opposite parts of the body, according to their location and size. 
The motor area is supposed to control sensory as well as motor functions ; and, therefore, with the disturbances of motor control there may be more or less anæsthesia.

New growths in the parietal lobe show the effects of various degrees of anæsthesia in the opposite extremities. It is only by pressure which is transmitted to other areas that further localizing symptoms arise.

In the occipital lobe tumors produce homonymous hemianopsia. Hore, also, indirect pressure on adjacent areas elicits corresponding symptoms.

In the temporo-occipital region tumors, which are situated near the base of the brain and above the petrous part of the temporal bone, produce optical aphasia or visual amnesia by pressing upon or destroying the association tract between the occipital and temporal region (Starr). New growths of the temporo-sphenoidal lobe may give no symptoms, or indefinite ones, involving the senses of hearing, smell, and taste.

Tumors developing in the lateral ventricles, island of Reil, and ganglia at the base, may give rise to a wide variety of symptoms because they impinge upon the internal capsule, which acts as a station wherein various tracts are received. Thus there may be both motor and sensory symptoms, according to the part which is affected.

Tumors in the crura may also produce motor and sensory symptoms of one or both sides. Among these are ataxic gait, alternate hemiplegia, homonymous hemianopsia, hemianæsthesia, and optic neuritis.

Tumors of the corpora quadrigemina give an ataxic gait, progressive double ophthalmoplegia, and some degree of paralysis. Pressure communicated to the aqueduct of Sylvius produces hydrocephalus, and when the crura are similarly involved motor and sensory symptoms result.

Tumors of the pons varolii produce the ataxic gait, and disorders of the third, fifth, sixth, seventh, and eighth cervical nerves, according to the size and location of the growth and transmitted pressure.

Growths of the medulla oblongata involve a very wide combination of symptoms on account of the many centres and tracts therein contained. Thus there may be disorders of motion and feeling, of respiration, and pulse; there may be vasomotor dis- 
turbances, difficulty in swallowing, projectile vomiting, glycosuria, polyuria, impeded sucking and articulation.

Tumors of the cerebellum give rise to sudden spasm of the extremities, which is most commonly shown on arising in the morning, the ataxic gait, and vertigo. Communicated pressure produces disorders of the pons varolii, cranial nerves, and medulla oblongata. Pressure upon the fourth ventricle may cause hydrocephalus.

Treatment. - The care of these cases is symptomatic, until the child is put into the hands of a surgeon for operation. If the tumor is deep-seated and the operation is inadvisable, one can do nothing but make the child as comfortable as possible. If the tumor is syphilitic, which is exceedingly rare, mercury and the iodide of potassium will cause an immediate amelioration of the condition, most of all if the drugs are prescribed in large and increasing doses.

Prognosis. - Unless the growth is syphilitic, or its situation allows removal, the outlook is absolutely bad. A tubercular tumor may set up a similar inflammation of the pia mater, and may kill the child before the pressure symptoms are very marked. Or a hæmorrhage may arise from a glioma, and produce the ordinary picture of a cerebral apoplexy. In a considerable number of cases the vitality of the child is so much affected that he dies from an intercurrent disease either before or after a diagnosis of intracranial tumor can be made. The disease is not supposed to last more than two years.

Differential Diagnosis. - The determination of this condition depends upon the recognition of the general symptoms plus the evidence of local pressure and destruction. The main items to keep in mind are the absence of a sufficient cause (excepting in tuberculosis and syphilis), the apyrexia, slow development, headache, and occasional tenderness of the scalp, and the gradual growth of focal symptoms. Macewen's symptom, " the elicitation of a differential cranial percussion note," should be sought for, but not with the expectation of regular success. It is practised by means of the finger or percussion hammer while a stethoscope is placed on the forehead, or on a shaved patch of the scalp on the top of the head. The diseases which may possibly be confused with intracranial tumor are tubercular meningitis, abscess of the brain, and hydrocephalus. 


\section{Tumors of the Spinal CoRd}

The same growths that occur in the brain may also be seen in the spinal cord and its meninges. Likewise, outside of tuberculosis, syphilis, and metastasis, there is no known cause for them. The number of cases recorded has hardly been large enough to found a reliable table of frequency of occurrence. As in cerebral tumor, the reader should consult a special work on nervous diseases if he desires an extended account of the disorder.

The tumor is usually unilateral, but may by extension invade the other side. Likewise, the growth may be in the cord itself or in the meninges, and from one may extend to the other. In rare cases there may be some external evidence of the growth, but in the large majority the symptoms are those of pressure grafted upon the signs of a degeneration of some section of the cord. Extra-medullary growths give pain in the back and the results of involving the posterior nerve roots. If the growth is on the anterior aspect atrophy and paralysis result.

In the symptomatology of all sorts of growths, vaso-motor disturbances occupy an important place; the skin is tender and painful, flushed and shining. There are muscular wasting and paralysis, in degree and locality according to the lesions. If the growth is in the cervical region, there will be pain in the arms, and especially in some special portions of the brachial plexus; if it is in the dorsal region, the pain is felt in the thorax and abdomen; if it is in the lumbar region, the pain radiates to any part of the lumbar and sacral plexuses. The natural progress of the disease is a series of increasing symptoms of vaso-motor disturbances, pain or paræsthesia, followed by complete anæsthesia, complete paralysis, and finally trophic disturbances.

The muscles or groups of muscles supplied by the affected segment of the cord will, as one can readily understand, become paralyzed and atrophied; but those muscles supplied by the lower segments will merely be affected with a spastic paralysis. As one would naturally suppose, the only muscles which show the reaction of degeneration are those supplied by the affected segment of the cord. As the disease of the lumbar region progresses, there will be a growing paralysis of the rectal and vesical sphincters. When the tumor is in the higher portions of the cord, there will be incontinence or retention. In all cases, but markedly 
with tubercular and syphilitic growths, there is a disposition toward the development of myelitis, whose progress is apt to be rapid.

The treatment, as in cerebral tumor, is surgical; but it is not often that one may expect a successful result. Before the case is operated upon, all one can do is to treat the symptoms. Of course this does not apply to syphilitic growths, which improve rapidly under the use of mercury and iodide of potassium in large and increasing doses.

The prognosis is the same as in cerebral tumor. To avoid confusion, one must keep in mind, as the principal characteristics of spinal tumor, the gradual development, apyrexia, unilateral involvement, and the gradually increasing localizing symptoms which correspond to the position of the neoplasm. There is usually no history of a sufficient cause, excepting in the case of tuberculosis, syphilis, or a pathological condition which permits metastasis, in which case there would be the evidences of previous disease.

\section{HYDROCEPHALUS}

An accumulation of serous fluid in one or more cavities of the cranium is called hydrocephalus, or water on the brain. This fluid may be in the subdural spaces, when it is designated external hydrocephalus. In other cases the ventricles contain the effusion, and this condition is called internal hydrocephalus. An acute form is rare, and occurs, as a rule, as one of the symptoms of meningitis. There is also an acute serous hydrocephalus, described by Quincke, whose origin is usually the result of systemic exhaustion, although in some cases it is unknown. There are, however, a few cases which are due to the venous stasis of marked cardiac disease, or new growths at the base of the brain and the posterior fossa. The chronic external form is also rare, and follows congenital or acquired atrophy, and congenital malformations of the brain, pachymeningitis, and hæmorrhage of the meninges. The chronic internal variety may be either congenital or acquired; in the latter case it results from neoplasms at the base of the brain or chronic meningitis, and is thus a rare manifestation of pressure ; in the former we have the most noteworthy variety, which, on account of its greater prevalence and greater importance, is usually called, to the exclusion of all forms, by the simple term 
"hydrocephalus." Its causes are unknown, although various suppositions have included tuberculosis, syphilis, heredity, prenatal malnutrition, maternal impressions, and alcoholism in the list of possibly responsible factors.

Lesions. - The brain tissue is pale, weak, and does not clearly show the distinction between the gray and white matter. The fluid is present in various amounts, which, as they increase, produce equally great changes in the brain. The convolutions are flattened and thin, so much so in certain cases that there is nothing left but a mere shell; at both top and sides this may occur. The brain at the base is flattened and softened in a less degree. The increased pressure stretches the sutures and fontanelles out; the bones of the skull are thin, pliable, and bulge at the forehead and vertex. The ependyma in mild cases is normal, in severe cases it is enlarged, congested, and covered with granulations. In some exceptional cases there are variations from the ordinary appearances; there may be a premature ossification of the skull with an equal amount of compression, although the quantity of fluid is not great. In other children there may be a condition of spina bifida, or meningocele, or encephalocele in connection with the hydrocephalus. The fluid is alkaline in reaction, of a specific gravity of from 1.003 to 1.008 , slightly albuminous, and sometimes contains a trace of sugar.

Symptoms. - In most cases the head begins to increase in size before birth. The fluid first accumulates in the lateral ventricles; later, on the aqueduct of Sylvius is stretched out into the shape of a funnel, but the fourth ventricle is not much influenced except in very marked cases. The fontanelles bulge, and through them a wave of pulsation is easily recognized. The shape of the head is roughly square, on account of the distension of the anterior and posterior horns of the lateral ventricles, which causes bulging of the frontal and occipital bones. The forehead is grotesquely high, and by its marked projection makes a sharp prominence at the root of the nose. As the head increases in size, the sutures are forced apart and the scalp is stretched tightly over the skull. The weight of the head may be so great that the child cannot support it without aid. If he lives long enough he may be able to acquire sufficient strength so that he can sit up ; but, in spite of this gain, the head is still too heavy for him, and topples over to one side or the other. The eyes project outward 
and downward, and are only partially covered by their lids. Muscular growth is retarded, and in the lower extremities some degree of rigidity may be remarked. The thumbs are often held within the closed fist, the eyes may have a convergent squint, and convulsions may at any time ensue. The mental development is much retarded, both in the direction of sense perceptions and intellectual effort, so that the child remains in some degree of imbecility.

Treatment. - Treatment by means of drugs gives no good results, excepting in some cases of syphilitic heredity, which improve under the use of mercury and the iodide of potassium. Surgical means have been tried in great variety, and likewise without success. This also has been the experience with strapping, aspiration, lumbar puncture, counter-irritation, craniotomy; and none has given such satisfactory results that it may be recommended as a remedy of wide application. On the other hand, since these cases are generally forlorn hopes, any method which appeals to the individual operator and is justified on good surgical principles may be tried. At the same time, one should plainly understand that the outlook is not good. Some cases, especially the congenital, die within a few months; from these rapid involvements nothing in the way of cure or improvement need be expected, even long before the fatal stage of the disease. There are, however, instances of very slow growth which generally are secondary; these by the use of tonics may be restrained from a full development of the disease, and eventually may enjoy a fair degree of health and mental ability. Cases of extreme enlargement followed by rupture of the head have been reported.

Differential Diagnosis. - Those few cases of premature ossification in which hydrocephalus is concealed cannot be certainly diagnosticated. With the other cases the diagnosis is largely a matter of discerning the increased size of the head. It is true that increase in the cranial measurements may occur in rickets and syphilis; but differentiation from two such diseases with their strongly marked symptomatology is rarely difficult in an appreciable degree.

\section{Asthina}

Asthma is a neurosis, one of whose main symptoms is a certain prolonged form of respiratory spasm. As a rule, the term is 
frequently misunderstood and misapplied. Physicians as well as laymen commonly think of it as applicable to almost any form of dyspncea without much regard to the exciting cause.

Causes. - In some cases of asthma, one can find antecedent examples of the disease in parents or ancestors. Nevertheless one can scarcely say that the disease is a matter of strict heredity. Rather one should regard the appearance of the symptoms as the evidence of a predisposition to a certain nervous irritability which in the presence of proper physical conditions and an exciting cause may develop in ways that are analogous to those of heredity. The transmission of this neurosis has not the sureness, the steady, inevitable character, of an hereditary trait.

As auxiliary active causes, many disorders of the respiratory track and its connections take a front rank. Doubtless the principal of these are enlargement of the bronchial and tracheobronchial glands, and bronchitis. It is commonly supposed that these causes act by irritating and pressing upon the vagus or its ramifications. In addition to disorders of the bronchi and bronchial glands, any abnormal condition of the larynx, throat, or nose may induce an attack of spasm. Hyperæsthesia of the mucous membrane covering the turbinated bones of the nose, or deviations and growths of the septum, are likewise efficient in creating the symptoms. Occasionally the disorder seems to arise from or to have an intimate connection with renal, cardiac, or thymic disease ; but it is practically impossible to make a minute classification of the relationship.

Besicles all this, there are many causes which act in a more remote way: a strong, harsh wind, certain odors, the pollen of plants, dust, even such obscure changes as those involved in sexual disease, skin diseases, intestinal disorders, - in short, almost any pathological state can, by nervous irritation, precipitate an attack. In some cases a purely psychical reaction may, in some mysterious fashion, have an immediate and active effect. Thus, in some rare cases a stream of light may suddenly change the norinal breathing to violent respiratory efforts, or, on the other hand, the approach of darkness may have the same effect. In a noteworthy case of a boy of fourteen, whom I observed for upward of three years, the touch of plush, velvet, or the velvety skin of a ripe peach, acted with characteristic promptness. Finally, one may mention the uric acid diathesis and enlargements of the thyreoid gland as possible causes. 
Lesions. - The changes in the nervous system, whatever they may be, are as yet impossible to define. That there is in some cases a hyperæsthetic condition of the nerve filaments in the nasal mucous membrane is beyond dispute; it is entirely within reason to expect that eventually we may know as surely of a nervous irritation which more or less directly produces the symptoms of the disorder. There have been many theories concerning the probable development of the lesions, all the way from the first utterances of Van Helmont, through the theory of bronchial spasm of Biermer, the theory of tonic spasm of the diaphragm put forth by Wintrich and Bamberger, to the Weber-Stoerk theory of vasomotor disturbances in the circulation. At all events, when the neurosis is once in active existence, we know that a bronchitis, an emphysema, and sometimes inflammatory changes in nose, pharynx, and throat develop. In such an event the ordinary pathological changes which belong to these diseases will naturally show themselves. On careful examination one is very apt to find a more or less extensive adenitis, or abnormalities in the inner conformation of the nose, or nasal polypi. After a prolonged series of attacks one is apt to find a marked absence of elasticity in the lung tissue, a chronic bronchitis, a dilated heart, and possibly an endocarditis with insufficiency.

Certain changes have been noticed, the exact significance of which is not understood. For instance, Von Noorden and Müller claim that the blood of asthmatic patients contains an increased number of eosinophile cells. Another noteworthy fact is Leyden's discovery of the elongated crystals, which Charcot noted in other diseases, associated with mucous shreds. Most renowned of all is Curschmann's isolation of the so-called spirals; these consist of threads of mucin wound in strands about a central axis, sometimes much twisted, sometimes puffed out with air so as to assume a fusiform shape. These spirals are dotted here and there with small round cells, spindle-shaped cells, and epithelium. Curschmann's spirals have been seen in the expectoration of various pulmonary diseases.

Symptoms. - Commonly the symptoms appear at night, frequently without warning. The child may wake from a quiet sleep with the signs of anxiety and fear. He feels as if he could not breathe, and straightway tries by all the aids of position and extreme respiratory effort to obtain more air. As the attack 
increases in severity, inspiration becomes spasmodic while expiration is prolonged, labored, and wheezing. More and more the child puts forth every effort to satisfy his air hunger, and every effort seems futile. On account of his failure he becomes more or less cyanotic, his extremities are cold, his face is clammy with a slight perspiration. The suffering is pitiful, and may continue for minutes or hours. As the attack lessens, the symptoms are apt to be replaced by those of bronchitis with the ordinary cough, its physical signs, and a thick, viscid expectoration.

A noteworthy fact is that the respiration, although so strenuous, is not increased in rapidity; on the contrary, in very severe attacks the rate may be lessened. With each short, spasmodic, and strong inspiration the upper part of the chest seems to rise in a convulsive movement, but the lower part appears scarcely at all to move. Expiration is as forceful as the physical powers of the patient allow. Percussion and auscultation will give the symptoms of acute emphysema, but after the attack has subsided the physical signs of acute bronchitis are apt to be superadded. The pulse is weaker, thinner, and somewhat more rapid than in health.

Treatment. - If the asthma is due to an antecedent pathological condition, such as abnormalities in the nose, or throat, or glands, then patient efforts must be directed to their cure. For the relief of the asthmatic attacks, one obtains most satisfaction from chloral hydrate or antipyrin, the latter given with a cardiac stimulant. During the day small doses of potassium iodide, gradually increased, will give benefit. Many drugs, such as stramonium, lobelia, amyl nitrite, opium, quebracho, and grindelia robusta, have been recommended and used. But on the whole, one is apt to obtain no more than an intermittent satisfaction from them. The main thing to keep in mind is that the neurosis has no specific cure, that the means first suggested will in almost every case be sufficient to alleviate the distressing symptoms, and that when the attack is fairly ended, every possible method of improving the general health must be used. The attendant has here the opportunity of displaying resourcefulness in the administration of tonics, wisely ordered sanitary arrangements, exercise, and climatic changes.

Prognosis. - The outlook, especially in young cases, is good. Nevertheless, there is danger of a dilated heart, an emphysema, 
a chronic bronchitis, or a bronchiectasis which may remain as a sequel.

\section{Hay Fever}

Hay fever, also called rose cold, and autumnal catarrh, is a neurosis that is closely allied to asthma. Indeed it has been called "a nasal form of asthma." The two disorders are so closely allied as at times to be indistinguishable.

Causes. - The main foundation of the disease seems to be an hereditary susceptibility that, under the impulse of sufficiently active irritants, suddenly starts the symptoms into activity. Locally the hyperæsthesia is situated in the mucous membrane of the nose. Upon this sensitive area various irritants have the most marked effect. For the most part these irritants are the dust and pollen of the goldenrod (Solidago odorata) and ragweed (Ambrosia artemisicefolia) in America, Anthoxantum odoratum in England, the rye blossom in Germany. There are isolated cases in which the pollen of various other grains and grasses have a similar effect. That there is, as in asthma, an undoubtedly neurotic element in the causation, is proved by the number of cases which have their ostensible starting-point in an hysterical impression. In addition, one should mention those cases of abnormality in the nose, such as deviations and growths of the septum, and disorders of the mucous membrane. Among the many individual and indefinite causes are sense-impression, the lithæmic disposition, and some drugs.

Lesions. - In this disorder the mucous membrane of the nose, eyes, and throat become acutely inflamed, showing the usual products of catarrhal inflammation. This process in typical cases is most apt to spread to the trachea and bronchi, and even to the finer bronchial ramifications. In a well-marked case the lesions finally become coincident with those of asthma.

Symptoms. - The common division of the symptoms into the so-called catarrhal and asthmatic types is not necessarily a logical one. By insisting upon it one opens the door to confusion between the real hay fever and an acute rhinitis. The disorder as a rule is prevalent in this country from the middle of August to the beginning of November, in England in June and July. It begins with gradually prolonged and violent sneezing, and a painfully acute tickling of the nose. The mucous membrane of the eyes, 
nose, and throat becomes inflamed, and secretes a characteristic discharge. There are apt to be pains in the face and head, and some general prostration.

The temperature, pulse, and respiration are not materially affected. Later on the bronchial structures may be involved, with possibly the final occurrence of an acute emphysema. The logical development of the disorder results in a variable degree of asthma, which is apt, with successive attacks, to increase in severity. And this element in a sharply differentiated case of hay fever should be present, possibly in very slight degree in the beginning of the disorder, progressively more and more with recurring attacks. In addition, the demonstration of some hypersensitive point in the nasal mucous membrane will make the diagnosis more clearly defined. Unless influenced by treatment, the disease lasts from one to two months.

Treatment. - The treatment must be directed to the control of the factors of the hypersensitive area and the active cause. The offensive pollen must be kept from the nose by one of the various methods of filtering the air ; the best and most radical means, however, is a change of locality and climate. Any structural abnormalities in the nose should be corrected, and the hypersensitive portion of the mucous membrane may be cauterized. For the immediate relief of the symptoms, a weak solution of cocaine may be used in the nose, and a saturated solution of boric acid in the eyes. The usual plan of treatment in asthma will be needed. Before an expected attack, every means should be used to promote the local and general health.

Prognosis. - The prognosis in children who are treated wisely and patiently is on the whole good. This is especially true if after the first attack the right precautions are observed before the next one is due.

\section{Sporadic Cretinism}

When Hilton Fagge described this condition in 1871, no cases were known to have occurred outside of the localities where it is endemic. Since then the number of cases that are recognized is constantly increasing. And now in almost any large clinic for children instances of myxœdematous athyreosis are usually seen every year. The cause of the disease is the action 
of some agent which, before or after birth, so affects the thyreoid gland as to induce myxœdematous degeneration and atrophy. Like results have followed the experimental removal of the gland in human beings and monkeys. The disease may occur in one or more children in a family where the parents are healthy. In some cases the parents were related, in others there was a history of precedent acute disease, but we are ignorant of the value of these facts as ætiological agents.

Symptoms. - These cases may occur congenitally, or may develop in the first year or two of life. Doubtless a larger number than was formerly believed is congenital, but on account of deficient observation has not been recognized. The disease is easily recognized by the dwarfed figure, the lack of development, and a peculiar brutish expression of face. The head is large, heavy, brachycephalic; the hair is dry, hard, and often coarse. The forehead is low and broad, the nose is flat, and about the eyes there is an odematous, puffy condition of the tissues. The skin is dry, thick, somewhat wrinkled, and has a greenish yellow hue that suggests a combination of anæmia and jaundice. The teeth are slow in appearing, the lower jaw is prognathous. The tongue is macroglossic, protrudes from the mouth, and the poor development of the cheek muscles permits the saliva to dribble and drain from the heavily flaccid lips. The voice is harsh, thick, and on inspiration there is often a crowing sound. The neck is short and thick, the hands flat and saucerlike, the abdomen is thick and heavy, the genitals may be large or undeveloped, and the legs undersized. The internal temperature is commonly subnormal, anæmia may be marked, hæmoglobin is reduced, and in some cases there is a considerable degree of leucocytosis. These children grow very slowly in body and mind, so that at the age of eight or ten they may present the appearance of being no more than two or three years of age. They make the impression of being idiots, in many cases they are unable to talk, or to rise much beyond the condition of brutes. They are liable to have fatty tumors, the seat of which is commonly confined to the upper part of the torso.

Treatment. - Remarkable progress is made by the use of thyreoid extract, the dose of which is 0.03 gramme (gr. $\frac{1}{2}$ ) given two and then three times a day, for a child under eight months of age. The dose will have to be increased as the child grows older. 
When the remedy is stopped, the patient shows a strong tendency to relapse into his original condition; the younger the child, the better may one expect the results to be, and the less difficulty is there in maintaining them at a good level. Nevertheless, it is doubtful whether these children, even under good circumstances, are able to attain more than a very ordinary grade of development.

\section{Amaurotic Family Idiocy}

This condition has been described as " a fatal disease of infancy with paresis or paralysis, accompanied by idiocy or imbecility and progressive blindness; with symmetrical changes in the macula lutea." The first traces of it were accidentally observed by Warren Tay in 1881. Since then we have been made familiar with the symptomatology by a number of writers, foremost among whom is B. Sachs of New York.

The cause is unknown, but the disease occurs in families. Almost all the cases occurred in the children of Eastern or Russian Jews who are well known to be peculiarly subject to neurotic degeneration.

Lesions. - The changes seem to represent, according to the opinion of Sachs and others, an arrest of development. The pia mater and arachnoid are thickened, the cortex of the brain is hardened, and the strata of pyramidal cells in the cortex are indistinct. The large and small pyramidal cells are somewhat rounded, the protoplasm is irregularly shrunken about the nucleus, and has lost its granular character. The upper portions of the cord are in a condition of ascending degeneration. In some cases there is atrophy of the optic disks.

Symptoms. - The children at birth seem normal, and may so continue for three, four, or five months. Then, instead of developing still further, their growth remains as it was, and in addition the child begins to lose vitality and activity. They become passive and stupid, their muscular strength fails progressively, so that, instead of trying to grasp objects, crawl about, or sit up, they remain flaccid and quiet. The head is not raised, the limbs are motionless, and the appearance of paralysis with or without spastic contractions may be counterfeited. As a rule the reflexes are increased, but in some cases may be absent. Joined to the relaxation and flabbiness of the muscular tissue there is a corre- 
sponding weakness of the mind. The child is without curiosity and lacks expression, and, unless irritated, takes no notice of what is going on about him. Gradually he becomes blind, the pupils contract and dilate without much cause, and there may be hyperacuity to near-by sound. Ophthalmological examination shows in the locality of the macula lutea a fairly large, rounded, white spot in the centre of which is a cherry-red or brownish red patch. The disks are of yellow-gray color, and present the appearance of atrophy. In two cases that I saw there was oscillatory nystagmus. The abdomen is large, the bowels constipated, and as the disease advances the child declines into a condition of malnutrition or marasmus from which, or from some intercurrent disease, he dies.

The disease may continue until the child is from one to six years of age. So far as we now know it is always fatal. The treatment is symptomatic. Even the requirements of the passing phases of the disease are at times difficult to meet; and the care of the case is uniformly unsatisfactory.

\section{IDIOCY}

There are no hard and fast lines that divide the various degrees of defective mentality. The full degree of deficiency we call idiocy, the lesser degrees, imbecility, and a weakening of normal powers, feeble-mindedness. The distinction between them is an arbitrary one, and each observer may call any one condition by the name that seems good to him. Just as it is impossible to draw a line between idiocy and imbecility, so one is unable to mark off the dividing line between imbecility and weak-mindedness. Between all these states there are innumerable gradations, and these steps lead so insensibly up to the wide threshold of health that one can never be quite sure of one's position. Moreover, the normal mental condition of childhood is not the normal mental condition of adult life, and the lack of equilibrium in the former partakes very much of the nature of weak-mindedness. Finally, we are thrown back upon the idea of infinite variation from an ideal norm, the exact dimensions of which no one has defined.

The causes have been generally stated to include almost every abnormal condition that can possibly affect children in their con- 
ception, intra-uterine development, and extra-uterine life. In many cases claims of atiology have been made upon insufficient grounds, the assumption being made that abnormal growth must result from abnormal conditions. At the same time there has always been a disposition to overlook the normal results that may succeed these same conditions. Thus, consanguineous marriages have been supposed to be a fruitful source of mental defects, and instances of this tendency are commonly related by laymen and medical men; on the other hand, if a careful summary of all such marriages were made, one would, I am sure, be surprised at the really small percentage of feeble-minded children that resulted from them. Injuries to the mother during pregnancy is another common causal factor; nevertheless, the large majority of women who bear normal children have at some period of gestation sustained injuries of more or less seriousness. If the child proves to be deficient, a search is made for some possible trauma that will serve as a hook upon which to hang the garment of responsibility. But the same injury, or greater ones, are passed over without a thought so long as the child appears to be fairly normal. The same idea may be applied to the various sicknesses which may fall upon the pregnant woman or upon the child in the first weeks, months, or years of life. The only places where we feel perfectly at ease are in the cases of epileptic and insane descent, and mixtures of them, of hereditary syphilis, cretinism, and actual, palpable injury to the skull and brain. For the rest, the balance seems to lie in a more or less fortuitous arrangement of the elements of nutrition, which may or may not be disarranged under various pathological conditions. And when more is definitely known about the elements of nutrition, a step will be taken in the direction of preventing or modifying the results of their unfavorable development.

It may be worth while to give one of the most satisfactory classifications that have been made; at the same time the reader must recognize the purely provisional character of this arrangement, and that it is given, not because it is fully competent to cover and explain the cases as we see them, but merely because as yet there is nothing better. The classification is Shuttleworth's.

Class A. Congenital.

1. Microcephalic.

2. Hydrocephalic (also non-congenital). 
3. Scrofulous (Mongol type).

4. Sensorial (also non-congenital).

5. Primarily neurotic.

6. Paralytic (also non-congenital).

7. Choreic (also non-congenital).

8. Cretinoid.

(a) Sporadic.

(b) Endemic.

Class B. Non-Congenital.

9. Eclamptic.

(a) Developmental.

10. Epileptic.

11. Syphilitic.

12. Post-febrile (also accidental).

(b) Accidental or Acquired.

13. Toxic.

14. Traumatic.

15. Emotional.

16. From Mixed Causes.

The pathology includes any and every form of cerebral deformity, injury, and degeneration, and none can be selected as typical. Likewise it is plainly impossible to go into the symptomatology, which may include all variations from normal action of nerve structures.

This subject, which has never been adequately treated, needs prolonged investigation and extended exposition.

\section{Insanity in ChILdhood}

Insanity, while rare, is not unknown in childhood. Cases of mania have been reported in children of two and three years of age. And as the children grow older the liability becomes greater. It is at the time of puberty that the tendency to unsoundness of mind becomes most pronounced.

Causes. - The main cause lies in heredity, too close inbreed ing in tainted families, the crossing of epileptic and insane stocks; brain disease and cerebral injuries may be followed by it. And the acute disease, masturbation, worry and mental strain, and pathological organic and functional conditions of almost all sorts may in the presence of a predisposition act as 
the starting-point. The principal factors are heredity, predisposition, and injury.

Forms of Insanity. - Imperative concepts quite commonly appear in childhood and may be of the widest variety, ranging from physical acts to pseudo-philosophical questions.

Mania is not rare as a post-febrile condition, but as a permanent state it is not often seen. There is in it an absolute lack of restraint; there are wild flights of fancy, indecency of action and speech, and violent demonstrations of all sorts. It may and often does follow a period of depression.

Melancholia carries with it reasonless and marked depression. The child wishes to be alone, cannot be amused or interested, and finds in his acts or surroundings some cause for brooding and humiliation. He may even try to injure himself or commit suicide, and herein he differs from the maniac who turns his violence against others. The melancholy child sees visions, hallucinations, and while he sees them his depression is worst. This disorder is probably the one most frequently seen.

Epileptic insanity is by some observers considered common, Wildermuth believing that eighty per cent of infantile epileptics are of unsound mind. While these children, as a rule, show a tendency to epileptic attacks alone, nevertheless they at times change them for seizures of idiocy and dementia.

Moral insanity may occur at almost any except the very earliest age, and with it is commonly associated some intellectual defect, or at any rate some of the stigmata of degeneration. It may occur in children who have not had moral training. The evil tendency may take almost any vicious direction, - kleptomania, pyromania, homicide, perverted sexuality, noteworthy lying.

Neurasthenia, hypochondriasis, periodic insanity, cataleptic insanity, paranoia, acute dementia, and divers psychoses are also seen.

The treatment of these cases is very much like that of the adult forms. If any change is indicated, it is in the direction of more individual attention and an exaggeration of the element of environment. Since this subject really belongs to special works, it is not possible to go into all details of how to care for the particular eases. While the prognosis is somewhat better than in adults, nevertheless the patient must, for years after recovery, be regarded as potentially unreliable. 


\section{Functional Aphasia}

As the result of some mental impression, such as fright, or in chorea, or after the acute infectious diseases when impoverishment of the nervous system is both possible and probable, a child may temporarily lose the power of speech. The condition, so long as there is no sufficient organic lesion at the bottom of it, is not a serious one, and will gradually improve as the patient becomes stronger.

\section{Deficiency of Speech from Peripheral Paralysis}

After diphtheria and other infectious diseases there may be some degree of pharyngeal or palatal paralysis. There may be a proportionate inability to enunciate clearly and well. In most cases there is a gradual improvement, especially if the child is patiently trained. Not every case is quite successful, but one should have a sufficiently sanguine view to encourage unremitting efforts at developing whatever power exists.

\section{LISPING}

Lisping is a defect of speech in which the person substitutes a certain soft sound for a certain hard one, as $z$ for $s$ or $d$ for $t$. The deficiency is commonly interpreted as a necessity for pronouncing $s$ as if it were $t h$; but as a matter of fact this is only one form of lisping. It may be caused by faulty position or faulty development of some of the speech organs, in other cases it is due to habit or imitation. It is often cured by patient and careful teaching.

\section{StutTering}

In early years the stuttering habit is easily acquired. It consists in an inability to connect the different sounds in a word, although each sound is perfectly produced. Sometimes children who are perfectly normal may acquire it as the result of imitation. In other cases it starts from confusion, self-consciousness, or fright that is often repeated. More commonly it comes in debilitated, choreic, and nervous conditions, and may be a sign of degeneration. Most children recover spontaneously; in practically all cases a cure can be obtained by careful instruction and effort. 


\section{StAMMERING}

This word is used to designate a speech-defect in which the child is unable properly to pronounce individual sounds. It may be due to habit, imitation, or faulty development or condition of some of the organs of speech. Children whose physical condition is poor are more liable to suffer from it than those who are robust. The cure lies in careful training and the betterment of the imperfect physical conditions.

\section{Backwardness in ACQUiRING Speech}

This may be the result of slow but sufficient development, or of insufficient development due to sickness. The usual limit of acquiring a fair amount of speech is two years. If after that time a child, who is not deaf, cannot articulate, he may be regarded as mentally deficient.

This condition finds its extreme in alalia or a total lack of speech-function. It is normally present in young infants, but in older children it signifies some degree of weak-mindedness, imbecility, or idiocy.

\section{Echolalia}

Echolalia is the term applied to an hysterical condition in which the child is forced by some nervous impulse to repeat in a violent tone the last word of a spoken sentence or phrase. It is associated with other hysterical manifestations, and it is apt to pass away as the patient's general condition improves.

\section{Coprolalia}

Coprolalia is another hysterical disorder in which the child is impelled to shout some profane, obscene, or foolish expression, with the accompaniment of a violent contortion or absurd twitching of a part or the whole of the body. It does not occur alone, but rather is merely a symptom of a general poor condition. It improves under general treatment.

\section{Disorders of Sleep}

A normal infant is an eating and sleeping animal; and of these two functions sleep is not the less important. For the first 
month or two he should sleep all the time that is not consumed in nursing, and being bathed and dressed. The waking time gradually increases until it measures about eight hours at onehalf year, and about ten hours at one year of age. This amount, usually divided into two parts in the first few years of life, gradually increases until at puberty a healthy child obtains about ten hours of undisturbed rest.

The cause of disturbed sleep in early childhood, outside of real sickness, is some disorder of the natural functions. In most cases this is an interference with normal digestion. Either the patient is fed too little or too much. A very common fault is the unduly short intervals between the feedings. In this way the stomach is unable to complete a given task before a new piece of work is imposed upon it. Exhaustion must necessarily result, and with it an inability to digest food, so that the child may practically be underfed, although the supply of food is excessive. Or the quality of food may be poor, so that a relative starvation ensues whether or not the actual quantity taken into the stomach is much or little. The cases of deficient quantity as well as quality are so rare and so evident as to need no mention. Usually the acute disorders are gastric or gastro-enteric, while the subacute are enteric. In other cases the cause may be defective ventilation, lack of quiet, the irritation of teething, lack of cleanliness, and the errors due to poorly arranged environment. In nervous or sensitive children, the reason may lie in domestic tumults, in exciting scenes and incidents, in the straining ambitions so commonly seen in competitive tests in school or home life, or in the keen anticipations of desired events. When such a condition becomes exaggerated, a careful search should be made not only for some functional cause, but also for some physical or environmental condition which may, although not suspected by the mother, be at the root of the trouble. Thus hypertrophy of the pharyngeal tonsil, a long or tight prepuce, or other condition which induces nocturnal enuresis, may be found; and often the rectification of the fault will remove the evil effects. When the disordered sleep occurs in children who are well past the age of infancy, it commonly takes the form of nightmares or night terrors, both of which come under the heading of pavor nocturnus. The first designates a condition brought on by non-nervous causes, such as overfeeding or indigestion, in which the child 
wakes up after or in the midst of a terrifying dream, and by it is thoroughly frightened. The second denotes nervous conditions in which hallucinations exist in sleep. The child may wake in terror with or without full recognition of circumstances and the people about him. No recollection remains the next day of what happened. This condition is often associated with known nervous disorders, such as hysteria or chorea or epilepsy.

The treatment in all these cases is as far as possible to remove every cause of irritation, to regulate all details of the child's life, and especially to arrange the diet and the possible causes of great excitement upon a fairly wise plan. When there are physical disabilities, such as adenoid vegetations or adherent prepuce, they should be removed. With corrective treatment the use of sedatives, such as the bromide of soda, may be combined. If the cause of the disorder is promptly diagnosticated, relief is almost sure to come.

\section{Adventitious Sucking}

The young infant in his waking moments obtains his only pleasure in nursing. The instinct of self-preservation leads him to suck on whatever comes or is put into his mouth. Since gratification of this desire is the most important thing that he desires, it will temporarily at least distract his attention from other impressions, even if they are moderately unpleasant. Restlessness from any cause is so regularly quieted, at all events for a short time, by sucking at the breast or the nipple that one can easily understand how a perversion comes to exist, and the mere symbol of taking food replaces the act. Mothers and nurses frequently take advantage of this, and in order to keep the child still, give him a rubber nipple, a "sugar-teat," a piece of ivory, or other foreign substance with which he may busy himself. Gradually the child becomes used to his "comforter," as I have heard it called, and insists upon having it whenever he is not nursing.

Sometimes children find out about the "comforter" without another's aid, and then use whatever object is within reach. It may be a thumb, a finger, the toes, the bed-clothes - in fact, any object upon which the attention has been fixed. In a few cases the practice may be accompanied by rubbing or scratching some part of the body. Under all circumstances the habit should be stopped at its inception. Mothers should understand that if a 
child is in perfect health, he needs no such aid to keep him quiet; and that if a comforter becomes necessary, not as the result of habit, but before the habit is formed, there must exist some cause of irritation which should promptly be removed. Among the penalties which may follow this habit are disorders of digestion, septic infection of the gastric contents, deformities of the thumb, finger, or toes, as well as lips, teeth, and jaws. Occasionally a tendency toward masturbation may result.

The treatment usually requires no more than a fair amount of intelligence and patience. The child must be restrained from the indulgence. No artificial "comforter" should be allowed, and if he selects the thumb or any other similar part, it should be so swathed as to prevent the act. The child may be cross and irritable for a few days; but if the treatment is regularly carried out, he will soon become resigned and forget his former longing. There are no circumstances which excuse or justify the habit.

\section{Masturbation}

This habit may be contracted and followed at any time of life, from the first few months of life. In infants the tendency to it may be spontaneous, or may be developed by a vicious nurse, who seeks thereby to keep her charge quiet. Every observer who treats large numbers of children, finds instances of this habit at all ages, in all classes, and in various forms. Sometimes the hand is used as the active agent, sometimes friction against furniture or bedding, sometimes thigh-friction, and I have seen in a baby girl the producing of the act by means of the heel. In spontaneous cases occurring in infancy, the tendency is usually a mark of sexual perversion or degeneracy. In older children it may be acquired by imitation, teaching, or accident. Local causes, such as a long, tight, or adherent prepuce, phimosis, adherent clitoris, or an inflammation of the skin in the neighborhood, may be the starting-point; in other cases the habit may be traced to intestinal parasites, especially thread-worms, to irritating urine, to certain exercises and amusements, such as tree-climbing.

In many children no especially bad results may be noticed in early years. In others there may be nervousness, irritability, lack of confidence, bashfulness, lack of energy and vitality ; occasionally some serious nervous disorder, such as hysteria, epilepsy, or even insanity, has seemingly resulted from the habit. 
The treatment consists in some form of restraint in combination with the removal of abnormal physical conditions. Thus, a long, tight, or adherent foreskin may be amputated; a phimosis may be reduced; an adherent clitoris may be freed; intestinal parasites should be removed; and disorders of the skin may be cured. Sometimes one's ingenuity will be severely taxed to find means which are sufficient against the low cunning of the occasional child. One must make a resolution that the habit is to be followed up and stamped out, even if much time and effort are thereby consumed. If there are local causes at work, they must be removed.

\section{Deaf-Mutism}

Deaf-mutism may be congenital or acquired. In the former it may or may not be associated with some degree of idiocy. When the mental condition is good, it may be caused by colloid degeneration of the labyrinth, absence or disease of the semicircular canal or related parts of the ear, diseases of the brain, imperfect development of the petrous bone, and diseases of the skull. In the acquired form it may follow the acute infectious diseases, especially those which are associated with purulent diseases of the ear; diseases of the brain and meninges, and atrophy of the auditory nerve, may bring about a like result. In general, one may say that conditions which destroy the hearing of children who are under five or six years of age will occasion deafmutism. Not all cases show the defect in equal degree; cranial conduction gives a means of hearing in a large proportion of the cases, while not a few preserve some part of the natural faculty.

In children who are not idiotic much may be done by proper methods of instruction to atone for the loss of hearing and speech. Normal children attain the ability to speak when they are two years old; if this time passes without the development of speech, artificial training should without much delay be instituted.

\section{Convulsions}

Convulsions, or eclampsia neonatorum, is a symptom that has many causes, and occurs with the most widely separated pathological conditions. Like other common manifestations, for example fever, its occurrence means very little in itself, and only comes to 
have a definite pathological connection when we distinguish its causal relations and the relative importance which they bear to the health of the patient.

Causes. - One of the most important factors is the age of the child : the younger he is, the greater is his liability to have convulsions. Leaving out of account any discussion of a possible convulsion-centre, we know that the development of the nervous system in infancy is partial and unbalanced, that all its elements are not present, and our experience demonstrates every day that full control over such undeveloped tissue is not to be expected or found. At such age any predisposition toward cortical irritation may find its expression in attacks whose severity is at times quite out of proportion to the immediate factor that started the seizure in motion. Under these circumstances reflex disturbances may be of the utmost importance. Such disturbances may include widely separated conditions : irritations of the skin, nasal obstructions, a tight or adherent foreskin, the presence of intestinal parasites, even possibly a difficulty in cutting teeth. All these items are of variable importance, and some of them - notably the eruption of the teeth - may be of very slight value or of rare occurrence. One must keep in mind that the child represents a phase in development, rather than a fixed state. His nervous system is gradually evolving into a well-poised functional activity. But in the condition of unstable equilibrium which is natural to the infantile brain, a small weight in one direction or another is sufficient to alter the balance.

Another instance of variable but real influence lies in disorders of the gastro-intestinal canal. There is no doubt that in the majority of cases this is more or less directly the cause. . The disorder may consist of indigestion or fermentation of food, with or without the absorption of toxic products. Doubtless in all cases some form of toxæmia or allied condition is at the root of the trouble. A related cause is the intoxication that accompanies acute infectious disease of microbic origin, such as measles, scarlet fever, lobar pneumonia, pertussis, malaria, chicken-pox, and others. In further cases a poison, whether it is organic or mineral, may excite the attack. Rarer conditions that are responsible for the eclamptic seizure are trauma, neoplasm, abscess, hæmorrhage, and hyperæmia of the brain. Sometimes one of these may start from a far-removed source, as hyperæmia from rickets, or capillary 
hæmorrhages from scurvy. Finally the convulsions may be of epileptic or unknown origin, or may accompany enlargement of the thymus gland. Most instances occur before the third year of age.

There is no characteristic set of lesions that fits all cases. In some autopsies there have been cerebral congestion (doubtless preceded by anæmia) and meningeal hyperæmia; there may be hæmorrhages of various sizes in the cortex, and in the viscera evidences of deficient aëration.

Symptoms. - The convulsive fit may be partial or general; the first occurs as a rule with organic changes in the brain, the second commonly is functional. Usually there is a sudden loss of consciousness which may be complete or incomplete. Sometimes it is preceded by a cry and followed by convulsive turning up of the eyes, and occasional movements of the bowels and bladder. The hands become clinched, the thumbs turned in, wrists and elbows flexed, and the legs stiff. The body may be still or rigid, or arched, and in the last case the head and neck seek to participate in the curve. The spasm involves the whole body, so that the muscles of respiration are temporarily fixed, and for the time the patient ceases to breathe, and turns blue. The color is noteworthy, because previous to the attack the skin is white and afterward it is flushed. The eyes are open, but the pupil does not react. Then the tonic convulsion, after a short duration, becomes resolved into a number of diminishing clonic spasms, and finally the fit passes away. It is succeeded by a period of stupor, or somnolence, that varies in length.

Treatment. - As soon as one sees such a case, one should apply dry heat to the body, cold to the head, and then administer an enema to empty the bowel. In the convulsions which occasionally occur in the acute fevers during a time of high fever, the dry heat may be changed to a warm bath. If the pyrexia is extreme, the temperature of the water may gradually be lowered until it is a few degrees below the normal heat of the body. The convulsion itself is best quieted by inhalations of chloroform, and in some cases amyl nitrite. After the child has recovered, the gastrointestinal track should be emptied by small and frequently repeated doses of calomel followed by a saline cathartic. To counteract any convulsive tendency that may remain, one may prescribe bromide of soda in large doses, or chloral, or antipyrine. The latter two drugs should be combined with a cardiac stimulant. 
The cause must be carefully sought for and, if possible, corrected. The diet especially must receive scrupulous attention, and the parents should be instructed concerning the importance which an unhealthy state of the stomach and intestines bears to convulsions.

The use of morphine by mouth or hypodermatically is not often necessary, and in most cases should be put aside. Nevertheless, some competent observers have used it. If it must be employed, hypodermatic injection is the best mode of administration, a safe dose being 0.002 gramme (gr. 1-30) for a child one year old.

Prognosis. - In most cases convulsions have no very serious import. With reflex irritations and gastro-intestinal disorders they pass away whon the cause is removed. In the acute infectious diseases they signify a severe invasion, and in very sensitive children their effects as well as their occasions are generally transitory. Nevertheless, one should be cautious in giving a prognosis until a comparatively harmless ætiology is ascertained ; for one should always remember that the seizure may be epileptic, or may follow an injury or organic disease of the brain.

Differential Diagnosis. - The majority of cases, being due to gastro-intestinal disorders, will be preceded or accompanied by a corresponding history of overfeeding or bad feeding, constipation, nausea, vomiting, and anorexia, and usually have some pyrexia. In the cases associated with acute infectious fevers, the temperature is high, the prostration is apt to be marked, and often prodromata may be made out. Convulsions with brain disease are usually not accompanied by pyrexia, are apt to recur, and generally are followed by focal manifestations. In epilepsy the attacks are repeated from time to time, there is no pyrexia, there may be an aura, a cry, biting of the tongue, so that if there is froth at the mouth it is blood-stained. An examination of the urine will show whether there is a nephritic element in the disorder.

\section{EPILEPSY}

This disorder, the old-time morbus sacer, is less often seen in childhood than is usually supposed, and before puberty it is not by any means a common complaint. Like the ordinary "convulsions" of infancy it doubtless is merely a symptom of some as yet 
unknown brain-condition. Its causes outside of injury, malformations, hereditary predisposition, tumors, and disease of the brain, are likewise unknown. It is believed that certain reflex and toxic conditions, such as auto-intoxication from the gastro-intestinal canal, may have a predisposing effect. Characteristic lesions and pathology have never as yet been ascertained, although many theories concerning them have been enunciated. Since these are matters of conjecture, they need not be touched upon.

Symptoms. - The seizures are usually divided according to their duration and severity into two classes, called petit mal and grand mal. The first named consists of slight, convulsive movements which last for a few seconds or half a minute. There is commonly no premonitory sign, and after the attack the child may be dazed and stupid. In other cases the only evidence of the seizure is a momentary loss of consciousness, a momentary stoppage of continuous speech or action, with a feeling of being stunned, of some confusion, of dizziness, or of fainting. Grand mal is much more distinctive and lasts for a much longer time, in some cases even as long as twelve or fifteen minutes. It is often preceded by a premonitory symptom, called an aura. This may be sensory or motor ; in the one case there is a sudden and inexplicable sense impression, such as a certain smell, taste, sound, flash of light, muscular sensation or disturbance ; in the other it consists of some spasmodic movement of a part of the body. The attack itself is often preceded with a cry, and begins with a convulsion which may be partial or general. The child falls to the ground in unconsciousness, and the tonic spasm gradually changes to clonic vibrations which gradually decrease and fade away. The face is pale, but slowly becomes cyanotic, and later is flushed. The eyes are open, the pupils are dilated and do not react to light. The set jaws may have caught the tongue between the teeth as they become clinched, and the resulting hæmorrhage will stain the froth which sometimes forms at the lips. The head may be drawn back or to one side, the elbows and wrists are flexed, the legs are rigid, and the respiratory muscles are set in spasm. There may be involuntary passage of urine and fæces, and the reflexes are often diminished. After the attack has passed, the patient is confused and drowsy.

A mong the most important symptoms or after effects of epilepsy are the psychical states which it may induce. These, broadly 
stated, are any abnormal deviation from ordinary manifestations of healthy, cerebral action. They include any degree of insanity, lack or distortion of memory, perverted moral sense, delusions of persecution, hallucinations, mania, chronic intellectual deterioration, and a host of abnormal or unnatural acts. The epileptic should be regarded as a potential infringer of any normal rule of conduct, feeling, or thought.

A rare form of epilepsy, called "procursive," in which the attack consists of or is preceded by violent attempts to run forward or backward, may for the sake of completeness be mentioned. A doubtful form is the so-called "masked epilepsy," where the convulsion is replaced by a momentary psychosis. In the exceptional cases, where one convulsion follows rapidly on the heels of another, we have the grave condition called epileptic status. It should be mentioned that seizures may occur at night and leave no evidence excepting, possibly, a bloody stain on the bed-clothes, or the results of loss of rectal and vesical sphincter control.

Treatment. - Epileptics, if they are able to select their place of residence, should not live in a large city. A wisely regulated life in the country, as has been demonstrated in the experience of the Craig Colony, may do much to remove causes of irritation. It is of the utmost importance that the diet, occupation, exercise, and general life be regulated in the most careful manner. Such oversight is of as much importance as specific, medicinal treatment. For the latter purpose the bromide of soda or the combined bromides of soda and ammonium are the most useful remedy. It is considered good practice to give the drug in large quantities shortly before an expected attack, while in the intervals the amount is reduced. In all cases it should be well cliluted. During a paroxysm all that one need do is to give inhalations of nitrate of amyl or chloroform, and to put a cork or similar article between the teeth to preserve the tongue from being bitten.

When the epilepsy is due to neoplasm of the brain or traumatic depression of the skull, much good may be obtained by surgical means which seek to remove the irritation. In some cases, however, the benefits are not permanent.

Prognosis. - It is very seldom that epilepsy involves danger to life. Nevertheless cases of cerebral hæmorrhage, of death from asphyxiation due to spasm of the respiratory muscles, or even of rupture of the heart muscle, have occurred. Such cases, however, 
are exceedingly unusual. A more constant menace is the inefficiency which the infirmity puts upon a child: he may not be alone, his ordinary vocations and avocations are limited, and the possibilities of a satisfactory development are lessened. In addition, his intellectual vigor and control are apt to be lessened, and a pathological condition of mind is possible at any time.

Differential Diagnosis. - In most cases the only question involved is whether the seizures are of traumatic, organic, or reflex origin. Rarely is there a question of identity; for the symptoms are so marked and characteristic that continued repetition of them gives sufficient opportunity for careful observation. The attacks which are caused by brain lesion are apt to be partial and focal; but each case must be carefully judged by itself, according to the manifest symptoms. Those which depend upon a reflex origin disappear or are greatly improved when the cause of such irritation is removed. The traumatic cases give a history and show the evidences of the injury. Usually it is not difficult to exclude uræmia and hysteria on the facts of the history.

\section{Chorea}

Chorea, chorea of Sydenham, chorea minor (in contra-distinction to the hysterical chorea major), and St. Vitus's dance are the names given to a neurosis of childhood that has irregular and involuntary twitchings of one or more parts of the body as its principal characteristic. It occurs most frequently at and under the age of puberty, and may even be congenital.

Causes. - Its causation has been a matter of much dispute. It has been put among the hereditary diseases, it has been attributed to fright, to reflex irritations, to conditions involving imperfect aëration of the blood, to exhaustion, and to the rheumatic diathesis. The growing opinion of to-day tends to emphasize the connection with rheumatism. Chorea certainly is associated in the majority of cases with rheumatic manifestations, such as articular pains, "growing pains," endocarditis, pericarditis, subcutaneous nodules, erythema nodosum, or a family tendency. These symptoms may precede, accompany, or follow the chorea. An additional fact of importance is that the only other acute disease with which chorea is connected is scarlet fever, which in turn is closely associated in some cases with a rheumatic 
sequel. Future investigation will doubtless prove the intimacy in ætiology of chorea and rheumatism ; even more, it may demonstrate that chorea is nothing more than a rheumatic manifestation, and that the other factors which were formerly considered causative are no more than coincidental or predisposing. Girls are oftener attacked than boys.

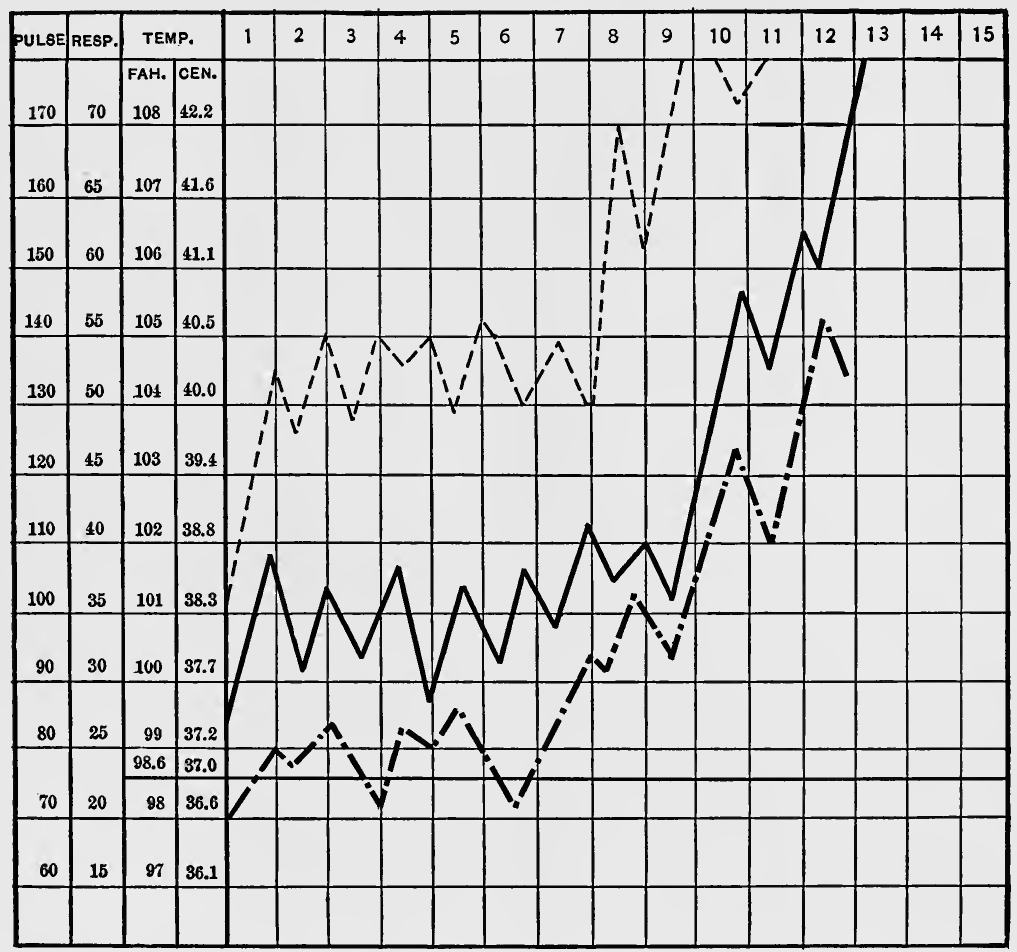

PULSE, RESPIRATION AND TEMPERATURE CHART OF CHOREA, LOBAR-PNEUMONIA. HYPERPYREXIA, DEATH.

AGE, 6 YEARS.

PULSE______- RESPIRATION _-____ TEMPERATURE

Frg. 121.

There are no known and characteristic lesions of constant occurrence.

Symptoms. - The twitchings of the affected parts are irregular, spasmodic, uncontrollable; but they subside during sleep. While any part of the body may be affected, the muscles of the face, arms, and hands are the seats of election. A noteworthy 
fact is that attempts to control the neurosis by the exercise of the patient's will-power result in a possible momentary quiet which is promptly followed by exaggerated movements. The twitchings are not constant in any except very rare cases, but occur in gusts or flurries. Usually they are not profound, although occasionally one may see a child who is unable to sit or stand up on account of the disturbance which they create. In most instances they may be noticed as an inability to keep the face quiet, to protrude the tongue easily and evenly, or to execute purposive movements with cleanness and celerity. Sometimes a group of muscles is affected, or a localized part of the body ; the involvement of half of the body has produced the name hemi-chorea. In all cases there is apt to be some weakness of the affected muscles which in unusual instances may be so severe as to simulate paralysis. These children have poor mental control, are irritable, hysterical, and easily excited, and bear restraint poorly. Very commonly they are anæmic, insufficiently nourished, have headaches, deficient appetite, and a tendency to functional disorders of the gastro-intestinal system. If complicating diseases occur, they are apt to run a much more severe course than otherwise. Speech may be sufficiently affected to produce a dysarthria, or the chorea may be laryngeal and characterized by peculiar, barking explosions of sound. The urine has an excess of urates and phosphates, the tendon reflexes are diminished, and electrical stimulation of the muscles produces overexcitation. A very significant fact is the frequent occurrence of a heart murmur that is usually indicative of endocarditis, but which occasionally may be hæmic. Other rheumatic manifestations of rheumatism, such as articular pain, "growing pains," subcutaneous nodules, may also be present.

Treatment. - The best thing that can be done for these children is to confine them to bed, at the same time providing for the administration of nutritious and easily digested foods, warm baths, an active condition of the bowels, and general massage. The more complete and continuous the rest, the sooner will the child recover. If this " rest-cure" is faithfully carried out, there will, in many cases, be little necessity for medication. Of all the drugs employed arsenic is the surest to give good results. It may be prescribed in the form of Fowler's solution, and given in increasing doses, dissolved in an alkaline water. A good method of administration is to begin with four or five drops three times a 
day; each succeeding day should increase each dose by one drop, until from fifteen to twenty drops are given at a time. It is then wise to begin at a smaller number, and again increase the amount. Usually a combination of the rest-treatment and arsenic will be efficient. At the same time the condition of the stomach and the conjunctivæ should be watched in order to guard against cumulative and poisonous effects. As the child becomes quiet, it may be necessary to treat complicating symptoms of anæmia, endocarditis, gastro-intestinal disorders, or malnutrition.

Prognosis. - The outlook is good and recovery may be predicted in almost all cases, except where complicating symptoms or diseases are grave enough to endanger life. It is impossible to foretell the probable duration of the disease. At the same time the parents should be informed that the attacks are liable to return, especially if the patient is allowed to fall back into a poor condition, or is permitted to undergo much exposure. A very few cases run a chronic course.

Differential Diagnosis. - There may be an hysterical imitation of chorea which is at times deceiving. It may be distinguished by its slower action, longer intervals between paroxysms, its more rhythmical character, and the presence of other signs of hysteria. Mere "nervousness," general convulsive tic, can be differentiated by its occurrence in periods of rest rather than during voluntary movements, by absence of awkwardness in action or muscular weakness, and finally by a chronic course. The choreiform movements, or hemi-chorea, that may follow hemiplegia have slow, irregular motions on voluntary exertion rather than spasmodic twitchings; moreover, there is a history of some organic lesion. The disorder should not be called choreic.

\section{Tetany}

This disease, called also tetanilla and pseudo-tetanus, occurs under a large variety of predisposing or caúsative circumstances. One of its most frequent methods of occurrence in children is during or after conditions of malnutrition or exhaustion. Such conditions may be rachitic, gastro-intestinal, or convalescence after acute. disease. Bad food and bad hygiene may predispose it, and in general one may say that whatever destroys the normal equilibrium of the body prepares the way for an attack of this 
disorder. The extirpation of the thyreoid gland produces typical symptoms, although the method of the production is not understood. The lesions and pathology are not known.

Symptoms. - The attack begins by a sensation of an indefinite disorder in the extremities, followed by a diffuse tingling and fragmentary painfulness. This is succeeded by a growing rigidity of the muscles, which finally develops into a tonic spasm that may be so severe as to prevent both voluntary and passive motion. The hands are oftener and sooner affected than the feet. The flexor muscles are the principal sufferers. The fingers assume the writer's or accoucheur's position so regularly as to give by this means a characteristic sign. In mild cases as little as one finger may be affected; but in severe attacks the forearm may be flexed at the elbow, and the arm adducted to the shoulder. In the same manner the toes become flexed, the arch of the foot cupped, and the legs extended to the thighs. These spasms may last for minutes or hours, may suddenly cease, or continue to recur. While they last the muscles are hard and tender on pressure, but in the intervals they may be no more changed than to feel somewhat stiff and sore. In unusual cases the trunk, neck, and face may be involved, with a resulting interference with the muscles of respiration, of the eyes, tongue, and the powers of articulation and deglutition. The patient may feel a constant desire to urinate, without being able to gratify the desire. There is commonly a deficient muscular sense, and the patient, if he is old enough, speaks of feeling as if he were walking on a velvet-like fabric whenever he moves. During the attacks there may be an elevation of temperature, sweating, headache, dizziness, and a sensation of hearing a roaring noise. The involved muscles are symmetrically situated, and often present a fibrillary twitching.

A noteworthy observation, called after the name of its maker, Trousseau, is the fact that a spasm may be caused during an interval, or exaggerated, if it is present, by pressing upon or constricting an affected part. This sign is not invariably present, but it is often supplemented by the so-called Chvostek symptom, which consists of an unusual sensitiveness of the nerves to mechanical irritations, such as light blows. It is also not invariably present, and may be best obtained in the face by striking with the finger, as in percussion, below the zygoma and in front of the ear. Erb's symptom consists in an exaggerated response 
of the nerves to electrical stimulation. Both anodal and cathodal opening and closing contractions may be obtained with a comparatively weak current.

Vaso-motor disturbances have been observed-such as a puffy redness in the neighborhood of the joints, and disturbances of the hair and nails. Atrophy of the muscles and mental derangements have in a few cases been noticed.

Treatment. - There is no specific treatment, although symptomatic care is usually efficient. First of all, the general environment must be improved. Diet, bathing, clothing, ventilation, and the gastro-intestinal functions must be carefully regulated, and when possible the patient should be removed to the fresh air of the country. He should under all circumstances be confined to bed. For the control of the spasms, antipyrine prescribed in connection with a cardiac stimulant will give satisfaction. Other useful drugs are bromide of soda, in large doses, and chloral. In exceedingly severe attacks it may be necessary to resort to temporary inhalations of chloroform.

Because the symptoms of tetany have followed extirpation of the thyreoid gland, the plan of prescribing the extract of the dried gland has been proposed. The value of the method is as yet unknown.

Prognosis. - Usually these cases, after a variable length of time, recover. There are a few fatal cases, most of which seem to occur in children who have a coincident complication of gastric dilatation.

Differential Diagnosis. - Tetany may be confused with tetanus or hysteria. In the former trismus is a common and early symptom, while in tetany it is a rare and late one. In tetanus and hysteria there will be an absence of the Trousseau, Chvostek, and Erb symptoms, which, with the general picture of the disease, constitute an easy and ready way of marking it off from other complaints.

\section{Hysteria}

Hysteria is a functional disorder of the nervous system, whose manifestations are so wide and irregular that every case is apt to vary in some detail or other from any strictly formed type. It is not so commonly seen in children as in adults; and, following a like rule, its frequency diminishes as we try to gather cases 
from younger and younger children. The possibility of becoming hysterical seems to depend upon the disturbance which follows a state of overpowering impressions, of being overmastered by some fixed idea or powerful impulse. In all cases, however, there seems to be a necessity of a predisposition, expressed or latent. Such a tendency may flow from hereditary taint, association with hysterical or imperfectly restrained persons, physical depression or asthenia, such as might follow poorly regulated lives, acute or chronic diseases, injury, fright, overexertion, sexual malformations, and perversions, vicious habits - in fact, any condition which makes for lack of nervous equilibrium. There are no known lesions that are characteristic of all cases.

In mild attacks the symptoms may be of almost any variety; there may be psychical, sensory, or motor disorders. In the first type we may see such symptoms as uncontrollable laughing or crying, occasionally followed by some degree of mania. In other cases there may be a longer period before the mania, made up of irritability, sleeplessness, lack of self-control, and functional disorders. In still other cases there may be cataleptic, somnambulistic, and trance-like states that may occasionally start from impulses which seemingly are petty. Again, there may be attacks of hystero-epilepsy (more properly called grand hysteria or hysteria major), although there is no real epilepsy present. In such cases there is an aura, often globus hystericus or clavus, in other instances ovarian or testicular pain. Closely following this there will be an epileptoid condition which gives way to a stage of "grand movement." This stage is characterized by violent postures or movements which may be complexly automatic, purposive, or atypical; they may even take the form of pronounced opisthotonos. The last stage that we usually see is one of delirium, in which the child cries and weeps incoherently, and then finally becomes quiet.

Under the heading of motor symptoms one may include practically every sort of clonic and tonic spasm or convulsion. These may vary from a mere twitching of a muscle or an extremity to elaborate attacks of chorea major. Under this division, again, may likewise be included hystero-epilepsy, whose manifestations are so manifold as to allow its being placed in any variety. Chorea major, which has no choreic symptoms, is really a partial or incomplete attack of hystero-epilepsy. Among the other possible 
symptoms are hysterical cough, wry neck, hiccough, laryngeal spasm, dyspnœa, œsophageal and gastric spasm, intestinal and vesical contractions, or even a pseudo-paralysis.

The sensory symptoms include any form of increased or diminished sensation. Hyperæsthesia is most commonly located over the ovaries, testicles, and spine; and pressure over these socalled hysterogenous zones is capable of producing typical attacks of hysteria. In some children the painful areas are confined to one or more joints, and occasionally their ability in magnifying these pains into the likeness of a serious organic disease is remarkable. Also, anæsthesia is comparatively common, and its atypical situation is really typical. It has no respect for anatomical distribution and may cover any spot on the surface - an extremity, a zone, or a half of the body. In the last-named case the anæsthesia is remarkably complete and may carry with it a loss of sight, hearing, smell, and taste on the affected side. In other children the blindness may be the only manifestation, or may be replaced by photophobia or other sense deprivation.

Among the conditions that are seen in conjunction with hysteria are various grades of anæmia, gastro-intestinal disorders, dilatations of the stomach and small intestine.

Treatment. - The first and often most important step lies in the direction of improving the child's environment. This will often necessitate separation from a parent who is hysterical or lacking in self-control. If possible, the patient should be placed in charge of a relative or nurse who has the blessed gift of common sense, with an admixture of sympathy. The child should be treated kindly but with firmness, and his custodian must be made to understand that the disorder thrives upon the large amount of fright, commotion, undue solicitude, and forced restraint which its manifestations elicit in the spectators. He should lead a regular life, with wisely regulated duties, exercises, and relaxations. Hydrotherapy is of great use, and the administration of general tonics is in all cases necessary. If there are any possible causative deformities, such as a tight or very long prepuce, or an hypertrophied pharyngeal tonsil, they should be rectified.

Prognosis. - While the chances of recovery are large, nevertheless children are apt to bear the marks of their disorder for a long time, as well as to preserve a tendency to repeated attacks. 
This especially is true of those cases that have an hysterical descent or the family association of hysterical persons.

Differential Diagnosis. - It is difficult to give an exact method of separating hysterical manifestations from all others. In certain minor degrees they are scarcely recognizable, or may be combined with the symptoms of organic disease in such a way that one must use the best judgment in drawing a line between the two. On the whole, one may say that by recognizing the hysterical stigmata, such as the physical, motor, and sensory disturbances, and the lack of definite or organic disease, one may arrive at a true view of the case in hand. It is only in the unusual case, if there is full opportunity for satisfactory observations, that the real state of affairs is hard to see.

\section{HEADACHES}

Pain in the head is a symptom that is seen in almost every diseased condition at some part or other of its course. The only safe way in which we can regard it is as a secondary matter, whose cause must carefully be sought for and isolated before a real cure can be obtained. Each observer may make his own classification, and one may be as good as another. The main thing is to insist upon a thorough sifting of the various facts in each case; for the physician should never be satisfied that the disorder is of no importance, until he has excluded every cause except a trivial one. In children, even more than in adults, a headache may be a danger signal to inform one of a pathological condition, existent or forming, which may finally become of considerable importance.

Anæmia and malnutrition are a fruitful source of headaches, which, if accurately located, are referred to the forehead and bregma. They are dull and heavy, and are apt to be most marked in the morning.

Gastric disturbances are one of the commonest causes of headaches in children. Usually they are frontal, but may also be located in the top of the head ; with them there will be associated other symptoms of the primary complaint. They have a tendency to recur, and also to run a course of remissions and exacerbations. Their mode of causation is doubtless associated with the absorption of the products of partial digestion, fermentation, 
and putrefaction of food stuffs. In this way they represent a form of poisoning, and are closely related to the so-called toxic causes.

These toxic causes cover a wide range of conditions. They arise from the absorption of poisonous substances in the acute infectious fevers, uræmic conditions, malaria, and the metallic poisons, such as lead. Each is apt to be accompanied by other symptoms by which its identity can be settled.

Disorders of the special senses, in the eyes, ears, and nose, are an important source. Eye-strain, and pathological conditions of any part of the eyes; abscess, otitis media, foreign bodies in the ear, and mastoid disease ; hypertrophy of the pharyngeal tonsil, deviations of the septum, nasal polypi, and acute rhinitis, all these are able to cause headaches which are variable in location and degree.

Less often in children some pathological condition of a part of the nervous system is at fault. Here again the range is very wide. There may be organic disease of the brain and its membranes, new growths, neuresthenic states, hyperæmia, induced by fright, excitement, strain, or physical malnutrition. In this class one might easily include reflex conditions, such as the headaches which follow genital malformations or disordered function. A tight or adherent prepuce has been known to cause severe pain in the head, which ceased when the glans penis was released. Delayed or disordered menstruation is frequently connected with headaches in young girls.

In all these cases the syndrome must be carefully analyzed, and if the analysis is correct, one will at once have obtained diagnosis, treatment, and prognosis. The headache should be regarded as a secondary consideration. The primary disease is the main factor. 


\section{CHAPTER XXIII}

\section{DISEASES OF OBSCURE ORIGIN}

\section{Diabetes Mellitus}

This disease is not often seen in children, but when it does attack them its course is severe and rapid, and its outcome is commonly fatal. Its causes are unknown; but I remember two cases, both in girls, where the condition occurred after acute disease of a severe type. Boys seem, according to statistics, to be somewhat oftener afflicted than girls. In some families there seems to be a marked hereditary influence, on account of which the disease has a liability to break out during some period of profound physical exhaustion.

The symptoms appear in the form of polyuria, polydipsia, disorders of the gastro-intestinal track, and progressive loss of flesh and strength. The child complains of many nervous symptoms, of irritability, lack of concentration, variable appetite, and noteworthy change of disposition. The urine may have a specific gravity of from 1.020 to 1.040 , the increase in quantity is large, and it may contain indican, acetone, and diacetic acid. There may be albumin, or even casts, which usually are the result of a superimposed nephritis. Coincidentally there may be small quantities of diacetic acid and acetone in the blood.

The treatment is unsatisfactory, and consists of strict dietetic regulation, with the use of large doses of strychnine. The bichloride of mercury has been advocated in New York for this disease; but the results are not encouraging. Also, the use of opium has been advised, and temporary amelioration of symptoms has in some cases followed it. But we have no reason to believe that the drug will make a definite cure. With the regulation of the diet according to the rules which we observe in treating adults, we combine a conscientious supervision of the hygiene, exercise, studies, and general mode of life.

The prognosis is bad, and commonly death results from gradual asthenia, diabetic coma, or intercurrent disease. 


\section{Rheumatism}

Rheumatism in children is a widely prevalent disease, much more so than is commonly believed. It occurs in such varied and at times obscure forms that its true nature is commonly overlooked. One more often hears of some of its manifold manifestations, such as endocarditis, pericarditis, "growing pains," tonsillitis, torticollis, and chorea, than of the typical arthritis that is so commonly seen in adults. The cardiac disorders in particular are so prominent and frequent, they exert so overmastering an influence in the course and outcome of the disease, that one is often in doubt whether they or the arthritic symptoms should be regarded as the typical characteristics of the sickness.

Causes. - The tendency of modern research is all in the direction of placing both on the plane of important but not absolutely essential symptoms. One cannot as in former times regard rheumatism as a "blood" disease, and progressively there is more and more difficulty in believing that it is a nervous or chemical disorder. Uric and lactic acids, while covering a multitude of diagnoses, cannot longer be held responsible for rheumatism, any more than the free melanin developed in the course of malaria can be regarded as the essential cause of paludism. On the other hand, the more one observes the disease the more is one struck by its resemblance to an acute infection of bacterial origin. It occurs in waves, as enteric fever or scarlet fever does ; a house in which a rheumatic person has lived is very apt to have other cases of the same sickness; its peculiarity of attacking a viscus that is removed from the ostensible seat of disorder is striking; and a person who is in poor physical condition, whose environment provides poor air, food, and habitation, is more apt to contract the disease than a well cared for child. Its more frequent occurrence at one time of the year, as in late autumn and early winter, than at others creates a still further resemblance. The fact that the disease seems to run in an hereditary strain through certain families should be regarded in the light of a predisposition, such as exists in tuberculosis. Under these circumstances a child of rheumatic parentage acts as a fertile culture medium for the germ; his body welcomes, especially in conditions of debility, instead of fighting against the contagion. It is a noteworthy fact that where both parents are rheumatic, the liability of the child 
to contract the disorder is much greater than if only one parent is tainted.

Rheumatism in the earliest years of life affects boys oftener than girls; from five to ten years the rate is nearly even; from ten to fifteen years girls are attacked more frequently than boys. The degree of severity seems to be about the same in both, and complicating conditions show no partiality for either. The explanation of these changes is hard to give, but the facts themselves are interesting.

Lesions. - The changes which are regularly present are not startling, and it is only in marked cases that they become noteworthy. In the joints the synovial membranes may be congested and infiltrated with lymphoid cells, and the superficial layers may be in a condition of desquamation and proliferation. There may be an effusion of serous fluid into the cavity, in the walls of which one may discern fine hæmorrhages. Fibrous tissues about the joint become infiltrated with products of inflammation, so that they are thickened, nodular, and hard. In some cases this thickening and hardening process attacks the fascial tendons entirely outside of and beyond the joint, and may especially in cases of serious cardiac complication be easily felt through the skin. These nodular formations are closely allied to the neoplastic roughnesses on an inflamed heart valve or the pericarditis of this disease. Endocarditis and pericarditis are very common, and are marked by interstitial changes. These lesions of the latter condition may extend to the connective tissue of the anterior mediastinum, so that a mass of matted adhesions may be formed which seriously impedes the growth and development of the immature heart. The ordinary complications and sequels of cardiac disease may follow.

Symptoms. - The attack may begin very quietly, with indisposition, restlessness, and slight or moderate fever. There is often pain in one or more joints, but at times it is so slight as to attract little attention; in some cases only one joint is attacked, and the pain may remain there or flit about to other joints. Thus the knees, hips, ankles, feet, elbows, and shoulders may be involved, together or separately. Occasionally we can discern no more than stiffness and a slight soreness in the tendons of the muscles, or muscular spasm may be so prominent as to overmaster the other objective signs. In some of these cases the child is with difficulty 
kept in bed, and insists on being about even if in doing so he is forced to hobble. The temperature does not often rise beyond $39^{\circ} \mathrm{C}$. $\left(102.2^{\circ} \mathrm{F}\right.$.), the stomach and intestines are not seriously disturbed, and the patient does not seem seriously sick.

On the other hand, one occasionally, most of all in older children, sees sharp and violent attacks where the joints are acutely swollen and very painful, the temperature high, the prostration marked. Instead of lasting for a week or two, the disease continues for nearly a month. The heart disturbance may, from the first, be startling, and the anæmia may be so sudden and profound that its hæmic murmurs are interpreted as being caused by an endocarditis. This impoverishment of the blood is so characteristic of rheumatism that it may be noticed in the mild as well as in the severe cases. However, when these violent cases occur their course is much the same as in adults.

The inflammations of the pericardium and endocardium as well as of the heart muscle are more frequent in their incidence, and proportionately severer in their course than similar attacks in adults. An endocarditis may be one of the first symptoms, or may come at any part of the disease. It commonly begins in a quiet fashion, and its presence may not be noticed until the heart is auscultated. In some cases, from its onset, there may be precordial pain, irregular heart action, embarrassed breathing, and the characteristic murmur. Most commonly the mitral valve is rendered insufficient; but any other pathological condition may also result, and one or both of the other valves be simultaneously involved. The severity of these inflammations may cover the widest range : they may be merely an accentuation of the two sounds, or the second alone may be accentuated or duplicated, with no worse symptoms than thin, hard pulse, dyspncea on exertion, slight cough, and nervous irritability. On the other hand, the endocarditis may become violent and give all the symptoms that belong to fatal cardiac disease. The ulcerative form is, excepting in older children, very rarely seen.

Pericarditis usually appears late in the sickness, with or without endocarditis. The severer the endocarditis the greater is the likelihood of an associated pericarditis, and in these grave cases the attack may come sooner than in the mild ones. The onset in most children is, like that of the endocarditis, gradual; the temperature is moderate, the pulse rapid, the heart action labored. 
The pericardial friction sound is plainly heard, usually near the apex. If effusion into the pericardial sac takes place, the area of cardiac dulness becomes correspondingly enlarged. The growth of adhesions may be considerable, so much so, in fact, that the ordinary signs are changed and made difficult of recognition. Nevertheless, the rapid, irregular, and straining heart action remains, no matter what remedies are administered. In some children the result may be obliteration of the whole pericardial cavity, the heart may be bound to the neighboring tissue, and the fibrosis may extend over a part of the anterior mediastinum. In this way the mediastinal glands, the pleura, and pericardium may become so changed and connected that normal action of both heart and lungs is seriously, or even fatally, impeded. In such severe cases of pericarditis and endocarditis there is generally an interstitial myocarditis as well. The added symptoms of weakened and irregular heart action are not plainly discernible, and the condition is usually ascertained on post mortem examination.

In these cases of grave cardiac changes one sometimes meets with the development of fibrous nodules near the skin in the fibrous tissue of fasciæ and tendons, and on autopsy they may be found in the periosteum, and sometimes on the pericardium. I have seen them along the spine, on the ankles, backs of the hands, and on the knees; also they may appear on the elbows and back of the head. They may be as small as small seeds, or as large as a small nut. They last for a variable time, from a few days to months. They give no especial symptoms, and their importance lies in the fact that they indicate a tendency to serious cardiac involvement. Such cases, more than all others, should be treated with the utmost caution.

The connection of chorea with rheumatism is a very close one - so close, in fact, that many observers believe chorea to be essentially rheumatic in its origin. Not only is rheumatism in children often followed by chorea, but also, on the other hand, chorea is commonly the forerunner of an attack of rheumatism. And even when it occurs without arthritic manifestations, its connection with cardiac lesions is striking enough to attract attention. The relationship between these three groups of symptoms is remarkable, so that one may safely regard a rheumatic child as potentially choreic and cardiopathic. The general predisposition so plainly runs through families that on seeing a marked case of chorea, one 
may expect in other children of the same family cardiac or rheumatic tendencies. In these cases the degree of anæmia is greater than in the milder ones; but all rheumatic children may be said to suffer in some measure a deterioration in the quality of the blood, whose signs are like those of simple secondary anæmia.

Another manifestation of the rheumatic disposition that is doubtless allied to the fibrous infiltrations of the tendons is the spasmodic torticollis which one occasionally sees. It may come without warning, or with a rheumatic attack. The sterno-cleidomastoid muscle is put on the stretch, sometimes to a very painful degree, so that the head is bent to the opposite shoulder. This is especially likely to happen in those children who easily suffer from inflammation of the tonsils and pharynx, which in itself may commonly be looked upon as another indication of rheumatism.

An unexplained phenomenon in this disease is the appearance of purpura hæmorrhagica. This may possibly be an indication of hæmophilic tendency, a simple purpura, or some special and severe form of infection which is peculiar to rheumatism. Doubtless one form exists in one child and another in the next. But there is good reason to believe that some cases have a distinct connection between the inflammation in the joint and the hæmorrhagic eruption that is generally located near to it. The lower extremities are affected more often than other parts of the body.

The only other skin eruption which we meet in rheumatism is erythema. The ordinary forms are all represented, and stated in the order of frequency would read: papulated, marginated, urticarious, and nodular. They may accompany cases in which the articular symptoms are predominant and, on the other hand, may appear where the cardiac disorder is supreme and the articular trouble slight. They have no especial significance.

Treatment. - At the first indication of rheumatism the gastro-intestinal track should be emptied by means of small and repeated doses of calomel, followed by the citrate of magnesia. The affected joints may be wrapped in cotton-wool, and, if the pain is great, protected by some sort of cradle to prevent pressure or injury from without. A few doses of salophen may be rapidly administered to relieve the pain; when the child's suffering has abated, this drug may be replăced by the natural oil of wintergreen given on a lump of sugar, salicin, or the salicylate of soda. In extremely painful cases it may be necessary to give a few doses 
of opium to quiet the patient. In children, even more than in adults, it is absolutely necessary to reduce the suffering as quickly and as thoroughly as possible. They, much more than their elders, are injured by the restlessness and exertion which come with suffering. It is in this way that cardiac disorders, to which they seem to have a strong tendency, are invited. Under such circumstances measures, which otherwise might not be looked upon as necessary or even justifiable, become highly desirable. The value of opium or morphine is often thus indicated, most of. all if endocarditis or pericarditis is imminent or existent. With this complex of symptoms there is no other drug of greater importance. Such a cardiac inflammation may, in addition, necessitate the application of an ice bag to the pericardium and the use of digitalis or strophanthus.

The child should receive a sponge bath every day, and if the temperature is high, he will be benefited by being placed in a bath of an approximate temperature of $37.5^{\circ} \mathrm{C}$. $\left(99.5^{\circ} \mathrm{F}\right.$.), which is rapidly reduced to from $16^{\circ}$ to $19^{\circ} \mathrm{C}$. $\left(60.8^{\circ}\right.$ to $66.2^{\circ} \mathrm{F}$.). During this bath the surface of the body, excepting the inflamed joint or joints, should be briskly rubbed with the hand. The diet should be milk, meat soups, and beef extracts; and a sufficiently large quantity of pure water must be given.

Until recovery is assured, or at least until all tenderness has left the joints and dangerous cardiac symptoms have disappeared, the child must be kept in bed. After this period has passed, he may be taken into the sunshine. It will then be necessary to treat his anæmia, to endeavor to promote compensation if he has had cardiac disease, and in general to build up his health and strength. For months after the attack he should be under medical supervision, even although active medical treatment be unnecessary. His diet should be carefully supervised, and it especially should not be allowed to include more than a minimum of starches and sugars.

Prognosis. - The principal danger in the rheumatism of children is not the joint as much as the cardiac affection. As far as the articular part is concerned, the outlook on the whole is not bad, and the cases generally recover within one or two weeks. A disturbing factor, however, is the well-marked tendency of the disease to recur; and the successive attacks progressively weaken the patient, and increase the dangers of cardiac involvement. 
These dangers are very serious ones, for at any moment an endocarditis that has seemed comparatively slight may burst out into extravagant violence.

Differential Diagnosis. - The diseases with which rheumatism may be confused are scurvy, hereditary syphilis, tuberculosis, pyæmia, and rachitis. But none of these disorders has the combination of family and personal history, involvement of joints, possible endocarditis, pericarditis, chorea, tonsillitis, torticollis, subcutaneous nodules, and tendency to erythematous eruptions. The association of comparatively slight articular disease and an ever present leaning to cardiac disorder is in itself often diagnostic. Instances of "growing pains" come under this head, and should always be looked upon as likely to foreshadow serious trouble. Finally, one must keep in mind that rheumatic patients are anæmic, that anæmia is often accompanied by soft hæmic murmurs, which one should not confuse with those of true endocarditis.

\section{Rheumatoid Arthritis}

This is a very rare disease in children, the cause of which is unknown. Most of the few cases on record have occurred in England and on the Continent of Europe. Almost all of them were in children under six years of age.

There is a chronic swelling of the joints, which gradually become anchylosed. The synovial membranes and ligaments are injected and thickened, the capsule may be swollen, and the synovial fluid may be increased in quantity. The spleen and lymphatic glands are enlarged as the result of simple hyperplasia, and may show minute ecchymoses.

The disease begins in a slow and gradual fashion, with slight or no fever. There may be some tenderness in the joints, which, however, is practically never very great. The joint gradually increases in size, at the same time slowly losing its power of movement. The disease very slowly involves the knees, elbows, wrists, fingers, toes, and cervical vertebræ. The articular enlargement is smooth and regular, therein differing from the irregularly knobbed contour of the adult form of the disease. When these joints are moved, one can feel the creaking and rubbing of the tendons and cartilages. 
As the child becomes helpless and is confined to bed, the muscles become atrophied, weak, and flabby. In some cases muscular contractures are formed. The glands are hard, swollen, not sensitive. The spleen is enlarged, there may be inflammations of the pleural and pericardial sacs. There is a marked loss of flesh and strength, a distinct anæmia, and a moderate febrile movement. The disease runs a slow and sure course, with periods of remissions and exacerbations.

There is no specific treatment, and all that one can do is to relieve the symptoms. Death comes from some intercurrent disease.

Another form of rheumatoid arthritis, that is quite similar to the disease in adults, may occur in older children. It needs no special description in this place.

\section{Acute Arthritis of Infants}

A form of arthritis, which is really an acute purulent disease of the bone, occurs in young infants as the result of pyogenic infection contracted through the umbilicus, a wound, or after the acute infectious fevers. It may attack any of the articulations, but oftenest affects the hip, knee, shoulder, wrist, elbow, ankle, the fingers and toes. The lesions are what ordinarily occur in purulent synovitis. The symptoms are the regular ones of pus infection, - a chill, irregular fever, prostration, local pain, heat, and swelling. The formation of pus leads to the sensation of deep fluctuation; and if the abscess is not opened the fluid, after destroying the joint, breaks through the capsule into the surrounding tissue, from which it may finally rupture.

The disease is really a surgical one, and demands the usual surgical treatment of any abscess cavity. The chances of life are very poor, as they are in all cases where infants have to contend against a pyæmic infection; in the cases that survive, the only hope resides in an undelayed operation. 


\section{CHAPTER XXIV}

\section{INFLAMMATIONS OF THE MIDDLE EAR}

Causes. - Otitis media is a common trouble in infants and children; the fact that it does not always end in a discharge from the ear helps to obscure its existence, and prevent a diagnosis. Evidence of this lies in the many cases where the physician learns with surprise that serum or pus has suddenly appeared, usually attended by relief of the symptoms which he had believed to be due to other causes. In some cases the disorder develops without any known reason ; in others it accompanies or follows complaints that often do not at all affect the ear. The lack of inevitable sequence is thus the main excuse for a failure to appreciate the facts of the situation. While injuries, blows, and foreign bodies can set up such an inflammation, they are, nevertheless, comparatively rare. Usually the trouble begins by extension or infection from the nose and Eustachian tube, and abnormal conditions in these parts must be regarded as the primary factor. Thus the various forms of rhinitis, of pharyngitis, of amygdalitis, of laryngitis, and hypertrophy of the pharyngeal tonsil are the real responsible elements, aided by the size, direction, and patency of the youthful Eustachian tube. These diseases may exist alone, or may accompany or follow some of the acute infectious diseases, such as measles, scarlet fever, epidemic influenza, diphtheria, enteric fever, and pneumonia. When the inflammation begins it may be simple or, in the presence of the proper micro-organisms, may become purulent. Either may be acute or chronic.

Lesions. - Commonly the changes begin in the mucous membrane of the tube, which becomes reddened, congested, and swollen. The process then extends into the ear, where, in addition, a small amount of serum or mucus is held. The swelling and consequent occlusion of the tube prevents an escape in that direction, so that the fluid is retained, with the natural results 
of fever and pain. When this fluid becomes contaminated by pyogenic organisms, pus is formed. The symptoms become more marked, especially in the face of the developing lesions. The pus may rupture through the membrane, as in an abscess formation; or, following the eccentricities of such processes, it may burrow through adjacent structures. Thus, in a few rare cases, it has made its way along the cartilages, and then through the skin. The serious results of purulent otitis may be mastoiditis, purulent inflammation of the ossicles and bony walls of the ear, of the petrous bone, meningitis, cerebral and cerebellar abscess, thrombosis and of the intra-dural sinuses.

Symptoms. - In the simple form the signs may not be very pronounced. There will be an irregular but moderate fever, pain in the ear on pressure or without pressure, headache, malaise, and, if a synchronous disease exists, an exaggeration of the subjective symptoms of that disease. In some cases no change in the tympanum can be noticed; in others it is congested, lacking its normal hue, and to a small extent bulges out. After a few hours or days a thin serous discharge may be noticed, and with its appearance the pain and fever disappear. The child, even in slight attacks, is left weak and exhausted. If the amount of fluid is large, the pain and pressure symptoms are greater.

In the purulent inflammation the symptoms are much severer. The disease may begin with a chill, there may be nausea and vomiting, and convulsions and delirium are not rare. The temperature runs higher, often reaching $40^{\circ} \mathrm{C}$. $\left(104^{\circ} \mathrm{F}\right.$.), and the pain is keen and tearing, giving rise to a peculiarly sharp cry. In times of consciousness the child puts his hand to the affected side of the head, is unwilling to lie upon it, and fears a foreign touch. The whole surface around the ear is tender, especially in front and behind it, and abnormal sounds may be heard. 'Otoscopic examination frequently shows a swollen and protruding membrane, most of all in the lower arc. Spontaneous perforation relieves the pain, pressure, and temperature, and either may be recognized directly, or may be inferred from a large amount of discharge, a whistling sound on blowing the nose or inflating the ear, air-bubbles in the canal, or pulsation of the membrane.

The complications are always serious. Mastoiditis may be marked by external rupture more easily in young children than in adults, on account of the thinness of the one cavity, the antrum. 
In most cases this does not follow; but the symptoms of pain, local swelling, which pushes the external ear away from the head, and pressure effects require treatment before they subside. In other cases a pachymeningitis or general meningitis may result from infection through the tegmen tympani; most rarely it follows, as a secondary effect, some other brain disease which originated in the same way. These diseases are abscesses of the cerebrum, or cerebellum, and thrombosis of the intra-dural sinuses. On the other hand, the meningitis, being the first infection, may be followed by these other infections as secondary complications. Facial paralysis may occur as an extension of the inflammation to the seventh nerve, which passes through the bony canal. The labyrinth is rarely involved.

Treatment. - The stomach and intestines should be thoroughly emptied. A rubber bag filled with hot water should be placed against the ear, while the cavity of the organ may be filled with a warm boracic acid solution, or a pledget of cotton soaked in such a solution. 'The warmed oils, the tinctures of opium and peroxide of hydrogen, supplemented by the application of leeches, which were formerly used, are now thought to do as much harm as good, some by means of producing an unclean surface, others by affecting the integrity of the delicate epithelium. Sometimes, especially in mild cases, considerable relief may be obtained by the instillation of a few drops of a one per cent solution of cocaine in boracic acid. But the main plan of treatment is to apply dry heat for the relief of pain, while the boracic solution will cleanse the canal preparatory to a possible paracentesis, and at the same time, by its soothing property, give a small added measure of comfort. Such a method is fully equal in efficiency to the use of an ice bag, the cold of which most children undoubtedly dislike. If the symptoms are not relieved within six hours, paracentesis of the membrane should be performed. No deep syringing is necessary, for there is always danger of thereby carrying a purulent discharge farther than it had hitherto progressed. The ear may be lightly washed with a saturated solution of boracic acid, and lightly packed with sterilized gauze for protection and drainage. The temperature falls almost immediately, unless there is an involvement of the bony structure. If it begins to mount again, the cause must be sought in imperfect drainage, the pocketing of pus, or a spread of the infection. 
During the whole attack the throat and the nose should be kept clean by an alkaline antiseptic solution, such as Seiler's or Dobell's, used in the form of a fine spray. Complications should be treated according to their needs. The tendency of modern practice lies in the way of a comparatively early resource to surgical help, rather than in that of a protracted delay with its possibly serious consequences. The mastoid, however, is often tender, although there may be no pus; we accordingly do no more than apply an icebag or a cold water coil. If, however, there are signs of pus in it, an operation should be performed without hesitation. If one keeps in mind the fact that all purulent diseases of the ear are instances of localized sepsis, the treatment immediately becomes plain.

After the acute attack has passed, the child is sufficiently debilitated to need the systematic administration of tonics for a considerable time.

Prognosis. - The simple cases are not at all serious, and usually respond quickly to treatment. An especially disagreeable feature is their tendency to recur. This doubtless is due to the existence of some abnormal condition of the naso-pharynx, pharynx, or tubes. The various forms of rhinitis, hypertrophied pharyngeal and faucial tonsils, and pharyngitis must be thoroughly cared for until a healthy condition returns. If spontaneous rupture occurs without much delay, or if paracentesis is done promptly, the dangers are lessened. Extensions of the purulent process are always alarming. 


\section{CHAPTER XXV}

\section{DISEASES OF THE SKIN}

\section{SCLERODERMA}

THIs unusual condition consists of a hardening of the skin, with subsequent atrophy and malnutrition. Its cause is unknown, and there are no characteristic lesions with which we are acquainted, excepting an increase in the connective tissue elements and the natural results of such a change. The disease may begin at any time of life after infancy, and may involve a part or the whole of the body. The face, neck, and trunk are oftener attacked than the extremities. The process may or may not begin with œedema; the affected area then becomes indurated, the skin cannot be picked up, and does not pit on pressure. The color may be pale and lifeless, and later on it may be sufficiently pigmented to assume a much darker hue. On account of the hardening, the muscles of the face and body become rigid, so that there is a progressive loss of expression and motion. Superficial sensation becomes diminished, but tenderness is increased. The skin and mucous membranes finally become so tense and rigid that circulation is impeded and muscular atrophy results ; this tenseness may be so much exaggerated that deformities are very apt to ensue. The evil effects may not only be rendered greater, but also may be hastened by disease of the heart, lungs, and gastro-intestinal track. When death occurs its immediate cause is marasmus.

There is no specific treatment; and all one's efforts are directed toward the maintenance of body-heat, the vigorous use of massage and inunctions of oil, the careful regulation of the patient's general circumstances, and the administration of tonics.

\section{SEBORRHGA}

When a child comes into the world he is covered by an oily secretion, the so-called vernix caseosa. This may be called a 
physiological seborrhœa. When the child is weak or suffers from anæmic, nutritional, or gastro-intestinal disorders, or is poorly cared for, a functional disease of the sebaceous glands may result, which is called by the same name. It consists of oily or scaly crusts upon the surface. These crusts are made of fat and free oil-globules, epithelial débris, and amorphous granular matter. It may affect any part of the surface excepting the palms and soles, but its most common locations are on the scalp, and in badly nourished children on limbs and body. It is likewise often seen in smaller degree at the umbilicus, under the prepuce of the penis, and about the clitoris and labia. In most cases the condition is not serious, nor does it involve organic changes in the scalp, skin, and mucous membranes; if, however, it is allowed to lie undisturbed for too long a time, an eczema may develop from it. It produces no itching nor infiltration of the skin.

To remove it one should soak the crusts with olive oil until they fall off; under no circumstances should they be roughly pulled off with the fingers or a fine comb. The parts should then be covered with vaseline, or a one per cent ointment of salicylic acid. Seborrhœa of the umbilicus or genital organs requires thorough washing with soap and water, which may be followed by the application of a solution of alum.

\section{HYPERIDROSIS}

When the sweat-glands are in a condition of over-activity, an excessive amount of perspiration may be secreted, caused by some functional disorder of the sympathetic nervous system. In some cases this is hereditary or congenital. Under such circumstances it is usually located on one part of the body, such as the hands or feet, or any part of them. With sweating of the feet, sometimes called bromidrosis, the odor may be very offensive. In other cases the sweating may be confined to the head or forehead, as in malnutrition and rickets. In still others it may cover the whole body and then is merely a temporary symptom, as in rickets, the acute fevers, and tuberculosis ; in still other instances it may result from overheated rooms and the wearing of clothes that are too warm. Thus it may be acute, subacute, or chronic.

The treatment varies according to circumstances. In the 
acute fevers nothing is needed beyond a liberal number of baths. In malnutrition and rickets, the sweating disappears as soon as the original disease is eliminated. In congenital or hereditary sweating of the feet and hands the treatment is more serious and less successful. Washing the parts two or three times a day with a mixture of water and alcohol, in which is dissolved one per cent of salicylic acid, is sometimes useful. Or a dusting powder consisting of one per cent salicylic acid with boracic acid and oxide of zinc will often give good results. The treatment must often be continued for months. If this powder is used for the feet, it may be sprinkled in the stockings; if the hands are affected, the patient must as continuously as possible wear gloves into which the powder is put. At the same time the general physical condition may need the regulation of the diet, exercise, and the continued use of tonics.

\section{ERYthema}

Erythema is a hyperæmic condition of the skin in which the influenced area may be circumscribed or diffuse ; it is always flat, and its heightened color fades on pressure. Usually there are few subjective symptoms, such as itching, burning, and tingling. The simple erythema, according to its cause, is divided into E. caloricum or the erythema of heat and cold ; E. traumaticum from mild injuries such as pressure or friction; E. venenatum, due to poisons; E. intertrigo, produced in fat children where two skin surfaces meet and rub, as in the groin ; E. pernio, commonly called chilblain. E. intertrigo is very common, is heightened in likelihood and severity by malnutrition, and is exaggerated by lack of cleanliness. Chilblains occur for the most on terminal parts of the body, such as fingers, toes, nose, and ears, especially in poorly nourished and anæmic children. For the sake of completeness one may mention the so-called symptomatic erythema that occurs as the specific eruption in the acute infectious diseases, such as scarlet fever, measles, rötheln. The form called infantile erythema is of very frequent occurrence in young children who are suffering from gastro-intestinal irritations. The treatment of all these conditions consists of the emptying and regulation of the gastro-intestinal track and the application of a bland dusting powder. In chilblains the inflamed skin may be painted with iodine and then protected. Most children suffering from erythema 
may be suspected of some degree of atonicity, and must be treated accordingly.

Erythema multiforme is usually described as an acute exudative inflammation of the skin, characterized by the separate or combined appearance of erythematous, vesicular, papular, nodose, tubercular, and bullous eruptions. The names are descriptive of the appearances, but do not characterize the nature of the lesion. In the same way we get the name E. annulare from an erythematous patch which has faded in the centre; E. gyratum when such rings meet and produce a broken figure; E. marginatum denotes the occurrence of separated patches with sharp edges. Tubercles, vesicles, and bullæ occurring in an erythematous eruption give an adjectival appellation to the disorder. The ætiology of these various forms is conjectured - not known. It has been commonly supposed that they are angio-neurotic in their nature, and that some septic condition in the body gives rise to them. An especial form, E. nodosum, whose name describes its appearance, is a frequent accompaniment of rheumatism. In this case, also, the manner of causation is unknown. These various varieties are apt to occur in connection with systemic disorder, such as fever and malaise with a local accompaniment of itching, burning, and a variable degree of tenderness.

The treatment is general and symptomatic. In most cases the erythema exists in connection with some general pathological condition, either trivial or serious. Free catharsis is always useful, combined with a strict regulation of the gastro-intestinal system. Bland dusting powders or ointments will relieve the local irritation. In all such cases the eruption should not be regarded as a distinct entity, but rather as the expression of disturbed nutritional equilibrium.

\section{Miliaria}

Prickly heat, otherwise known as miliaria and lichen tropicus, is an acute irritation or inflammation of the sweat-glands, the outward expression of which is a vesicular or papular eruption; under proper conditions of irritation and infection the papules and vesicles may become pustules. The main ætiological factor seems to be a combination of unusual heat and a condition of debility. Two varieties are described : one is inflammatory and is represented by two types, M. rubra, or vesiculose ("red gum "), and 
M. papulosa ("prickly heat"); the other is non-inflammatory, and is called M. crystallina, or sudamina. In miliaria the changes consist in a congestion of the capillary vessels about the ducts with resulting effusion about and into the sweat-glands, to which is added a variable but always excessive amount of normal excretion. In sudamina an excess of secretion is dammed up in the deep strata of the horny layer, on account of which the duct becomes filled. The result is a destruction of the wall, followed by the development of a small, white vesicle.

Besides the eruption, there is a keen sensation of itching, burning, and tingling. The rash may be scattered all over the body, but the face and extremities are oftener attacked than the trunk. The vesicles are set close together, are not large, and unless infected have no discharge.

The simplest treatment is usually the best. The bowels should be thoroughly cleansed, the diet should be very plain and light, and considerable water should be given to drink. The child may be bathed once or twice a day, preferably in alkaline water, and powdered after the bath with a simple dusting powder, such as talcum powder or a combination of oxide of zinc and starch. If the child is badly nourished, he may need tonic treatment before the eruption will be permanently cured.

\section{ECZEMA}

Eczema is the most important, and, potentially, is the commonest skin disease of infancy and childhood. Its forms are numerous, not merely because there are many distinct varieties, but rather because there are many degrees of severity complicated by different intensities of adventitious infection. It should primarily be regarded as a catarrhal inflammation of the skin with manifold developmental changes.

Causes. - In children the skin is very sensitive, and its relation to the gastro-intestinal and excretory systems is very intimate, so that disorders of them are very apt to engender simultaneous or consequent irritations of the surface. We see this especially well demonstrated in children who have by inheritance a sensitive skin, as well as in those who are weak, poorly nourished, and poórly cared for. After exhausting disease and the acute infectious fevers, such disorders are matters of common 
complaint, most of all when there are disorders of the gastrointestinal track. Indeed, this last factor may in many ways be regarded as the main source of eczema in its various forms. The more serious and prolonged the digestive disorder, the greater is the likelihood of an eczematous outbreak. Even where the former seems not especially serious, as in chronic constipation or the sub-acute or chronic enteritis which runs through a tiresome series of remissions and exacerbations, the danger to the skin is definite and pronounced. As one would naturally expect, artificially fed children and those older ones whose diet is poorly arranged are more frequently attacked than the breast-fed and wisely nourished. Certain foods, such as oatmeal and potatoes, are commonly blamed as being causative of this disease, but the charge is not entirely logical. We should go farther back and ascertain whether the children in question were really receiving in their food too great a proportion of these articles, and whether other and similarly one-sided dietaries could not produce the same tendency to general and gastro-intestinal inefficiency, and, therefore, to this skin disease.

In addition, almost any irritating and injurious influence from without, especially when the victims are not strong and well nourished, may start the inflammation. Thus scratches, wounds, excessive heat and cold, harsh winds, bad soaps and water, lack of cleanliness, dirty or coarse clothing, may be the starting-point. The milder and non-inflammatory dermal disorders frequently develop into an eczema. And when the exposed rete becomes infected with septic micro-organisms, the severer forms of the disease may be expected.

Lesions. - The changes resemble those which are so commonly seen in the mucous membranes. The upper layers of the epithelium become degenerated, leaving the rete exposed and unprotected. The vessels are congested, and there may be an exudation of serum and fibrin, in which are mixed some white blood-cells, pus-cells, epithelial débris, and micrococci. Infiltration may exist to a variable extent, and the formation of scales and crusts is reasonable and easily understood. Thus the surface may be dry or wet, fairly clear, or covered with foul crusts ; and hardness, stiffness, and fissured and roughened conditions follow mechanically.

Symptoms. - The commonest variety of eczema is the ordinary chronic form or eczema rubrum. Its usual location is any part of 
the head or face, but it may extend all over the body. The first signs, as a rule, are small scattered papules which break down and expose a raw, moist surface. These areas spread and meet, their exudate thickens and hardens, and the whole surface itches and burns to a most irritating degree. Usually the child scratches it, and thus causes some bleeding. The skin becomes swollen, hard, thickened, and irregular. With it is associated hyperplasia of the lymph nodes, which may finally break down and suppurate. Both dermal and adenitic disorders may spread to an indefinite degree.

Seborrhœic eczema is really a combination of a seborrhœa and areas of eczema, caused according to Unna by a certain " mulberry coccus." It usually begins on the scalp and face, from which it spreads to the neck, chest, back, and parts where the skin is tender. The condition of the head is seborrhœic, that of the body is seborrhœic and eczematous. "There is less infiltration in the skin, less true inflammation, and less pain.

Intertrigo, frequently classified as eczematous, is really so in a potential sense only. It is caused by uncleanliness, by wearing unclean napkins, by the influence of two moist surfaces upon each other when they are in contact. It is most commonly seen about the buttocks, genitals, and anus, but often it attacks the groin, the axillæ, the neck, and behind the ears. At first there is a reddened patch of skin which may pass away or become exaggerated. In the latter case the upper layers of the epithelium are thrown off, the rete becomes exposed, and the beginning of an eczema exists. In this disorder the formation of thick crusts need not be expected, but the itching, tenderness, and pain are present.

As has been said above, an eczematous surface may become infected with septic micro-organisms, in which case a pyogenic process may naturally result. The most favorable conditions for the propagation of such a process exist in the hairy scalp, and there one finds the so-called pustular form. The crusts grow in and through the mass of hair, so that ideal conditions for pusformation exist. Either a part or the whole scalp may be covered, for the spread of the disease is rapid. Contagion is likewise easy, or a series of auto-infections may prolong the disease for weeks. The lymph-nodes are enlarged and tender, and may finally suppurate. 
Treatment. - As in every other disease where there is an ascertainable cause, the first and most important step is to recognize and remove it. In many cases this alone is enough to bring about a cure. Especially it is necessary to regulate the condition of the gastro-intestinal track and the kidneys. An equally important factor is the diet, from which every component part that is at all irritating or difficult of digestion must be removed. Children who are being fed artificially must have their milk sufficiently modified and diluted to secure its easy and satisfactory absorption. The details of such modification may be referred to in the section on Feeding. The diet of older children should be largely nitrogenous. When the disease is acute, the food should be made up of milk and soup, which, as the patient improves, may be supplemented by eggs, dark meat, toast, and finally fresh vegetables and fruit. The details of exercise and ventilation should be rigidly administered. While the healthy skin should be carefully washed in the usual manner, the eczematous areas should first be freed from crusts and scales by means of olive oil or sweet oil, and then may be cleansed with bran water or starch water.

The medicinal treatment need not be complicated. Disorders of the gastro-intestinal track and the kidneys should be cared for according to their indications until their action is fully and efficiently restored. As soon as possible, tonic treatment should be begun. The affected surface may at the beginning be so sensitive and the itching so disturbing that one may need a carbolic wash, usually a one per cent or one and a half per cent solution, to give relief. Where there are exudation and crusts a simple salve made of bismuth subgallate and rose water ointment, eight to sixty, will give good service. With this and all other salves the rule should be made to remove the mixture daily by means of sweet or olive oil, to wash the surface as mentioned above, and then apply a fresh quantity of the ointment. In the sub-acute or chronic cases an ordinary zinc and tar ointment may be prescribed. Where there is little or no crust formation Lassar's paste is in most cases efficient; and in the eczematous intertrigo no more than a good dusting powder is required. In the pustular form nothing curative can be expected until the crusts have been entirely removed and the hair clipped close; the surface should then be washed with a weak carbolic solution, and covered with Lassar's paste or a resorcin 
mixture. The annoying eczema of the ears generally improves if pieces of gauze soaked in zinc and calamine lotion are placed on it and between the ear and the opposing surface of the head. In all cases, however, the external treatment must not be regarded as the most important factor in the cure. The internal care comes first, and by a long interval at that.

Prognosis. - The general outlook is thoroughly good. Cases which persist in a discouraging series of remissions and exacerbations are usually those in which the diet is poorly arranged, and the alimentary and excretory functions are in debilitated condition. This applies to a large proportion of the so-called chronic and incurable cases.

\section{Simple Herpes}

Herpes simplex, called fever blisters and cold sores, is a mild, inflammatory disorder that appears on any part of the face below the forehead, usually on the upper lip, and sometimes on the mucous membranes. It is caused by febrile conditions, exposure, and irritation. It consists of one or more vesicles with a reddened base. At first they are clear, later they are cloudy and contain a small quantity of pus, and finally they heal under a crust formation which scales off, leaving a faintly reddened surface. In a week or ten days the skin appears normal.

The stomach and intestines should be emptied and any precedent febrile conditión must receive its appropriate care. The vesicles may be protected by a layer of Lassar's paste or flexible collodion.

\section{Herpes Zoster}

This disease, also called shingles, is a descending interstitial neuritis of the spinal ganglion; it may likewise originate from a cerebral or peripheral source. Its cause is unknown, and some observers believe that it should be classified among the acute infectious diseases. It consists of a group of vesicles with a surrounding reddened zone, and its location lies along the course of the cutaneous nerves. While it is oftenest seen on the body, it may likewise occur on the face, head, or neck. The vesicles are light colored and filled with a clear serum, which after the lapse of a few days becomes puriform. After running a course of from one to three weeks, the vesicles desiccate, a brown crust falls off, 
and the delicate reddened skin is seen to be almost normal. These vesicles may coalesce and form blebs of considerable size, but their separate structure can nevertheless be recognized. Very rarely they are hæmorrhagic. At the beginning of the eruption there may be pain, malaise, and a rise of temperature, which in a short time, as a rule, pass away.

The treatment, outside of free catharsis and regulation of the diet, calls for no more than protection of the vesicles. So long as they are not irritated or ruptured, no destruction of the skin need be feared. Ordinary dusting powder covered with absorbent cotton, which is held in place by a roller bandage, gives sufficient relief. On the face a convenient dressing is flexible collodion. In children the pain is so slight as rarely to call for sedatives.

\section{Impetigo Contagiosa}

Impetigo contagiosa is an acute contagious disease, oftenest seen in dirty children during warm weather. There are practically no symptoms except the eruption which in most cases begins on the face, neck, and hands, whence it may spread to the body by auto-infection. It appears as a series of small vesicles, each of which is surrounded by a reddened zone. These vesicles grow until they may be as large as one's finger nail, or even larger. At first they are filled with a clear serum which gradually becomes purulent. Simultaneously the vesicles become umbilicated, and finally they rupture. The crusts then drop off and the skin returns to its normal condition. The disease lasts from one and a half to three weeks, unless auto-infection takes place.

For treatment one should remove the crusts and apply a mild, antiseptic salve, such as Lassar's paste.

\section{Dermatitis Exfoliativa Neonatorum}

A severe form of exfoliative dermatitis occurs in children under a month of age. Its origin has been the subject of much conjecture, and is not certainly known. The only known lesion as well as symptom is a dermatitis that begins about the mouth, whence it spreads to the rest of the body. Fluid may collect under the epidermis, which flakes off and leaves a raw surface. The eruption develops in various ways and may resemble various other skin diseases, and also may be followed by eczema, furun- 
cles, or superficial gangrene. The treatment is symptomatic, and in about fifty per cent of the cases is unable to prevent death. The disorder was first described by Ritter, after whom it is sometimes called.

\section{Dermatitis Gangranosa Infantum}

As a result of marked debility superficial gangrene may occur in weak children. Usually it attacks marantic, syphilitic, or tuberculous babies, or those who have gone through an acute and exhausting sickness. Rarely it may follow a septic infection. The eruption occurs as a superficial slough, or a pustular process that becomes gangrenous. With these lesions there are the symptoms of debility, high and variable temperature, and severe systemic depression. The treatment consists of local antiseptics and the administration of tonics.

The prognosis is not good.

\section{URTICARIA}

This disorder, commonly called hives or nettle-rash, is a common and transitory eruption, which in most cases follows disturbed gastro-intestinal function. It may be caused by eating strawberries, pastry, and meats that are hard to digest. It occurs in malaria, and as the result of taking certain drugs, notably quinine. It consists of wheals or streaks which vary in size from a seed to one's finger nail. These pomphi are slightly elevated, white in the centre, and pink at the periphery. There is a coincident itching and tingling, which may pass away within one or more hours, followed in a short time by the subsidence of the swelling. The eruption sometimes is papular, and the effusion remains for a variable time after all other symptoms have passed away. Any part of the face and body may be affected. On account of the intense itching, the child is apt to scratch the parts with enough vigor to produce excoriations and superficial infections. The course of the disease is generally very short, excepting in the papular form, which may persist for weeks.

The treatment is mainly directed to the care of the alimentary system. The bowels should be thoroughly emptied, the diet should be made very simple and light, and a generous amount of drinking water should be ordered. Functional disorders of the 
stomach and intestines must be carefully followed up, under penalty of a recurrence of the rash. For the eruption little need be done. Warm alkaline baths are grateful to the patient, and the good effects may be prolonged by sponging the affected areas with a one and a half per cent or two per cent carbolic solution. If the wheals are so situated that the clothing rubs against them, they should be sprinkled with a dusting powder, and covered with cotton and a roller bandage.

\section{Furunculosis}

A furuncle, commonly called a boil, is a local inflammation about a lair follicle or gland of the skin which follows infection by staphylococcus aureus. In most cases there is an antecedent condition of debility. The affected spot at first tingles and feels uncomfortable, then a small, highly colored papule forms which gives way to a hard, dull-red pyogenic process, in the centre of which is the so-called core. If left alone pus forms, and may then escape by spontaneous rupture of the skin, although occasionally the process aborts and is followed by absorption. The furuncles may be located in any part of the body, but usually affect the face, neck, and back; they may occur singly or in large numbers, in one or several crops.

As soon as the furuncle is forming, the hair in the centre of the follicle, if it can be seen, should be pulled out. A moist dressing, composed of gauze moistened with bichloride of mercury solution (1-2000) and covered with rubber tissue, should be held in place over the inflamed area by a roller bandage. If the process does not then stop, the furuncle must be freely incised and packed. The boil may often at the beginning be aborted by touching its centre with a sharpened tooth-pick, dipped in pure carbolic acid. Various salves and plasters have been recommended at various times, but their action is not reliable. I have obtained most satisfaction from ordinary surgical methods.

\section{Lentigo}

Lentigo, or freckles, is a localized pigmentation deposited in the rete. It occurs in older children, most profusely in those of a light complexion, and is usually, but not exclusively, situated on the face and hands. Exposure to the sun seems to be the 
cause. They are hard to remove; and recur easily and repeatedly. In most cases it is best to leave them undisturbed; but if there is any good reason why they should be eliminated, an ointment of equal parts of ammoniated mercury and bismuth subgallate plus ten times as much rose-water ointment may be prescribed.

\section{ICHTHYOSIS}

Ichthyosis is a foetal, congenital, or infantile disease in which the skin is dry, scaly, and horny. The cause of it is unknown; nor is the fact that the condition improves in warm and becomes worse in cold weather susceptible of exact explanation. It may be on a localized area, usually an extensor surface, or may cover the whole body. The condition may vary from a mere dryness and tendency to desquamation, to the formation of large, thick scales, which occasionally are diamond-shaped. A variety, called ichthyosis hystril, consists of an exaggeration of this appearance, so that the skin may be very rough, or have small spines.

The hair and nails are dry and brittle, the skin cracks easily, and the margins of the orifices are distorted. The epithelium shrivels off in small or large flakes, and the tender tissue underneath gradually grows hard, contracted, and deformed.

There is no treatment except warm baths and continued inunctions with lanolin. At the same time the general health must be promoted in all possible ways.

If the condition appears at birth, the prognosis is bad. If it comes on in infancy or childhood, it may yield in part to treatment, but does not entirely disappear. In older children it seems to have no deleterious influence upon vitality or life.

\section{VERRUCA}

Verruca is the common excrescence called warts, which usually occurs on children's hands. Other locations, such as the head, body, and extremities, are possible, but much less frequent. It may be congenital or acquired, and in a few cases seems contagious. Its cause is unknown. The growth consists of a hypertrophy of the papillæ, as large as the seed of an orange, and covered with a thickened epidermis. Its surface may be smooth or rough, or divided complexly, as if made up of several factors. The main 
interest in it lies in the method of easy and painless removal. For this one may use an alcoholic saturated solution of salicylic acid, or a mixture of this acid and collodion in the proportion of one to fifteen. The first may be used twice a day, the second only every two days.

\section{Nevus Pigmentosus}

Nævus pigmentosus, or mole, is a localized hyperpigmentation in the rete of the skin, with or without an increase of the connective tissue. It may be congenital, or acquired at any period of life ; its cause in either case is unknown. It may be as small as a pin's head and as large as a lima bean, its color may vary from a light to a dark brown or black, and its surface may be smooth (N. spilus), warty (N. verrucosus), or covered with hair (N. pilosus). While in children it is usually harmless, it may, in older persons, be the seat of a malignant growth. Removal in children is usually harmless, although I saw one case in which sarcoma developed in the scar within seven months after operation. If removal is desired, nothing more than the ordinary surgical measures is needed.

\section{Alopecta Areata}

This condition is doubtless trophoneurotic, but there is no fixed certainty in the opinion. It has been attributed to parasites, to malnutrition, to neuralgia, to trauma, and the debility that follows acute disease. Any portion of the scalp may be affected, with or without the symptom of pain. As a rule, the disease begins with a sudden falling of the hair in a fairly circular area, the size of which may be large or small ; or more than one area may exist, and when they coalesce - as they sometimes do-a large part of the hairy surface may be involved. The bald spots are white, shining, and slightly depressed. After a time, varying from months to years, the hair returns, at first thin and white, later full and of normal color.

No special treatment is needed besides attention to the general health, and friction with a stimulating lotion, such as alcohol or a weak solution of the tincture of cantharides; other useful remedies are the tincture of green soap and bichloride of mercury. 


\section{RINGWORM}

Ringworm is a parasitic disease caused by trichophyton, a fungus which may grow in the skin, hair, and nails. When it occurs on the skin it is called tinea circinata, and on the scalp tinea tonsurans. In most cases the disease can be recognized at a glance. On the body the favorite locations are the face, neck, and hands. It begins as a small, pink, and elevated spot that grows larger at the margin while the centre heals. The rings seldom become larger than a watch glass, and may then remain stationary, and finally disappear. In some cases there may be several adjacent patches which spread until they meet. At the points of junction the outline is broken; in others the centre refuses to heal; in still others the periphery may be sufficiently irritated to produce a crop of papules or vesicles.

Ringworm of the scalp is somewhat frequent, and on account of its contagiousness spreads very rapidly from one child to another. Like the disease on the body, it may appear on one or several areas. They are fairly circular, and are studded with short and broken hairs. The capillary destruction may be so complete that not a hair is left, and the skin is left as smooth as in alopecia areata. In some severe cases pyogenic infection may take place around the hairs, with a consequent pus-formation in and under the follicles. Usually the disease is readily recognized by the combination of the round patch in which are a few ragged hairs, the scales on the skin, and the lustreless hairs in the periphery. The diagnosis may be confirmed by microscopical examination, which shows the spores in the hairs and the mycelium in the scales. There are no subjective symptoms.

The preventive treatment is exceedingly important. If convenient, the child may be isolated; in all cases he should be taken from school, should not be allowed to sleep with other children, and until the disease is cured should wear a close-fitting cap which will not permit a spread of the disease. As soon as the ringworm is discovered, a wide zone around it should be cleared of hair, and the diseased hairs should be pulled out. Germicides, in as strong a mixture as the skin will bear, should be continuously kept on the affected area. Tincture of iodine was formerly used for this purpose, as also was kerosene oil. The practice nowadays is to use bichloride of mercury dissolved and mixed 
in lanoline and olive oil in the proportion of one to five hundred. A further method consists of the continued use of moist dressings, composed of gauze saturated with a solution of bichloride of mercury and covered with rubber protective. Toward the end of the disease these dressings may be replaced by ointments of salicylic acid.

\section{Favus (Tinea Favosa)}

This is a parasitic disease which gets its name from the resemblance that its characteristic crusts or scutula bear to a honeycomb. The responsible parasite may occur in five species of achorion: A. Schonleini, A. atakton (Unna), A. enthytrix, and the two unnamed species of Bodin. The usual microscopical picture is a mass of small, jointed mycelia, growing approximately at right angles with the horny layers of the epidermis (Unna), and associated with many conidia. The disease appears for the most part in dirty children, who become infected from other children, or from the domestic animals.

Generally the scalp is first infected, but the skin surface of the body and even the nails may be attacked. The first sign of the invasion is the appearance of a small, yellow crust or scutulum about a hair. Many areas may gradually become covered, and finally the crusts may coalesce, thus forming a large scaly surface. These crusts give off a peculiar mouse-like odor that is quite characteristic of the disease. If a dry scutulum is pulled off, a smooth, dull, and slightly irregular surface is seen. But if these crusts are allowed to remain so long that they dry up and fall off spontaneously, a dry, weak looking, scar-like area remains. The hairs which are caught in the crusts become starved, dry, brittle, and finally fall out. Favus is commonly associated with pediculosis, and the combination of the two produces much itching.

On the skin surface the attack begins with the formation of a small pimple, surrounded by a red and inflamed zone. Within this the crust begins to form. If the disease attacks the nails they become dry, harsh, irregular, and easily friable. Scutula may form on them, and if the process is not stopped the whole nail may be lost.

In addition to the local symptoms, the general condition of the child is usually poor. 
The first step in treatment is to soak the crusts for a day with sweet oil, after which they must be removed, and the entire area must be epilated. Germicidal ointments or moist dressings (bichloride of mercury), should then be applied, and their use should be continued until the disease has quite disappeared. If this treatment is begun promptly, the disease may be checked and the parts restored to their normal condition. But if the crusts have been allowed to remain for weeks, a new growth of hair need not be expected. Under such circumstances, healthy, new-plucked hairs have been implanted with a fair amount of success. A recent treatment consists in the use of moist bichloride of mercury dressings under a coil of Leiter's tubes, through which a stream of hot water $\left(52-53^{\circ} \mathrm{C}\right.$.) flows.

Favus is very tenacious, and demands long and patient treatment before it is eradicated. All crusts and dressings should be burned as soon as removed, in order to prevent further contagion. The differential diagnosis is made by the mouse-like odor, the yellow crusts and the microscopic appearance.

\section{SCABIES}

Scabies is a parasitic disease caused by the burrowing into the skin of the female acarus or itch mite. The male remains on the surface, and has no active part in the disease. The localities most frequently affected are those where the epithelium is tenderest, and where the mite has least difficulty in making a burrow for her eggs. This burrow is most readily seen between the fingers, in the armpits, about the genitals, and in the soft flexures of the joints. They may be recognized as short, black, irregular lines which lie near the surface epithelium. As a result of their presence, the skin may be sufficiently inflamed to be studded with vesicles, papules, and pustules. These are exaggerated by the vigorous scratching which the child is forced to make, most of all on the portions of the skin which are kept moist and warm. The blood-marked scratches are often the first sign that attracts the observer's attention.

The active treatment must be supplemented by preventive care. The clothing which the child has been wearing should be thoroughly boiled or destroyed. The patient should be given a warm bath of a quarter to a half hour in duration, and then should 
be scrubbed with a mixture of equal parts of green soap and blue ointment in the same manner that one would use soft soap. After being taken from the bath and dried, his body should be anointed with pure balsam of Peru. The bedding should be thoroughly cleansed, and he should not be allowed to sleep with other children until he is cured. A few nights of this treatment are sufficient to bring about thorough cleanliness.

\section{Pediculosis}

Contamination of the hair with pediculi capitis is so common that it scarcely needs description. One may be justified in mentioning that the irritation which they bring about, added to the scratching and infection produced by the child's finger nails, is often sufficient to set up a dermatitis, crust formation, or even a pustular eczema. Examination will show the lice on the head and the nits on the sides of the hairs. When the irritation of the scalp is severe, there will be an enlargement of the cervical glands.

Rarely the child's eyebrows and lashes may be infected by the pubic louse. In this case, besides the louse and nits, there may be minute hæmorrhages in the near-by tissue.

To cleanse the child the hair should be cut, the crusts, if any, removed, and the scalp should be thoroughly scrubbed with a mixture of green soap and blue ointment, in the same way that one would use soft soap. One or two applications are efficient. 


\section{INDEX}

Abdomen, palpation of, 32 .

Abscess of the brain, 566 ; causes, 566 ; differential diagnosis, 567 ; lesions, 566 ; symptoms, 567 ; treatment, 567.

Abscess, peritonsillar, 241 ; prognosis, 242 ; treatment, 242.

Abscess, rectal, 199.

Abscess, retro-pharyngeal, 246-247.

Abscess, subphrenic, 148.

Accoucheur's hand, 602 .

Acetone, 34.

Achondroplasia, 214.

Acid, diacetic, 34.

Acute arthritis of infants, 616 .

Acute ascending paralysis, 561 .

Acute pyogenic infection, 62 ; symptoms, 63 ; treatment, 63.

Adenitis, lymph-, retro-œsophageal, 115.

Adenitis, lymph-, retro-pharyngeal, 246.

Adenoid vegetations of pharyngeal vault, 234.

Adventitious sucking, 590 .

Alalia, 588.

Albuminuria, functional, 34 .

Alcohol, use of, 37.

Alopecia areata, 634.

Amaurotic family idiocy, 582 ; lesions, 582 ; treatment, 582.

Amazia, 50.

Amœbic infection, ileo-colitis due to, 164.

Amyelia, 39.

Amyloid degeneration of kidney, 381 .

Anæmia,infantile pseudo-leucæmic, 337 ; differential diagnosis, 338; lesions, 338 ; prognosis, 338 ; symptoms, 338 ; treatment, 345.

Anæmia, pernicious, 334 ; differential diagnosis, 337 ; lesions, 335 ; prognosis, 337 ; symptoms, 336 ; treatment, 344.

Anæmia, simple secondary, 329 ; symptoms, 330 ; treatment, 344.
Anæmic murmurs, 321.

Analysis of infant foods, 91 .

Anencephalia, 38.

Animal parasites of the intestines, 181; symptoms, 184, 185, 186 ; treatment, 184, 185, 186, 187.

Ankle, congenital dislocation of, 52 .

Annulus tympanicus, growth of, 9 .

Anterior poliomyelitis, acute, 549 .

Antipyretics, use of, 35 .

Antipyrine, use, 35-36.

Anuria, 33; causes, 33 ; treatment, 33.

Anus, fissure of, 201.

Anus, prolapse of, 197.

Aorta, congenital malformation of, 42 .

Aortic insufficiency in chronic endocarditis, 312 .

Aortic stenosis, in chronic endocarditis, 313.

Apex beat, location of, in infant, 13 .

Aphasia, functional, 587.

Appendicitis, 191 ; causes, 192 ; differential diagnosis, 196; lesions, 193; prognosis, 196 ; symptoms, 194 ; treatment, 195.

Arrhythmia, 301.

Arthritis in scarlet fever, 401.

Arthritis of infants, acute, 616 .

Arthritis, rheumatoid, 615.

Artificial respiration in asphyxia, 59.

Ascarida, 184.

Ascaris lumbricoides, 185 ; symptoms, 185 ; treatment, 185.

Ascaris mystax, 187.

Ascaris vermicularis, 186.

Ascites, 202.

Ascites, chylous, 202.

Asphyxia, 58 ; treatment, 59.

Ass's milk, analysis of, 79.

Asthma, 575 ; causes, 576 ; lesions, 577 ; prognosis, 578 ; symptoms, 577 ; treatment, 578.

Ataxia, hereditary, 558.

Ataxia, hereditary, cerebellar, 559. 
Ataxia, hereditary, spinal, 558.

Atelectasis, 292 ; causes, 292 ; lesions, 293 ; prognosis, 294 ; symptoms, 294 ; treatment, 294.

Atelomyelia, 39.

Atrophy, acute yellow, of liver, 143.

Atrophy, simple, 211.

Auditory canal, in infancy, course of, 9.

Auscultation, methods of, 31 .

Auscultation, of heart in childiren, 32

Backwardness in acquiring speech, 588.

Bacteriology of milk, 76 .

Balanitis, 357.

Baner's formulas for modifying milk, 38 .

Barlow's disease, 225.

Bathing in infancy, 25.

Beef extract, 96.

Beef juice, 96 .

Belladonna, use of, 36 .

Bell's paralysis, 554 .

Bifid uvula, 102.

Bilharzia hæmatobia, 387 .

Biliary calculi, 149.

Bladder, ${ }^{\cdot}$ changes in position during growth, 19.

Bladder, congenital deformities of, 48 .

Bladder, functional derangement of, 352.

Blisters, use of, 37.

Blood, diseases of the, 329 .

Boil, 632.

Boiled milk, 86.

Bone, comparative analysis of, 4.

Bone-marrow in infant and adult, 4 .

Bothriocephalus latus, 183.

Bottles, nursing, care of, 88 .

Brachycardia, 301.

Brain, abscess of, 566 .

Brain, character of, in infants, 6 .

Brain, congenital malformation of, 38 .

Brain, cysts of, 569 .

Brain, development of, 6, 7.

Brain, fœtal, 6.

Brain, glioma of, 569 .

Brain, heruia of, 38.

Brain, myxo-glioma of, 569.

Brain, sarcoma of, 569.

Brain, tumor of, 568 .

Breathing, puerile, 31.

Bromide of soda, use of, 36.

Bronchi, diseases of, 255.

Bronchiectasis, 277 ; causes, 278 ; lesions, 278 ; symptoms, 278 ; treatment, 279.
Bronchitis, acute, 255 ; causes, 255 ; differential diagnosis, 261 ; lesions, 256 ; prognosis, 261 ; symptoms, 257 ; treatment, 259.

Bronchitis, chronic, 262 ; lesions, 262 ; symptoms, 262 ; treatment, 263.

Broncho-pneumonia, acute, 263 ; causes, 263-264 ; differential diagnosis, 271272 ; lesions, 265 ; prognosis, 271 ; symptoms, 267 ; treatment, 270.

Broncho-pneumonia, chronic, 272 ; causes, 272 ; differential diagnosis, 274 ; lesions, 272 ; prognosis, 274 ; symptoms, 273 ; treatment, 273.

Broncho-pneumonia, special types of, 266.

Buhl's disease, 66 .

Cajal's figures, 8.

Calculi, biliary, 149.

Calculi, renal, 388 ; symptoms, 388 ; treatment, 389.

Calculi, vesical, 356.

Cancer aquaticus, 103.

Cancrum oris, 103.

Caput succedaneum, 53.

Cardiac disorders, functional, 301.

Cardiac hypertrophy and dilatation, 318 ; prognosis, 321 ; symptoms, 319 ; treatment, 321.

Care of premature infants, $91,92$.

Cartilages, character of, in infants, 4.

Cataleptic insanity, 586.

Cavernous sinus, thrombosis of, 566 .

Cephalhæmatoma, 53.

Cephalhæmatoma, false, 54 .

Cephalhæmatoma, true, 53.

Cephalocele, 38.

Cerebral convolutions, development of, 6 .

Cerebral ganglion cells, development of, 6 .

Cerebral paralysis, infantile, 561 .

Cerebral paralysis, obstetric, 57 .

Cerebro-spinal meningitis, epidemic, 478.

Cestoda, 181.

Chapin's formulas for modifying milk, 84,85 .

Cheilitis, 101.

Chicken-breast, 220.

Chicken-pox, 415.

Chilblains, 623.

Child, the normal, 1 .

Chloral, use of, 36 . 
Chlorosis, 331 ; causes, 332 ; differential diagnosis, 334 ; lesions, 332 ; prognosis, 334 ; symptoms, 333 ; treatment, 344.

Chondro dystrophia, 214.

Chorea, 598 ; causes, 598 ; differential diagnosis, 601 ; prognosis, 601 ; symptoms, 599 ; treatment, 600.

Chorea in connection with rheumatism, 612.

Chorea major, 604.

Chorea minor, 598.

Chorea of Sydenham, 598.

Chromatin granules, 8.

Chronic functional derangement of intestines, 173 ; causes, 173 ; lesions, 174 ; prognosis, 176 ; symptoms, 174 ; treatment, 175.

Chvostek's symptom in tetany, 602, 603.

Chylous ascites, 202.

Circulation, change from antenatal to postnatal, 13.

Circulation, fœtal, 12, 13.

Clavicle, congenital dislocations of, 52.

Clavicle, relative positions of, 12 .

Clavus, 604.

Cleft palate, 44, 101-103.

Cleft palate, effects of, 102.

Cleft palate, time of operation for, 103 .

Clitoris, enlarged, 48.

Clothing of infant, 25, 26.

Club-hand, 52.

Coccyx, development of, 9 .

Cold sore, 629.

Colic, intestinal, in infants, 187.

Colon, congenital malformations of, 46 .

Colon, growth of, 18.

Colon, transverse portion, changes in position, 18.

Colostrum, analysis of, 73 .

Compensation in heart disease, 311.

Compression of spinal cord, 546 ; differential diagnosis, 548; lesions, 546; prognosis, 548 ; symptoms, 547 ; treatment, 548.

Conducting fibres of brain and cord, 7.

Congenital atelectasis, 60 ; symptoms, 61 ; treatment, 61.

Congenital dislocations, 51-52.

Congenital mucous polypus of umbilicus, 67.

Congenital rachitis, 222.

Congenital subluxations, 52.
Congestion of the kidneys, acute, 365 ; causes, 365 ; lesions, 365 ; symptoms, 365 ; treatment, 365.

Congestion of the kidneys, chronic, 372 ; causes, 372 ; lesions, 372 ; prognosis, 374 ; symptoms, 373 ; treatment, 374.

Congestion of liver, 138.

Congestion of spleen, 149.

Constipation, habitual, 188.

Conus arteriosus, position of, in infants, 13.

Convulsions, 592 ; causes, 593 ; differential diagnosis, 595; prognosis, 595 ; symptoms, 594 ; treatment, 594.

Coprolalia, 588.

Cord, compression of spinal, 548.

Cord, spermatic, absence of, 51 .

Cord, treatment of, after birth, 24 .

Cord, tumors of spinal, 572 .

Corpora quadrigemina, medullation of, 7 .

Costal cartilages, position of, 12.

Cows, breeds of, 79 .

Cow's milk, analysis of, 79.

Cow's milk, modification, 80 .

Craig Colony, 597.

Craniotabes in inherited syphilis, 517.

Craniotabes in rachitis, 217.

Cretinism, sporadic, 580.

Crying, in abdominal disorders, 30 ; in meningitis, 30 ; in otitis, 30 ; in pneumonia, 30 .

Crying in infants, 30.

Cryptorchidism, 51.

Curschmann's spirals in asthma, 577.

Cyclopia, 38.

Cystercercus, 182.

Cystitis, acute, 352.

Cystitis, chronic, 353.

Cystoidei, 183.

Cysts of the brain, 569 .

Deaf-mutism, 592.

Deficiency of speech from peripheral paralysis, 587.

Degeneration, amyloid, of kidney, 381 .

Degeneration, fatty, of new-born, 66 .

Degeneration of kidneys, acute, 366 ; lesions, 366 ; symptoms, 366 ; treatment, 367.

Degeneration of kidney, amyloid, 381 .

Degeneration of kidney, chronic, 372 ; causes, 372 ; lesions, 372 ; prognosis, 374 ; symptoms, 373 ; treatment, 374. Delicate children, feeding of, 89,90 . 
Delicate children, partial peptonization of food for, 90 .

Dementia, acute, 586.

Dendrons, nerve, growth of, 7 .

Dentition, 96-100.

Dentition and sickness, 98.

Dentition, supposed effects of, 97 .

Derangement, functional, of liver, 137.

Dermatitis exfoliativa neonatorum, 630 .

Dermatitis gangrænosa infantum, 631.

Diabetes mellitus, 608 .

Diaphragm, hernia of the, 70 .

Diet for children of two and three years, 93,94 .

Difficult dentition, 97 .

Difficult dentition, lancing gums in, 98.

Dilatation, cardiac, 318.

Diphtheria, 428 ; cause, 428 ; differential diagnosis, 440 ; lesions, 429 ; paralysis in, 435 ; prognosis, 439 ; symptoms, 432 ; treatment, 436.

Diplomyelia, 39.

Dislocations, congenital, 51-52.

Disorders of sleep, 588.

Distoma crassum, 181.

Drugs not well tolerated by children, $36,37$.

Drugs well tolerated by children, 37 .

Drying up of milk supply, 93.

Duodenitis, 132.

Duodenitis, chronic, 133 ; causes, 133 ; lesions, 134 ; prognosis, 135 ; symptoms, 134 .

Dura mater, character of, in infants, 5.

Dura mater, path of infection of, 5

Dwarfism, 214.

Dysentery, 154-155.

Ear, development of, 9.

Echinococcus cysts, 182.

Echinococcus of kidney, 387.

Echinococcus of liver, 147.

Echinococcus of spleen, 151.

Echolalia, 588.

Eczema, 625 ; causes, 625 ; lesions, 626 ; prognosis, 629 ; symptoms, 626 ; treatment, 628.

Eczema of lip, 101.

Elbow, congenital dislocation of, 52 .

Elbow, congenital dislocation, varieties, 52.

Elongated uvula, 242.
Emphysema, 294 ; causes, 294 ; lesions, 296 ; prognosis, 297 ; symptoms, 296 ; treatment, 297.

Emphysema, interlobular, 295.

Emphysema, substantive, 295.

Emphysema, vesicular, 295.

Empyema, 284.

Endocarditis, acute, 305 ; causes, 305 ; differential diagnosis, 309 ; lesions, 305 ; prognosis, 309 ; symptoms, 307 ; treatment, 308.

Endocarditis, chronic, 309 ; causes, 309 ; lesions, 310 ; prognosis, 315 ; symptoms, 310 ; treatment, 314.

Endocarditis, chronic, aortic insufficiency in, 312.

Endocarditis, chronic, aortic stenosis in, 313.

Endocarditis, chronic, mitral stenosis in, 312 .

Endocarditis, chronic, mitral insufficiency in, 311.

Endocarditis, chronic, tricuspic insufficiency in, 313.

Endocarditis in rheumatism, 611 .

Endocarditis, malignant, 315 .

Endocarditis, mycotic, 315 ; causes, 315 ; differential diagnosis, 318 ; lesions, 316 ; prognosis, 318 ; symptoms, 317 ; treatment, 318.

Enteric fever, 464 ; cause, 464 ; differential diagnosis, 471 ; lesions, 465 ; prognosis, 471 ; symptoms, 467 ; treatment, 470.

Epidemic cerebro-spinal meningitis, 478; causes, 478; differential diagnosis, 482 ; lesions, 478; prognosis, 482 ; symptoms, 479 ; treatment, 481.

Epidemic hæmoglobinuria, 65.

Epidemic infectious parotitis, 440 ; differential diagnosis, 443 ; symptoms, 441 ; treatment, 442.

Epidemic influenza, 449 ; cause, 449 ; differential diagnosis, 453 ; lesions, 449 ; prognosis, 452 ; symptoms, 449 ; treatment, 452.

Epididymis, absence of, 51.

Epididymitis, 363.

Epiglottis, relative position of, in infant and adult, 11.

Epilepsy, 595; differential diagnosis, 598 ; prognosis, 597 ; symptoms, 596 ; treatment, 597.

Epilepsy, masked, 597. 
Epilepsy, procursive, 597.

Epileptic insanity, 586.

Epispadias, 50.

Epistaxis, 232 ; causes, 232 ; symptoms, 232 ; treatment, 232.

Erb's symptom in tetany, 602, 603.

Erosions, hæmorrhagic, of the gastric mucous membrane, 131.

Erosions, simple, of the mouth, 106.

Eruption of permanent teeth, order of, 100.

Eruption of temporary teeth, order of, 100.

Erysipelas, 472 ; differential diagnosis, 476 ; lesions, 473 ; prognosis, 475 ; symptoms, 473 ; treatment, 474 .

Erysipelas, ambulans, 474.

Erysipelas, migrans, 474.

Erythema, 623.

Erythema in rheumatism, 613.

Essential paralysis of children, 549 .

Eustachian tube, growth of, 9, 10.

Examination, methods, 29.

Exercise, outdoor, during infancy, 27.

Eye, growth of, in infancy, 9 .

Eyes, care of, after birth, 24.

Face, expression of infantile, in health and disease, 30.

Facial paralysis, 554 .

Facial paralysis, obstetric, 57.

Fallopian tubes, congenital deformities of, 49,50 .

Family idiocy, amaurotic, 582.

Fat in human milk, composition of, 73.

Fats, excessive, effects of, 89.

Fatty degeneration of heart, 303 .

Fatty degeneration of kidneys, 373 .

Fatty degeneration of liver, 144.

Fatty degeneration of new-born, 66 .

Favus, 636.

Feeding, 72.

Feeding directly after birth, 25.

Feeding during second year, and its continuance, 93-94.

Feeding during third year, 94 .

Feeding, effects of attenuated milk, 89.

Feeding for delicate children, 89, 90.

Feeding in acute sickness, 95.

Feeding in first year, intervals and quantities, 88.

Feeding, length of each, 89.

Feeding of premature infants, 92.

Feeding, scheme for, 88.
Feeding, substitute, 77 .

Fever blisters, 629 .

Fever, scarlet, 392.

Fever, typhoid, 464.

Filaria sanguinis hominis, 387.

Fingers, congenital dislocations of, 52 .

Fingers, supernumerary, 52 .

Fingers, webbed, 52.

Fissure of the anus, 201.

Flukes, 181.

Flushing the lower bowel, 179.

Follicles, lingual, growth of, 11.

Fontanelles, closure of, 5 .

Foods, infant, 90, 91.

Foods, infant, analysis of, 91.

Foramen cæcum, closure of, 6 .

Foramen magnum, 4.

Foramen ovale, patent, 42 .

Forest's method of artificial respiration, 59.

Formulas for modifying milk, Baner's, 83.

Formulas for modifying milk, Chapin's, 84,85 .

Formulas for modifying milk, Holt's, 81:

Freckles, 632.

Fremitus in infants, 32.

Friedreich's disease, 558 .

Frontal bone, ossification of, 5 .

Functional aphasia, 587.

Functional cardiac disorders, 301.

Furuncle of the lips, 101.

Furunculosis, 632 .

Gaertner's bacillus, 56 .

Gall bladder, changes in position and activity during growth, 15 .

Gangrene of the cheek, 103 ; causes, 104; lesions, 104 ; prognosis, 105 ; symptoms, 104 ; treatment, 104.

Gangrene of the lungs, 298 ; causes, 298 ; lesions, 298 ; prognosis, 300 ; symptoms, 299 ; treatment, 300.

Gastralgia, 131 ; treatment, 131.

Gastritis, acute, 119 ; causes, 119 ; lesions, 120 ; symptoms, 120 ; treatment, 121.

Gastritis, chronic, 124 ; causes, 124 ; differential diagnosis, 124 ; lesions, 124 ; prognosis, 128 ; symptoms, 128 ; treatment, 126.

Gastritis, croupous, 122.

Gastritis, toxic, 123 ; lesions, 123 ; treatment, 123. 
Genitals, cleanliness of, in infants, 28.

Genito-urinary system, diseases of, 352 .

Gingivitis, ulcerative, 106 ; causes, 106 ; lesions, 106 ; prognosis, 107 ; symptoms, 107 ; treatment, 107.

Glands, lymphatic, location and drainage areas, 390.

Glioma of brain, 569 .

Globus hystericus, 604 .

Glomerulo-nephritis, 367.

Glossitis, acute, 113.

Glossitis, simple superficial, 113.

Glottis, œdema, 253 ; lesions, 254 ; symptoms, 254 ; treatment, 254.

Glycosuria, 33.

Goat's milk, analysis of, 79 .

Goltz's experiment, 7.

Gram's method of decolorization, 359.

Grand hysteria, 604 .

Grand mal, 596.

Grand movement, 604.

Grippe, la, 449.

Growing pains, 598, 609, 615.

Habitual constipation, 188 ; causes, 188 ; prognosis, 191 ; symptoms, 190 ; treatment, 190.

Hæmatemesis, 132 ; differential diagnosis, 132.

Hæmatoma of sterno-cleido-mastoid, 54.

Hæmaturia, 34.

Hæmoglobinuria, 34 ; symptoms, 346 ; treatment, 346.

Hæmophilia, transmission of, 345-346.

Hæmorrhage, visceral, in new-born, 55 .

"Hæmorrhagic disease" of the newborn, 56 .

Hæmorrhagic erosions of the gastric mucous membrane, 131.

Hæmorrhoids, 198.

Hare-lip, 44, 101-103.

Hare-lip, effects of, 102.

Hare-lip, time of operation, 103.

Harrison's Groove, 218.

Hay fever, 579 ; causes, 579 ; lesions, 579 ; prognosis, 580 ; symptoms, 579 ; treatment, 580 .

Headaches, 606.

Heart, comparative size, to that of arteries, 13-14.

Heart, congenital malformations, 41.

Heart, development of, 12-13.

Heart disease, congenital, symptoms, 43.
Heart disease, prognosis in congenital, 43.

Heart, diseases of, 301.

Heart, fatty, 393.

Heart, increase of size and weight, 14.

Heart, murmurs in childhood, 32.

Heart, relative size in infant and adult, 13.

Heart, relation of volume to aorta at different stages, 14 .

Henoch's purpura, 348-350.

Hepatitis, interstitial, 141 ; causes, 141 ; lesions, 142 ; symptoms, 143 ; treatment, 143.

Hepatitis, parenchymatous, 139.

Hepatitis, purulent, 139 ; differential diagnosis, 140 ; symptoms, 140 ; treatment, 141.

Herd-milk, 79.

Hereditary ataxias, 358.

Hereditary ataxic paraplegia, 560 .

Hereditary cerebellar ataxia, 559.

Hereditary spinal ataxia, 558 .

Hermaphroditism, 50-51.

Hernia of the diaphragm, 70.

Hernia, umbilical, 68.

Herpes of lips, 101.

Herpes simplex, 629.

Herpes Zoster, 629.

Heteropia, 39.

Hip, congenital dislocations of, 51.

History of disease as obtained from mother or nurse, 29.

Hives, 631.

Hodgkin's disease, 342.

Holt's cream gauge, 75 .

Holt's formulas for modifying milk, 81 .

Human milk, analysis of, 72,74 .

Hydrocele, 363 ; varieties of, 363-364.

Hydrocephalus, 573 ; differential diagnosis, 575 ; lesions, 574 ; symptoms, 574 ; treatment, 575 ; varieties of, 573.

Hydromyelia, 544.

Hydromyelia externa, 39.

Hydromyelocele, 39.

Hydronephrosis, 386 .

Hydrorrachis externa, 39.

Hydrotherapy for children, 37.

Hygiene of new-born child, 24.

Hymen, irregular formation of, 48.

Hyperidrosis, 622.

Hypertrophy, cardiac, 318.

Hy pochondriasis, 586.

Hypospadias, 50. 


\section{INDEX}

Hysteria, 603 ; differential diagnosis, 606 ; prognosis, 605 ; symptoms, 604 ; treatment, 605 .

Hysteria major, 604.

Hystero-epilepsy, 604.

Icterus, 136.

Icterus neonatorum, 61-62.

Icthyosis, 633 .

Idiocy, 583.

Idiocy, amaurotic family, 582.

Idiocy, Shuttleworth's classification of, 584.

Ileo-colitis, acute, 153 ; causes, 154 ; differential diagnosis, 161 ; lesions, 155 ; prognosis, 161 ; symptoms, 157 ; treatment, 159.

Mleo-colitis, chronic, 161; differential diagnosis, 164 ; lesions, 161 ; prognosis, 164 ; symptoms, 162 ; treatment, 163.

Ileo-colitis due to amœbic infection, 164 ; lesions, 164 ; prognosis, 165 ; symptoms, 165 ; treatment, 165.

Impetigo contagiosa, 630 .

Incontinence of urine, 354 .

Incubator, Rotch's, 92.

Incubators, 92.

Indican, 34.

Infant foods, 90, 91.

Infant foods, analyses of, 91 .

Infantile cerebral paralysis, 561 ; causes, 562 ; differential diagnosis, 565 ; lesions, 562 ; prognosis, 564 ; symptoms, 563 ; treatment, 564.

Infantile pseudo-leucæmic anæmia, 337.

Infantile scurvy, 225 ; causes, 225 ; differential diagnosis, 227 ; lesions, 225 ; prognosis, 227 ; symptoms, 226 ; treatment, 227.

Infantile spinal paralysis, 549 ; differential diagnosis, 551 ; lesions, 549; prognosis, 551 ; symptoms, 550 ; treatment, 550 .

Infantilism, 214.

Infants, premature, care of, 91, 92 .

Infants, premature, feeding of, 92.

Infarction of kidney, 383.

Infection, acute pyogenic, 62 .

Infectious derangements of the intestines, 166 ; causes, 166 ; differential diagnosis, 169 ; lesions, 166 ; prognosis, 170 ; symptoms, 167 ; treatment, 169.
Infectious hæmoglobinæmia, 65.

Infectious hæmoglobinæmia, lesions, 65.

Infectious hæmoglobinæmia, symptoms, 65.

Inflammations of the middle ear, 617 ; causes, 617 ; complications, 618 ; lesions, 617 ; prognosis, 620 ; symptoms, 618 ; treatment, 619.

Influenza, epidemic, 449.

Inherited syphilis, 515.

Inhibitory centres, development of, 7.

Insanity, cataleptic, 586 .

Insanity, epileptic, 586.

Insanity, in childhood, 585 ; causes, 585 ; forms of, 586 .

Insanity, moral, 586 .

Insanity, periodic, 586 .

Insufficiency in heart disease, 311.

Intellectual impulses, comparative development of, 7 .

Intertrigo, 627 .

Intestinal colic in infants, 187.

Intestinal obstruction in the new-born, 69.

Intestine, changes in glands during growth, 18.

Intestine, congenital malformations, 45 .

Intestine, growth and development, 18.

Intestine, large, diseases of, 153.

Intestine, small, diseases of, 117.

Intestines, chronic functional derangements of, 173.

Intestines, infectious derangements of, 166.

Intestines, subacute infection of, 170 .

Intracranial sinuses, thrombosis of, 565 .

.Intubation, feeding in, $437-439$.

Intussusception, 177 ; causes, 177 ; differential diagnosis, 179 ; lesions, 177 ; prognosis, 179 ; symptoms, 178 ; treatment, 179.

Jaundice, 136.

Jaws, development of, 5 .

Kernig's sign in epidemic cerebro-spinal meningitis, 481, 482.

Kidney, blended, 47.

Kidney, horseshoe, 47.

Kidney, increase in calibre of arterial system, 17.

Kidney, infarction of, 383.

Kidney, new growths of, 387.

Kidney, parasites of, 387. 
Kidney, uric acid in infants, at birth, 17.

Kidneys, acute congestion of, 365 .

Kidneys, acute degeneration of, 366 .

Kidueys, amyloid degeneration of, 381 .

Kidneys, chronic congestion of, 372 .

Kidneys, chronic degeneration of, 372 .

Kidneys, congenital absence of, 47.

Kidneys, congenital deformities of, 47.

Kidneys, diseases of, 365 .

Kidneys, growth and development of, 16.

Kleptomania, 586.

Knee, congenital dislocations of, 52 .

Kumyss, 95.

Laborde's lingual traction, 60.

Lachrymal glands, growth of, 9.

Lancing gums in difficult dentition, 98.

Landry's paralysis, 561.

Large intestine, diseases of, 153.

Laryngismus stridulus, 209 ; causes, 209 ; lesions, 210 ; prognosis, 211 ; symptoms, 210 ; treatment, 211.

Laryngitis, acute, 249 ; prognosis, 250 ; symptoms, 249 ; treatment, 250.

Laryngitis, chronic, 250 ; causes, 250 ; lesions, 251 ; prognosis, 251 ; symptoms, 251 ; treatment, 251.

Laryngitis, pseudo-membranous, 251.

Laryngitis, spasmodic, 252 ; causes, 252 ; lesions, 252 ; prognosis, 253; symptoms, 252 ; treatment, 253.

Larynx, congenital malformations of, 40.

Larynx, development of, 11.

Late rachitis, 222.

Lateral sinus, thrombosis of, 566 .

Lentigo, 632.

Leptomeningitis, acute, 537 ; causes, 537 ; differential diagnosis, 539 ; lesions, 537 ; prognosis, 538 ; symptoms, 538 ; treatment, 538.

Leptomeningitis, chronic, 539 ; differential diagnosis, 540; lesions, 539; prognosis, 540 ; symptoms, 539 ; treatment, 539.

Leucocythæmia, 338 ; causes, 339 ; differential diagnosis, 342 ; lesions, 339 ; prognosis, 342 ; symptoms, 341 ; treatment, 344.

Leyden's crystals in asthma, 577.

Lichen tropicus, 624.
Lips, diseases of, 101.

Lips, eczema of, 101.

Lips, furuncle of, 101.

Lips, herpes of, 101.

Lisping, 587.

Lithuria, 34.

Liver, acute yellow atrophy of, 143 .

Liver, amyloid degeneration of, 145.

Liver, changes during growth, 14.

Liver, changes in position, 15.

Liver, congenital malformation of, 46.

Liver, congestion of, 138.

Liver, diseases of, 136.

Liver, echinococcus, infection of, 147 ; differential diagnosis, 148 ; symptoms, 167 ; treatment, 148.

Liver, fatty, 144.

Liver, functional derangements of, 137.

Liver, waxy, 145.

Liver, wounds of, 148.

Lobar pneumonia, 453 ; causes, 454 ; differential diagnosis, 463 ; lesions, 455 ; prognosis, 463 ; symptoms, 457 ; treatment, 461.

Longet's experiment, 7.

Lungs, congenital malformation, 41.

Lungs, development of, 16.

Lungs, diseases, 255.

Lungs, gangrene of, 299.

Lungs, size relative to heart at different ages, 16.

Lymph-adenitis, retro-œsophageal, 115. Lymph-adenitis, retro-pharyngeal, 246, Lymphatic glands, location and drainage areas, 390.

Macrocheilia, 101.

Macroglossia, 47, 101.

Macula lutea, growth of, 9 .

Malaria, 528; causes, 528 ; differential diagnosis, 534 ; lesions, 528 ; prognosis, 534 ; symptoms, 531 ; treatment, 533.

Malnutrition, diseases of, 209.

Mamma, deformities, 50.

Mamma, supernumerary, 50.

Mania, 586.

Mare's milk, analysis of, 79.

Marie's disease, 559.

Masked epilepsy, 597.

Mastitis in infants, 68.

Mastoid cells, development of, $\boldsymbol{9}$.

Mastoiditis, 618.

Masturbation, 591. 
Materna milk-modifying apparatus, 84. Matzoon, 96.

Maxilla, inferior, congenital dislocation of, 52 .

Measles, 405 ; causes, 405 ; differential diagnosis, 413; incubation, 406; lesions, 406; prognosis, 413; treatment, 411.

Measles, German, 414.

Meckel's diverticulum, 45, 67 .

Medulla oblongata, medullation of, 7 .

Melancholia, 586.

Meningitis, epidemic cerebro-spinal, 478.

Meningitis, spinal, 542.

Meningocele, spinal, 39.

Meningo-myelocele, 39.

Microcephalus, 38.

Microglossia, 44.

Miliaria, 624 .

Milk, ass's, analysis of, 79 .

Milk, bacteriology of, 76 .

Milk, boiled, 86 .

Milk, cow's, analysis of, 79 .

Milk, cow's, for modification, 79-80.

Milk, cow's, modification of, 80 .

Milk, goat's, analysis of, 79 .

Milk, human, analysis of, 72, 74 .

Milk, human, instability of, 76 .

Milk, human, regulation of, 77 .

Milk laboratories, 86.

Milk, mare's, analysis of, 79 .

Milk, pasteurized, 87.

Milk, practical asepsis of, 87 .

Milk spot, 13.

Milk, sterilized, 86.

Milk supply, drying up of, 93.

Milk, unboiled, value of, 87.

Milk, variations in, 76 .

Mineral matters in human milk, composition of, 74 .

Mitral insufficiency in chronic endocarditis, 311.

Mitral stenosis in chronic endocarditis, 312.

Moist dressings, use of, 37 .

Mole, 634.

Moral insanity, 586.

Morbilli, 405.

Morbus sacer, 495.

Morbus virgineus, 331.

Mouth, cleansing of, in infants, 25.

Mouth, congenital malformation, 44.

Mouth, diseases of, 101.

Mouth, simple erosions of, 106.
Multiple neuritis, 552 ; causes, 552 ; differential diagnosis, 555; lesions, 552 ; prognosis, 555 ; symptoms, 552 ; treatment, 554.

Mumps, 440.

Murmur, in congenital heart disease, 43.

Murmurs, anæmic, 321.

Muscles, character of, in infants, 4.

Myelitis, 544 ; differential diagnosis, 546 ; lesions, 544 ; prognosis, 546 ; symptoms, 545 ; treatment, 545.

Myelosyringosis, 543 .

Myocarditis, 302 ; causes, 302 ; lesions, 303 ; symptoms, 304 ; treatment, 304.

Myocarditis, purulent form of, 303 .

Myxo-glioma of brain, 369 .

Nævus of the rectum, 199.

Nævus pigmentosus, 634 .

Nasal polypi, 233.

Nemathelminthes, 181, 184.

Nephritis, acute diffuse, 369 ; causes, 369 ; lesions, 369 ; prognosis, 372 ; symptoms, 369 ; treatment, 371 .

Nephritis, acute exudative, 367 ; lesions, 367 ; treatment, 368.

Nephritis, catarrhal, 367:

Nephritis, chronic diffuse, with exudation, 377 ; lesions, 377 ; symptoms, 378.

Nephritis, chronic diffuse, without exudation, 374 ; lesions, 374 ; prognosis, 376 ; symptoms, 374 ; treatment, 376.

Nephritis, croupous, 367.

Nephritis, desquamative, 367.

Nephritis, glomerulo-, 367 .

Nephritis, parenchymatous, 367 .

Nephritis, pyelo-, chronic, 381.

Nephritis, suppurative, 379; lesions, 380; prognosis, 381 ; symptoms, 380 ; treatment, 381 .

Nerve medullation, development of, 7 .

Nettle rash, 631.

Neurasthenia, 586 .

Neurons, growth of, 7.

New growths of kidney, 387.

Nightmare, 589.

Night terror, 589.

Nipples, nursing, care of, 88.

Nipples, supernumerary, 50.

Nodules, subcutaneous, in rheumatism, 610-612.

Noma, 103. 
Nose, diseases of, 229.

Nurse, selection of, 28.

Nursing bottles, care of, 88 .

Nursing, frequency and duration of, 27.

Nursing, intervals between, 77 .

Nursing nipples, care of, 88 .

Obstetric paralyses, 56.

Obstruction, intestinal, in the new-born, 69.

Edema, in infant, 71.

(Edema of the glottis, 253.

Esphagitis, acute, 114.

Esophagus, congenital malformation, 45.

Esophagus, diseases of, 101.

Ophthalmia neonatorum, 63 ; symptoms, 64 ; trea+ment, 64.

Opium, use of, in children, 35 .

Orchitis, 363.

Order of eruption of permanent teeth, 100.

Order of eruption of temporary teeth, 100.

Otitis media, 617.

Outdoor exercise, during infancy, 27.

Ovary, congenital deformities of, 49-50.

Oxyuris vermicularis, 186 ; symptoms, 186 ; treatment, 186.

Pachymeningitis, 540 ; symptoms, 540541 ; treatment, 541.

Pachymeningitis, acute, external, 540.

Pachymeningitis, acute, internal, 540.

Pachymeningitis, chronic, 541.

Palate, soft, development of, 11.

Pancreas, congenital deformities of, 47.

Pancreas, diseases of, 136.

Paralyses, obstetric, 56.

Paralysis, acute, 561.

Paralysis, Bell's, 554.

Paralysis, essential, of children, 549.

Paralysis, facial, 554.

Paralysis, false, of inherited syphilis, 524.

Paralysis, infantile cerebral, 561.

Paralysis, Landry's, 561.

Paranoia, 586.

Paraplegia, hereditary ataxic, 560.

Paraphimosis, 358.

Parasites of kidney, 387.

Parenchymatous degeneration of kidney, 366.
Parenchymatous inflammation of kidney, 366.

Parotitis, 440.

Pasteurization of milk, 87.

Pavor nocturnus, 589.

Pediculosis, 638.

Peliosis rheumatica, 348.

Peliosis rheumatica of Schœnlein, 550.

Pelvic viscera, changes in position during growth, 19.

Pelvis, changes during growth, 19.

Pemphigus in infants, 66 ; symptoms,

67 ; treatment, 67.

Penis, deformities of, 50.

Peptonization of food, 95.

Peptonization, partial, for delicate children, 90.

Percentage of water in fœtus, 3.

Percentage of water in new-born infant, 3 .

Percussion, method and results of, 32 .

Pericarditis, acute, 321 ; causes, 322 ; differential diagnosis, 327 ; lesions, 323 ; prognosis, 327 ; symptoms, 324 ; treatment, 326.

Pericarditis, chronic, 327 ; lesions, 327 ; prognosis, 328 ; symptoms, 327 ; treatment, 327.

Pericarditis, fibrinous, 323.

Pericarditis, hæmorrhagic, 324.

Pericarditis in rheumatism, 611 .

Pericarditis, purulent, 323.

Pericarditis, serous, 323.

Pericarditis, simple, 323.

Perihepatitis, in chronic peritonitis, 206.

Perinephritis, 384 ; causes, 384 ; differential diagnosis, 384 ; lesions, 384 ; prognosis, 385 ; symptoms, 385 ; treatment, 385.

Perinephritis in chronic peritonitis, 206.

Periodic insanity, 586.

Periosteum, in infant and adult, 4.

Peripheral nerves, medullation of, 7 .

Peripheral paralysis, obstetric, 57.

Perisplenitis in chronic peritonitis, 206.

Peritonæum, congenital malformation of, 46.

Peritonæum, diseases of, 202.

Peritonitis, acute, 202; causes, 202 ; differential diagnosis, 205; lesions, 203 ; prognosis, 205 ; symptoms, 203 ; treatment, 204.

Peritonitis, chronic, 206 ; lesions, 206 ; prognosis, 208 ; symptoms, 207 ; treatment, 207. 
Peritonitis, chronic, laparotomy in, 207.

Peritonsillar abscess, 241.

Pernicious anæmia, 337.

Pertussis, 443 ; causes, 443 ; differential diagnosis, 448 ; lesions, 443 ; prognosis, 448 ; symptoms, 444 ; treatment, 446.

Petit mal, 596.

Petrosal sinus, thrombosis of, 597.

Petro-squamosal suture, closure of external, 9.

Pharyngeal vault, adenoid vegetations of, 234 ; causes, 234 ; lesions, 234 ; prognosis, 236 ; symptoms, 235 ; treatment, 236.

Pharyngitis, acute, 243 ; causes, 243 ; lesions, 243 ; symptoms, 244 ; treatment, 244.

Pharyngitis, chronic, 244 ; causes, 244 ; lesions, 245 ; symptoms, 245 ; treatment, 245.

Phimosis, 358.

Physiological icterus, 61.

Physiological mastitis, 69.

Platyhelminthes, 181.

Pleura, diseases of, 255.

Pleurisy, acute, 279 ; causes, 280 ; complications, 288 ; differential diagnosis, 289 ; lesions, 281 ; prognosis, 289; symptoms, 284 ; treatment, 288.

Pleurisy, chronic, 290 ; causes, 290 ; lesions, 290 ; symptoms, 290 ; treatment, 290.

Pleurisy, dry, 282.

Pleurisy, fibrinous, 282.

Pleurisy, purulent, 284.

Pleurisy with effusion, 283.

Pleuritic fluid, 282.

Pneumonia, croupous, 453.

Pneumonia, fibrinous, 453.

Pneumonia, interstitial, 274 ; causes, 274 ; differential diagnosis, 276; lesions, 274 ; prognosis, 276 ; symptoms, 275 ; treatment, 276.

Pneumonia, lobar, 453.

Pneumonia, secondary, 276 ; causes, 276 ; lesions, 277 ; prognosis, 277 ; symptoms, 277 ; treatment, 277.

Pneumotomy in bronchiectasis, 279.

Poliomyelitis, acute anterior, 549.

Polymazia, 50.

Polypi, nasal, 233.

Polypus of rectum, 200.
Polypus, umbilical, 67.

Polythelia, 50.

Polyuria, 34 ; treatment, 34.

Porencephalus, 38.

Portal vein, injury of, 148.

Post-hemiplegic polymyo-clonus of $\mathrm{Pe}$ terson, 564 .

Poultices, use of, 37.

Pons Varolii, medullation of, 7.

Predigestion of food, 95.

Premature infants, care of, 91-92.

Premature infants, feeding of, 92.

Prescription writing for children, 36.

Prickly heat, 624 .

Proctitis, 196 ; membranous form, 197 ; ulcerative form, 197.

Procursive epilepsy, 597.

Progressive muscular atrophies, 556; Aran-Duchenne type, 556; CharcotMarie-Tooth type, 557; Erb's type, 557 ; Landouzy-Dejerine type, 558.

Prolapse of anus and rectum, 197 ; causes, 197 ; treatment, 198.

Proportions of infant as compared with those of adult, 3 .

Proteids, determination of, in milk analysis, 75 .

Proteids, excessive, effects of, 89.

Proteids in human milk, composition of, 73.

Pseudo-hermaphroditism, 51.

Pseudo-leucæmia, 342; differential diagnosis, 344 ; lesions, 342 ; prognosis, 344 ; symptoms, 343 ; treatment, 344 . Pseudo-tetanus, 601.

Ptyalin-forming glands in infancy, 11.

Pulmonary artery, relation of pressure to that of aorta at different stages, 14.

Pulmonary artery, stenosis, 42.

Pulse, character of, in infant, 3.

Purkinje's cells, development of, 6 .

Purpura, 347; differential diagnosis, 351; prognosis, 351; treatment, 351.

Purpura, hæmorrhagic, 348-349.

Purpura, hæmorrhagic, in rheumatism, 613.

Purpura, infective group of, 348 .

Purpura, mechanical group of, 348.

Purpura, nervous group of, 348.

Purpura, primary, 348.

Purpura, toxic group of, 348.

Pyelo-nephritis, chronic, 381 .

Pyromania, 586.

Pyuria, 34. 
Quincke's lumbar puncture in epidemic cerebro-spinal meningitis, 482.

Rachitic girdle, 218.

Rachitic rosary, 219.

Rachitis, 214 ; causes, 214 ; differential diagnosis, 214 ; lesions, 216 ; prognosis, 224 ; symptoms, 18 ; treatment, 222.

Rachitis, congenital, 222.

Rachitis, craniotabes in, 217.

Rachitis, late, 222.

Recessus opticus, 9.

Rectal abscess, 199.

Rectal polypus, 200.

Rectum, changes in, during growth, 19.

Rectum, nævus of, 199.

Rectum, prolapse of, 197.

Red gum, 624 .

Renal calculi, 388 .

Respiration, artificial, Forest's method of, $5 \dot{9}$; Schultze's method of, 59 ; Sylvester's method of, 60 .

Respiration in infants, 3,30 .

Retro-œsophageal lymph-adentis, 115 ; prognosis, 116 ; treatment, 115.

Retro-pharyngeal abscess, 246-247.

Retro-pharyngeal lymph-adenitis, 246, 248 ; causes, 246 ; prognosis, 248 ; symptoms, 247 ; treatment, 248.

Rheumatism, 609 ; causes, 609 ; differential diagnosis, 615 ; lesions, 610 ; prognosis, 614 ; symptoms, 610 ; treatment, 613.

Rheumatism, in connection with chorea, 612.

Rheumatoid arthritis, 615 .

Rhinitis, acute, 229 ; causes, 229 ; lesions, 229 ; prognosis, 230 ; symptoms, 229 ; treatment, 230.

Rhinitis, chronic, 230 ; causes, 230 ; lesions, 231 ; prognosis, 232 ; symptoms, 231; treatment, 231.

Ribemont's laryngeal inflation, 60 .

Ribs, development of, 12.

Ringworm, 635.

Rolando, development of fissure of, 6 .

Romberg's symptom in multiple neuritis, 553.

Rose cold, 579.

Rötheln, 414.

Rubella, 414 ; cause, 414 ; differential diagnosis, 415 ; symptoms, 414 ; treatment, 415.
Salivary glands in infancy, 11.

Sarcoma of brain, 569 ; varieties of, 569.

Scabies, 637.

Scarlatina, 392.

Scarlet fever, 392 ; causes, 392 ; complications, 399-401 ; differential diagnosis, 404; lesions, 393 ; prognosis, 403 ; symptoms, 394, treatment, 402.

Scarlet fever, arthritis in, 401.

Scarlet fever, cerebral, 400.

Schœnlein's peliosis rheumatica, 350 .

Schultze's method of artificial respiration, 60 .

Sclerema neonatorum, 70.

Scleroderma, 621.

Scurvy, infantile, 225.

Seborrhœa, 621.

Seminal vesicles, absence of, 51 .

Shingles, 629.

Shoulder joint, congenital dislocation of, 52 ; subacromial, 52 ; subcoracoid, 52 ; subglenoid, 52 ; subspinous, 52.

Simple atrophy, 211 ; causes, 211 ; differential diagnosis, 213 ; prognosis, 213 ; symptoms, 212 ; treatment, 213.

Simple herpes, 629 .

Sinus, thrombosis of cavernous, 566 .

Sinus, thrombosis of lateral, 566 .

Sinus, thrombosis of petrosal, 566 .

Sinus, thrombosis of superior longitudinal, 566 .

Sinuses, development of frontal, 6.

Sinuses, thrombosis of intra-cranial, 565 .

Skin, diseases of the, 621 .

Skoda's resonance, 285.

Skull, comparative proportions in infant and adult, 5 .

Skull, development of, 4 .

Sleep, best position for infant, 26 .

Sleep, disorders of, 588 .

Sleep, necessity of, for infant, 26.

Sleep, regular hours for, 27.

Small-pox, 417.

Somatic impulses, comparative development of, 7.

Soxhlet's method of milk analysis, 74 .

Speech, backwardness in acquiring, 588.

Speech, deficiency of, from peripheral paralysis, 587.

Spermatic cord, absence of, 51 .

Sphenoid and occipital bones, closure of, 5 .

Spina bifida, 39; treatment, 40. 
Spinal cord, comparative length of, 8 .

Spinal cord, compression of, 546 .

Spinal cord, congenital malformation of, 39.

Spinal cord, medullation of, 7.

Spinal cord, tumors of, 572.

Spinal meningitis, 542.

Spleen, amyloid degeneration of, 151 .

Spleen, changes during growth, 15.

Spleen, congenital deformities of, 47 .

Spleen, congestion of, 149.

Spleen, diseases of, 136.

Spleen, echinococcus of, 151.

Spleen, in diaphragmatic hernia, 47.

Spleen, parasites of, 151.

Spleen, transposition of, 47 .

Spleen, waxy, 151.

Spleen, wounds of, 151.

Sporadic cretinism, 580 ; symptoms, 581 ;

treatment, 581.

Sprue, 110.

Stammering, 588.

Stenosis of orifices in heart disease, 311.

Stenosis of pulmonary artery, 42 .

Sterilization of milk, 86.

Sterno-cleido-mastoid, hæmatoma of, 54 .

Stomach, acute functional derangement of, 117,118 ; lesions, 118 ; prognosis, 119 ; symptoms, 118 ; treatment, 118.

Stomach, changes in glands of, during growth, 17.

Stomach, changes in shape and size during growth, 17.

Stomach, congenital malformations of, 45.

Stomach, dilatation of, 128 ; prognosis, 129 ; symptoms, 128 ; treatment, 129.

Stomach, diseases of, 117.

Stomach, ulcer of, 129 ; causes, 129 ; lesions, 130 ; prognosis, 131 ; symptoms, 130 ; treatment, 131.

Stomatitis, acute, simple, 108 ; causes, 108 ; lesions, 108 ; symptoms, 108; treatment, 109.

Stomatitis, croupous, 111 ; causes, 111 ; lesions, 112 ; prognosis 112 ; symptoms, 112 ; treatment, 112.

Stomatitis, gangrænosa, 103.

Stomatitis, gonorrhœal, 112.

Stomatitis, herpetoid, 109 ; lesions, 109 ; prognosis, 110 ; symptoms, 109 ; treäment, 110.

Stomatitis, mycetogenetic, 110 ; causes,
110 ; lesions, 110 ; prognosis, 110 ; symptoms, 110.

Strawberry tongue in scarlet fever, 397.

Stricture of the urethra in boys, 362 .

Strongylus gigas, 387 .

Stuttering, 587.

St. Vitus dance, 598.

Subacute intestinal infection, 170 ; lesions, 170 ; prognosis, 173 ; symptoms, 171 ; treatment, 172.

Subarachnoid space in infant and adult, 5-6.

Subluxations, congenital, 52.

Subphrenic abscess, 148.

Substitute feeding, 77.

Sucking, adventitious, 590.

Sudamina, 625.

Sugar, deficient, effects of, 89.

Sugar in human milk, 74.

Superior longitudinal sinus, thrombosis of, 566 .

Supernumerary fingers, 52 .

Supernumerary mammæ, 50 .

Supernumerary nipples, 50.

Supernumerary toes, 52 .

Suprarenal capsules, congenital deformities of, 47.

Suprarenal capsules, development of, 17.

Suture, petro-squamous, patency of, 5 .

Sylvester's method of artificial respiration, 59.

Sylvian fissure, development of, 6 .

Symptoms of congenital heart disease, 43.

Syphilis, inherited, 515; causes, 515 ; differential diagnosis, 527 ; lesions, 516 ; prognosis, 527 ; symptoms, 520 ; treatment, 526.

Syphilis, late hereditary, 525 .

Syringomyelia, 543.

Syringomyelocele, 40 .

Table (Boas) of weight and height, 22-23.

Table of differential diagnosis between scarlet fever, measles, varicella, variola, and rubella, 424 .

Table of dimensions of boys, 20.

Table of dimensions of girls, 21 .

Table (Quetelet) of height and weight, 22.

Tænia canina, 183.

Tænia cucumerina, 183.

Tænia echinococcus, 183. 
Tænia elliptica, 183.

Tænia flavo-punctata, 183.

Tænia inermis, 182.

Tænia madagascariensis, 183.

Tænia mediocanellata, 182.

Tænia nana, 183.

Tænia saginata, 182.

Tænia solium, 182.

Tæniarhynchus mediocanellatus, 182.

Tachycardia, 301.

Tapeworms, 181.

Teeth, development of, 10, 99 .

Teeth, permanent, order of eruption, 100 .

Teeth, temporary, order of eruption, 100 .

Teething, 96-100.

Teething, supposed effects of, 97 .

Temperature, elevation of, in infants and children, 30 .

Temporal bone, relation of squamous and parietal portions in infant and adult, 5 .

Testicles, deformities of, 51 .

Tetanilla, 601 .

Tetanus, 476 ; prognosis, 477 ; symptoms, 476 ; treatment, 477.

'Tetanus, pseudo-, 601.

Tetany, 601 ; differential diagnosis, 608 ; prognosis, 603 ; symptoms, 602 ; treatment, 603 .

Therapeutic suggestions, 35.

Thorax, comparative dimensions of infant and adult, 12.

Throat, diseases of, 229.

Thrombosis of cavernous sinus, 566 .

Thrombosis of lateral sinus, 566 .

Thrombosis of petrosal sinus, 566 .

Thrombosis of superior longitudinal sinus, 566.

Thrombosis of the intracranial sinuses, 565.

Thrush, 110.

Thymus gland, atrophy of, 11 .

Thymus gland, at birth, 11 .

Thyroid gland, atrophy of, 11.

Tinea favosa, 636 .

Toes, supernumerary, 52.

Toes, webbed, 52.

Tongue, coated, 113.

Tongue, geographical, 113.

Tongue, growth of, 11.

Tongue, bairy, 113.

Tongue, strawberry, 113.

Tongue, strawberry, in scarlet fever, 397.

Tonsil, Luschka's, 234.
Tonsil, pharyngeal, at birth, 11 .

Tonsil, pharyngeal, hypertrophy of, 234 .

Tonsil, third, 234.

Tonsillitis, acute follicular, 238 ; differential diagnosis, 239; lesions, 238 ; prognosis, 239 ; symptoms, 238 ; treatment, 239.

Tonsillitis, acute simple, 236; lesions, 237 ; prognosis, 238 ; symptoms, 237 ; treatment, 237.

Tonsils, chronic inflammation and hypertrophy of, 240 ; lesions, 240 ; symptoms, 240 ; treatment, 241.

Torticollis, congenital, 55 ; from hæmatoma, 55 .

Trachea, relative position of, 11-12.

Trematoda, 181.

Trichina cystica, 387.

Trichodectes canis, 183.

Tricuspic insufficiency in chronic endocarditis, 313.

Trousseau's symptom in tetany, 602-603. Tubes, Fallopian, deformities of, 50 .

Tuberculosis, 484 ; differential diagnosis, 513 ; heredity of, 484 ; inoculation of, 485.

Tuberculosis, lesions, 486; lesions in cerebral, 497 ; lesions in gastric, 495 ; lesions in glandular, 491; lesions in hepatic, 494 ; lesions in intestinal, 495 ; lesions in mesenteric 495 ; lesions in nephritic, 493 ; lesions in osseous, 493 ; lesions in peritoneal, 496 ; lesions in pleuritic, 490 ; lesions in pulmonary, 487 ; lesions in splenic, 494 ; lesions in placental, 484 .

Tuberculosis, prognosis in, 512.

Tuberculosis, symptoms, 498 ; symptoms in adenitis, 502 ; symptoms in cerebral, 506 ; symptoms in gastric, 505 ; symptoms in miliary, 499 ; symptoms in nephritic, 504 ; symptoms in osseous, 503; symptoms in peritoneal, 505 ; symptoms in pleuritic, 502 ; symptoms in pulmonary, 499.

Tuberculosis, treatment of, 509 .

Tuberculosis, treatment in adenitis, 512 ; treatment, diet, 511 ; treatment, special methods of, 511 .

Tumor of brain, cystic, 569 .

Tumor of brain, gliomatous, 569 .

Tumor of brain, myxo-gliomatous, 569.

Tumor of brain, sarcomatous, 569 .

Tumor of brain, tubercular, 566 . 
Tumor of the brain, 568; differential diagnosis, 571 ; prognosis, 571 ; symptoms, 569 ; treatment, 571.

Tumors of the spinal cord, 572.

Typhoid fever, 464.

Ulcerative gingivitis, 106 .

Ulcerative stomatitis, 106.

Umbilical hernia, 68.

Umbilical polypus, 67.

Umbilicus, diverticulum, tumor of, 67 .

Urachus, patent, 48.

Ureters, congenital deformities of, 47.

Urethra, congenital deformities of, 48.

Urethritis, in male children, 359 ; symptoms, 360 ; treatment, 360 .

Uric acid, quantity of, at different ages, 32.

Urination, regulation of, in infancy, 27.

Urine, collection of, in infants, 32 .

Urine, different constituents, at different ages, 33.

Urine, incontinence of, 354 ; prognosis, 356 ; treatment, 355.

Urine, quantity in $\mathbf{2 4}$ hours, at different ages, 33.

Urticaria, 631.

Uterus, congenital deformities of, 49.

Uvula, bifid, 602.

Uvula, elongated, 242.

Uvula, growth of, 11.

Uvulitis, 242.

Vaccinia, 424.

Vagina, congenital deformities of, 49.

Varicella, 415; differential diagnosis, 417 ; symptoms, 416 ; treatment, 417.

Variola, 417 ; causes, 418 ; differential diagnosis, 424 ; prognosis, 423 ; symp- toms, 418 ; treatment, 422 ; varieties of, 419.

Varioloid, 424-427.

Ventilation, of nursery, for infant, 28.

Verruca, 633.

Vertebræ, congenital dislocations of, 52 .

Vertebræ, consolidation of, 9.

Vertebræ, development of, 8.

Vertebral column, pliability of, in infants, 8 .

Vertebral column, proportions of, in infants, 8.

Vesical calculi, 356 .

Vesicles, seminal, absence of, 51 .

Vierordt's table of percentage of weights of organs, 2.

Visceral hæmorrhage, in new-born, 55.

Volvulus, 180.

Vomer, 4.

Vulva, congenital deformities of, 48.

Vulvo-vaginitis, 361.

Wahl's sign, 180, 181.

Warts, 633.

Waxy liver, 145.

Waxý spleen, 151.

Weaning, 93.

Webbed fingers, 52 .

Webbed toes, 52.

Weil's disease, 535 ; differential diagnosis, 536 ; lesions, 535 ; symptoms, 535 ; treatment, 535 .

Werlhof's disease, 349 .

Werner-Schmidt method of cream analysis, 75 .

Wet-nurse, 77.

Whooping-cough, 443.

Winckel's disease, 65 .

Wrist, congenital dislocations of, 52 .

Wrist, congenital dislocations, varieties of, 52 .

Yellow atrophy, acute, of liver, 143. 


\section{THE DEVELOPIMENT OF THE CHILD}

BY

NATHAN OPPENHEIM

Attending Physician to the Children's Department of Mt. Sinai Hospital Dispensary

12mo. Cloth. \$1.25 net

"This is one of the best Child-Study books that has ever appeared. . . No student of the child can afford to be without this book. It contains much that is new, but each proposition is so well authenticated by an array of actual facts that conviction of the author's position on any question discussed is bound to ensue. The author deserves the highest possible credit for giving us so much that is valuable in the way of scientific ChildStudy." - Child-Study Monthly.

"This book should be read by all who are interested in the proper education and training of children. They will find in it a good deal of original thought and many valuable suggestions." - New York Herald.

"It is difficult to restrain one's enthusiasm when speaking of this book. The chapter 'Heredity and Environments' might well be printed as a mother's tract for general circulation." - The Outlook.

"This is an extremely helpful book. It is a book with a mission for mankind. The author has a great purpose, and his treatment is both scholarly and original." - Journal of Education.

The book is for any one who has in any capacity the care of a growing child, for the primary school teacher, for the physician who advises its parents, but most of all for every parent.

"A lucid, well-reasoned, and trustworthy guide."-Sat. Evening Gazette, Boston.

"Remarkably interesting and thoughtful work." - Boston Transcript.

\section{THE MACMILLAN COMPANY}

66 FIFTH AVENUE, NEW YORK 


\section{RENAL GROWTHS}

Their Pathology, Diagnosis, and Treatment. By T. N. Kelynack, M.D. (Vict.), M.R.C.P. (London), Pathologist, Manchester Royal Infirmary; Demonstrator and Assistant Lecturer in Pathology, The Owens College, Manchester. 8vo. Cloth. With 96 illustrations. $\$ 4.00$.

“Dr. Kelynack has presented us with an interesting monograph upon a subject which, from the standpoint of the pathologist, is one of the highest interest, but of extreme difficulty. This is the first systematic treatise upon tumors of the kidney which has yet appeared in English, and the author's main pretension is 'to indicate the work already accomplished, and to suggest lines for further research.' This he has done exceedingly well. His interesting and suggestive book is a welcome addition to our meagre knowledge. It is beautifully published, and profusely illustrated with photographic reproductions which show gross appearances unusually well. There is an exhaustive bibliography." $-N$. Y. Medical Journal.

\section{DISEASES OF WOMEN}

A text-book for students and practitioners, by J. C. WebsTer, B.A., M.D. (Edin.), F.R.C.P., Ed.; Professor of Gynæcology, Rush Medical College, Chicago; late Demonstrator of Gynæcology, McGill University, Montreal, etc. Illustrated with 24I figures. Crown 8vo. $\$ 3 \cdot 50$.

\section{DISEASES OF THE HEART AND AORTA}

By George Alexander Gibson, M.D., I.Sc., F.R.C.P. (Edin.), Senior Assistant Physician to and Lecturer on Clinical Medicine at the Royal Infirmary, Edinburgh, etc. With 2 ro illustrations. 8 vo. $952 \mathrm{pp}$. Cloth, $\$ 6.00$; sheep, $\$ 7.00$.

"We have only words of praise for the admirable monograph which is destined to have a permanent place in medical literature. It is refreshing, in these days of ceaseless activity in the making of books, to come across one characterized by the completeness and comprehensiveness, the forcefulness of presentation, and the authoritative accuracy of this. Dr. Gibson has, besides having done a fine piece of literary and scientific work, rendered a distinct service to the profession, for which he is deserving of its gratitude and commendation." - Philadelphia Medical Journal.

\section{MANUAL OF BACTERIOLOGY}

By Robert Muir, M.D., F.R.C.P., Ed., University of Edinburgh ; Pathologist, Edinburgh Royal Infirmary; and James Ritchie, M.D., B.Sc., Lecturer in Pathology, University of Oxford, with 108 illustrations. Crown 8vo. New edition. \$3.25.

"A well-digested, well-arranged, and wisely and clearly expressed epitome of the medical phases of bacteriology and of the bacteriological phases of disease. The book is altogether excellent, and is really a modern epitome of a difficult and complex theme, a safe and stimulating guide to the student, and a boon to the busy practitioner." - Science.

\section{THE MACMILLAN COMPANY}




\section{THE PRACTITIONER'S HANDBOOK OF TREATMENT; OR, THE PRINCIPLES OF THERAPEUTICS}

By the late J. Milner Fothergill, M.D., M.R.C.P., Foreign Associate Fellow of the College of Physicians of Philadelphia. Fourth edition. 8vo. Cloth. \$5.00. Edited, and in great part rewritten, by WILLIAM MURRELL, M.D., F.R.C.P.

The enormous progress in all departments of medicine during the last ten years has necessitated a thorough revision of the work. Considerable additions have been made, but Dr. Fothergill's original design, and, above all, his characteristic style, have as far as possible been preserved.

In the Preface to the First Edition of this work, Dr. Milner Fothergill points out that it is not " an imperfect practice of physic, but an attempt of original character to explain the rationale of our therapeutic measures... and "is a work on medical tactics for the bedside rather than the examination table."

The Lancet, in its obituary notice of Dr. Fothergill, states that " in his profession he exhibited great natural skill in interpreting the indications for treatment of disease, and in many cases of difficulty he would clear up the lines of treatment with a hand that was felt to be masterly. . . . He always wrote what was instructive in a vivacious and interesting, oftentimes original and pungent, style."

\section{INTRODUCTION TO THE OUTLINES OF THE PRINCIPLES OF DIFFERENTIAL DIAGNOSIS}

With Clinical Memoranda, by Fred J. Smith, Senior Pathologist to the London Hospital. Ex. Cr. 8vo. \$2.00.

\section{DIABETES MELITUS AND ITS TREATMENT}

By R. T. Williamson, M.D. (Lond.), M.R.C.P., Medical Registrar, Manchester Royal Infirmary; Hon. Med. Officer, Pendleton Dispensary (Salford Royal Hospital) ; Assistant to the Professor of Medicine, Owens College, Manchester. With 18 illustrations (two colored). Royal 8vo. Cloth. \$4.50.

"The study of diabetes, which formed the basis of the author's discovery of the discoloration of methylene blue by blood taken from a diabetic subject, has made his name well known in connection with this disease. A contribution from his pen, in the form of a monograph upon diabetes, is bound to be interesting. In the work before us, we find a more thorough consideration of the subject than has yet appeared in the English language. The chapters devoted to symptomatology and complications are particularly full and thorough. The treatment of the disease is excellently handled, and closes the work in a thoroughly practical manner. The bibliography attached to each chapter and an appendix on diabetic dietetics add value to a work which in completeness and didactic worth is unexcelled." - N. Y. Medical Nerws.

THE MACMILLAN COMPANY 


\section{CONSTIPATION IN ADULTS AND CHILDREN}

With special reference to Habitual Constipation and its most Successful Treatment by the Mechanical Methods, by H. Illoway, M.D., formerly Professor of the Diseases of Children, Cincinnati College of Medicine and Surgery; with many plates and illustrations. 8 vo. $\$ 4.00$; sheep, $\$ 5.00$.

"The work is not large, but is replete with facts which are of practical value to the practitioner of medicine." - The Canadian Journal of Medicine and Surgery.

\section{ATLAS OF EXTERNAL DISEASES OF THE EYE}

By A. Maitland Ramsay, Ophthalmic Surgeon, Glasgow Royal Infirmary; Professor of Ophthalmology, St. Mungo's College, Glasgow ; and Lecturer on Eye Diseases, Queen Margaret College, University of Glasgow. IVith 30 full-page colored plates, and I 8 fullpage photogravures. Sold only by subscription. 4to. Half morocco, gilt top. $\$ 20.00$.

"A work of great beauty. The illustrations are unrivalled, many of them masterpieces in their kind. The text gives connected descriptions of the cliseases, supplementing the stages and phases not presented in the illustrations. It is prepared with the utmost care as to precision and comprehensiveness of language. The book is written for the observing student, describing the etiology, symptomatology, and pathology of the diseases, but omitting the treatment. The whole work, which in care of preparation and elegance of getting up, appeals, in contrast with the book of Haab, to a select class of reaclers, is an ornament to Scotch ophthalmology, and in particular to Glasgow, the place from which emanated the best 'practical treatise on the diseases of the eye' before the discovery of the ophthalmoscope - the classical text-book of William Mackensie." - H. K., Archives of Ophthalmology, New York, Dr. H. KNapP, Editor.

\section{THE FUNDUS OCULI}

With an ophthalmoscopic atlas illustrating its physiological and pathological conditions, by W. Addas Frost, F.R.C.S., Ophthalmic Surgeon, St. George's Hospital; Surgeon to the Royal Westminster Ophthalmic Hospital. 4to. Half morocco. $\$ 20.00$. Sold by subscription only.

"A work which is a pleasure to look upon and an equally great pleasure to read. The book is a folio of 220 pages of letterpress, illustrated by 46 figures in black and white, of exquisite workmanship, representing macroscopically and microscopically those parts of the eye which we see with the ophthalmoscope. Bound up in the same volume are 47 large colored plates, containing 107 figures, beautifully drawn and colored, representing the fundus of the eye as seen with the ophthalmoscope. The discussion of the different conditions observed in the fundus bears evidence of very careful observation and research. The direct, concise, and lucid manner in which the des iptions of the various conditions are given is truly admirable." $-N$. Y. Medical Record.

"We venture the assertion that of all Ophthalmoscopic Atlases which have been produced in the last forty years, Mr. Frost's book is facile princeps. We wish that it might be found in the library of every physician and surgeon." - Professor James Moore BALl, Editor The State Medical Journal and Practitioner.

\section{THE MACMILLAN COMPANY}






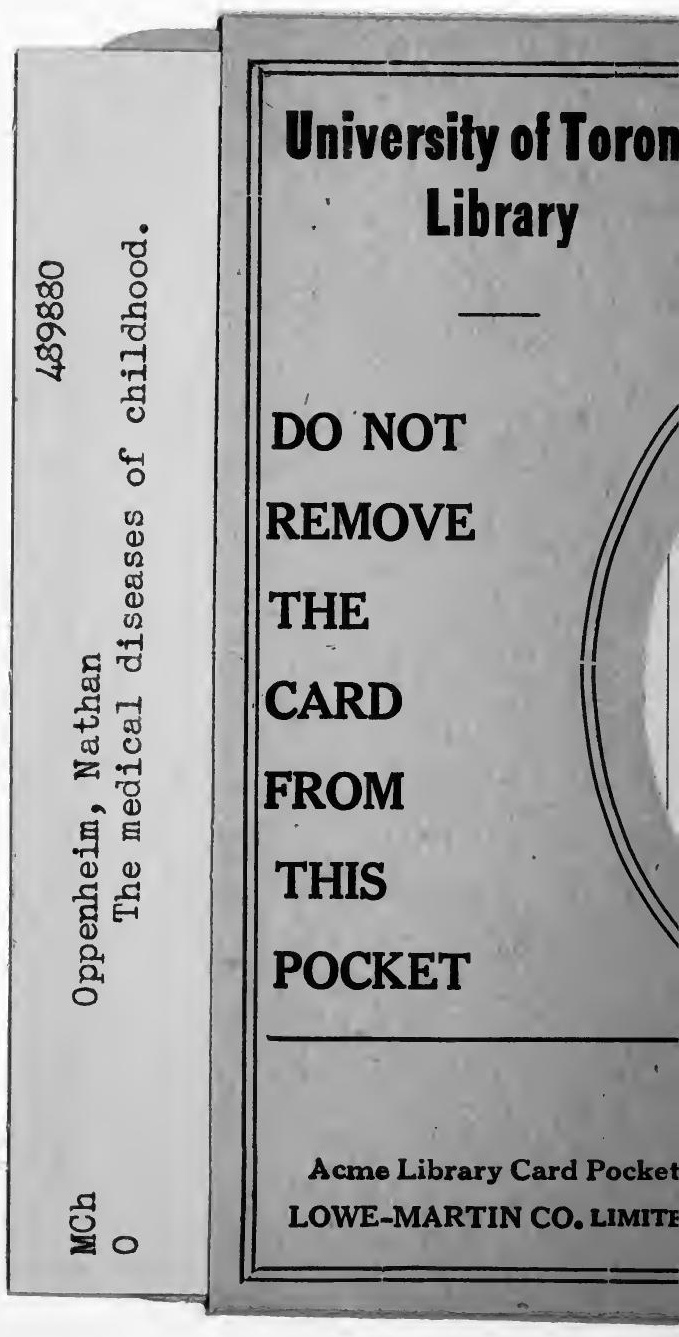


H

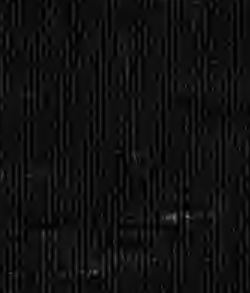

